\title{
High-Resolution
}

\author{
Mass Spectrometry for the
}

Analysis of Interfacial Kinetics

of Organic Surface Reactions

\section{Rickdeb Sen}




\section{Thesis committee}

\section{Promotor}

Prof. Dr H. Zuilhof

Professor of Organic Chemistry

Wageningen University \& Research

\section{Other members}

Prof. Dr F.A.M. Leermakers, Wageningen University\& Research

Prof. Dr J. Huskens, University of Twente

Prof. Dr K. Lammertsma, VU Amsterdam

Dr T. F. A. de Greef, Eindhoven University of Technology

This research was conducted under the auspices of the Graduate School of VLAG

(Advanced studies in Food Technology, Agrobiotechnology, Nutrition and Health Sciences). 


\section{High-Resolution}

\section{Mass Spectrometry for the}

\section{Analysis of Interfacial Kinetics \\ of Organic Surface Reactions}

\section{Rickdeb Sen}

\section{Thesis}

submitted in fulfillment of the requirements for the degree of doctor

at Wageningen University

by the authority of the Rector Magnificus

Prof. Dr A.P.J.Mol,

in the presence of the

Thesis Committee appointed

by the Academic Board

to be defended in public

on Wednesday 20 September 2017

at 4 p.m. in the Aula. 


\section{Rickdeb Sen}

High-Resolution Mass Spectrometry for the Analysis of Interfacial Kinetics of Organic Surface Reactions

308 pages

PhD thesis, Wageningen University, Wageningen, the Netherlands (2017)

With references, with summary in English

DOI: $10.18174 / 419479$

ISBN: $978-94-6343-624-3$ 


\section{Table of Contents}

$\begin{array}{llr}\text { Chapter } 1 & \text { Introduction } & 7\end{array}$

Chapter 2 Use of Ambient Ionization High-Resolution 27

Mass Spectrometry for the Kinetic Analysis

of Organic Surface Reactions.

Chapter 3 Rapid and Complete Surface Modification with

Strain-Promoted Oxidation-Controlled

Cyclooctyne-1,2-Quinone Cycloaddition (SPOCQ).

Chapter 4 Approach Matters: The Kinetics of Interfacial

Inverse-Electron Demand Diels-Alder Reactions.

Chapter 5 Ultrathin Covalently Bound Organic Layers on Mica. $\quad 89$

$\begin{array}{lll}\text { Chapter } 6 & \text { General Discussion } & 103\end{array}$

$\begin{array}{ll}\text { Appendix } 1 & 115\end{array}$

$\begin{array}{lr}\text { Appendix 2 } & 139\end{array}$

$\begin{array}{lr}\text { Appendix } 3 & 179\end{array}$

$\begin{array}{ll}\text { Appendix } 4 & 251\end{array}$

$\begin{array}{ll}\text { List of Publications } & 297\end{array}$

$\begin{array}{ll}\text { Overview of completed training activities } & 299\end{array}$

$\begin{array}{ll}\text { Acknowledgements } & 301\end{array}$

$\begin{array}{ll}\text { About the author } & 307\end{array}$ 


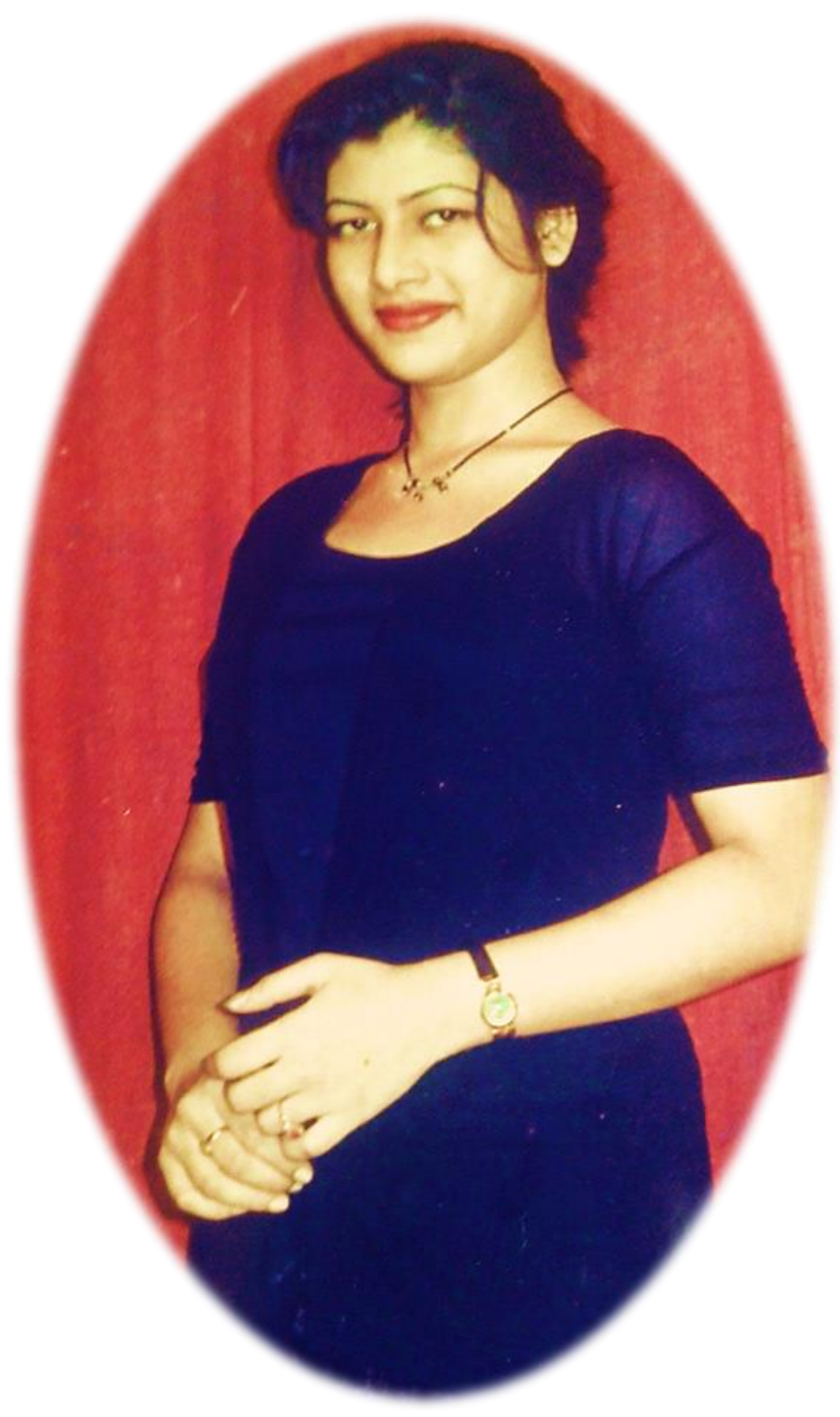

Dedicated to my dearest sister, Samriddi Saha (21 ${ }^{\text {st }}$ August, 1981-14 $4^{\text {th }}$ July, 2001). 


\title{
Chapter 1:
}

\author{
Introduction
}

\section{Table of Contents}

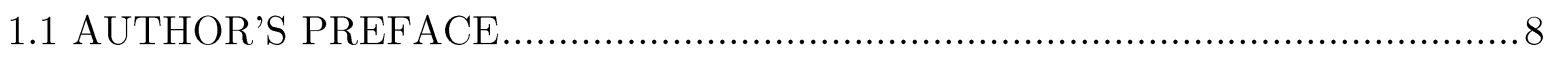

1.2 DEVELOPMENT OF KINETICS....................................................... 9

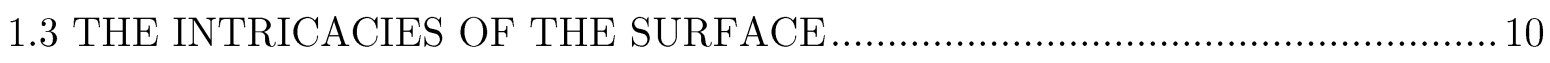

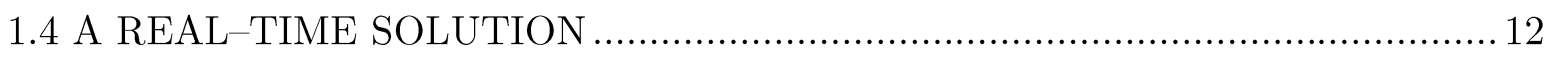

1.5 METHODOLOGY OF DETERMINATION OF INTERFACIAL KINETICS..... 17

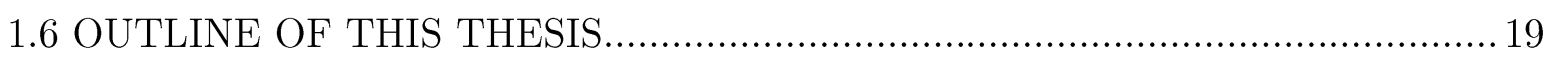

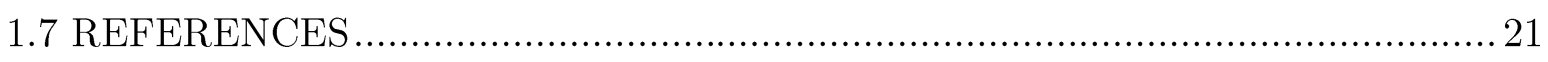




\subsection{AUTHOR'S PREFACE}

The very human desire to investigate and comprehend is a principle driving force for innovation. We have since time immemorial tried to decipher mysteries and disseminate the acquired knowledge to future generations. Being similarly curious, the author of this thesis began his quest to understand reaction rates on surfaces.
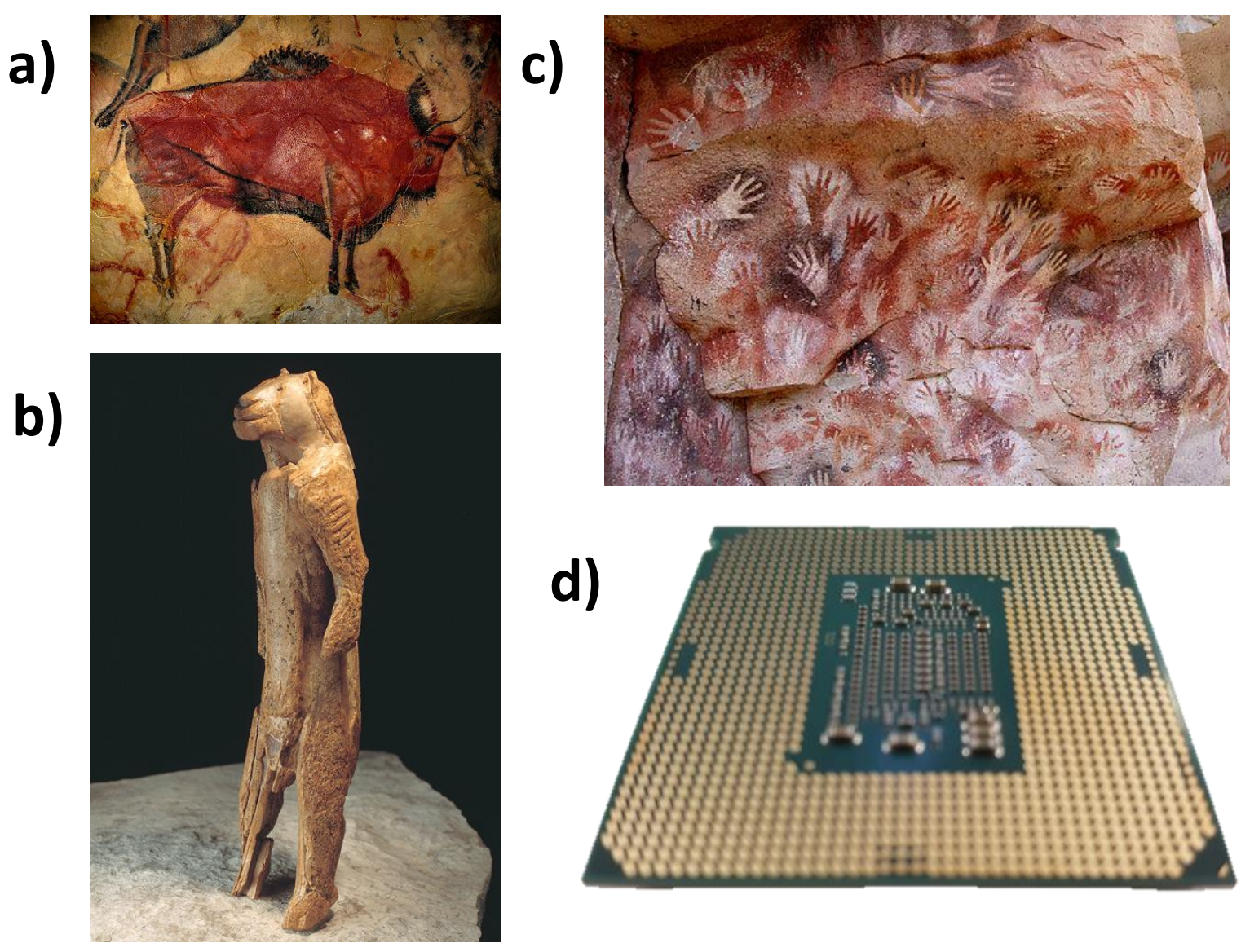

Figure 1. a) Ancient rock paintings at cave of Altamira, Spain b) the Lowenmensch (lion man), Germany c) a hello from the past, the cave paintings at Cueva de las Manos, Argentina and d) a modern computer chip.

Surface modification has had a rather long history, from the earliest rock paintings ${ }^{[1]}$ to modern day body implants, ${ }^{[2]}$ aerospace materials, ${ }^{[3]}$ wearable sensors ${ }^{[4]}$ and hybrid materials ${ }^{[5]}$ (Figure 1). The greatest improvement might be the greatly decreased layer thickness ${ }^{[6]}$ and very high precision we have achieved since and a transition from non-functional to functional surfaces. ${ }^{[7]}$ We have become quite good 
at it too, with incredible volume-to-surface ratios obtained in recent times along with a much higher level of control over resolution. ${ }^{[8]}$ We have come a long way, but as put by Helmut Schmid most ornately: "The biggest room in the world is the room for improvement."

\subsection{DEVELOPMENT OF KINETICS}

German chemist, Carl Friedrich Wenzel in 1777, while studying the rate of dissolution of metals in acid provided the first empirical correlation between rate and concentration. $^{[9]}$ The beginnings of our quantitative understanding about chemical kinetics can be traced back to 1850s with Ludwig Ferdinand Wilhelmy elucidating the rate of inversion of sucrose by means of a differential equation. ${ }^{[10]}$ As happens often with several scientific discoveries, his work unfortunately went unnoticed until its revival by Ostwald almost three decades later. ${ }^{[1]}$ Analogously, the discoverer of hydrogen peroxide, Louis Jacques Thénard also studied its decomposition. ${ }^{[12]}$ However, an overall understanding of the issue of reaction kinetics was (unfortunately) not the focus of chemical community at that time.

A wider understanding of rate equations as a product of reactant concentration was introduced by Marcelin Berthelot and Léon Pean de Saint-Gilles

in their study of the esterification reaction between ethanol and acetic acid. ${ }^{[13]} \mathrm{As}$ chemists became more proficient in synthesis and the overall synthetic repertoire increased, a concomitant desire to understand such processes (and with that: the reaction kinetics thereof) arose proportionally, and substantial efforts were made for elucidating reaction kinetics of several chemical transformations in solution.

Currently, chemists are quite adept at understanding chemical kinetics in solution using techniques such as nuclear magnetic resonance (NMR), ultravioletvisible spectroscopy (UV-Vis), infrared (IR), mass spectrometry (MS), etc. However, determining the same for interfacial reactions has been an uphill task. Certain specific 
cases exist and have been discussed later in greater detail. The importance of surface science is evident in the Nobel Prize awarded to Gerhard Ertl, who provided novel tools for the understanding of surface reactions. ${ }^{[14]}$ Such investigation clearly substantiated the claim that studying kinetics at the surface has remained a significant challenge.

\subsection{THE INTRICACIES OF THE SURFACE}

Surface irregularities at the microscopic level are no simple parameters to comprehend let alone predict effects thereof on substrate reactivity. ${ }^{[15]}$ As eloquently put by Hasselbrink "as there are many coordinates, the "surface" is really a "hypersurface" in multidimensional space".[16] The unbalanced interactions experienced by the surface atoms or molecules- as compared to the bulk- are the driving force for interactions which help lower its energy-aggravated state. This is the basis for surface modification and the basic tenet of surface chemistry. The driving forces for surface modification usually become rapidly absent after the formation of the monolayer unless a far more energetically favorable secondary interaction/ process is present namely, bio-fouling ${ }^{[17]}$, auto-polymerization ${ }^{[18]}$ and zeta potential. $^{[19]}$

However, the chemical modification of the surface is just a stepping stone upon which more complicated and chemically interesting architectures can be built. Analogously, the reaction kinetics of such interfacial reactions can be determined. To study interfacial reactions on the surface, one of the reaction counterparts has to be tethered to the surface and reacted with its solution counterpart. There should be enough driving force for the reaction to occur and there should be a clearly identifiable tag to confirm the occurrence of the event. The detection of this tag will provide an indirect handle for understanding the rate of consumption of the surface counterpart and thus provide kinetics of the reaction. However, due to increasing 
complexities of reactions, the task becomes increasingly complex as well. Coupled with it is the fact that analytes on the surface are restricted to an atomic scale. Also, the added challenge of extremely small limits of detection (LOD) in the picomole range presents further complications. Thus, not only a unique approach is required for solving of the significant challenge, but we also require an analytical tool which specializes in the robust and quick detection of minute analyte quantities. This thesis presents a straight-forward method addressing the above challenges and elucidates kinetic information for several "metal-free" click reactions. Additionally, we present the activation parameters and effect of microenvironment (ME) in addition to several dependencies and intricacies of surface chemistry.

To understand each point fully we deal with each of the challenges separately. Firstly, due to evident reasons approach of an organic reactant from the surface side is not possible. Additional complications from in situ generation of additional species (say $\mathrm{H}_{2}$ and $\mathrm{O}_{2}$ ) from the surface (for example, during electrochemistry) is a serious hindrance and is avoided in this thesis. The approach is also somewhat restricted along the monolayer for steric reasons. Also the diffusion effects and interactions with the already attached/reacted products on the surface play an important role. To put it succinctly, the incoming moiety faces significant difficulty during its approach. Other complicating factors are side-reactions or non-specific interactions. 


\subsection{A REAL-TIME SOLUTION}

Several studies have aimed (and painstakingly succeeded) to study and understand organic reactions at a surface. They provide a thorough ground-work for further exploration of interfacial kinetics. Studying the decay of some optical or electrical signal from surface-specific groups gives a direct indication of the progress of its reaction with a solution counterpart. Alternatively, one can also study the increase in the signal from a product forming on the surface. Disparate surface analytical techniques are widely used for this purpose utilizing a broad range of surface phenomena namely, hydrophobicity, electrochemical-activity, core electron binding energy, functional group stretching frequencies, interaction with AFM tips, etc.

Reaction rates of copper-catalyzed azide alkyne (CuAAC) click reactions have been studied on germanium substrates using attenuated total reflection infrared

spectroscopy (ATR-IR) by Koberstein. ${ }^{[20]}$ The yield and kinetics of post-synthetic modification of highly oriented molecular organic frameworks (MOF) thin films utilizing SPAAC were studied by Wang et al. ${ }^{[21]}$ Both studies relied on the strong and specific infrared frequencies from azide groups moieties, at $2114 \mathrm{~cm}^{-1}$. However, they have contradictory claims on which click reaction is more efficacious. Infrared (IR) vibrations in the fingerprint region $\left(1500-500 \mathrm{~cm}^{-1}\right)$ contain valuable structural information and can be directly correlated to the structure, bonds and configuration. ${ }^{[22]}$ Kinetics of different alkynes and polymer brushes functionalized with azide groups mediated by both metal and metal-free click reactions were studied by Orski and co-workers based on the change of UV-Vis spectra of azide group (190-700 $\mathrm{nm}) .{ }^{[23]}$ However, full structural information is not easily obtained by either UV-Vis or IR spectroscopy. In all aforementioned cases, emphasis is on a specific signal which only provides partial information of the molecule without a complete molecular 
picture. Nonetheless the works are significant in their own right as they demonstrate the fundamental concept of kinetics on a surface.

Buriak and co-workers have studied the self-assembly of photoinitiated hexadecyl monolayers on H-terminated silicon [H-Si (111)] utilizing the increasing surface hydrophobicity by water static contact angle (SCA) as the reaction progressed. ${ }^{[24]}$ Kinetic studies of monolayer growth by water contact angle on chromium nitride $(\mathrm{CrN})$ surfaces $^{[25]}$ have been pursued in our laboratory too. Additionally, ellipsometry is a valuable tool for following the increase of selfassembled monolayers as a function of time and thus unravelling the kinetics of formation. This has been used as a complimentary method along with SCA by our group. ${ }^{[26]}$

$\mathrm{X}$-ray photoelectron spectroscopy (XPS) is an ultrahigh vacuum (UHV) technique based on detection of photoelectrons ejected upon X-ray excitation, routinely used for the characterization of surfaces. The specificity of binding energies measured for a particular type of photoelectron makes possible the identification of the atomic composition of solid surfaces and to characterize their local chemical environment, in particular their oxidation state. ${ }^{[27]}$ Since the binding energies of core electrons of elements is highly characteristic and the signal intensity obtained is proportional to surface concentration, XPS can also be used for surface kinetics determination. Certain elements, such as fluorine are used prominently due to their high relative sensitivity factor $(\mathrm{RSF} F 1 \mathrm{~s}=4.43$, w.r.t. $\mathrm{C} 1 \mathrm{~s}=1.00)$ and concomitant higher signal-to-noise ratio and can provide a handle to study their change on the surface. However, this is by no means trivial as shown by the works of $\mathrm{Yang}^{[28]}$ and $\mathrm{Li}^{[29]}$ who struggled with the non-specificity arising from lack of exact structural resolution. Density Functional Theory (DFT) calculations may be used to assign binding energies based on the chemical environments around elements, and this can 
facilitate XPS assignments tremendously. ${ }^{[30]}$ However, this may not be done reliably for all kinds of surfaces.

Hamers et al., have also used XPS to study the photochemical grafting of different alkenes on single-crystal rutile (110) $\mathrm{TiO}_{2} \cdot{ }^{[31]}$ Additionally, they have also demonstrated the photochemical grafting of alkenes on flat $\mathrm{ZnO}$ surfaces. Both studies utilized XPS to unravel the kinetics of monolayer formation. ${ }^{[32]}$ Multilayers for some alkenes were reported and efficient techniques to circumvent the above through subsequent post attachment modification were outlined with each step being thoroughly characterized by XPS. The ease of applicability and ability to extend XPS to a disparate number of substrates highlights the importance of this method. In addition to that XPS has been rigorously demonstrated to be quantitative by the group of Allongue. ${ }^{[33]}$ Manova et al. have used the SPAAC reaction as an effective method for oriented immobilization of biomolecules on silicon nitride surfaces, and they have followed the reaction progress by XPS based on increase in number of $\mathrm{F}$ atoms. ${ }^{[34]}$ The conclusions drawn from these works are that XPS is an established technique for surface analysis and should serve as an independent technique to supplement our surface modification and interfacial kinetics data. However, a recurrent impediment is to obtain the structural information in a reliable fashion.

A direct (and real time) method to study in situ the inductive effects of various substituents on thermal grafting rate of aryl iodides on anatase $\mathrm{TiO}_{2}$ was studied by Hamers. ${ }^{[35]}$ For this purpose, special $\mathrm{TiO}_{2}$ coated MS vials were exposed to different aryl iodides and mass fragments formed were studied. This gave direct structural information on the groups generated and the activation parameters were determined by extending the study to different temperatures. However, such a study cannot be extended to a system where the bonds would be strongly covalent with the surface tethers remaining intact. A technique which is gaining increasing traction for imaging and surface analysis is time-of-flight secondary ion mass spectrometry 
$(\text { ToF-SIMS })^{[36]}$ for its high resolution. However, the energy of the beam used causes extensive fragmentation of the parent ion which is especially undesirable for complex biological media which consist of hundreds if not thousands of distinct components. Thus, a significant body of empirical data has been accrued ${ }^{[37]}$ and new primary ion sources are optimized regularly after significant engineering and development. ${ }^{[38]}$ However, most fragments obtained using ToF-SIMS are either neutral (making them undetectable by the mass spectrometer) or too fragmented to be readily interpreted. In addition to that, the dependence on high vacuum, radioactive elements and high operational costs restrict swift application to any emerging field. Thus, ToF-SIMS was not a viable option for our purposes.

A high-potential application to monolayer-based kinetics was, however, reported by the group of Mrksich. ${ }^{[39]}$ Their work delved into the effect that neighboring inert alkyl groups exert on the reaction site i.e. the microenvironment (ME). They investigated the kinetics of a Diels-Alder reaction between surface tethered 1, 4-diquinones and cyclopentadiene in solution phase using cyclic voltammetry $(\mathrm{CV})$. Although being an easy approach, the method is restricted to highly conducting materials, such as gold $(\mathrm{Au})$. In addition, the reaction to be studied should involve redox active compounds. Thus, the study, although highly important and instructive to our setup, is greatly hampered by many limitations. Change of a single parameter like a non-conducting surface and/or nonelectrochemically active groups will completely preclude CV as a rational analytical tool of choice.

Direct Analysis in Real Time High Resolution Mass Spectrometry (DARTHRMS) is a rapid, non-contact ambient mass spectrometry technique ${ }^{[40]}$ which utilizes preheated meta-stable He species $\left({ }^{3} \mathrm{~S}_{1}\right)$ with an energy of $19.8 \mathrm{eV} \cdot{ }^{[41]}$ Developed by Cody et al. in 2005, it has become a complimentary technique to matrix-assisted laser desorption/ionization (MALDI) ${ }^{[42]}$ because of its excellence in 
a)

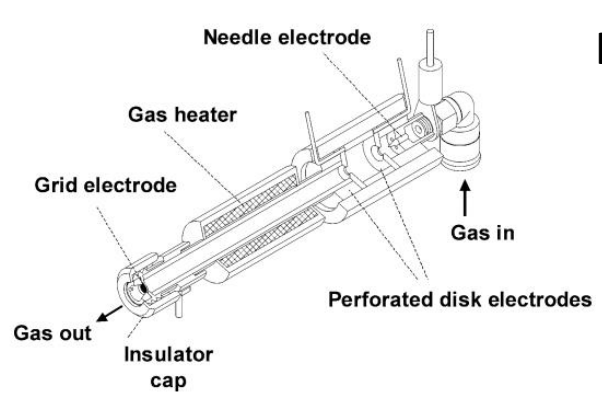

c)

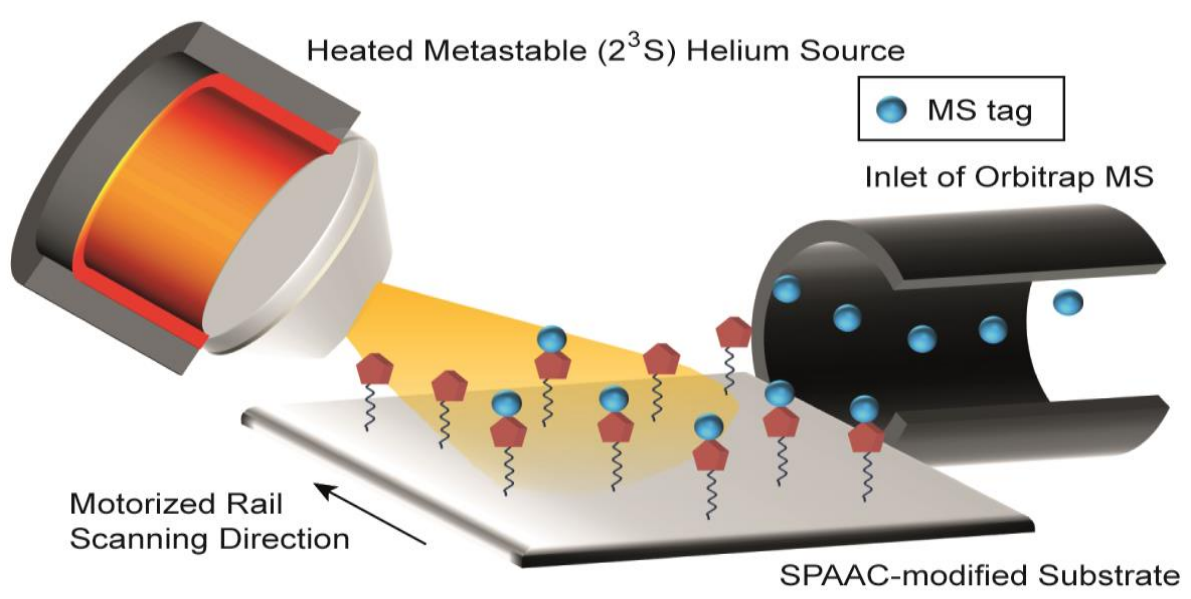

Figure 2. a) Initial DART gun setup ${ }^{[40 a]}$ b) setup of DART at an angle for surface analysis and c) schematic representation of the DART setup used for monitoring the progress of "metal-free click reactions" on surfaces.

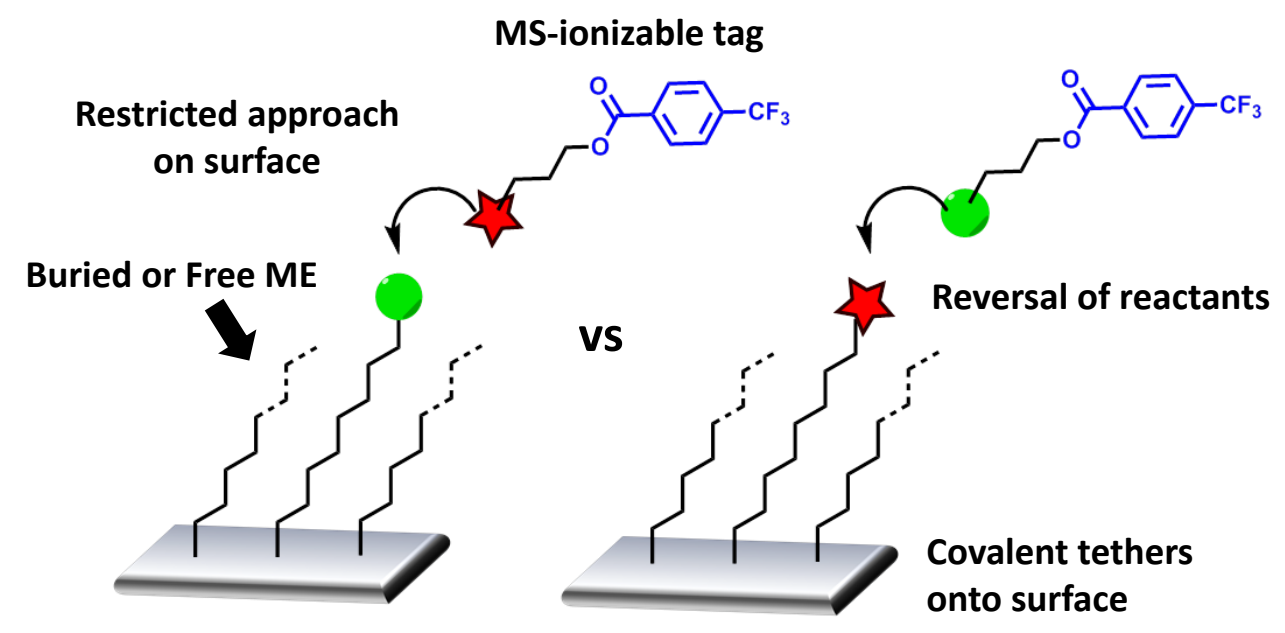

Figure 3. Our general setup for interfacial reaction between the green ball and the red star depicting the parameters under current study. Increasing the length of surrounding inert alkyl chains allow for generation of different microenvironments (ME). 
detection of low mass fragments and non-requirement of any matrix. DART-HRMS is a plasma-based technique and most suitable for ions within the upper limit of $\mathrm{m} / \mathrm{z}$ $2000^{[40 a]}$ and can be suitably employed for both surface and solution analyte detection.

Since, the vast majority of organic chemistry and its allied sciences, happens in the small-molecule range $(<400$ Da), DART-HRMS provided an optimal solution to "visualize" the growth of a tag molecule which is also within that mass range. A close cousin would be the Low Temperature Plasma (LTP ${ }^{[43]}$ which (as the name suggests) does not involve heating. In DART, however, software-controllable temperatures up to $550{ }^{\circ} \mathrm{C}$ allow for desorption of analytes from surface or solution and detection by a mass spectrometer. All these plasma based methods, especially DART provide significant advantages, namely 1) uncomplicated instrumentation, 2) minimal to nonexistent sample preparation, 3) easily interpretable spectra and 4) minimum need of high purity and therefore expensive solvents.

Significant effort has been expended by the group of Bartmess to understand and clearly rationalize the mechanisms of ionization by DART-HRMS in both positive $^{[44]}$ and negative mode. ${ }^{[45]}$ Throughout this thesis, the negative mode has been used heavily, specifically for the cleavage of aromatic esters to yield negatively charged benzoate anions (e.g. $p-\mathrm{CF}_{3}$ benzoate anion and $p-\mathrm{C}_{4} \mathrm{~F}_{9}$ benzoate anion, $m / z$ 189.0016 and 339.0062, respectively). The spacial resolution and orientation of the DART gun ${ }^{[46]}$ was found to be in the order of a few $\mathrm{mm}^{2}$ and was perfectly suited for our $2 \times 1 \mathrm{~cm}^{2} \mathrm{Al}$ samples modified with varying degrees of reaction completion. Although high-resolution mass spectrometry imaging (MSI) is a rapidly emerging field, ${ }^{[47]}$ it was not found necessary for our case. Alternatively, desorption electrospray ionization mass spectrometry (DESI-MS) was also not a convenient choice given the complications arising from the electrospray of organic solvents onto our substrates. ${ }^{[48]}$ 


\subsection{METHODOLOGY OF DETERMINATION OF INTERFACIAL KINETICS}

Since, DART is a relatively "soft ionization technique" (19.8 eV as compared to $70 \mathrm{eV}$ for electron impact MS), only certain bonds can be cleaved effectively. ${ }^{[49]}$ To this end, we utilized the aromatic ester bond which was found to cleave with sufficient signal-to-noise while being relatively straightforward to synthesize. An implicit assumption was that the depth of the penetration of the He metastable species would be unaffected by the depth of the ester tag in our monolayer. This is indeed not out of hand, as depths of overlaid paint layers have been probed by low temperature plasma techniques up to depths which are far greater than in our case. ${ }^{[4]}$ In addition to that, the introduction of significant number of $\mathrm{F}$ atoms also helped in an independent orthogonal detection by XPS.

Since, a picture says a thousand words... (Figure 3). Our setup for determination of interfacial kinetics was thus set. One of the reaction partners would be covalently tethered onto a surface and surrounded by inert alkyl chains of various lengths. Our project significantly draws upon the concept of monolayers pioneered by Zisman $^{[50]}$ and Sagiv. ${ }^{[51]}$ In this manner, the microenvironment (ME) around the reactive site could be systematically tuned (freely accessible/ partially accessible or buried). Phosphonic acid chemistry on aluminium (Al) was chosen given ease of modification, stability and rigorous reproducibility. ${ }^{[52]}$ The orientation of such monolayers is generally in the range of $37-42^{\circ}$ with respect to the surface and directed by the geometry around the adsorbate-adsorbate bonds $(\mathrm{O}-\mathrm{P}(=\mathrm{O})$-alkyl bond in this case). ${ }^{[53]}$ The bonds are irreversibly formed and thus a ratio of two or more adsorbates in the solution is reflected on the surface. To avoid the deposition of micelles on the surface it is necessary to carry out the early phase of the surface modification at an elevated temperature. This was indeed found to be the case for 
our system and modification at $50{ }^{\circ} \mathrm{C}(5 \mathrm{~min})$ followed by room temperature $(\sim 5 \mathrm{~h})$ gave homogenous alkyl monolayers on aluminum oxide without any evidence of granules or domains formation.

The other reaction partner coupled to a MS-ionizable tag bound (aromatic ester) would thus be in the solution phase. The tag was separated from the main reacting species by a methylene group to insulate it from any inductive and resonance effects. Given the very large excess $\left(>10^{7}\right)$ of solution reactant ( $\sim \mathrm{mM}$ range) with respect to the surface counterpart (picomole range), we expect the concentration of the former to remain essentially constant through the kinetic regime. The solution was constantly stirred to avoid product build-up and any concurrent complications in kinetics. To undermine the adverse effects of non-specific interactions and unnecessary by-product buildup, we chose a rapid and efficient model reaction strategy, the so-called "metal-free click reactions" for our purpose. Since the seminal paper by Sharpless and co-workers defining "click" reactions, they have become extremely popular in chemistry and are continually reinforced by new click strategies being discovered regularly. ${ }^{[54]}$

\subsection{OUTLINE OF THIS THESIS}

The following four chapters will focus on a greater understanding of chemical kinetics followed by our novel approach for modification of mica. Chapter 2 describes the determination of kinetics and activation parameters of strain-promoted azide alkyne click reaction $(\mathrm{SPAAC}) .\left[{ }^{54 \mathrm{~b},} 55\right]$ This reaction has recently achieved prominence $^{[56]}$ as compared to its copper-catalyzed counterpart, i.e. copper catalyzed azide-alkyne cycloaddition (CuAAC). The main reason for this preference is the cytotoxicity $^{[57],[58]}$ of copper. In "strain-promoted" click reactions, the release of ring strain rather than an external catalyst is the main driving force of the reaction. ${ }^{[59]}$ In Chapter 3, we focused on the interfacial kinetics and quantitative nature of the 
strain-promoted oxidation controlled cyclooctyne quinone cycloaddition (SPOCQ) developed by van Delft and co--workers. ${ }^{[54 a, 60]}$ We also elucidate the surface activation parameters for this reaction and explore its usability for rapid fluorophore attachment onto surfaces.

Thus far, in all studies of surface-bound organic conversions one of the reactant counterparts was bound on the surface while the other remained in solution. However, the reactivity of the inverse reaction (i.e. couple the solution-phase structure to a surface and decouple the previously surface-bound species from the surface and have it in solution phase) had never been systematically studied for any reactions. Yet, that a difference exists upon reversal of reactants was already known in literature. ${ }^{[61]}$ Therefore, a careful study was in order.

Hence in Chapter 4, we studied the effect of the approach of a solution reactant towards a surface-immobilized counterpart for an inverse electron demand Diels-Alder (IEDDA) reaction, more specifically the norbornene-tetrazine ligation. ${ }^{[62]}$ The effects of stereochemistry of the reactants on reaction rate were also investigated for the first time, in addition to the effect of the microenvironment on the approach of different reactant combinations. We believe this lays the groundwork for future studies which shall establish the choice of surface-immobilized partner in any interfacial reaction.

In Chapter 5, we utilized our experience in surface chemistry by developing a novel strategy for the modification of an otherwise difficult to modify substrate, namely mica. Mica with its exceptional flatness is a substrate of choice for microscopy of interesting biomolecules such as DNA. However, prior to our work the above process is not possible in a step wise manner. This was due to the inability to rapidly modify mica with covalent links with the resulting surface still retaining high flatness. To this end, we utilized a mussel-inspired approach as shown by Messersmith. ${ }^{[63]}$ Butler $^{[64]}$ and Huang ${ }^{[65]}$ based their modification approach on the vital 
catechol moieties, however, neither left a route for post-attachment modification. We addressed the problem in a "two-punch" approach, by using a molecule with a catechol linker for easy surface attachment and an amine linker which could help with surface attachment while also acting as a chemical handle for further

modification. Despite the high propensity for catechols to auto-polymerize, we obtained ultrathin layers with very low roughness. A step further is the possibility of reducing the reactivity of the catechol group by attachment of a nitro group which is the current focus of our and other laboratories. ${ }^{[6]}$

In Chapter 6, we summarize the most salient aspects of this thesis. Furthermore, we deliberate and pen the author's personal thoughts regarding further development of this methodology.

\subsection{REFERENCES}

[1] A. W. G. Pike, D. L. Hoffmann, M. García-Diez, P. B. Pettitt, J. Alcolea, R. De Balbín, C. González-Sainz, C. de las Heras, J. A. Lasheras, R. Montes, J. Zilhão, Science 2012, 336, 1409.

[2] A. Kurella, N. B. Dahotre, J. Biomater. Appl. 2005, 20, 5-50.

[3] J. I. Kleiman, in Encyclopedia of Aerospace Engineering, John Wiley \& Sons, Ltd, 2010.

[4] M. Melzer, J. I. Mönch, D. Makarov, Y. Zabila, G. S. Cañón Bermúdez, D. Karnaushenko, S. Baunack, F. Bahr, C. Yan, M. Kaltenbrunner, O. G. Schmidt, Adv. Mater. 2015, 27, 1274-1280.

[5] K. Hu, R. Xiong, H. Guo, R. Ma, S. Zhang, Z. L. Wang, V. V. Tsukruk, Adv. Mater. 2016, 28, 3549-3556.

[6] C. Tan, X. Cao, X.--J. Wu, Q. He, J. Yang, X. Zhang, J. Chen, W. Zhao, S. Han, G.-H. Nam, M. Sindoro, H. Zhang, Chem. Rev. 2017, DOI: 10.1021/acs.chemrev.1026b00558. 
[7] J. Escorihuela, M. Á. González-Martínez, J. L. López-Paz, R. Puchades, Á. Maquieira, D. Gimenez-Romero, Chem. Rev. 2015, 115, 265-294.

[8] J. O. Zoppe, N. C. Ataman, P. Mocny, J. Wang, J. Moraes, H.-A. Klok, Chem. Rev. 2017, 117, 1105-1318.

[9] C. F. Wenzel, Lehre von der Verwandtschaft der Körper 1777, Dresden.

[10] L.Willhelmy, Pogg. Ann. 1850, 81, 499.

[11] F. W. Ostwald, J. Prakt. Chem. 1884, 29, 385.

[12] L. J. Thénard, Ann.Chim. Phys. 1818, 9, 314.

[13] P. E. M. Berthelot, L. P. d. Saint-Gilles, Ann. Chim. Phys. 1862, 63, 385.

[14] J. Phys. Chem. B 2004, 108, 14197-14215.

[15] H. S. Taylor, Proceedings of the Royal Society of London. Series A 1925, 108, 105.

[16] E. Hasselbrink, Science 2009, 326, 809.

[17] D. Rana, T. Matsuura, Chem. Rev. 2010, 110, 2448-2471.

[18] S. Sánchez-Cortés, O. Francioso, J. V. García-Ramos, C. Ciavatta, C. Gessa, J. Col. Surf. A 2001, 176, 177-184.

[19] A. P. R. Johnston, E. S. Read, F. Caruso, Nano Lett. 2005, 5, 953-956.

[20] S. Zhang, J. T. Koberstein, Langmuir 2012, 28, 486-493.

[21] Z. Wang, J. Liu, H. K. Arslan, S. Grosjean, T. Hagendorn, H. Gliemann, S. Bräse, C. Wöll, Langmuir 2013, 29, 15958-15964.

[22] R. Meier, In Handbook of Vibrational Spectroscopy; Chalmers, J., Griffiths, P. R., Eds.; John Wiley \& Sons Ltd.: Chichester, UK, 2003.

[23] S. V. Orski, G. R. Sheppard, S. Arumugam, R. M. Arnold, V. V. Popik, J. Locklin, Langmuir 2012, 28, 14693-14702.

[24] L. A. Huck, J. M. Buriak, Langmuir 2012, 28, 16285-16293.

[25] S. P. Pujari, L. Scheres, B. van Lagen, H. Zuilhof, Langmuir 2013, 29, 1039310404. 
[26] B. Rijksen, S. P. Pujari, L. Scheres, C. J. M. van Rijn, J. E. Baio, T. Weidner, H. Zuilhof, Langmuir 2012, 28, 6577-6588.

[27] a) Handbook of X-ray and Ultraviolet Photoelectron Spectroscopy; Briggs, D., Ed.; Heyden: London, 1978; b) Modern Techniques of Surface Science, 2nd ed.; Cambridge University Press: Cambridge, U.K., 1994.

[28] M. Yang, R. L. M. Teeuwen, M. Giesbers, J. Baggerman, A. Arafat, F. A. de Wolf, J. C. M. van Hest, H. Zuilhof, Langmuir 2008, 24, 7931-7938.

[29] Y. Li, H. Zuilhof, Langmuir 2012, 28, 5350-5359.

[30] M. Giesbers, A. T. M. Marcelis, H. Zuilhof, Langmuir 2013, 29, 4782-4788.

[31] R. Franking, R. J. Hamers, J. Phys. Chem. C 2011, 115, 17102-17110.

[32] R. E. Ruther, R. Franking, A. M. Huhn, J. Gomez-Zayas, R. J. Hamers, Langmuir 2011, 27, 10604-10614.

[33] X. Wallart, C. Henry de Villeneuve, P. Allongue, J. Am. Chem. Soc. 2005, $127,7871-7878$.

[34] R. K. Manova, S. P. Pujari, C. A. G. M. Weijers, H. Zuilhof, T. A. van Beek, Langmuir 2012, 28, 8651-8663.

[35] C. R. English, R. J. Hamers, J. Phys. Chem. C 2015, 119, 27972-27981.

[36] S. G. Boxer, M. L. Kraft, P. K. Weber, Annual review of biophysics 2009, 38, 53-74.

[37] a) C. M. McQuaw, A. G. Sostarecz, L. Zheng, A. G. Ewing, N. Winograd, Langmuir 2005, 21, 807-813; b) A. G. Sostarecz, D. M. Cannon, C. M. McQuaw, S. Sun, A. G. Ewing, N. Winograd, Langmuir 2004, 20, 4926-4932.

[38] L.-J. Chen, S. S. Shah, S. V. Verkhoturov, A. Revzin, E. A. Schweikert, Surface and interface analysis : SIA 2011, 43, 555-558.

[39] Y. Kwon, M. Mrksich, J. Am. Chem. Soc. 2002, 124, 806-812.

[40] a) R. B. Cody, J. A. Laramee, H. D. Durst, Anal. Chem. 2005, r7, 2297-2302;

b) X. Ding, Y. Duan, Mass Spectrom. Rev. 2015, 34, 449-473. 
[41] a) J. H. Gross, Anal Bioanal. Chem. 2014, 406, 63-80; b) M. E. Monge, G. A. Harris, P. Dwivedi, F. M. Fernández, Chem. Rev. 2013, 113, 2269-2308.

[42] a) M. Karas, D. Bachmann, U. Bahr, F. Hillenkamp, International Journal of Mass Spectrometry and Ion Processes 1987, 78, 53-68; b) P. Juhasz, C. E. Costello, K. Biemann, J. Am. Soc. Mass Spectrom. 1993, 4, 399-409.

[43] S. Martínez-Jarquín, R. Winkler, Trends in Analytical Chemistry 2017, 89, $133-145$.

[44] L. Song, S. C. Gibson, D. Bhandari, K. D. Cook, J. E. Bartmess, Anal. Chem. 2009, 81, 10080-10088.

[45] L. Song, A. B. Dykstra, H. Yao, J. E. Bartmess, J. Am. Soc. Mass Spectrom. 2009, 20, 42-50.

[46] E. S. Chernetsova, A. I. Revelsky, G. E. Morlock, Rapid Commun. Mass Spectrom. 2011, 25, 2275-2282.

[47] C. Wu, A. L. Dill, L. S. Eberlin, R. G. Cooks, D. R. Ifa, J. Am. Soc. Mass Spectrom. 2013, 32, 218-243.

[48] Y. Liu, X. Ma, Z. Lin, M. He, G. Han, C. Yang, Z. Xing, S. Zhang, X. Zhang, Angew. Chem. Int. Ed. 2010, 49, 4435-4437.

[49] R. K. Manova, S. Joshi, A. Debrassi, N. S. Bhairamadgi, E. Roeven, J. Gagnon, M. N. Tahir, F. W. Claassen, L. M. W. Scheres, T. Wennekes, K. Schroën, T. A. van Beek, H. Zuilhof, M. W. F. Nielen, Anal. Chem. 2014, 86, $2403-2411$.

[50] W. C. Bigelow, D. L. Pickett, W. A. Zisman, J. Col. Sci. 1946, 1, 513-538.

[51] J. Sagiv, J. Am. Chem. Soc. 1980, 102, 92-98.

[52] M. Kosian, M. M. J. Smulders, H. Zuilhof, Langmuir 2016, 32, 1047-1057.

[53] a) D. K. Schwartz, Annu. Rev. Phys. Chem. 2001, 52, 107-137; b) J. C. Love, L. A. Estroff, J. K. Kriebel, R. G. Nuzzo, G. M. Whitesides, Chem. Rev. 2005, 105, 1103-1170. 
[54] a) A. Borrmann, O. Fatunsin, J. Dommerholt, A. M. Jonker, D. W. P. M. Löwik, J. C. M. van Hest, F. L. van Delft, Bioconjugate Chem. 2015, 26, 257261; b) D. A. MacKenzie, A. R. Sherratt, M. Chigrinova, L. L. W. Cheung, J. P. Pezacki, Current Opinion in Chemical Biology 2014, 21, 81-88; c) X. Ning, R. P. Temming, J. Dommerholt, J. Guo, D. B. Ania, M. F. Debets, M. A. Wolfert, G.--J. Boons, F. L. van Delft, Angew. Chem. Int. Ed. 2010, 49, 30653068; d) B. C. Sanders, F. Friscourt, P. A. Ledin, N. E. Mbua, S. Arumugam, J. Guo, T. J. Boltje, V. V. Popik, G.-J. Boons, J. Am. Chem. Soc. 2011, 133, 949-957; e) S. Wallace, J. W. Chin, Chem. Sci. 2014, 5, 1742-1744.

[55] E. M. Sletten, C. R. Bertozzi, Angew. Chem. Int. Ed. 2009, 48, 6974-6998.

[56] S. T. Laughlin, J. M. Baskin, S. L. Amacher, C. R. Bertozzi, Science 2008, 320, 664-667.

[57] P. B. Tchounwou, C. Newsome, J. Williams, K. Glass, Met Ions Biol Med. 2008, 10, 285-290.

[58] a) J. E. Hein, V. V. Fokin, Chem. Soc. Rev. 2010, 39, 1302-1315; b) V. V. Rostovtsev, L. G. Green, V. V. Fokin, K. B. Sharpless, Angew. Chem. Int. Ed. 2002, 41, 2596-2599; c) C. W. Tornøe, C. Christensen, M. Meldal, J . Org. Chem. 2002, 67, 3057-3064; d) T. Plass, S. Milles, C. Koehler, C. Schultz, E. A. Lemke, Angew. Chem. Int. Ed. 2011, 50, 3878-3881.

[59] J. C. Jewett, C. R. Bertozzi, Chem. Soc. Rev. 2010, 39, 1272-1279.

[60] a) A. M. Jonker, A. Borrmann, E. R. H. van Eck, F. L. van Delft, D. W. P. M. Löwik, J. C. M. van Hest, Adv. Mater. 2015, 27, 1235-1240; b) J. J. Bruins, A. H. Westphal, B. Albada, K. Wagner, L. Bartels, H. Spits, W. J. H. van Berkel, F. L. van Delft, Bioconjugate Chem. 2017.

[61] a) N. S. Bhairamadgi, S. Gangarapu, M. A. Caipa Campos, J. M. J. Paulusse, C. J. M. van Rijn, H. Zuilhof, Langmuir 2013, 29, 4535-4542; b) J. 
Escorihuela, M. J. Banuls, R. Puchades, A. Maquieira, Chem. Commun. 2012, 48, 2116-2118.

[62] N. K. Devaraj, R. Weissleder, S. A. Hilderbrand, Bioconjugate Chem. 2008, 19, 2297-2299.

[63] H. Lee, S. M. Dellatore, W. M. Miller, P. B. Messersmith, Science 2007, 318, 426.

[64] G. P. Maier, M. V. Rapp, J. H. Waite, J. N. Israelachvili, A. Butler, Science 2015, 349, 628 .

[65] C. Lim, J. Huang, S. Kim, H. Lee, H. Zeng, D. S. Hwang, Angew. Chem. Int. Ed. 2016, 55, 3342-3346.

[66] M. Rodenstein, S. Zürcher, S. G. P. Tosatti, N. D. Spencer, Langmuir 2010, 26, 16211-16220. 


\section{Chapter 2:}

\section{Use of Ambient Ionization High-Resolution Mass}

\section{Spectrometry for the Kinetic Analysis of Organic}

\section{Surface Reactions}

In contrast to homogeneous systems, studying the kinetics of organic reactions on solid surfaces remains a difficult task due to the limited availability of appropriate analysis techniques that are general, high-throughput and capable of offering quantitative, structural surface information. Here, we demonstrate how Direct Analysis in Real Time Mass Spectrometry (DART-MS) complies with above considerations and can be used for determining interfacial kinetic parameters. The presented approach is based on the use of a MS tag that allows application to any reaction of interest. To show the potential of DART-MS we selected widely applied strain-promoted alkyne-azide cycloaddition (SPAAC) as a model reaction to elucidate the effects of the microenvironment on the interfacial reaction rate.

This Chapter has been published as:

"Use of Ambient Ionization High-Resolution Mass Spectrometry for the Kinetic Analysis of Organic Surface Reactions”. Rickdeb Sen, Jorge Escorihuela, Maarten M. J. Smulders and Han Zuilhof. Langmuir 2016, 32 (14), 3412-3419. 


\section{Table of Contents}

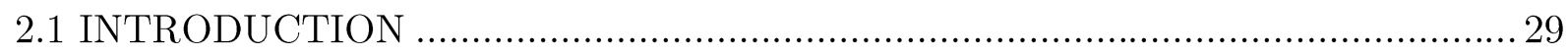

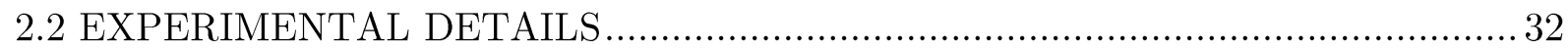

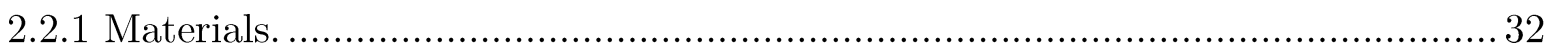

2.2.2 General procedure for the preparation of phosphonic acid monolayers.............. 32

2.2.3 General procedure for interfacial SPAAC reaction.................................... 32

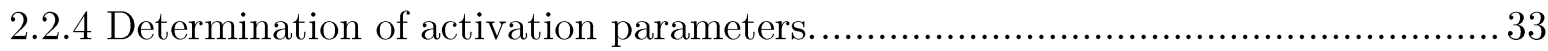

2.3 ANALYTICAL INSTRUMENTS. ............................................................... 33

2.3.1 Static Water Contact Angle (SCA) Measurements.......................................... 33

2.3.2 Infrared Reflection Absorption Spectroscopy (IRRAS) ............................... 33

2.3.3 X-ray Photoelectron Spectroscopy (XPS) Measurements................................ 34

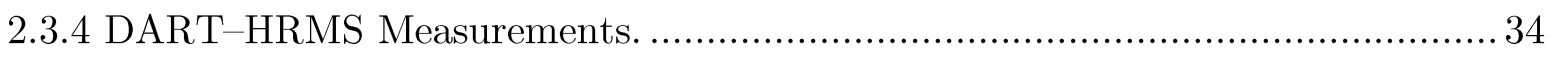

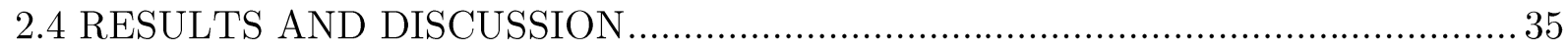

2.4.1 Monolayer Formation and Characterization. ........................................... 35

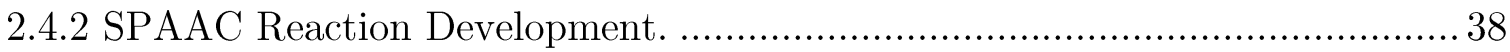

2.4.3 DART-Based Reaction Rate and Activation Parameters Determination....... 40

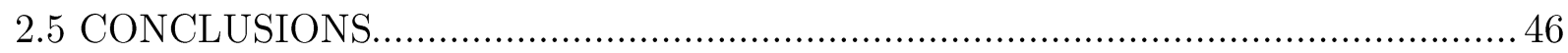

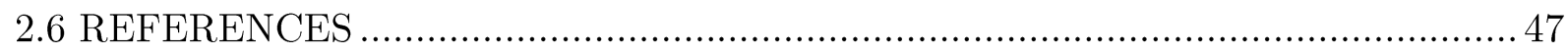




\subsection{INTRODUCTION}

Chemical reactions on solid surfaces play critical roles in many fields, such as the functionalization of devices used in catalysis, solar energy conversion, nanotechnology and biosensing. ${ }^{1}$ The advancement of such devices is hampered by the absence of general, cheap, fast and quantitative analytical techniques to probe the kinetics of organic reactions at the solid-liquid interface. Although interfacial reactions kinetics have been studied by different techniques along the past years, ${ }^{2-7}$ their applicability so far is limited as many of these techniques can only be used for specific functional groups, conducting surfaces and/or require high-vacuum. Accordingly, the development of rapid and easy-to-use analytical techniques to measure the rates of interfacial reactions is of utmost interest.

Ambient desorption/ionization (ADI) mass spectrometry techniques offer a viable alternative to overcome the above limitations. ${ }^{8}$ Particularly for thiol-on-gold selfassembled monolayers matrix-assisted laser desorption ionization time-of-flight mass spectrometry (SAMDI) has been efficiently applied over the last decade. ${ }^{9-12}$ This technique has e.g. recently been used for high-throughput optimization of reaction conditions. ${ }^{13}$ On the other hand, this technique displays also significant boundary conditions, such as the required use of a matrix, the concomitant restriction to fragments with $m / z$ values higher than 450 , while it is also limited to the cleavage of relatively weak bonds, like the $\mathrm{Au}-\mathrm{S}$ one. ${ }^{13}$ Alternatively, DART-HRMS is a promising technique, ${ }^{14}$ due to its ability to detect small molecules $(m / z<400)$ without either the use of a matrix or solvents or the need for weak, non-covalent surface chemistries, unlike matrix-assisted laser desorption/ionization mass spectrometry (MALDI) and desorption ionization electrospray (DESI). ${ }^{15}$ DART-MS is an atmospheric pressure ionization (API) technique that uses high voltage to generate hot and electronically excited He species $\left({ }^{3} \mathrm{~S}_{1}, 19.8 \mathrm{eV}\right)$, which can 


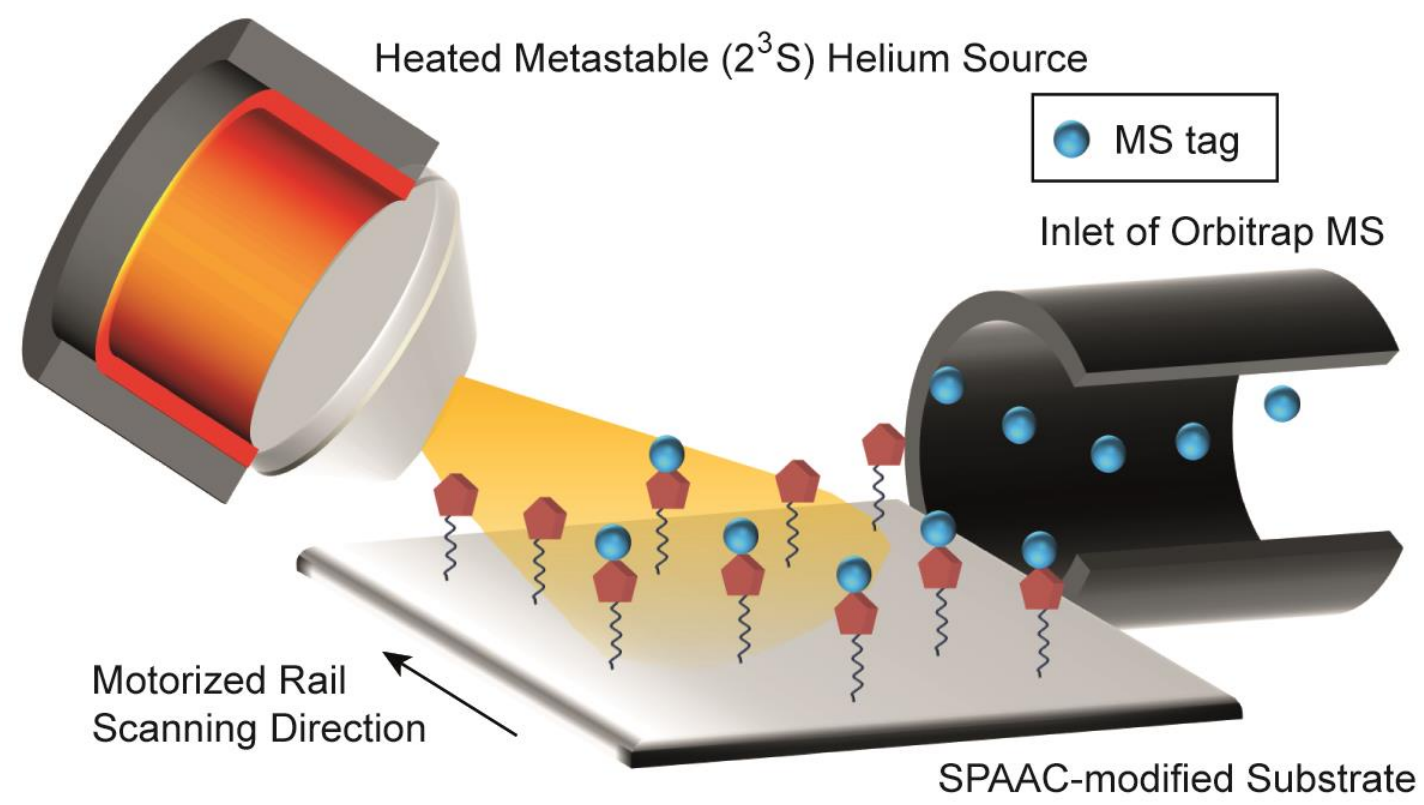

Figure 1. Schematic representation of the DART setup used for monitoring the progress of chemical reactions on surfaces.
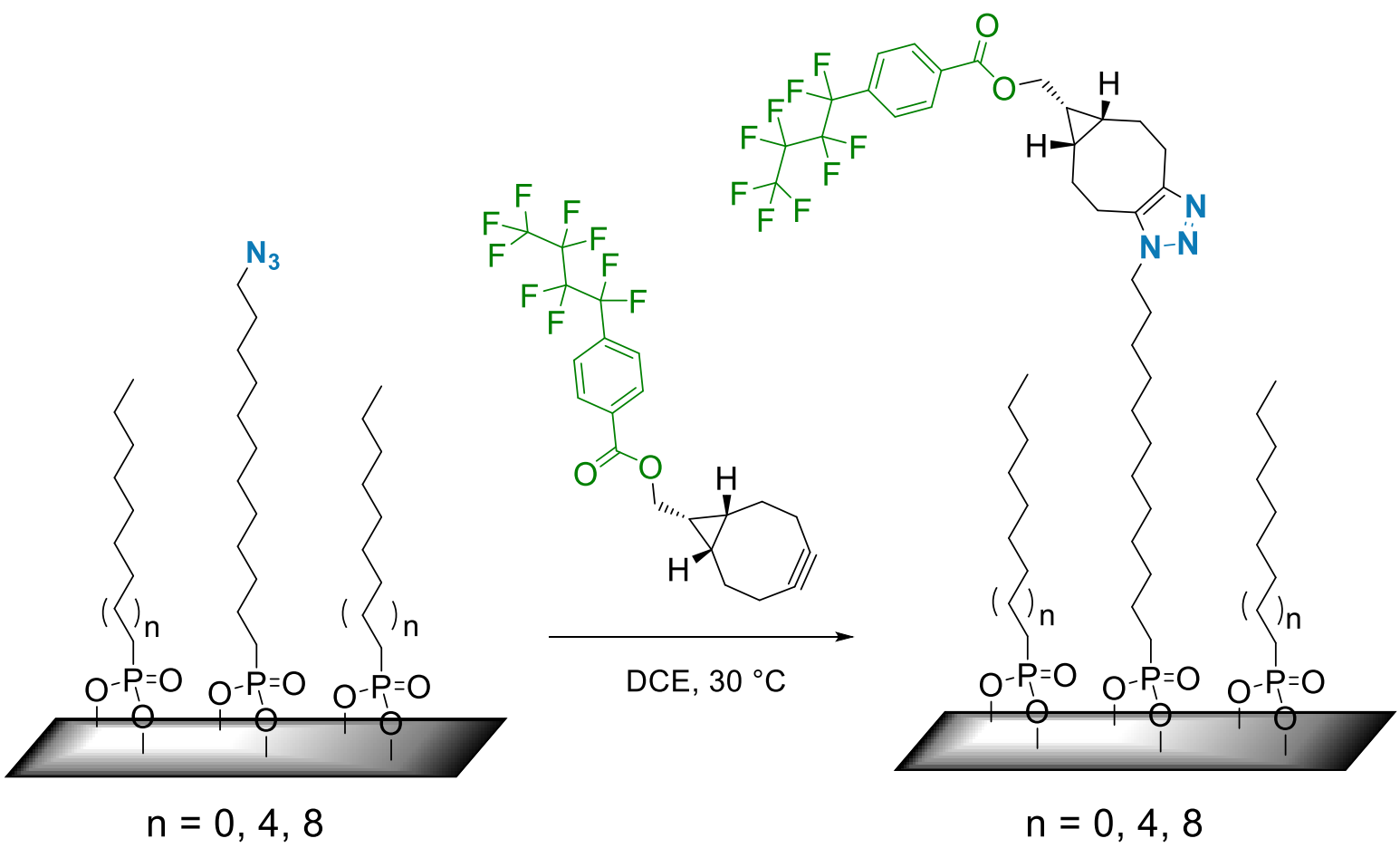

Scheme 1. SPAAC reaction of BCN derivative 1 with immobilized azides present in three different microenvironments: freely accessible $(n=0, \mathbf{C 8})$, partially accessible $(\mathrm{n}=4, \mathbf{C 1 2})$ and buried $(\mathrm{n}=8, \mathbf{C 1 6})$. The MS tag $\left(\left[\mathrm{C}_{11} \mathrm{H}_{4} \mathrm{O}_{2} \mathrm{~F}_{9}\right]^{-}\right)$is highlighted in green. 
simultaneously ionize and desorb surface-bound species under ambient conditions. In addition, this metastable He generates in its passage through the atmosphere also highly reactive protonated water clusters and $\mathrm{O}_{2}{ }^{\cdot-}$ species that also react with the surface. ${ }^{16}$ As a result, DART is capable of generating ionized fragments from a very wide range of functional groups, including amides and esters. Recently, DART-MS has also been successfully applied for molecules up to $m / z 5000$, e.g. ionic liquids, ${ }^{17}$ polydimethylsiloxanes, ${ }^{18}$ and oligomers. ${ }^{19}$ The effectiveness of this method has been shown for both solution and surface substrates for routine high-throughput analysis, as analysis times vary from seconds to at most minutes. ${ }^{20}$ In recent years, this technique (Figure 1) has been successfully applied for the fast, qualitative analysis of monolayers on a wide variety of surfaces ${ }^{21-23}$ and recently for the study of gas-surface heterogeneous reactions. ${ }^{24}$

In this work, we present for the first time the use of DART-HRMS as a general and fast analytical method to obtain quantitative kinetic insights into chemical reactions at the solid-liquid interface. As a model reaction we selected the widely used strain-promoted alkyne-azide cycloaddition (SPAAC) metal-free click reaction $^{25-26}$ on a non-conducting surface. This 1,3-dipolar cycloaddition is based on the use of strained cyclooctynes ${ }^{27-32}$ and has been successfully applied for bioconjugation, ${ }^{3337}$ in vivo imaging ${ }^{38}$ and in the field of surface functionalization..$^{39}$ While the kinetics of SPAAC have been well-studied in solution, ${ }^{44-46}$ no such effort has been made for the corresponding surface reaction. To this end, we investigated the reaction of a fluorine-tagged bicyclo[6.1.0]nonyne (BCN) derivative with mixed monolayers on an aluminum oxide surface that present azide moieties in sterically different microenvironments in which the SPAAC reaction takes place (Scheme 1). SPAAC was particularly selected as the model reaction, because it is such a wellstudied reaction in solution, thereby making it an ideal model reaction to validate our 
new DART method for getting quantitative kinetic information of interfacial reactions.

\subsection{EXPERIMENTAL DETAILS}

2.2.1 Materials. Unless otherwise specified, all chemicals were used as received without further purification. Octylphosphonic acid, hexadecylphosphonic acid, hydrochloric acid, methanol, hexane, acetone, dichloromethane, 2-propanol were purchased from Sigma-Aldrich. 12-Azidododecylphosphonic acid, dodecylphosphonic acid were purchased from SiKÉMIA. Aluminium pieces (99.5\% purity, mirror polished, Staalmarkt Beuningen BV) were cut using a mechanical cutter into pieces of $2 \times 1 \mathrm{~cm}$. For surface modification reactions, the samples were loaded onto a specially constructed PTFE wafer holder able to hold up to 16 samples at a time thus ensuring rigorous reproducibility between samples (See Appendix 1, Figure S13).

\subsubsection{General procedure for the preparation of phosphonic acid} monolayers. $2 \times 1 \mathrm{~cm} \mathrm{Al} \mathrm{slides} \mathrm{were} \mathrm{sonicated} \mathrm{in} \mathrm{hexane} \mathrm{for} 15 \mathrm{~min}$ followed by wiping with lint-free cotton swabs (Texwipe, NC, USA) to remove polymer protection layer on top and remove any residual glue. The surfaces were chemically activated by immersing in 1:1 (v/v) 37\% HCl-MeOH mixture for $5 \mathrm{~min}$, followed by washing with water and 2-propanol. The activated surfaces were then immersed into $\mathrm{N}_{2}$ filled vials of $5 \mathrm{mM}$ solution of corresponding phosphonic acid derivative mixture in 2-propanol, heated to $50{ }^{\circ} \mathrm{C}$ for $5 \mathrm{~min}$, and then left undisturbed for $5 \mathrm{~h}$ at room temperature to obtain self-assembled mixed monolayers. Then surfaces were taken out and sonicated successively for 5 min with 2 -propanol, acetone and $\mathrm{CH}_{2} \mathrm{Cl}_{2}$. The surfaces were then cleaned with $\mathrm{CH}_{2} \mathrm{Cl}_{2}$, air dried and stored under $\mathrm{N}_{2}$ atmosphere.

2.2.3 General procedure for interfacial SPAAC reaction. Azide-functionalized slides were immersed in a dichloroethane $3 \mathrm{mM}$ of (1) solution under $\mathrm{N}_{2}$ atm and 
allowed to react. After completion of reaction for the desired time, the $\mathrm{Al}$ pieces were taken out, sonicated and washed with acetone to remove physisorbed moieties, dried under $\mathrm{N}_{2}$ stream and immediately used for XPS and DART analysis.

2.2.4 Determination of activation parameters. The activation parameters (enthalpy of activation $\left(\Delta H^{\dagger}\right)$ and entropy of activation $\left(\Delta S^{\ddagger}\right)$ ) were obtained using Eyring equation by a least-squares fit of plots of $\ln (\mathrm{k} / \mathrm{T})$ versus $1 / \mathrm{T}$. Values for $\Delta H^{\ddagger}$ and $\Delta S^{\ddagger}$ were calculated from the slope and intercept of the aforementioned plot (Equation 1).

$$
\ln (k / T)=\ln \left(K k_{B} / h\right)+\Delta S^{\ddagger} /{ }_{R}-\Delta H^{\ddagger} / R T
$$

where $k^{\prime}$, is the observed rate constant (in $\mathrm{s}^{-1}$ ), $T$ is the absolute temperature (in $\mathrm{K}$ ), $K$ is the transmission coefficient (set to be 1 ), $k_{B}$ is the Boltzmann's constant, $h$ is Planck's constant, $R$ is the gas constant, $\Delta S^{4}$ is the entropy of activation, (in $\left.\mathrm{J} \cdot(\mathrm{K} \cdot \mathrm{mol})^{-1}\right)$ and $\Delta H^{\ddagger}$ is the enthalpy of activation, in $\left(\mathrm{kJ} \cdot \mathrm{mol}^{-1}\right)$.

\subsection{ANALYTICAL INSTRUMENTS.}

2.3.1 Static Water Contact Angle (SCA) Measurements. The wettability of the modified surfaces was determined by automated static water contact angle measurements with a Krüss DSA 100 goniometer (volume of the drop of deionized water was $3.0 \mu \mathrm{L}$ ). The reported values are the average of at least five droplets, and the relative error is less than $\pm 1^{\circ}$.

2.3.2 Infrared Reflection Absorption Spectroscopy (IRRAS). IRRAS spectra were obtained with a Bruker Tensor 27 FT-IR spectrometer equipped with a liquid nitrogen cooled MCT-detector, using a commercial variable-angle reflection unit (Auto Seagull, Harrick Scientific). A Harrick grid polarizer was installed in front of 
the detector and was used for measuring spectra with $\mathrm{p}$-polarized radiation with respect to the plane of incidence at the sample surface. A Ge ATR crystal was used to obtain the spectra. Single channel spectra were collected at $35^{\circ}$ using 256 scans in each measurement. The raw data were divided by the spectrum recorded on a freshly etched reference $\mathrm{Al}$ oxide surface, after which a baseline correction was applied to give the reported spectra.

\subsubsection{X-ray Photoelectron Spectroscopy (XPS) Measurements. The XPS} analysis of surfaces was performed using a JPS-9200 photoelectron spectrometer (JEOL, Japan). Survey and high-resolution spectra were obtained under UHV conditions using monochromatic $\mathrm{Al} \mathrm{K \alpha} \mathrm{X}$-ray radiation at $12 \mathrm{kV}$ and $20 \mathrm{~mA}$, and an analyzer pass energy of $50 \mathrm{eV}$ for wide scans and $10 \mathrm{eV}$ for narrow scans. The emitted electrons were collected at $10^{\circ}$ from the surface normal (take-off angle relative to the surface normal $10^{\circ}$ ). All XPS spectra were evaluated by using Casa XPS software (version 2.3.15). Survey spectra were corrected with linear background before fitting, whereas high-resolution spectra were corrected with linear background. Atomic area ratios were determined after a baseline correction and normalizing the peak area ratios by the corresponding atomic sensitivity factors $(1.00$ for $\mathrm{C} 1 \mathrm{~s}, 1.80$ for N1s, 2.93 for O1s, 4.43 for F1s, 1.18 for P2s and 0.54 for Al2p).

2.3.4 DART-HRMS Measurements. Analysis of the SPAAC reacted Al surfaces were performed using a DART-SVP ion source (Ion-Sense, Saugus, MA, USA) coupled to an Exactive orbitrap high-resolution mass spectrometer (Thermo Fisher Scientific, San Jose, CA, USA), mounted on a motorized rail travelling at $0.2 \mathrm{~mm} / \mathrm{s}$. Thermo Scientific Xcalibur software (V2.1.0.1139) was used for data acquisition and processing. The measurements were performed in negative mode at $450{ }^{\circ} \mathrm{C}$ using a scan range of $m / z 330.0-350.0$, a mass resolution of 100,000 (FWHM) at a scan rate of $1 \mathrm{~Hz}$. The ion trap was tuned with $0.1 \mathrm{mg} / \mathrm{mL}$ methanolic solution of quinine $(\mathrm{m} / \mathrm{z}$ 323.41 in negative mode) to optimize capillary temperature and voltage (Figure S16). 
The DART source was positioned $6.1 \mathrm{~cm}$ on the horizontal scale, $7 \mathrm{~cm}$ on the vertical scale with an angle of $45^{\circ}$, such that it is around $1 \mathrm{~mm}$ above the surface (See Appendix 1, Figures S13-S16). The distance from the surface to the ceramic tube is minimized by placing them at the edge of the moving rail so that maximum of the $p$ $\mathrm{C}_{4} \mathrm{~F}_{9}$ benzoate ions would enter the MS.

\subsection{RESULTS AND DISCUSSION}

\subsubsection{Monolayer Formation and Characterization.}

The proposed strategy to control the steric microenvironment around the azide moieties on the monolayers relies on the introduction of non-reactive alkyl chains of variable length around the reactive azide, which was linked to the surface using a C12 linker, as schematically depicted in Figure 2. In this regard, with a C8 alkyl chain (C8), the azide is freely accessible layer, whereas with a longer alkyl chain, the reactive group is only partially accessible $(\mathbf{C 1 2})$ or is buried within the monolayer (C16).
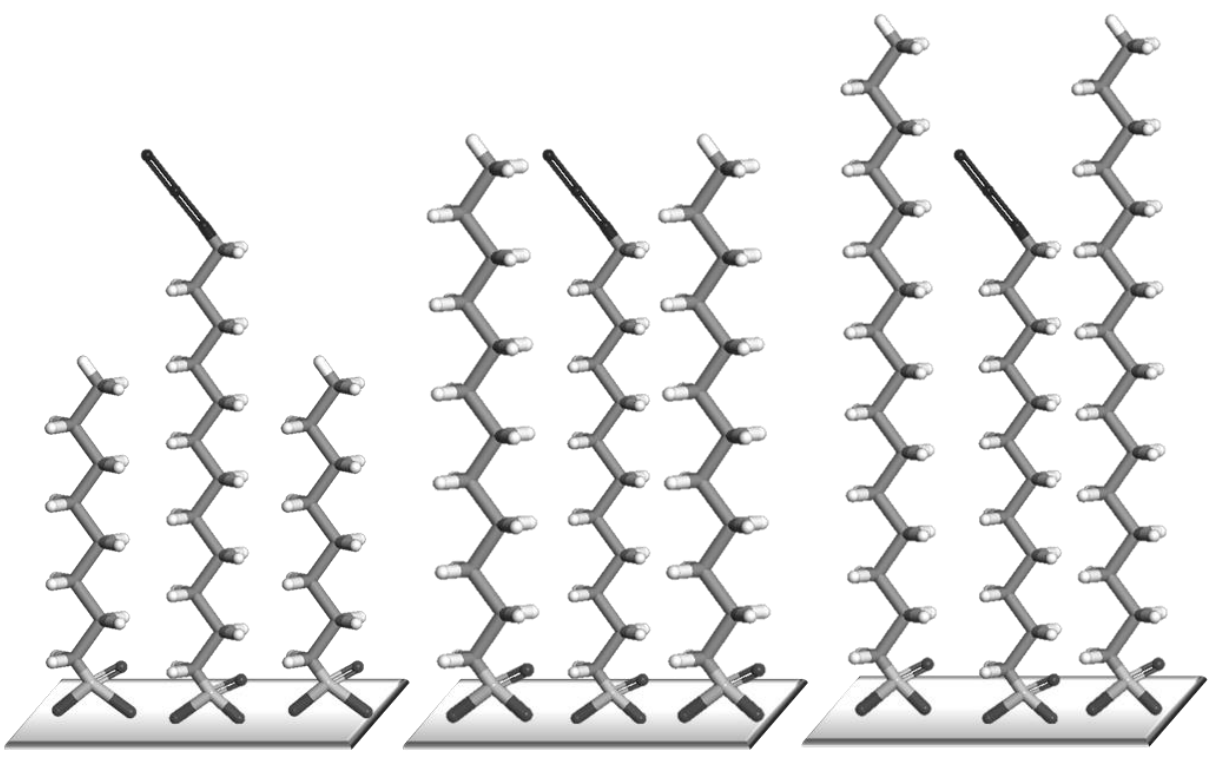
Figure 2. Schematic model of the mixed monolayer used for the SPAAC with immobilized azide groups present in the three different microenvironments: freely accessible (C8), partially accessible (C12) and buried (C16).

For surface modification, a well-established, robust attachment chemistry, relying on phosphonic acids (PA) was used to covalently anchor the monolayer onto hydroxylated aluminum oxide surfaces. ${ }^{47-49}$ Freshly etched aluminium substrates were immersed for $5 \mathrm{~h}$ in 2-propanol solutions of 12-azidododecyl PA and the appropriate alkyl-terminated PA (in different ratios), yielding monolayers with 28-30\% C content as determined by XPS (See Appendix 1, Figure S1). From static water contact angle (SCA) measurements, it was found that the reaction was complete after $5 \mathrm{~h}$ (See Appendix 1, Figure S4). Remarkably, substantially longer reaction times (16 h) contributed to the formation of undesirable multilayers $(42-44 \%$ C). Contrary to previous reports, ${ }^{50-52}$ the ratio of azides in a mixed monolayer was found to be identical to the ratio in the solution (within experimental error) as confirmed by XPS (See Appendix 1, Figure S2). After reaction with the PA mixture, the static water contact angle (SCA) increased from $35^{\circ}$ (-OH terminated aluminum oxide) to $106^{\circ}$ due to the hydrophobic nature of the alkyl chain (See Appendix 1, Table S1 and Figure S3), in accordance with similar PA monolayers on aluminium oxide surfaces. ${ }^{53}$ The XPS N1s narrow scan showed two bands with a ratio 2:1 (399.6 and $403.4 \mathrm{eV}$, respectively), in accordance with the presence of an $\mathrm{N}_{3}$ group (Figure 3a), ${ }^{54}$ and IRRAS confirmed the presence of the $\mathrm{N}_{3}$ group (Figure 3c), showing the characteristic azide peak at $2102 \mathrm{~cm}^{-1} .^{55}$ The XPS C1s narrow scan (Figure 3b) shows the peak deconvolution into different components corresponding to the carbon atoms having different environments in the mixed 3:1 C8 monolayer.

As shown, IRRAS gives evidence of the presence of different functional groups in the monolayer and also provides information about the mode of attachment of the monolayer onto the surface. The positions of the antisymmetric and symmetric 
methylene stretching $\left(v_{\mathrm{a}}\right.$ and $v_{\mathrm{s}} \mathrm{CH}_{2}$, respectively) can be used to distinguish between monolayers with a short-range order $\left(2918 / 2850 \mathrm{~cm}^{-1}\right)$ or disorder $\left(2928 / 2854 \mathrm{~cm}^{-1}\right)$. The presence of sharp signals for the antisymmetric $\mathrm{C}-\mathrm{H}$ stretch vibration (Figure 3c) at $2925 \mathrm{~cm}^{-1}(\mathbf{C 8}), 2922 \mathrm{~cm}^{-1}(\mathbf{C 1 2})$, and $2924 \mathrm{~cm}^{-1}$ (C16) and for the symmetric $\mathrm{C}-\mathrm{H}$ stretch vibration at $2854 \mathrm{~cm}^{-1}$ (C8), $2852 \mathrm{~cm}^{-1}$ (C12) and 2953 $\mathrm{cm}^{-1}(\mathbf{C 1 6})$, in combination with the XPS and contact angle data above, indicate the formation of densely packed monolayers without a high degree of short-range order. ${ }^{56}$

\subsubsection{SPAAC Reaction Development.}

For the study of the SPAAC reaction, the endo-BCN diastereomer (1, Scheme 1) was selected. The design of the BCN derivative was guided by two criteria; firstly (part of) the molecule should be detectable by DART, and secondly the molecule should also be observable by XPS (for independent validation of the DART results). Previous research in our group showed DART to be effective in cleaving ester bonds both in solution as well as on surfaces, to release a suitable MS tag that can be detected in negative ion mode (See Appendix 1, Figures S7-S8). ${ }^{23}$ Thus, the ester bond was selected as a labile linker that couples the reactive BCN group with a MS tag, which was chosen to be a para-substituted benzoic acid derivative with a fluorinated butyl chain (highlighted in green in Scheme 1). Such a labeling approach has been widely studied, ${ }^{57-58}$ however, to the best of our knowledge this is the first time an ionizable MS tag is used for interfacial kinetics elucidation. This design has three additional advantages: 1) the fluorinated alkyl chain increases the volatility of the benzoic acid group, allowing a rapid surface desorption; 2) the electronwithdrawing per-fluorobutyl group weakens the ester bond and stabilizes the carboxylate anion; 3) F signals are easily detected by XPS, thus providing a label for an independent quantitative analysis. 
The SPAAC reaction was performed using a $3 \mathrm{mM}$ solution of $\mathrm{BCN}$ derivative (1) on the azide-terminated monolayer in dichloroethane (DCE) at $30{ }^{\circ} \mathrm{C}$. Under these conditions, the $\mathrm{BCN}$ derivative is present in large excess over the immobilized

(a)

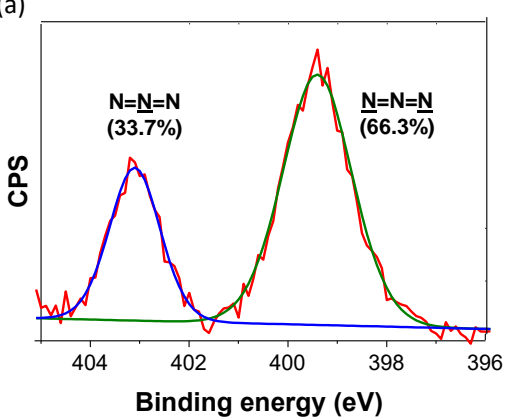

(b )

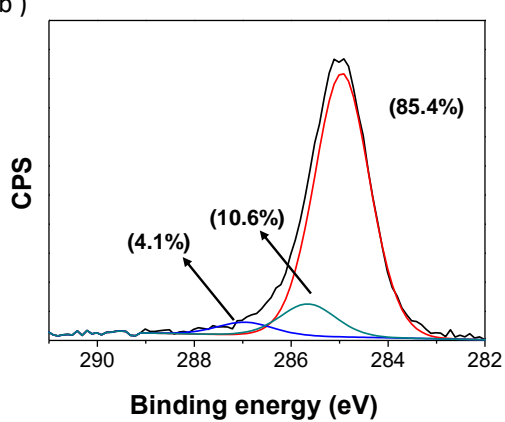

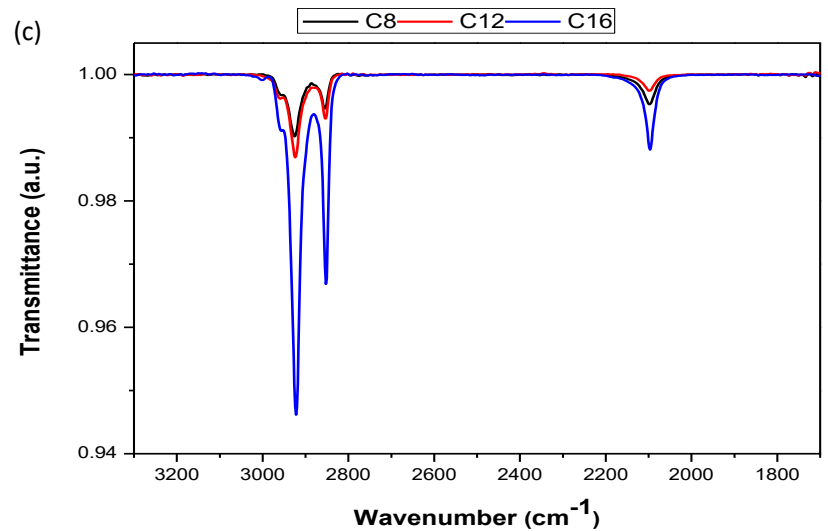

Figure 3. High-resolution XPS N1s (a) and C1s (b) spectra of a 3:1 mixture of octylphosphonic and 12-azidododecylphosphonic acid monolayer (C8) onto oxidized aluminum. (c) IRRAS spectra of 3:1 mixed monolayers of C8, C12 and C16 onto oxidized aluminum.
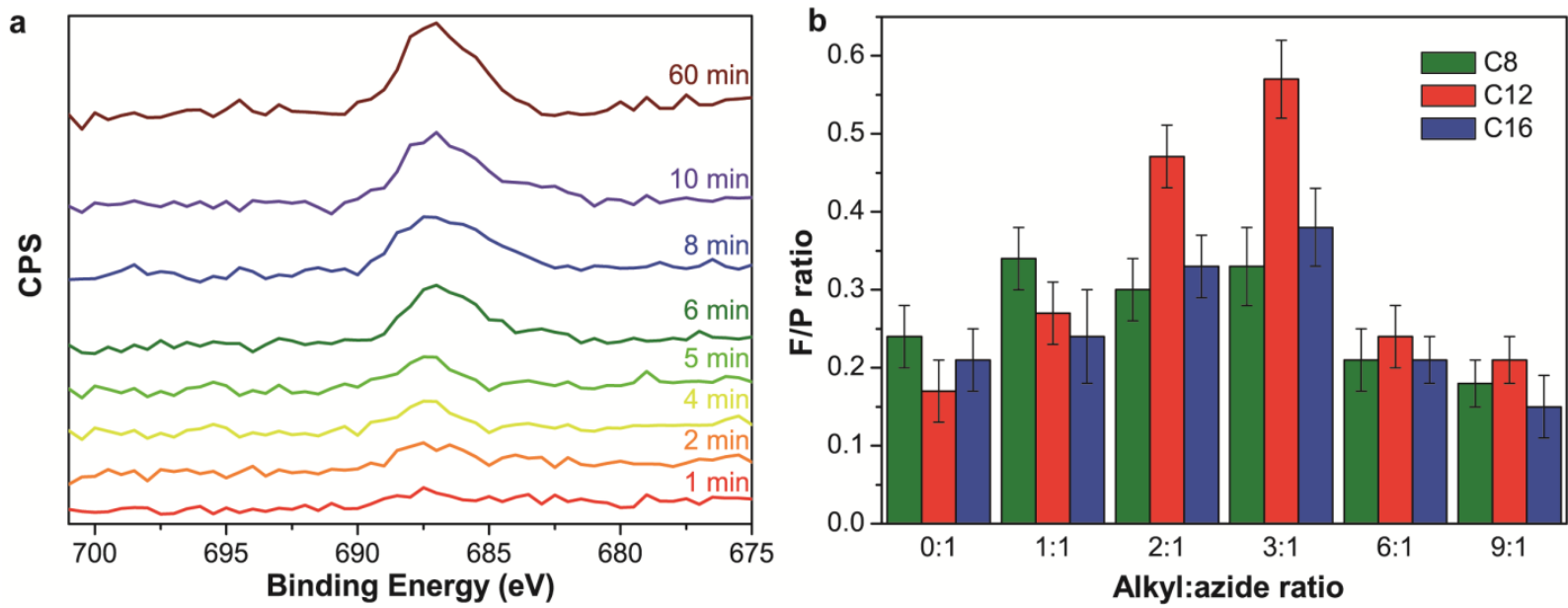
Figure 4. (a) XPS F1s spectra after SPAAC at different reaction times. (b) F/P ratio for the three different mixed monolayers with varying $\mathrm{C}$-alkyl environment after SPAAC reaction $(60 \mathrm{~min}$; as in rest of the paper: all results from six-fold repetition).

azides, therefore pseudo first-order kinetics can be assumed. After reaction with 1, XPS measurements showed a significant increase of $\mathrm{F}$ content on the surface (Figure 4a). As control experiment, treating 100\% alkyl-terminated surfaces with a $3 \mathrm{mM}$ solution of 1 for $24 \mathrm{~h}$ yielded no incorporation of fluorine, as determined by XPS. It is worth mentioning that no F signal was detected in a typical F1s narrow scan, and XPS wide scans were carried out under more sensitive conditions to obtain an acceptable signal-to-noise $(\mathrm{S} / \mathrm{N})$ ratio for the $\mathrm{F} 1 \mathrm{~s}$ region (Figure $4 \mathrm{a})$.

In order to study the optimal microenvironment for the SPAAC reaction, different monolayers with varying alkyl:azide ratios (from 0:1 to 9:1) were prepared and reacted with the $\mathrm{BCN}$ derivative for $60 \mathrm{~min}$. Results clearly showed that increasing the spacing between the azide groups, gave a substantial improvement in BCN incorporation: despite the reduction of $50 \%$ of azides on the surface, the $\mathrm{F} / \mathrm{P}$ ratio clearly increased when going from 0:1 to 1:1 (Figure 4b). Further variation afforded a maximum surface coverage after 60 min reaction time for the $3: 1$ monolayer (for the more diluted surfaces it was possible to reach higher conversion only at longer reaction times). Interestingly, the $3: 1$ ratio showed that the incorporation of the BCN derivative was greatest for the slightly accessible C12 monolayer, followed by the buried C16 and the accessible C8. In view of these results, the 3:1 mixed monolayer was selected for all further kinetics studies.

In the XPS N1s spectrum of the 3:1 mixed monolayers, signals corresponding to both the azide as well as the triazole moiety were observed, showing the presence of the unreacted azides even after long reaction times, as also confirmed by IRRAS (See Appendix 1, Figure S9). In particular, the maximum $\mathrm{F} / \mathrm{N}$ ratio was around 1, 
showing that only one third of the azide groups had been converted. This finding was corroborated for all three monolayers by separate experiments in which the reaction was continued for 5 days, and is consistent with other incomplete conversions reported in literature. ${ }^{59}$ We may attribute this incomplete conversion primarily to steric hindrance provided by the BCN moiety and the attached fluorinated tail.

\subsubsection{DART-Based Reaction Rate and Activation Parameters}

\section{Determination.}

Despite its applicability for routine surface analysis, XPS is not a very suitable technique to determine reaction rates as a consequence of the time-consuming data acquisition, as a typical narrow scan requires several hours (total measurement time for hexaplet data point typically $16 \mathrm{~h}$ ). Additionally, the comparatively low sensitivity hampers study at small conversions, requiring in our case at least six-fold repetitions to obtain usefully small experimental errors (Figure 4b). Alternatively, DART-HRMS is an atmospheric pressure ionization technique with high sensitivity and a much higher $\mathrm{S} / \mathrm{N}$ ratio than XPS), which allows fast measurements, as analysis times vary from seconds to minutes (total measurement time for hexaplet data point typically $18 \mathrm{~min}$ ). Under the studied conditions, the use of high resolution in the MS analysis was found to be necessary because of the presence of random contaminating

species (e.g. from the ambient atmosphere) with near-identical masses (See Appendix 1, Figure S17). A closer inspection of the mass spectra obtained during our measurements revealed unassigned signals for a minority of samples, close to the ion of interest (within $30 \mathrm{mmu}$ ). Therefore, to rule out any contributions of these contaminants and ensure to be analysing only the correct ion of interest, HR-MS was needed and used throughout (10 mmu mass window). To appreciate the strength of DART-HRMS, it is instructive to consider the minute amount of tag that is reacted 
on the surface and is desorbed from it: surface concentrations as low as $\sim 1 \mathrm{pmol} \mathrm{\textrm {cm } ^ { - 2 }}$ can be measured without the requirement of sample pretreatment. ${ }^{24}$

Figure 5a shows a typical time-dependent mass spectrometric signal recorded for a SPAAC-modified Al slide, where the extracted ion chromatogram (EIC) of the

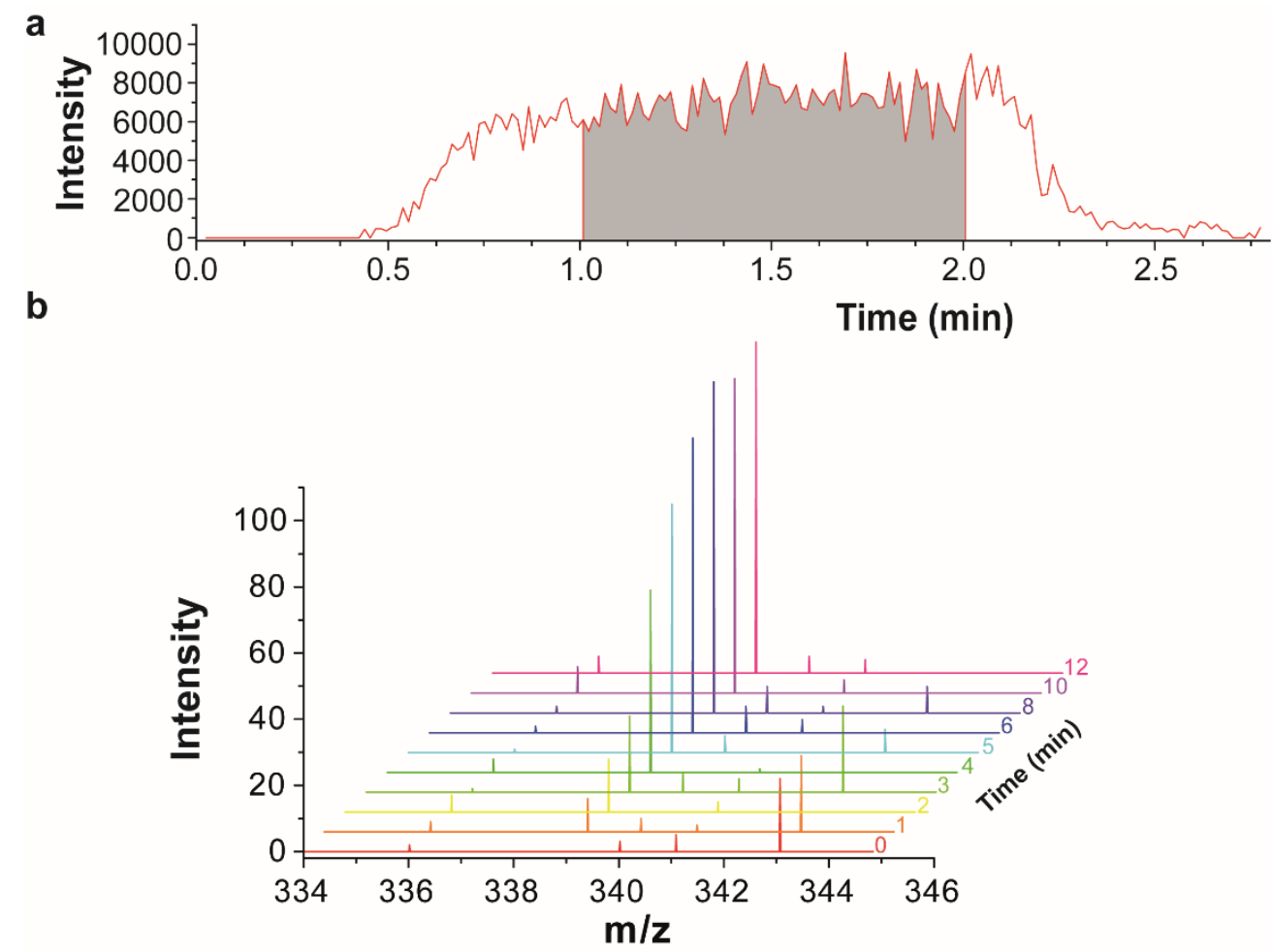

Figure 5. (a) Extracted ion chromatogram $(\mathrm{m} / z$ 339.0052-339.0072) from the labile group $\left(\left[\mathrm{C}_{11} \mathrm{H}_{4} \mathrm{O}_{2} \mathrm{~F}_{9}\right]^{-}\right)$and integrated peak area (in gray). (b) Mass spectra for the incorporation of $\mathrm{BCN}$ compound 1 to the azide-functionalized surface $\mathbf{C 1 2}$ at different times (from 0 to $12 \mathrm{~min}$ ).

labile group is shown in the red traces and was quantified by integrating the peak area over a fixed time interval (in gray). The intensity of MS tag $\left(\left[\mathrm{C}_{11} \mathrm{H}_{4} \mathrm{O}_{2} \mathrm{~F}_{9}\right]^{-}\right)$ monitored by DART over time is directly proportional to the extent of reaction, as this tag can only be on the surface as the result of the reaction between surfacebound azide and solution-based BCN 1. Control measurements without azide moieties showed no physisorption of unreacted BCN molecules onto the surface. A great advantage of DART-HRMS is the much higher sensitivity, compared to e.g. 
XPS: while with XPS it proved challenging to obtain discernible F signals for lower reaction times, with DART-HRMS intense signals with excellent $\mathrm{S} / \mathrm{N}$ ratios could be obtained routinely for reaction times as short as $15 \mathrm{~s}(\sim 3 \%$ conversion, Figure $5 \mathrm{~b})$.

A kinetic study was performed for each of the three mixed monolayers $(\mathbf{C 8}$, C12 and C16) using the intensity of the MS tag obtained by DART analysis as a measure for the conversion. In accordance to a first-order process, the rate of $\mathrm{BCN}$ incorporation (due to SPAAC) became progressively slower with increasing time, converging to a limiting value, which was different for each monolayer (Figure 6a). Particularly, the extent of the reaction was highest for the C12 monolayer, in line with the results of the XPS measurements previously discussed (Figure 6b). The lower extent of $\mathrm{BCN}$ incorporation in case of the $\mathbf{C 8}$ monolayer can be rationalized by considering that for this system the $\mathrm{BCN}$-containing alkyl chain is free to move above the microenvironment created by the octyl chains, meaning that this bulky group can fold back on the monolayer, thus blocking access to unreacted azide groups in its vicinity. This freedom is reduced in case of the $\mathbf{C 1 2}$ monolayer, where the BCN is forced to stand more upright on the surface. Similarly, the lower extent of BCN incorporation found for the $\mathbf{C 1 6}$ microenvironment can be explained by the fact that the SPAAC reaction needs to occur inside the microenvironment of the long hexadecyl chains, which means that as the reaction progresses the monolayer density will increase as a result of incorporation of the bulky BCN group within the monolayer. This can make it impossible for incoming BCN molecules to react with remaining azide groups that are buried within the $\mathbf{C 1 6}$ monolayer.

The first-order rate constants $\left(k^{\prime}\right)$ were obtained from the slope of plotting $\ln$ $\left[\left(\mathrm{I}_{\text {inf }}-\mathrm{I}_{0}\right) /\left(\mathrm{I}_{\mathrm{inf}}-\mathrm{I}_{\mathrm{t}}\right)\right]$ as a function of time (Figure $6 \mathrm{~b}$ and Table 1$)$. In the case under study, $\mathrm{I}_{\text {inf }}$ is the asymptotic (integrated) EIC intensity that is reached as the reaction progress stops, and this was determined by curve fitting of the data to an exponential (decay) function. For monolayers presenting freely accessible azide groups (C8), the 
rate constant at $30{ }^{\circ} \mathrm{C}$ was $0.0057 \mathrm{~s}^{-1}$. However, for the mixed monolayers presenting partially accessible (C12), or buried (C16) azide groups, the rate constants were 0.0037 and $0.0031 \mathrm{~s}^{-1}$ respectively, ca. two- to three-fold lower than the monolayer presenting the azide in a more accessible environment. From this, it can be concluded that as the azide becomes sterically less accessible, the reaction rate decreases, which can understood by considering that the large BCN derivative has to diffuse towards its azide counterpart in order for the reaction to occur. It is thus worth noting that the effect of microenvironment on the SPAAC reaction as could be elucidated by DART-HRMS, is manifested in different ways for the rate of the reaction and for the extent of the reaction. Whereas the rate simply decreases upon increasing the steric bulk around the azide group (see Figure 7 below for a schematic representation), for the extent of the reaction an optimum was found for the intermediate $\mathbf{C 1 2}$ monolayer.

Table 1. First-order rate constants and activation parameters (determined by DART-HRMS) for the interfacial SPAAC reaction of BCN and azido moieties on the three microenvironments (results from six-fold repetitions).

\begin{tabular}{|c|c|c|c|c|}
\hline Monolayer & $\begin{array}{c}k_{\text {DART }}^{\prime} \\
\left(\times 10^{-3} \mathrm{~s}^{-1}\right)\end{array}$ & $\begin{array}{c}k_{\mathrm{XPS}}^{\prime} \\
\left(\times 10^{-3} \mathrm{~s}^{-1}\right)\end{array}$ & $\begin{array}{c}\Delta H^{\ddagger} \\
\left(\mathrm{kcal} \cdot \mathrm{mol}^{-1}\right)\end{array}$ & $\begin{array}{c}\Delta S^{\ddagger} \\
\left(\mathrm{cal} \cdot \mathrm{K}^{-1} \cdot \mathrm{mol}^{-1}\right)\end{array}$ \\
\hline $\mathrm{C} 8$ & $5.7 \pm 0.4$ & $5.3 \pm 0.3$ & $2.86 \pm 0.24$ & $-0.05 \pm 0.01$ \\
\hline $\mathrm{C} 12$ & $3.7 \pm 0.3$ & $3.3 \pm 0.4$ & $5.76 \pm 0.48$ & $-0.05 \pm 0.02$ \\
\hline C16 & $3.1 \pm 0.3$ & $2.8 \pm 0.5$ & $5.02 \pm 0.72$ & $-0.05 \pm 0.02$ \\
\hline
\end{tabular}

It is worth mentioning that we observed relatively small differences between rate constants for the different microenvironments. While this observation is by itself already interesting from a mechanistic point of view, it -importantly- also clearly illustrates the potential of our DART measurements: we can accurately and reliably 
observe such small differences that are with conventional approaches, like XPS, well beyond the limits of observation. The validity of the DART method was confirmed independently by XPS (See Appendix 1, Figures S10-S11), which yielded similar rate constants for the three monolayers. This shows the potential use of DART-HRMS as a rapid and easy-to-use tool for the accurate measurement of the rates of surfacebound reactions.
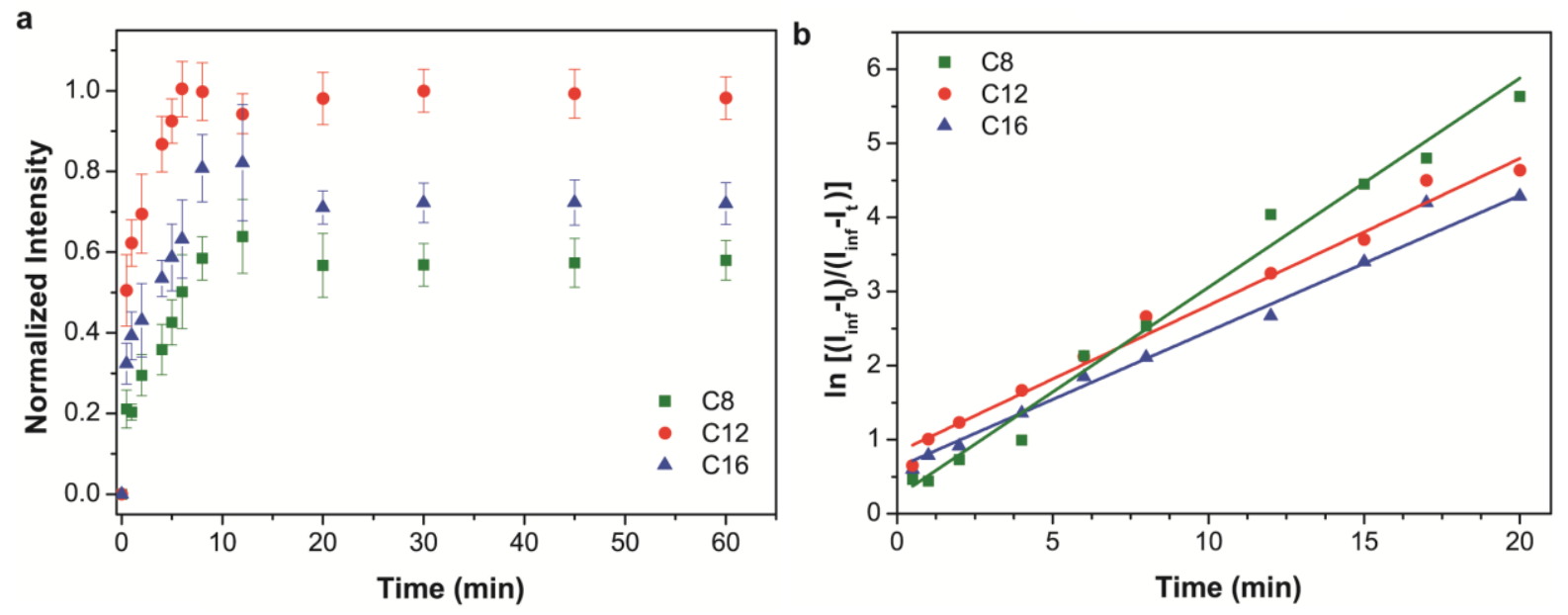

Figure 6. (a) Normalized intensity (w.r.t. C12) for the SPAAC reaction of BCN derivative 1 with immobilized azides in the three microenvironments at $30{ }^{\circ} \mathrm{C}$ (each data point from six-fold repetition). (b) Plot showing $\ln \left[\left(\mathrm{I}_{\text {inf }}-\mathrm{I}_{0}\right) /\left(\mathrm{I}_{\text {inf }}-\mathrm{I}_{\mathrm{t}}\right)\right]$ vs time, used to obtain the first-order rate constants.
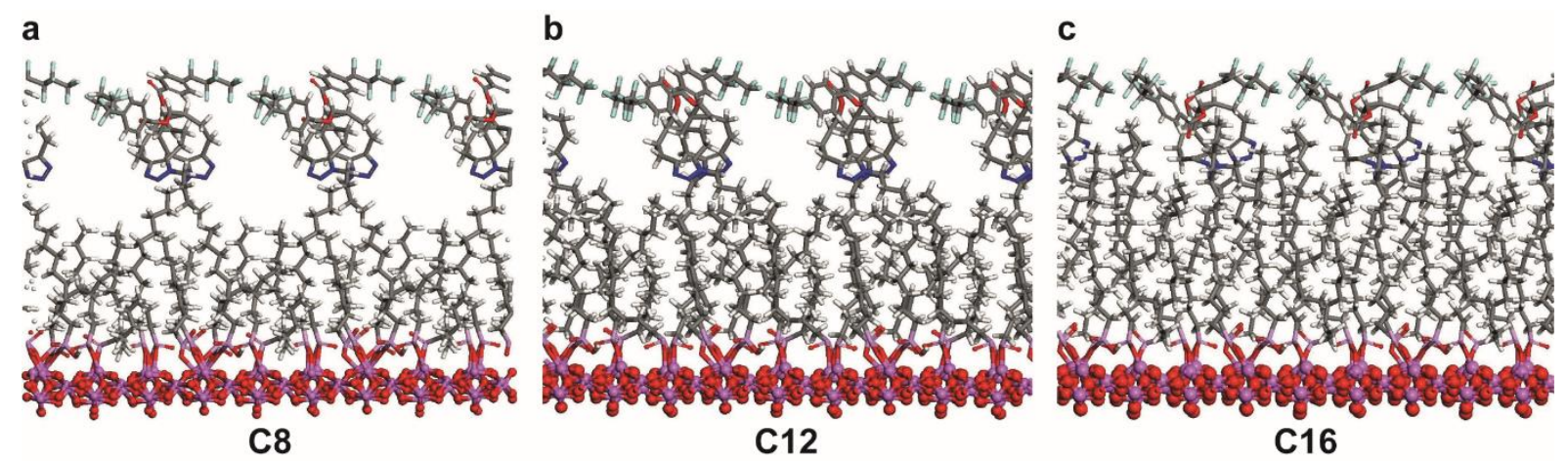

Figure 7. Schematic representation of the three monolayers after the SPAAC reaction: (a) freely accessible $\mathbf{C 8}$, (b) partially accessible $\mathbf{C 1 2}$ and (c) buried $\mathbf{C 1 6}$. 
To further illustrate the convenience of DART-HRMS the effect of temperature on the reaction rates was studied at four different temperatures in the range of 20 to $50{ }^{\circ} \mathrm{C}$, allowing us to extract the activation parameters by means of an Eyring analysis (See Appendix 1, Table S2). Whereas the entropy of activation is basically the same for all three cases within experimental error, the enthalpy of activation varies significantly in dependence of the microenvironment. In line with previously reported results, ${ }^{50}$ we observed that for the unhindered C8 monolayer the lowest enthalpy of activation $\left(5.3 \pm 0.3 \mathrm{kcal} \cdot \mathrm{mol}^{-1}\right)$ was found, which can be understood by an optimal overlap between the two $\pi$ - systems involved in the $[3+2]$ cycloaddition during the transition state for the $\mathbf{C 8}$ system. For both the $\mathbf{C 1 2}$ and the C16 monolayers significantly higher enthalpies of activation were determined $\left(5.76 \pm 0.48\right.$ and $5.02 \pm 0.72 \mathrm{kcal} \cdot \mathrm{mol}^{-1}$, respectively). Apparently, the increased sterics of the reaction with higher surrounding alkyl chains are quite significant in this reaction. Two factors likely contribute to this, namely the reduced flexibility in the orientation of the azide moieties, and the increased bulk close to the monolayer surface due to the BCN ring and its freely rotating fluoroalkyl tail (Figure 7). These values are all slightly smaller than the theoretically determined activation energies of $6.5-7.4 \mathrm{kcal} \cdot \mathrm{mol}^{-1} .60$

While the mechanistic insights into the SPAAC reaction are relevant by themselves, it should be emphasized that these insights are the direct consequence of the availability of DART-HRMS as a fast, quantitative surface analysis technique. Further studies on the application of DART-HRMS for determining the interfacial kinetic parameters of other click reactions are ongoing in our laboratory. 


\subsection{CONCLUSIONS}

The successful unraveling of the effects of the microenvironment on the rate of the commonly employed SPAAC highlights the power of DART-HRMS as a general, fast and cheap technique to study interfacial reaction kinetics in great detail. In addition it has been shown that tailor-made control over the microenvironment of the reactive moieties via tuning of the accessibility of the immobilized azide within the monolayer can steer both the reaction rates as well as the extent of the surfacebound SPAAC reaction. We anticipate DART-HRMS provides a powerful analytical platform for the kinetic study of many other reactions on surfaces.

\subsection{REFERENCES}

(1) Yates, Jr., J. T.; Campbell, C. T. Proc. Surface Chemistry: Key to Control and Advance Myriad Technologies. Natl. Acad. Sci. U.S.A. 2011, 108, 911-916.

(2) Gawalt, E. S.; Mrksich, M. Substituent Effects Study Reveals the Kinetic Pathway for an Interfacial Reaction. J. Am. Chem. Soc. 2004, 126, 15613-15617.

(3) Williams, J. M.; Rowland, B.; Jeffery, M. T.; Groenewold, G. S.; Appelhans, A. D.; Gresham, G. L.; Olson, J. E. Degradation Kinetics of VX on Concrete by Secondary Ion Mass Spectrometry. Langmuir 2005, 21, 2386-2390.

(4) Li, J.; Thiara, P. S.; Mrksich, M. Rapid Evaluation and Screening of Interfacial Reactions on Self-Assembled Monolayers. Langmuir, 2007, 23, 11826-11835.

(5) Orski, S.; Sheppard, G. R.; Arumugam, S.; Arnold, R. M.; Popik, V. V.; Locklin, J. Rate Determination of Azide Click Reactions onto Alkyne Polymer Brush Scaffolds: a Comparison of Conventional and Catalyst-Free Cycloadditions for Tunable Surface Modification. Langmuir 2012, 28, 14693-14702. 
(6) Han, C.; Liu, Y.; Ma, J.; He, H. Key Role of Organic Carbon in the SunlightEnhanced Atmospheric Aging of Soot by $\mathrm{O}_{2}$. Proc. Natl. Acad. Sci. U.S.A. 2012, $109,21250-21255$.

(7) Näslund, L. $-\AA$. Reaction Kinetics for the Oxygen Hydrogenation Process on Pt(111) Derived from Temperature-Programmed XPS. Surf. Sci. 2013, 618, 42-48.

(8) Montavon, T. J.; Li, J.; Cabrera-Pardo, J. R.; Mrksich, M.; Kozmin, S. A. Three-Component Reaction Discovery Enabled by Mass Spectrometry of SelfAssembled Monolayers. Nat. Chem. 2011, 4, 45-51.

(9) Min, D.-H.; Tang, W.-J.; Mrksich, M. Chemical Screening by Mass Spectrometry to Identify Inhibitors of Anthrax Lethal Factor. Nat. Biotechnol. 2004, 22, 717-720.

(10) Patrie, S. M.; Mrksich, M. Self-Assembled Monolayers for MALDI-TOF Mass Spectrometry for Immunoassays of Human Protein Antigens. Anal. Chem. 2007, 79, $5878-5887$.

(11) Gurard-Levin, Z. A.; Scholle, M. D.; Eisenberg, A. H.; Mrkisch, M. HighThroughput Screening of Small Molecule Libraries using SAMDI Mass Spectrometry. ACS Comb. Sci. 2011, 13, 347-350.

(12) Kuo, H.-Y.; DeLuca, T. A.; Miller, W. M;. Mrksich, M. Profiling Deacetylase Activities in Cell Lysates with Peptide Arrays and SAMDI Mass Spectrometry. Anal. Chem. 2013, 85, 10635-10642.

(13) Diagne, A. B.; Li, S.; Perkowski, G. A.; Mrksich, M.; Thomson, R. J. SAMDI Mass Spectrometry-Enabled High-Throughput Optimization of a Traceless Petasis Reaction ACS Comb. Sci. 2015, 17, 658-662.

(14) Cody, R. B.; Laramée, J. A.; Durst, H. D. Versatile New Ion Source for the Analysis of Materials in Open Air Under Ambient Conditions. Anal. Chem. 2005, 7\%, $2297-2302$.

(15) Su, J.; Mrksich, M. Using MALDI-TOF Mass Spectrometry to Characterize Interfacial Reactions on Self-Assembled Monolayers. Langmuir 2003, 19, 4867-4870. 
(16) Song, L.; Dykstra, A. B.; Yao, H.; Bartmess, J. E. Ionization Mechanism of Negative Ion-Direct Analysis in Real Time: a Comparative Study with Negative IonAtmospheric Pressure Photoionization. J. Am. Soc. Mass. Spectrom. 2009, 20, 4250.

(17) Gross, J. H. High-Mass Cluster Ions of Ionic Liquids in Positive-Ion and Negative-Ion DART-MS and their Application for Wide-Range Mass Calibrations. Anal. Bioanal. Chem. 2014, 406, 2853-2862.

(18) Gross, J. H. Analysis of Silicones Released from Household Items and Baby Articles by Direct Analysis in Real Time-Mass Spectrometry. J. Am. Soc. Mass Spectrom. 2015, 26, 511-521.

(19) El-Baba, T. J.; Lutomski, C. A.; Wang, B.; Trimpin, S. Characterizing Synthetic Polymers and Additives using New Ionization Methods for Mass Spectrometry. Rapid Commun. Mass Spectrom. 2014, 28, 1175-1184.

(20) Gross, J. H. Direct Analysis in Real Time-a Critical Review on DART-MS. Anal. Bioanal. Chem. 2014, 406, 63-80.

(21) Kpegba, K.; Spadaro, T.; Cody, R. B.; Nesnas, N.; Olson, J. A. Analysis of SelfAssembled Monolayers on Gold Surfaces using Direct Analysis in Real Time Mass Spectrometry. Anal. Chem. 2007, 79, 5479-5483.

(22) Manova, R. K.; Claassen, F. W.; Nielen, M. W. F.; Zuilhof, H.; van Beek, T. A. Ambient Mass Spectrometry of Covalently Bound Organic Monolayers. Chem. Commun. 2013, 49, 922-924.

(23) Manova, R. K.; Joshi, S.; Debrassi, A.; Bhairamagdi, N. S.; Roeven, E.; Gagnon, J.; Tahir, M. N.; Claassen, F. W.; Scheres, L. M. W.; Wennekes, T.; Schröen, K.; van Beek, T. A.; Zuilhof, H.; Nielen, M. W. F. Ambient Surface Analysis of Organic Monolayers using Direct Analysis in Real Time Orbitrap Mass Spectrometry. Anal. Chem. 2014, 86, 2403-2411. 
(24) Zhou, S.; Forbes, M. W.; Abbatt, J. P. D. Application of Direct Analysis in Real Time-Mass Spectrometry (DART-MS) to the Study of Gas-Surface Heterogeneous Reactions: Focus on Ozone and PAHs. Anal. Chem. 2015, 87, 4733-4740.

(25) Escorihuela, J.; Marcelis, A. T. M.; Zuilhof, H. Metal-Free Click Chemistry Reactions on Surfaces. Adv. Mater. Interfaces 2015, 1500135 (DOI: 10.1002/admi.201500135).

(26) Manova, R. K.; van Beek, T. A.; Zuilhof, H. Surface Functionalization by Strain-Promoted Alkyne-Azide Click Reactions. Angew. Chem. Int. Ed. 2011, 50, $5428-5430$.

(27) Agard, N. J.; Prescher, J. A.; Bertozzi, C. R. Strain-Promoted $[3+2]$ AzideAlkyne Cycloaddition for Covalent Modification of Biomolecules in Living Systems. J. Am. Chem. Soc. 2004, 126, 15046-15047.

(28) Codelli, J. A.; Baskin, J. M.; Agard, N. J.; Bertozzi, C. R. Second-Generation Difluorinated Cyclooctynes for Copper-Free Click Chemistry. J. Am. Chem. Soc. 2008, 130, 11486-11493.

(29) Ning, X.; Guo, J.; Wolfert, M. A.; Boons, G.-J. Visualizing Metabolically Labeled Glycoconjugates of Living Cells by Copper-Free and Fast Huisgen Cycloadditions. Angew. Chem. Int. Ed. 2008, 47, 2253-2255.

(30) Sletten, E. M.; Bertozzi, C. R. A Hydrophilic Azacyclooctyne for Cu-Free Click Chemistry. Org. Lett. 2008, 10, 3097-3099.

(31) Jewett, J. C.; Sletten, E. M.; Bertozzi, C. R. Rapid Cu-Free Click Chemistry with Readily Synthesized Biarylazacyclooctynones. J. Am. Chem. Soc. 2010, 132, 3688-3690.

(32) Friscourt, F.; Ledin, P. A.; Mbua, N. E.; Flanagan-Street, H. R.; Wolfert, M. A.; Street, R.; Boons, G.--J. Polar Dibenzocyclooctynes for Selective Labeling of Extracellular Glycoconjugates of Living Cells. J. Am. Chem. Soc. 2012, 134, 53815389. 
(33) Beatty, K. E., Fisk, J. D.; Smart, B. P.; Lu, Y. Y.; Szychowski, J.; Hangauer, M. J.; Baskin, J. M.; Bertozzi, C. R.; Tirrell, D. A. Live-Cell Imaging of Cellular Proteins by a Strain-Promoted Azide-Alkyne Cycloaddition. ChemBioChem 2010, 11, 2092-2095.

(34) Debets, M. F.; van Berkel, S. S.; Schoffelen, S.; Rutjes, F. P. J. T.; van Hest, J. C. M.; van Delft, F. L. Aza-dibenzocyclooctynes for Fast and Efficient Enzyme Pegylation via Copper-Free (3+2) Cycloaddition. Chem. Commun. 2010, 46, 97-99.

(35) Marks, I. S.; Kang, J. S.; Jones, B. T.; Landmark, K. J.; Cleland, A. J.; Taton, T. A. Strain-Promoted "Click" Chemistry for Terminal Labeling of DNA. Bioconjugate Chem. 2011, 22, 1259-1263.

(36) Singh, I.; Freeman, C.; Heaney F. Efficient Synthesis of DNA Conjugates by Strain-Promoted Azide-Cyclooctyne Cycloaddition in the Solid Phase. Eur. J. Org. Chem. 2011, 6739-6746.

(37) Wang, C.-F.; Mäkilä, E. M.; Kaasalainen, M. H.; Liu, D.; Sarparanta, M. P.; Airaksinen, A. J.; Salonen, J. J.; Hirvonen, J. T.; Santos, H. A. Copper-free azidealkyne cycloaddition of targeting peptides to porous silicon nanoparticles for intracellular drug uptake. Biomaterials 2014, 35, 1257-1266.

(38) Laughlin, S. T.; Baskin, J. M.; Amacher, S. L.; Bertozzi C. R. In Vivo Imaging of Membrane-Associated Glycans in Developing Zebrafish. Science 2008, 320, 664667.

(39) Canalle, L. A.; van Berkel, S. S.; de Haan, L. T.; van Hest, J. C. M. CopperFree Clickable Coatings. Adv. Funct. Mater. 2009, 19, 3464-3470.

(40) Orski, S. V.; Poloukhtine, A. A.; Arumugam, S.; Mao, L.; Popik, V. V.; Locklin, J. High Density Orthogonal Surface Immobilization via Photoactivated Copper-Free Click Chemistry. J. Am. Chem. Soc. 2010, 132, 11024-11026. 
(41) Manova, R. K.; Pujari, S. P.; Weijers, C. A. G. M.; Zuilhof, H.; van Beek, T. A. Copper-Free Click Biofunctionalization of Silicon Nitride Surfaces via StrainPromoted Alkyne-Azide Cycloaddition Reactions. Langmuir 2012, 28, 8651-8663

(42) Wendeln, C.; Singh, I.; Rinnen, S.; Schulz, C.; Arlinghaus, H. F.; Burley, G. A.; Ravoo, B. J. Orthogonal, Metal-Free Surface Modification by Strain-Promoted Azide-Alkyne and Nitrile Oxide-Alkene/Alkyne Cycloadditions. Chem. Sci. 2012, 3, $2479-2484$.

(43) Gobbo, P.; Novoa, S.; Biesinger, M. C.; Workentin, M. S. Interfacial StrainPromoted Alkyne-Azide Cycloaddition (I-SPAAC) for the Synthesis of Nanomaterial Hybrids. Chem. Commun. 2013, 49, 3982-3984.

(44) Debets, M. F.; van Berkel, S. S.; Dommerholt, J.; Dirks, A. J.; Rutjes, F. P. J. T.; van Delft, F. L. Bioconjugation with Strained Alkenes and Alkynes. Acc. Chem. Res. 2011, 44, 805-815.

(45) Debets, M. F.; Prins, J. S.; Merkx, D.; van Berkel, S. S.; van Delft, F. L. Synthesis of DIBAC Analogues with Excellent SPAAC Rate Constants. Org. Biomol. Chem. 2014, 12, 5031-5037.

(46) Wijdeven, M. A.; Nicosia, C.; Borrmann, A.; Huskens, J.; van Delft, F. L. Biomolecular Patterning of Glass Surfaces via Strain-Promoted Cycloaddition of Azides and Cyclooctynes. RSC Adv. 2014, 4, 10549-10552.

(47) Liu, D.; Xu, X.; Su, Y.; He, Z.; Xu, J.; Miao, Q. Self-Assembled Monolayers of Phosphonic Acids with Enhanced Surface Energy for high-Performance SolutionProcessed N-Channel Organic Thin-Film Transistors. Angew. Chem. Int. Ed. 2013, 52, 6222-6227.

(48) Pujari, S. P.; Scheres, L.; Marcelis, A. T. M.; Zuilhof, H. Covalent Surface Modification of Oxide Surfaces. Angew. Chem. Int. Ed. 2014, 53, 6322-6356.

(49) Bhairamadgi, N. S.; Pujari, S. P.; Trovela, F. G.; Debrassi, A.; Khamis, A. A.; Alonso, J. M.; Al Zahrani, A. A.; Wennekes, T.; Al-Turaif, H. A.; van Rijn, C.; 
Alhamed, Y. A.; Zuilhof, H. Hydrolytic and Thermal Stability of Organic Monolayers on Various Inorganic Substrates. Langmuir 2014, 30, 5829-5839.

(50) Houseman, B. T.; Mrksich, M. The Microenvironment of Immobilized Arg-GlyAsp Peptides is an Important Determinant of Cell Adhesion. Biomaterials 2001, 22, $943-955$.

(51) Kwon, Y.; Mrksich, M. Dependence of the Rate of an Interfacial Diels-Alder Reaction on the Steric Environment of the Immobilized Dienophile: an example of Enthalpy-Entropy Compensation. J. Am. Chem. Soc. 2002, 124, 806-812.

(52) Collman, J. P.; Devaraj, N. K.; Eberspacher, T. P. A.; Chidsey, C. E. D. Mixed Azide-Terminated Monolayers: a Platform for Modifying Electrode Surfaces. Langmuir 2006, 22, 2457-2464.

(53) Bauer, T.; Schmaltz, T.; Lenz, T.; Halik, M.; Meyer, B.; Clark, T. Phosphonateand Carboxylate-Based Self-Assembled Monolayers for Organic Devices: a Theoretical Study of Surface Binding on Aluminum Oxide with Experimental Support. ACS Appl. Mater. Interfaces 2013, 5, 6073-6080.

(54) Radhakrishnan, C.; Lo, M. K. F.; Warrier, M. V.; Garcia-Garibay, M. A.; Monbouquette, H. G. Photocatalytic Reduction of an Azide-Terminated SelfAssembled Monolayer using CdS Quantum Dots. Langmuir 2006, 22, 5018-5024.

(55) Levine, I.; Weber, S. M.; Feldman, Y.; Bendikov, T.; Cohen, H.; Cahen D.; Vilan A. Molecular Length, Monolayer Density, and Charge Transport: Lessons from Al-AlOx/Alkyl-Phosphonate/Hg Junctions. Langmuir 2012, 28, 404-415.

(56) Kosian, M.; Smulders, M. M. J.; Zuilhof, H. Structure and Long-Term Stability of Alkylphosphonic Acid Monolayers on SS316L Stainless Steel. Langmuir 2016, 32, $1047-1057$.

(57) Szewczyk, J.W.; Zuckerman, R. L.; Bergman, R. G.; Ellman, J. A. A Mass Spectrometric Labeling Strategy for High-Throughput Reaction Evaluation and Optimization: Exploring C-H Activation. Angew. Chem. Int. Ed. 2001, 40, 216-219. 
(58) Cabrera-Pardo, J. R.; Chai, D. I.; Liu, S.; Mrksich, M.; Kozmin, S. A. Label-Assisted Mass Spectrometry for the Acceleration of Reaction Discovery and Optimization. Nat. Chem. 2013, 5, 423-427.

(59) Gouget-Laemmel, A. C.; Yang, J.; Lodhi, M. A.; Siriwardena, A.; Aureau, D.; Boukherroub, R.; Chazalviel, J.-N.; Ozanam, F.; Szunerits, S. Functionalization of Azide-Terminated Silicon Surfaces with Glycans using Click Chemistry: XPS and FTIR Study. J. Phys. Chem. C 2013, 117, 368-375.

(60) Garcia-Hartjes, J.; Dommerholt, J.; Wennekes, T.; van Delft, F. L.; Zuilhof, H. Electronic Effects Versus Distortion Energies during Strain-Promoted Alkyne-Azide Cycloadditions: a Theoretical Tool to Predict Reaction Kinetics. Eur. J. Org. Chem. 2013, 3712-3720. 


\section{Chapter 3:}

\section{Rapid and Complete Surface Modification with}

\section{Strain-Promoted Oxidation-Controlled}

\section{Cyclooctyne-1,2-Quinone Cycloaddition (SPOCQ)}

Strain-promoted oxidation-controlled cyclooctyne-1,2-quinone cycloaddition (SPOCQ) between functionalized bicycle[6.1.0]non-4-yne (BCN) and surface-bound quinones revealed an unprecedented $100 \%$ conjugation efficiency. In addition, detailed analysis by Direct Analysis in Real Time Mass Spectrometry (DART-MS) revealed the underlying kinetics and activation parameters of this immobilization process in dependence on its microenvironment.

This Chapter has been published as:

"Rapid and Complete Surface Modification with Strain-Promoted OxidationControlled Cyclooctyne-1,2-Quinone Cycloaddition (SPOCQ)". Rickdeb Sen, Jorge Escorihuela, Floris L. van Delft and Han Zuilhof. Angew. Chem. Int. Ed. 2017, 56, $3299-3303$. 


\section{Table of Contents}

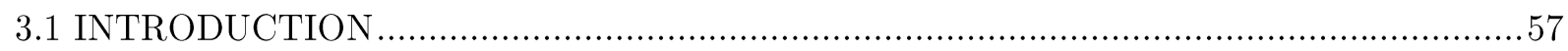

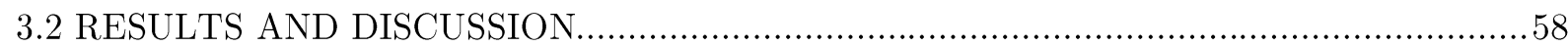

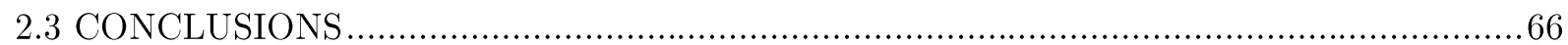

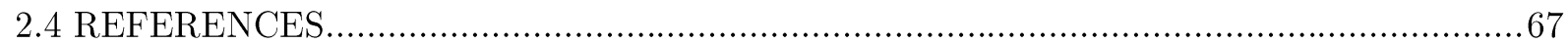




\subsection{INTRODUCTION}

Since the term 'click' chemistry was coined by Sharpless and co-workers in $2001,{ }^{[1]}$ this class of transformations has become of crucial importance in organic synthesis and materials science. ${ }^{[2]}$ In the last decade, this field has focused more on novel click reactions that have an improved kinetic profile without the necessity of toxic metal catalysts like copper. ${ }^{[3]}$ Among the reactions that emerged from this endeavor, the strain-promoted alkyne-azide cycloaddition (SPAAC) proved a particularly versatile alternative owing to its bioorthogonality. ${ }^{[4]}$ Recently, van Delft and coworkers reported the strain-promoted oxidation-controlled cyclooctyne-1,2quinone cycloaddition (SPOCQ) for selective protein conjugation ${ }^{[5]}$ and hydrogel formation $^{[6]}$ with a reaction rate around three orders of magnitude higher than those observed for SPAAC. This combination of high yield and high reaction rates prompted us to study the analogous surface reaction, since SPAAC on a surface typically stalls at $15-80 \%$ efficiency, ${ }^{[7]}$ thereby limiting its practical applicability as unreacted surface sites cannot be removed by purification as in solution-based chemistries. Importantly, solving the issue of variable 'clicking' efficiency on surfaces is severely hampered by a poor understanding of the kinetics of the solution-tosurface conjugation reactions. Apart from a handful of cases in which electrochemical methods have been used, ${ }^{[8]}$ and the recent measurement of SPAAC reactivity ${ }^{[7 c]}$ no rigorously measured kinetics on interfacial reactions involving self-assembled monolayers are available.

In this work, we report the first example of a surface-bound metal-free click reaction with complete conversion of all immobilized groups. We show that SPOCQ is a far superior surface click-type reaction that yields fast and $100 \%$ efficiency for the modification of surfaces. In particular, we studied the application of SPOCQ in the reaction of a bicyclo[6.1.0]non-4-yne (BCN) derivative bearing a MS tag with a monolayer presenting 1,2-quinone groups. 
In addition, we determined the activation parameters $\left(\triangle H^{\ddagger}\right.$ and $\left.\triangle S^{\ddagger}\right)$ by DART-HRMS to clarify, for the first time, the roles of enthalpy and entropy in this surface-bound reaction.

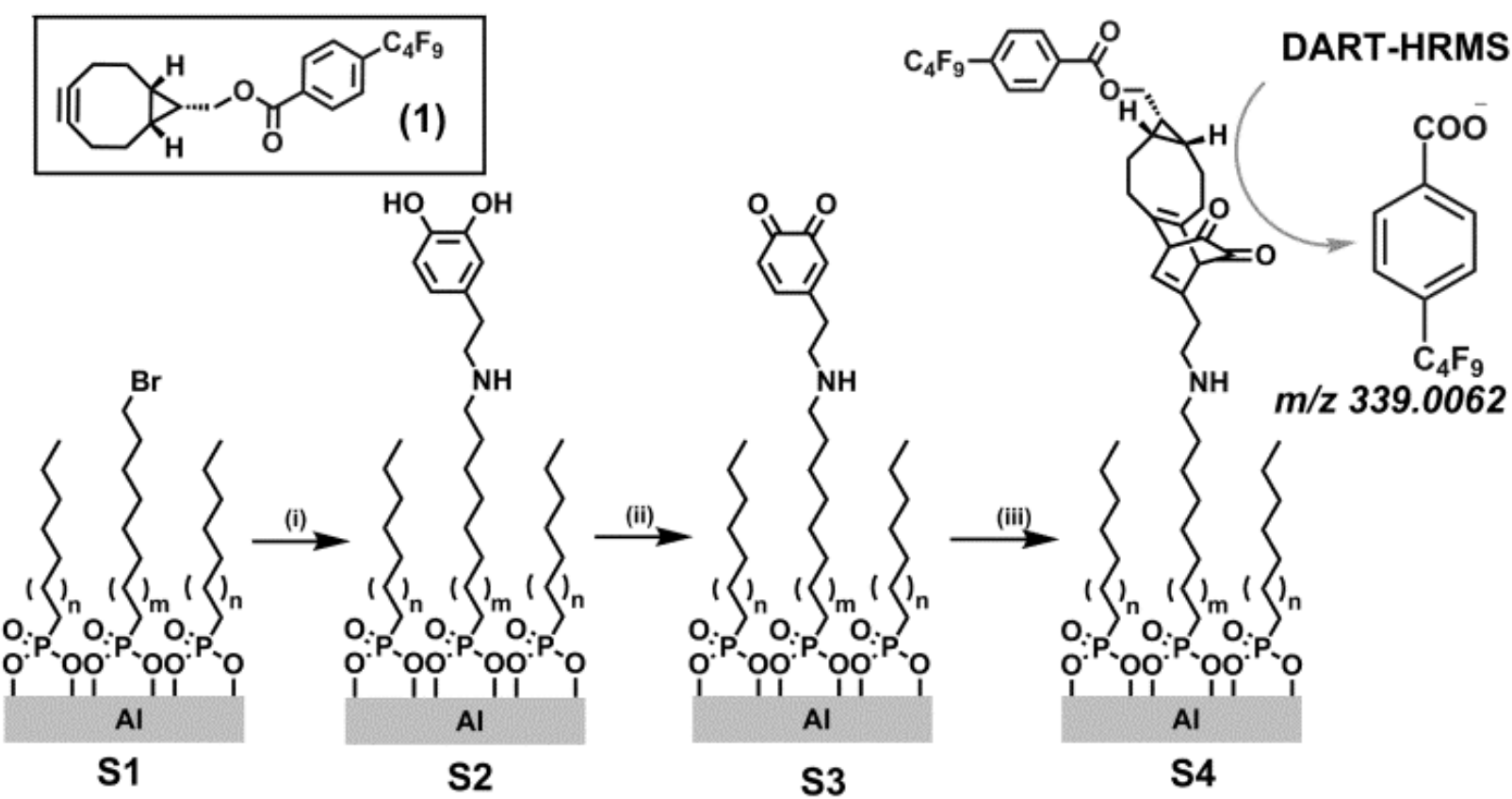

Figure 1. Schematic representation of SPOCQ on quinone-functionalized aluminum substrates. (i) Nucleophilic substitution with dopamine, (ii) Oxidation with $\mathrm{NaIO}_{4}$, and (iii) SPOCQ with BCN derivative 1.

\subsection{RESULTS AND DISCUSSION}

The surface chemistry described in this work is based on the use of phosphonic acids (PA) as anchoring groups for hydroxylated aluminum surfaces (Figure 1). ${ }^{[9]}$ Monolayer-coated aluminum surfaces were obtained from a reaction with an octyl/12-bromododecyl PA mixture (3:1 ratio, in isopropanol), as shown by an increase in the static water contact angle (SCA) from $35^{\circ}$ to $101^{\circ}$, in accordance with data reported for similar PA monolayers. ${ }^{[10]}$ The presence of $\mathrm{Br}$ was confirmed by $\mathrm{XPS}$, showing a $\mathrm{Br} / \mathrm{P}$ ratio of 4.1 (theoretical ratio $=4$ ), also correlating with a mixed 3:1 monolayer. Next, dopamine was immobilized on the surface via nucleophilic substitution of the Br-terminated monolayer with dopamine (50 $\mathrm{mM}$ in 
methanol; $1 \mathrm{~h}$ ). Dopamine attachment was reflected in the decrease of the SCA from $101^{\circ}$ to $91^{\circ}$, and in the complete disappearance and appearance of Br3d and N1s XPS peaks, respectively (Figure S2). Finally, treatment with an aqueous sodium periodate solution for $30 \mathrm{~min}$ led to efficient and quantitative oxidation of catechol to $1,2-$ quinone as corroborated by a decrease in SCA $\left(91^{\circ}\right.$ to $\left.74^{\circ}\right),{ }^{[11]}$ and by GATR-FTIR via the appearance of a peak at $1669 \mathrm{~cm}^{-1}$, assigned to the carbonyl stretch of the 1,2-quinone. Atomic force microscopy imaging showed that the roughness as measured for the unmodified surface (rms $\sim 4.5 \mathrm{~nm}$ ) remained constant upon monolayer formation. No evidence of granules was observed on modified surfaces, supporting the presence of a fully formed monolayer. Table 1. Atomic percentages (\%) of the elements on $\mathrm{Al}$ surface functionalized with $\mathrm{Br}$ (S1), dopamine (S2), 1,2quinone (S3) and BCN derivative $\mathbf{1}(\mathbf{S} 4)$.

\begin{tabular}{cccccccc}
\hline Surface & A12p & O1s & P2p & C1s & Br3p & N1s & F1s \\
\hline S1 & 28.1 & 45.0 & 3.1 & 23.0 & 0.8 & - & - \\
S2 & 29.5 & 42.8 & 4.4 & 22.1 & - & 1.2 & - \\
S3 & 30.8 & 44.0 & 3.0 & 21.2 & - & 1.0 & - \\
S4 $^{[a]}$ & 27.6 & 39.8 & 4.0 & 24.1 & - & 1.0 & 3.5 \\
\hline
\end{tabular}

[a] SPOCQ reaction for $1 \mathrm{~h}$ at $22^{\circ} \mathrm{C}$.

C1s narrow scan XPS spectra provided chemical state information of the different functionalized surfaces (Figure S7). S3 surfaces showed three bands centered at $285.0(\mathrm{C}-\mathrm{C}), 286.9(\mathrm{C}-\mathrm{O}$ and $\mathrm{C}-\mathrm{N})$ and $289.0 \mathrm{eV}(\mathrm{C}=\mathrm{O})$, in accordance with the presence of an immobilized 1,2-quinone group on the surface (Figure 2A). ${ }^{[12 a]}$ After SPOCQ reaction with derivative $\mathbf{1}$, the peak deconvolution also showed components corresponding to the fluorinated tag (Figure $2 \mathrm{~B}$ ), such as a $-\mathrm{CF}_{3}$ peak centered at $294.3 \mathrm{eV}$ and a larger peak from $-\mathrm{CF}_{2}-$ at $291.6 \mathrm{eV} \cdot{ }^{[12 \mathrm{~b}]} \mathrm{M} 11 / 6-311+\mathrm{G}(\mathrm{d}, \mathrm{p})-$ derived simulated C1s XPS spectra agreed well with the experimental spectra (Figure S6). ${ }^{[12 c]}$ 

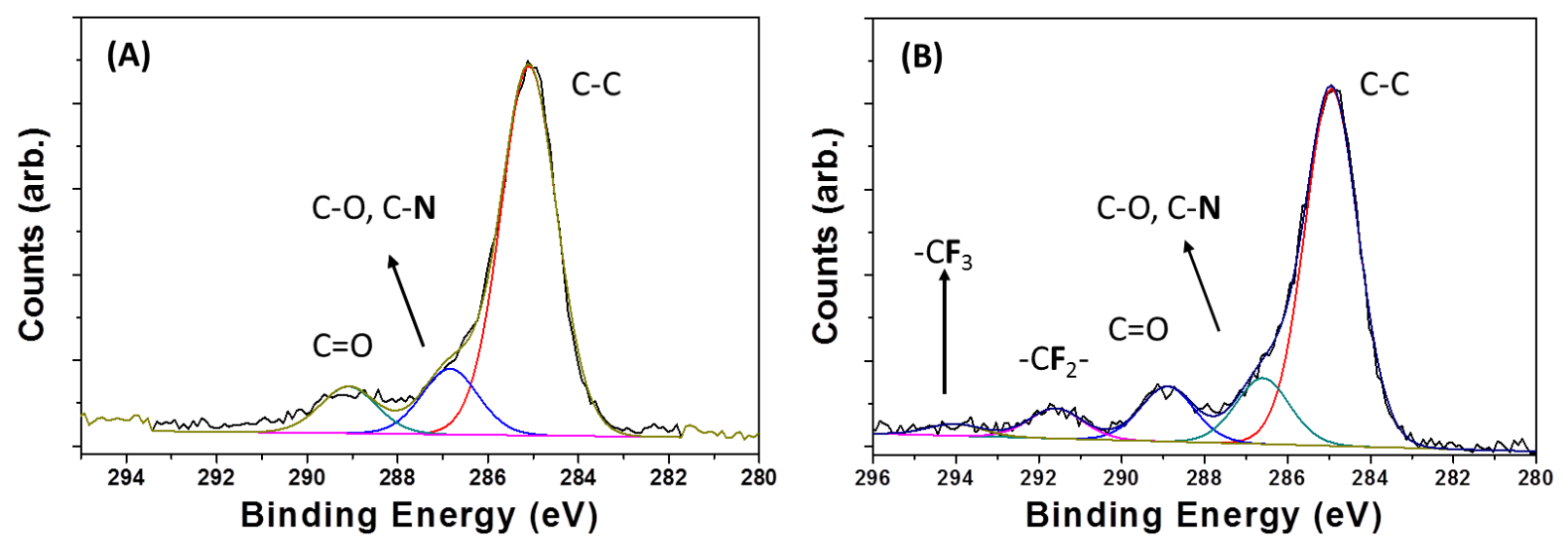

Figure 2. XPS C1s of a 3:1 mixture of octylphosphonic and 1,2-quinone-terminated surface (A) before SPOCQ reaction (S3) and (B) after SPOCQ reaction (S4) with BCN derivative 1 (3 $\mathrm{mM}$ in dichloroethane at $\left.30{ }^{\circ} \mathrm{C}, 8 \mathrm{~h}\right)$.

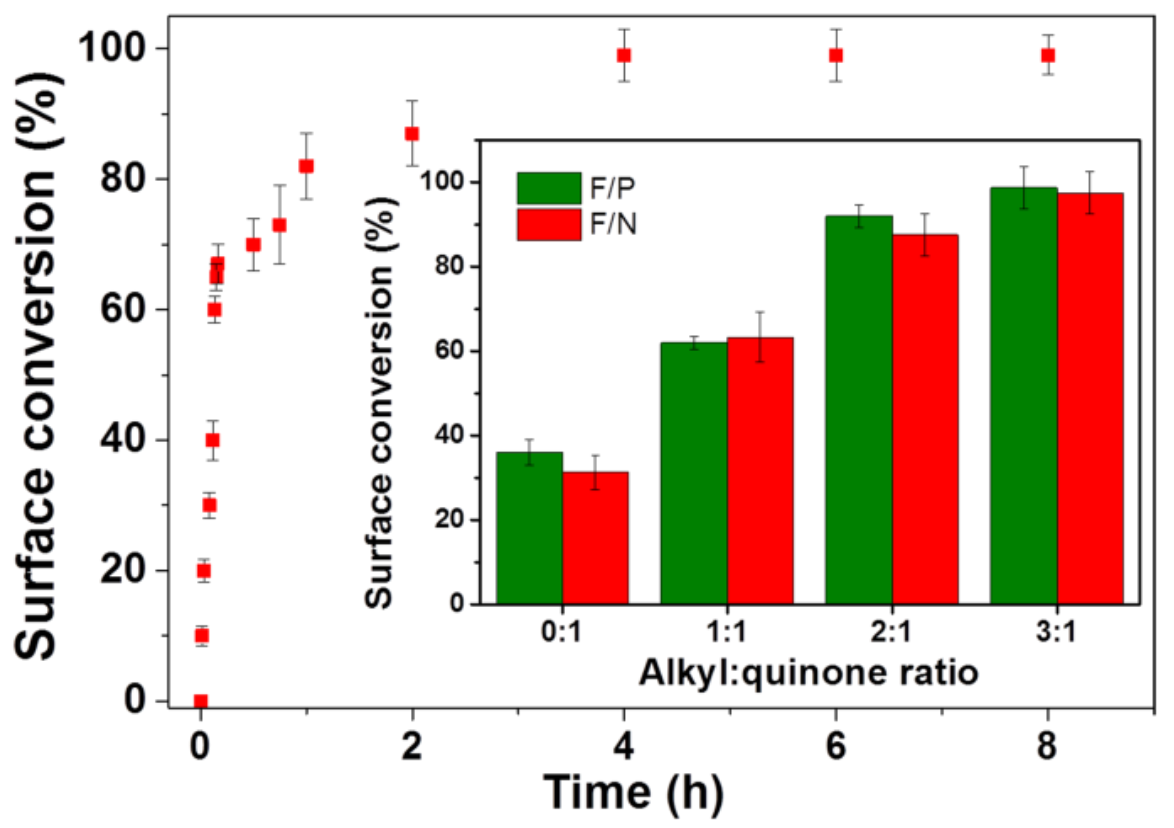

Figure 3. Reaction efficiency (determined by F/N XPS ratio) vs time for the SPOCQ reaction for the 3:1 mixed monolayer. (Insert) Surface conversion (determined by $\mathrm{F} / \mathrm{P}$ (green) or $\mathrm{F} / \mathrm{N}$ (red) XPS ratio) for the SPOCQ reaction of BCN derivative 1 with immobilized 1,2-quinones in different mixed monolayers.

Next, the strain-promoted cycloaddition with BCN derivative 1 (3 mM in 1,2dichloroethane) was investigated. As shown in previous studies, steric factors from the microenvironment surrounding the reacting site play an important role in 
interfacial reactions. ${ }^{[7,13]}$ Therefore, SPOCQ was studied for a series of mixed monolayers with variable alkyl:quinone ratios (from 0:1 to $3: 1$ ). Not surprisingly, the surface coverage of the attached BCN groups was related to surface density of the quinone, with the highest conversion observed for the 3:1 mixed (i.e. most diluted) monolayer (Figure 3, insert). For this monolayer, we found that the yield of incorporation of $\mathrm{BCN}$ was up to $82 \%$ after only $1 \mathrm{~h}$, while after $4 \mathrm{~h} 100 \%$ incorporation of $\mathrm{BCN}$ (according to both the $\mathrm{F} / \mathrm{P}$ and $\mathrm{F} / \mathrm{N}$ ratio by $\mathrm{XPS}$ ) was achieved (Appendix 2, Figure S12 and Table S1). The reaction efficiency was determined by comparing the atomic ratio determined by XPS with the theoretical value of 9:1 (for $\mathrm{F} / \mathrm{N}$ ) or 9:4 (for $\mathrm{F} / \mathrm{P}$ ), which corresponds to the $100 \%$ surface conversion of the 1,2-quinone-functionalized monolayer.

This quantitative conversion stands in contrast to surface functionalization with several other popular metal-free click chemistries (Appendix 2, Table S2), including SPAAC, ${ }^{[7 c]}$ inverse electron-demand Diels-Alder, ${ }^{[14]}$ and thiol-ene ${ }^{[15]}$ and thiol-yne couplings, ${ }^{[3,16]}$ where surface analyses invariably indicate incomplete conversion (typically 15-90\%) of the reactive groups.

The SPOCQ reaction was also studied (under exactly the same conditions as used for hydroxylated aluminium surfaces) using other surfaces (silicon and stainless steel), as well as other linkage chemistries to attach dopamine to the surface (see Appendix 2). XPS measurements ( $\mathrm{F} / \mathrm{N}$ ratio) also showed a full conversion of quinone groups in all cases, although Si (111) and stainless steel surfaces took slightly longer $(6-8 \mathrm{~h})$.

The specificity of the SPOCQ reaction was demonstrated by generation of a pattern of $1,2-$ quinone moieties via oxidation by microcontact printing of a sodium periodate solution over a catechol-terminated surface. After reaction with a dyelabelled BCN solution (DY649p1), fluorescence was exclusively visualized on the 
oxidized regions, confirming the absence of SPOCQ in areas where no oxidation was induced (Figure S19).

Intrigued by the efficiency of SPOCQ implemented on a surface, we performed an in-depth investigation of the kinetics of this cycloaddition by DART-HRMS, using the intensity of the MS tag as a measure for conversion. Among the different techniques that can be used to study the kinetics of interfacial reactions, ${ }^{[17]}$ Direct Analysis in Real Time (DART) has been shown to be particularly useful due to its ability to detect small molecules $(m / z<400)$ without the use of a matrix. Furthermore, DART shows excellent compatibility with robust covalent surface chemistries, ${ }^{[18]}$ unlike self-assembled monolayers for matrix-assisted laser desorption ionization mass spectrometry (SAMDI) and desorption ionization electrospray (DESI). Recently, we have shown that DART-HRMS accurately and rapidly offers quantitative rate information for interfacial reactions. ${ }^{[7]}$

The intensity of the MS tag is directly proportional to the extent of reaction, as this moiety can only be on the surface as a result of a successful reaction between surface-bound 1,2-quinone and BCN. To ensure pseudo-first order kinetics, a large excess of BCN reagent was used. As shown in Figure 4, the reaction proceeds extremely fast and the conversion reaches an asymptotic limit after only 5 min ( $\sim 70 \%)$. After that time, the remaining $30 \%$ of unreacted surface sites is converted using more complex kinetics, over a $4 \mathrm{~h}$ period.

In order to determine pseudo-first-order rate constants, the rate of reaction was modeled as the change in MS intensity relative to the asymptotically obtained maximum value. For that, the pseudo-first order rate equation (1) was used,

$$
\ln \left(\frac{\mathrm{I}_{\infty}-\mathrm{I}_{\mathrm{t}}}{\mathrm{I}_{\infty}-\mathrm{I}_{0}}\right)=-\mathrm{k}^{\prime} \mathrm{t}
$$

where, $\mathrm{k}^{\prime}$ is the pseudo-first order rate constant, and $I_{0}, I_{t}$, and $I_{\infty}$ are the MS intensities initially, at time interval t, and at the end of reaction (average of 5-15 min). Based on this model, the rate constant for SPOCQ was found to be $(10.1 \pm$ 
$0.3) \times 10^{-3} \mathrm{~s}^{-1}$ (Figure 4B, inset), two times faster than the interfacial SPAAC of BCN with azides and about 15 times faster than other reported surface-SPAAC reactions. ${ }^{[19]}$ This finding differs dramatically from the 1000-fold larger magnitude difference observed for SPOCQ and SPAAC in protic solutions (methanol/water $1: 1) \cdot[5]$

It was reasoned that the solvent plays a crucial role in the observed relatively small difference in surface-bound SPOCQ and SPAAC reaction rates (in 1,2dichloroethane) compared to those reported in solution (protic solvents). Therefore, we measured pseudo-first order reaction rates (in 1,2-dichloroethane at $25{ }^{\circ} \mathrm{C}$ ) for both SPAAC and SPOCQ of BCN with benzyl azide and 4-tert-butyl-1,2-quinone, respectively. A significant solvent effect seems to influence the reaction rate for the SPOCQ reaction, as the rate constant in 1,2-dichloroethane was found to be only $10 \times$ higher $\left(0.098 \pm 0.002 \mathrm{~s}^{-1}\right)$ than that for $\operatorname{SPAAC}\left(0.009 \pm 0.001 \mathrm{~s}^{-1}\right)$. A direct competition experiment between SPOCQ and SPAAC using ${ }^{1} \mathrm{H}$ NMR quantification in $\mathrm{CDCl}_{3}$ yielded a similar rate difference (Figures S25-27), close to the rate difference observed for the interfacial reaction.

Next, we studied the interfacial SPOCQ reaction at $22{ }^{\circ} \mathrm{C}$ in two additional microenvironments, where the 1,2-quinone was either partially accessible (M2), or 

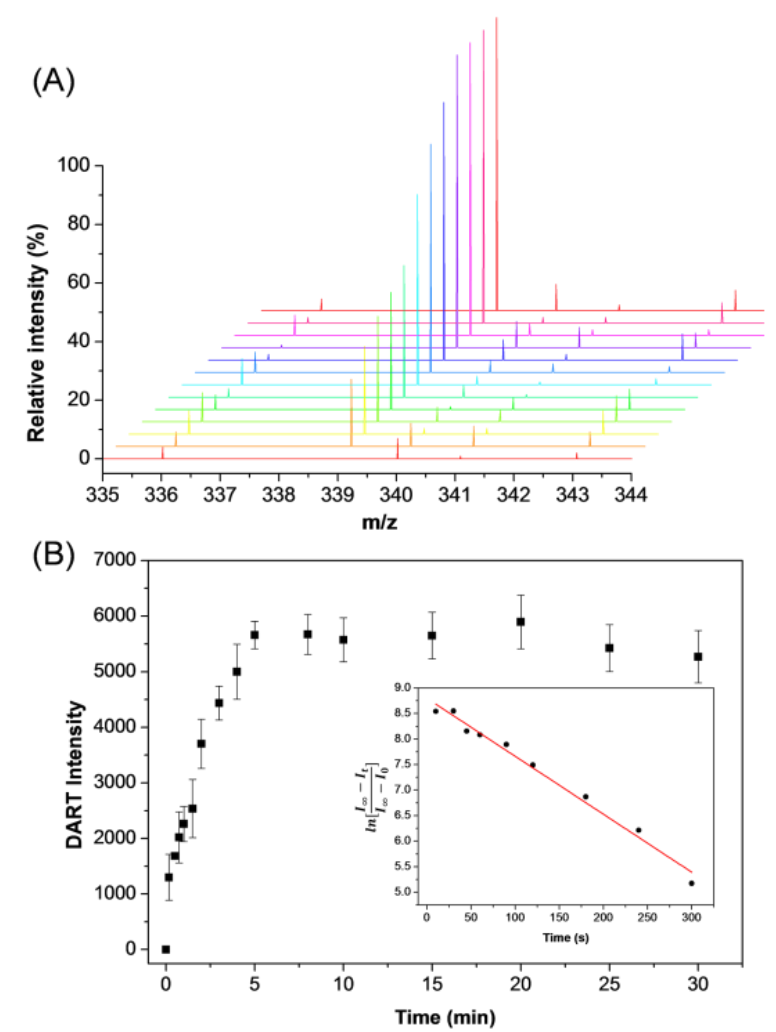

Figure 4. (A) Relative intensity for the for MS tag at different reaction times (from 0 to $15 \mathrm{~min}$ ). (B) Normalized intensity for MS tag for the SPOCQ reaction. (Insert) Plot of $\ln \left[\left(\mathrm{I}_{\infty}-\mathrm{I}_{\mathrm{t}}\right) /\left(\mathrm{I}_{\infty}-\mathrm{I}_{0}\right)\right]$ vs time, used to obtain the rate constant.

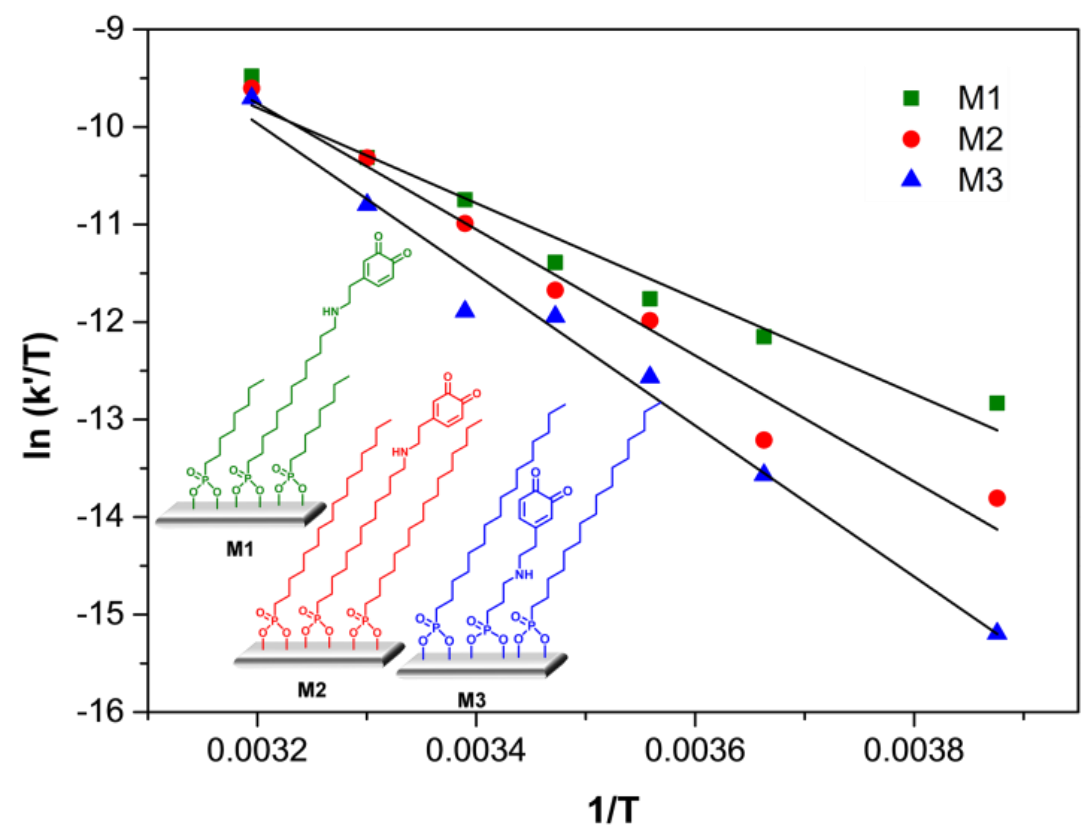

Figure 5. Plot of $\ln (\mathrm{k} / \mathrm{T})$ versus $1 / \mathrm{T}$ for the SPOCQ with monolayers presenting 1,2-quinone groups in either a freely accessible environment (M1), partially accessible (M2) or a buried environment (M3). 
completely buried within the monolayer (M3). For that, the mixed monolayers (in a 3:1 ratio, containing hexadecyl and 12-bromododecyl PA or hexadecyl and 3bromopropyl PA) were prepared and the quinone was introduced as described previously. Although the use of mixed monolayers is unlikely to be homogeneous if very short and long adsorbates are combined (e.g. as in M3), ${ }^{[20]}$ no evidence of the formation of micro domains was observed in the studied surfaces. Under the optimized conditions, the pseudo-first order reaction rate constants obtained by DART for the partially accessible (M2) and the buried 1,2-quinone (M3) monolayers were $(4.98 \pm 0.32) \times 10^{-3} \mathrm{~s}^{-1}$ and $(2.92 \pm 0.15) \times 10^{-3} \mathrm{~s}^{-1}$, respectively. In other words, the significant variation in steric hindrance (between e.g. M1 and M3) only yields a factor three difference in reaction rate.

Since these rates are quickly measurable using DART-HRMS, the effect of temperature on the reaction rate was studied at different temperatures (from -15 to $40{ }^{\circ} \mathrm{C}$; Table S2, Figures S28-30), to extract the thermodynamic parameters of activation by Eyring plot analysis. Values for $\Delta H^{\ddagger}$ and $\Delta S^{\ddagger}$ were calculated from the slope and the intercept, respectively, of the least-squares fit of plots of $\ln \left(\mathrm{k}^{\prime} / \mathrm{T}\right)$ versus $1 / \mathrm{T}$. As shown in Table 2, the reaction of the $\mathrm{BCN}$-derivative with the freely accessible 1,2-quinone (M1) had an enthalpy of activation of $9.6 \mathrm{kcal} / \mathrm{mol}$. With the partially accessible $1,2-$ quinone $(\mathbf{M} 2) \Delta H^{\ddagger}=12.8 \mathrm{kcal} / \mathrm{mol}$, while $\Delta H^{\ddagger}$ of the buried 1,2-quinone (M3) was $15.4 \mathrm{kcal} / \mathrm{mol}$, which may be readily explained by the increased steric hindrance imposed by the surrounding alkane chains. In contrast, the loss of disorder $\left(\Delta S^{\ddagger}\right)$ is smallest for the buried systems, suggesting some preorganization prior to the rate-determining step. These trends in $\Delta H^{\ddagger}$ and $\Delta S^{\ddagger}$ are clearly compensating one another, to give rather similar free energies of activation $\left(\triangle G^{\ddagger}\right)$, explaining the modest three-fold difference in reaction rate around room temperature. 
The observed activation parameters also shed light on the two-fold difference between the interfacial SPOCQ and SPAAC reactions. The activation enthalpy of the SPOCQ reaction is, for the sterically most accessible mixed monolayer (M1), about $6 \mathrm{kcal} / \mathrm{mol}$ higher than for the SPAAC reaction. ${ }^{[7 c]}$ However, since SPOCQ is still faster than SPAAC, the reaction rate is determined by the entropy of activation, and indeed the loss of disorder is much less for the transition state of SPOCQ than of SPAAC. More extensive studies of the activation parameters for a range of surfacebound organic reactions are underway to shed light onto the differences between different reactions, and to explain in more detail the differences between solutionphase and surface-bound reactions.

Table 2. Thermodynamic parameters of activation (determined by DARTHRMS) for the interfacial SPOCQ reaction with monolayers presenting 1,2-quinone groups in different microenvironment (M1, M2 and M3).

\begin{tabular}{ccc}
\hline Microenvironment & $\Delta \boldsymbol{H}^{\ddagger}\left(\mathbf{k c a l} \cdot \mathbf{~ m o l}^{\mathbf{- 1}}\right)$ & $\Delta \boldsymbol{S}^{\ddagger}\left(\mathbf{c a l} \cdot \mathbf{K}^{-\mathbf{1}} \cdot \mathbf{~ m o l}^{-\mathbf{1}}\right)$ \\
\hline M1 & $9.7 \pm 0.8$ & $-35.9 \pm 2.9$ \\
M2 & $12.8 \pm 0.7$ & $-25.5 \pm 2.4$ \\
M3 & $15.4 \pm 0.8$ & $-18.8 \pm 3.0$ \\
\hline
\end{tabular}

\subsection{CONCLUSIONS}

In conclusion, we have demonstrated the superior behavior of SPOCQ for the functionalization of surfaces, as shown by an unprecedented complete reaction of all immobilized quinones on the surface. The reaction rate of interfacial SPOCQ was successfully measured using DART-HRMS and it was established that interfacial cycloaddition proceeds with high reaction rates. We additionally determined the activation parameters $\left(\Delta H^{\ddagger}\right.$ and $\left.\Delta S^{\ddagger}\right)$, which help to understand the mechanistic basis 
for the variation in rate constants observed within the series of monolayers. We expect this facile and quantitative surface attachment using SPOCQ chemistry to be of particular value for the immobilization of small and large (bioactive) molecules on a wide variety of surfaces, which may find useful application in material sciences, life sciences and health care (next-generation diagnostics).

\subsection{REFERENCES}

(1) H. C. Kolb, M. G. Finn, K. B. Sharpless, Angew. Chem. Int. Ed. 2001, 40, 2004-2021; Angew. Chem. 2001, 113, 2056-2075.

(2) a) M. King, A. Wagner, Bioconjugate Chem. 2014, 25, 825-839; b) W. Xi, T. F. Scott, C. J. Kloxin, C. N. Bowman, Adv. Funct. Mater. 2014, 24, 2572-2590; c) G. Delaittre, A. S. Goldmann, J. O. Mueller, C. Barner-Kowollik, Angew. Chem. Int. Ed. 2015, 54, 11388-11403; Angew. Chem. 2015, 127, 11548-11564.

(3) Escorihuela, A. T. M. Marcelis, H. Zuilhof, Adv. Mater. Interfaces 2015, 2, 1500135 (DOI: 10.1002/admi.201500135).

(4) a) N. J. Agard, J. A. Prescher, C. R. Bertozzi, J. Am. Chem. Soc. 2004, 126, 15046-15047; b) S. T. Laughlin, J. M. Baskin, S. L. Amacher, C. R. Bertozzi, Science 2008, 320, 664-667.

(5) A. Borrmann, O. Fatunsin, J. Dommerholt, A. M. Jonker, D. W. P. M. Löwik, J. C. M. van Hest, F. L. van Delft, Bioconjugate Chem. 2015, 26, 257-261.

(6) A. M. Jonker, A. Borrmann, E. R. H. van Eck, F. L. van Delft, D. W. P. M. Löwik, J. C. M. van Hest, Adv. Mater. 2015, 27, 1235-1240.

(7) a) A. Kuzmin, A. Poloukhtine, M. A. Wolfert, V. V. Popik, Bioconjugate Chem. 2010, 21, 2076-2085; b) J. Guo, G. Chen, X. Ning, M. A. Wolfert, X. Li, B. Xu, G.-J. Boons, Chem. Eur. J. 2010, 16, 13360-13366; c) R. Sen, J. Escorihuela, M. M. J. Smulders, H. Zuilhof, Langmuir 2016, 32, 3412-3419. 
(8) a) Y. Kwon, M. Mrksich, J. Am. Chem. Soc. 2001, 124, 806-812; b) E. S. Gawalt, M. Mrksich, J. Am. Chem. Soc. 2004, 126, 15613-15617.

(9) S. P. Pujari, L. Scheres, A. T. M. Marcelis, H. Zuilhof, Angew. Chem. Int. Ed. 2014, 53, 6322-6356; Angew. Chem. 2014, 126, 6438-6474.

(10) a) T. Bauer, T. Schmaltz, T. Lenz, M. Halik, B. Meyer, T. Clark, ACS Appl. Mater. Interfaces 2013, 5, 6073-6080; b) T. V. A. G. de Oliveira, A. Eleta, L. E. Hueso, A. M. Bittner, J. Mat. Chem. C 2015, 3, 1181-1186.

(11) Y.-C. Lin, Y.-N. Hsu, Y.-C. Chung, RSC Adv. 2014, 4, 22931-22937.

(12) a) R. A. Zangmeister, T. A. Morris, M. J. Tarlov, Langmuir 2013, 29, 86198628: b) M. J. Hawker, A. Pegalajar-Jurado, E. R. Fisher, Langmuir 2014, 30, 12328-12336; c) M. Giesbers, A. T. M. Marcelis, H. Zuilhof, Langmuir 2013, 29, $4782-4788$.

(13) A. G. Larsen, K. V. Gothelf, Langmuir 2005, 21, 1015-1021.

(14) L. I. Willems, M. Verdoes, B. I. Florea, G. A. van der Marel, H. S. Overkleeft, ChemBioChem 2010, 11, 1769-1781.

(15) a) M. A. Caipa Campos, J. M. J. Paulusse, H. Zuilhof, Chem. Commun. 2010, 46, 5512-5514; b) J. Escorihuela, M. J. Bañuls, R. Puchades, A. Maquieira, Chem. Commun. 2012, 48, 2116-2118.

(16) J. Escorihuela, M. J. Bañuls, R. Puchades, A. Maquieira, J. Mater. Chem. B 2014, 2, 8510-8517.

(17) a) J. M. Williams, B. Rowland, M. T. Jeffery, G. S. Groenewold, A. D. Appelhans, G. L. Gresham, J. E. Olson, Langmuir 2005, 21, 2386-2390; b) M. Mrkisch, ACS Nano, 2008, 2, 7-18; c) A. A. Gurard-Levin, M. D. Scholle, A. H. Eisenberg, M. Mrkisch, ACS Comb. Sci. 2011, 13, 347-350; d) S. V. Orski, G. R. Sheppard, S. Arumugam, R. M. Arnold, V. V. Popik, J. Locklin, Langmuir 2012, 28, 14693-14702; e) C. Han, Y. Liu, J. Ma, H. He, Proc. Natl. Acad. Sci. 2012, 109, 21250-21255; f) L.-A. Näslund, Surf. Sci. 2013, 618, 42-48; g) W. Xie, R. Grzeschik, 
S. Schlgcker, Angew. Chem. Int. Ed. 2016, 55, 13729-13733; Angew. Chem. 2016, 128, 13933-13937.

(18) R. K. Manova, S. Joshi, A. Debrassi, N. S. Bhairamagdi, E. Roeven, J. Gagnon, M. N.; Tahir, F. W.; Claassen, L. M. W. Scheres, T.; Wennekes, K. Schröen, T. A. van Beek, H. Zuilhof, M. W. F. Nielen, Anal. Chem. 2014, 86, 2403-2411.

(19) S. V. Orski, G. R. Sheppard, S. Arumugam, R. M. Arnold, V. V. Popik, J. Locklin, Langmuir 2012, 28, 14693-14702.

(20) a) C. D. Bain, G. M. Whitesides J. Am. Chem. Soc. 1989, 111, 7164-7175; b) J. P. Collman, N. K. Devaraj, T. P. A. Eberspacher, C. E. D. Chidsey, Langmuir 2006, 22, 2457-2464. 


\section{Chapter 4:}

\section{Approach Matters: The Kinetics of Interfacial}

\section{Inverse-Electron Demand Diels-Alder Reactions}

Rapid and quantitative click functionalization of surfaces remains an interesting challenge in surface chemistry. In this regard, inverse electron demand Diels Alder (IEDDA) reactions represent a promising metal-free candidate. Herein, we reveal quantitative surface functionalization within $15 \mathrm{~min}$. Furthermore, we report the comprehensive effects of substrate stereochemistry, surrounding microenvironment and substrate order on the reaction kinetics as obtained via surface-bound mass spectrometry (DART-HRMS).

This Chapter has been published as:

"Approach Matters: The Kinetics of Interfacial Inverse-Electron Demand DielsAlder Reactions". Rickdeb Sen, Digvijay Gahtory, Jorge Escorihuela, Judith Firet, Sidharam P. Pujari and Han Zuilhof.

Chem. Eur.J. 2017, doi:10.1002/chem.201703103. 


\section{Table of Contents}

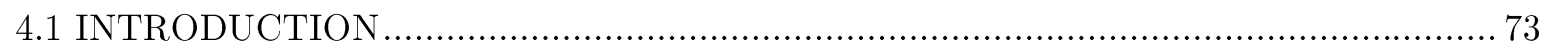

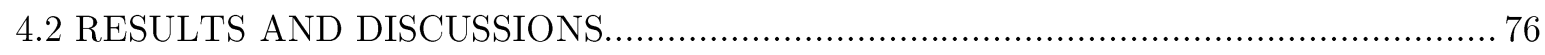

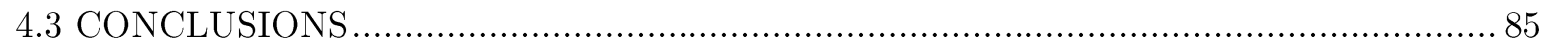

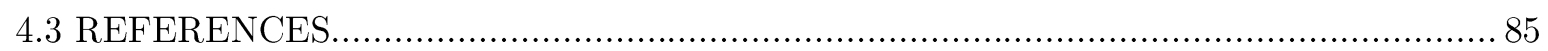




\subsection{INTRODUCTION}

The excellent kinetics, high yields, lack of by-products and high stereoselectivity of click reactions have led to their extensive use in total synthesis, ${ }^{[1]}$ and biorthogonal ${ }^{[2]}$ and site-specific ${ }^{[3,4]}$ labelling of biomolecules. The utility of these reactions for surface modification has blossomed in recent times as evident from a significant number of reports, ${ }^{[5]}$ specifically their use in biomolecular attachment and patterning. ${ }^{[6]}$ Crucial for surface modification are the rate of reactions (for effective implementation) and complete conversion of the surface-bound moiety to the product of interest. The latter aspect is of importance, as surface-bound moieties cannot be removed afterwards by the standard purifications techniques so central to solutionphase chemistry (column chromatography, HPLC, etc.).

In these regards, the inverse electron demand Diels-Alder reaction (IEDDA) between 1,2,4,5-tetrazine and strained alkenes/alkynes holds great promise. ${ }^{[7]}$ IEDDA reactions have been extensively studied in solution with particular focus on tuning the steric and electronic effects of a wide variety of dienes and dienophiles. ${ }^{[8]}$ Such studies indicated highly interesting features such as very fast reaction kinetics ${ }^{[8,9]}$ (among the highest for metal-free click reactions) and high chemoselectivity. Surprisingly, this facile reaction has been largely underexplored for surface functionalization with a few examples in literature using the highly reactive, but somewhat unstable trans-cyclooctene (TCO) reacting with tetrazine, ${ }^{[2,10]}$ or a more stable but less reactive reactant such as norbornene. ${ }^{[11]}$ In studies of click reactions at surface, both metal-catalyzed and metal-free a recurring but hitherto largely unresolved issue is the question whether it matters which component is better to be surface-bound or better to be in solution, i.e. in this case norbornene (surf.) $_{+}+$ tetrazine $_{(\text {soln.) }}$ versus tetrazine $_{(\text {surf.) }}+$ norbornene $_{(\text {soln.). }}$. For example, for both interfacial strain-promoted azide-alkyne cycloadditions ${ }^{[12]}$ and surface-bound thiol-ene click 
reactions $^{[13]}$ the choice of the surface-bound reaction partner, i.e. overall reaction orientation, is important, but published results are inconclusive in this regard.

We have recently shown that the microenvironment around the reactive site on the surface plays an important role in the kinetics of strain-promoted click reactions, as determined by highly accurate and facile rate studies using direct analysis in real time-high resolution mass spectrometry (DART-HRMS). ${ }^{[1]}$ Those studies allowed and stimulated us to investigate whether the microenvironment for the interfacial IEDDA using the more stable norbornene could be optimized to further improve the reaction rates, and possibly direct the yield of surface-bound IEDDA reactions towards $100 \%$.

Scheme 1. a) Overall tetrazine-norbornene IEDDA reaction and b) schematic depicting the three parameters (in parentheses) under current study.

a)

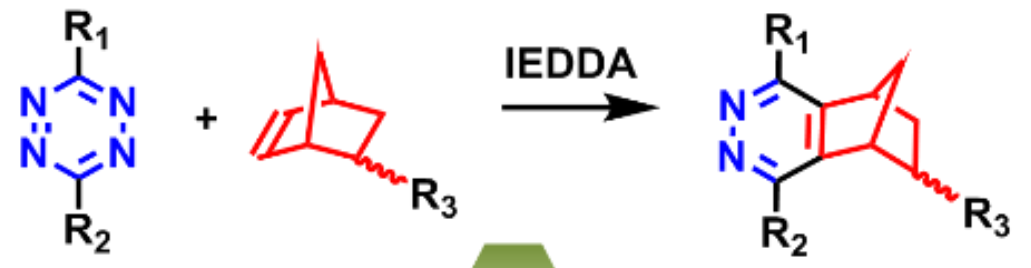

Tag
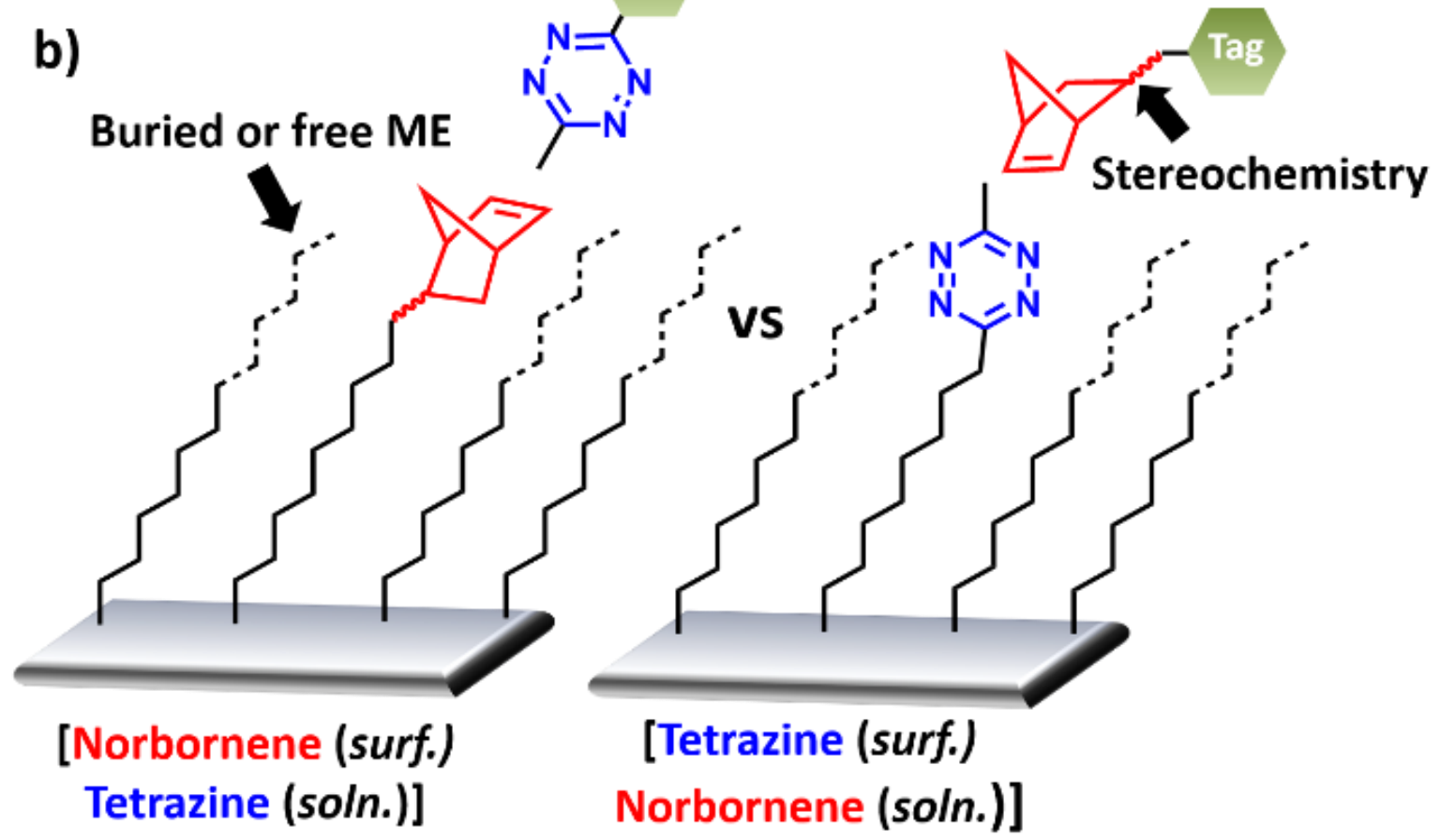
Given the high signal/noise $(\mathrm{S} / \mathrm{N})$ ratio of DART-HRMS, for example: about two orders of magnitude better than XPS, we are in this study for the first time able to systematically tune three aspects relevant for surface-bound organic conversions in detail (Scheme 1): surface-induced sterics (are the groups buried in a monolayer, or sticking out above it), stereochemistry (endo/exo-norbornene, as a means to investigate the effects of approach of the reactant in solution) and orientation (which reactant is in the solvent and which one surface-bound). ${ }^{[15]}$ Optimization of these factors yielded conditions in which the reaction is quantitative and complete within $15 \mathrm{~min}$, showing the potential of both this systematic approach and of this reaction.

Scheme 2. General scheme for the surface modification followed by interfacial IEDDA reaction.

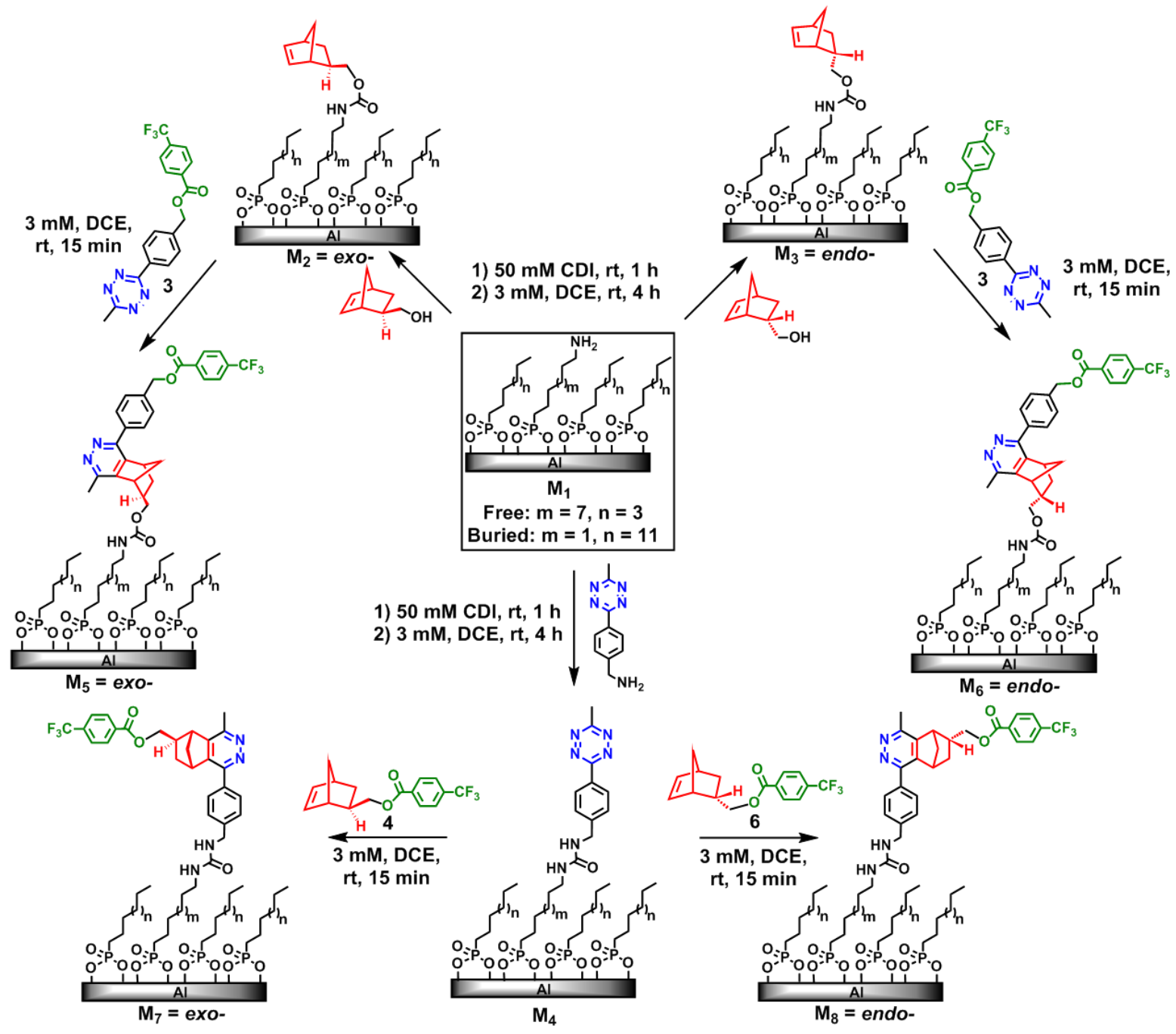




\subsection{RESULTS AND DISCUSSION}

To this end, first the surface of aluminum (Al) slides with its natural aluminum oxide coating were covalently modified by phosphonic acid-based monolayers via a well-established methodology. ${ }^{[5 a]}$ For this study, we prepared 3:1 alkyl to amine-terminated monolayers (M1; see Scheme 2), as previous studies indicated this to be the optimum between high density of functionalization and reactivity. In our experience, increasing the dilution of the reactive sites in the monolayers higher than $33 \%$ leads to an overall decreased reaction kinetics and reaction yields. ${ }^{[14]}$ The microenvironment of the reacting groups was tuned by modifying the lengths of the surrounding alkyl chains, so as to bury it in the monolayer (4 carbon atoms below the surface), or make it stick out (4 carbon atoms above the surface). The monolayer composition was confirmed by the $\mathrm{N} / \mathrm{P}$ ratio $(1: 4)$ in XPS wide scans (Figure S5.1, Appendix 3).

In order to obtain a better understanding of surface coverage, a molecular mechanics study was performed that studied the average packing energy per chain in dependence of the packing density. ${ }^{[16]}$ This was performed by creating schematic models of varying degrees of alkyl monolayer attachment with different random attachment at the available sites followed by optimization of the corresponding molecular models. For more detailed understanding of the randomization process, see section 7.1-7.9, Appendix 3. We found that roughly 50\% coverage corresponded to the lowest packing energy per molecule (Appendix 3, section 7.10 and 7.11). This means that ideally about half the surface sites available result in monolayer attachment.

Norbornene surfaces $\left(\mathbf{M}_{2} / \mathbf{M}_{3}\right)$ were prepared by coupling 5-norbornene-2methanol (exo-/endo-) to $\mathbf{M}_{\mathbf{1}}$ surfaces by a carbamate linkage (Scheme 2 and Figure S4.4, S5.2 and S5.3). For preparation of tetrazine surfaces $\left(\mathbf{M}_{4}\right)$, we chose unsymmetrical tetrazine with benzyl amine and methyl substitution, again via a 
carbamate linkage to ensure the same freedom/buried nature as in $\mathbf{M}_{2} / \mathbf{M}_{3}$. As shown by the groups of Hilderbrand and Chen, unsymmetrical tetrazines provide a better balance between stability and reactivity than their symmetric counterparts, ${ }^{[8 b, 17]}$ while also ensuring enough rigidity on a surface. Complete surface attachment for $\mathbf{M}_{4}$ surfaces was obtained for this reaction as confirmed by $\mathrm{N} / \mathrm{P}$ ratios $(1.5 \pm 0.1)$ in XPS analysis (Figure S5.4, Appendix 3). After confirmation of surface attachment of norbornenes and tetrazines, respectively, we embarked on exploring the IEDDA reaction.

For easy analysis of the reaction progress, we synthesized compounds with fluorinated tags 3, 4 and $\mathbf{6}$ that facilitate analysis by both XPS and DART-HRMS. The reactions were performed for both the reaction partners under stringently similar conditions (Scheme 2) and followed by monitoring the $\mathrm{F} / \mathrm{P}$ ratio in XPS wide spectra (Figure 1).

a)

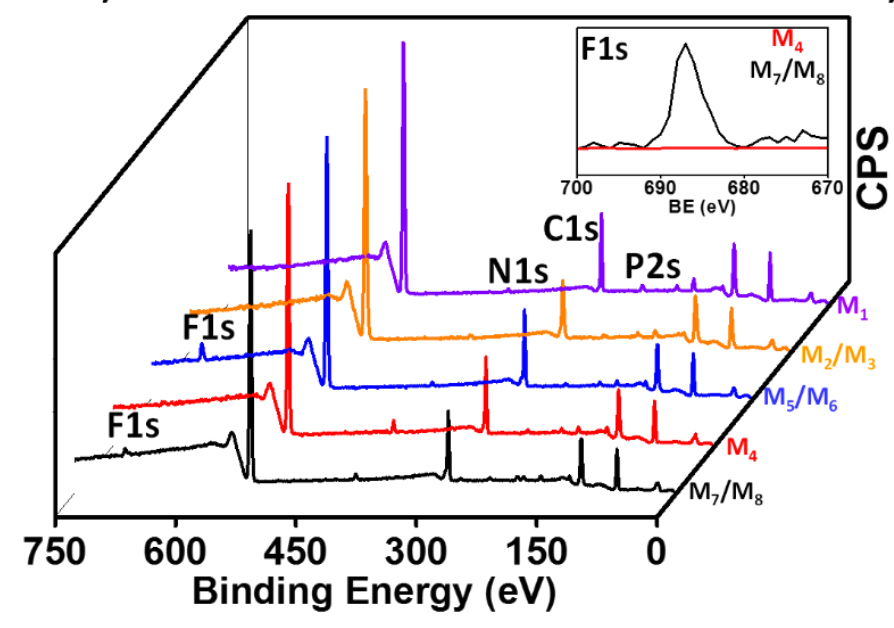

b)

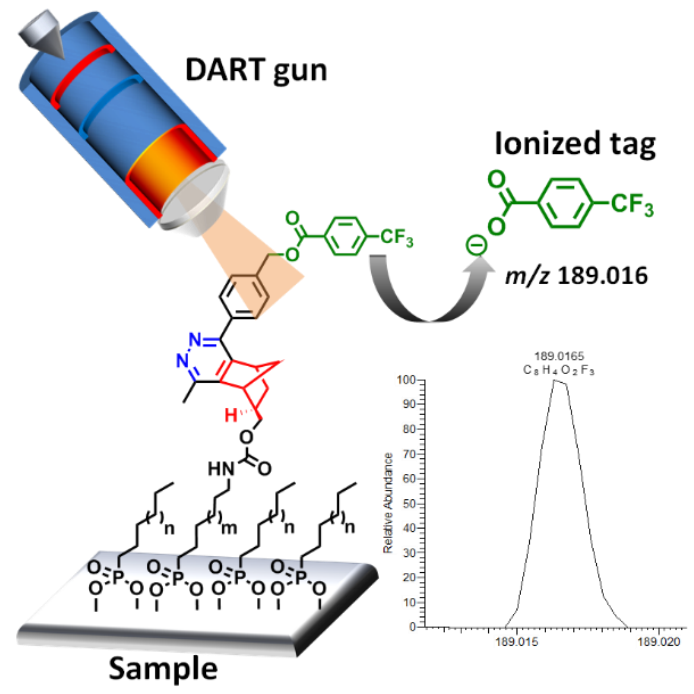

Figure 1. a) XPS wide range spectra for $M_{1}-M_{8}$ surfaces showing emergence of $F 1 s$ signal after the interfacial IEDDA reaction. b) Schematic impression of ionization of MS-tag (m/z 189.0163) by DART-HRMS.

Interestingly, we found that for the "free" $\mathbf{M}_{2}, \mathbf{M}_{3}$ and $\mathbf{M}_{4}$ systems, a quantitative surface reaction yield was obtained within $\mathbf{1 5}$ min irrespective of the 
orientation of the reaction (Figure S5.5 and S5.6, Appendix 3). In case of the "buried" system, the crowded microenvironments around the reactive sites slowed the reaction down, yielding a slightly lower reaction yield $(\sim 80 \%)$ after $15 \mathrm{~min}$ for "buried" exo-norbornene ${ }_{(\text {surf.) }}+$ tetrazine $_{(\text {soln.) }}$ system (Figure S5.10, Appendix 3), but $>90 \%$ conversion was also observed for such crowded microenvironments after $1 \mathrm{~h}$ (Figure S5.11, Appendix 3). See Appendix 3, for a more detailed description of the different calculations used for reaction quantification using XPS wide and narrow spectra.

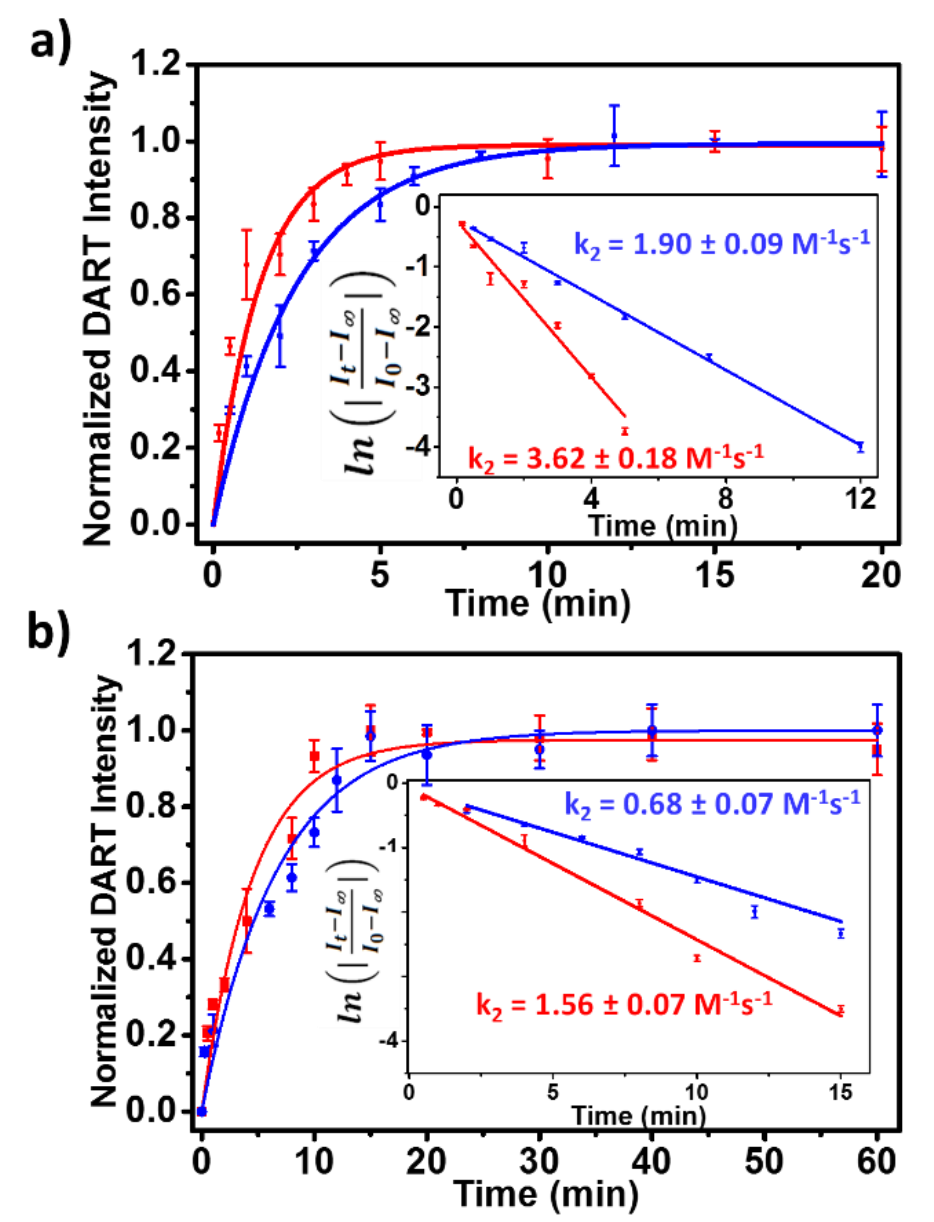

Figure 2. Normalized DART-HRMS intensity vs time (min) for IEDDA in a "free" microenvironment: a) exo-Norbornene surfaces (M2) reacting with $\mathbf{3}$ (red) and tetrazine surfaces (M4) reacting with $\mathbf{4}$ (blue). b) endo-Norbornene surfaces (M3) reacting with $\mathbf{3}$ (red) and tetrazine surfaces (M4) reacting with $\mathbf{6}$ (blue). Inserts: Linear plots of $\ln \left|\left(I_{\infty}-I_{t}\right) /\left(I_{\infty}-I_{0}\right)\right|$ vs time (min) to obtain the pseudo-first order 
constants, and using solute concentrations of $3.0 \mathrm{mM}$ the subsequently derived 2nd order rate constants $\left(\mathrm{k}_{2}\right)$.

To obtain accurate reaction kinetics, we reacted our samples for different time intervals (up to $20 \mathrm{~min}$ ) and followed the signal intensity of the corresponding MS$\operatorname{tag}(m / z$ 189.016) in DART-HRMS. Using such high $\mathrm{S} / \mathrm{N}$ data, the pseudo-firstorder rate constant $\left(\mathrm{k}^{\prime}\right)$ was calculated as the slope of the plot of $\ln \mid\left(I_{t}-I_{\infty}\right) /\left(I_{0}-\right.$ $\left.I_{\infty}\right) \mid$ versus time $(\mathrm{t})$, where $I_{\infty}$ corresponds to the asymptotic integrated extracted ion chronogram (EIC) intensity at obtained by exponential decay curve fitting of the data (Figure S3.1-S3.8, Appendix 3); the second-order rate constants were derived from there. The highest second-order reaction rate constant $\left(3.62 \mathrm{M}^{-1} \mathrm{~s}^{-1}\right.$ at a solute concentration of $3.0 \mathrm{mM}, 30^{\circ} \mathrm{C}$, DCE) was observed for exo-norbornene ${ }_{(\text {surf.) }}$ with tetrazine $_{(\text {soln.) }} \mathbf{3}$ in a "free" microenvironment (Figure 2).

\begin{tabular}{|c|c|c|c|}
\hline \multirow{2}{*}{$\begin{array}{l}\text { Surface } \\
\text { group }\end{array}$} & \multicolumn{3}{|c|}{ Reactant in solution } \\
\hline & \multicolumn{3}{|c|}{ Tetrazine } \\
\hline \multirow[t]{2}{*}{ Norbornene } & Free & $\begin{array}{c}(10.86 \pm 0.54) \times 10^{-3} \mathrm{~s}^{-1}(\text { exo- }) \\
3.62 \pm 0.18 \mathrm{M}^{-1} \mathrm{~s}^{-1}(\text { exo- })\end{array}$ & $\begin{array}{c}(4.68 \pm 0.21) \times 10^{-3} \mathrm{~s}^{-1}(\text { endo- }) \\
1.56 \pm 0.07 \mathrm{M}^{-1} \mathrm{~s}^{-1}(\text { endo }-)\end{array}$ \\
\hline & Buried & $\begin{array}{c}(2.61 \pm 0.18) \times 10^{-3} \mathrm{~s}^{-1}(\text { exo- }) \\
0.87 \pm 0.06 \mathrm{M}^{-1} \mathrm{~s}^{-1}(\text { exo- })\end{array}$ & $\begin{array}{c}(1.70 \pm 0.30) \times 10^{-3} \mathrm{~s}^{-1} \text { (endo-) } \\
0.57 \pm 0.08 \mathrm{M}^{-1} \mathrm{~s}^{-1} \text { (endo-) }\end{array}$ \\
\hline Tetrazine & \multicolumn{3}{|c|}{ Norbornene } \\
\hline & Free & $\begin{array}{c}(5.79 \pm 0.27) \times 10^{-3} \mathrm{~s}^{-1}(\text { exo- }) \\
1.90 \pm 0.09 \mathrm{M}^{-1} \mathrm{~s}^{-1}(\text { exo- })\end{array}$ & $\begin{array}{c}(2.05 \pm 0.20) \times 10^{-3} \mathrm{~s}^{-1}(\text { endo- }) \\
0.68 \pm 0.07 \mathrm{M}^{-1} \mathrm{~s}^{-1}(\text { endo- })\end{array}$ \\
\hline & Buried & $\begin{array}{c}(1.74 \pm 0.24) \times 10^{-3} \mathrm{~s}^{-1}(\text { exo- }) \\
0.58 \pm 0.08 \mathrm{M}^{-1} \mathrm{~s}^{-1}(\text { exo- })\end{array}$ & $\begin{array}{c}(1.83 \pm 0.15) \times 10^{-3} \mathrm{~s}^{-1}(\text { endo- }) \\
0.61 \pm 0.05 \mathrm{M}^{-1} \mathrm{~s}^{-1}(\text { endo- })\end{array}$ \\
\hline
\end{tabular}

Table 1. Pseudo-first $\mathrm{k}^{\prime}\left(\mathrm{s}^{-1}\right)$ and second-order rate constants $\mathrm{k}_{2}\left(\mathrm{M}^{-1} \mathrm{~s}^{-1}\right)$ of the tetrazine-norbornene IEDDA reaction in "free" and "buried" microenvironments.

Using the inverse situation, tetrazine on a surface surrounded by lower alkyl chains reacting with exo-norbornene 4 in solution, afforded a two-fold slower rate (Figure 2). The IEDDA reaction efficiency can be determined by comparing the atomic ratio determined by XPS with the theoretical value of 3:4 (for $\mathrm{F} / \mathrm{P}$ ), which corresponds to the $100 \%$ surface conversion. Interestingly, recently we reported the 
first 100\%-yielding surface-bound metal-free click reaction (a strain-promoted cyclooctyne-quinone or SPOCQ reaction), which was further characterized by a second order rate constant $\left(\mathrm{k}^{\prime}\right)$ of $3.3 \mathrm{M}^{-1} \mathrm{~s}^{-1} \cdot{ }^{[14 a]}$ While thus displaying virtually the same rate, in that case, we observed two distinct kinetic regimes: an initial fast regime followed by a slower, more complex one; the rate constant refers to the initial well-behaved kinetic regime only. The SPOCQ reaction was eventually also quantitative, but reaches full conversion only in $4 \mathrm{~h}$. Here, the IEDDA reaction turned out to be a significant improvement, reaching an unprecedented complete conversion within $15 \mathrm{~min}$, while the entire kinetic regime could be followed using one exponent, i.e. the IEDDA reaction displays clean kinetics.

In addition, excellent yields were obtained both in 'free' and 'buried' conditions, and independent of the orientation of the reaction, thereby showing the scope of this strategy for surface modifications. Changing the stereochemistry of the immobilized norbornene to endo- in a "free" microenvironment halved the reaction rate (Figure S3.3) which is consistent with the rate differences observed in solution. ${ }^{[9 \mathrm{~b}]}$ Interestingly, also in this case a kinetic preference (two-fold difference) was observed for immobilized norbornene reacting with tetrazine in solution than its reverse, i.e. tetrazine on the surface reacting with endo-norbornene $\mathbf{6}$ in solution (Table 1). This result outlines that the slowing down of reaction rate for tetrazine immobilization occurs irrespective of norbornene stereochemistry.

Finally, we wanted to know the kinetic effects of doing the reaction 'above' the monolayer versus 'within' the monolayer. Therefore we studied the reaction in a "buried" state: surfaces were prepared with either norbornene or tetrazine moieties bound to the surface that are surrounded by long alkyl chains, and used for IEDDA reaction (Scheme 2). The rate differences again amounted to about two folds in favor of norbornene immobilization (Table 1). The highest reaction rate in this microenvironment $\left(0.87 \mathrm{M}^{-1} \mathrm{~s}^{-1}\right)$ was observed for exo-norbornene attached on the 
surface reacting with tetrazine while the lowest $\left(0.58 \mathrm{M}^{-1} \mathrm{~s}^{-1}\right)$ was observed for its reverse. Comparing the best and worst possibility, the rate constant for the exonorbornene immobilization in "free" microenvironment was 6.2 fold higher than that for tetrazine immobilization in a "buried" state. It is of relevance to state that only with high $\mathrm{S} / \mathrm{N}$-techniques like DART-HRMS such relatively small rate differences can come into view and thus be rationalized.

To this latter aim, we also applied quantum chemical and molecular mechanics calculations. The reaction between unsubstituted ${ }^{[18]}$ and substituted tetrazines ${ }^{[19]}$ and various alkenes has been studied theoretically by Houk, Devaraj and co-workers. In our case, we modeled the IEDDA reaction between a substituted tetrazine and exo/endo-norbornene (substituted so as to mimic their surface attachment or solution functionalities, see Figure 3). In line with previous findings, density functional theory (DFT) calculations (at M06-2X/6-311+G(d,p) level) revealed that the Diels-Alder cycloaddition, rather than the subsequent $\mathrm{N}_{2}$ expulsion, is the rate-determining step in this reaction. In accordance with experimental results, exo-norbornene has a lower activation barrier than endo-norbornene (Figure 3a). Also, our calculations show that

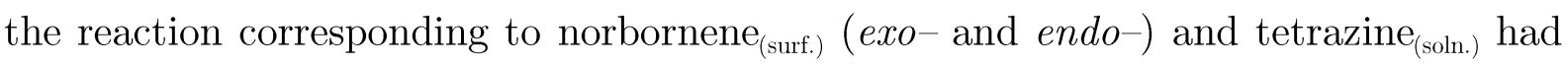
a lower energy barrier (by $\sim 1 \mathrm{kcal} \mathrm{mol}^{-1}$ ) than the reverse orientation (Figure 3b and c). This energy difference is in line with the observed rate difference for the interfacial IEDDA in the "free" microenvironment. 
a)

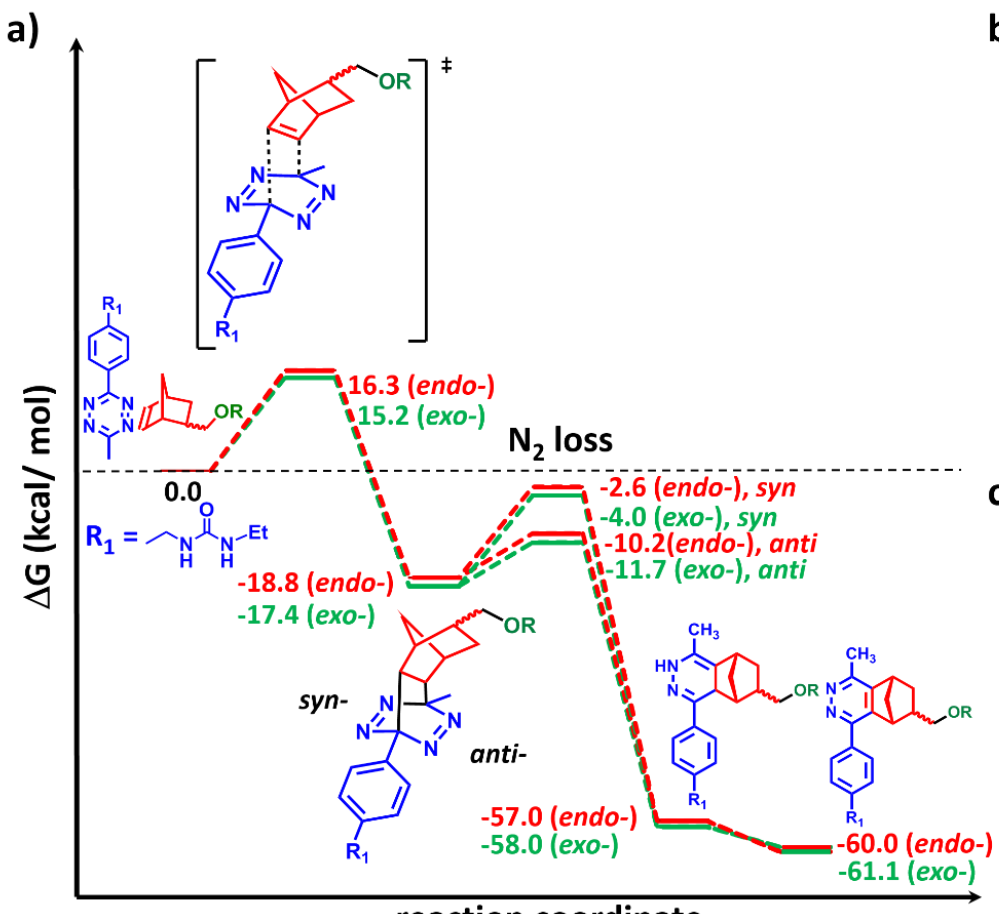

reaction coordinate
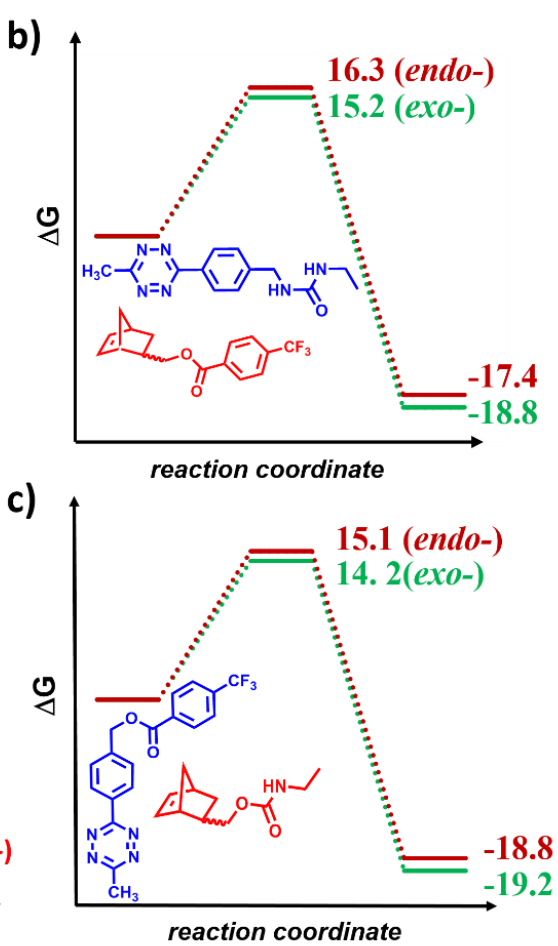

Figure 3. a) DFT calculation for the full mechanism of the multistep IEDDA reaction between an unsymmetrical tetrazine and exo- and endo-norbornene mimicking molecules used in our work, b) and c) reaction coordinate diagram for the IEDDA reaction mimicking our reaction condition showing tetrazine and exo-/endonorbornene on surface, respectively

A visual representation of the orientation of immobilized groups was obtained by performing sequential molecular dynamics and molecular mechanics optimizations of the IEDDA cycloadducts on aluminum oxide surface. The modeling was performed on large supercells obtained by attachment of the norbornene or tetrazine moieties in a random pattern followed by subsequent energy minimization (see section 7.13-7.14, Appendix 3). Next, a series of molecular dynamics runs at $773.0 \mathrm{~K}$ were performed to 'shake up' the conformations of the chains, and get truly random orientations of the surface-bound moieties. Finally, those geometries were optimized by molecular mechanics, to indicate the most stable orientations of reactive groups with respect to the surface (see section 7.15-7.19, Appendix 3 and two movies with rotating 3D structures of the surface available online). Representative geometries, from a much 
large set, are shown in Figure 4. For surface-bound norbornenes (Figure 4a), the surface-bound molecules prefer to be apart (no specific attractions; significant steric repulsions) with the double bond (highlighted in green) facing outwards. In contrast, tetrazines $_{(\text {surf.) }}$ in a "free" microenvironment showed a higher propensity to clump/cluster together due to additional stabilization attributable to $\pi-\pi$ stacking (Figure 4b).
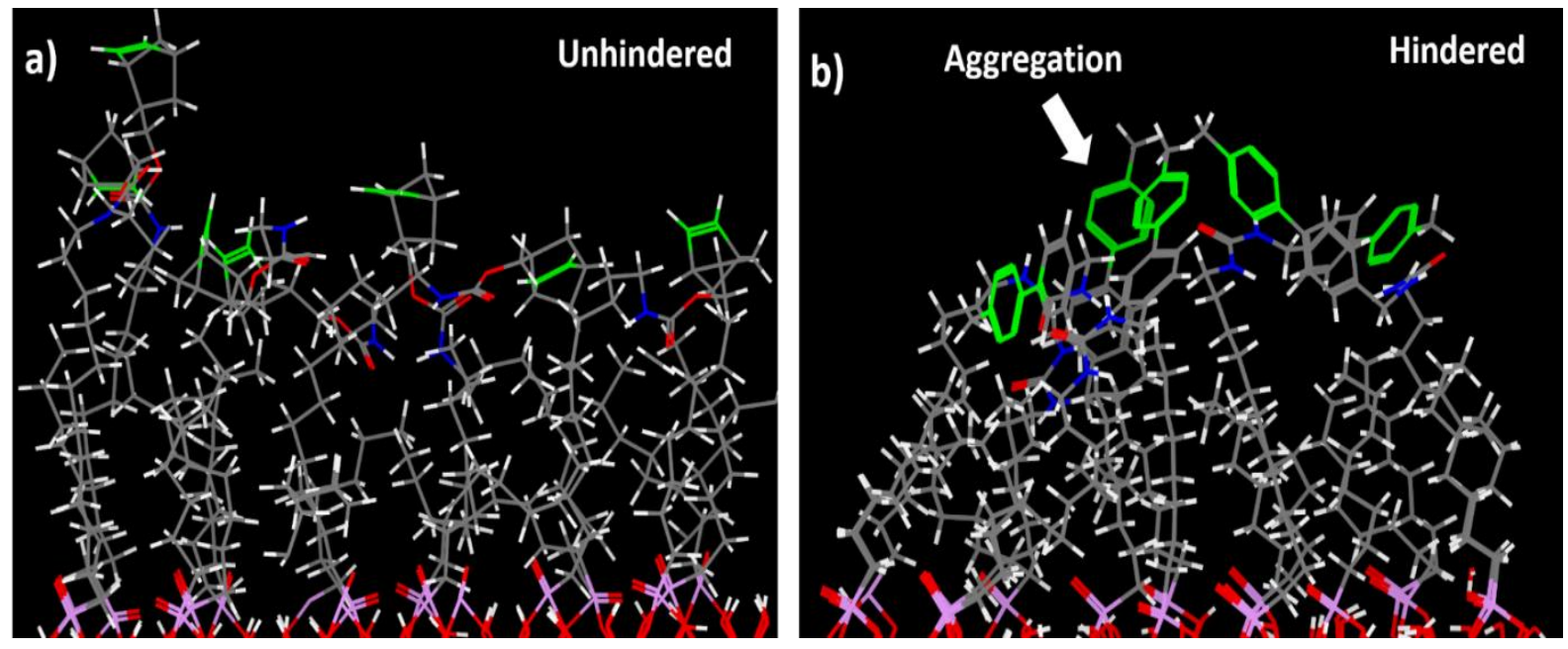

Figure 4. Comparison of surface disposition of IEDDA reactants in "free" ME after molecular dynamics of a) norbornene $_{(\text {surf.) }}$ and b) tetrazine ${ }_{(\text {surf.) }}$.

These orientations and clustering (or lack thereof) will influence the reactivity and angle of approach of any reacting solute. Finally, those geometries were optimized by molecular mechanics, to indicate the most stable orientations of reactive groups with respect to the surface (see section 7.15-7.19, Appendix 3 and two movies with rotating 3D structures of the surface). Representative geometries, from a much large set, are shown in Figure 4. For surface-bound norbornenes (Figure 4a), the surface-bound molecules prefer to be apart (no specific attractions; significant steric repulsions) with the double bond (highlighted in green) facing outwards. In contrast, tetrazines $_{\text {(surf.) }}$ in a "free" microenvironment showed a higher propensity to clump/cluster together due to additional stabilization attributable to $\pi-\pi$ stacking 
(Figure 4b). These orientations and clustering (or lack thereof) will influence the reactivity and angle of approach of any reacting solute.

Based on the TS geometry obtained by DFT calculations we deduce that the approach of tetrazine ${ }_{(\text {soln.) }}$ moieties preferentially occurs in a "top-down" manner or perpendicular to the surface (Figure 5a). In contrast, the approach of the incoming norbornene $_{(\text {soln.) }}$ should be "side-ways" or parallel to the surface (Figure 5b), and the reactant in solution thus encounters a lot more steric hindrance along the reaction path.
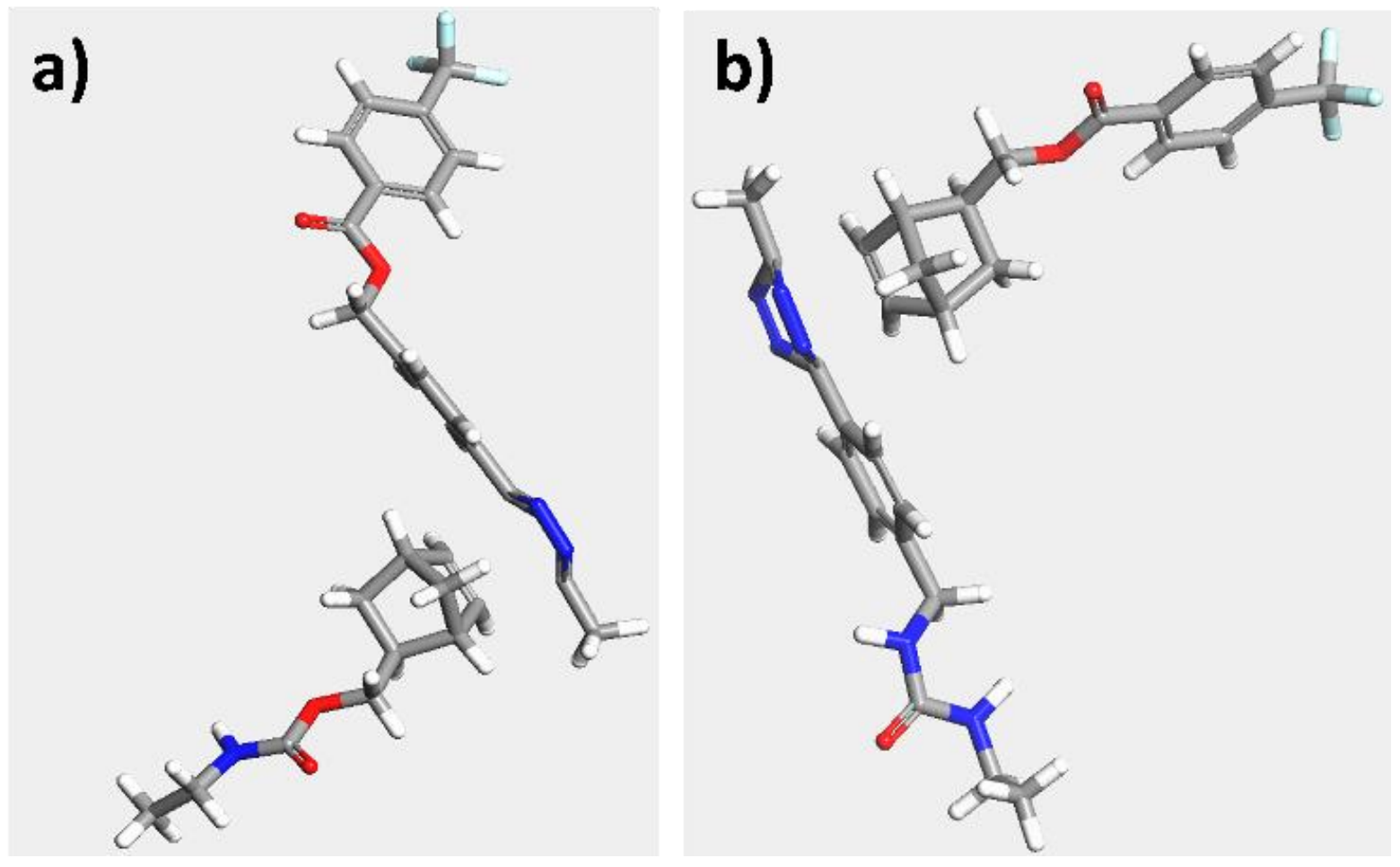

Figure 5. DFT calculation of approach of a) Norbornene $_{(\text {surf.) }}$ with tetrazine (soln.) $_{\text {. }}$ and b) vice-versa, clearly showing different angles of approach to surface groups.

In addition, the aggregation of surface-bound tetrazines also lowers the available number of tetrazines in statistical terms by masking one or both the available faces. Both these factors will likely contribute to the overall slower kinetics of tetrazine (surf.) + norbornene $_{(\text {soln.) }}$ reaction. These findings point to the overall relevance of choosing prior to immobilization which agent is surface-bound and which comes in from solution. 


\subsection{CONCLUSIONS}

We have achieved an expeditious (within $15 \mathrm{~min}$ ) and quantitative surface functionalization using inverse electron demand Diels-Alder reactions. The reaction displays clean pseudo-first order kinetics over the full conversion range. We found that the approach of the solution reactant towards the surface-bound counterpart plays an important role in the course of the reaction, supporting the significance of the orientation in surface-bound reactions. Such detailed insights into surface-bound organic reactions are both required and becoming available by combining techniques like XPS and DART-MS to further improve surface modification procedures for a wide range of applications.

\subsection{REFERENCES}

[1] W. Tang, M. L. Becker, Chem. Soc. Rev. 2014, 43, 7013-7039.

[2] F. Ehret, H. Wu, S. C. Alexander, N. K. Devaraj, J. Am. Chem. Soc. 2015, $137,8876-8879$.

[3] C. D. Spicer, B. G. Davis, Nat. Commun. 2014, 5, 4740-4754.

[4] E. M. Sletten, C. R. Bertozzi, Angew. Chem. Int. Ed. 2009, 48, 6974-6998; Angew. Chem. 2009, 121, 7108-7133.

[5] a) S. P. Pujari, L. Scheres, A. T. M. Marcelis, H. Zuilhof, Angew. Chem. Int. Ed. 2014, 53, 6322-6356; Angew. Chem. 2014, 126, 6438-6474; b) H. C. Kolb, M. G. Finn, K. B. Sharpless, Angew. Chem. Int. Ed. 2001, 40, 2004-2021; Angew. Chem. 2001, 113, 2056-2075; c) J. Escorihuela, A. T. M. Marcelis, H. Zuilhof, Adv. Mater. Interfaces 2015, 2, 1500135.

[6] a) K. Godula, D. Rabuka, K. T. Nam, C. R. Bertozzi, Angew. Chem. Int. Ed. 2009, 48, 4973-4976; Angew. Chem. 2009, 121, 5073-5076; b) B. Uszczynska, T. 
Ratajczak, E. Frydrych, H. Maciejewski, M. Figlerowicz, W. T. Markiewicz, M. K. Chmielewski, Lab on a Chip 2012, 12, 1151-1156.

[7] a) M. L. Blackman, M. Royzen, J. M. Fox, J. Am. Chem. Soc. 2008, 130, 13518-13519; b) N. K. Devaraj, R. Upadhyay, J. B. Haun, S. A. Hilderbrand, R. Weissleder, Angew. Chem. Int. Ed. 2009, 48, 7013-7016; Angew. Chem. 2009, 121, $7147-7150$.

[8] a) D. Wang, W. Chen, Y. Zheng, C. Dai, K. Wang, B. Ke, B. Wang, Org. Biomol. Chem. 2014, 12, 3950-3955; b) M. R. Karver, R. Weissleder, S. A. Hilderbrand, Bioconjugate Chem. 2011, 22, 2263-2270.

[9] a) A.-C. Knall, M. Hollauf, C. Slugovc, Tet. Lett. 2014, 55, 4763-4766; b) M. Vrabel, P. Kölle, K. M. Brunner, M. J. Gattner, V. López-Carrillo, R. de VivieRiedle, T. Carell, Chem. Eur. J. 2013, 19, 13309-13312.

[10] P. Wang, Z. Na, J. Fu, C. Y. J. Tan, H. Zhang, S. Q. Yao, H. Sun, Chem. Commun. 2014, 50, 11818-11821.

[11] a) N. K. Devaraj, R. Weissleder, S. A. Hilderbrand, Bioconjugate Chem. 2008, 19, 2297-2299; b) J. Schoch, M. Wiessler, A. Jäschke, J. Am. Chem. Soc. 2010, 132, 8846-8847; c) H. S. G. Beckmann, A. Niederwieser, M. Wiessler, V. Wittmann, Chem. Eur. J. 2012, 18, 6548-6554; d) O. Roling, A. Mardyukov, S. Lamping, B. Vonhoren, S. Rinnen, H. F. Arlinghaus, A. Studer, B. J. Ravoo, Org. Biomol. Chem. 2014, 12, 7828-7835.

[12] R. Sen, D. Gahtory, R. R. Carvalho, B. Albada, F. L.van Delft, H. Zuilhof, Angew. Chem. Int. Ed. 2017, 129, 4194-4198.

[13] a) N. S. Bhairamadgi, S. Gangarapu, M. A. Caipa Campos, J. M. J. Paulusse, C. J. M. van Rijn, H. Zuilhof, Langmuir 2013, 29, 4535-4542; b) J. Escorihuela, M. J. Banuls, R. Puchades, A. Maquieira, Chem. Commun. 2012, 48, 2116-2118. 
[14] a) R. Sen, J. Escorihuela, M. M. J. Smulders, H. Zuilhof, Langmuir 2016, 32, 3412-3419; b) R. Sen, J. Escorihuela; F. L. van Delft, H. Zuilhof, Angew. Chem. Int. Ed. 2017, 56, 3299-3303.

[15] Y. Kwon, M. Mrksich, J. Am. Chem. Soc. 2002, 124, 806-812.

[16] B. Rijksen, S. P. Pujari, L. Scheres, C. J. M. van Rijn, J. E. Baio, T. Weidner, H. Zuilhof, Langmuir 2012, 28, 6577-6588.

[17] X. Fan, Y. Ge, F. Lin, Y. Yang, G. Zhang, W. S. Ngai, Z. Lin, S. Zheng, J. Wang, J. Zhao, J. Li, P. R. Chen, Angew. Chem. Int. Ed. 2016, 55, 14046-14050.

[18] L. Törk, G. Jiménez-Osés, C. Doubleday, F. Liu, K. N. Houk, J. Am. Chem. Soc. 2015, 13\%, 4749-4758.

[19] J. Yang, Y. Liang, J. Seckute, K. N. Houk, N. K. Devaraj, Chemistry 2014, 20, 3365-3375.

[20] Gaussian 09, Revision D.01, M. J. Frisch, G. W. Trucks, H. B. Schlegel, G. E. Scuseria, M. A. Robb, J. R. Cheeseman, G. Scalmani, V. Barone, B. Mennucci, G. A. Petersson, H. Nakatsuji, M. Caricato, X. Li, H. P. Hratchian, A. F. Izmaylov, J. Bloino, G. Zheng, J. L. Sonnenberg, M. Hada, M. Ehara, K. Toyota, R. Fukuda, J. Hasegawa, M. Ishida, T. Nakajima, Y. Honda, O. Kitao, H. Nakai, T. Vreven, J. A. Montgomery Jr., J. E. Peralta, F. Ogliaro, M. J. Bearpark, J. Heyd, E. N. Brothers, K. N. Kudin, V. N. Staroverov, R. Kobayashi, J. Normand, K. Raghavachari, A. P. Rendell, J. C. Burant, S. S. Iyengar, J. Tomasi, M. Cossi, N. Rega, N. J. Millam, M. Klene, J. E. Knox, J. B. Cross, V. Bakken, C. Adamo, J. Jaramillo, R. Gomperts, R. E. Stratmann, O. Yazyev, A. J. Austin, R. Cammi, C. Pomelli, J. W. Ochterski, R. L. Martin, K. Morokuma, V. G. Zakrzewski, G. A. Voth, P. Salvador, J. J. Dannenberg, S. Dapprich, A. D. Daniels, Ö. Farkas, J. B. Foresman, J. V. Ortiz, J. Cioslowski, D. J. Fox, Gaussian, Inc., Wallingford, CT, USA, 2009.

[21] Y. Zhao, D. G. Truhlar, Theor. Chem. Acc. 2008, 120, 215-241. 
[22] a) F. Liu, R. S. Paton, S. Kim, Y. Liang, K. N. Houk, J. Am. Chem. Soc. 2013, 135, 15642-15649; b) L. Törk, G. Jiménez-Osés, C. Doubleday, F. Liu, K. N. Houk, J. Am. Chem. Soc. 2015, 137, 4749-4758.

[23] C. Gonzalez, H. B. Schlegel, J. Phys. Chem. 1990, 94, 5523-5527.

[24] J. Yang, M. R. Karver, W. Li, S. Sahu, N. K. Devaraj, Angew.Chem. Int. Ed. 2012, 51, 5222-5225. 


\section{Chapter 5:}

\section{Ultrathin Covalently Bound Organic Layers on}

\section{Mica: Formation of Atomically Flat Bio- functionalizable Surfaces}

Mica is the substrate of choice for microscopic visualization of a wide variety of intricate nanostructures. Unfortunately, the lack of a facile strategy for its modification has prevented the on-mica assembly of nanostructures. Herein, we disclose a convenient catechol-based linker that enables various surface-bound metal-free click reactions, and an easy modification of mica with DNA nanostructures and a horseradish peroxidase mimicking hemin/G-quadruplex DNAzyme.

This Chapter has been published as:

"Ultrathin Covalently Bound Organic Layers on Mica: Formation of Atomically Flat Bio-functionalizable Surfaces". Rickdeb Sen, Jorge Escorihuela, Floris van Delft and H. Zuilhof. Angew. Chem. Int. Ed. 2017, 56, 4130-4134. 


\section{Table of Contents}

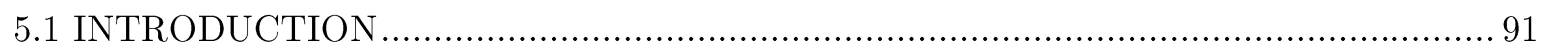

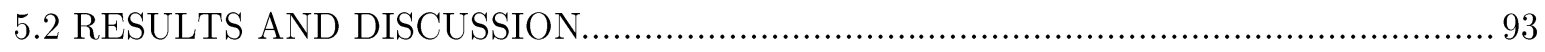

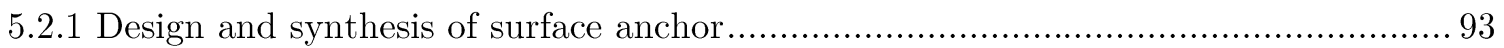

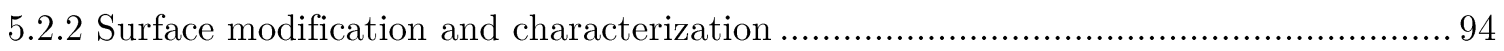

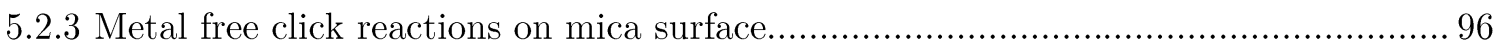

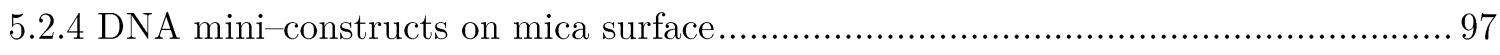

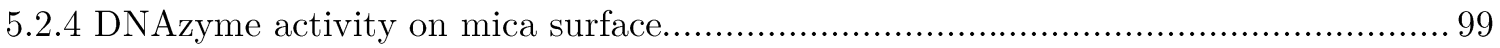

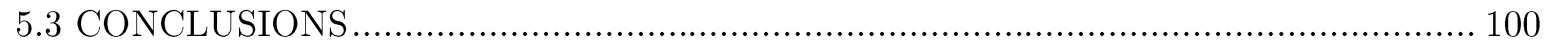

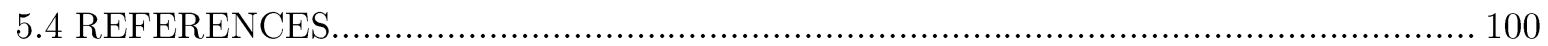




\subsection{INTRODUCTION}

The atomically flat nature of mica has made it the substrate of choice for microscopic visualization of dimensional parameters of various pre-fabricated nanomaterials such as DNA origami ${ }^{[1]}$ and protein conjugates, ${ }^{[2],[3]}$ among others. Mica is an hydrophilic aluminosilicate, which in saline solution is covered by a hydration layer with $\mathrm{K}^{+}$ions that are tightly bound to the anionic silicate. While widely used for atomic force microscopy $(\mathrm{AFM})$ studies, ${ }^{[4]}$ the surface modification and chemical functionalization of mica itself has received surprising little attention. ${ }^{[5]}$

Lately, catechol-based coatings including mussel adhesive poly-dopamine proteins have found increasing attention for mica surface modification. ${ }^{[6]}$ Despite a general acceptance that the catechol moiety in mussel proteins is central to their adhesion ability, ${ }^{[7]}$ its exact adhesion mechanism is still unknown. ${ }^{[8]}$ Butler et al. recently showed that a pending amine functionality is central to binding. ${ }^{[9]}$ To demonstrate this, a symmetric trichrysobactin was synthesized that bears three 2,3dihydroxybenzoyl moieties and a lysine tail. In this 'two-punch' approach, the $\mathrm{NH}_{3}{ }^{+}$ moiety displaced the $\mathrm{K}^{+}$ions, followed by surface attachment of the catechol-moiety. Unfortunately, the six-step synthesis required for the scaffold hampers its widespread application. Even more, post-attachment functionalization of mica substrates with interesting biomolecules such as DNA and proteins is still uncharted domain. Such an approach would require both facile and strong adhesion, combined with the presence of a moiety that can be routinely used for post-attachment functionalization. The current approaches ${ }^{7,8}$ do not provide such a handle, and as such there is still need for a platform that allows various surface-modification strategies and a stepwise observation of the assembly processes of biological nanostructures in a controlled fashion. ${ }^{[10]}$ 


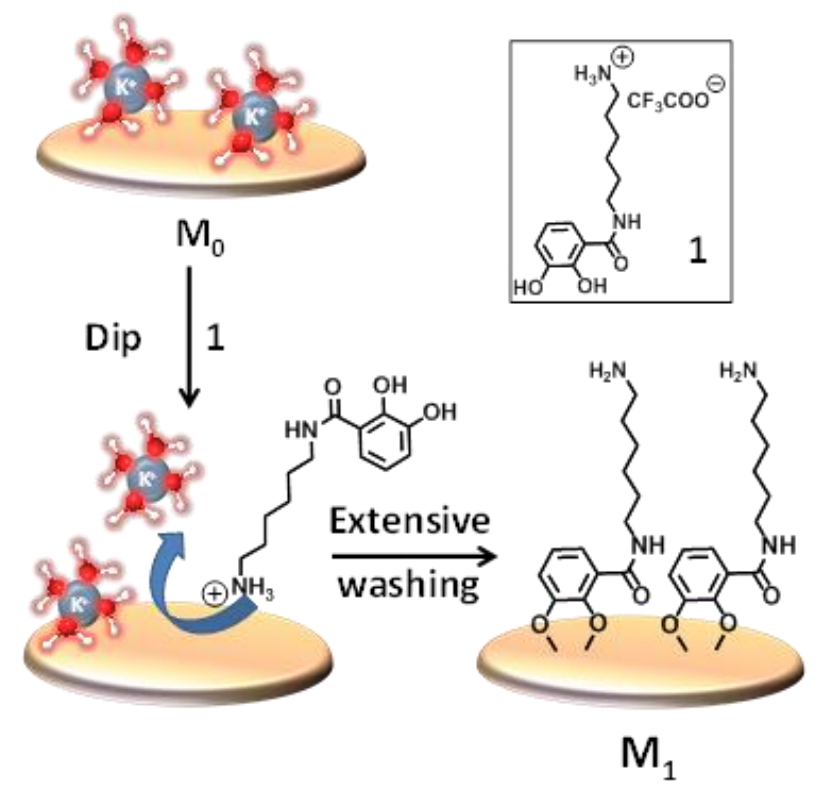

Figure 1. Tentative mechanism of mica modification by surface anchor $\mathbf{1}$.

a)
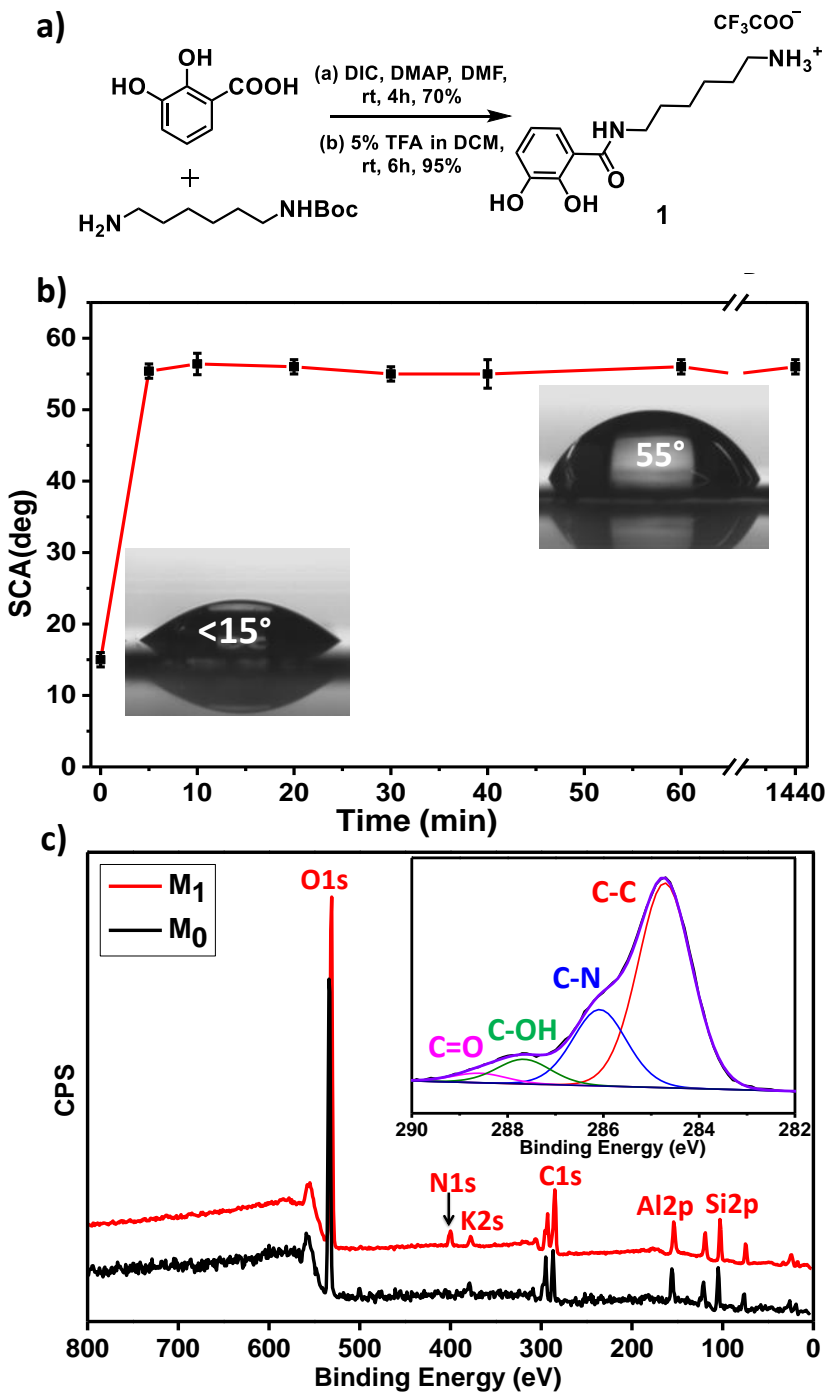
Figure 2. a) Synthesis of surface anchor 1 b) Increase of water SCA during modification process. c) XPS wide range spectra of bare $\left(\mathbf{M}_{0}\right)$ and modified $\left(\mathbf{M}_{1}\right)$ mica (inset: narrow scan of C1s signal).

In this paper, we address this unresolved problem through a dual approach via the development of a simple molecule that allows both (i) covalent modification of mica, and (ii) post-modification stepwise growth and study of molecular assemblies. To this end, we envisaged and synthesized a surface anchor (1) that possesses the structural characteristics required to combine optimal adhesion characteristics with a handle for modular functionalization (Figure 1). We extensively characterized the modified surfaces $\left(\mathbf{M}_{\mathbf{1}}\right)$ by static water contact angle (SCA), X-ray photoelectron spectroscopy (XPS) and AFM measurements. To illustrate the potential for surface functionalization, we explored several metal-free strain-promoted click reactions. Finally, to demonstrate bio-functionalization we pursued the stepwise formation of functional DNA constructs, such as $\mathrm{G}$-quadruplex (GQ, G = guanine) structures on covalently modified surfaces by AFM.

\subsection{RESULTS AND DISCUSSION}

\subsubsection{Design and synthesis of surface anchor.}

As shown by Butler et al. ${ }^{[9]}$ and Hwang et al.. ${ }^{[11]}$ mica adhesive molecules should likely contain both catechol and amino groups. However, in their case this resulted in a poorly defined polymeric layer possibly due to intramolecular Michaeltype addition reactions between the catechol and free amino groups. ${ }^{[12]}$ Spencer et al. have tackled this issue of polymerization by inclusion of a strongly electronwithdrawing nitro group in the catechol motif to minimize auto-oxidation. ${ }^{[13]}$ Using this information, the design of our surface anchor 1 was based on the retention of the 
key components viz. a catechol moiety linked proximally via an electron-withdrawing amide group to a protonated amine (Figure 1).

A first step forward was the synthesis of surface anchor 1: 2,3dihydroxybenzoic acid (DHBA) and mono-Boc-protected 1,6-hexanediamine were linked via a conventional amide coupling, followed by subsequent Boc-deprotection (Figure 2a) to afford 1 in an overall yield of $66 \%(0.78 \mathrm{~g})$.

\subsubsection{Surface modification and characterization.}

A $1 \mathrm{mM}$ solution of 1 in PBS buffer ( $\mathrm{pH} 7.4$ ) allowed for the simultaneous modification of large numbers of freshly cleaved mica surfaces in $24 \mathrm{~h}$. A constant low water contact angle $\left(55^{\circ}\right)$ (Figure $2 \mathrm{~b}$ ), the emergence of N1s signal in XPS (Figure 2c), and the XPS analysis of subsequent modifications on $\mathbf{M}_{\mathbf{1}}$ confirmed modification of the mica surfaces with surface anchor 1 (see Appendix 4). Importantly, molecules similar to surface anchor 1, but lacking one of the mentioned components, performed inferior than anchor molecule $\mathbf{1}$ (Table S1).

XPS analysis of modified surfaces also showed the overall chemical composition of $\mathbf{M}_{\mathbf{1}}$ to fit the molecular composition of $\mathbf{1}$ within experimental error $\left(\mathrm{C} / \mathrm{N}_{\mathrm{XPS}}=6.8 ; \mathrm{C} / \mathrm{N}_{\text {Theor }}=6.5\right)$ (see Appendix 4, section S4.2). As shown by Hwang et $a l$. , the formation of polymeric layers on mica surfaces leads to the disappearance of the Al2p, K2s and Si2p signals in the XPS spectrum. ${ }^{[11]}$ Interestingly in our case, the continued high intensity of these substrate-specific signals hinted at formation of ultrathin layers (Figure 2c).

The deconvolution of the C1s narrow scan clearly revealed carbons in distinct environments, namely, $\mathrm{C}-\mathrm{C}, \mathrm{C}-\mathrm{N}, \mathrm{C}-\mathrm{O}$ and $\mathrm{C}=\mathrm{O}$ (Figure 2c, inset). Experimental XPS binding energy results correlated nicely with simulated values obtained from DFT calculations (Section 6.1). ${ }^{[14]}$ AFM analysis of modified surfaces $\left(\mathbf{M}_{1}\right)$ displayed 


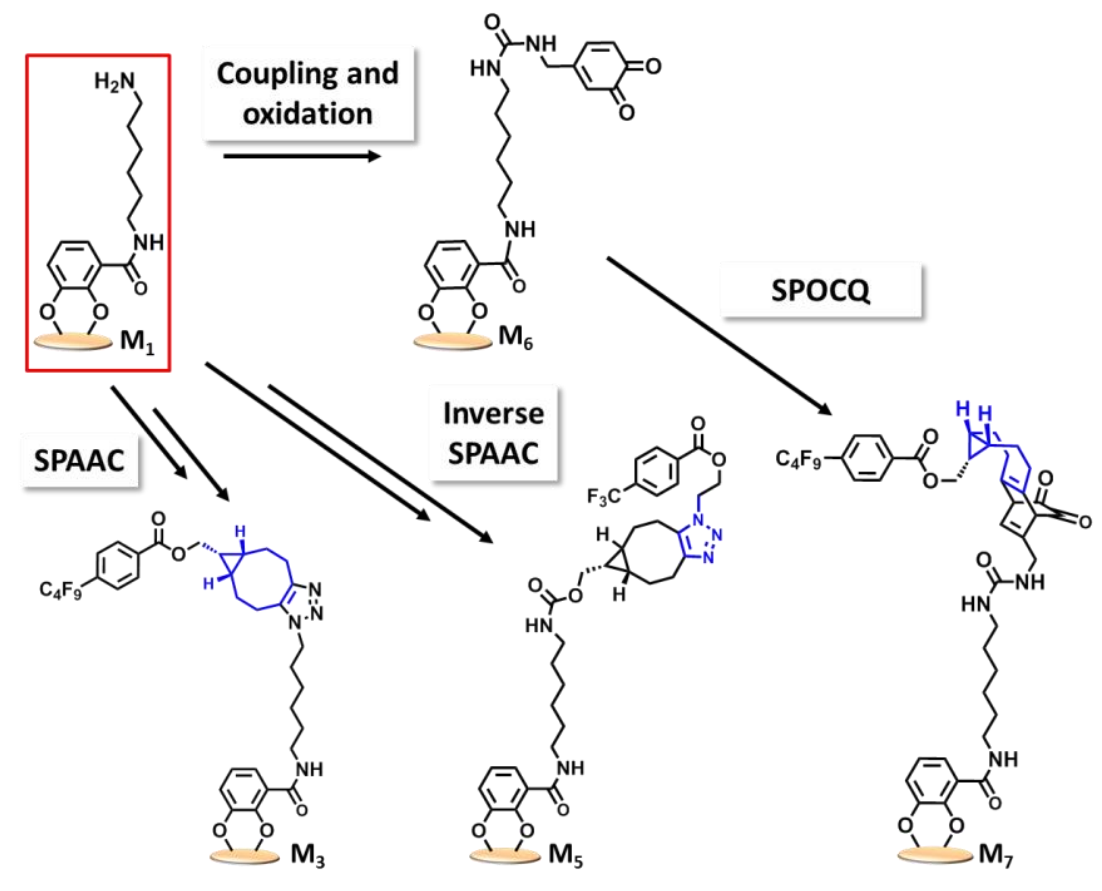

Figure 3. Functionalized mica as building block: General scheme showing the cycloaddition adducts of various metal-free click reactions on $\mathbf{M}_{\mathbf{1}}$.
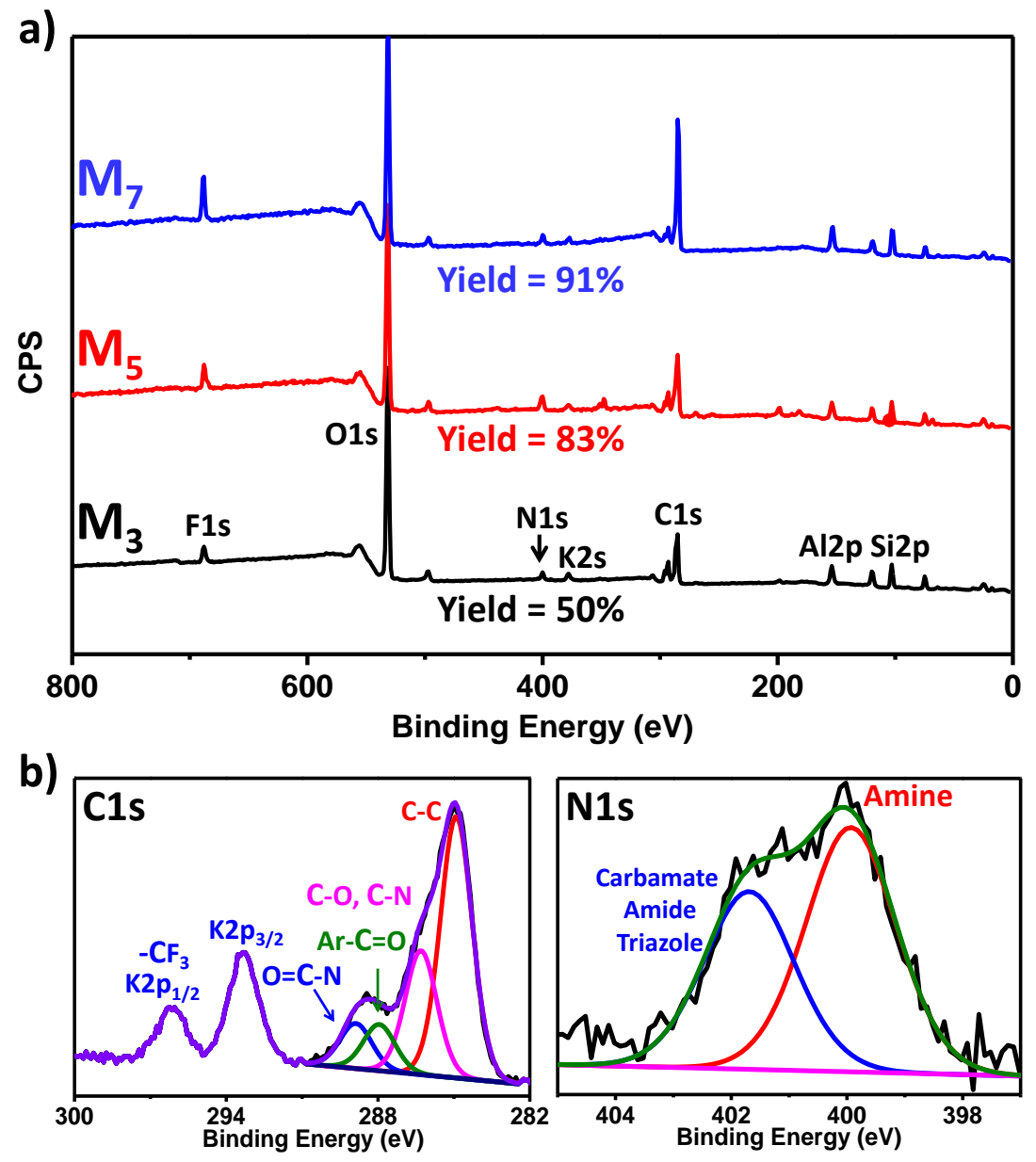
Figure 4. a) Stacked XPS wide scans of final cycloaddition adducts of SPAAC with azide on surface $\left(\mathbf{M}_{3}\right.$; bottom), BCN on surface $\left(\mathbf{M}_{5} ;\right.$ middle) and SPOCQ ( $\mathbf{M}_{7}$; top). The F1s and N1s peaks were used for determining the yield of these reactions. b) XPS narrow scan C1s region (left) and N1s region (right) of SPAAC cycloaddition adducts $\left(\mathbf{M}_{5}\right)$

a remarkably low surface roughness of $0.4 \mathrm{~nm}$ with a layer thickness of $(2.5 \pm 0.5)$ nm (Appendix 4, Figure S1-S3). This confirms exclusive formation of ultrathin layers (length of 1 in stretched-out fashion is ca. $1.3 \mathrm{~nm}$ ) on mica surfaces. We note that the low $(0.4 \mathrm{~nm})$ roughness makes our modified surfaces amenable for reliable microscopic visualization of various covalently attached nanostructured biomolecules.

\subsubsection{Metal free click reactions on mica surface.}

In view of the importance of metal-free click chemistry in bioconjugation chemistry, we performed various state-of-the-art "metal-free" strain-promoted click reactions (Figure 3). For this, amine-terminated layers $\mathbf{M}_{\mathbf{1}}$ were converted to azidefunctionalized coatings $\mathbf{M}_{2}$ (see Appendix 4) by a $\mathrm{Cu}$-catalyzed azide transfer reaction. ${ }^{[15]}$ The resulting azide-terminated layers underwent a facile SPAAC reaction with a fluorinated labile BCN aromatic ester. This allowed easy characterization of the resulting surfaces $\mathbf{M}_{2}$ by XPS and direct analysis in real time high-resolution mass spectrometry (DART-HRMS) ${ }^{[16]}$ which showed the characteristic $\mathrm{m} / z$ peak of the surficial 4-perfluorinated butyl benzoate anion (339.0062) in the extracted ion chronogram (EIC; Appendix 4 Figure S4). A clear XPS F1s signal was evident at $686.0 \mathrm{eV}$, indicating a successful reaction (50\%) (Figure 4a). An inverse strategy for the surface-bound SPAAC reaction was also pursued with BCN immobilized on the surface $\left(\mathbf{M}_{4}\right)$ and a reaction with an azide coupled to a fluorinated tail (Figure 3), to yield surface $\mathbf{M}_{5}$. The XPS F1s peak at $686.0 \mathrm{eV}$ was used to demonstrate the extent of reaction (83\%) (Figure 4a and Appendix 4, Section S4.10), while also the C1s and N1s spectra confirm the success of this reaction (Figure $4 \mathrm{~b}$ ). 
The strain-promoted click reaction between $\mathrm{BCN}$ and ortho-quinones $(\mathrm{SPOCQ})^{[17]}$ was investigated as a second example on mica surfaces modified by our method. To this end, 2,3-dihydroxybenzyl amine was attached to surface $\mathbf{M}_{\mathbf{1}}$ via a DIC coupling, and subsequently oxidized to its corresponding 1,2-quinone $\left(\mathbf{M}_{\mathbf{6}}\right)$ using $\mathrm{NaIO}_{4}$. XPS analysis confirmed the presence of a modified anchor on mica (Appendix 4, Section S4.13). The quinone underwent a facile SPOCQ reaction producing in excellent yield (91\%) surface $\mathbf{M}_{7}$ in $4 \mathrm{~h}$, e.g. as evidenced by the $\mathrm{F} / \mathrm{N}$ ratio in XPS (Figure 4a and Appendix 4, Section S4.14).

\subsubsection{DNA mini-constructs on mica surface.}

Having established prominent bioconjugation reactions on mica, we switched our attention to our other objective: the formation of AFM-observable covalently linked DNA nanostructures. Building on the insights from Famulok et al., a 168-bp DNA minicircle consisting of 8 distinct DNA fragments ${ }^{30}$ was assembled in a stepwise fashion after one of the components was tethered covalently onto mica surface $\mathbf{M}_{1}$. Covalent attachment of strand D1 (Table S2) allowed its hybridization to a preformed assembly of oligomers D2-D8 (Table S2), forming circular structures. As shown in Figure 5a, 3D height images of DNA-containing surfaces revealed circular structures that possess an internal cavity (Appendix 4, Figure S8-S11). A large number of contiguous shapes with circular features was obtained, with an internal diameter of $20 \pm 2 \mathrm{~nm}$ (Appendix 4, Figure S12), in accordance with literature (18.2 nm). ${ }^{[18]}$ When significantly lower concentrations of strands D2-D8 were applied (100-fold dilution, i.e. $0.1 \mathrm{M}$ ), we observed mostly open structures, and circles of which the larger internal diameter hinted to the formation of semi-closed dimeric and trimeric structures (Figures 5b, 5c, Appendix 4, S13 and S14). Control experiments with bare mica surfaces, i.e. without the anchored DNA, did not show any circles or other (linear) structures, proving the need for a covalently attached anchor. 


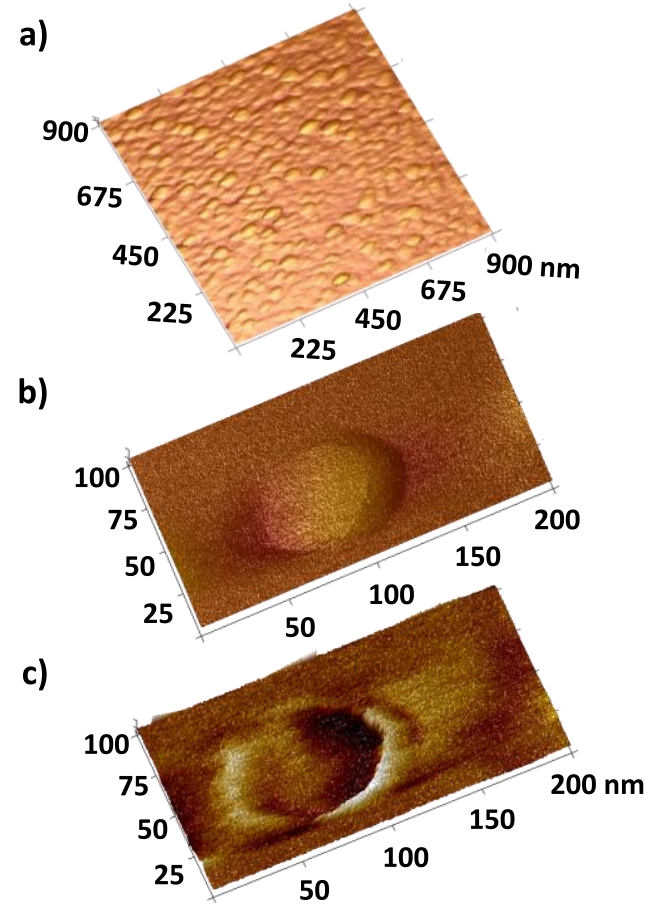

Figure 5. a) 3D height images of DNA circles assembled on mica. b) 3D height and c) quadrature image of single DNA circle.
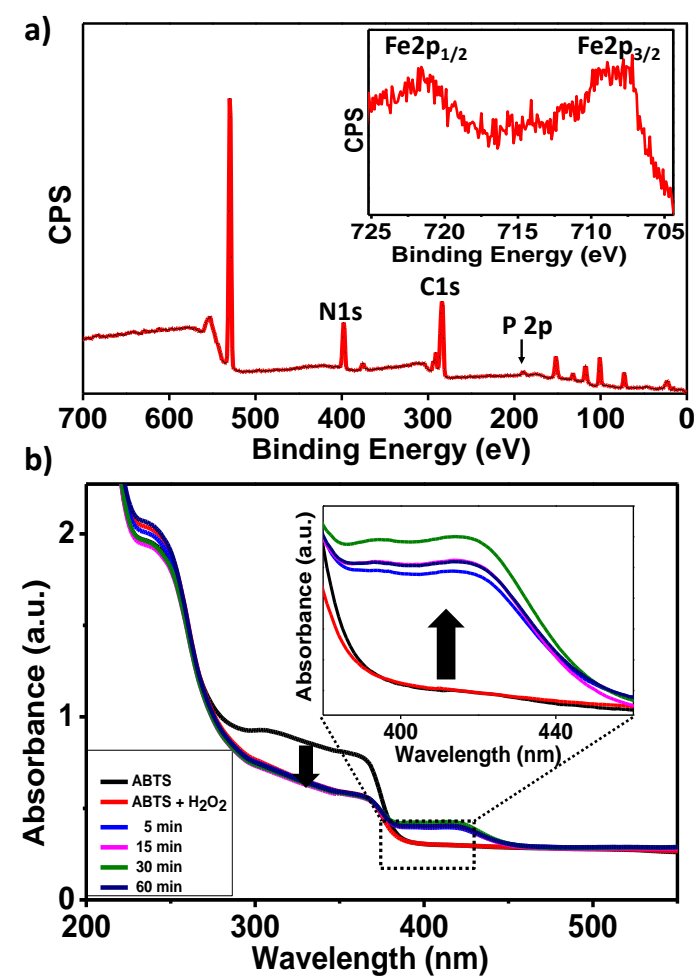

Figure 6. a) Wide scan XPS spectra of EAD2 immobilized on mica surface, (inset: Fe2p narrow spectra of the EAD2-based hGQ DNAzyme-functionalized mica). b) 
UV-vis spectrum of ABTS oxidation catalyzed by EAD2-hemin DNAzyme, inset: changes in the spectrum upon ABTS oxidation.

\subsubsection{DNAzyme activity on mica surface.}

Lastly, we added another dimension to our method by the attachment of functional DNA, namely the horseradish peroxidase-mimicking hemin/G-quadruplex (hGQ) DNAzyme. ${ }^{[19]}$ Such a hGQ DNAzyme can oxidize a variety of organic substrates in the presence of $\mathrm{H}_{2} \mathrm{O}_{2} \cdot{ }^{[20]}$ To demonstrate the feasibility of hGQ catalytic activity of DNAzymes on mica surfaces, we attached the guanine-rich sequence EAD2 (Table S2) covalently to $\mathbf{M}_{\mathbf{1}}$ mica surfaces. Covalent attachment of DNA was confirmed with XPS analysis by the emergence of P2s and P2p signals in the XPS wide spectra at $189.0 \mathrm{eV}$ and $133.0 \mathrm{eV}$, respectively (Figure 6a). Furthermore, complexation of hemin [Fe(III)-protoporphyrin IX chloride] to the formed GQ under $\mathrm{K}^{+}$-rich conditions was confirmed by the emergence of characteristic XPS Fe2p $\operatorname{peaks}^{[20 b]}(710.0$ and $721.0 \mathrm{eV})$ (Figure 6a, inset).

After validation of the covalent attachment of DNA, we assessed its HRPmimicking ability using 2,2'-azino-bis(3-ethylbenzothiazoline-6-sulfonate) (ABTS ${ }^{2-}$, which is converted into $\mathrm{ABTS}^{--}$in the presence of the hGQ DNAzyme and $\mathrm{H}_{2} \mathrm{O}_{2} \cdot{ }^{[20 \mathrm{a}]}$ Indeed, upon addition of $\mathrm{H}_{2} \mathrm{O}_{2}$ to an ABTS-solution that contained just a single hGQ-functionalized mica slide (12 mm diameter), within $5 \mathrm{~min}$ a green-colored solution with $\max _{\text {max }}=414 \mathrm{~nm}$ (Figure 6b) was formed. Control experiments (without ABTS, without modified mica, or without $\mathrm{H}_{2} \mathrm{O}_{2}$ ) did not show formation of this oxidation product. Therefore, the oxidation of $\mathrm{ABTS}^{2-}$ was solely attributable to the presence of catalytically active hGQ DNAzyme EAD2 on the surface.

\subsection{CONCLUSIONS}

To summarize, we demonstrate facile and covalent modification of mica using a catechol-based surface anchor with a flexibly linked amino group. This yielded 
robustly bound, low-roughness and ultrathin layers that are amine-terminated. We demonstrate that this approach allows for a highly flexible surface modification, using a range of metal-free click reactions as points in case. In addition, we display the potential of our strategy for microscopic imaging of functional DNA constructs, and the potential for following the formation of such constructs in a stepwise fashion. We thus believe our work opens up new avenues for the immobilization and successive visualization of a wide variety of biomolecule conjugates on mica and related surfaces.

\subsection{REFERENCES}

[1] P. W. K. Rothemund, Nature 2006, 440, 297-302.

[2] P. Vongchan, Y. Wutti-In, W. Sajomsang, P. Gonil, S. Kothan, R. J. Linhardt, Carbohydr. Polym. 2011, 85, 215-220.

[3] J. F. Rusling, C. V. Kumar, J. S. Gutkind, V. Patel, Analyst 2010, 135, 24962511.

[4] H. Schönherr, ACS Nano 2015, 9, 12-15.

[5] a) Z.-H. Liu, N. M. D. Brown, A. McKinley, Appl. Surf. Sci. 1997, 108, 319332; b) B. Lego, M. François, W. G. Skene, S. Giasson, Langmuir 2009, 25, 53135321.

[6] a) T. H. Anderson, J. Yu, A. Estrada, M. U. Hammer, J. H. Waite, J. N. Israelachvili, Adv. Funct. Mater. 2010, 20, 4196-4205; b) Y. Li, M. Qin, Y. Li, Y. Cao, W. Wang, Langmuir 2014, 30, 4358-4366.

[7] a) B. P. Lee, P. B. Messersmith, J. N. Israelachvili, J. H. Waite, Annu. Rev. Mater. Res. 2011, 41, 99-132; b) C. E. Brubaker, P. B. Messersmith, Langmuir 
2012, 28, 2200-2205; c) T. Kang, D. X. Oh, J. Heo, H.-K. Lee, S. Choy, C. J. Hawker, D. S. Hwang, ACS Appl. Mater. Interfaces 2015, 7, 24656-24662.

[8] H. Lee, N. F. Scherer, P. B. Messersmith, Proc. Natl. Acad. Sci. U. S. A. 2006, 103, 12999-13003.

[9] G. P. Maier, M. V. Rapp, J. H. Waite, J. N. Israelachvili, A. Butler, Science 2015, 349, 628-632.

[10] K. E. Dunn, F. Dannenberg, T. E. Ouldridge, M. Kwiatkowska, A. J. Turberfield, J. Bath, Nature 2015, 525, 82-86.

[11] C. Lim, J. Huang, S. Kim, H. Lee, H. Zeng, D. S. Hwang, Angew. Chem. Int. Ed. 2016, 55, 3342-3346; Angew. Chem. 2016, 128, 3403-3407.

[12] a) S. Hong, J. Kim, Y. S. Na, J. Park, S. Kim, K. Singha, G.-I. Im, D.-K. Han, W. J. Kim, H. Lee, Angew. Chem. Int. Ed. 2013, 52, 9187-9191; Angew. Chem. 2016, 128, 13933-13937 b) J. Zhou, C. Wang, P. Wang, P. B. Messersmith, H. Duan, Chem. Mater. 2015, 27, 3071-3076.

[13] a) M. Rodenstein, S. Zürcher, S. G. P. Tosatti, N. D. Spencer, Langmuir 2010, 26, 16211-16220; b) T. Gillich, C. Acikgöz, L. Isa, A. D. Schlüter, N. D. Spencer, M. Textor, ACS Nano 2013, 7, 316-329; c) S. P. Pujari, L. Scheres, A. T. Marcelis, H. Zuilhof, Angew. Chem. Int. Ed. 2014, 53, 6322-6356; Angew. Chem. 2014, 126, 6438-6474.

[14] M. Giesbers, A. T. M. Marcelis, H. Zuilhof, Langmuir 2013, 29, 4782-4788.

[15] E. D. Goddard-Borger, R. V. Stick, Org. Lett. 2007, 9, 3797-3800.

[16] a) M. E. Monge, G. A. Harris, P. Dwivedi, F. M. Fernandez, Chem. Rev. 2013, 113, 2269-2308; b) J. H. Gross, Anal. Bioanal. Chem. 2014, 406, 63-80; c) R. 
B. Cody, J. A. Laramée, H. D. Durst, Anal. Chem. 2005, 7\%, 2297-2302; d) R. Sen, J. Escorihuela, M. M. J. Smulders, H. Zuilhof, Langmuir 2016, 32, 3412-3419; e) R. Sen, J. Escorihuela; van Delft, F. L.; Zuilhof, H., 2017, Angew. Chem. Int. Ed. 10.1002/anie.201612037; Angew. Chem. 2017, 10.1002/ange.201612037.

[17] A. Borrmann, O. Fatunsin, J. Dommerholt, A. M. Jonker, D. W. P. M. Löwik, J. C. M. van Hest, F. L. van Delft, Bioconjugate Chem. 2015, 26, 257-261.

[18] G. Rasched, D. Ackermann, T. L. Schmidt, P. Broekmann, A. Heckel, M. Famulok, Angew. Chem. Int. Ed. 2008, 47, 967-970 ; Angew. Chem. 2008, 120, 981984.

[19] Y. Li, D. Sen, Nat. Struct. Biol. 1996, 3, 743-747.

[20] a) P. Travascio, Y. Li, D. Sen, Chem. Biol. 1998, 5, 505-517; b) R. R. Carvalho, S. P. Pujari, D. Gahtory, E. X. Vrouwe, B. Albada, H. Zuilhof, Langmuir 2017, 10.1021/acs.langmuir.6b03931. 


\section{Chapter 6:}

\section{General Discussion}

Surface chemistry holds increasing promise in our ever-miniaturizing world allowing for extraordinary surface-to-volume ratios. The drive to decorate molecules on a surface with a controlled architecture and to know their reaction rates is the main issue addressed in this thesis. If the above is addressed beyond reasonable doubt then the author deems it a job well done and worth his modest efforts.

The road taken was a tough one. One that required standing up and staying upright after errors or experimental troubles. This was probably the most reproducible element of my thesis: when things almost seemed to work, something caved in, broke down, turned out to be more complex. Way more complex... The starting-up phase of this work thus took about 2.5 years (of the total of 4): a way had to be built, but rather than focusing on a narrow path, we aimed for a highway towards interfacial kinetics and composition, and we did not stop till it was there, to be driven on many times by ourselves, and hopefully in the future also by others.

To recap, the determination of interfacial kinetics of various reactions posed a significant challenge to the scientific community. This stemmed from the absence of a general analytical technique that can be applied to a wide variety of reactions independent of their nature. In addition to that impediment, the method had to be highly sensitive with very low limits of detection given the minute amount of analytes present on the surface (picomole range). The problem is especially compounded in the early part of the reaction regime where the kinetic information actually often can be found. Being able to follow the initial \% conversion with great precision and high signal-to-noise ratio is perhaps the greatest challenge addressed herein. 
In this thesis, XPS and DART-HRMS have been used in close conjugation to supplement each other, since the latter is a relatively new addition to surface chemist's repertoire that - after development - needed a firm comparison to build up a reputation of its own. The strength of our approach has been underlined by the high correlation between these two independent analytical techniques.

Central to our approach has been the formation of mixed monolayers in case of aluminum oxide substrates. As presented in chapters $\mathbf{2}, \mathbf{3}$ and $\mathbf{4}$, we have succeeded in the rapid formation of a range of stable, covalently bound mixed monolayers. The subsequent development of a general and fast analytical technique to determine the interfacial reaction kinetics, including the activation parameters $\Delta H^{\ddagger}$ and $\Delta S^{\ddagger}$, provided unparalleled insights. We have developed a "MS-ionizable tag" technique, which has been applied for the analysis of surface-bound organic reactions, to the best of our knowledge, for the first time. Thus began the journey...

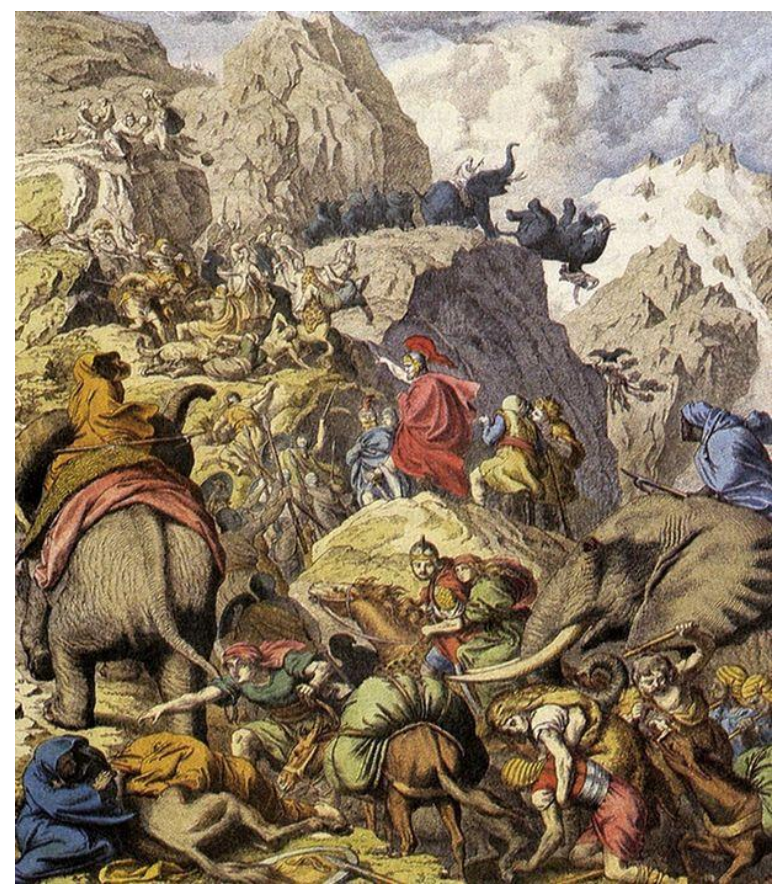

Figure 1: Carthaginian general Hannibal Barca (247 - 181 BCE) crosses the Alps.

The Strain-Promoted Alkyne-Azide Cycloaddition (SPAAC) reaction was chosen as a model reaction given the fact that its kinetics had been well-studied in 
solution. As shown in Chapter 2, the microenvironment around the reactive surface group was carefully controlled by the length of the inert alkyl chains surrounding it.

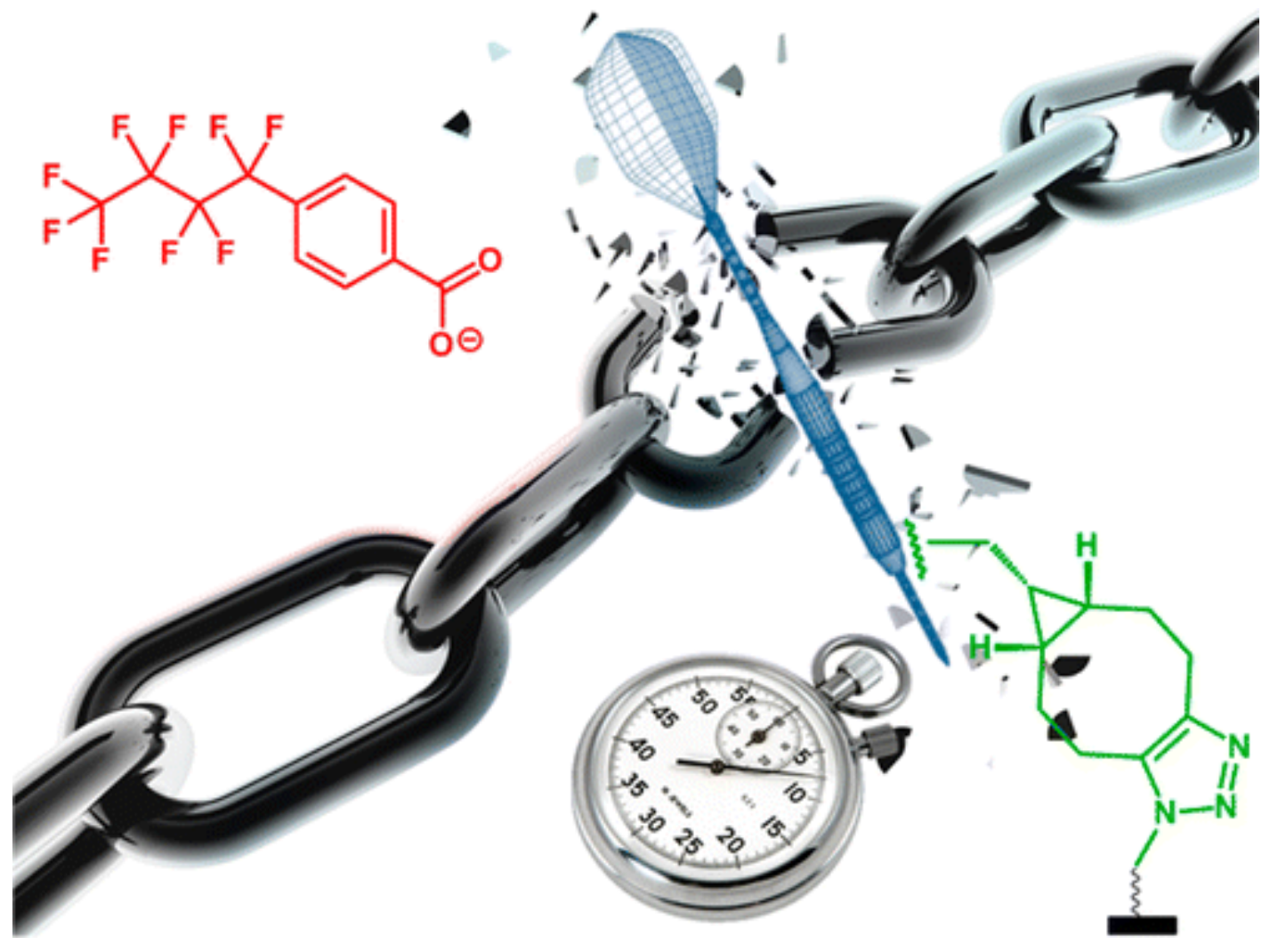

Figure 2: TOC art for Chapter 2.

We observed a few interesting trends which could be of great interest to future surface chemists. First, the SPAAC reaction - which is a click reaction in solution does not retain this nature on the surface (It does not proceed to full conversion and converges sluggishly to around $37 \%$ yield after significant temporal passage). A partially accessible microenvironment, where the motion of reactive groups is slightly restricted, was found to provide a high rate with the highest surface yield. In contrast, a freely accessible reactive moiety afforded a lower surface yield albeit with the highest overall rate. Finally, a buried microenvironment led to the highest overall rate albeit with a lower surface yield. As a corollary, for the surface-bound SPAAC reaction we can compare the partially accessible microenvironment to a marathon runner who is able to run further but at a pace 
slower than a sprinter (free microenvironment). This provides the surface chemist with a handle for tuning the monolayer as per her/his reaction goals.

Harnessing the valuable insights gained from the SPAAC reaction, our concept of ionizable MS tag coupled with DART-HRMS was further extended to a more novel and yet unstudied interfacial reaction in Chapter 3 .

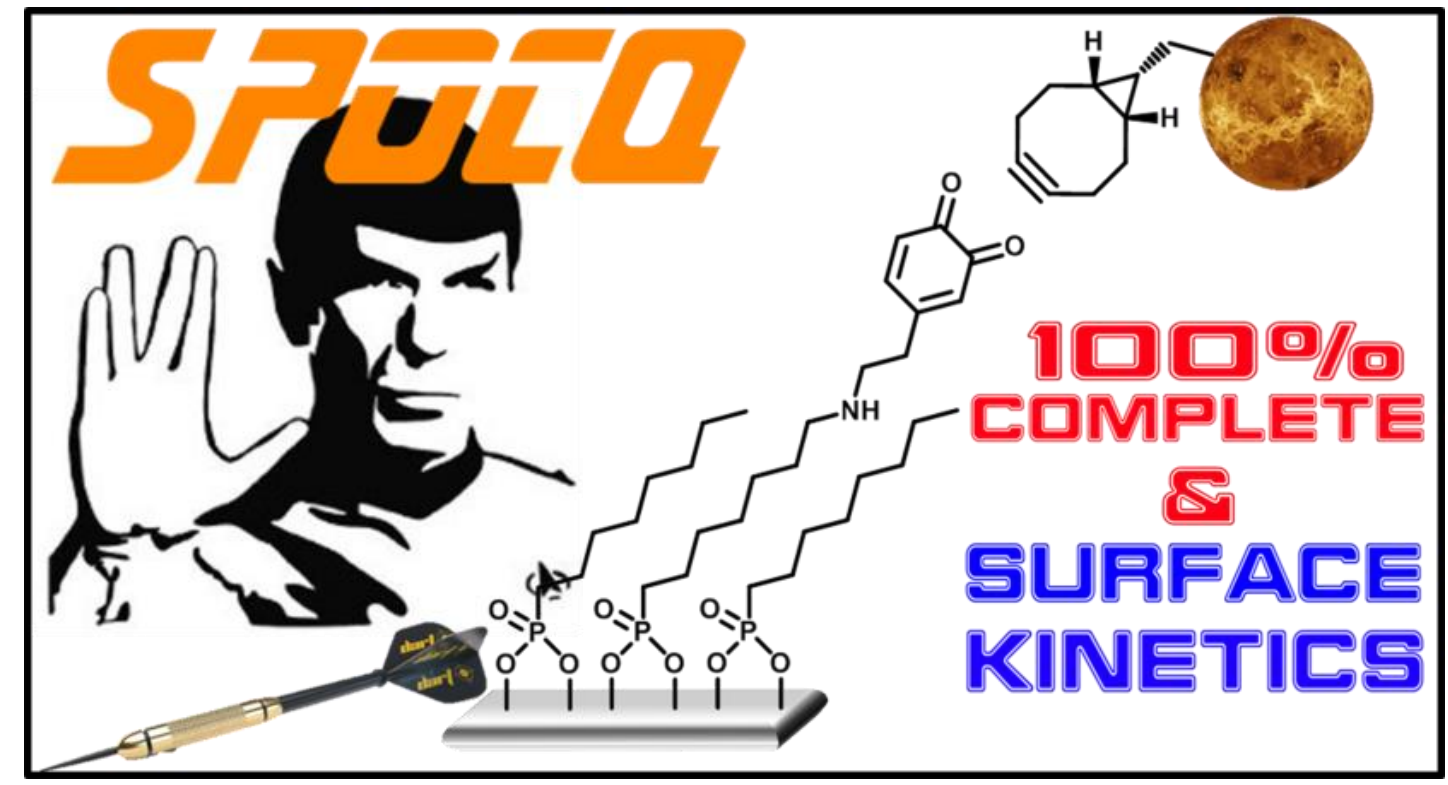

Figure 3: TOC art for Chapter 3.

The Strain-Promoted Oxidation-Controlled cycloalkyne-1,2-Quinone (SPOCQ) cycloaddition was applied for the first time on a surface and afforded a quantitative yield for a free microenvironment in under $4 \mathrm{~h}$. It is to be noted here, that for the first time a $\mathbf{1 0 0 \%}$ (quantitative) metal-free click reaction was observed at a surface. This proved that our approach of engineering the microenvironment around the reactive site provides a distinct edge needed to attain quantitative yields. Quinones are hard to synthesize/store/use in solution given their high propensity to polymerize. However, we demonstrated that on the surface, quinones can be easily generated and stored over-extended period of time by a facile periodate oxidation. Auto-polymerization of surface-bound quinones is precluded by their tether and enforced distal separation by surrounding inert alkyl chains (3:1 ratio). The wider application of this interesting mixture has been further rigorously 
demonstrated in later chapters too. The bioorthogonality of the SPOCQ reaction coupled with its higher speed and its quantitative yields on the surface are definitely its most salient features. Combining the ideas of Chapter $\mathbf{2}$ and $\mathbf{3}$, we can make a mixed monolayer containing azide and quinone termination interspersed with inert alkyl chains to create a well-defined "two click surface". Imaginative thinking can easily increase the number of click partners through careful planning. The strong covalent tethers and controlled architecture of aluminium-phosphonic acid chemistry can even surpass similar work (restricted up to four orthogonal 'clicks') on Au-thiol surface, in which case the low monolayer strength remains an encumbrance.

After studying strain-promoted click reactions on the surface (culminating for SPOCQ in quantitative conversion within $4 \mathrm{~h}$ ), the question arose if DART-HRMS could also be used to reproducibly and precisely determine a different class of cycloadditions, for which we selected the interfacial inverse electron demand Diels-Alder (IEDDA) reaction as this reaction was reported to be really fast -at least for click reactions- in solution.

\begin{tabular}{|c|c|c|}
\hline Name of reaction & $\begin{array}{c}\mathrm{k}^{\prime}\left(\times 10^{-3} \mathrm{~s}^{-1}\right) \\
\text { "Free" } \\
\text { microenvironment }\end{array}$ & $\begin{array}{c}\mathrm{k}^{\prime}\left(\times 10^{-3} \mathrm{~s}^{-1}\right) \\
\text { "Buried" } \\
\text { microenvironment }\end{array}$ \\
\hline SPAAC & $5.7 \pm 0.4$ & $3.1 \pm 0.3$ \\
\hline SPOCQ & $6.4 \pm 0.1$ & $2.9 \pm 0.2$ \\
\hline${\text { IEDDA } e x o-\text { Norbornene }_{(\text {surf.) }}}$ & $10.9 \pm 0.5^{[\mathrm{a}]}$ & $4.7 \pm 0.2^{[\mathrm{a}]}$ \\
\hline IEDDA Tetrazine $_{(\text {surf.) }}$ & $5.8 \pm 0.3^{[\mathrm{a}]}$ & $2.1 \pm 0.2^{[a]}$ \\
\hline
\end{tabular}

Table 1. Concise summary of reaction rates for different metal-free click reactions on the surface for "free" or "buried" microenvironments at $25{ }^{\circ} \mathrm{C}\left({ }^{[a]}\right.$ at $\left.30{ }^{\circ} \mathrm{C}\right)$ 


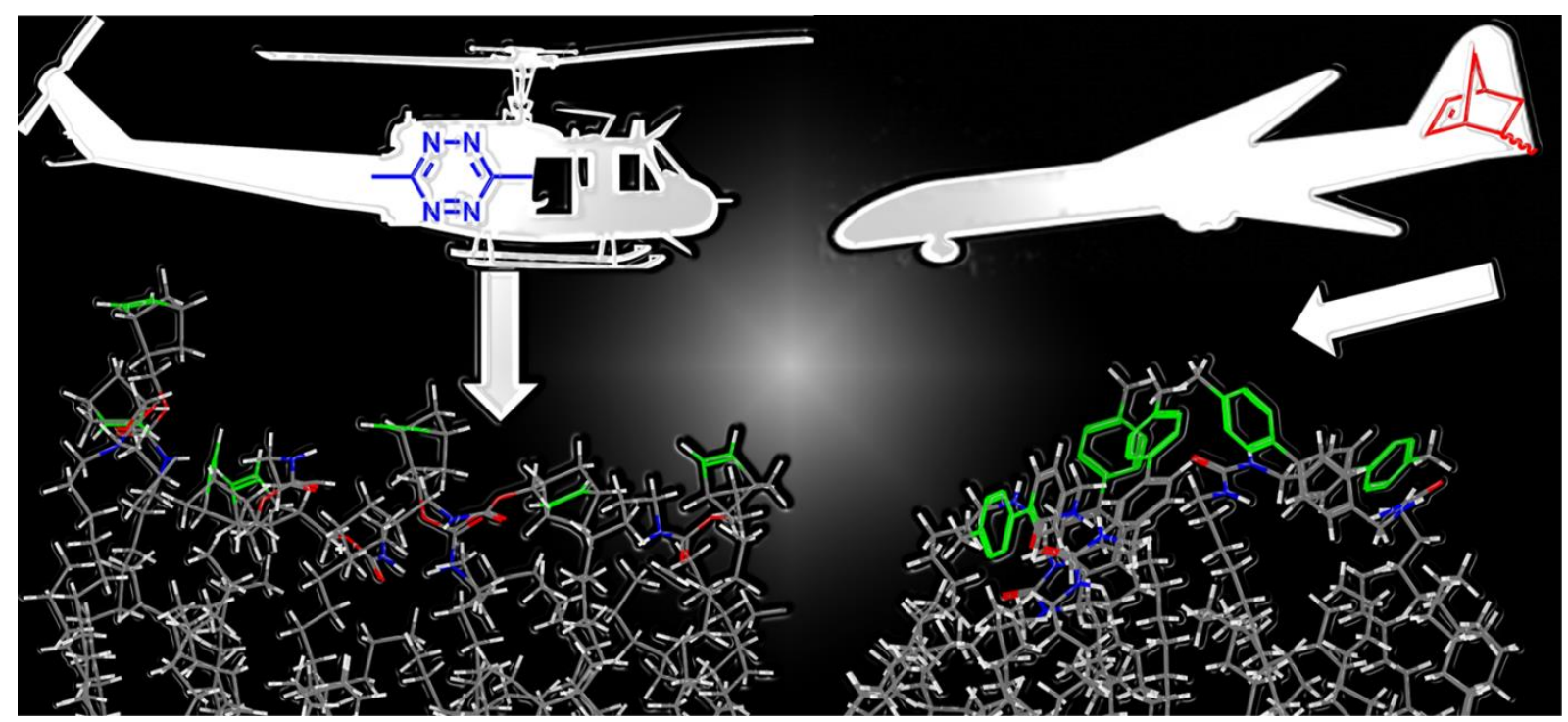

Figure 4: TOC art for Chapter 4.

This was studied in Chapter 4 extensively and we surpassed our previous kinetic record (SPOCQ) by obtaining a quantitative yield in a mere $15 \mathrm{~min}$. The other interesting observation of this study was that reversing the reaction counterparts on the surface produced a discernible reaction rate difference. We found that one of the reactants when tethered in a particular stereochemistry (exo-form) gave the highest surface coverage $(100 \%)$ within the shortest amount of time. This was also the first time that the effect of diastereomerism on interfacial reaction rates was studied. We believe this should open up a whole new avenue for "large scale surface-directed synthesis". An effective insight into surface disposition of stereoisomers on the surface might help in favoring/increasing enantiomeric excess (ee). If one of the reactant is oriented in such a way that the surface, the neighboring inert chains and the target group itself, work in conjunction to achieve approach of reacting species from only one chirally active face (Re or $\mathrm{Si}$ ), in principle a high ee could be achieved.

The above three chapters lend credence to our belief that mixing adsorbates of differing lengths during monolayer assembly does not necessarily form distinct domains. If there was indeed a clustering of similar chains (azides/ quinones/ norbornenes and tetrazines) surrounded by a "sea" of inert alkyl chains, then the 
reaction yield should have an inverse relationship with increasing dilution of the monolayer. In contrast, we observed that such relationship was not found in our case. Instead, we consistently found an optimum dilution of $3: 1$ ratio of alkyl to reactive group that provided the best surface yields. Based on these observations, we hypothesize that in our case mixed monolayer formation occurs in a random fashion with 3:1 being the best scenario i.e. provides adequate space for the final cycloadduct while being sufficiently concentrated for the reaction to be completed within a measureable time frame.

In Chapter 5, covalent modification of native non-activated mica has been carried out utilizing catechol linkers. Previous studies for mica modification produced poorly defined polymeric structures on the surface or required extensive and tedious organic synthesis. We have addressed both these issues head-on in this thesis. Welldefined and characterized ultrathin layers were constructed on mica using a catecholbased molecule involving a two-step synthesis. This opens the pathway for the attachment of more complex architectures on the surface with higher functionality along with the ability to study their formation in a step-wise controlled fashion. Mica being atomically flat provides an ideal surface upon which to study various phenomena by AFM and other forms of microscopy. However, most research until now was restricted to simply drop-casting the pre-fabricated moieties followed by studying their final structures. Our method now allows for the step-wise formation and characterization of these very interesting structures. Along with it, we also performed several click attachment chemistries on these ultrathin layers which can be harnessed by surface chemists to put various functional and structurally complex moieties on the surface. The above said research was also prominently featured as a "Hot Paper" with accompanying frontispiece of Angewandte Chemie International Edition, 2017, Volume 56, Issue 15 as shown in Figure 5. 


\begin{tabular}{|c|c|c|c|}
\hline GDCh & \multicolumn{2}{|c|}{ Communications } & Angewandte \\
\hline Surface Chemistry & Hot Paper & & $\begin{array}{l}.1002 / \text { anie. } 20170130 \\
.1002 / \text { ange. } 20170130\end{array}$ \\
\hline
\end{tabular}

\section{Ultrathin Covalently Bound Organic Layers on Mica:} Formation of Atomically Flat Biofunctionalizable Surfaces

Rickdeb Sen ${ }^{+}$, Digvijay Gahtory ${ }^{+}$, Rui Rijo Carvalho, Bauke Albada, Floris L. van Delft, and Han Zuilhof*

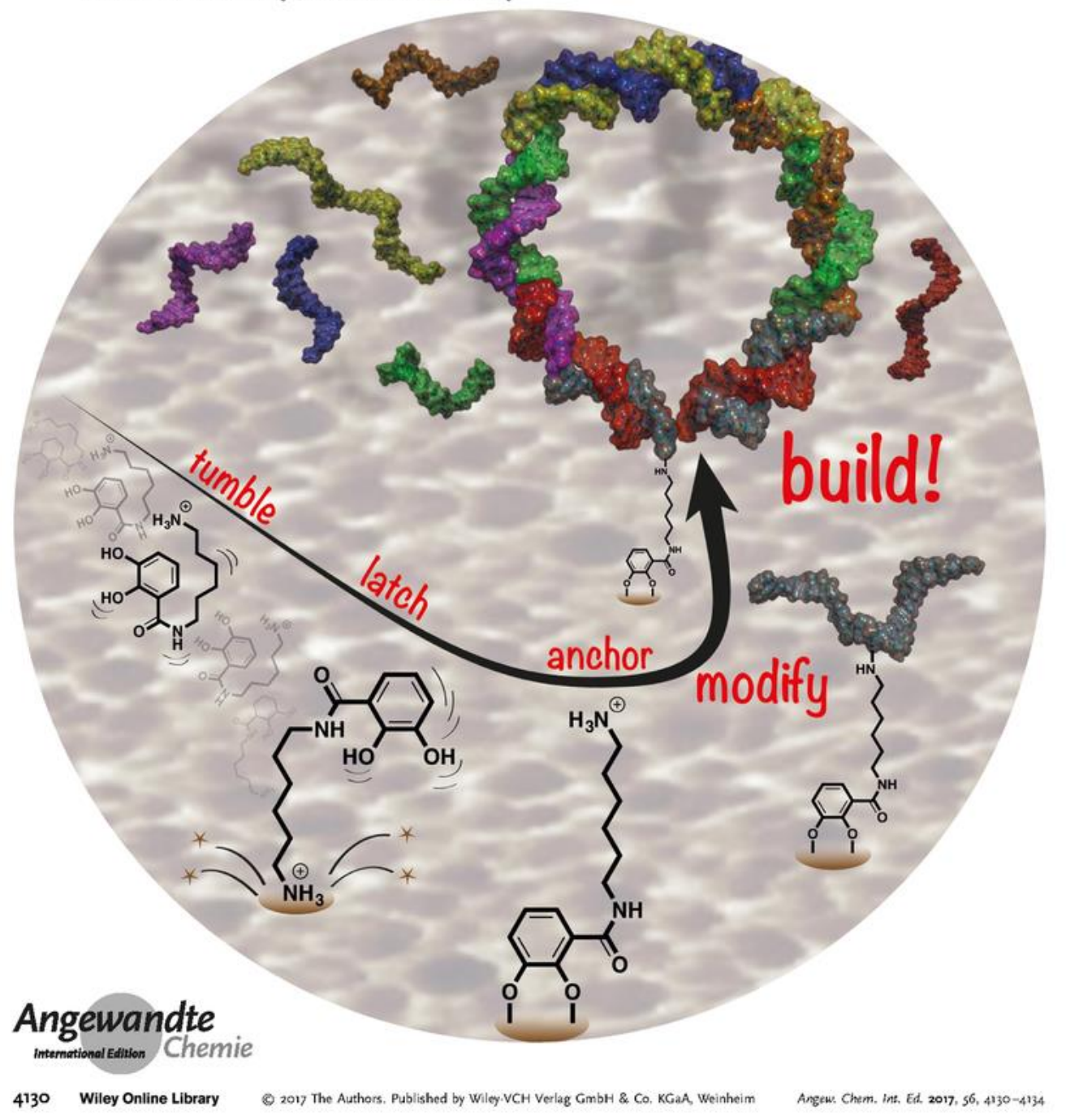

Figure 5: Frontispiece in Angewandte Chemie International Edition for Chapter 5.

\section{WHAT WOULD BE AN IDEAL TAG AND FUTURE PERSPECTIVES.}

Since this thesis almost entirely focuses on "indirect" or tag-based interfacial kinetics it pays to contemplate on the virtues of a good tag. An implicit question 
which arises at the end of our investigations is the role the tag itself plays in influencing the reaction kinetics especially in the latter stages of the reaction where diffusion plays a much more significant role. Since, the valuable kinetic information often lies mostly in the initial kinetic regime (i.e. first $10 \%$ of a conversion) we can assume this effect to be minor. However, one can argue from a steric perspective that a smaller tag would alleviate this conundrum. We have already tested this hypothesis in our own research in terms of moving away from a bulkier $p-\mathrm{C}_{4} \mathrm{~F}_{9}$ tag to a $p-\mathrm{CF}_{3}$ tag. Technically, even a single $\mathrm{F}$ atom used as tag would be detectable by mass spectrometry but independent corroboration by XPS would be near-impossible due to the extremely low signal-to-noise ratio in our diluted microenvironment.

An interesting avenue that can be considered while using such tags for mass spectrometry on surfaces is the use of independent and orthogonal mass fragments during the course of a kinetic measurement. Let us consider a reaction, where a surface-bound component (A) is able to react simultaneously with 2 different solution counterparts (B and C) albeit at different rates. Affixing different mass tags on each of the solution counterparts (B-MS tag 1 and C-MS tag 2) should in theory allow one to independently follow their incorporation on the surface. Now this requirement of great similarity of tags raises a great predicament for most conventional surface analysis techniques which would fail to differentiate between them (AFM, GATR-IR, $\mathrm{UV}$-vis etc.). For example, $-\mathrm{CF}_{3}$ and $-\mathrm{C}_{2} \mathrm{~F}_{5}$ tag-based kinetics would be difficult to be follow using XPS, as their cumulative signal will not give useful information about the rates of individual incorporation and hence their individual kinetics. Thus a solution has to be found. This is where mass spectrometry shines compared to other analytical techniques as we can use two mass tags with the same number of atoms but with an isotope thrown in for differentiation (say, $-\mathrm{CH}_{3}$ and ${ }^{13} \mathrm{CH}_{3}$ or say, $-\mathrm{CH}_{3}$ and $\left.-\mathrm{CH}_{2} \mathrm{D}\right)$. We can assume them to behave identically in terms of ionization, 
sterics, approach parameters albeit with easy distinction in mass spectrometric analysis.

In my opinion, this is a fundamental change one can incorporate in a tag while getting meaningful signal by DART-HRMS. This kind of isotope labeling on the surface would also allow for greater understanding of how different reactions compete on a molecular scale with the limited surface reaction sites. Perhaps another factor one can think of is tuning the microenvironment on the surface whereby providing an edge to an otherwise sluggish reaction. This could result from more favorable hydrophobic interactions between the microenvironment and one of the solution counterparts. This further opens up a new avenue to determine how we can better tailor our surfaces and the extremely vital microenvironment to produce an intricate pattern of intermingling reagents on the surface.

Next, I would like to go a step further and argue: do we not need a tag at all? Precluding the tag would bypass any of the aforementioned parameters associated with it and should indeed be a better solution. So would tag-less monitoring of reaction rates on surfaces be feasible? Together with Digvijay Gahtory I have demonstrated in our study "Surface-bound quadruple H-bonded dimers: Formation and exchange kinetics", (List of publications, Faraday Discussions, 2017, accepted) that no tag is required altogether with the entire molecule being used for following kinetics. In this work, we have cleaved off of the surface $\mathrm{H}$-bound ureidopyrimidinone (UPy) moieties "as a whole" and thus the kinetics of its surface incorporation could be elucidated in a "direct" manner. In our initial studies where an independent analytical technique required strong XPS signals we restricted ourselves to an "indirect" approach.

However, one has to keep in mind the surface attachment has to be weak (non-covalent, reversible) or hydrolyzable so that the elevated temperatures of the DART metastable He beam will suffice to get the analytes desorbed and ionized and 
hence detected by mass spectrometric analysis. Thus, our DART HRMS-based approach would be a very efficient technique to especially study supramolecular interactions. If the host or guest is tailor-made to be easily ionizable and volatile, while not reverting to its non-charged native form during the ambient desorption/ionization process, then we can ensure higher signal-to-noise ratio in the studies. This would allow us to understand supramolecular kinetics with high precision on surfaces. We have laid the groundwork for such studies in the aforementioned work, which has focused on "metal-free click reactions", and would argue that a much wider field of applications is just around the conceptual corner.

Overall, this thesis wishes to understand organic surface chemistry and several of its intricate mysteries. It clearly outlines several modification techniques and unravels interfacial kinetics of several interesting "metal-free click reactions". It strives to rationalize the activation parameters in conjunction with classical organic chemistry and gives details on how surrounding "inert" alkyl chains can play a profound role in reaction rates. Lastly, we have striven to and achieved rapid and quantitative reactions on the surface by virtue of optimization of this microenvironment. Personally I believe, we have treaded on a road seldom traveled and unraveled a new understanding about molecular interactions on the everinteresting and an infinitely-complex surface...

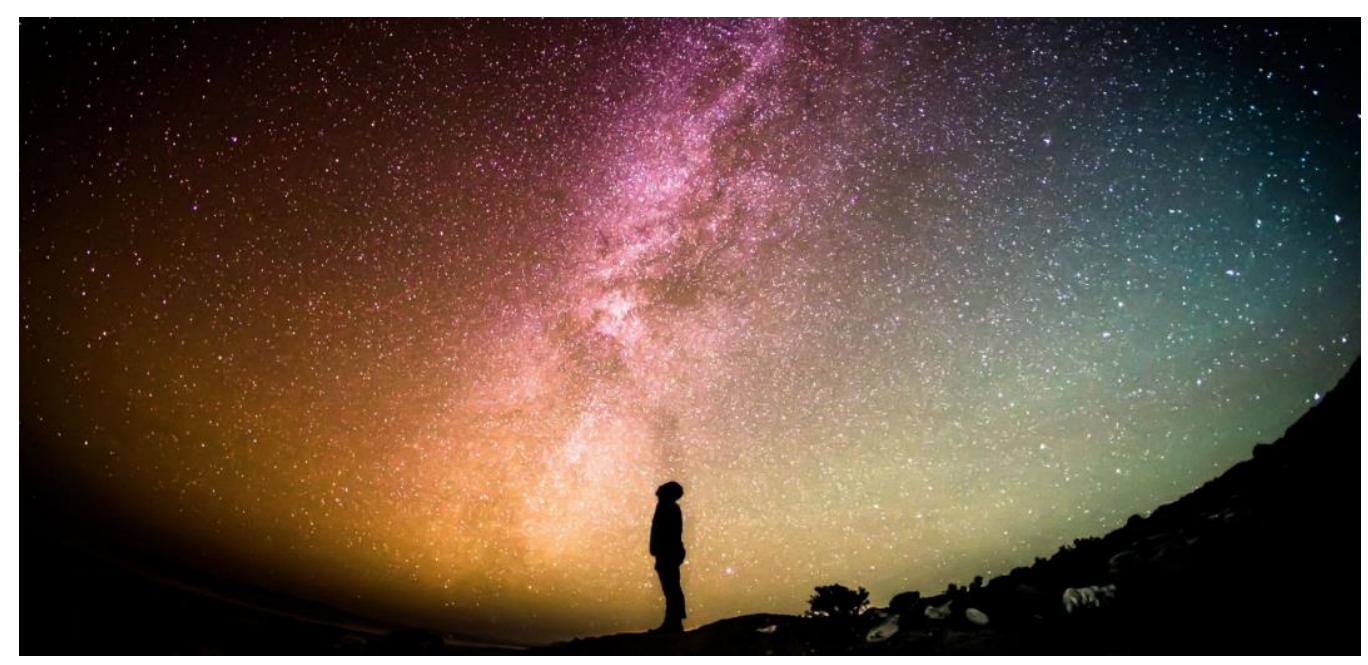

Figure 6: Staring at the stars helps understand how modest this effort was. 


\section{Appendix 1}

Use of Ambient Ionization High-Resolution Mass

Spectrometry for the Kinetic Analysis of Organic

Surface Reactions

For full Supporting Information look at online ESI available in ACS Langmuir website at la6b00427 si $001 . p d f(2.17 \mathrm{MB})$

\section{Table of Contents}

1.1. Methods

1.2. Chemical Synthesis.

1.3. Supplementary Figures

1.4. Supplementary Tables ....

1.5. NMR spectra 


\subsection{Methods}

1.1.1. General. Unless otherwise specified, all chemicals were used as received without further purification. Octylphosphonic acid, hexadecylphosphonic acid, hydrochloric acid, methanol, hexane, acetone, dichloromethane, 2-propanol were purchased from Sigma-Aldrich. 12-Azidododecylphosphonic acid, dodecylphosphonic acid were purchased from SiKÉMIA. Aluminium pieces (99.5\% purity, mirror polished, Staalmarkt Beuningen BV) were cut using mechanical cutter into exactly $2 \times 1 \mathrm{~cm}$ dimension. For surface modification reactions, the samples were loaded onto a specially constructed PTFE wafer holder able to hold up to 16 samples at a time thus ensuring rigorous reproducibility between samples.

1.1.2. Preparation of phosphonic acid monolayers. $2 \times 1 \mathrm{~cm}$ Al slides were sonicating in hexane for $15 \mathrm{~min}$ followed by wiping with lint-free cotton swabs (Texwipe, NC, USA) to remove polymer protection layer on top and remove any residual glue. The surfaces were chemically activated by immersing in 1:1 (v/v) $37 \%$ $\mathrm{HCl}-\mathrm{MeOH}$ mixture for $5 \mathrm{~min}$, followed by washing with water and 2-propanol. The activated surfaces were then immersed into $\mathrm{N}_{2}$ filled vials containing a $5 \mathrm{mM}$ solution in 2-propanol of the appropriate alkylphosphonic acid (with $\mathrm{C}_{8}, \mathrm{C}_{12}$ or $\mathrm{C}_{16}$ alkyl chain) and 12-azidododecylphosphonic acid, in the required ratio, which was varied from 0:1 up to 9:1. The solution was heated to $50{ }^{\circ} \mathrm{C}$ for $5 \mathrm{~min}$, and then left undisturbed for $5 \mathrm{~h}$ at room temperature to obtain self-assembled mixed monolayers. Then surfaces were taken out and sonicated successively for 5 min with 2 -propanol, acetone and $\mathrm{CH}_{2} \mathrm{Cl}_{2}$. The surfaces were then cleaned with $\mathrm{CH}_{2} \mathrm{Cl}_{2}$, air dried and stored under $\mathrm{N}_{2}$ atmosphere. From static water contact angle (SCA) measurements, it was found that the reaction was complete after $5 \mathrm{~h}$, yielding monolayers with 28 $30 \% \mathrm{C}$ as determined by XPS. Remarkably, substantially longer reaction times (as overnight) contributed to the formation of undesirable multilayers ( $42-44 \% \mathrm{C})$. 


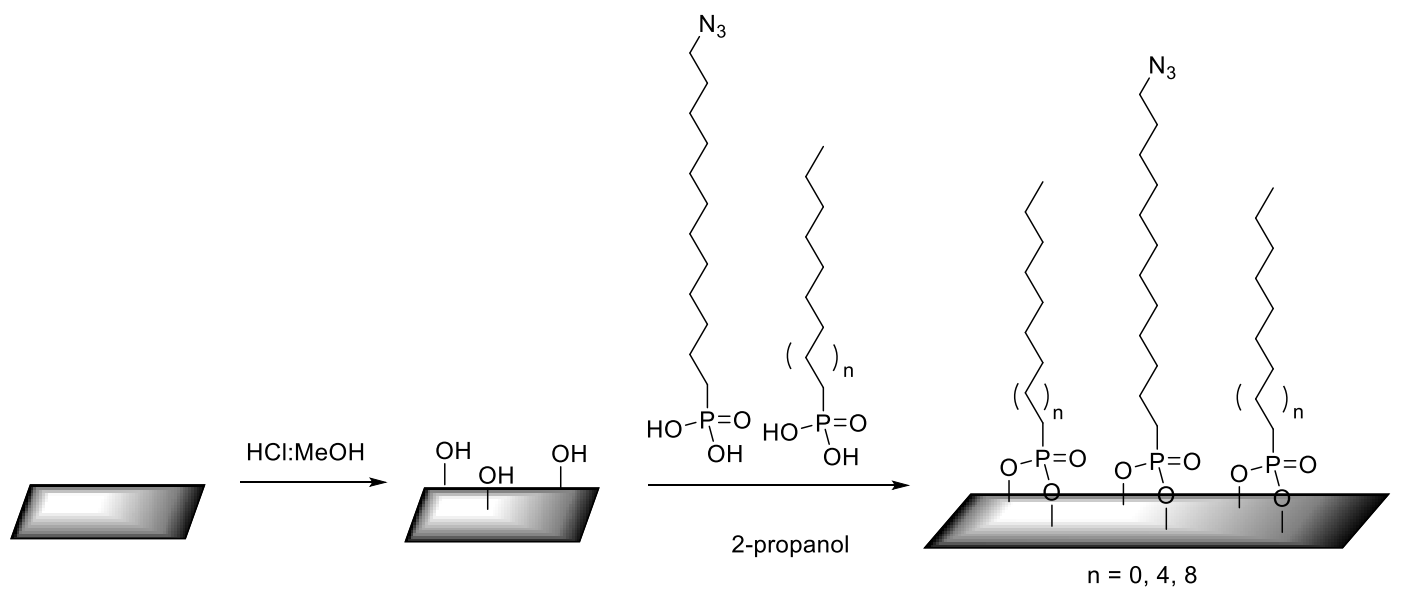

Scheme S1. Preparation of mixed alkyl:azide monolayers.

1.1.3. Interfacial SPAAC reaction. Azide-functionalized slides were immersed in a DCE $3 \mathrm{mM}$ solution of 1 under $\mathrm{N}_{2}$ atm and kept reacting (see Section 1.2.6 for synthesis). After completion of reaction for the desired time, the $\mathrm{Al}$ pieces were taken out, sonicated and washed with acetone to remove physisorbed moieties, dried under $\mathrm{N}_{2}$ stream and immediately used for XPS and DART analysis.
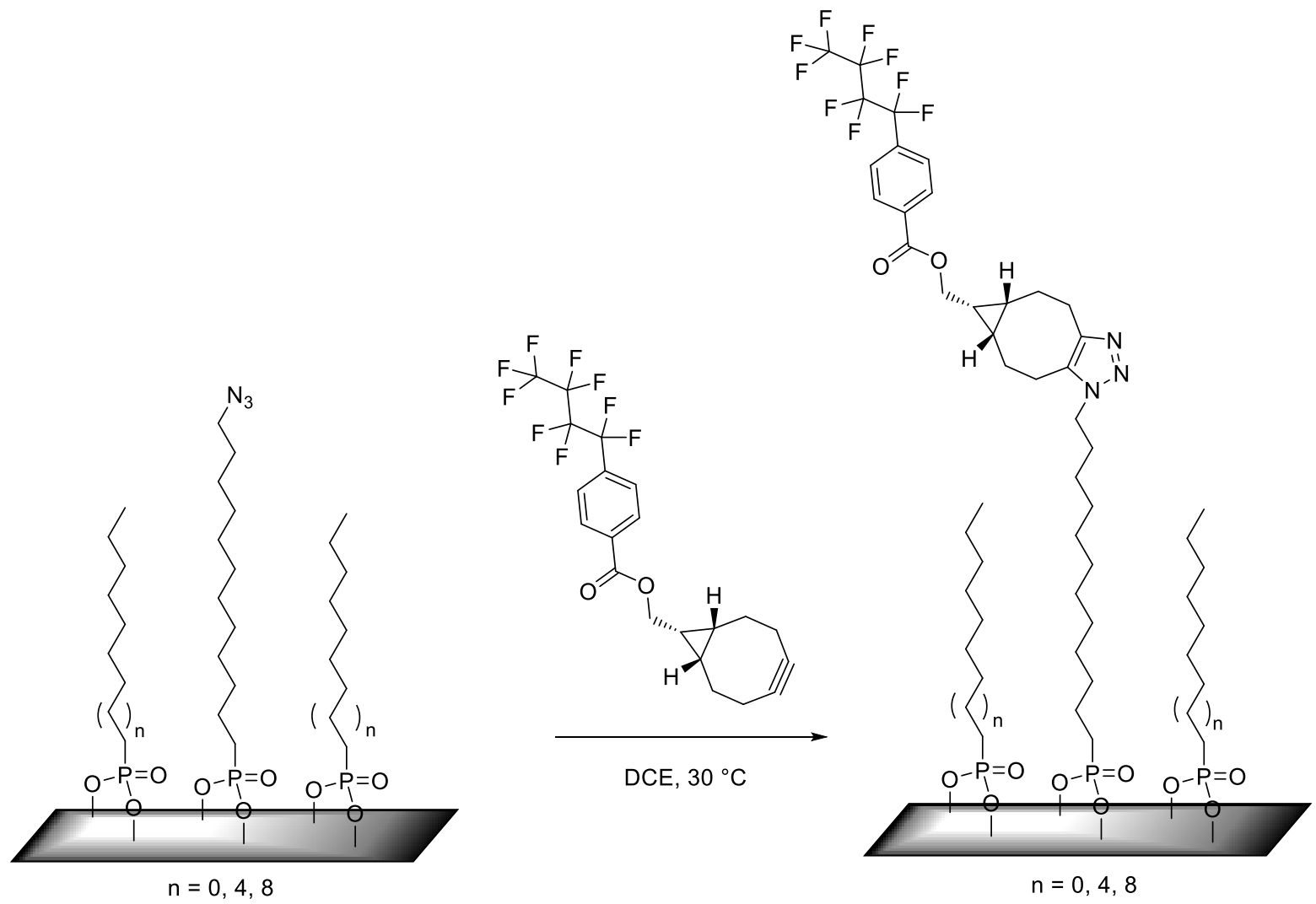

Scheme S2. SPAAC reaction on mixed alkyl:azide monolayers. 
1.1.4. Rate constant determination. Equation 1 describes the rate for the studied reaction $\left(\mathrm{mol} \cdot \mathrm{cm}^{-2} \cdot \mathrm{s}^{-1}\right)$, where $\Gamma_{N}$ is the density of azide groups on the surface $\left(\mathrm{mol} \cdot \mathrm{cm}^{-2}\right)$ and $k$ is the second-order rate constant $\left(\mathrm{M}^{-1} \cdot \mathrm{s}^{-1}\right)$. Because these experiments were carried out under pseudo first-order conditions the amount of BCN derivative $(3 \mathrm{mM})$ is in large excess over the immobilized azides on the surface $\left(\Gamma_{N}=\right.$ $\sim 6 \mathrm{pmol} \cdot \mathrm{cm}^{-2}$ ), the apparent rate constant $k^{\prime}$ can be estimated as described in equation (2), as $k[\mathbf{1}]$. Therefore, from plots of $\ln \left[\left(I_{\infty}-I_{t}\right) /\left(I_{\infty}-I_{0}\right)\right]$ versus time the resulting first-order rate constants $\left(k^{\prime}\right)$ can be obtained from the slope, as shown in equation (3). For determining every rate constant, DART-HRMS measurements for every time data point were performed on six independent surfaces.

$$
\begin{gathered}
v=k[\mathbf{1}] \Gamma_{N}=\frac{d \Gamma_{N}}{d t} \\
v=k[\mathbf{1}] \quad\left([\mathbf{1}] \gg \Gamma_{N}\right) \\
\ln \left(\frac{I_{\infty}-I_{t}}{I_{\infty}-I_{0}}\right)=k^{\prime} t
\end{gathered}
$$

$I_{\infty}$ is the asymptotic (integrated) EIC intensity that is reached as the reaction progress stops. It was determined by curve fitting of the data to an exponential (decay) function, which has two parameters: the rate constant $k^{\prime}$ and $I_{\infty}$. In XPS, $I_{\infty}$ was obtained from the $\mathrm{F} / \mathrm{P}$ ratio after infinite reaction time, when reaching the plateau; $I_{t}$ refers to the $\mathrm{F} / \mathrm{P}$ ratio after a defined reaction time; and $I_{0}$ is the $\mathrm{F} / \mathrm{P}$ ratio at a reaction time $(\mathrm{t}=0)$ and it is equal to zero.

\subsection{Chemical Synthesis}

1.2.1 General Remarks. Unless stated otherwise, solvents and dry solvents like dichloromethane, DMSO, perfluorinated hexane and diethyl ether were purchased from Sigma-Aldrich. Unless stated otherwise all of these chemicals were used without further purification. 
1.2.2. Reaction Handling. Unless stated otherwise all non-aqueous reactions were performed in dried glassware under an atmosphere of argon. All flasks were equipped with rubber septa and reactants were handled using standard Schlenk techniques. Temperatures above the room temperature refer to oil bath temperatures which were controlled by a thermostat. Reactions were magnetically stirred.

1.2.3. ${ }^{1} \mathbf{H}-\mathbf{N M R}$ spectra were recorded at room temperature on a Bruker AVB-400 spectrometer with ${ }^{1} \mathrm{H}$ operating frequency of $400 \mathrm{MHz}$. Unless stated otherwise all spectra were recorded at room temperature in $\mathrm{CDCl}_{3}$ and all chemical shifts are given in $\delta$ units relative to the residual $\mathrm{CHCl}_{3}$ (central line of singlet: $\delta_{\mathrm{H}}=7.27 \mathrm{ppm}$ ). Analyses followed first order and the following abbreviations were used throughout: s $=$ singlet, br. $\mathrm{s} .=$ broad singlet, $\mathrm{d}=$ doublet, $\mathrm{t}=$ triplet, $\mathrm{q}=$ quartet, quin $=$ quintet, $\mathrm{sxt}=$ sextet, sept $=\mathrm{spt}, \mathrm{dd}=$ doublet of doublet, $\mathrm{dt}=$ doublet of triplet, $\mathrm{m}$ $=$ multiplet, $\mathrm{mc}=$ centered multiplet. Coupling constants $(J)$ are given in Hertz $(\mathrm{Hz})$.

1.2.4. ${ }^{13} \mathbf{C}-\mathbf{N M R}$ spectra were recorded at room temperature on a Bruker AVB400 spectrometer with ${ }^{13} \mathrm{C}$ operating frequency of $101 \mathrm{MHz}$. Unless stated otherwise all spectra were recorded at room temperature in $\mathrm{CDCl}_{3}$ and all chemical shifts are given in $\delta$ units relative to the $\mathrm{CDCl}_{3}$ (central line of triplet: $\delta_{\mathrm{C}}=77.0 \mathrm{ppm}$ ). The following abbreviation was used throughout: $\mathrm{s}=$ singlet, $\mathrm{d}=$ doublet, $\mathrm{dd}=$ doublet of doublet. If no coupling constants are given, the multiplicity refers to the ${ }^{1} \mathrm{H}-$ decoupled spectra.

1.2.5. Synthesis of 4-(perfluorobutyl) benzoic acid.

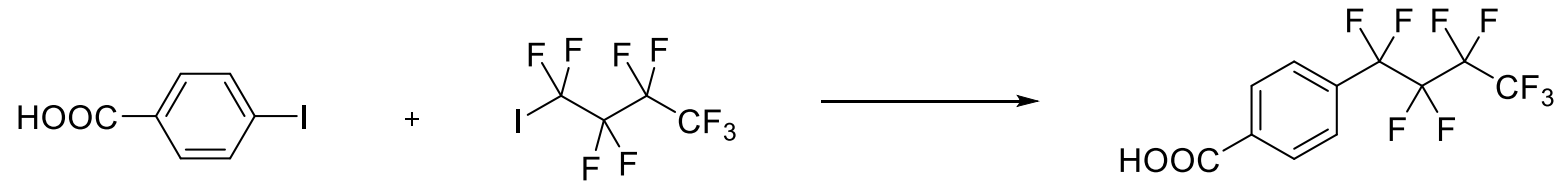

Commercially available nonafluorobutyl iodide was coupled with $p$-iodobenzoic acid using $\mathrm{Cu}$-nanopowder in anhydrous DMSO using established literature procedure. Fluorous phase extraction with perfluorinated hexane by Soxlet continuous extraction 
was used to yield the pure product). Yield: 49\% (GC-MS>99.4\%). IR-ATR ${ }_{\text {max }} 1725$ $(\mathrm{C}=\mathrm{O}) \mathrm{cm}^{-1} \cdot{ }^{1} \mathrm{H}$ NMR (DMSO-d6, $\left.400 \mathrm{MHz}\right) \delta=7.81-7.83(\mathrm{~d}, J=7.8 \mathrm{~Hz}, 2 \mathrm{H}), 8.13$ $-8.15(\mathrm{~d}, J=8.1 \mathrm{~Hz}, 2 \mathrm{H}), 13.48(\mathrm{~s}, 1 \mathrm{H}) \mathrm{ppm} .{ }^{13} \mathrm{C}$ NMR (DMSO-d6, $\left.100 \mathrm{MHz}\right) \delta=$ $114.9(\mathrm{~m}), 118.0(\mathrm{~m}), 120.6(\mathrm{~m}), 123.5(\mathrm{~m}), 132.2,135.1,136.0(\mathrm{t}), 140.0,171.3 \mathrm{ppm}$. ${ }^{19} \mathrm{~F}$ NMR (DMSO-d6, $\left.377 \mathrm{MHz}\right) \delta=-81.0,-11.4,-122.7,-125.5 \mathrm{ppm}$.

1.2.6. Synthesis of $((1 \mathrm{R}, 8 \mathrm{~S}, 9 \mathrm{~S})-$ bicyclo[6.1.0]non-4-yn-9-yl)methyl 4(perfluorobutyl)benzoate (1).

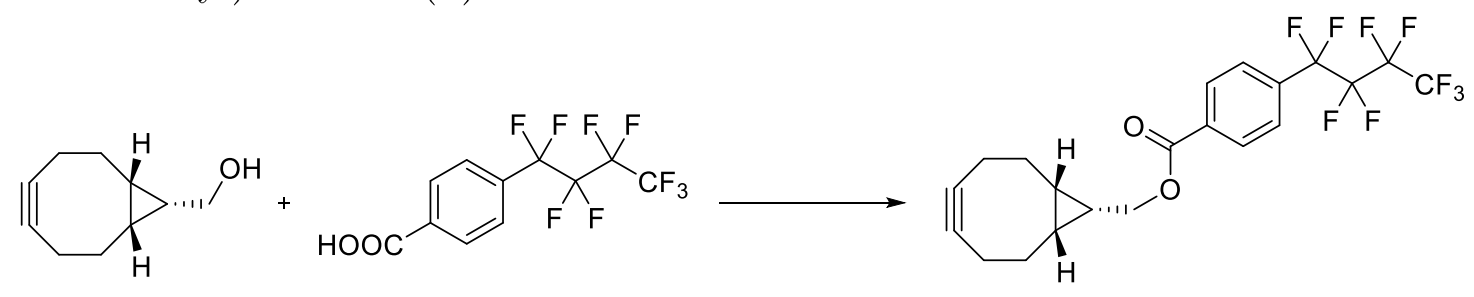

4-(perfluorobutyl) benzoic acid $(0.505 \mathrm{~g}, 2.66 \mathrm{mmol})$ was dissolved in $40 \mathrm{~mL}$ of anhydrous $\mathrm{CH}_{2} \mathrm{Cl}_{2}$ and stirred for $30 \mathrm{~min}$ at room temperature with DCC $(0.550 \mathrm{~g}$, $2.67 \mathrm{mmol})$ and DMAP $(0.162 \mathrm{~g}, 1.33 \mathrm{mmol})$ under $\mathrm{N}_{2}$ atm. To this mixture, endoBCN (0.333 g, $2.217 \mathrm{mmol}$ ) was added under $\mathrm{N}_{2}$ atm and refluxed overnight at 30 ${ }^{\circ} \mathrm{C}$. The DCU was filtered out and product purified by column chromatography $(1: 9$ ethyl acetate: hexane) to afford a colourless oil. Yield: 49\% (GC-MS>99.5\%). IRATR $_{\max } 1719(\mathrm{C}=\mathrm{O}) \mathrm{cm}^{-1} .{ }^{1} \mathrm{H}$ NMR $\left(\mathrm{CDCl}_{3}, 400 \mathrm{MHz}\right) \delta=8.16-8.18(\mathrm{~d}, 2 \mathrm{H}), 7.70$ $7.73(\mathrm{~d}, 2 \mathrm{H}), \quad 4.45-4.47(\mathrm{~d}, 2 \mathrm{H}), 2.29(\mathrm{~m}, 6 \mathrm{H}), 1.67(\mathrm{~m}, 2 \mathrm{H}), 1.58(\mathrm{~m}, 1 \mathrm{H}), 1.05(\mathrm{~m}$, 1H) ppm. ${ }^{13} \mathrm{C} \mathrm{NMR}\left(\mathrm{CDCl}_{3}, 100 \mathrm{MHz}\right) \delta=17.4,20.4,21.4,29.2,63.8,95.8,110.6$, 112.5, 115.4, 121.5, 127.2, 129.8, 132.8, 133.8, 165.5 ppm. ${ }^{19} \mathrm{~F}$ NMR $\left(\mathrm{CDCl}_{3}, 377\right.$ $\mathrm{MHz}) \delta=-83.5(\mathrm{t}, 3 \mathrm{~F}),-113.9(\mathrm{t}, 2 \mathrm{~F}),-125.3(\mathrm{t}, 2 \mathrm{~F}),-128.0(\mathrm{dt}, 2 \mathrm{~F}) \mathrm{ppm}$. HRMS (ESI+): Calculated for $\mathrm{C}_{20} \mathrm{H}_{16} \mathrm{~F}_{9} \mathrm{O}_{2}[\mathrm{M}+\mathrm{H}]^{+} 459.1007$; found 459.1009. 


\subsection{Supplementary Figures}

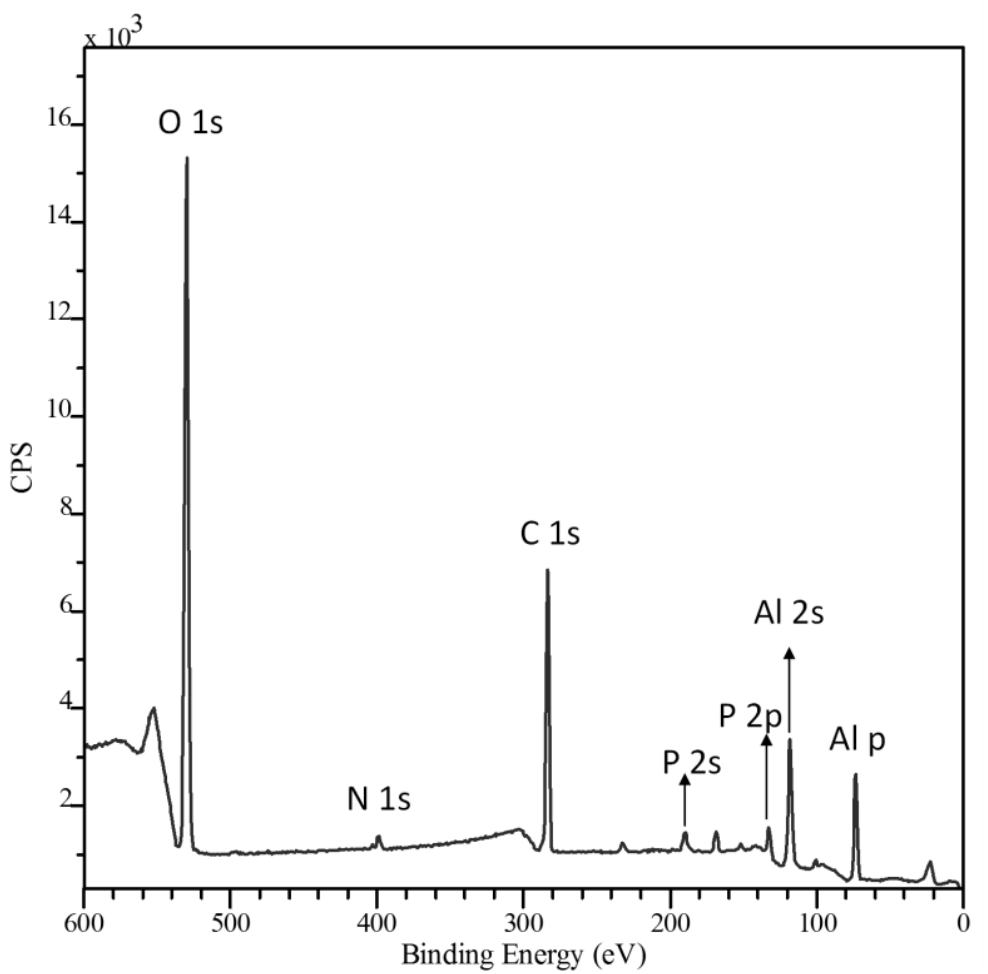

Supplementary Figure S1. XPS spectrum (wide scan) of a 3:1 mixture of octylphosphonic and 12-azidododecylphosphonic acid monolayer onto oxidized aluminium.

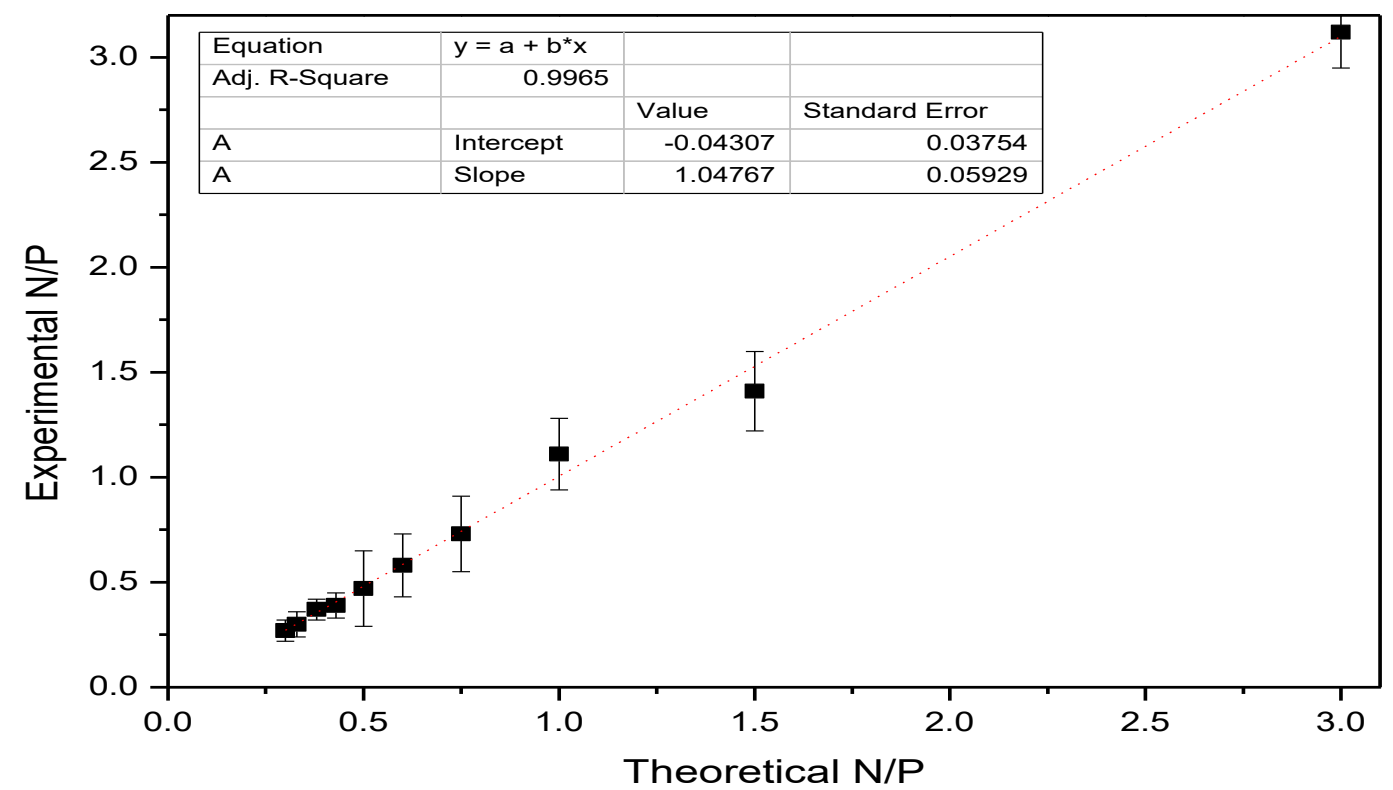

Supplementary Figure S2. Experimental N/P ratio versus theoretical N/P ratio.

The results show that the ratio of azides in a mixed monolayer is identical to the 
ratio of azides in the solution from which the monolayer assembled (within experimental error).

\section{AFTER ETCHING}

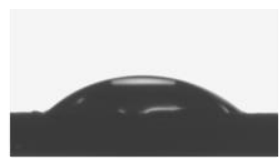

$35.0 \pm 0.9$

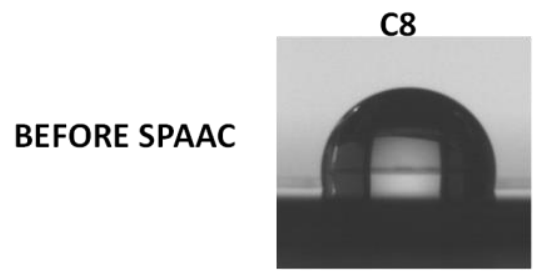

$98.6 \pm 1.0$

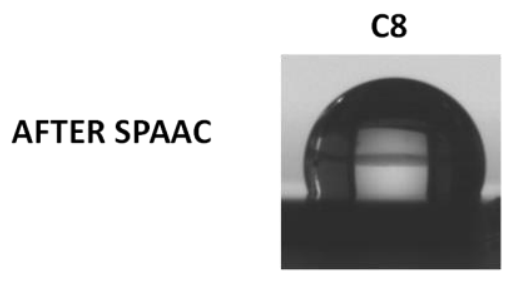

$107.7 \pm 1.2$

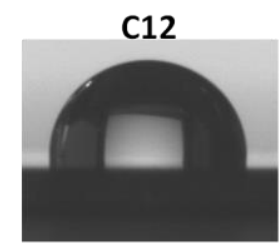

$95.8 \pm 0.8$

C12

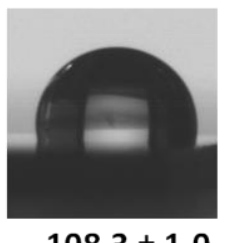

$108.3 \pm 1.0$

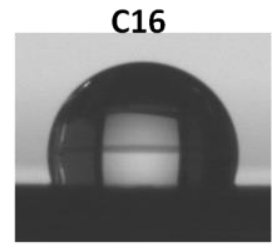

$103.1 \pm 1.1$

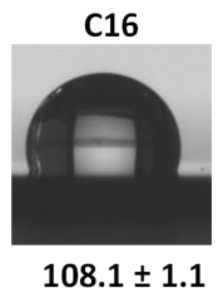

Supplementary Figure S3. SCA for different functionalized surfaces.

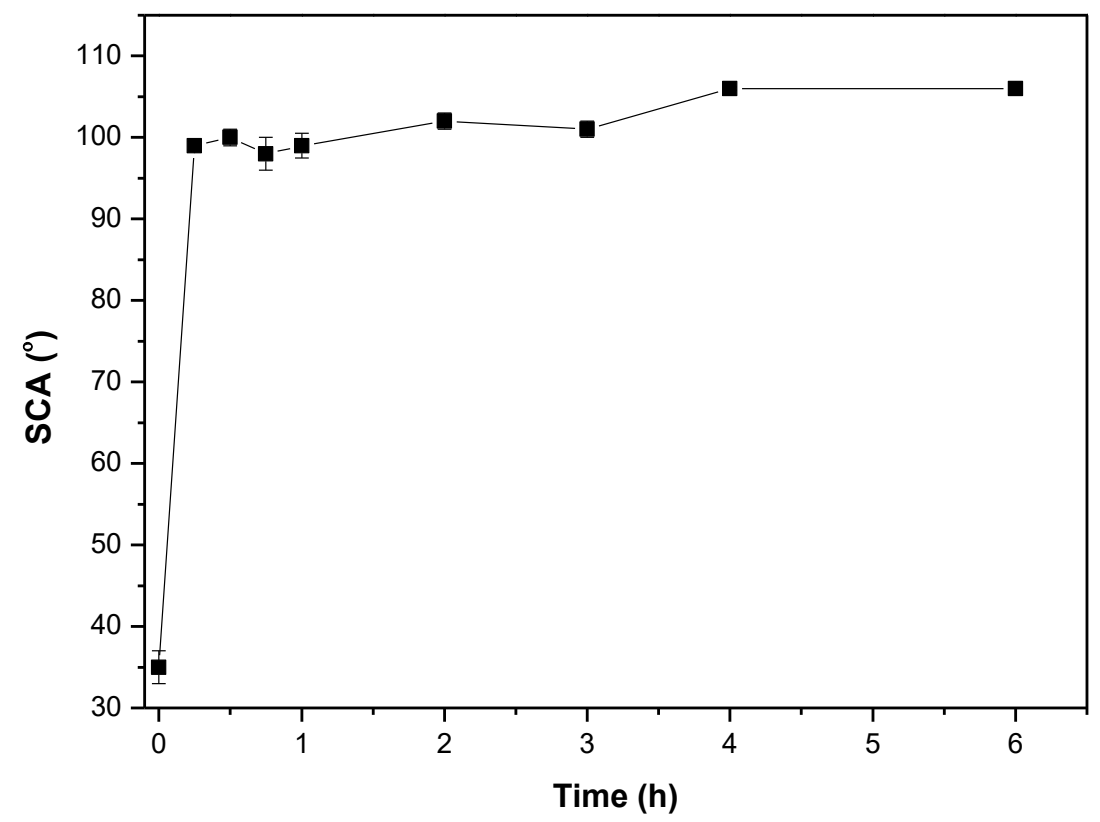

Supplementary Figure S4. Variation of SCA of functionalized 3:1 C8 monolayer. The values are the average of five droplets. 
(a)

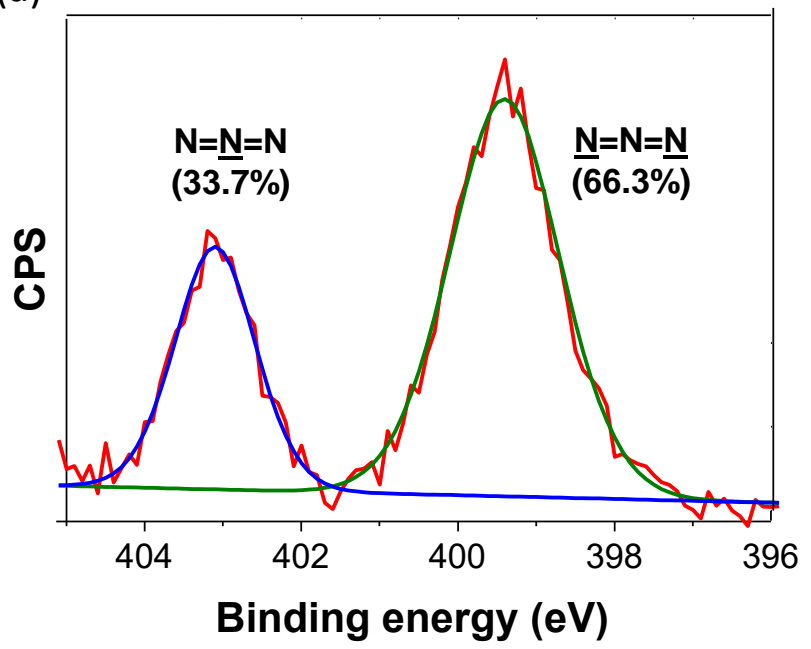

(b)

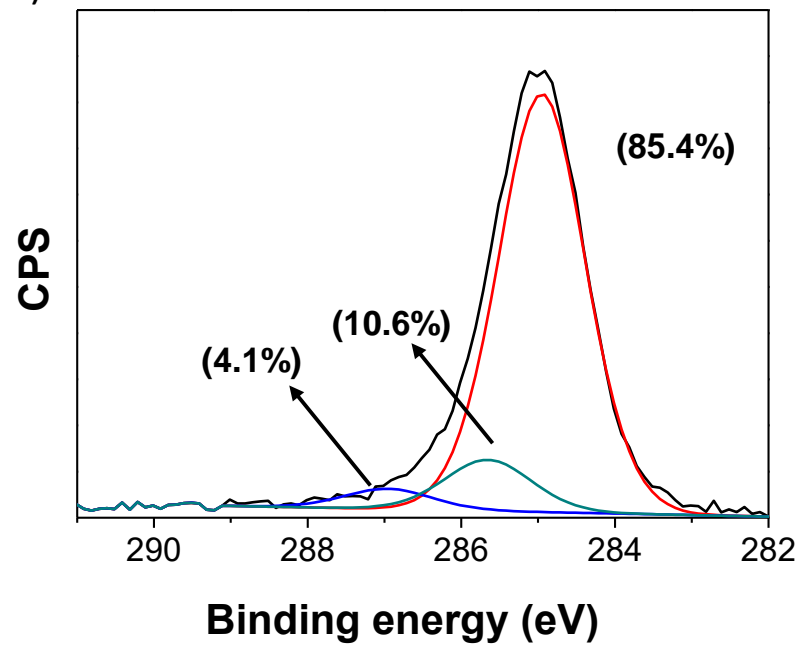

Supplementary Figure S5. High resolution N1s (a) and C1s (b) spectra of a 3:1 mixture of octylphosphonic and 12-azidododecylphosphonic acid monolayer (C8) onto oxidised aluminium.

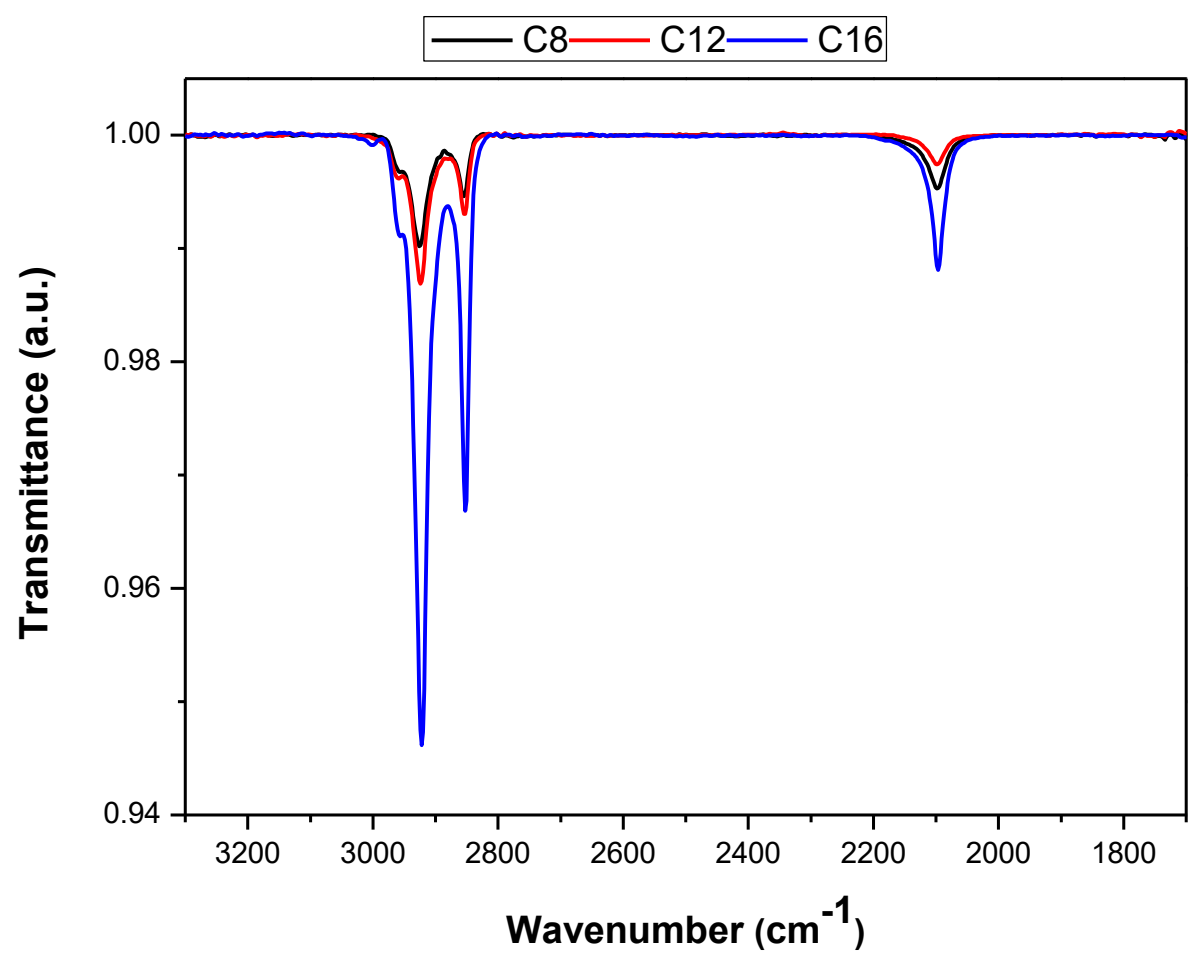

Supplementary Figure S6. IRRAS spectra of 3:1 mixed monolayers of C8, C12 and $\mathbf{C 1 6}$ onto oxidised aluminium. 


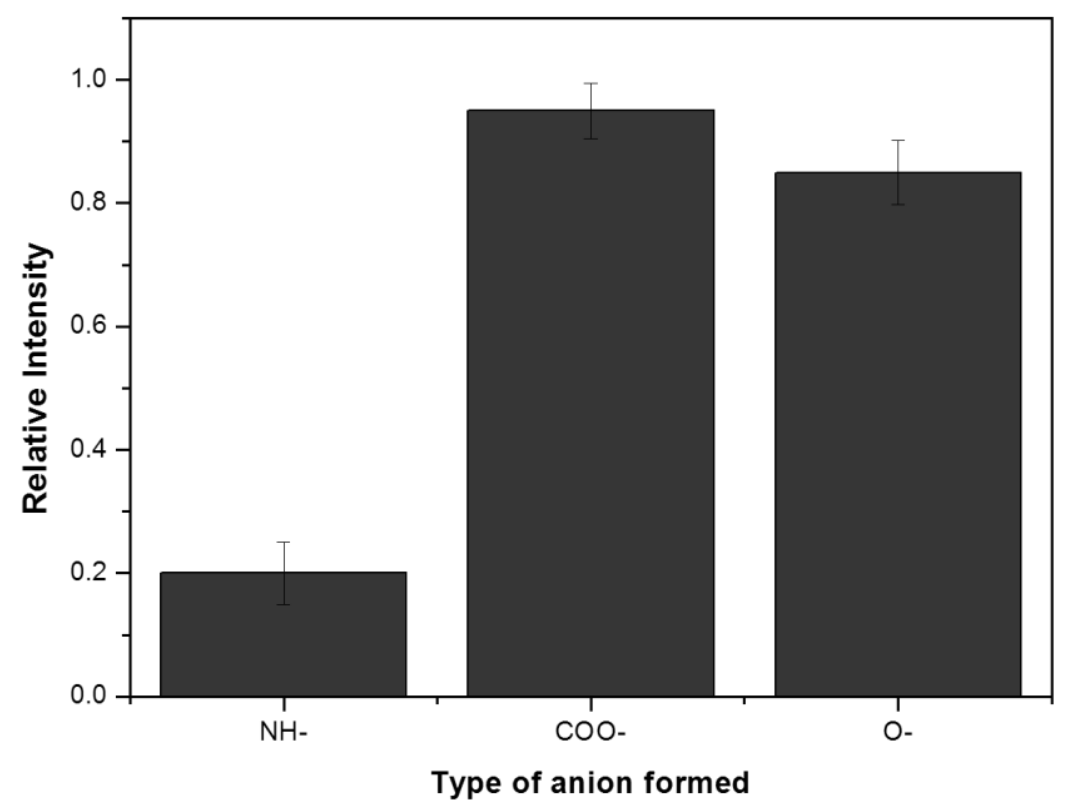

Supplementary Figure S7. Intensities of different anionic derivatives after DART ionization.

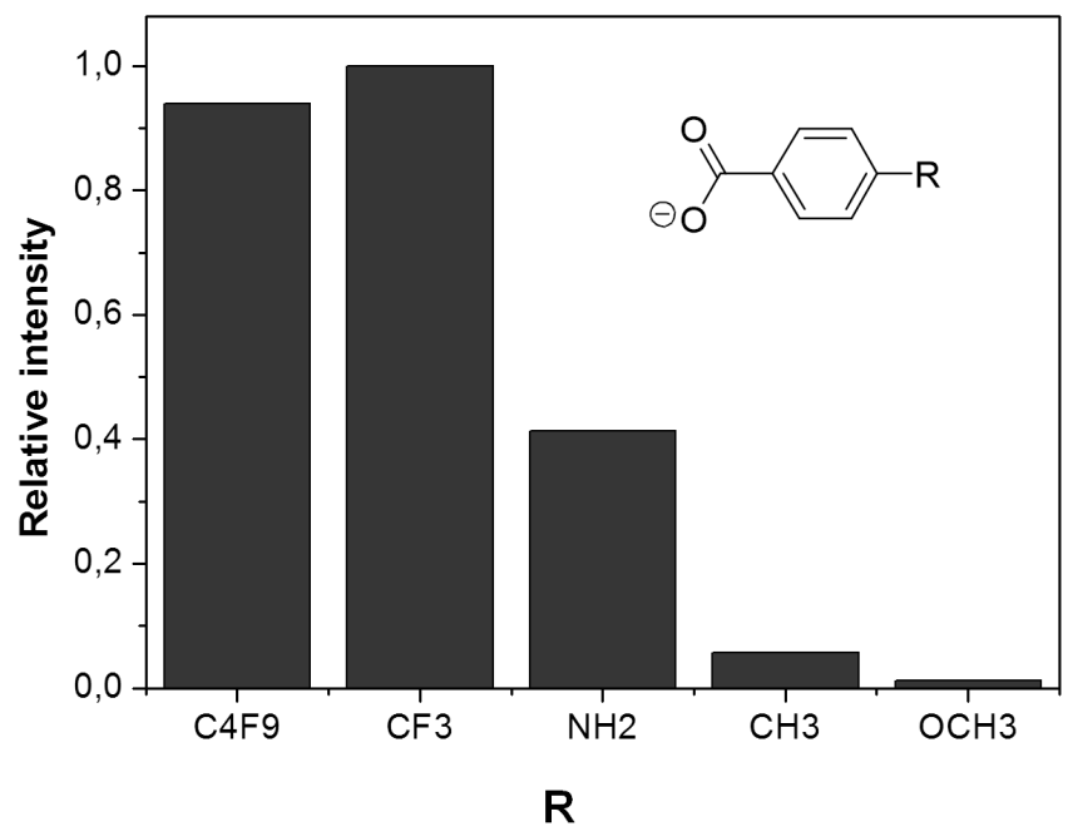

Supplementary Figure S8. Intensities of different benzoate derivatives after DART ionization. 


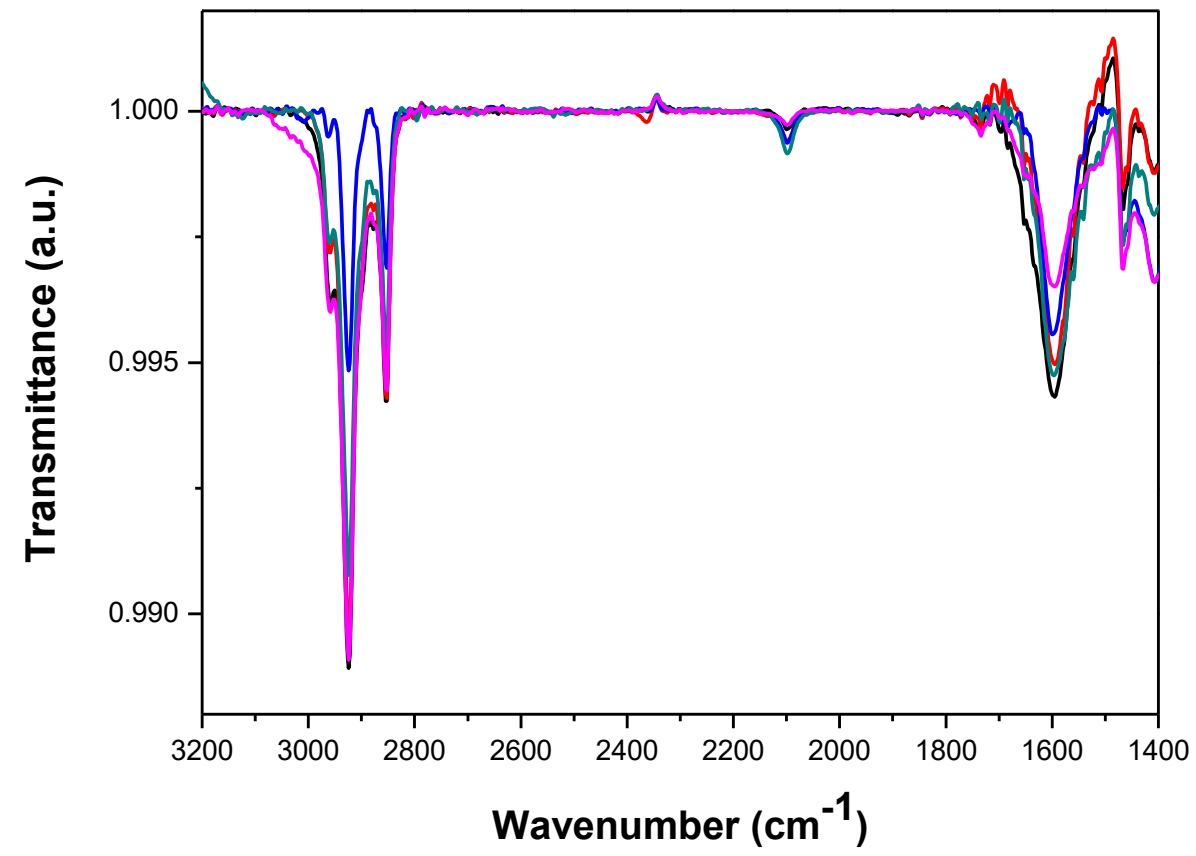

Supplementary Figure S9. IRRAS spectra of 3:1 mixed monolayers of C8 after different SPAAC reaction times.

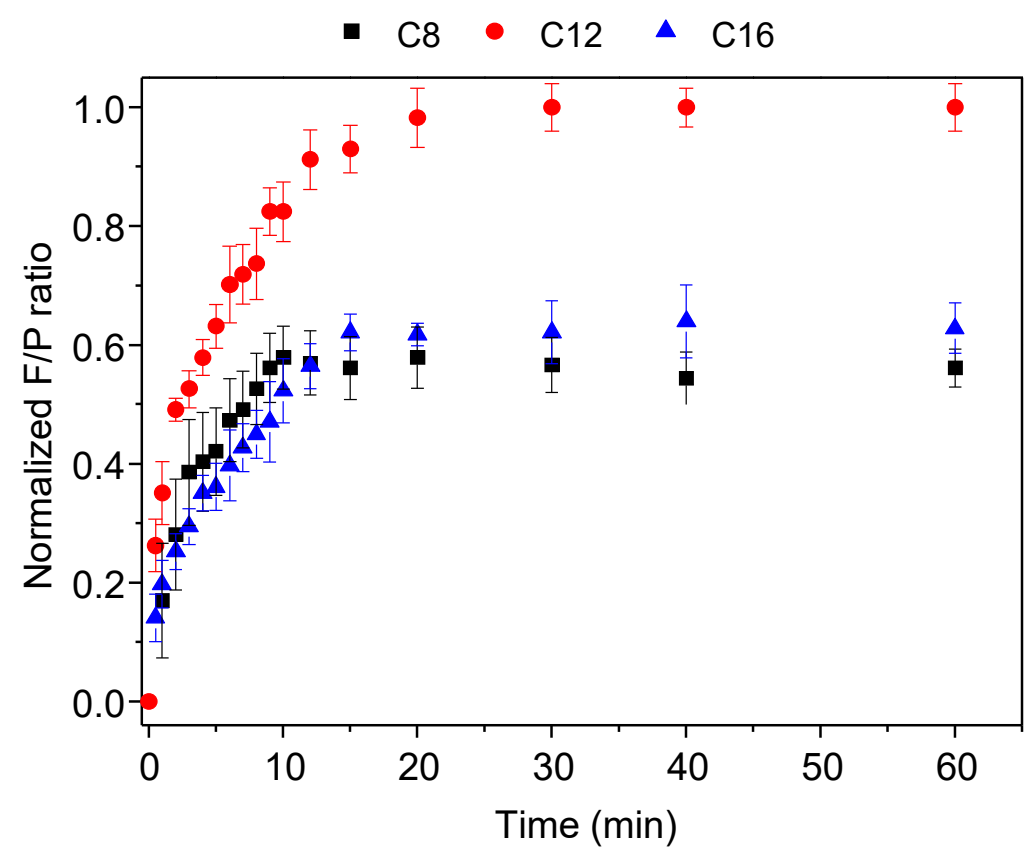

Supplementary Figure S10. Normalized F/P ratio for the SPAAC reaction of BCN derivative 1 for the three ME. Each time value was obtained as the average of 2 measurements on 3 independent surfaces. Normalized $\mathrm{F} / \mathrm{P}$ values were obtained by normalization each $\mathrm{F} / \mathrm{P}$ ratio (for a given time) to the maximum $\mathrm{F} / \mathrm{P}$ ratio after 60 min for the $\mathbf{C} 12$ monolayer. 


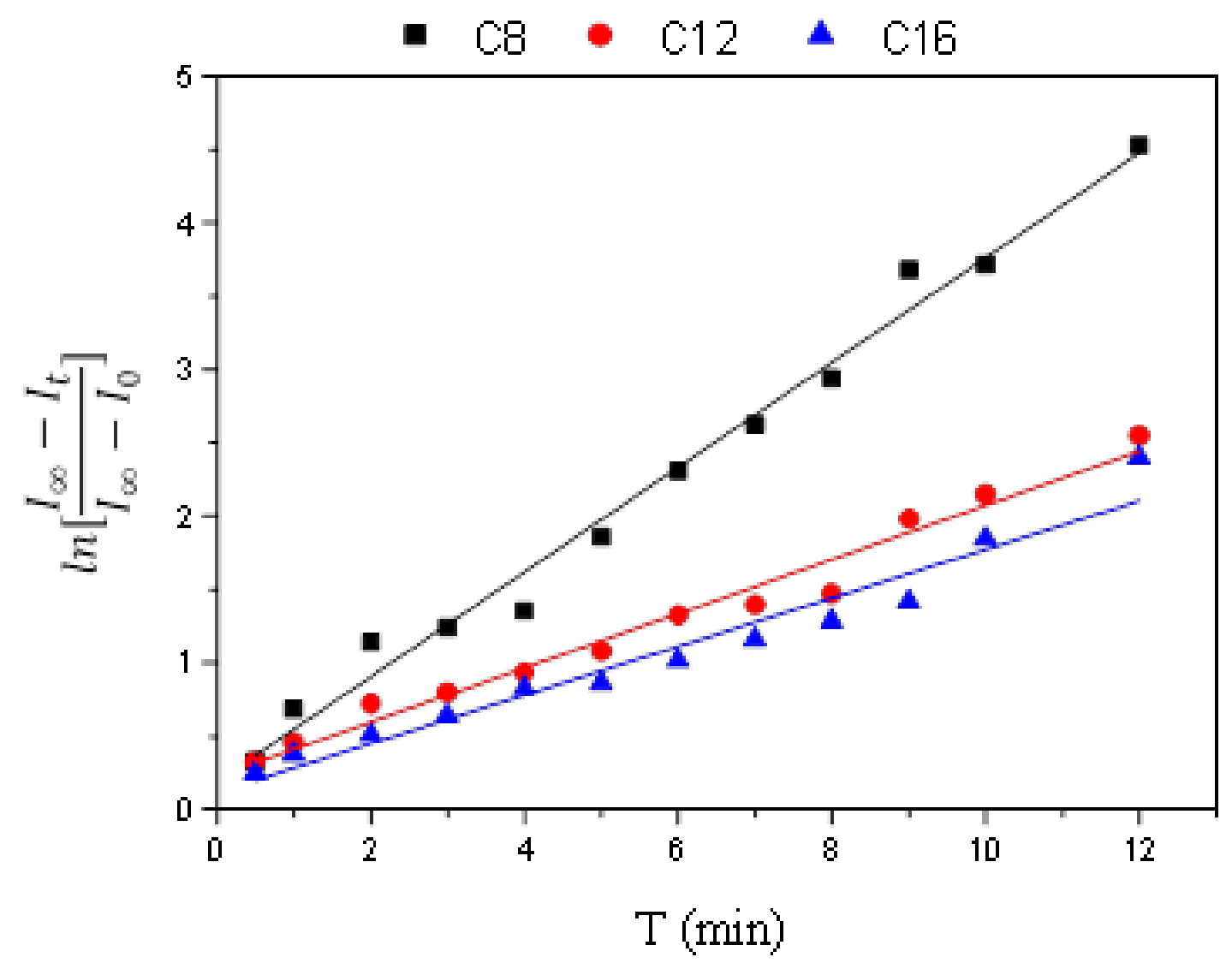

Supplementary Figure S11. Plot of $\ln \left[\left(I_{\infty}-I_{t}\right) /\left(I_{\infty}-I_{0}\right)\right]$ versus time to obtain first-order rate constants by XPS.
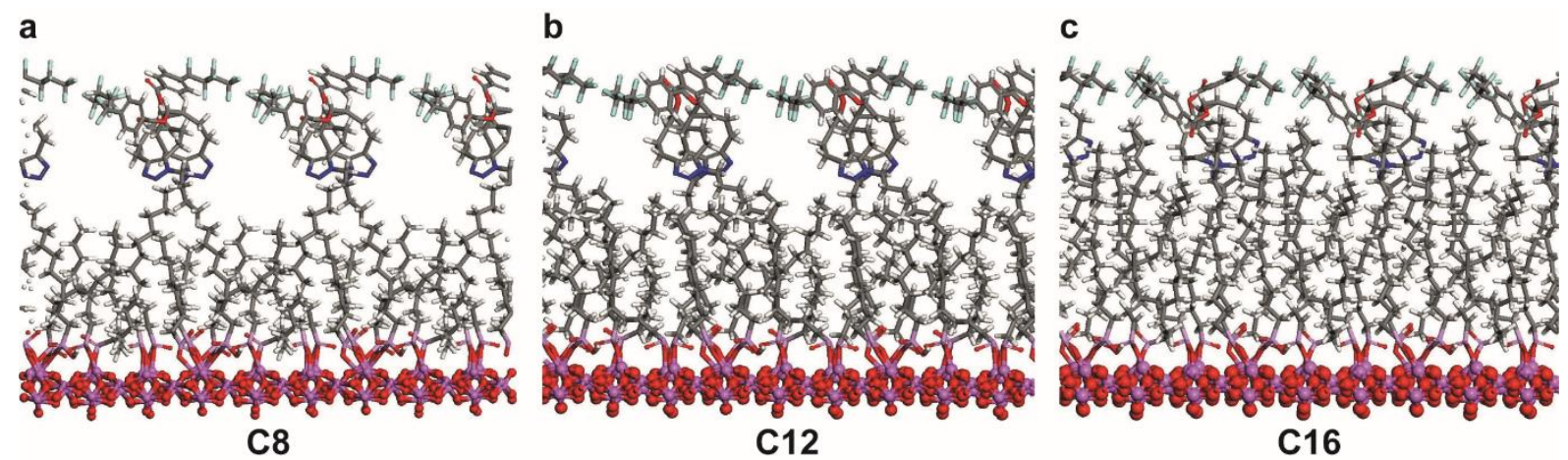

Supplementary Figure S12. Schematic representation of the three monolayers after the SPAAC reaction. 


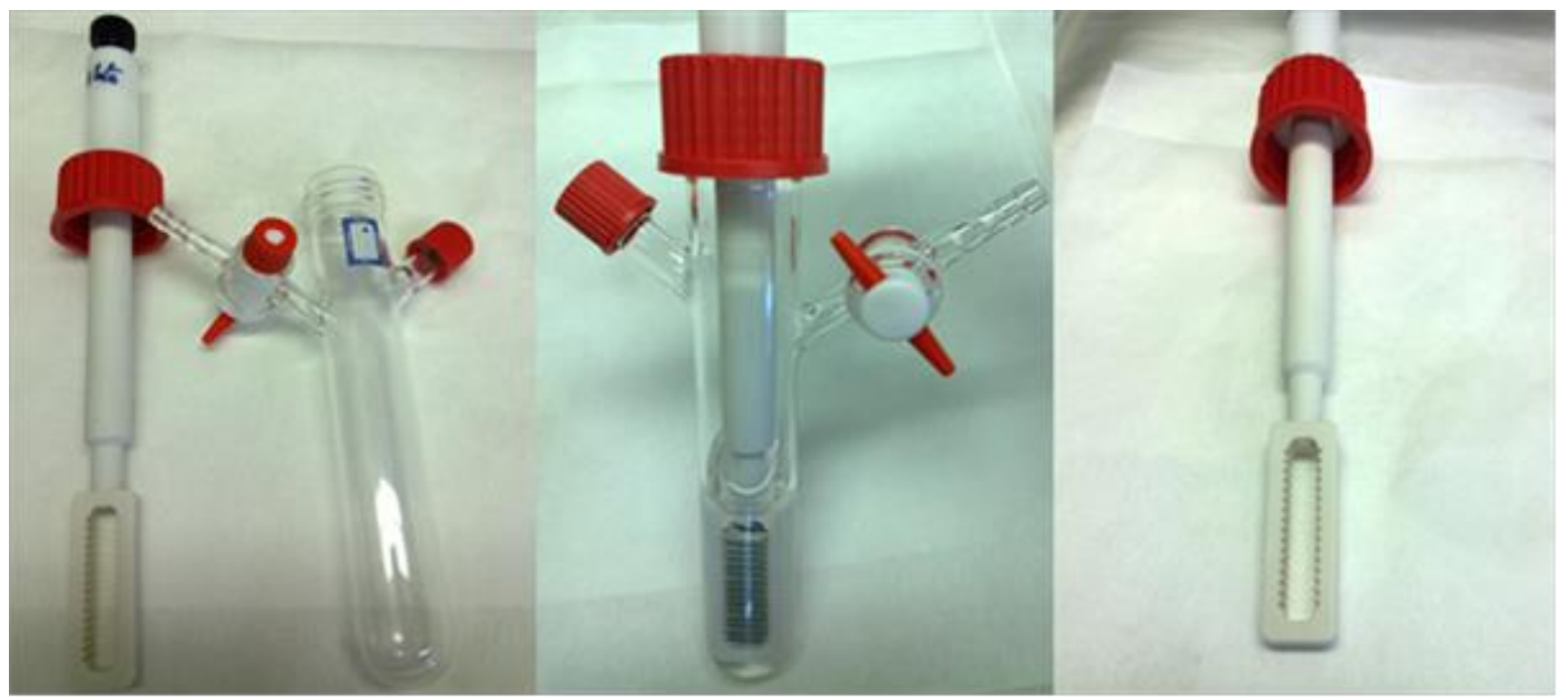

Supplementary Figure S13. Image of the sample holder.

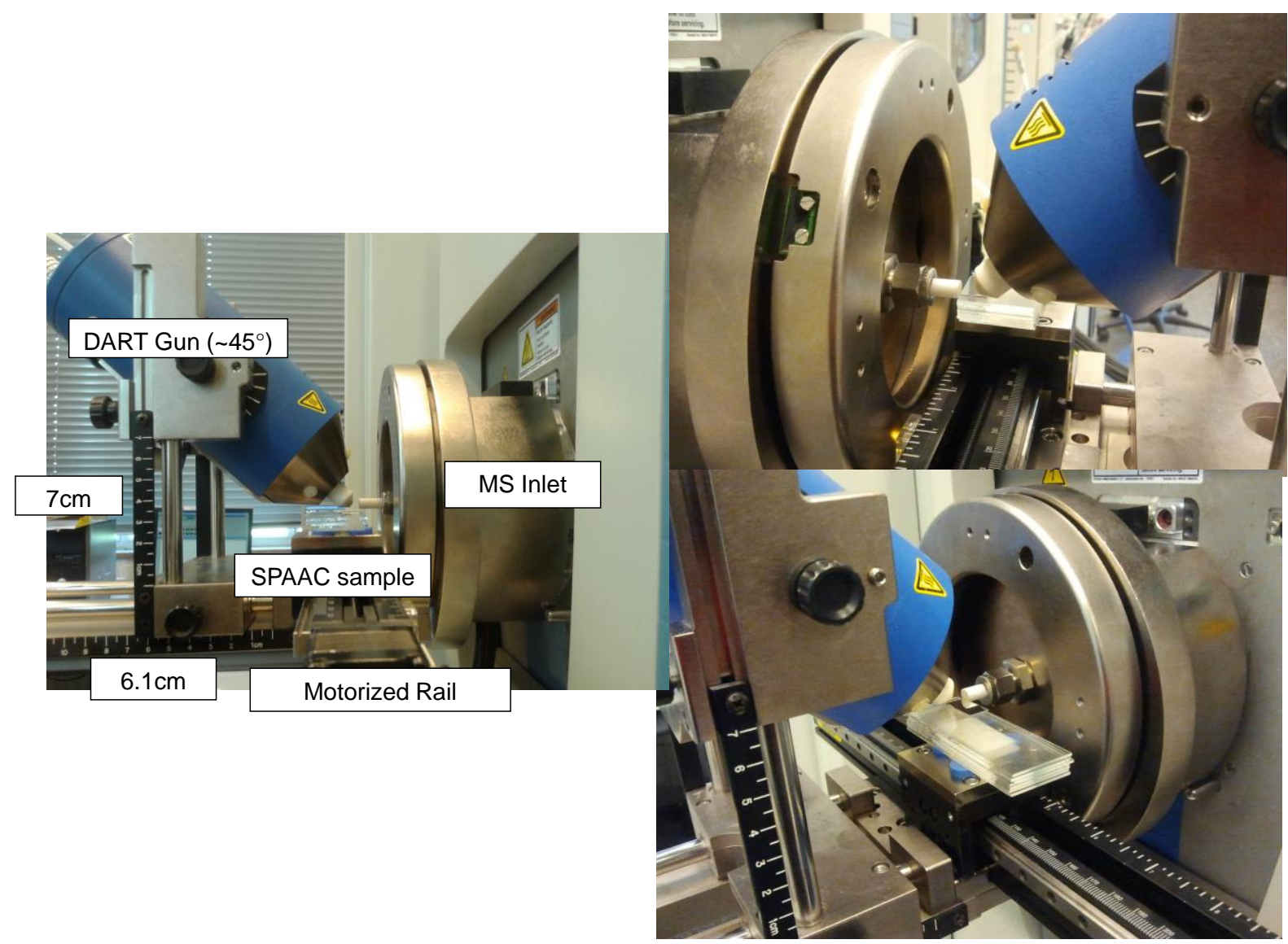

Supplementary Figure S14. Pictures of DART setup for the kinetic analysis of organic surface reactions. 


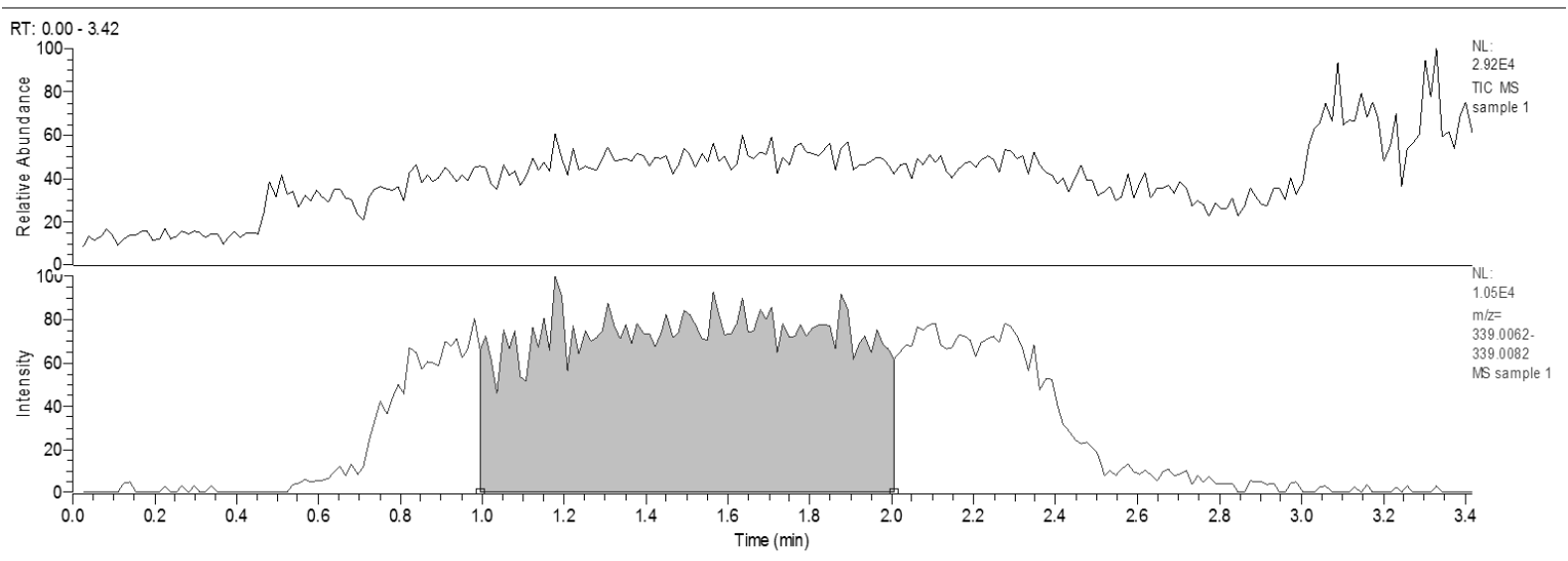

sample 1 \#69-140 RT: 0.99-2.01 AV: $72 \quad$ NL: $7.58 E 3$

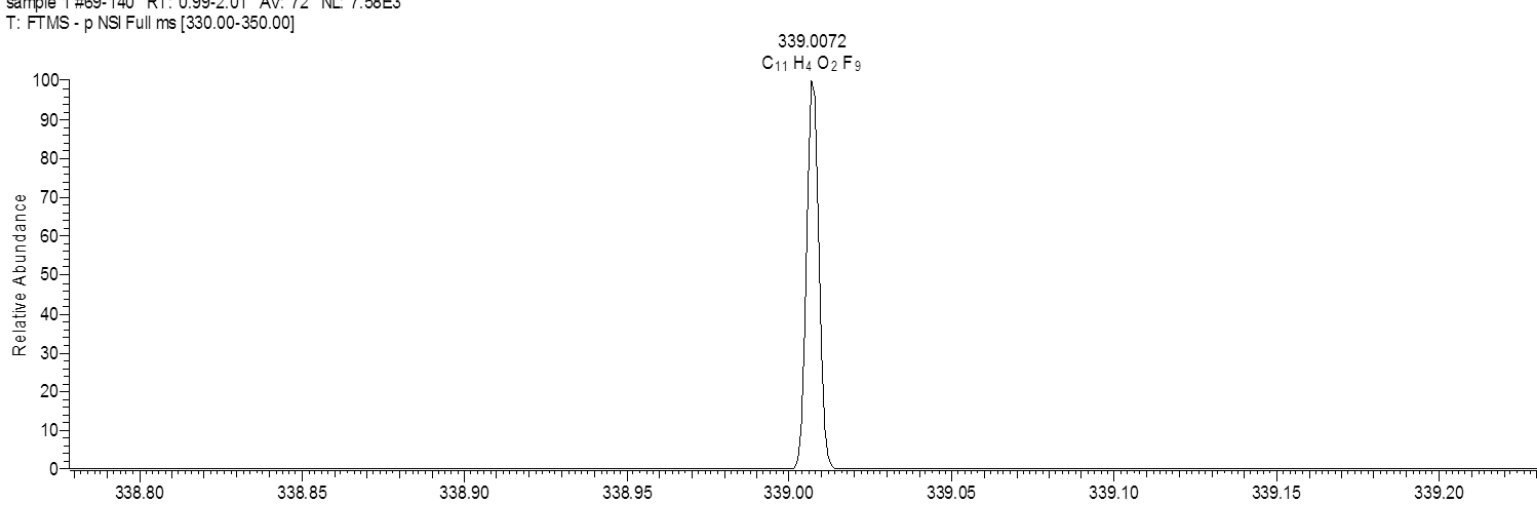

$\begin{array}{lllll}338.80 & 338.85 & 338.90 & 338.95 & 339.00\end{array}$

$\mathrm{m} / \mathrm{z}$

Elemental composition search on mass 339.0072

$\mathrm{m} / \mathrm{z}=334.0072-344.0072$

\begin{tabular}{|c|c|c|c|c|}
\hline $\mathrm{m} / \mathrm{z}$ & $\begin{array}{r}\text { Theo. } \\
\text { Mass }\end{array}$ & $\begin{array}{r}\text { Delta } \\
\text { (mmu) }\end{array}$ & $\begin{array}{c}\text { RDB } \\
\text { equiv. }\end{array}$ & Composition \\
\hline 339.0072 & 339.0062 & 1.01 & 5.5 & $\mathrm{C}_{11} \mathrm{H}_{4} \mathrm{O}_{2} \mathrm{~F}_{9}$ \\
\hline & 339.0226 & -15.39 & 5.5 & $\mathrm{C}_{12} \mathrm{H}_{5} \mathrm{~F}_{10}$ \\
\hline & 338.9862 & 21.00 & 6.5 & $\mathrm{C}_{11} \mathrm{H} O \mathrm{~F}_{10}$ \\
\hline & 339.0426 & -35.38 & 4.5 & $\mathrm{C}_{12} \mathrm{H}_{8} \bigcirc \mathrm{F} 9$ \\
\hline & 339.0437 & -36.52 & 0.5 & $\mathrm{C}_{9} \mathrm{H}_{9} \mathrm{O}_{2} \mathrm{~F}_{10}$ \\
\hline & 339.0626 & -55.36 & 3.5 & $\mathrm{C}_{12} \mathrm{H}_{11} \mathrm{O}_{2} \mathrm{~F}_{8}$ \\
\hline & 339.0801 & -72.90 & -0.5 & $\mathrm{C}_{10} \mathrm{H}_{13} \mathrm{OF}_{10}$ \\
\hline
\end{tabular}

Supplementary Figure S15. Typical DART HRMS measurement. 


\begin{tabular}{|c|c|c|}
\hline \multicolumn{3}{|c|}{ v] Instrument Control } \\
\hline \multicolumn{2}{|c|}{ ANl scan parameters } & (2) \\
\hline History & $\Rightarrow$ & \\
\hline Scan range & \multicolumn{2}{|l|}{330.0 to $350.0 \mathrm{~m} / \mathrm{z}$} \\
\hline Frogmentation & \multicolumn{2}{|l|}{ None } \\
\hline Resolution & \multicolumn{2}{|l|}{ Ultra high } \\
\hline Polsrity & \multicolumn{2}{|l|}{ Negative } \\
\hline Mcroscans & \multicolumn{2}{|l|}{1} \\
\hline Lock masses & \multicolumn{2}{|l|}{ off } \\
\hline AGC target & \multicolumn{2}{|l|}{ Ultimate mass accuracy } \\
\hline Maximum inject time : & \multicolumn{2}{|l|}{$50 \mathrm{~ms}$} \\
\hline Apply Help & \multicolumn{2}{|l|}{$\square$ Hok Ink } \\
\hline \multicolumn{3}{|l|}{ Fi:9 NSI source } \\
\hline \multirow{2}{*}{\multicolumn{2}{|c|}{ Sheath gas flow rate 0}} & actual \\
\hline & 0 & 0 \\
\hline Aux gas flow rate & 0 & 0 \\
\hline Sweep gas flow rate & o & 0 \\
\hline Spray voltoge ( $|\mathrm{KV}|)$ & 0.00 & 0.00 \\
\hline Spray current (uA) & & 0.00 \\
\hline Capilary temp. $\left({ }^{\circ} \mathrm{C}\right)$ & 250 & 273 \\
\hline Caplary voltage (V) & -27.50 & \\
\hline Tube lens voltage (V) & -65.00 & \\
\hline Skimmer volkage (V) & -20.00 & \\
\hline Apply Help & Vot link & \\
\hline
\end{tabular}

Supplementary Figure S16. Typical DART HRMS setup.
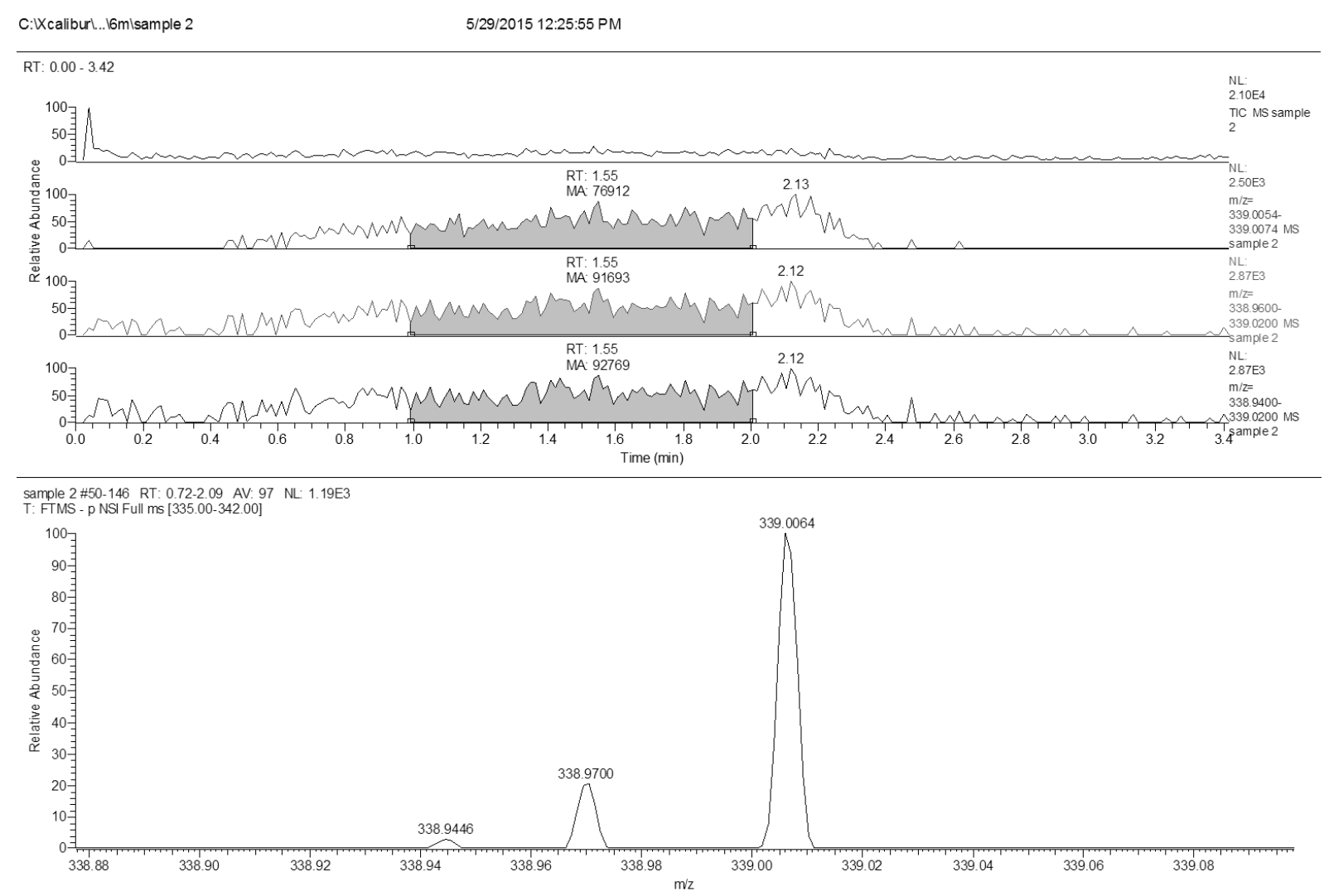

Supplementary Figure S17. Top: the total ion count (TIC) is shown for experiment where the reaction for the azide in the $\mathrm{C} 12$ nanoenvironment, stopped after 6 minutes. In subsequent panels, the extracted ion current (EIC) of the ion of interest with increasing mass window (10 $\mathrm{mu}, 30 \mathrm{mu}$ and $40 \mathrm{mu}$, respectively). As is 
clear from the EIC traces, widening the mass window leads to integration of unwanted signals of background species of highly similar mass, which can also be seen in the bottom mass spectrum of the same sample. Apart from the target analyte peak at $m / z=339.0064$, two distinct other peaks can be seen at $m / z=338.9446$ and 338.9700. To avoid including the intensity of these undesired species in the overall integration, the DART measurements were performed in High-Resolution only (with a mass window of $10 \mathrm{mu}$ ). 


\subsection{Supplementary Tables}

Supplementary Table S1. First-order rate constants (determined by DARTHRMS) for the interfacial SPAAC reaction of BCN and azido moieties at selfassembled monolayers containing methylene spacers of varying lengths. For determining every rate constant, DART-HRMS measurements were performed on 6 independent surfaces.

\begin{tabular}{|c|c|c|c|}
\hline & \multicolumn{3}{|c|}{$k^{\prime}\left(\times \mathbf{1 0}^{-3} \mathbf{s}^{-\mathbf{1}}\right)$} \\
\hline $\mathbf{T}\left({ }^{\circ} \mathbf{C}\right)$ & $\mathbf{C 8}$ & $\mathbf{C 1 2}$ & $\mathbf{C 1 6}$ \\
\hline 20 & $3.7 \pm 0.3$ & $2.3 \pm 0.3$ & $2.7 \pm 0.2$ \\
\hline 30 & $5.7 \pm 0.4$ & $3.7 \pm 0.3$ & $3.1 \pm 0.3$ \\
\hline 40 & $5.6 \pm 0.3$ & $4.6 \pm 0.4$ & $4.5 \pm 0.3$ \\
\hline 50 & $6.8 \pm 0.4$ & $6.5 \pm 0.4$ & $6.4 \pm 0.5$ \\
\hline
\end{tabular}

Supplementary Table S2. Comparison of the activation parameters for the interfacial SPAAC reaction of $\mathrm{BCN}$ derivative 1 with monolayers that present the azide group in a buried environment (C8), an accessible environment (C12 and C16).

\begin{tabular}{|c|c|c|}
\hline Monolayer & $\Delta \mathbf{H}^{\ddagger}\left[\mathrm{kcal} \cdot \mathbf{~ m o l}^{-\mathbf{1}}\right]$ & $\Delta \mathbf{S}^{\ddagger}\left[\times \mathbf{1 0}^{\mathbf{2}} \mathbf{c a l} \cdot(\mathbf{K} \cdot \mathbf{~ m o l})^{-\mathbf{1}}\right]$ \\
\hline $\mathbf{C 8}$ & $2.86 \pm 0.24$ & $-0.05 \pm 0.01$ \\
\hline $\mathbf{C 1 2}$ & $5.76 \pm 0.48$ & $-0.05 \pm 0.02$ \\
\hline $\mathbf{C 1 6}$ & $5.02 \pm 0.72$ & $-0.05 \pm 0.02$ \\
\hline
\end{tabular}

The activation parameters (enthalpy of activation $\left(\Delta H^{+}\right)$and entropy of activation $\left.\left(\Delta S^{\ddagger}\right)\right)$ were obtained using Eyring equation by a least-squares fit of plots of $\ln (\mathrm{k} / \mathrm{T})$ versus $1 /$ T. Values for $\Delta H^{\ddagger}$ and $\Delta S^{\ddagger}$ were calculated from the slope and intercept of the aforementioned plot. 


$$
\ln (k / T)=\ln \left(K k_{B} / h\right)+\Delta S^{\ddagger} / R T-\Delta H^{\ddagger} / R
$$

Where: $k=$ Observed rate constant, in $\left[\mathrm{s}^{-1}\right] ; T=$ Absolute temperature, in $[\mathrm{K}] ; K=$ Transmission coefficient, set to be $1 ; k_{B}=$ Boltzmann's constant: $1.381 \times 10^{-23} \mathrm{~J} \mathrm{~K}^{-1} ; h$ $=$ Planck's constant: $6.626 \times 10^{-34} \mathrm{~J} \mathrm{~s} ;\left(\Delta H^{\ddagger}\right.$ and $\Delta S^{\ddagger}$ converted to calorie units using factor 4.184$) S^{\ddagger}=$ Entropy of activation, in cal $\cdot(\mathrm{K} \cdot \mathrm{mol})^{-1} ; \quad R=$ Gas constant: $8.3145 \mathrm{~J} \cdot(\mathrm{K} \cdot \mathrm{mol})^{-1} ; H^{\ddagger}=\quad$ Enthalpy of activation, in $\left[\mathrm{kcal} \mathrm{mol}^{-1}\right]$. 


\subsection{NMR spectra}

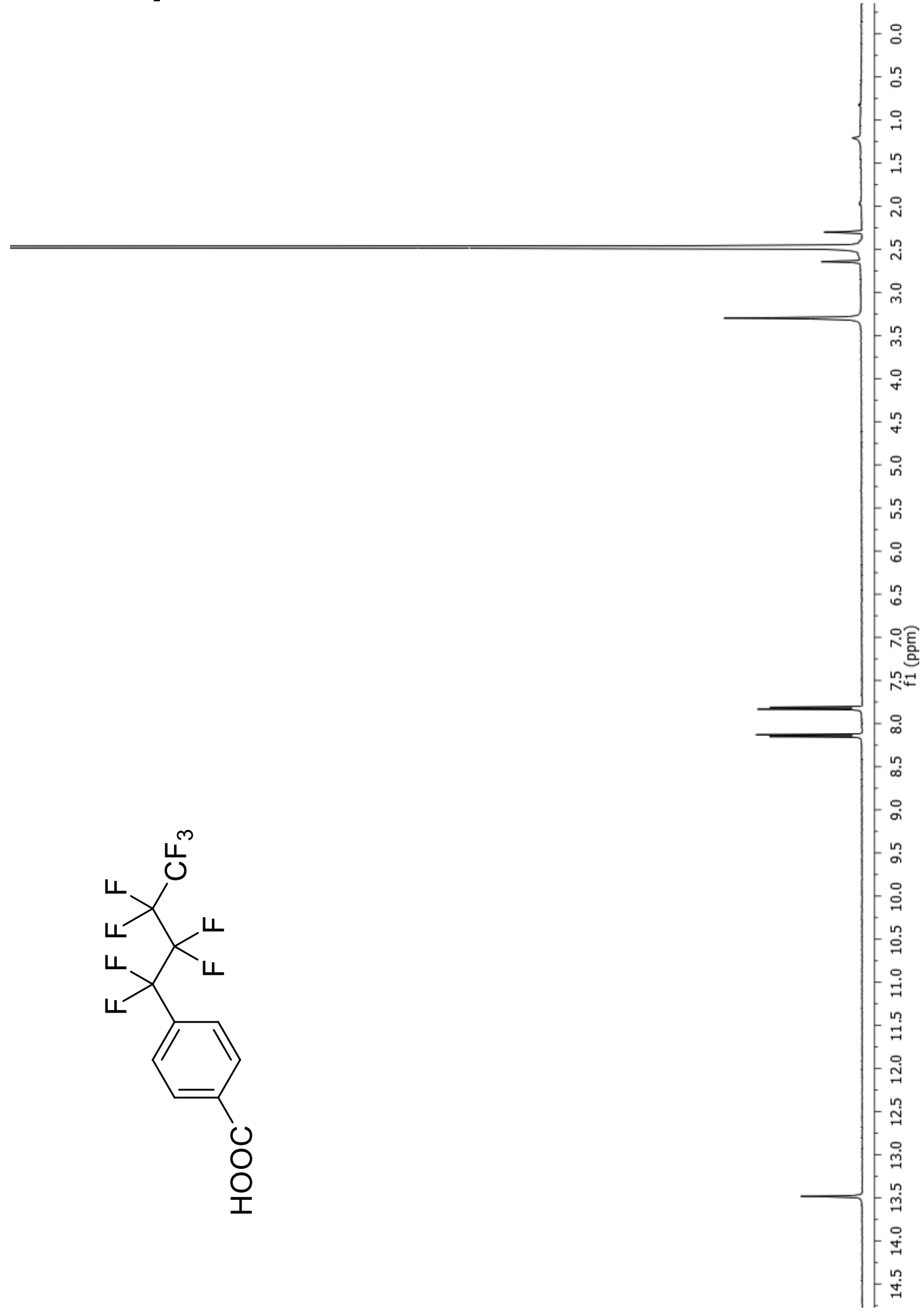




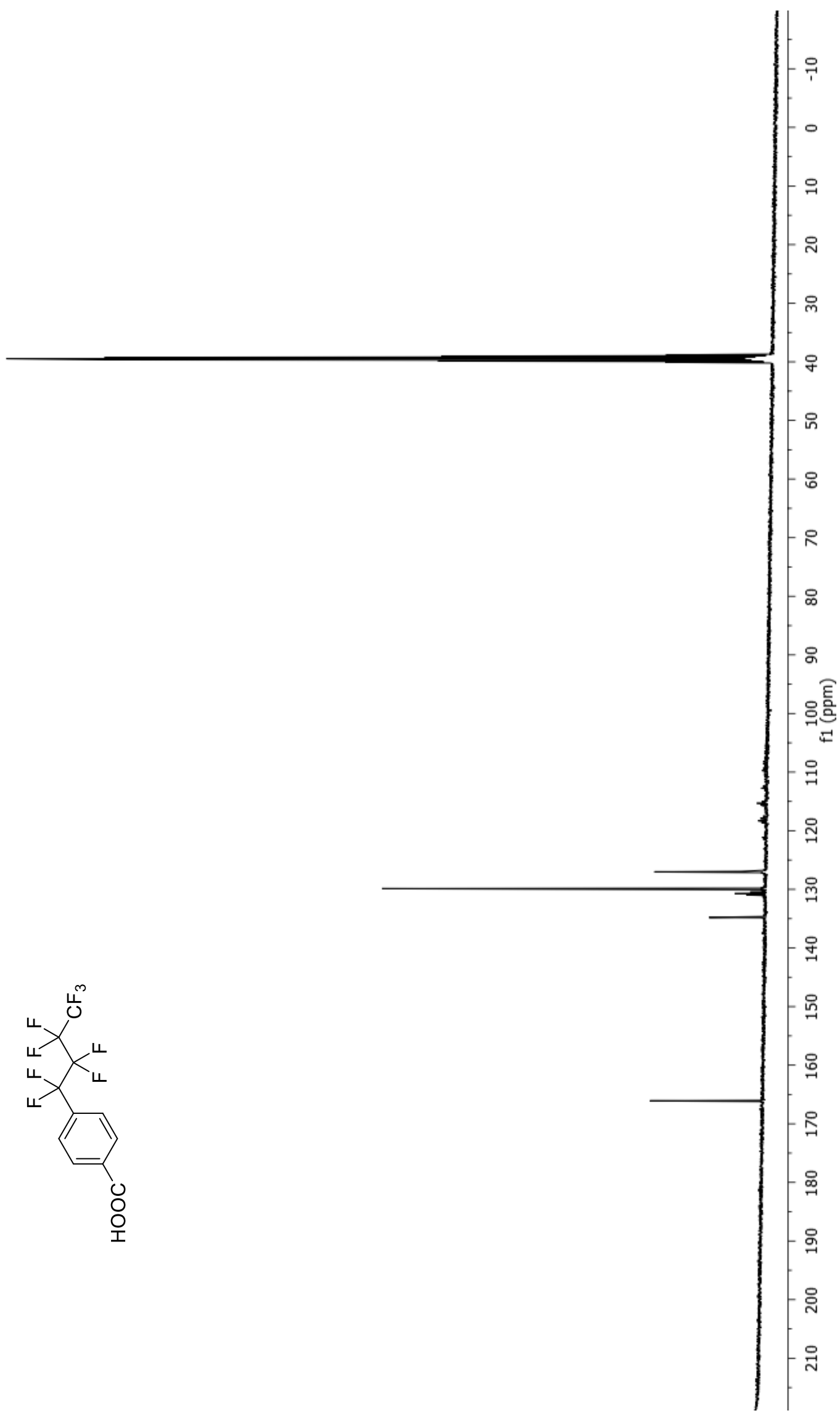




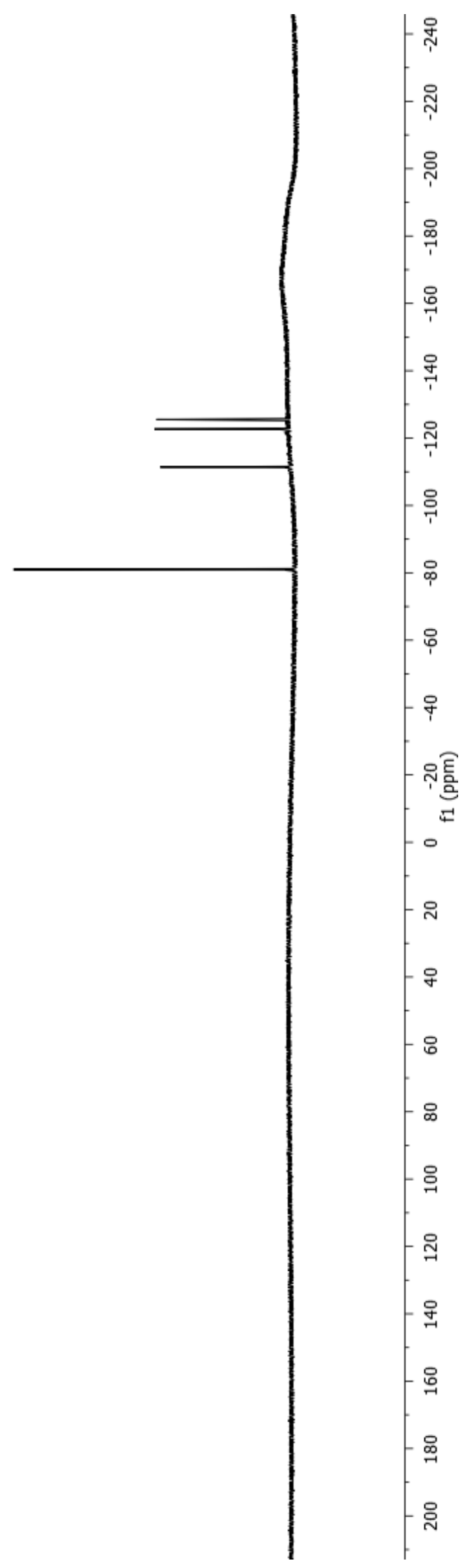




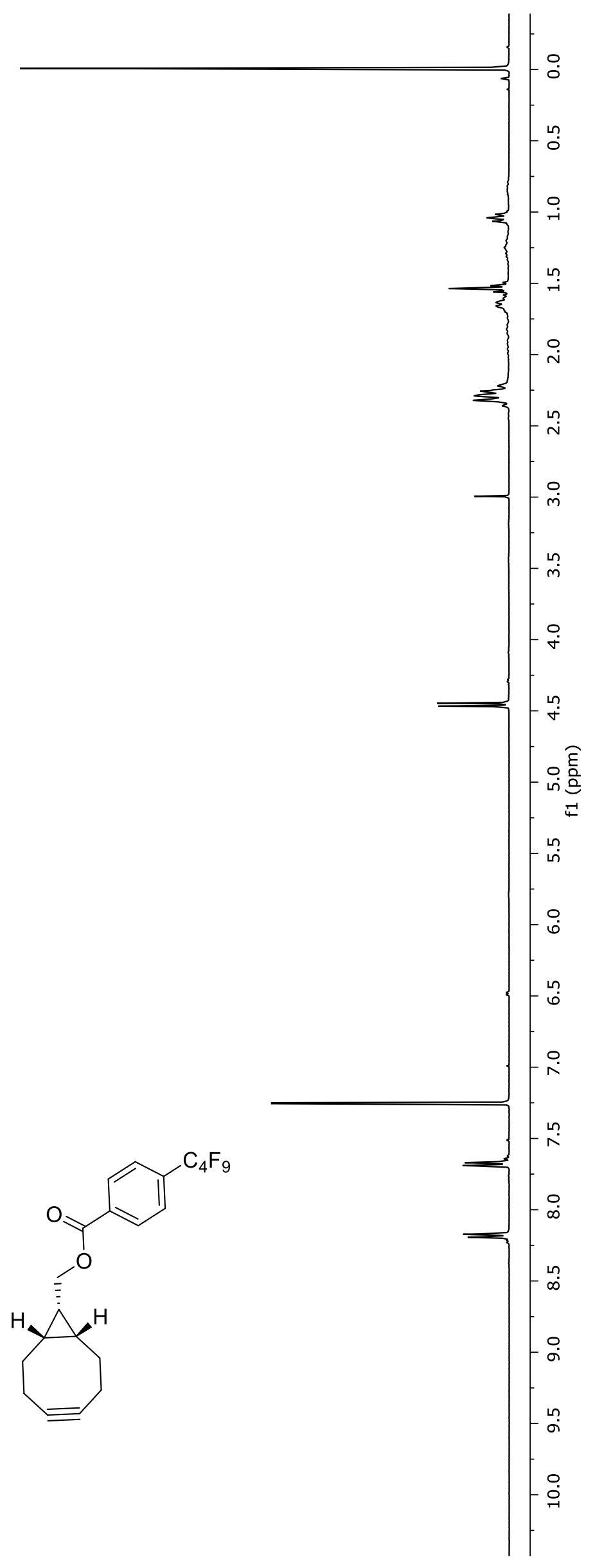




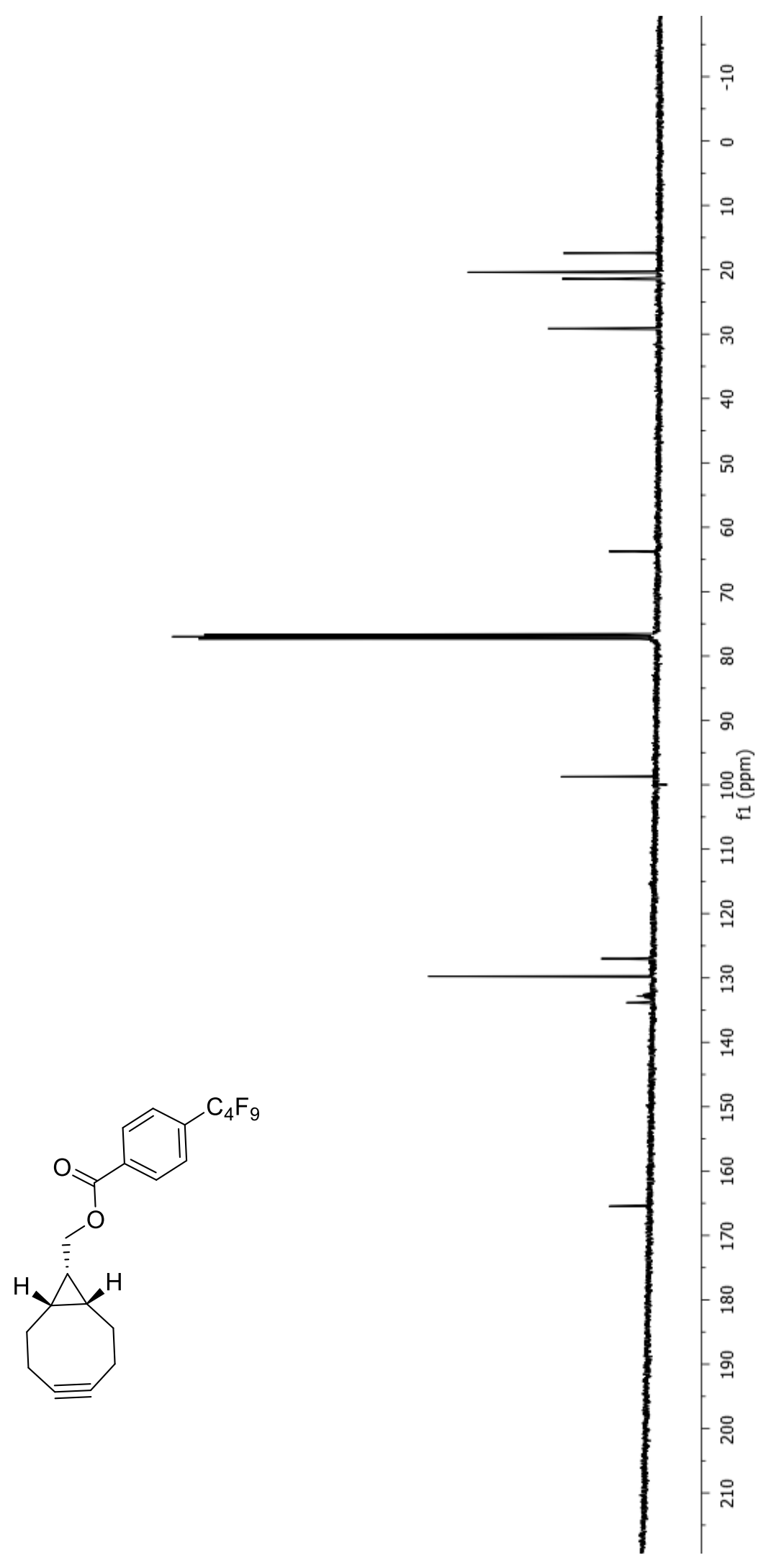




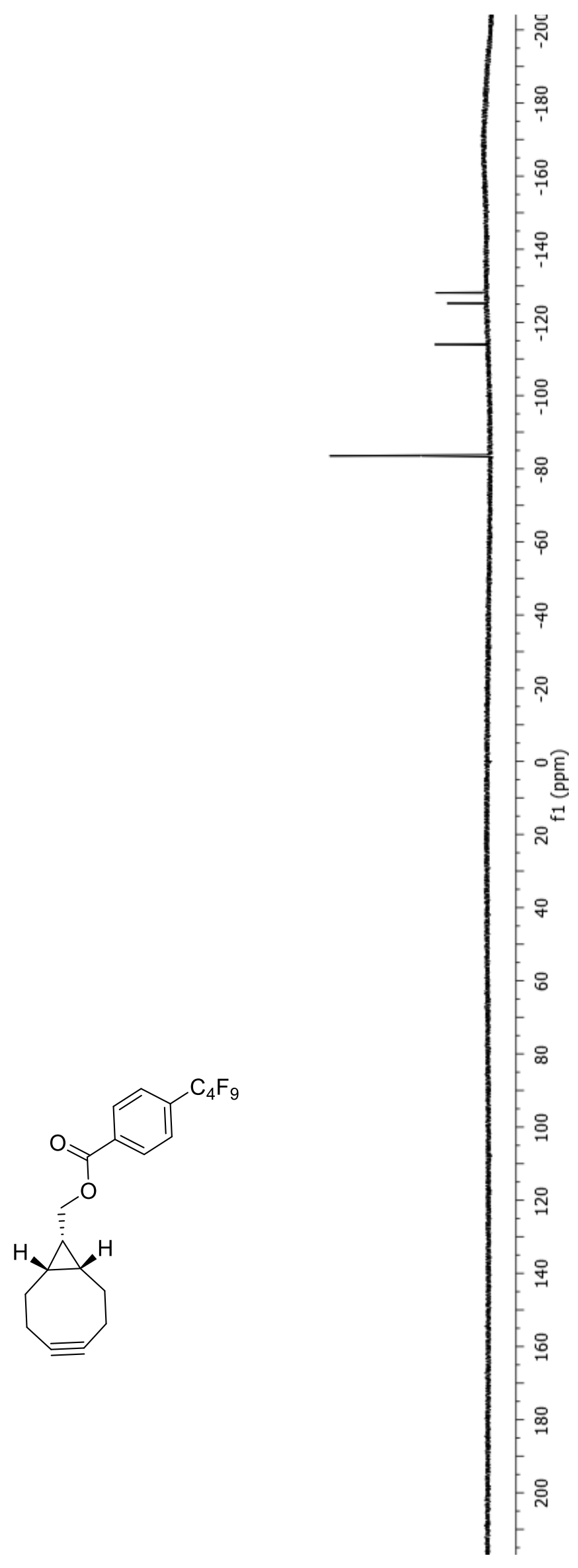




\section{Appendix 2}

Rapid and Complete Surface Modification with Strain-Promoted Oxidation-Controlled

Cyclooctyne-1,2-Quinone Cycloaddition (SPOCQ)

For full Supporting Information including Cartesian coordinates look at online ESI available in Angewandte Chemie International website at anie201612037-sup-0001-misc_information.pdf

\section{Table of Contents}

2.1 Material and Methods.

2.2. Supplementary Figures

2.3. Supplementary Tables.

2.4 References 178 


\subsection{Material and Methods}

\section{Materials}

Unless otherwise specified, all chemicals were used as received without further purification. Octylphosphonic acid, hexadecylphosphonic acid, dopamine hydrochloride, sodium periodate, hydrochloric acid, methanol, hexane, acetone,

dichloromethane, 2-propanol, trimethoxy(propyl)silane and (3bromopropyl)trimethoxysilane) were purchased from Sigma-Aldrich. 12Bromododecylphosphonic acid and 3-bromopropylphosphonic acid were purchased from SiKÉMIA. Aluminum pieces $(99.5 \%$ purity, mirror polished, Staalmarkt Beuningen BV) were cut using a mechanical cutter into $2 \times 1 \mathrm{~cm}$ dimensions. For surface modification reactions, the samples were loaded onto a specially constructed PTFE wafer holder able to hold up to 16 samples at a time, thus ensuring rigorous reproducibility between samples.

Static Water Contact Angle (CA) Measurements. The wettability of the modified surfaces was determined by automated static water contact angle measurements with a Krüss DSA 100 goniometer (volume of the drop of deionized water was $3.0 \mu \mathrm{L}$ ). The reported values are the average of at least six droplets, and the relative error is less than $\pm 1^{\circ}$.

Infrared Reflection Absorption Spectroscopy (IRRAS). IRRAS spectra were obtained with a Bruker Tensor 27 FT-IR spectrometer equipped with a liquid nitrogen cooled MCT-detector, using a commercial variable-angle reflection unit (Auto Seagull, Harrick Scientific). A Harrick grid polarizer was installed in front of the detector and was used for measuring spectra with $\mathrm{p}$-polarized radiation with respect to the plane of incidence at the sample surface. A Ge ATR crystal was used to obtain the spectra. Single channel spectra were collected at $55^{\circ}$ using 256 scans in each measurement. The raw data were divided by the spectrum recorded on a freshly 
etched reference $\mathrm{Al}$ oxide surface, after which a baseline correction was applied to give the reported spectra.

X-ray Photoelectron Spectroscopy (XPS) Measurements. The XPS analysis of surfaces was performed using a JPS-9200 photoelectron spectrometer (JEOL, Japan). Survey and high-resolution spectra were obtained under UHV conditions using monochromatic $\mathrm{Al} \mathrm{K} \alpha \mathrm{X}$-ray radiation at $12 \mathrm{kV}$ and $20 \mathrm{~mA}$, and an analyser pass energy of $50 \mathrm{eV}$ for wide scans and $10 \mathrm{eV}$ for narrow scans. The emitted electrons were collected at $10^{\circ}$ from the surface normal (take off angle relative to the surface normal $10^{\circ}$ ). All XPS spectra were evaluated by using Casa XPS software (version 2.3.15). Survey spectra were corrected with linear background before fitting, whereas high-resolution spectra were corrected with linear background. Atomic area ratios were determined after a baseline correction and normalizing the peak area ratios by the corresponding atomic sensitivity factors (1.00 for C1s, 1.80 for N1s, 2.93 for O1s, 4.43 for F1s, 1.18 for P2s and 0.54 for Al2p, 5.03 for Br3p).

DART-HRMS Measurements. Analysis of the SPOCQ reacted Al surfaces were performed using a DART-SVP ion source (Ion-Sense, Saugus, MA, USA) coupled to an Exactive Orbitrap high-resolution mass spectrometer (Thermo Fisher Scientific, San Jose, CA, USA), mounted on a motorized rail travelling at $0.2 \mathrm{~mm} / \mathrm{s}$. Thermo Scientific Xcalibur software (V2.1.0.1139) was used for data acquisition and processing. The measurements were performed in negative mode at $450{ }^{\circ} \mathrm{C}$ using a scan range of $m / z$ 335.0-345.0, a mass resolution of 100,000 (FWHM) at a scan rate of $1 \mathrm{~Hz}$. The ion trap was tuned with $0.1 \mathrm{mg} / \mathrm{mL}$ methanol solution of quinine $(\mathrm{m} / z$ 323.41 in negative mode) and optimized. The DART source was positioned $6.1 \mathrm{~cm}$ on the horizontal scale, $7 \mathrm{~cm}$ on the vertical scale with an angle of $45^{\circ}$, such that it is around $1 \mathrm{~mm}$ above the surface (see Appendix 2, Fig. S1). The distance from the surface to the ceramic tube is minimized by placing them at the edge of the moving rail so that maximum of the $p-\mathrm{C}_{4} \mathrm{~F}_{9}$ benzoate ions (MS-tag) would enter the MS. 
2.1.1 Preparation of phosphonic acid monolayers. $2 \times 1 \mathrm{~cm} \mathrm{Al}$ slides were sonicating in hexane for 15 min followed by wiping with lint-free cotton swabs (Texwipe, NC, USA) to remove polymer protection layer on top and remove any residual glue. The surfaces were chemically activated by immersing in $1: 1(\mathrm{v} / \mathrm{v}) 37 \% \mathrm{HCl}-\mathrm{MeOH}$ mixture for $5 \mathrm{~min}$, followed by washing with water and 2-propanol. The activated surfaces were then immersed into $\mathrm{N}_{2}$ filled vials of $5 \mathrm{mM}$ solution of corresponding phosphonic acid derivative mixture in 2-propanol, heated to $50{ }^{\circ} \mathrm{C}$ for $5 \mathrm{~min}$, and then left undisturbed for $5 \mathrm{~h}$ at room temperature to obtain self-assembled mixed monolayers. Then surfaces were taken out and sonicated successively for 5 min with 2-propanol, acetone and $\mathrm{CH}_{2} \mathrm{Cl}_{2}$. The surfaces were then cleaned with $\mathrm{CH}_{2} \mathrm{Cl}_{2}$, air dried and stored under $\mathrm{N}_{2}$ atmosphere. From static water contact angle (SCA) measurements, it was found that the reaction was complete after $5 \mathrm{~h}$, yielding monolayers with $28-30 \% \mathrm{C}$ as determined by XPS. Remarkably, substantially longer reaction times $(16 \mathrm{~h})$ contributed to the formation of undesirable multilayers $(42-44 \% \mathrm{C}){ }^{1}$

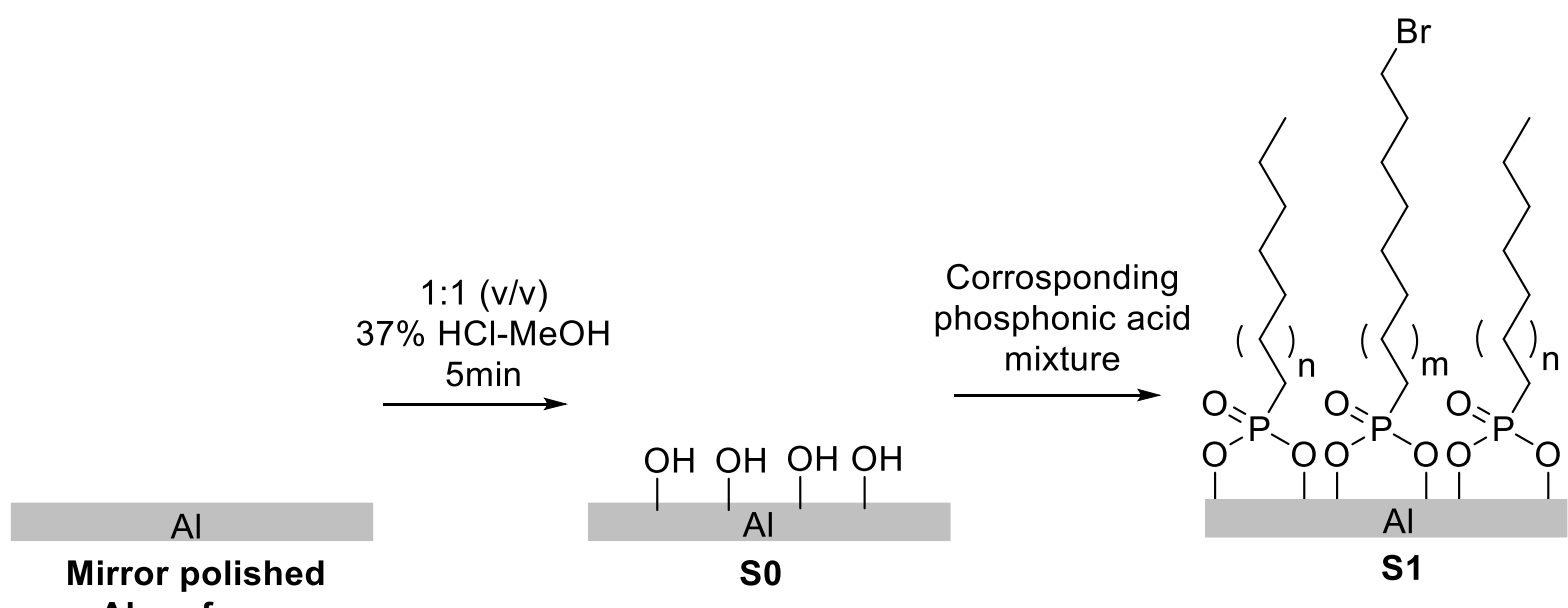

Scheme S2. Preparation of mixed bromo: alkyl monolayers (M1 in this case).

2.1.2 Preparation of catechol-terminated monolayers. [S1 to S2] Dopamine hydrochloride $(162.5 \mathrm{mg}, 0.86 \mathrm{mmol})$ was neutralized with freshly distilled triethylamine $(0.155 \mathrm{~mL}, 1.12 \mathrm{mmol})$ in methanol. Bromo terminated Al chips were loaded onto PTFE holder and stirred for $1 \mathrm{~h}$. For samples in M3 microenvironment a 
stirring time of $1.5 \mathrm{~h}$ was found optimal. The disappearance of the Br $3 \mathrm{p}$ signal and replacement by the N1s signal from dopamine in XPS was used to optimize the stirring time. The samples were sonicated with methanol, washed with copious amounts of water followed by methanol, dried and stored under nitrogen atmosphere.

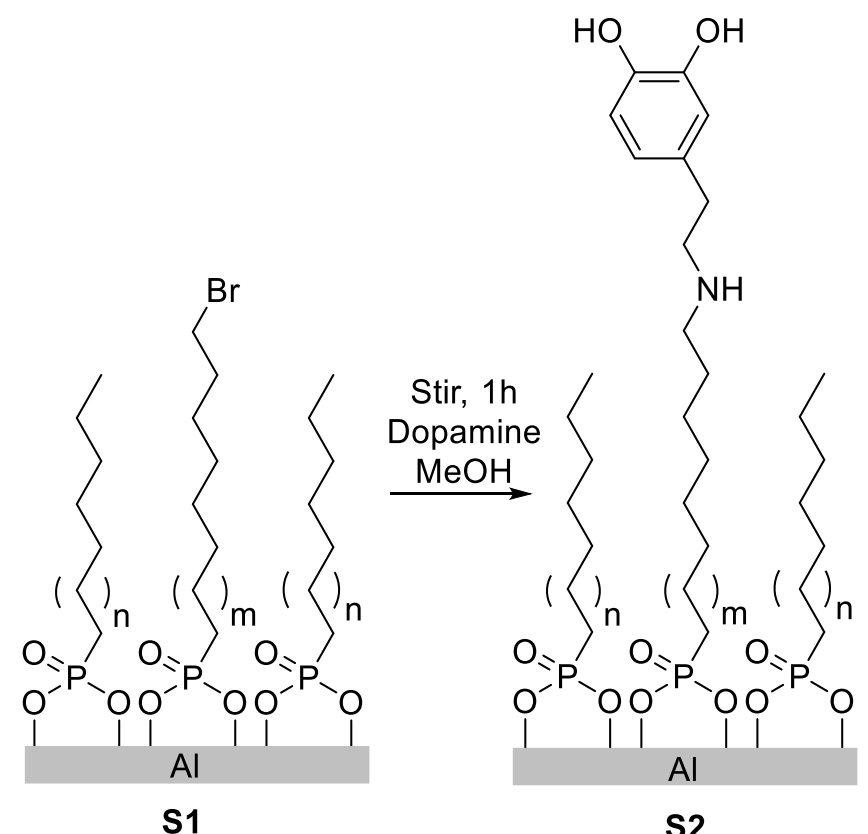

Scheme S3. Preparation of catechol-terminated (S2) from bromo-terminated monolayer $(\mathbf{S} 1)$

2.1.3 Preparation of quinone-terminated monolayers. [S2 to S3] Catecholterminated surface (S2) prepared above were stirred with $\mathrm{NaIO}_{4}(213 \mathrm{mg}, 50 \mathrm{mM})$ for $30 \mathrm{~min}$, to yield quinone-terminated monolayers (S3). The samples were then washed with copious amounts of water, followed by 15 min sonication in methanol, dried and stored under nitrogen atmosphere. 


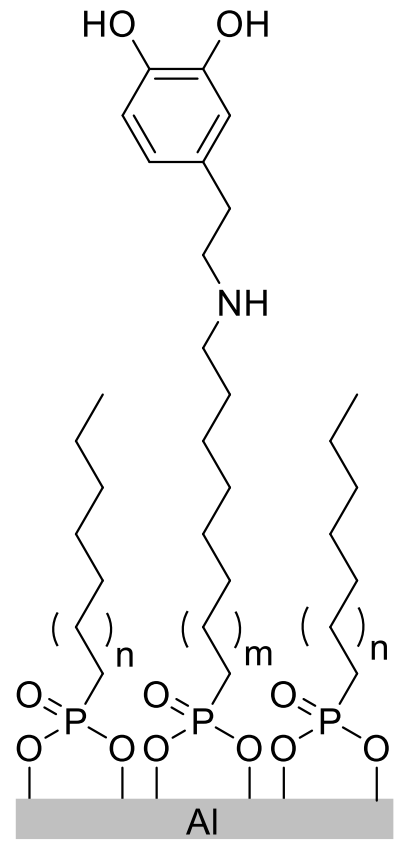

S2

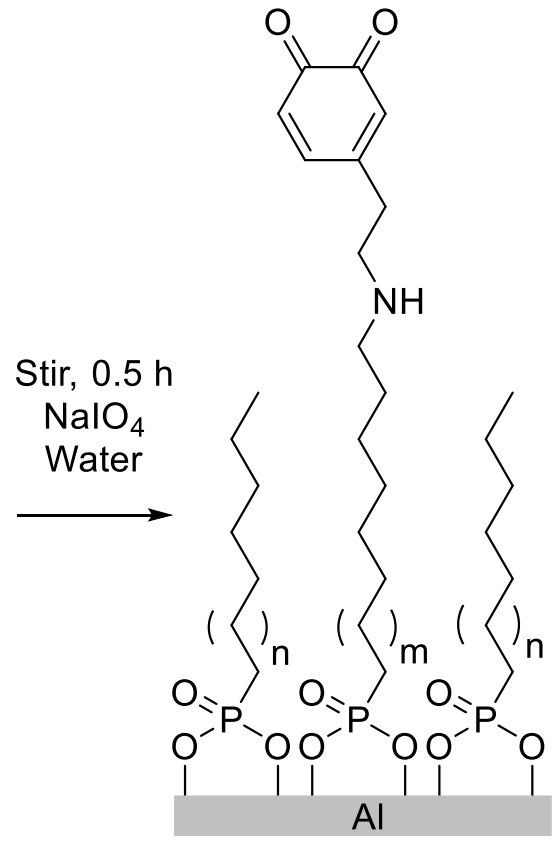

S3

Scheme S4. Preparation of quinone-terminated monolayer S3 from bromoterminated monolayer S2.

\subsubsection{Optimization of quinone-terminated monolayer (S3) preparation.}

Periodate oxidation for longer period of time, led to detrimental effect as evidenced by lower intensity of MS-tag after $1 \mathrm{~h}$ of SPOCQ reaction or lower signal due to incomplete conversion of catechol to quinone.

2.1.5 Interfacial SPOCQ reaction. [S3 to S4] Quinone-functionalized slides (S3) were immersed in $3 \mathrm{mM}$ BCN derivative (1) in DCE solution under $\mathrm{N}_{2}$ atm and kept reacting. After completion of reaction $(\mathbf{S} 4)$ for the desired time, the Al pieces were taken out, sonicated and washed with acetone to remove physisorbed moieties, dried under $\mathrm{N}_{2}$ stream and immediately used for DART analysis. Hexaplet measurements were carried out for each time point and average plotted along with standard deviation for each set. Thus, a statistical number of samples were measured with relative standard deviation (RSD) between $2-15 \%$ for any time point. 


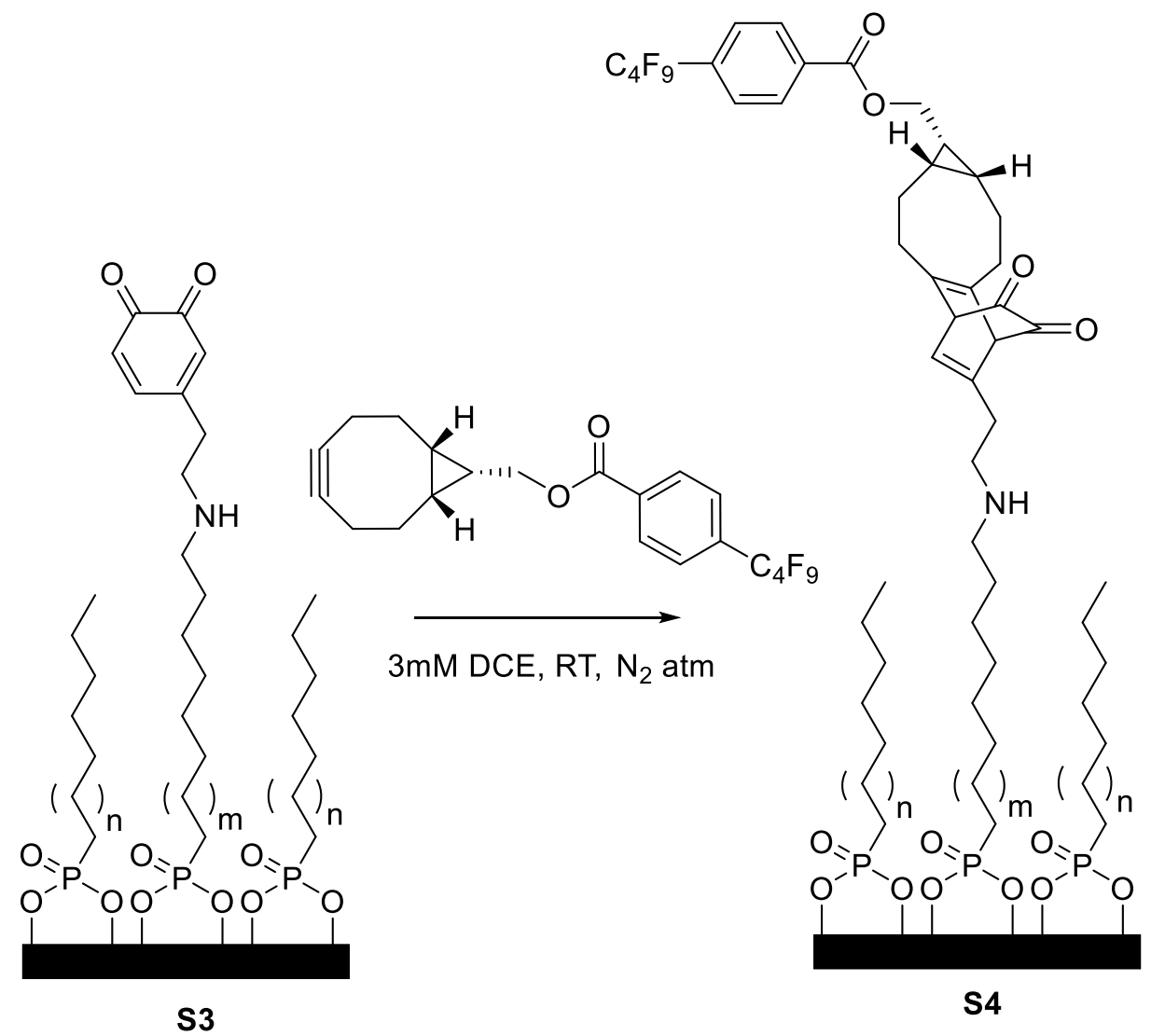

Scheme S5. SPOCQ reaction between surface bound 1,2-quinone (S3) and strained alkyne moiety of (1) on Al surface.

2.1.6 Computational procedures. All of the DFT calculations reported herein were carried out using performed using Gaussian'09. ${ }^{2}$ All geometries were fully optimized using density functional theory (DFT) by means of the M11L functional and the $6-311+\mathrm{G}(\mathrm{d}, \mathrm{p})$ basis set. Analytical frequencies were calculated at the same optimization level in all cases, and the natures of the stationary points were determined in each case according to the proper number of imaginary frequencies. Initially, a Monte Carlo conformational search using conformer distribution option available in Spartan'14 was used. ${ }^{3}$ With this option, a search without constraints was performed for every structure. The torsion angles were randomly varied and the obtained structures were fully optimized using the MMFF force field. Thus, 100 minima of energy within an energy gap of $10 \mathrm{kcal} \cdot \mathrm{mol}^{-1}$ were generated. These structures were analysed and ordered considering the relative energy, being the 
repeated geometries eliminated. In all cases, molecules with the lowest energy and an energy gap of $3.0 \mathrm{kcal} \cdot \mathrm{mol}^{-1}$ were selected and studied at the M11L level.

2.1.7 Interfacial SPOCQ reaction on stainless steel. Stainless steel substrates were obtained from Goodfellow Inc. and further cut into $1 \times 1 \mathrm{~cm}^{2}$ pieces using an industrial metal cutter. The bare surface was initially cleaned by 15 min of sonication in methanol, followed by $15 \mathrm{~min}$ of sonication in dichloromethane. Final removal of adsorbed carbon impurities was effected by a 3 min plasma cleaning. To introduce reactive functional groups, the chip was immersed under an argon atmosphere into a solution of a 3:1 mixture of octylphosphonic acid and 12-bromododecylphosphonic acid (1 $\mathrm{mM}$ in ethanol) at room temperature overnight. After that, the substrates were then rinsed with ethanol and placed in a curing oven at $120{ }^{\circ} \mathrm{C}$ for $4 \mathrm{~h}$. The modified surfaces were allowed to cool to room temperature, rinsed twice with isopropyl alcohol and twice with dichloromethane, and dried under a stream of $\mathrm{N}_{2}$ to yield the modified surface (S9). Next, functionalized surfaces (S9) were immersed in a dopamine hydrochloride solution (neutralized with freshly distilled triethylamine) in methanol for $30 \mathrm{~min}$. The samples were sonicated with methanol, washed with copious amounts of water followed by methanol, dried and stored under nitrogen atmosphere to yield the catechol terminated surface (S10). Then, surfaces were oxidised with periodate during $30 \mathrm{~min}$ (S11). Finally, quinone-functionalized slides (S11) were immersed in $3 \mathrm{mM}$ BCN derivative (1) in DCE solution under $\mathrm{N}_{2}$ and kept reacting. After completion of reaction for the desired time $(8 \mathrm{~h})$, the pieces (S12) were taken out, sonicated and washed with acetone to remove physisorbed moieties, dried under $\mathrm{N}_{2}$ stream and immediately. As shown in Figure S14, a $\mathrm{F} / \mathrm{N}$ ratio around 9 indicated the full conversion of quinone groups immobilized on the silicon surfaces.

2.1.8 Interfacial SPOCQ reaction on oxidized silicon (Strategy A, epoxide ring opening with dopamine under basic conditions). Si (111) wafers were cut 
into pieces of $1 \times 1 \mathrm{~cm}^{2}$ and cleaned with piranha solution $\left(\mathrm{H}_{2} \mathrm{SO}_{4}: 30 \% \mathrm{H}_{2} \mathrm{O}_{2} 3: 1 \mathrm{v} / \mathrm{v}\right)$ for $1 \mathrm{~h}$ at $50{ }^{\circ} \mathrm{C}$ to remove organic contaminants. Caution: Piranha solutions react violently with organic materials and should be handled with extreme care. Then the pieces were washed with deionized water and air-dried. To introduce reactive functional groups, the chip was immersed under an argon atmosphere into a $2 \%$ in toluene solution (containing a 3:1 mixture of trimethoxy(propyl)silane and 3glycidoxypropyl trimethoxysilane) for $2 \mathrm{~h}$ at room temperature. After $2 \mathrm{~h}$, the pieces were washed with 2 - propanol and air-dried. Finally, the chips were baked for 10 $\min$ at $150{ }^{\circ} \mathrm{C}(\mathbf{S 1 3})$. Next, functionalized surfaces $(\mathbf{S 1 3})$ were inmersed in a dopamine hydrochloride solution (neutralized with freshly distilled triethylamine) in methanol for $30 \mathrm{~min}$. The samples were sonicated with methanol, washed with copious amounts of water followed by methanol, dried and stored under nitrogen atmosphere to yield the catechol terminated surface (S14). Then, surfaces were oxidised with periodate during $30 \mathrm{~min}$ (S15). Finally, quinone-functionalized slides (S15) were immersed in $3 \mathrm{mM}$ BCN derivative (1) in DCE solution under $\mathrm{N}_{2}$ and kept reacting. After completion of reaction for the desired time $(8 \mathrm{~h})$, the pieces (S16) were taken out, sonicated and washed with acetone to remove physisorbed moieties, dried under $\mathrm{N}_{2}$ stream and immediately. As shown in Figure $\mathrm{S} 14$, a $\mathrm{F} / \mathrm{N}$ ratio around 9 indicated the full conversion of quinone groups immobilized on the silicon surfaces. 


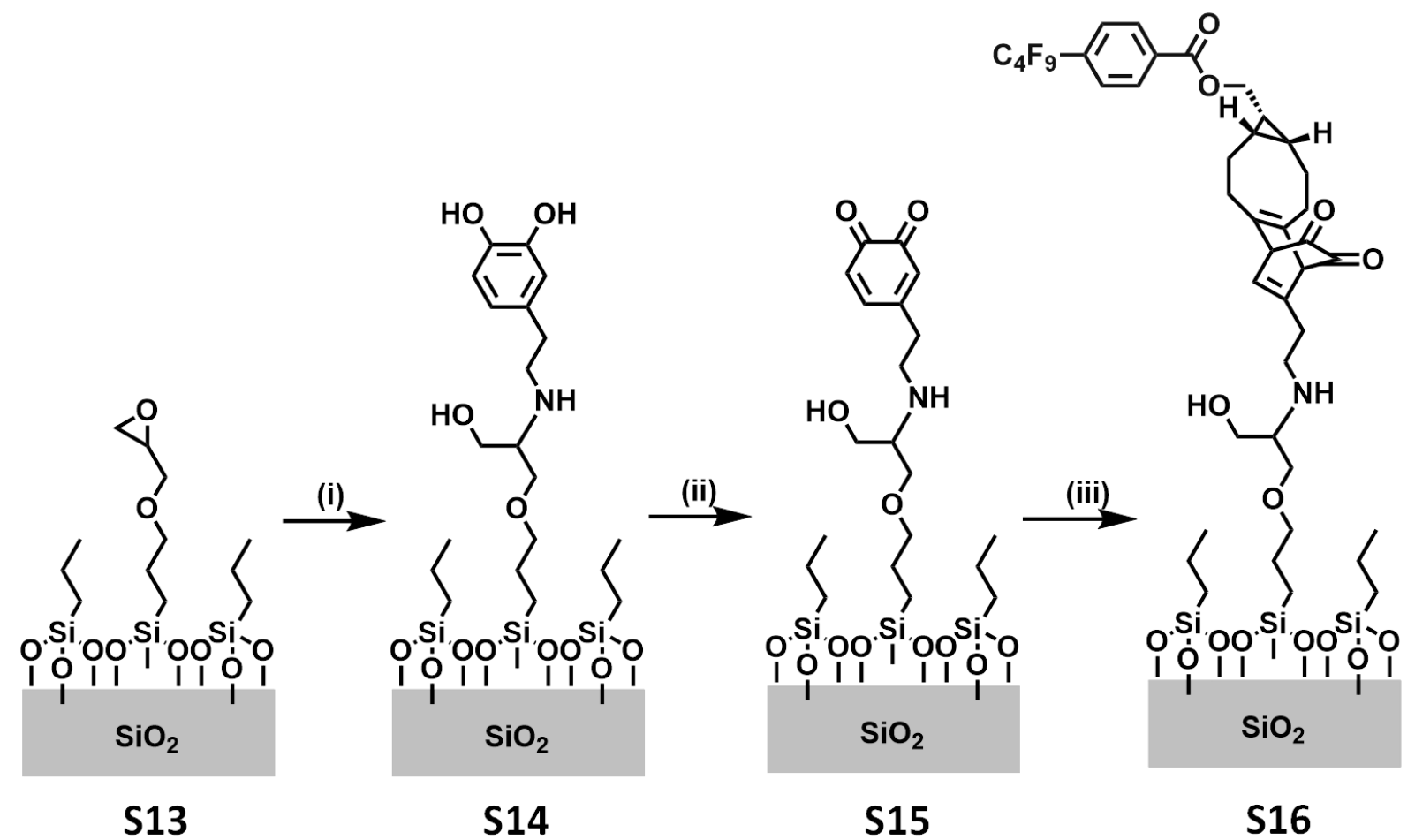

Scheme S6. Strategy A: epoxide ring opening with dopamine under basic conditions.

2.1.9 Interfacial SPOCQ reaction on oxidized silicon (Strategy B, nucleophilic substitution with dopamine under basic conditions). Si (111) wafers were cut into pieces of $1 \times 1 \mathrm{~cm}^{2}$ and cleaned with piranha solution $\left(\mathrm{H}_{2} \mathrm{SO}_{4}: 30 \% \mathrm{H}_{2} \mathrm{O}_{2} 3: 1 \mathrm{v} / \mathrm{v}\right)$ for $1 \mathrm{~h}$ at $50{ }^{\circ} \mathrm{C}$ to remove organic contaminants. Caution: Piranha solutions react violently with organic materials and should be handled with extreme care. Then the pieces were washed with deionized water and air-dried. To introduce reactive functional groups, the chip was immersed under an argon atmosphere into a $2 \%$ in toluene solution (containing a $3: 1$ mixture of trimethoxy(propyl)silane and (3-bromopropyl)trimethoxysilane) for $2 \mathrm{~h}$ at room temperature. After $2 \mathrm{~h}$, the pieces were washed with $2-$ propanol and air-dried. Finally, the chips were baked for $10 \mathrm{~min}$ at $150{ }^{\circ} \mathrm{C}(\mathbf{S 1 7})$. Next, functionalized surfaces $(\mathbf{S 1 7})$ were inmersed in a dopamine hydrochloride solution (neutralized with freshly distilled triethylamine) in methanol for $30 \mathrm{~min}$. The samples were sonicated with methanol, washed with copious amounts of water followed by methanol, dried and stored under nitrogen atmosphere to yield the catechol terminated surface (S18). 
Then, surfaces were oxidised with periodate during $30 \mathrm{~min}$ (S19). Finally, quinonefunctionalized slides (S19) were immersed in $3 \mathrm{mM}$ BCN derivative (1) in DCE solution under $\mathrm{N}_{2}$ and kept reacting. After completion of reaction for the desired time $(8 \mathrm{~h})$, the pieces $(\mathbf{S 2 0})$ were taken out, sonicated and washed with acetone to remove physisorbed moieties, dried under $\mathrm{N}_{2}$ stream and immediately. As shown in Figure S15, a $\mathrm{F} / \mathrm{N}$ ratio around 9 indicated the full conversion of quinone groups immobilized on the silicon surfaces.

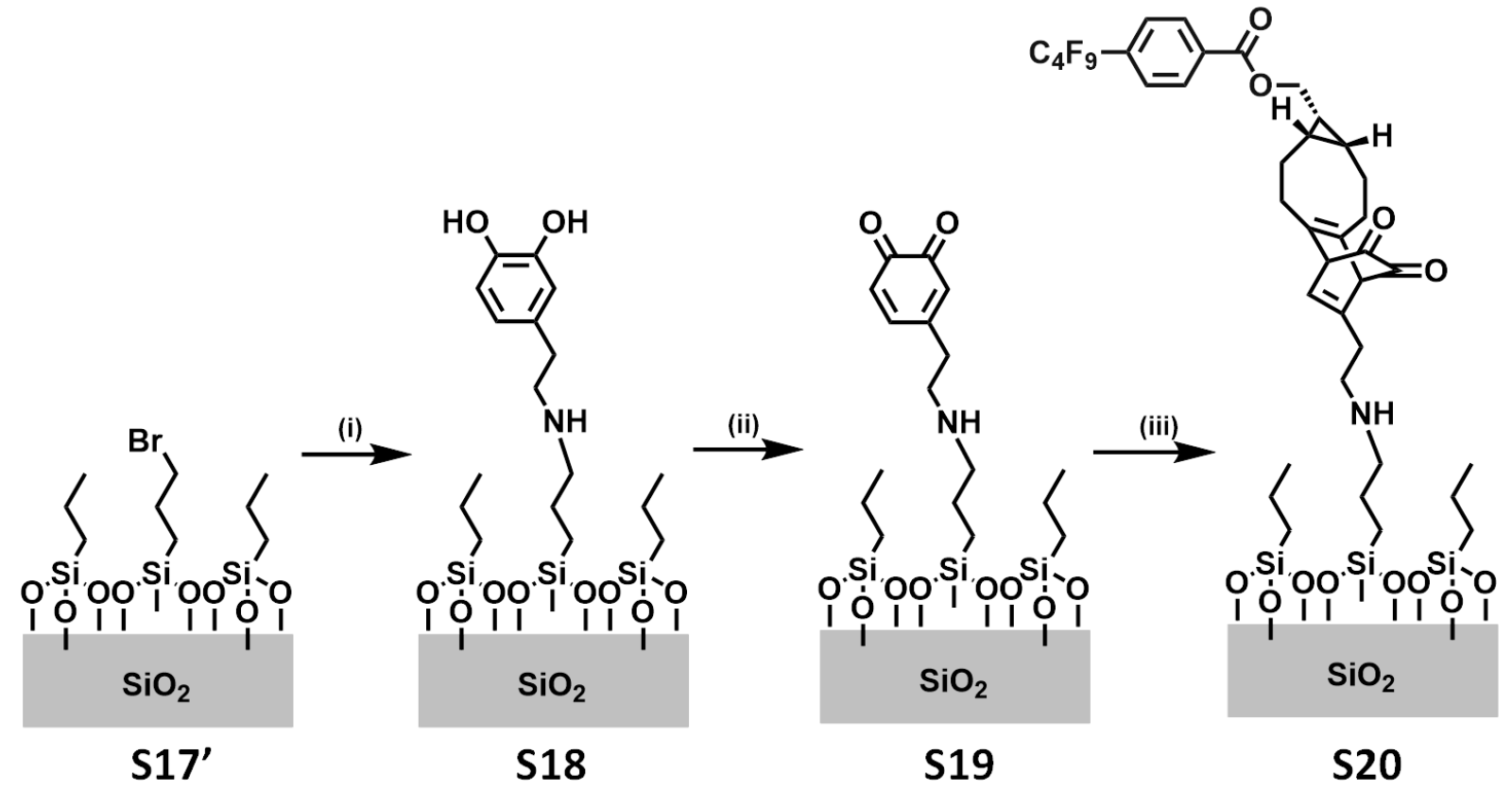

Scheme S7. Strategy B: nucleophilic substitution with dopamine under basic conditions,

2.1.10 Rate constant determination. Equation 1 describes the second order rate for the studied reaction $\left(\mathrm{mol} \cdot \mathrm{cm}^{-2} \cdot \mathrm{s}^{-1}\right)$, where $\Gamma_{Q}$ is the density of quinone groups on the surface $\left(\mathrm{mol} \cdot \mathrm{cm}^{-2}\right)$ and $\mathrm{k}_{2}$ is the second-order rate constant $\left(\mathrm{M}^{-1} \mathrm{~s}^{-1}\right)$. Since, the amount of $\mathrm{BCN}$-derivative $(3 \mathrm{mM})$ is in very large excess compared to the amount of surfacial quinone $\left(\Gamma_{Q}=\sim 6 \mathrm{pmol} \cdot \mathrm{cm}^{-2}\right)$ it can be assumed to be essentially constant throughout the entire kinetics experiment. Thus, the interfacial SPOCQ reaction can be reduced to pseudo-first order kinetics, with rate constant, k'. Therefore, from 
plots of $\ln \left[\left(I_{\infty}-I_{t}\right) /\left(I_{\infty}-I_{0}\right)\right]$ versus time the resulting pseudo first-order rate constant $\left(\mathrm{k}^{\prime}\right)$ can be obtained directly from the slope, as shown in equation (3).

$$
\begin{aligned}
& v=k_{2}[\mathbf{1}] \Gamma_{Q}=\frac{d \Gamma_{Q}}{d t} \\
& v=k \Gamma_{Q} \quad\left(\text { since },[\mathbf{1}] \gg \Gamma_{N}\right) \\
& \ln \left(\frac{I_{\infty}-I_{t}}{I_{\infty}-I_{0}}\right)=k^{\prime} t
\end{aligned}
$$

2.1.11 Determination of activation parameters. The activation parameters (enthalpy of activation $\left(\Delta H^{\ddagger}\right)$ and entropy of activation $\left(\Delta S^{\ddagger}\right)$ ) were obtained using Eyring equation by a least-squares fit of plots of $\ln \left(\mathrm{k}^{\prime} / \mathrm{T}\right)$ versus $1 / \mathrm{T}$. Values for $\Delta H^{\ddagger}$ and $\Delta S^{\ddagger}$ were calculated from the slope and intercept of the aforementioned plot (Equation 1).

$$
\ln \left(k^{\prime} /_{T}\right)=\ln \left(\kappa k_{B} /_{h}\right)+\Delta S^{\ddagger} /_{R}-\Delta H^{\ddagger} /_{R T}
$$

where, $k^{\prime}=$ observed rate constant $\left(\right.$ in $\mathrm{s}^{-1}$ ), $T=$ absolute temperature (in $\mathrm{K}$ ), $\kappa$ $=$ transmission coefficient $(=1), k_{B}=$ Boltzmann's constant, $h=$ Planck's constant, $R=$ gas constant, $\Delta S^{\dagger}=$ entropy of activation (in cal $\cdot \mathrm{K}^{-1} \cdot \mathrm{mol}^{-1}$ ), and $\Delta H^{\ddagger}=$ enthalpy of activation in $\left(\mathrm{kcal} \cdot \mathrm{mol}^{-1}\right)$. 


\subsection{Supplementary Figures.}
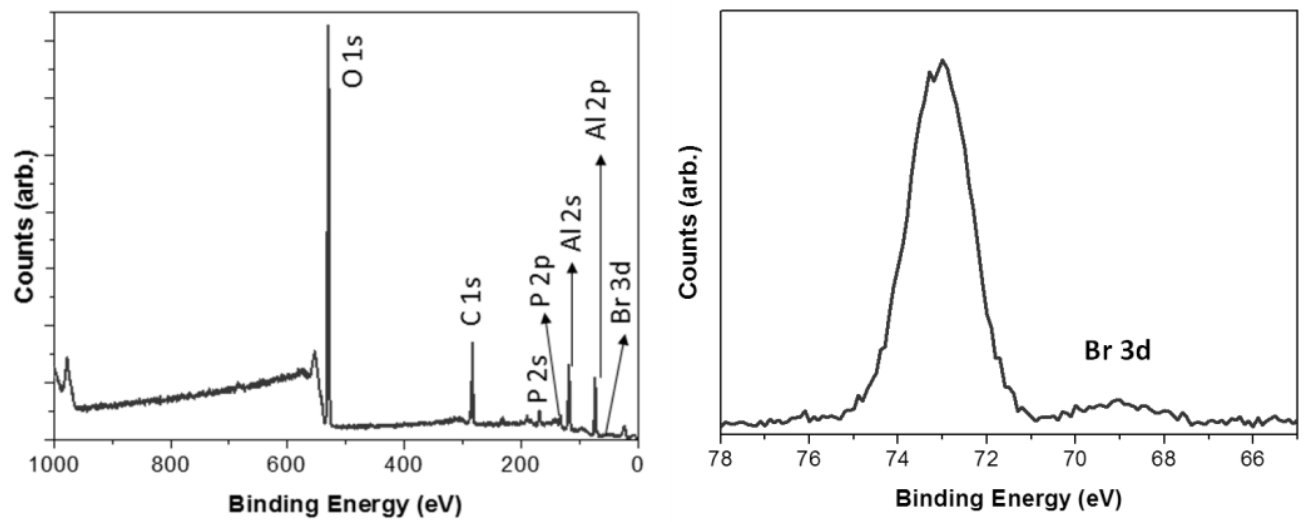

Supplementary Figure S1. XPS wide scan of Br-terminated monolayer (S1) with distinct $\mathrm{Br}$ peak visible in narrow scan.
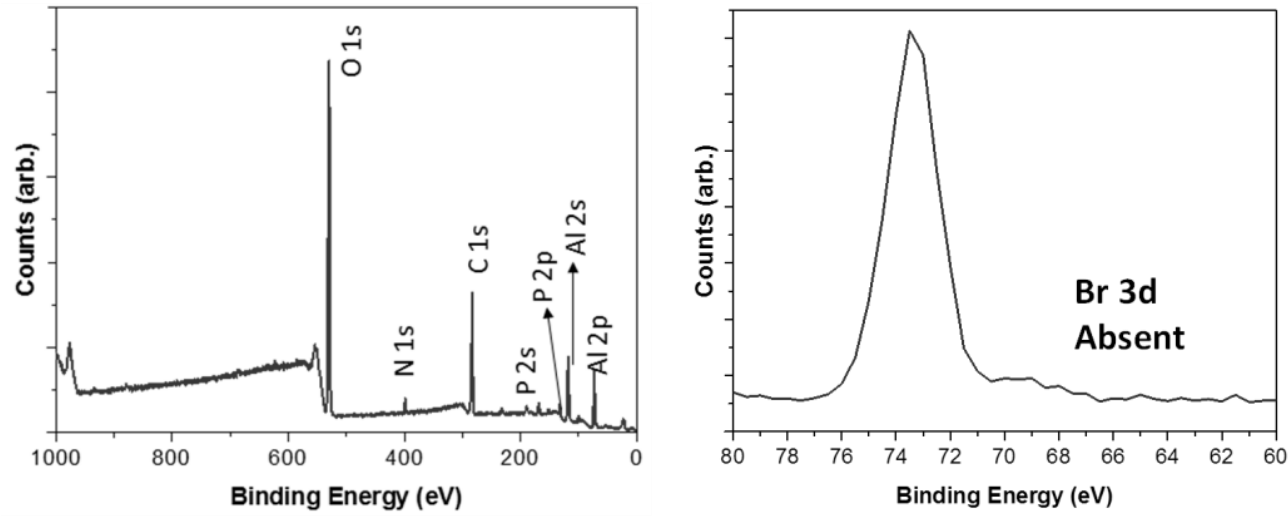

Supplementary Figure S2. XPS wide scan of catechol-terminated monolayer (S2) with now absent Br peak after $1 \mathrm{~h}$ stirring with dopamine.
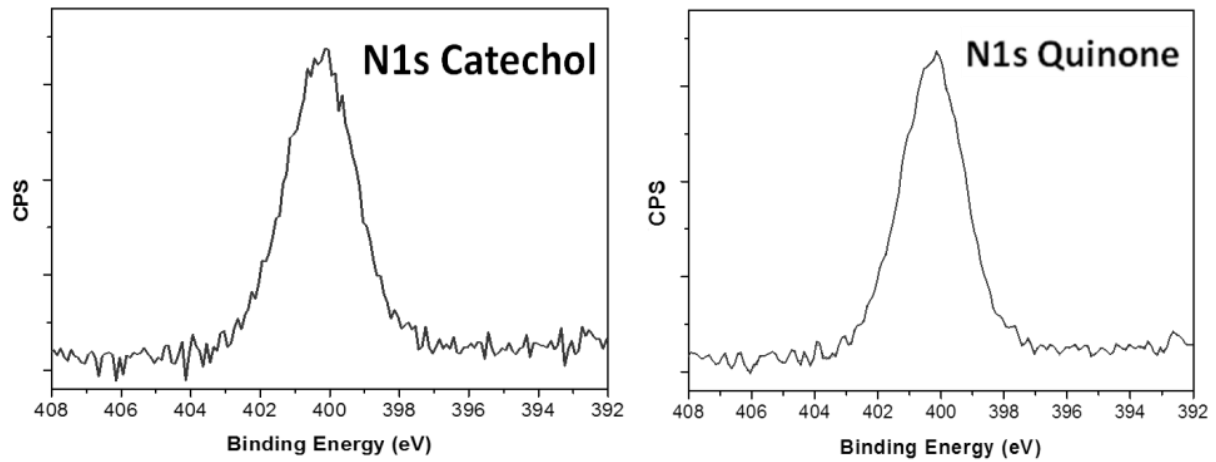

Supplementary Figure S3. XPS N1s narrow scan of catechol-terminated monolayer (S2) and quinone-terminated monolayer (S3) shows that the dopamine nitrogen essentially remains unchanged, before and after periodate oxidation. 

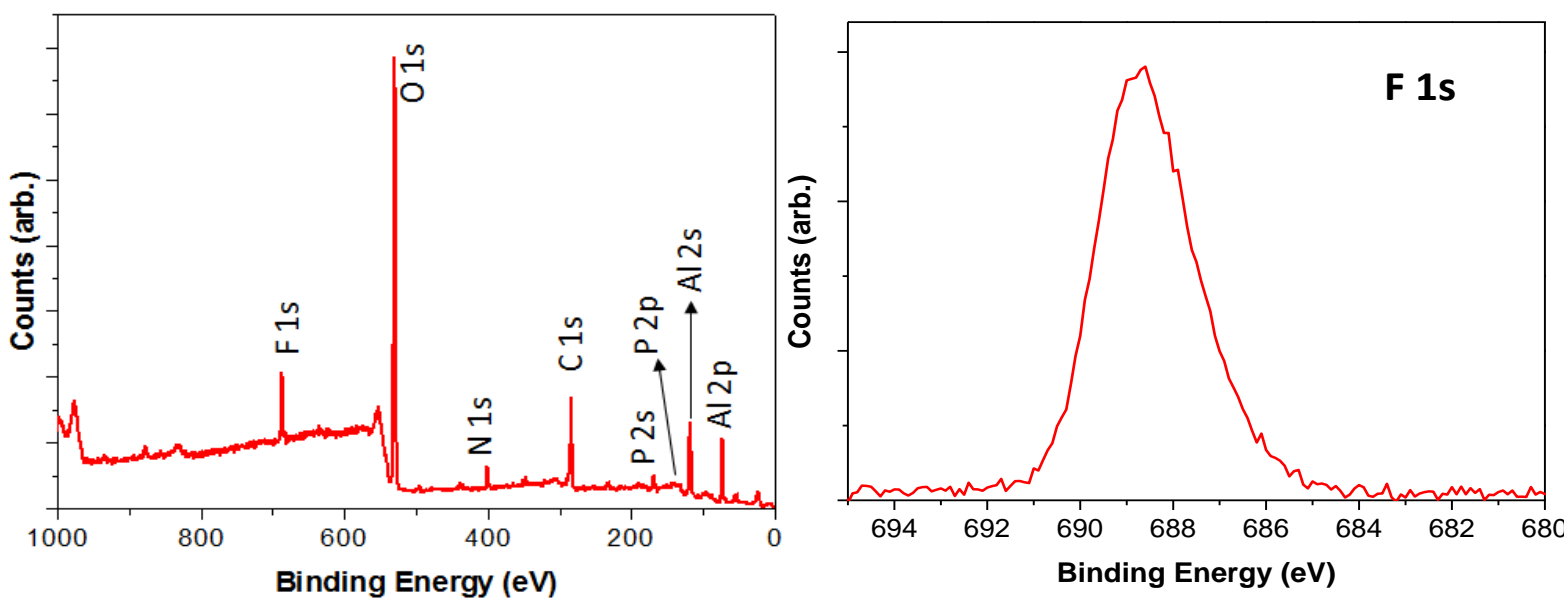

Supplementary Figure S4. XPS wide and F1s narrow scans after SPOCQ reaction (S4) for 30 mins clearly showing visible F1s peak at $686 \mathrm{eV}$.

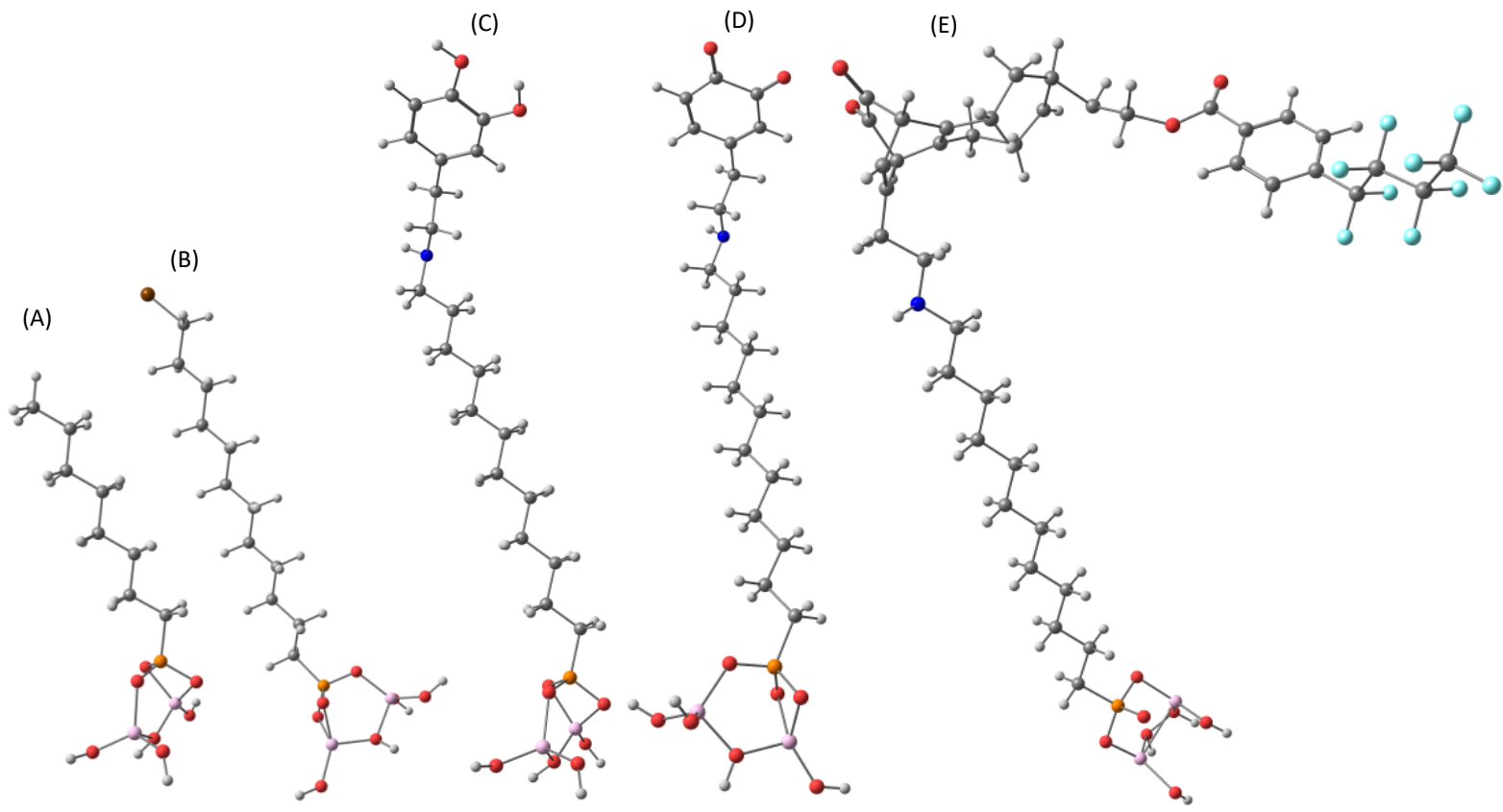

Supplementary Figure S5. Optimized geometries of models used to simulate the XPS spectra of the different monolayers attached to the aluminium oxide surface. (A) octyl phosphonic acid, (B) 12-bromododecyl phosphonic acid (S1), (C) dopaminefunctionalized surface (S2), (D) 1,2-quinone-functionalized surface (S3) and (E) SPOCQ cycloadduct after reaction of BCN derivative $\mathbf{1}$ with 1,2-quinonefunctionalized surface (S4). 


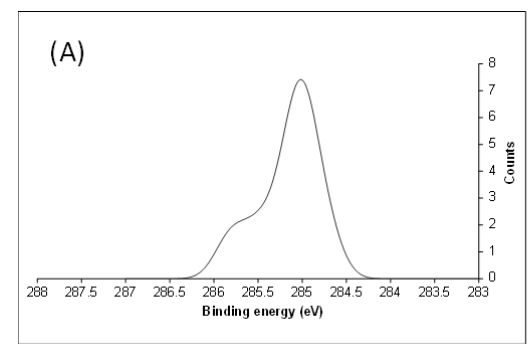

(B)

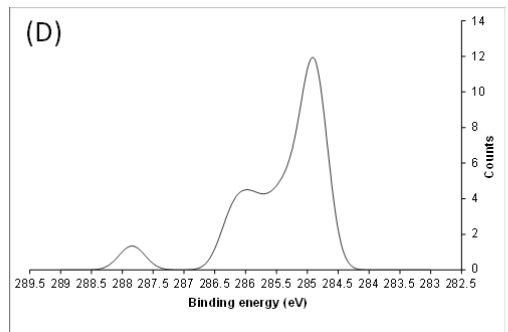

(C)
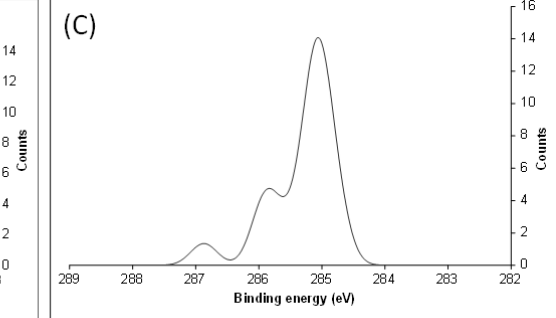

Supplementary Figure S6. Simulated C1s XPS narrow of aluminium oxide surfaces containing (A) octyl phosphonic acid monolayer, (B) 12-bromododecyl phosphonic acid monolayer (S1), (C) dopamine-functionalized monolayer (S2), (D) 1,2-quinonefunctionalized monolayer (S3) and (E) SPOCQ cycloadduct after reaction of BCN derivative $\mathbf{1}$ with 1,2-quinone-functionalized surface $(\mathbf{S 4})$. Simulations were performed by DFT calculations at M11L/6-311g(d,p) theory level with Gaussian 09. ${ }^{2}$
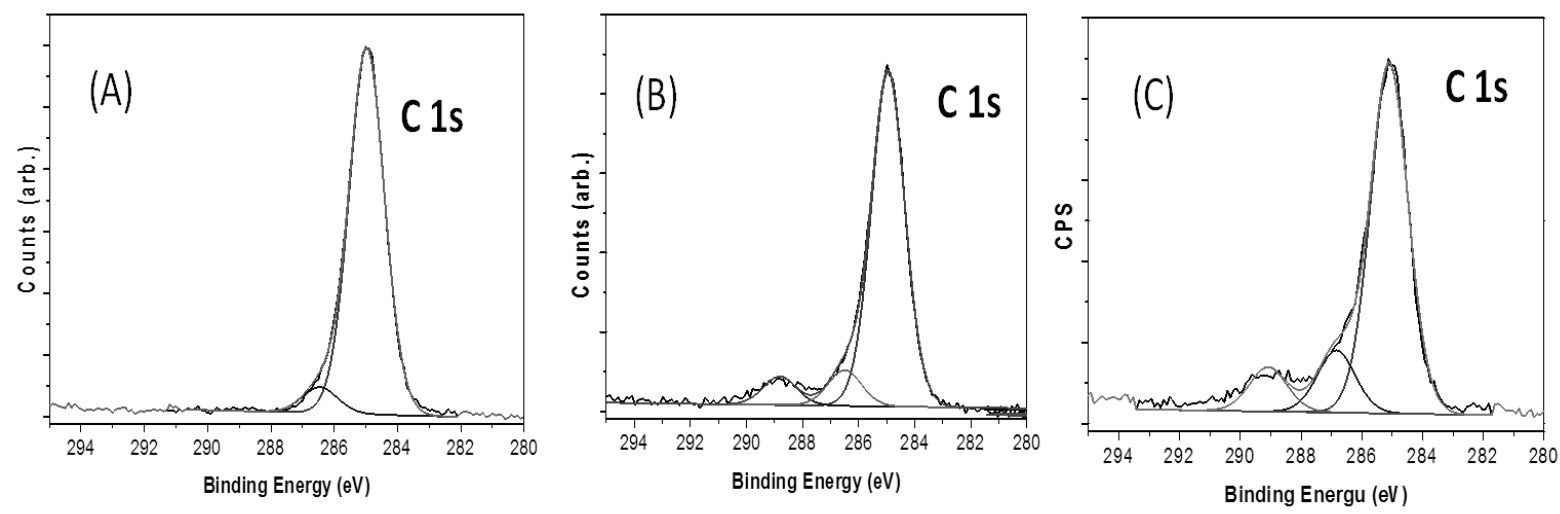

Supplementary Figure S5. C1s XPS narrow scan after SPOCQ reaction for (A) S1, (B) S2 and (C) S3 of M1 type of microenvironment. 


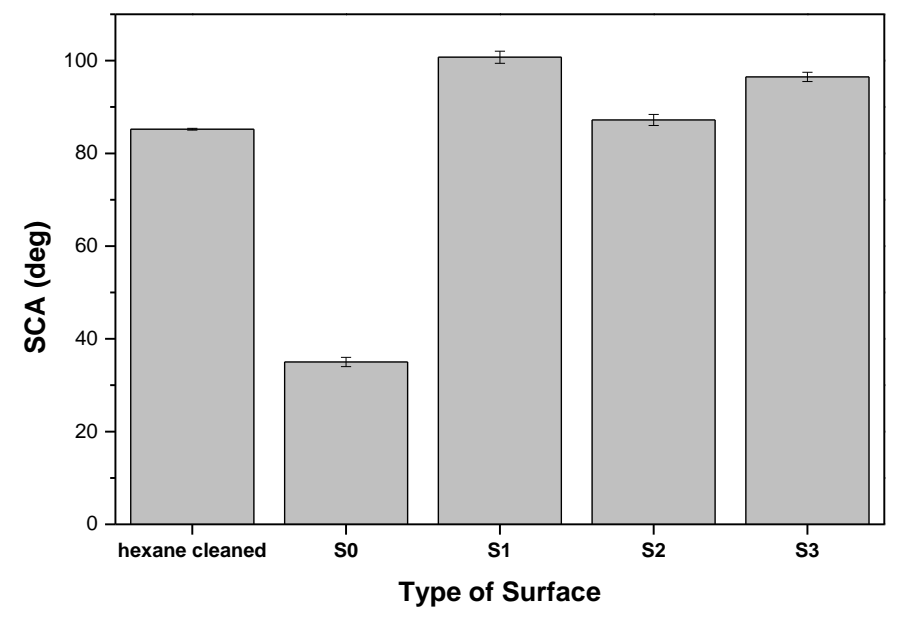

Supplementary Figure S6. Water SCA of different type of surface. All measurements and errors are for hexaplet droplets.

(A)

$$
101 \pm 0.9
$$

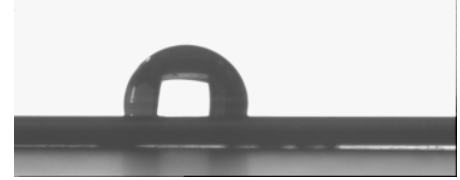

(C)

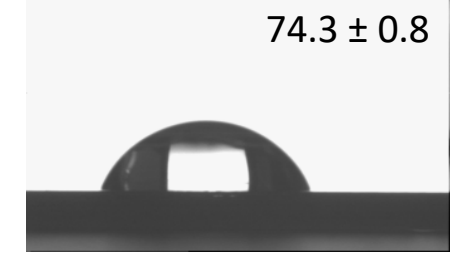

(B)

$91.4 \pm 0.9$

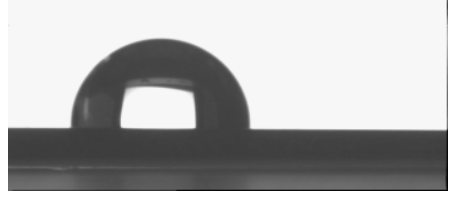

(D)

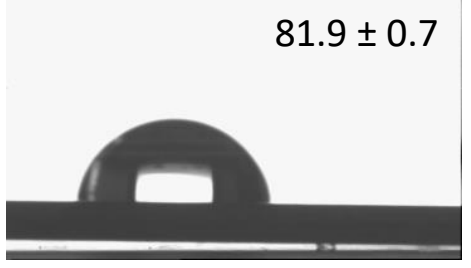

Supplementary Figure S7. Water SCA images for 3:1 mixed monolayers with (A) Br functionalization, (B) dopamine functionalization, (C) 1,2-quinone functionalization and (D) after SPOCQ reaction with compound $1\left(1 \mathrm{~h}, 22^{\circ} \mathrm{C}\right)$. 


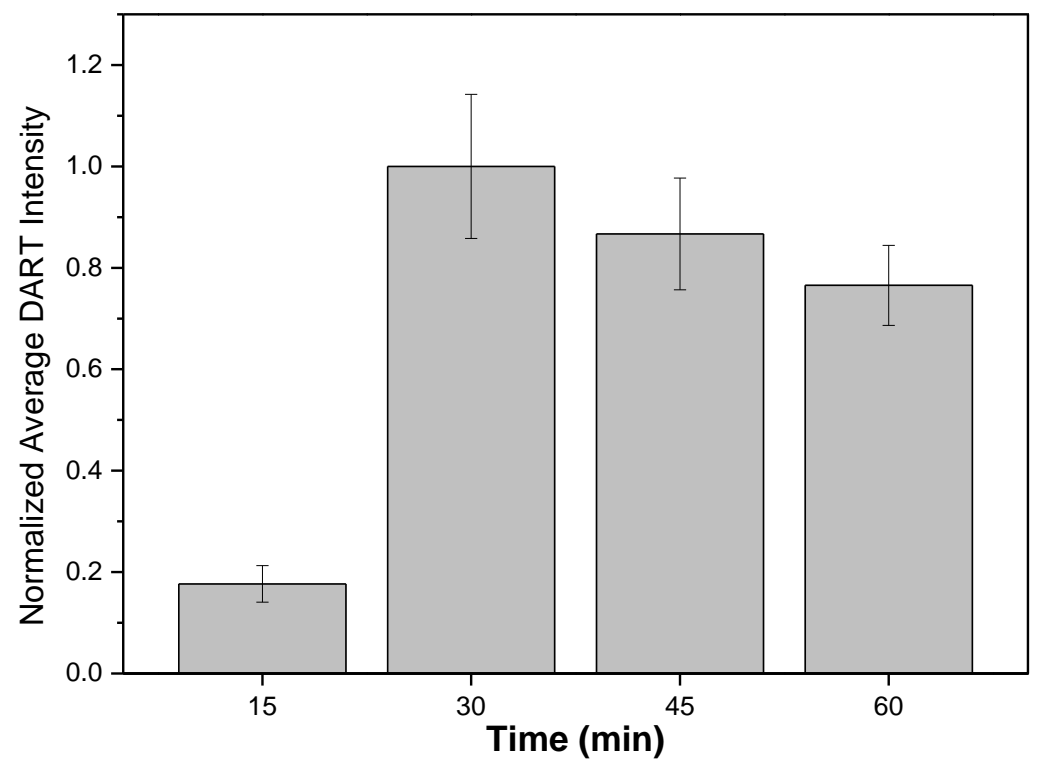

Supplementary Figure S8. Normalized average DART intensity of MS-tag signal $(\mathrm{m} / \mathrm{z} 339.0062)$ for varying periodate oxidation time followed by $1 \mathrm{~h}$ SPOCQ reaction.

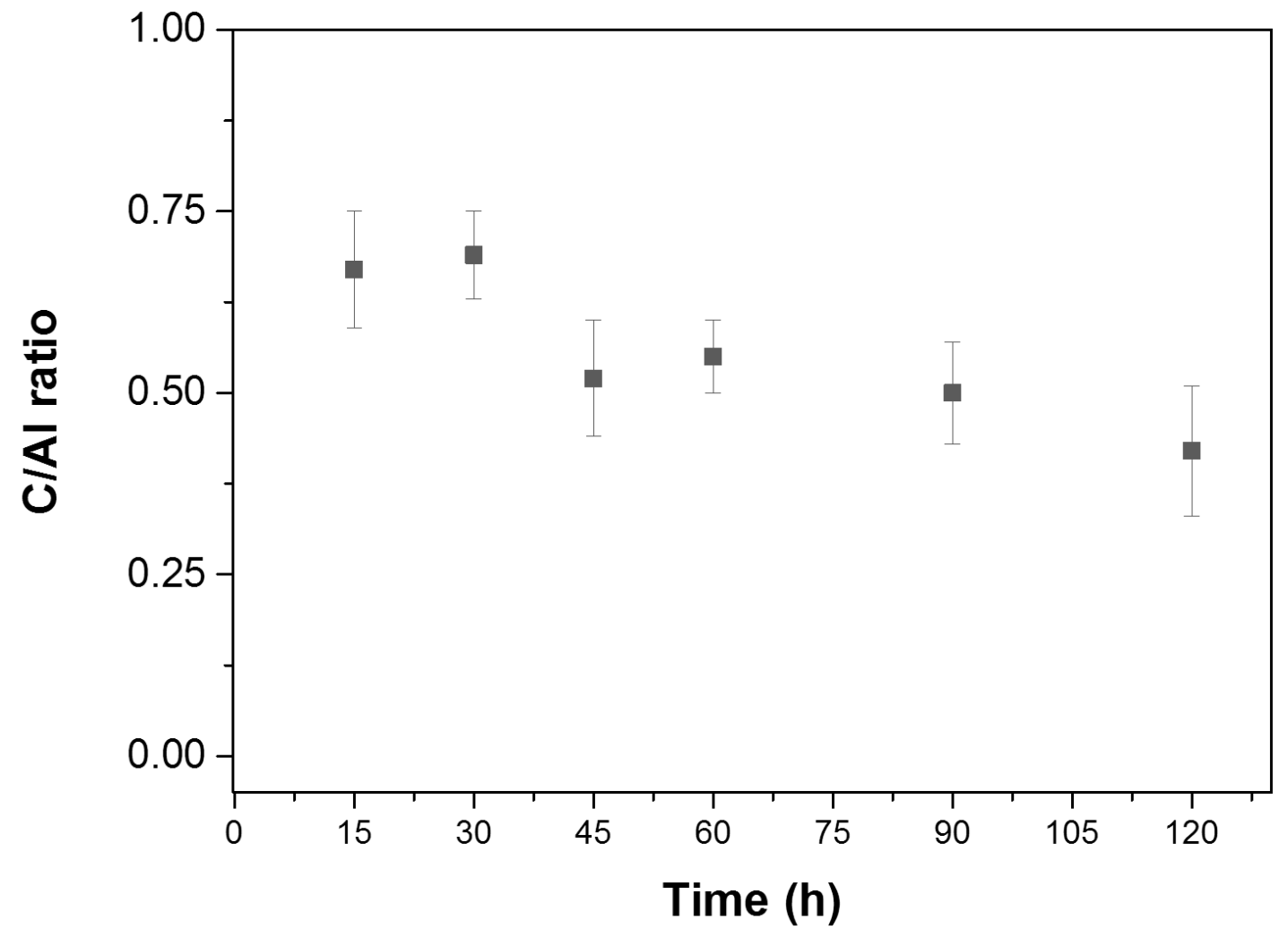

Supplementary Figure S9. C/Al ratio determined by XPS for different periodate oxidation times followed by $1 \mathrm{~h}$ SPOCQ reaction with compound $\mathbf{1}\left(1 \mathrm{~h}, 22{ }^{\circ} \mathrm{C}\right)$. 


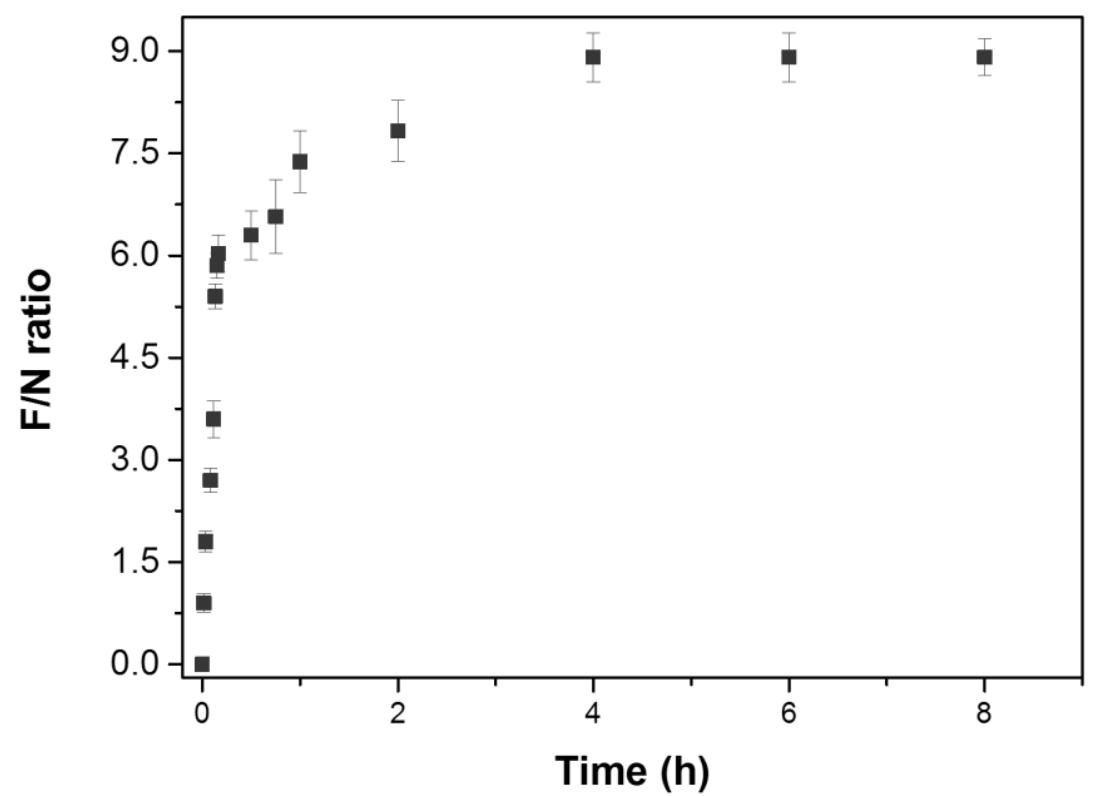

Supplementary Figure S10. F/N ratio determined by XPS after SPOCQ reaction with compound $1\left(1 \mathrm{~h}, 22{ }^{\circ} \mathrm{C}\right)$. Note that a $\mathrm{F} / \mathrm{N}$ ratio of 9 would correspond to a $100 \%$ conversion of catechol moieties into the corresponding SPOCQ adduct.

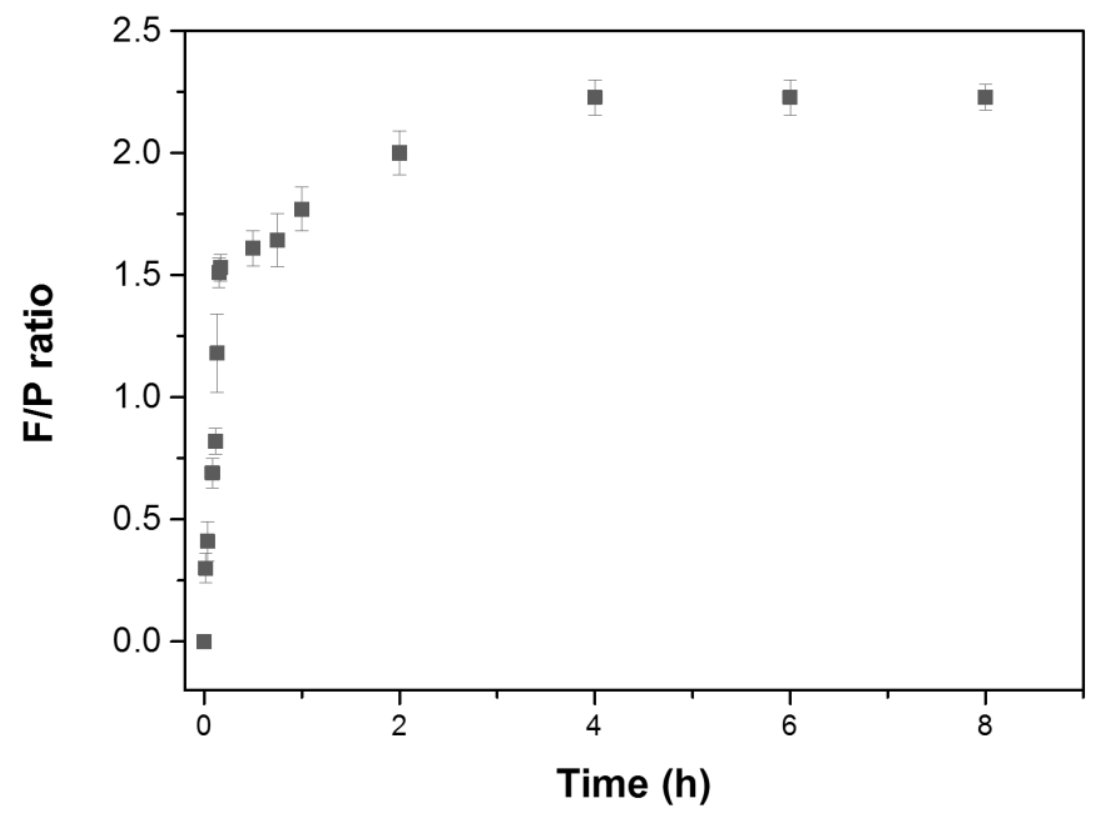

Supplementary Figure S11. F/P ratio determined by XPS after SPOCQ reaction with compound $1\left(1 \mathrm{~h}, 22{ }^{\circ} \mathrm{C}\right)$. Note that a $\mathrm{F} / \mathrm{P}$ ratio of 2.25 would correspond to a $100 \%$ conversion of catechol moieties into the corresponding SPOCQ adduct. 


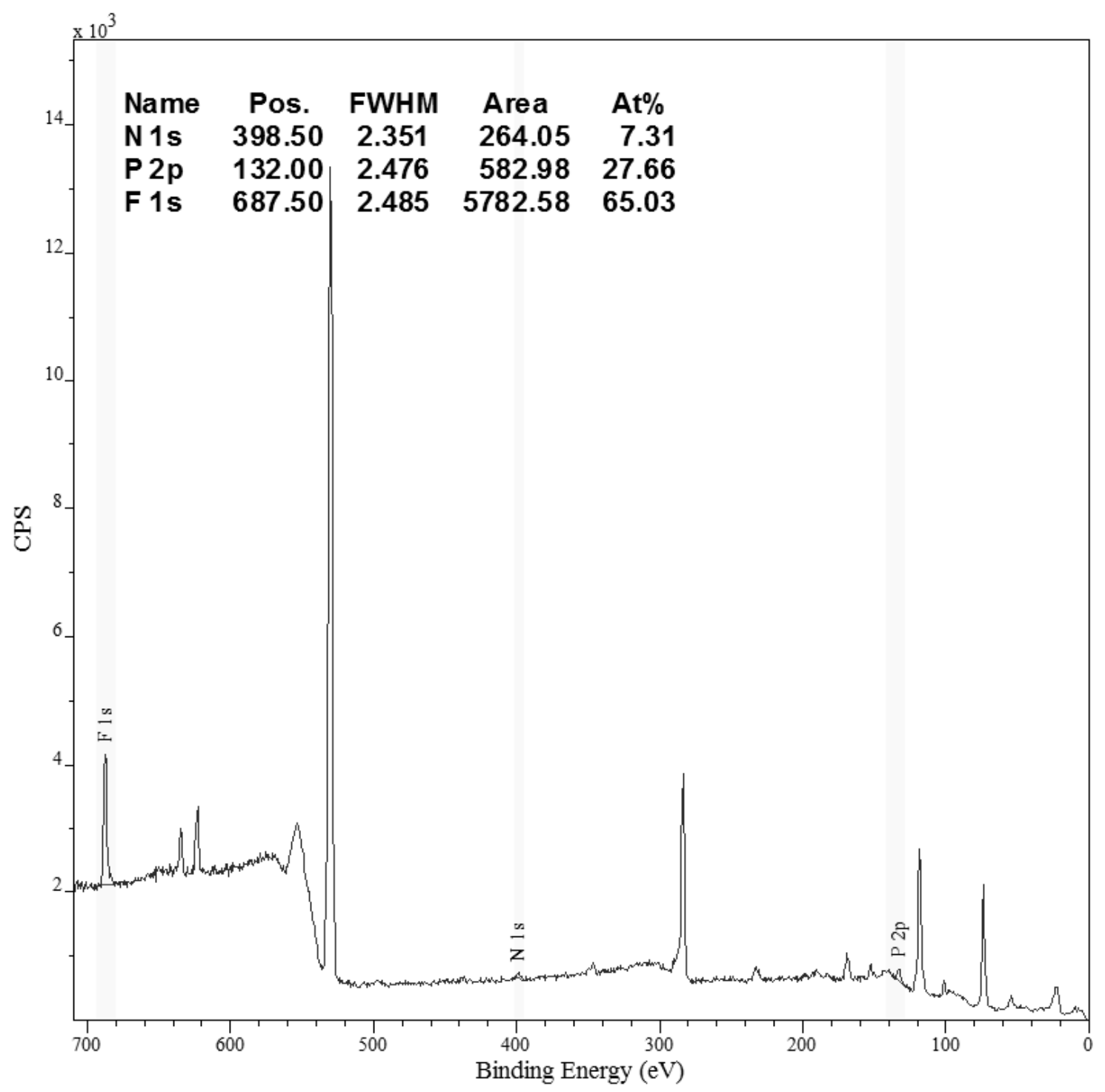

Supplementary Figure S12. Representative XPS wide range spectrum of a 3:1 monolayer after SPOCQ reaction showing full conversion of 1,2-quinone groups. The reaction efficiency can determined by comparing the experimentally determined $\mathrm{F} / \mathrm{N}$ or $\mathrm{F} / \mathrm{P}$ ratios with the theoretical values of $9 / 1$ or $9 / 4$, respectively, which corresponds to the effective click of the fluorine-labelled BCN with the 1,2-quinonefunctionalized monolayer. 


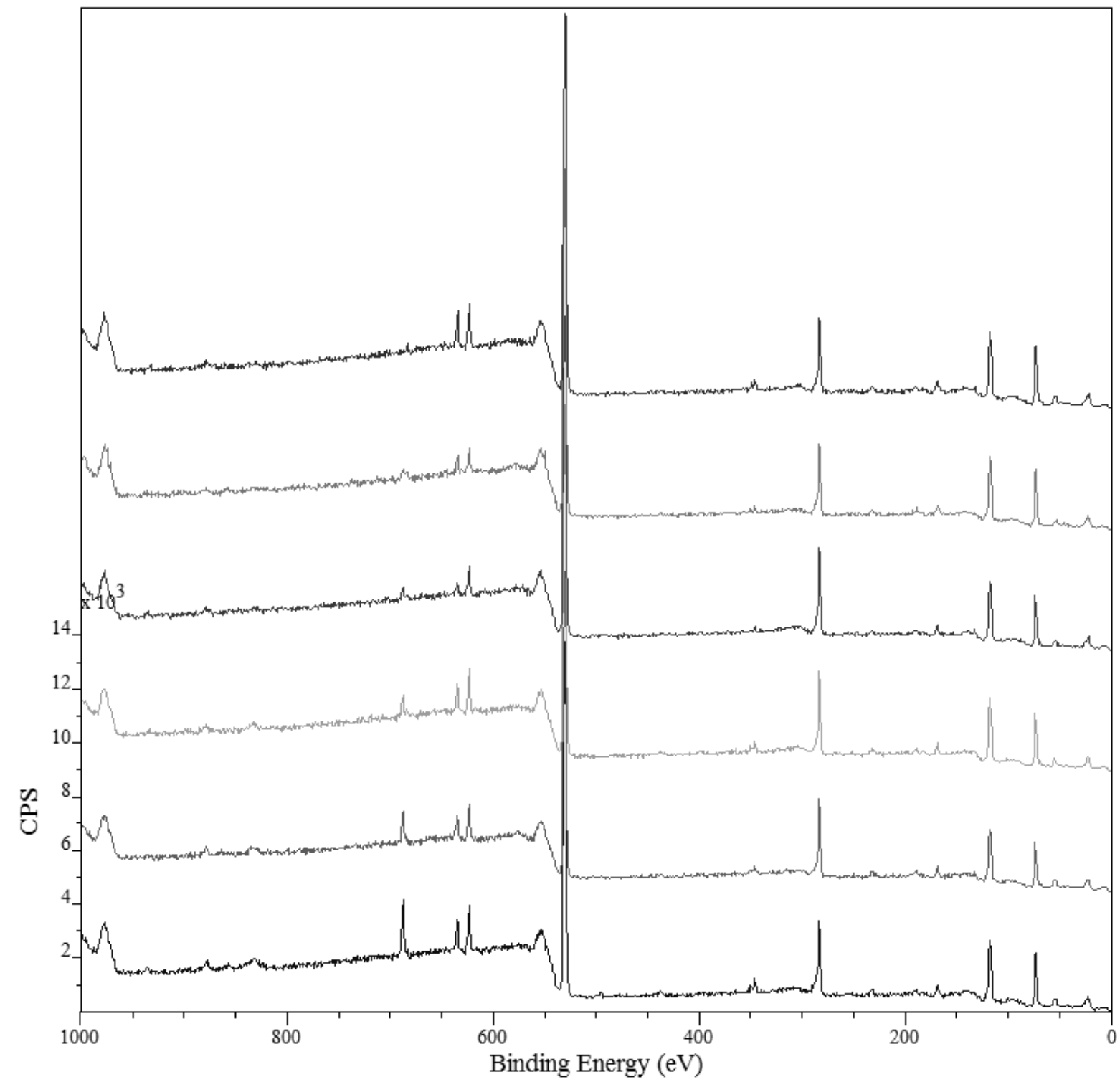

Supplementary Figure S13. Representative XPS wide range spectra of a 3:1 monolayer after SPOCQ reaction at different reaction times (from top to bottom: 0, 1, 2, 510 and $30 \mathrm{~min})$. 


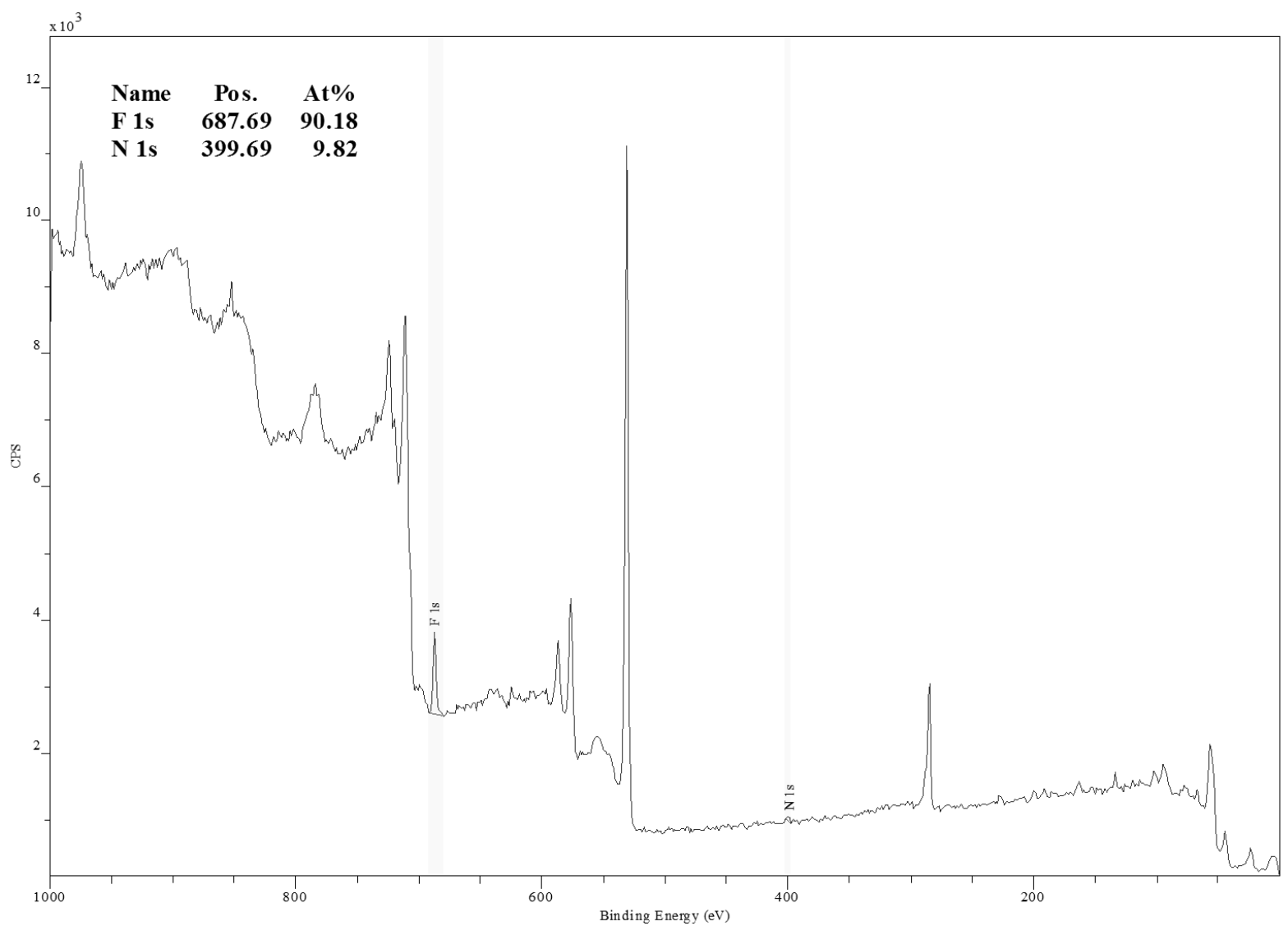

Supplementary Figure S14. Representative XPS wide range spectrum of a $3: 1$ monolayer after SPOCQ reaction in stainless steel showing full conversion of $1,2-$ quinone groups. Reaction efficiency can determined by comparing the $\mathrm{F} / \mathrm{N}$ ratio with the theoretical value of $9 / 1$, which corresponds to the effective click of the fluorinelabelled BCN with the 1,2-quinone-functionalized monolayer. 


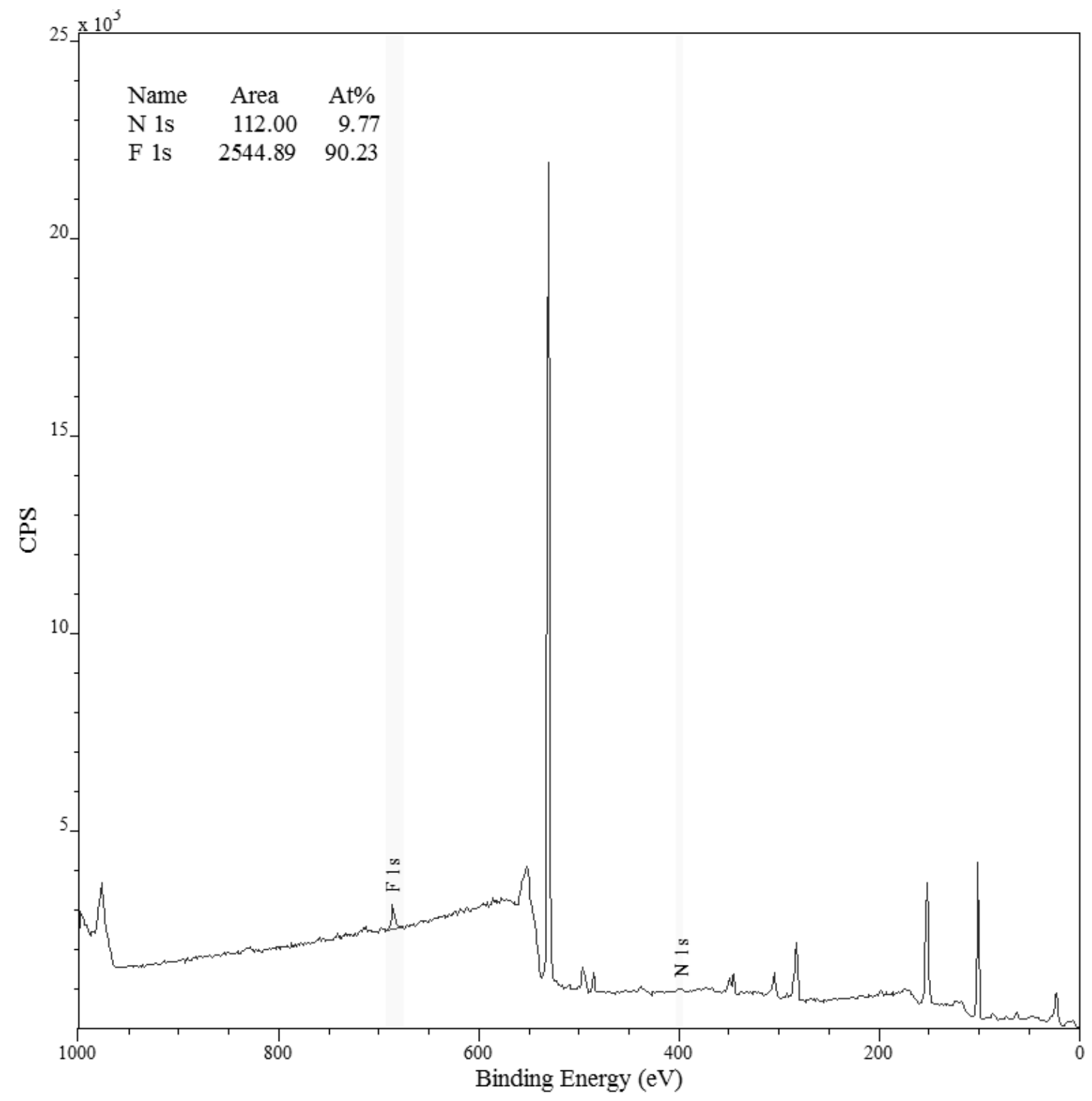

Supplementary Figure S15. Representative XPS wide range spectrum of a 3:1 monolayer after SPOCQ reaction in oxidized Si (111) modified via strategy A, showing full conversion of 1,2-quinone groups. Reaction efficiency can determined by comparing the $\mathrm{F} / \mathrm{N}$ with the theoretical value of $9 / 1$, which corresponds to the effective click of the fluorine-labelled $\mathrm{BCN}$ with the 1,2-quinone-functionalized monolayer. 


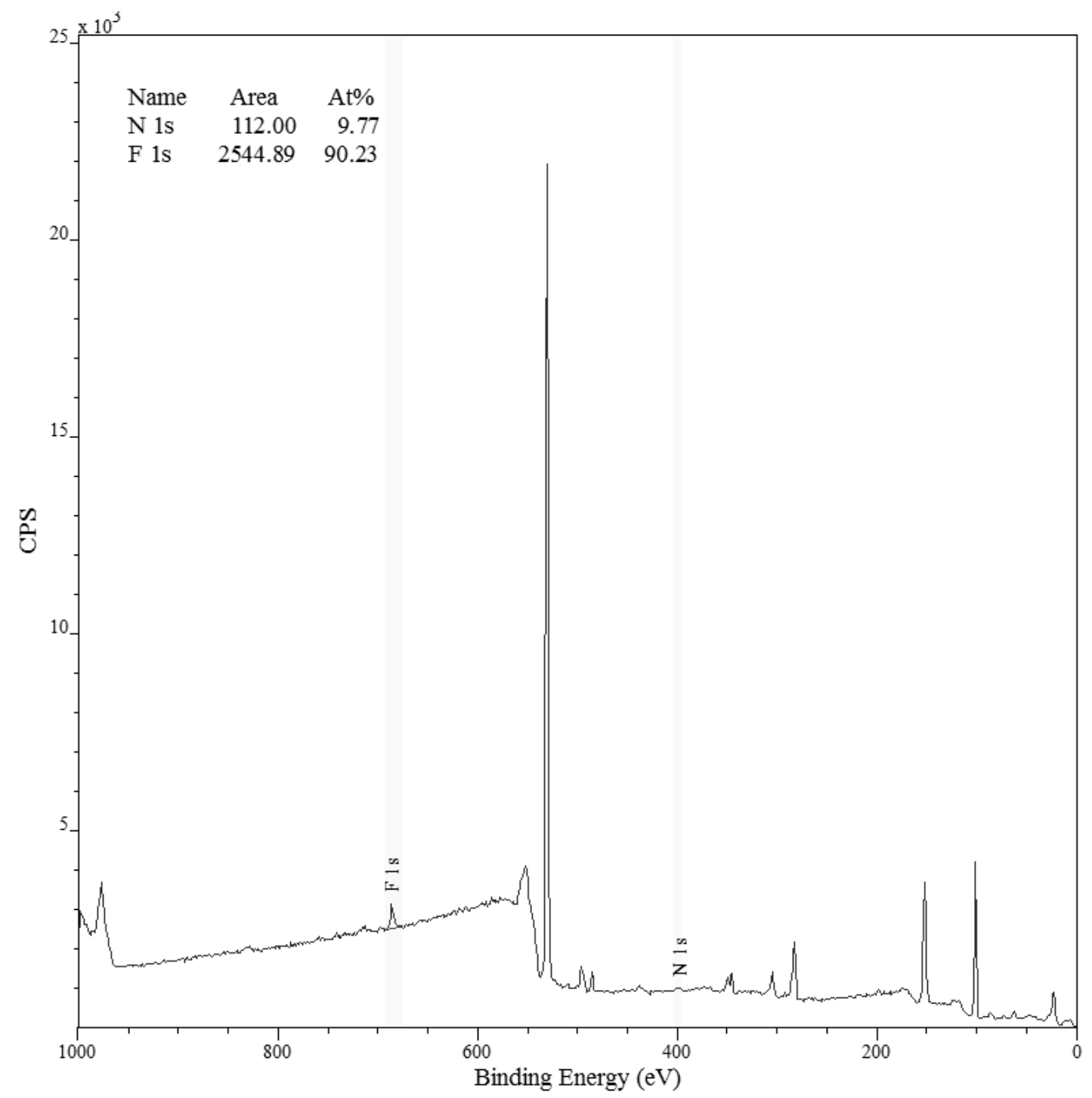

Supplementary Figure S15. Representative XPS wide range spectrum of a $3: 1$ monolayer after SPOCQ reaction in oxidized Si (111) modified via strategy B, showing full conversion of 1,2-quinone groups. Reaction efficiency can determined by comparing the $\mathrm{F} / \mathrm{N}$ ratio with the theoretical value of $9 / 1$, which corresponds to the effective click of the fluorine-labelled BCN with the 1,2-quinone-functionalized monolayer. 


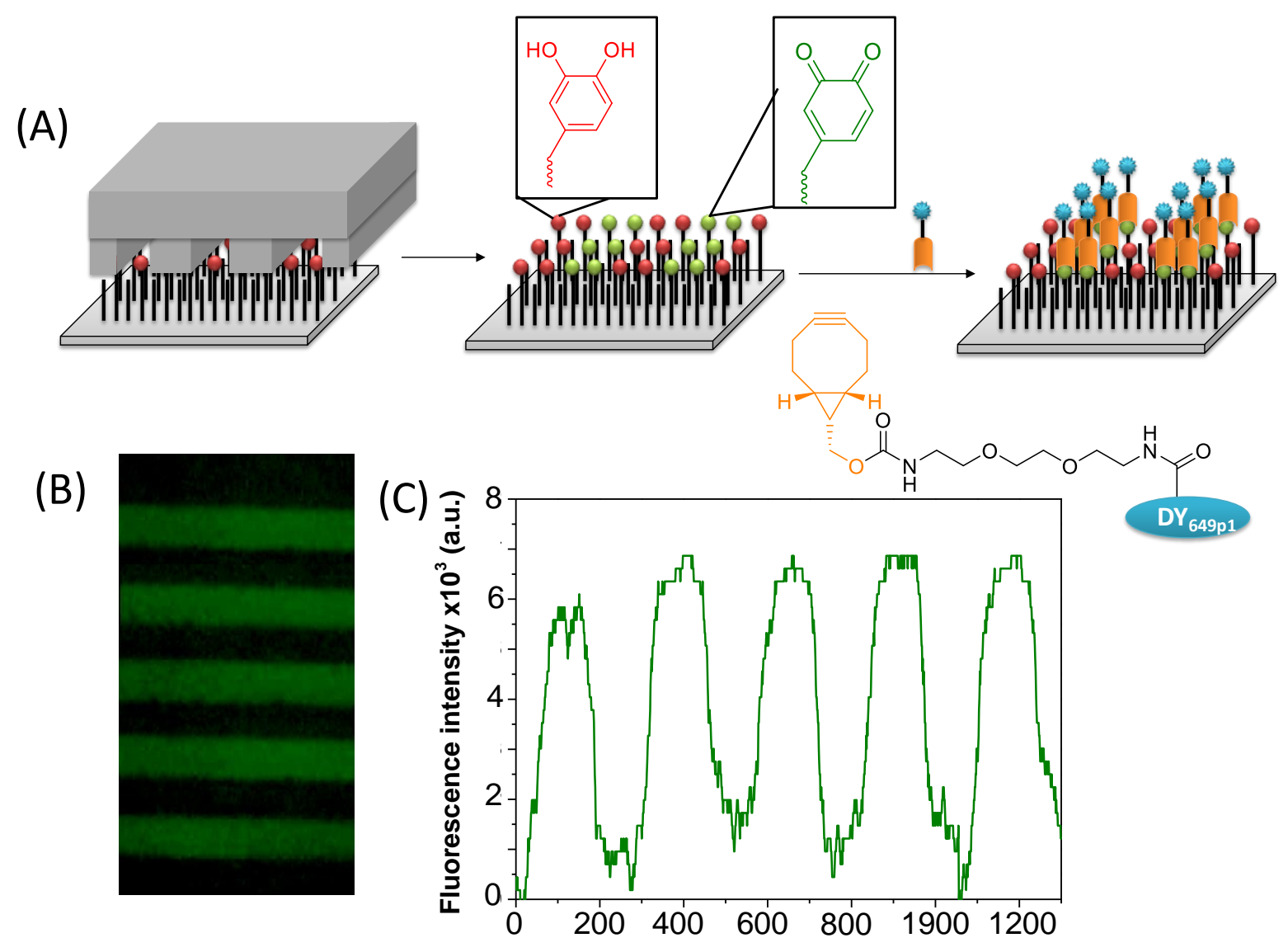

Supplementary Figure S16. (A) Schematic representation of locally generated 1,2quinones from catechol-functionalized $\mathrm{Al}$ surfaces via microcontact printing and (B) fluorescence microscopy images of locally oxidized surfaces after reaction with BCNDY649p1. 


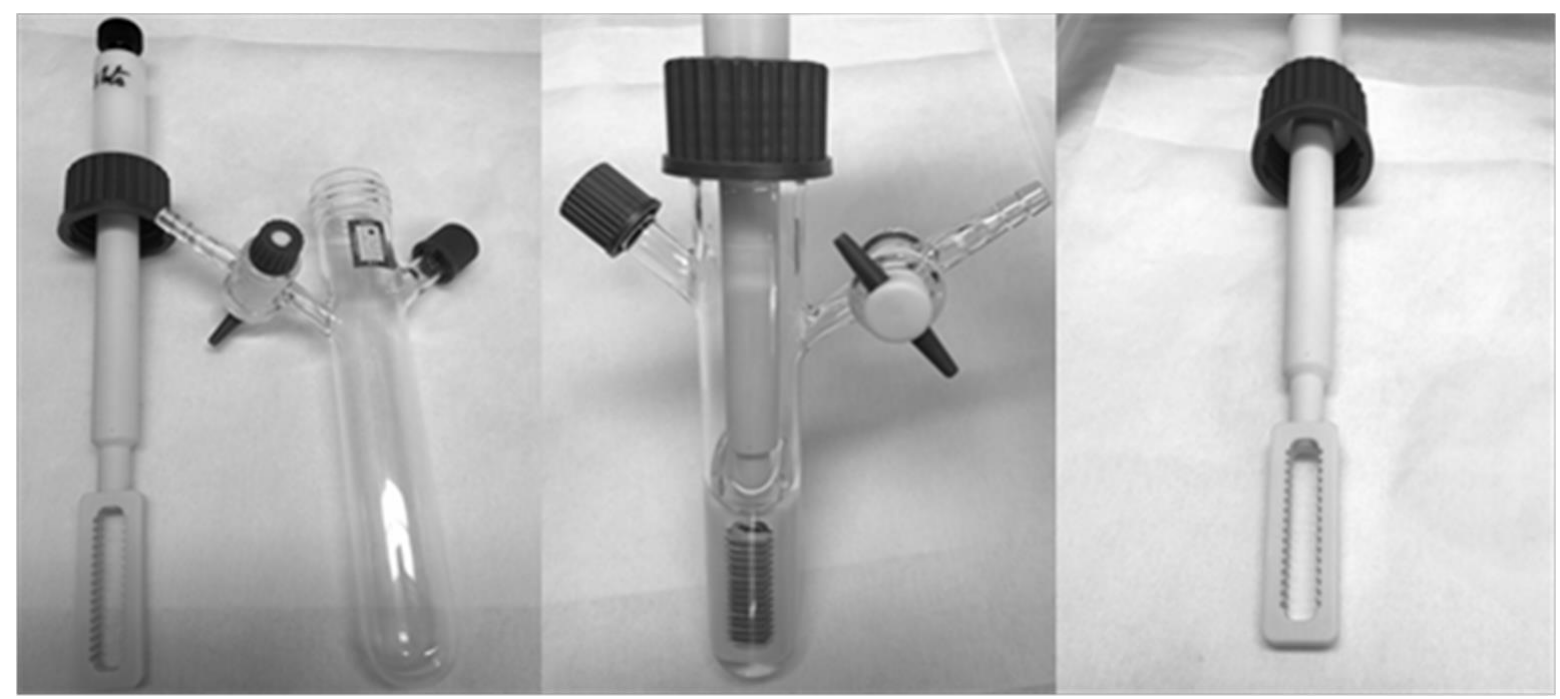

Supplementary Figure S17. Image of the sample holder.

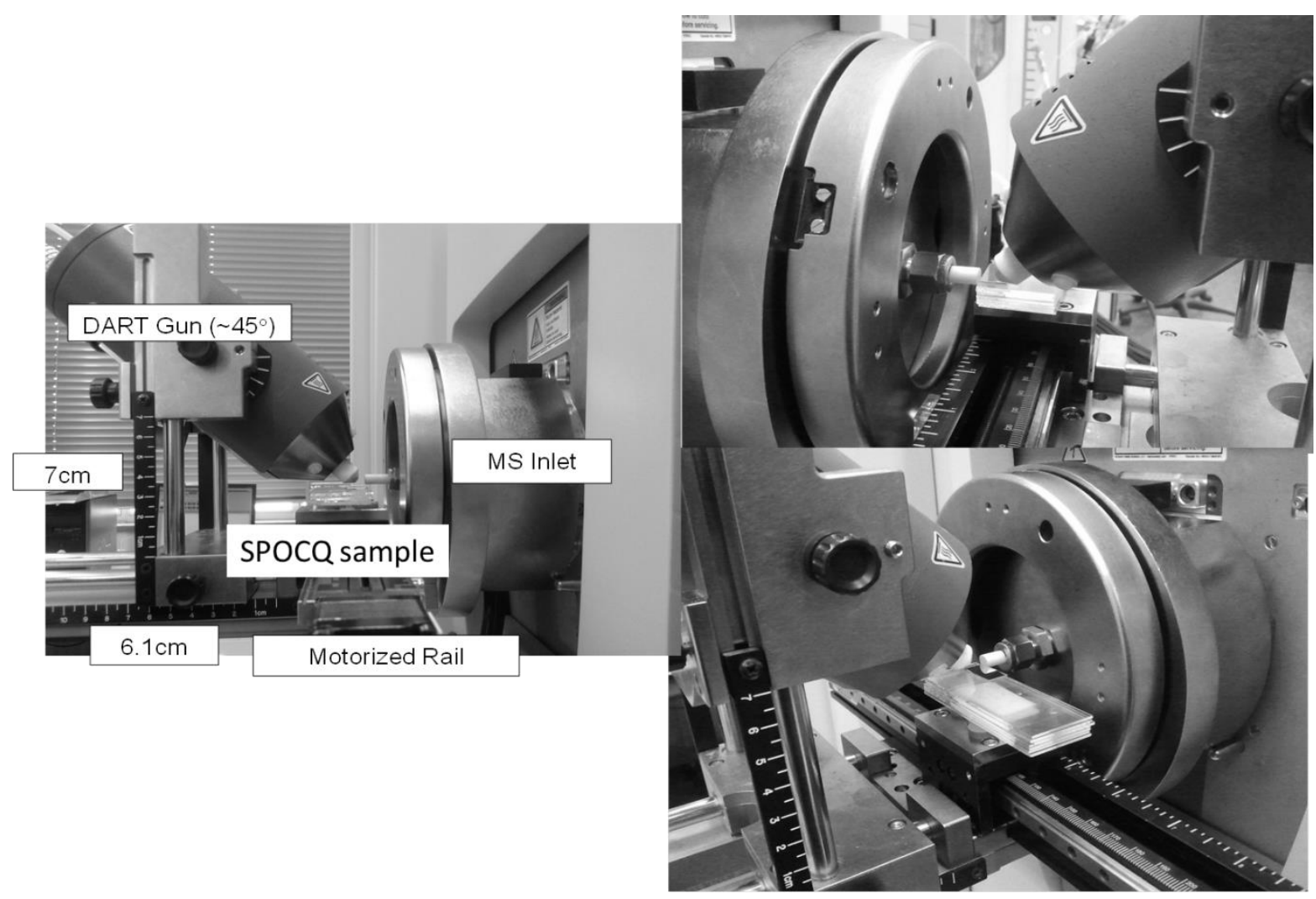

Supplementary Figure S18. Pictures of DART setup for the kinetic analysis of organic surface reactions. For clarity: the sample that is analyzed is $2 \times 1 \mathrm{~cm}$. 

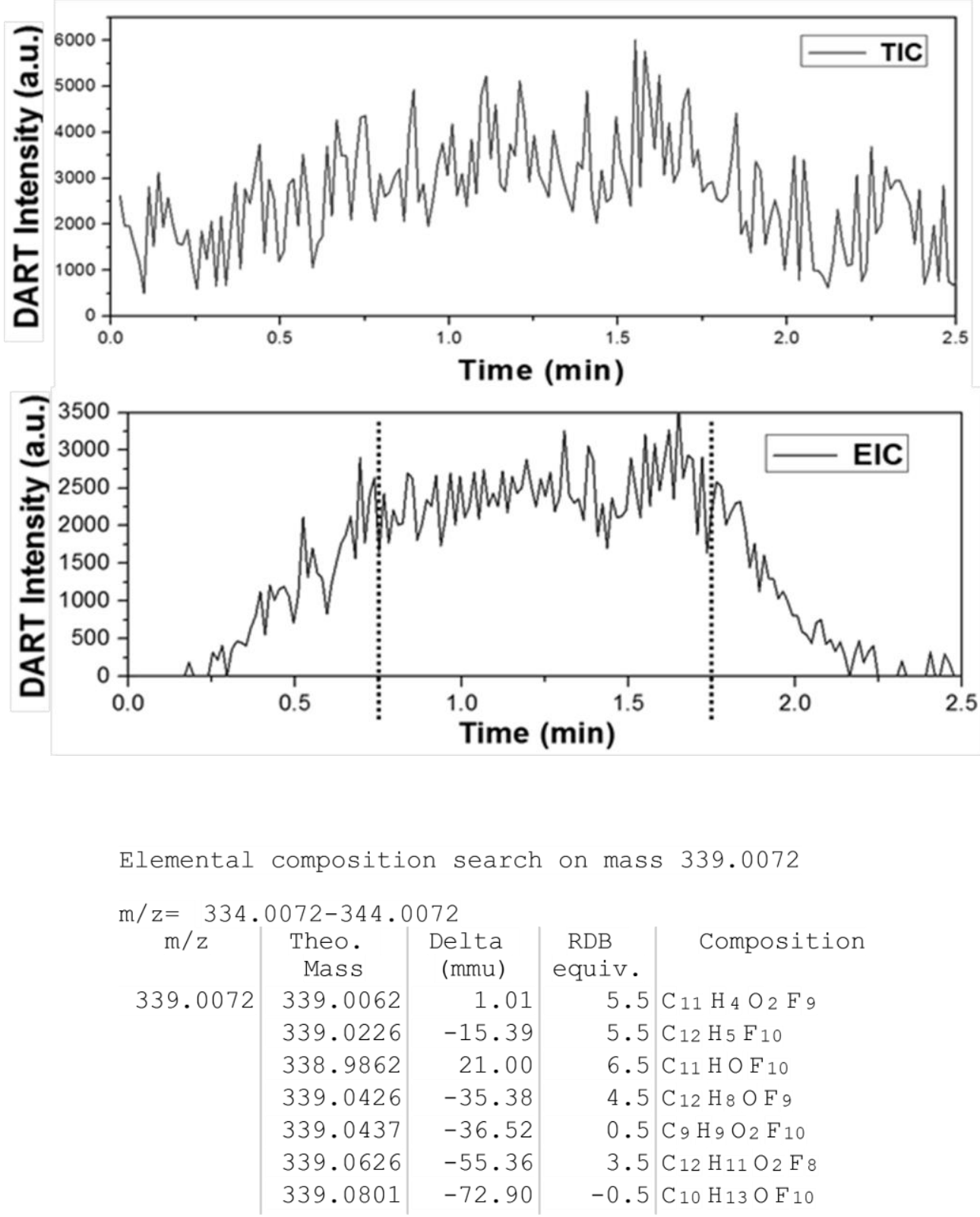

Supplementary Figure S19. Typical DART HRMS measurement of Total Ion Current (TIC), Extracted Ion Chromatogram (EIC) and error based on predicted elemental composition. 

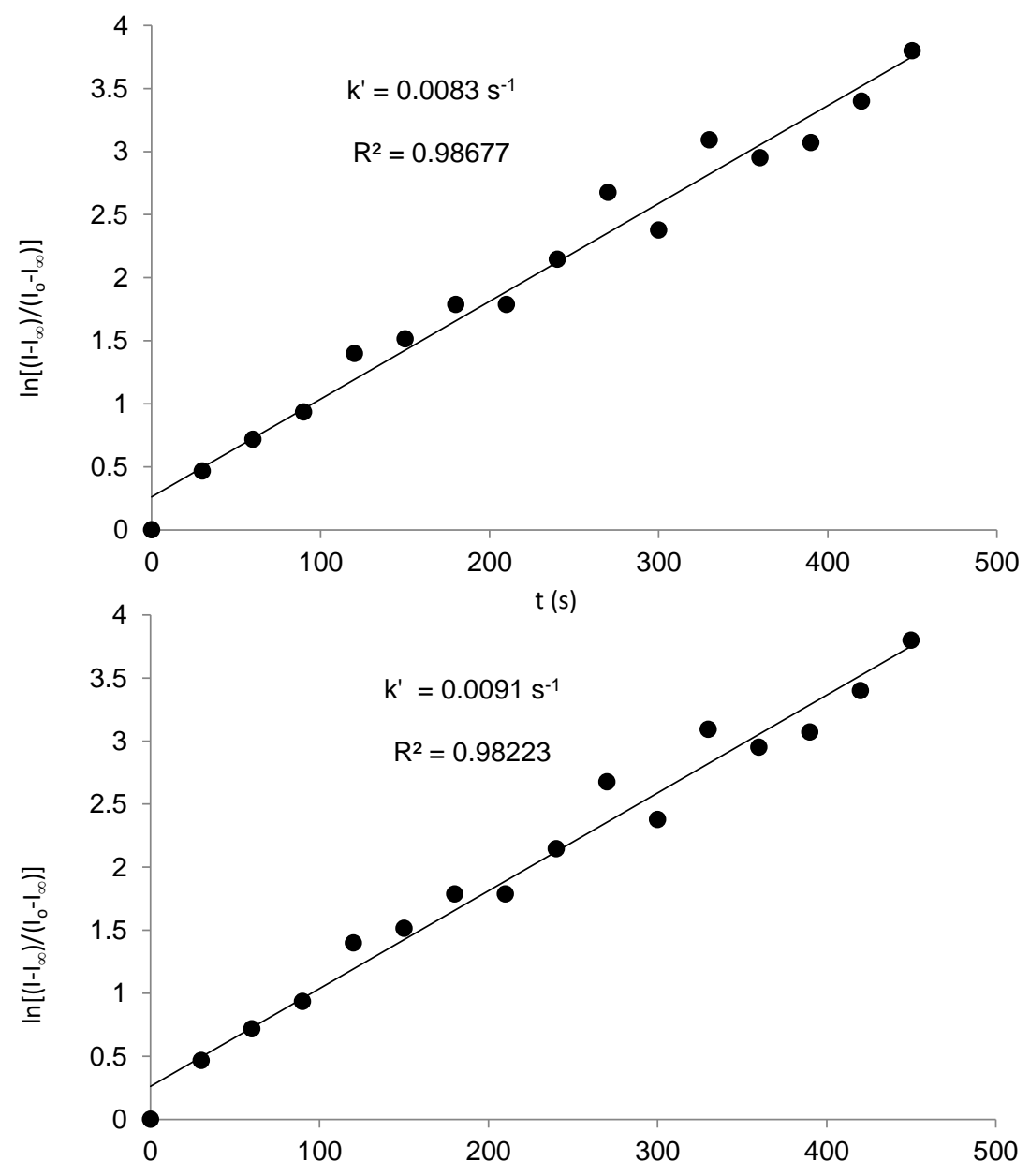

$t(s)$

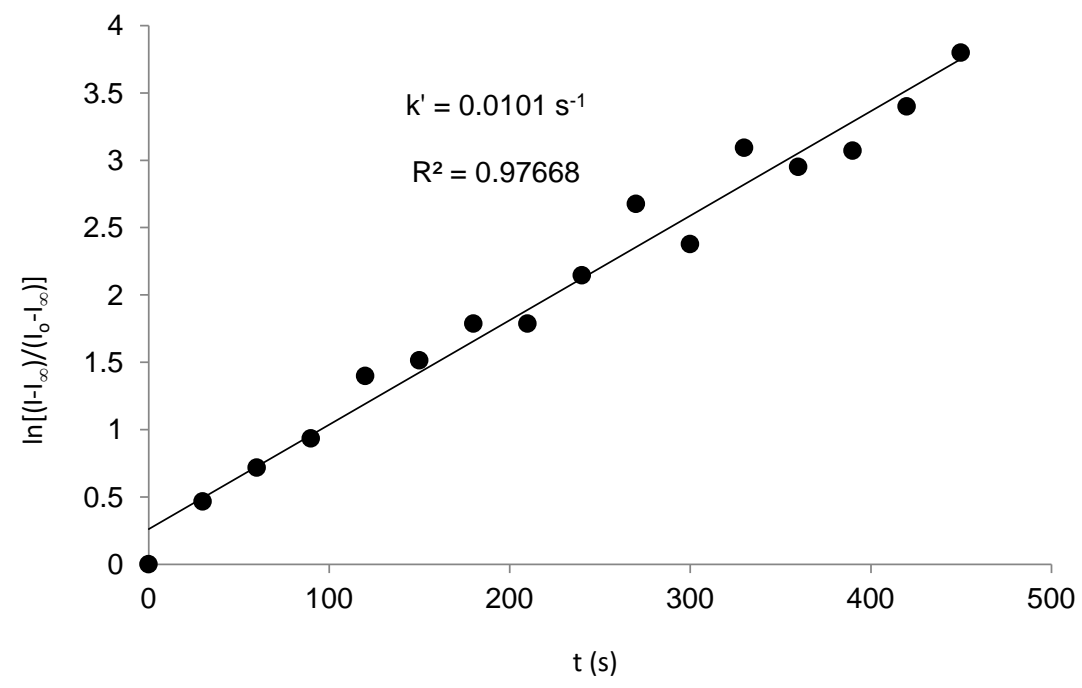

Supplementary Figure S20. Determination of the rate reaction constant for SPAAC reaction of benzyl azide $(0.1 \mathrm{mM})$ and $\mathrm{BCN}(5.5 \mathrm{mM})$ in 1,2 -dichloroethane at $25^{\circ} \mathrm{C}$, as followed by the decrease of the azide IR band at $2099 \mathrm{~cm}^{-1}$. 

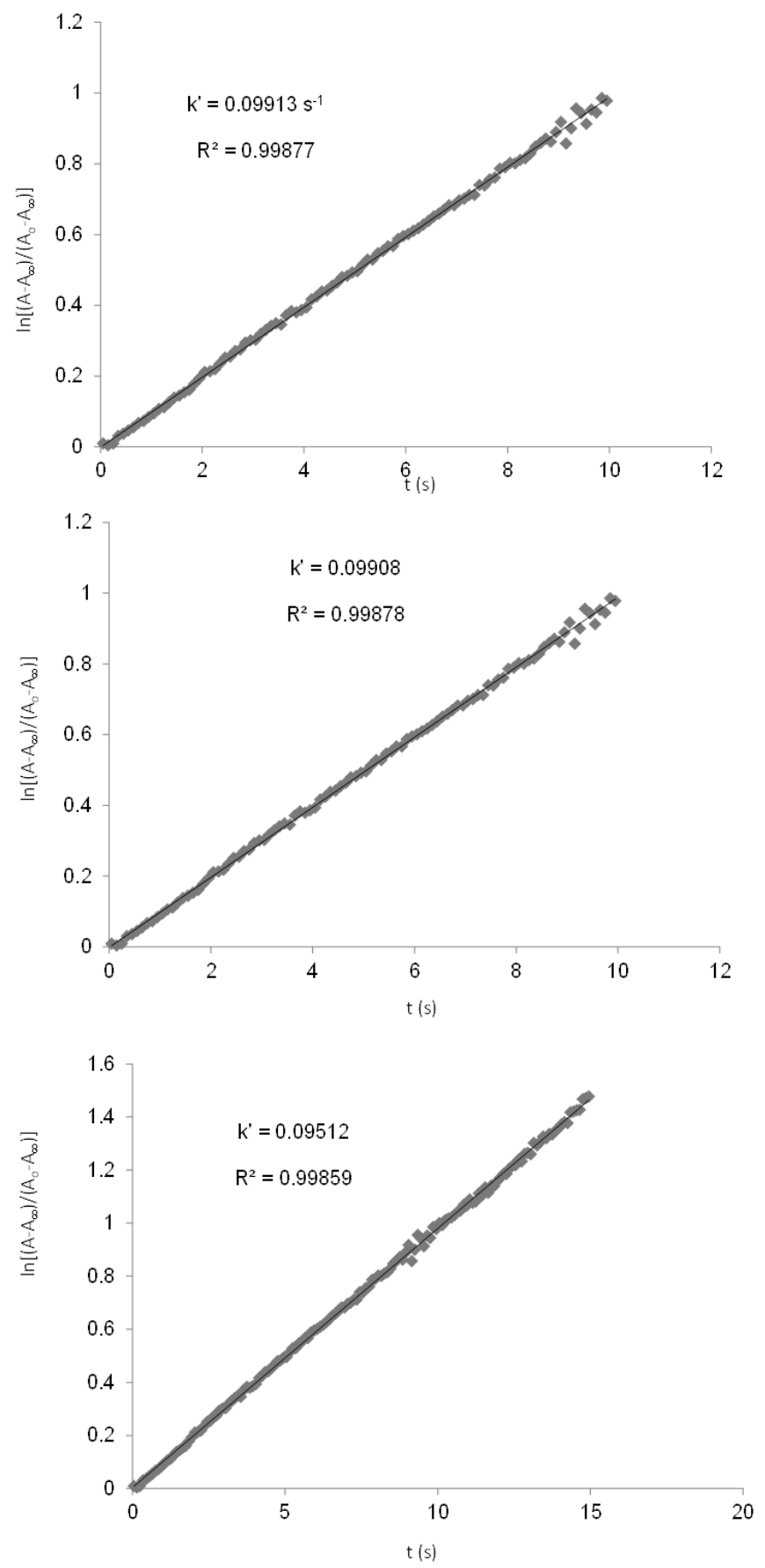

Supplementary Figure S21. Determination of the rate reaction constant for the SPOCQ reaction of 4 -tert-butyl-1,2-quinone $(0.1 \mathrm{mM})$ and $\mathrm{BCN}(5.5 \mathrm{mM})$ in $1,2-$ dichloroethane at $25{ }^{\circ} \mathrm{C}$, followed by the decrease of the UV-vis absorption band at $384 \mathrm{~nm}$ corresponding to the quinone. 


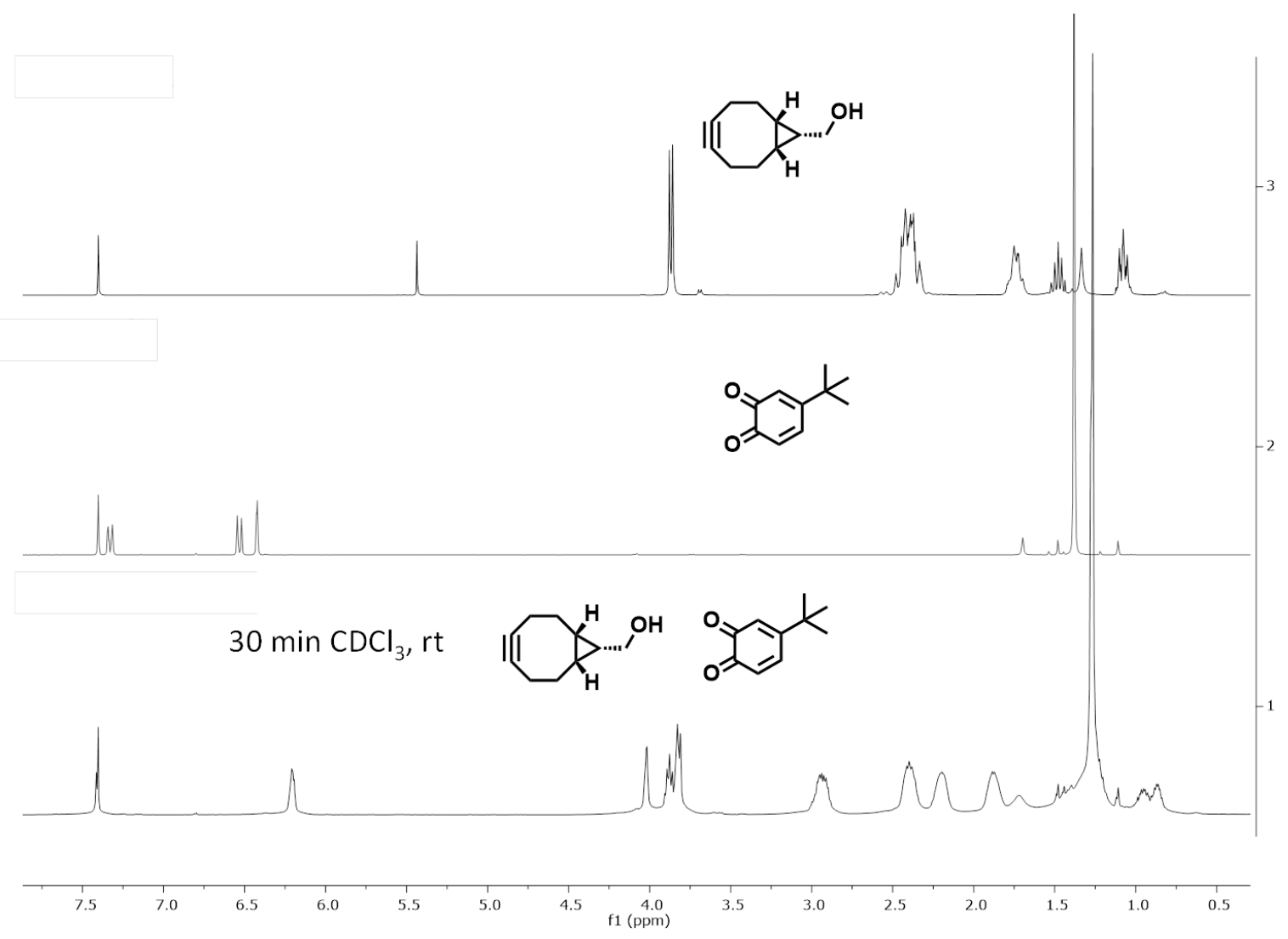

Supplementary Figure S22. NMR solution phase study of SPOCQ reaction of BCN and 4 -tert-butyl-quinone in $\mathrm{CDCl}_{3}\left(22{ }^{\circ} \mathrm{C}\right)$. 


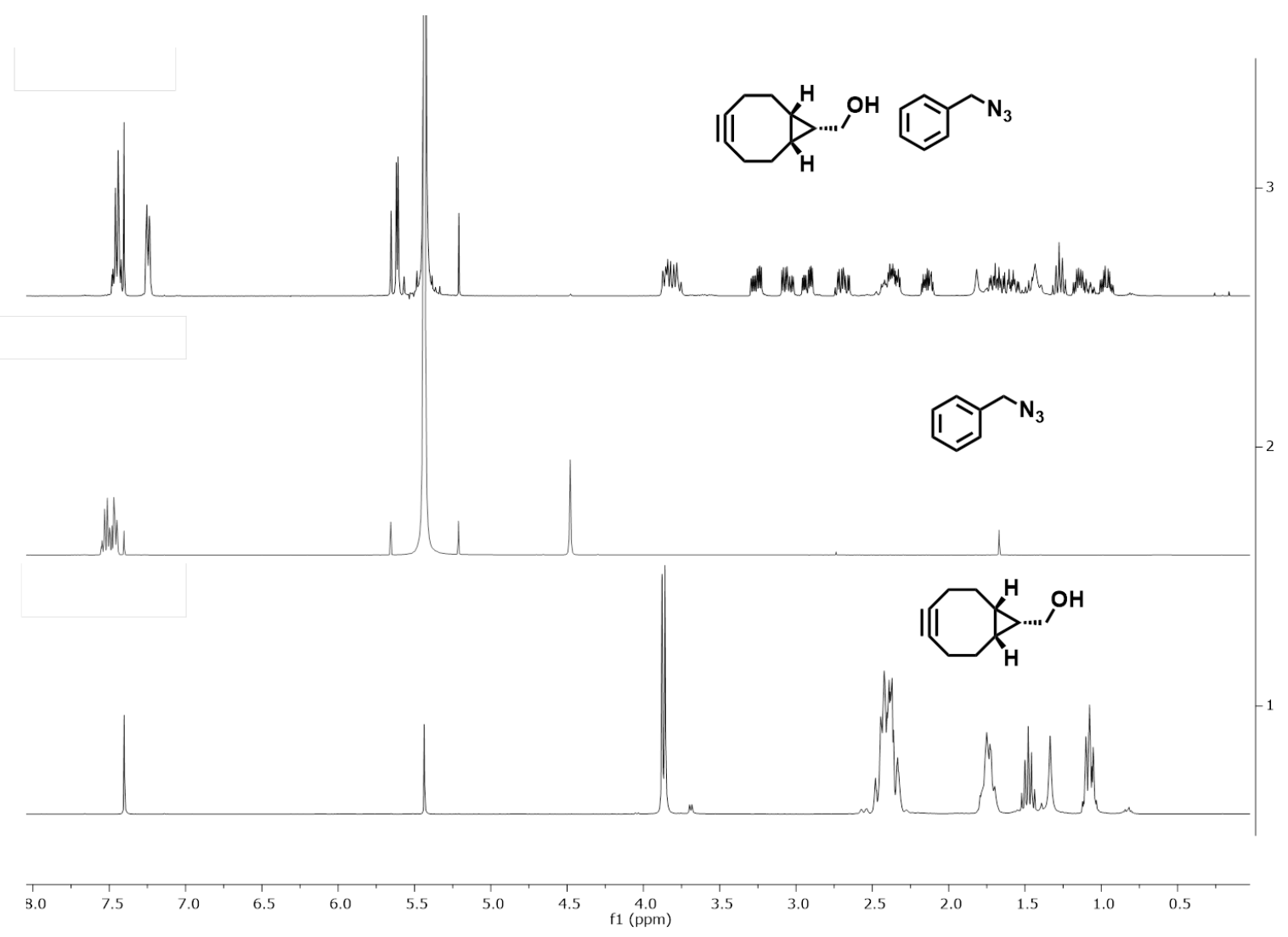

Supplementary Figure S23. NMR solution phase study of SPAAC reaction of BCN and benzyl azide $\left(\mathrm{CH}_{2} \mathrm{Cl}_{2}\right.$ commercial solution) in $\mathrm{CDCl}_{3}\left(22{ }^{\circ} \mathrm{C}\right)$. 


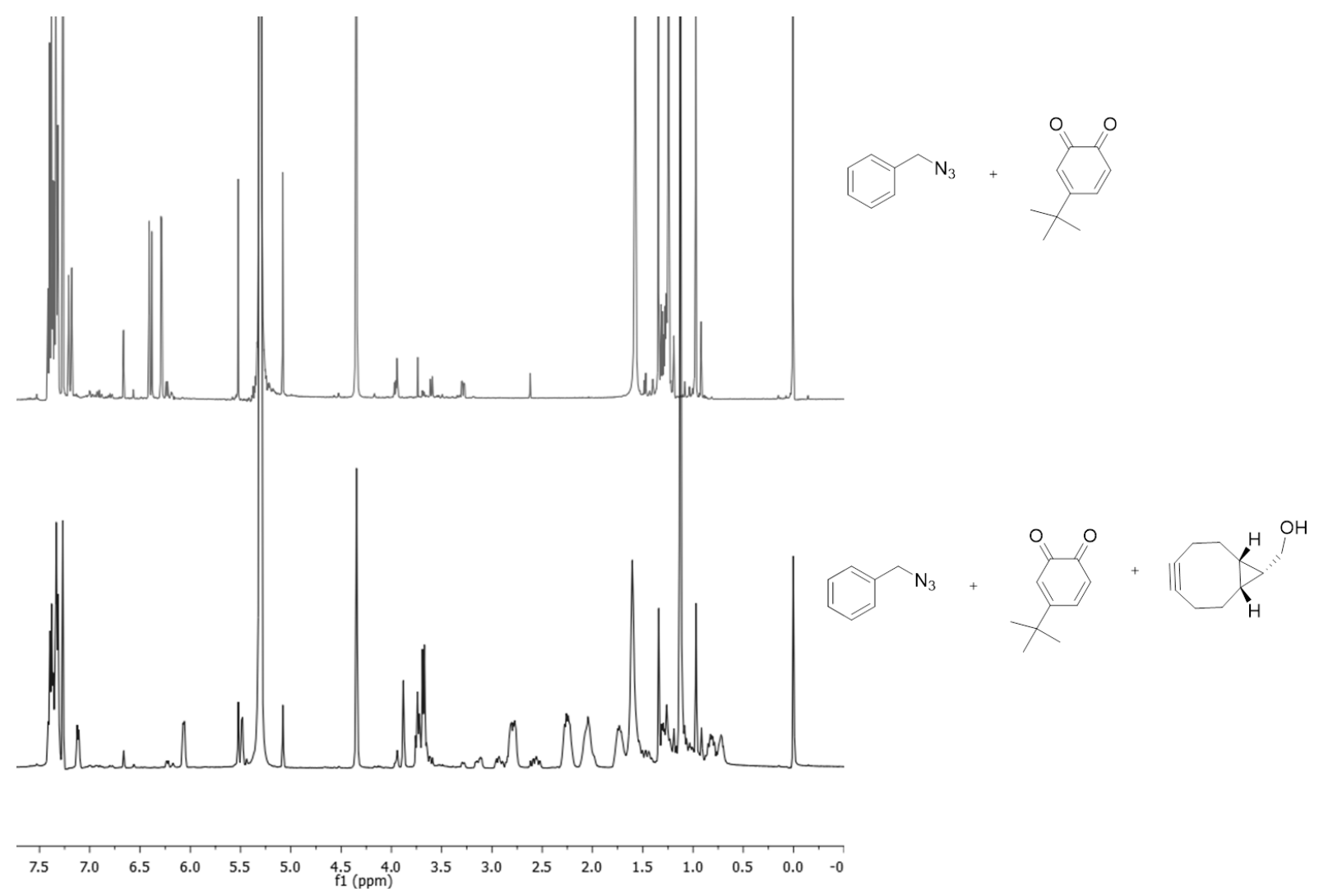

Supplementary Figure S24. Competition experiment for SPOCQ and SPAAC using benzyl azide $\left(\mathrm{CH}_{2} \mathrm{Cl}_{2}\right.$ commercial solution) and 4-tert-butyl-quinone in $\mathrm{CDCl}_{3}(22$ $\left.{ }^{\circ} \mathrm{C}\right)$. 
SPOCQ M1 $\left(40^{\circ} \mathrm{C}\right)$

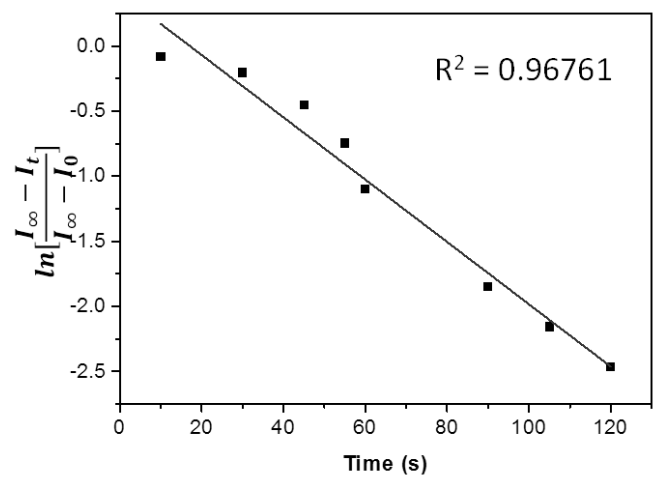

SPOCQ M1 $\left(22^{\circ} \mathrm{C}\right)$

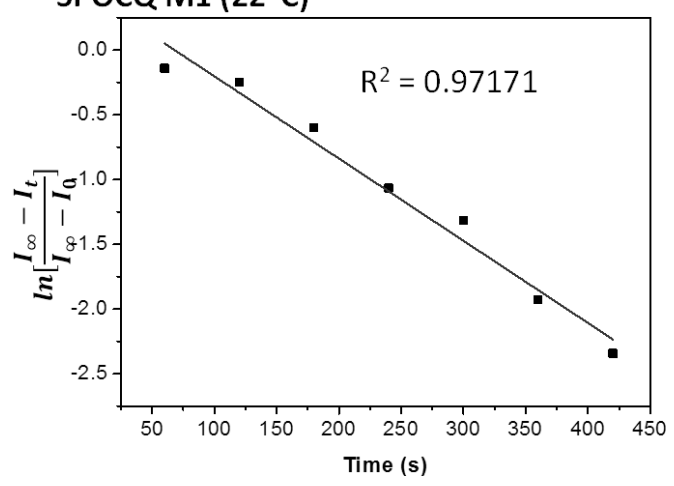

SPOCQ M1 $\left(8^{\circ} \mathrm{C}\right)$
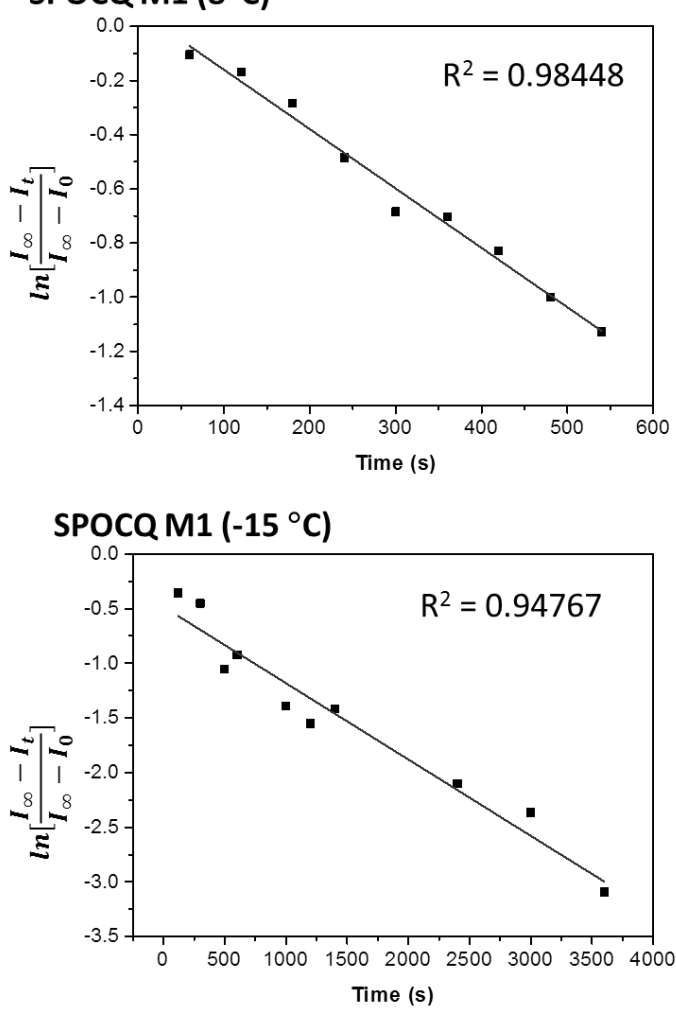
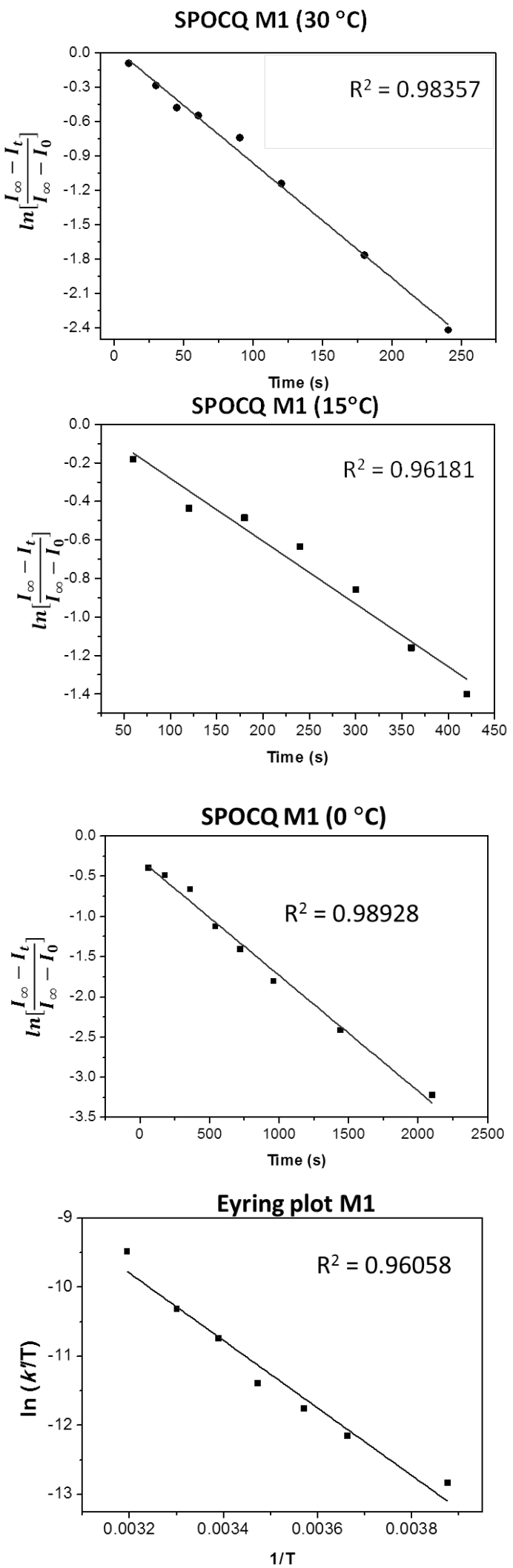

Supplementary Figure S25. Linear kinetics plot for M1 type microenvironment at 7 different temperatures along with Eyring Plot. (Fitting by least squares analysis) 
SPOCQ M2 $\left(40^{\circ} \mathrm{C}\right)$

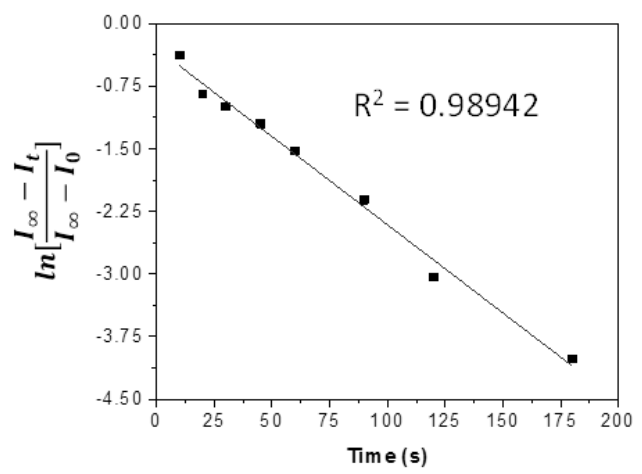

SPOCQ M2 $\left(22^{\circ} \mathrm{C}\right)$

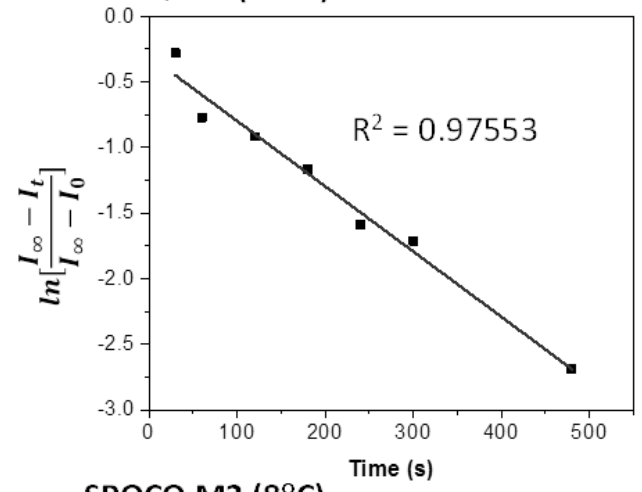

SPOCQ M2 $\left(8^{\circ} \mathrm{C}\right)$

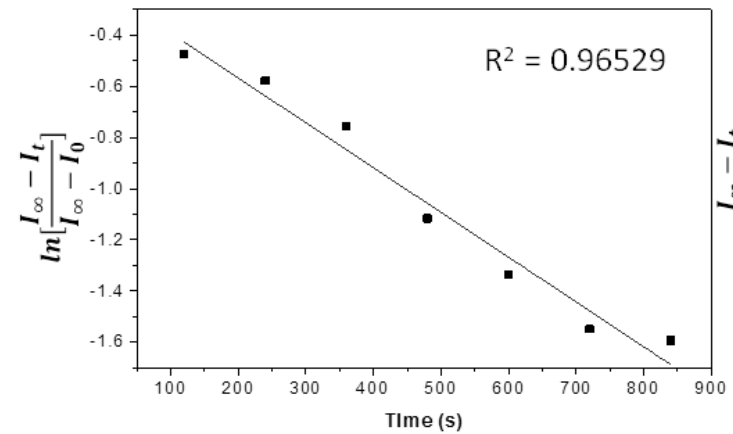

SPOCQ M2 $\left(-15^{\circ} \mathrm{C}\right)$

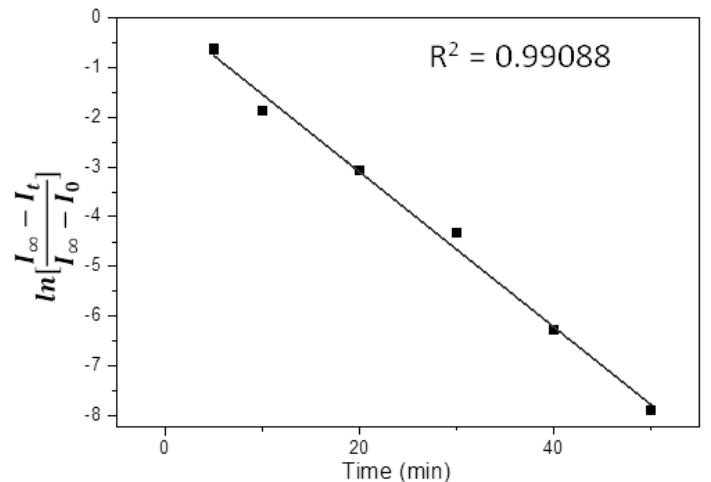

SPOCQ M2 $\left(30^{\circ} \mathrm{C}\right)$

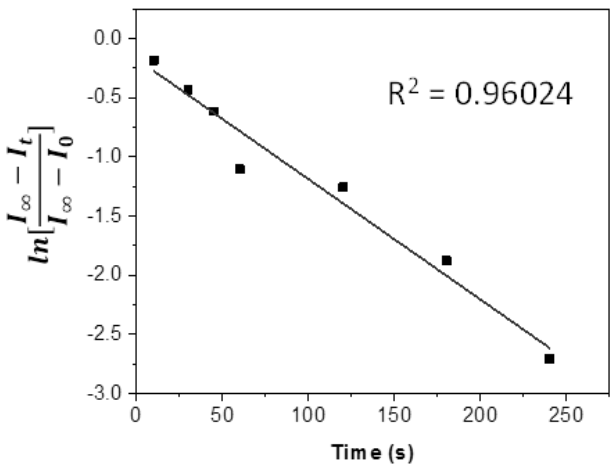

SPOCQ M2 $\left(15^{\circ} \mathrm{C}\right)$

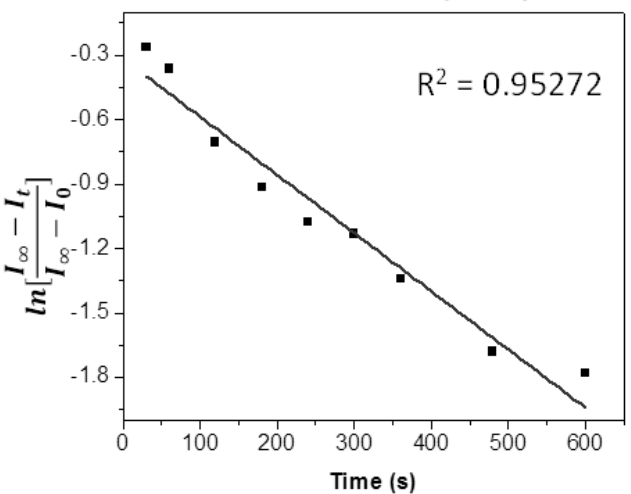

SPOCQ M2 $\left(0^{\circ} \mathrm{C}\right)$

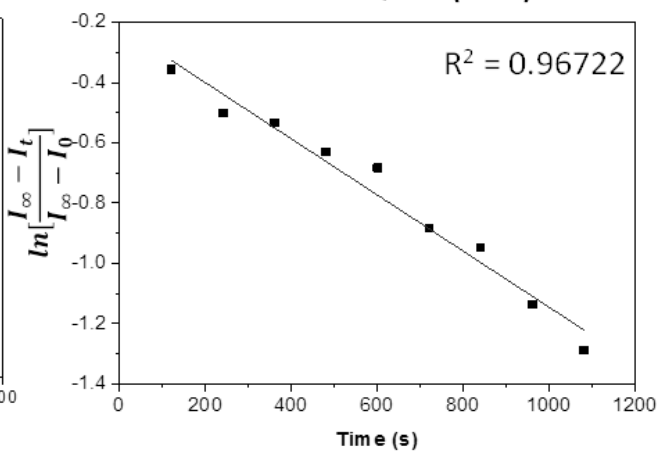

Eyring plot M2

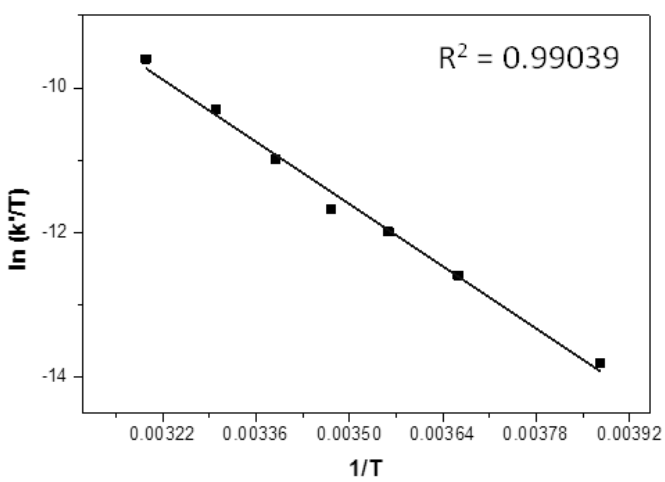

Supplementary Figure S26. Linear kinetics plot for M2 type microenvironment at 7 different temperatures along with Eyring Plot. (Fitting by least squares analysis) 
SPOCQ M3 $\left(40^{\circ} \mathrm{C}\right)$

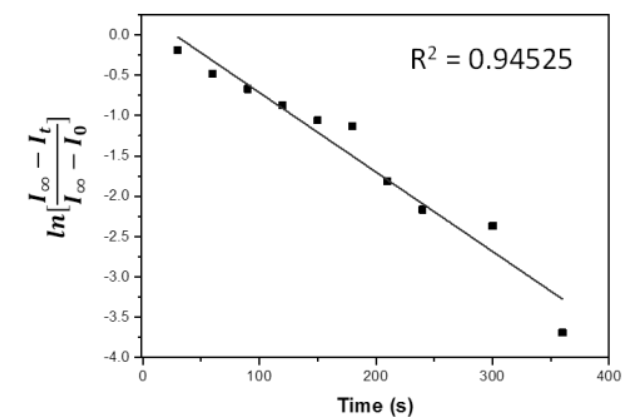

SPOCQ M3 $\left(22^{\circ} \mathrm{C}\right)$

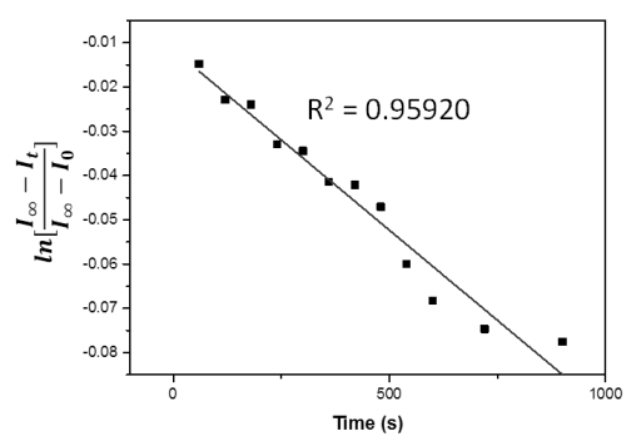

SPOCQ M3 $\left(8^{\circ} \mathrm{C}\right)$
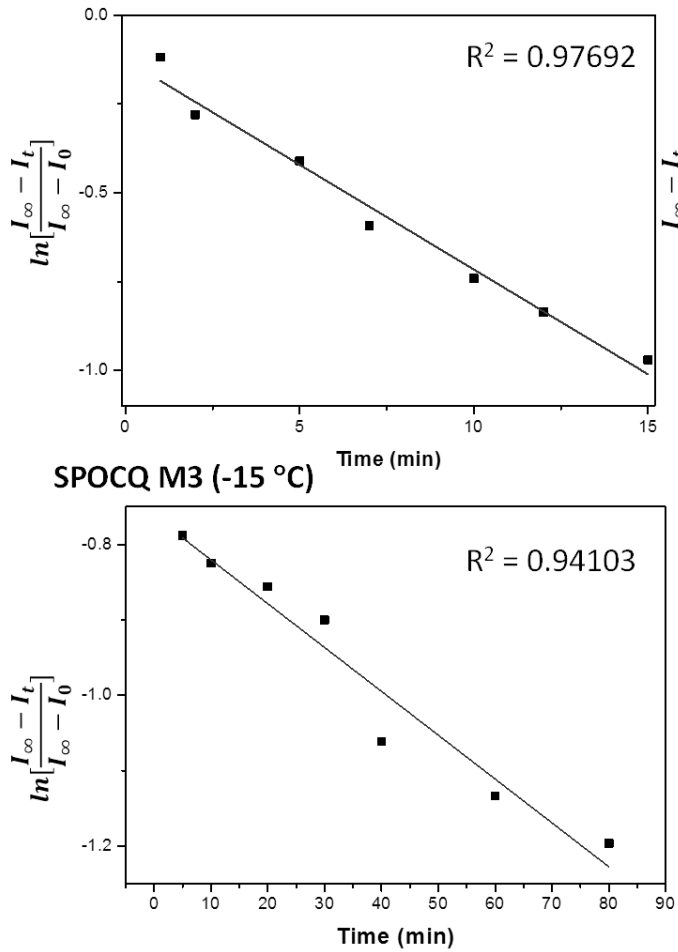

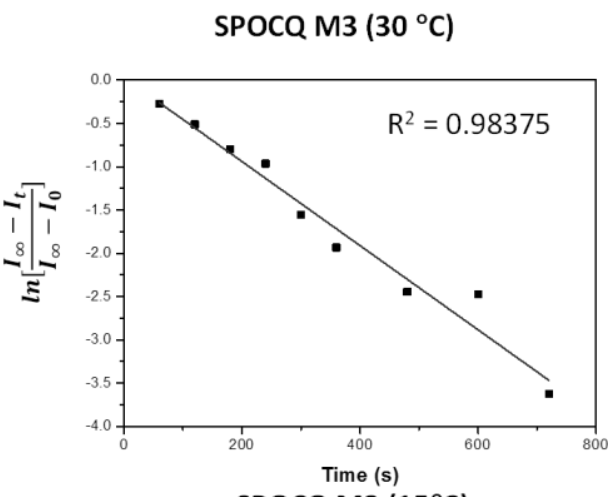

SPOCQ M3 $\left(15^{\circ} \mathrm{C}\right)$

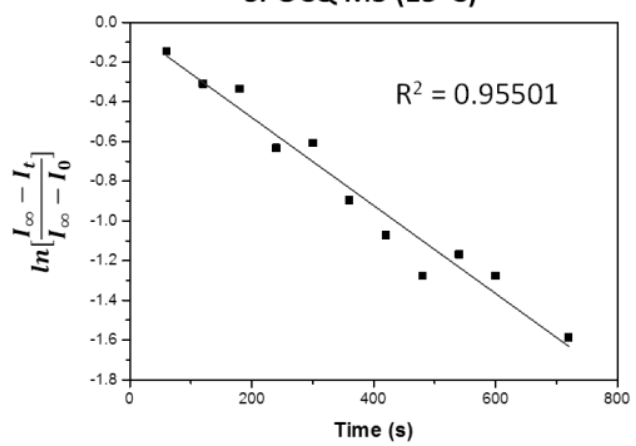

SPOCQ M3 $\left(0^{\circ} \mathrm{C}\right)$

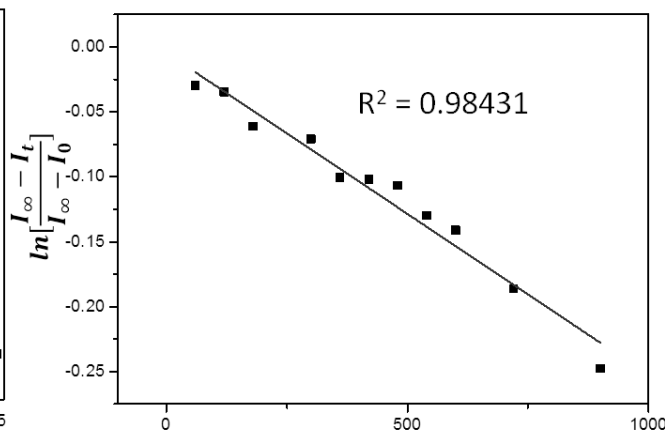

Eyring plot M3

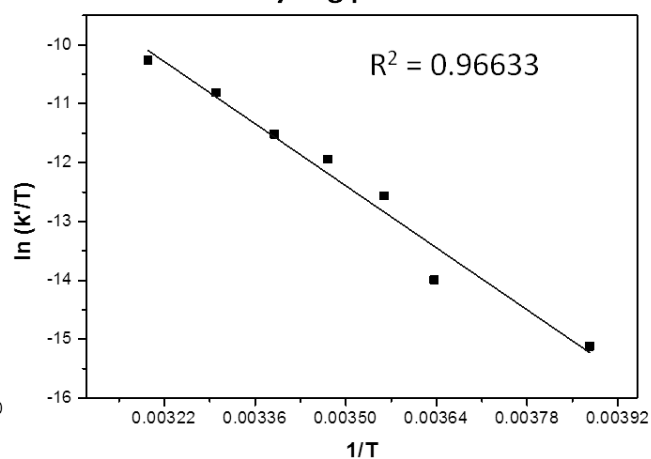

Supplementary Figure S27. Linear kinetics plot for M3 type microenvironment at 7 different temperatures along with Eyring Plot. (Fitting by least squares analysis) 


\subsection{Supplementary Tables.}

2.3.1 Supplementary Table S1. Evolution of F/N determined by XPS ratio and reaction efficiency (determined by $\mathrm{F} / \mathrm{N}$ ) vs time for the SPOQC reaction for the 3:1 mixed monolayer.

\begin{tabular}{|c|c|c|}
\hline Time (h) & F/N determined by $\mathbf{X P S}^{[\mathrm{a}]}$ & ${\text { Reaction efficiency }(\%)^{[\mathbf{b}]}}$ \\
\hline 0.00 & 0.00 & 0 \\
\hline 0.02 & $0.91 \pm 0.14$ & 10.1 \\
\hline 0.03 & $1.70 \pm 0.18$ & 29.5 \\
\hline 0.08 & $2.66 \pm 0.15$ & 39.6 \\
\hline 0.12 & $3.56 \pm 0.27$ & 58.4 \\
\hline 0.13 & $5.26 \pm 0.19$ & 64.8 \\
\hline 0.15 & $5.83 \pm 0.28$ & 68 \\
\hline 0.17 & $6.12 \pm 0.30$ & 71.2 \\
\hline 0.50 & $6.41 \pm 0.32$ & 73.4 \\
\hline 0.75 & $6.61 \pm 0.49$ & 82.1 \\
\hline 1.00 & $7.39 \pm 0.54$ & 87.7 \\
\hline 2.00 & $7.89 \pm 0.45$ & 99.1 \\
\hline 4.00 & $8.92 \pm 0.24$ & 99.5 \\
\hline 6.00 & $8.96 \pm 0.36$ & 99.1 \\
\hline 8.00 & $8.92 \pm 0.27$ & \\
\hline
\end{tabular}

[a] Average value of three separately prepared monolayers.

[b] Reaction efficiency was determined by comparing the $\mathrm{F} / \mathrm{N}$ ratio determined by XPS at a certain time with the theoretical value of $9 / 1$ which corresponds to the effective click of the fluorine-labelled BCN with the 1,2-quinone-functionalized monolayer. See that in that case, the final adduct attached to the monolayer would 
contain $9 \mathrm{~F}$ atoms (from the $\mathrm{C}_{4} \mathrm{~F}_{9}$ tag) and one $\mathrm{N}$ atom (which comes from the dopamine attachment to the monolayer).

2.3.2 Supplementary Table S2. Reported surface yields for metal-free click chemistry reactions.

\begin{tabular}{|c|c|c|c|}
\hline Type of Reaction & $\begin{array}{c}\text { Reaction time } \\
\text { (h) }\end{array}$ & \% yield & Reference \\
\hline Hetero-Diels Alder & 2 & 90 & 4 \\
\hline Thiol-ene Reaction & 1.5 & $45-70$ & 5 \\
\hline Thiol-yne & 4 & 90 & 7 \\
\hline SPAAC & 18 & 80 & 8 \\
\hline SPAAC & 2 & 63 & 9 \\
\hline SPAAC & 14 & & 70 \\
\hline
\end{tabular}


2.3.3 Supplementary Table S3. First-order rate constants (k') determined by DARTHRMS for the interfacial SPOCQ reaction of BCN and 1,2-quinone moieties in varying microenvironment M1, M2 and M3. For determining every rate constant, DART-HRMS measurements were performed on 6 independent surfaces.

\begin{tabular}{|c|c|c|c|}
\hline & M1 & M2 & M3 \\
\hline Temp & $\boldsymbol{k}^{\prime}\left(\times \mathbf{1 0}^{-\mathbf{3}} \mathbf{s}^{-}\right.$ & $\boldsymbol{k}^{\prime}\left(\times \mathbf{1 0}^{-\mathbf{3}} \mathbf{s}^{-}\right.$ & $\boldsymbol{k}^{\prime}\left(\times \mathbf{1 0}^{-\mathbf{3}} \mathbf{s}^{-}\right.$ \\
$\left({ }^{\circ} \mathbf{C}\right)$ & $\left.\mathbf{1}^{\mathbf{1}}\right)$ & $\left.\mathbf{1}^{\mathbf{1}}\right)$ \\
\hline-15 & $0.69 \pm 0.11$ & $0.26 \pm 0.05$ & $0.07 \pm 0.03$ \\
\hline 0 & $1.44 \pm 0.06$ & $0.93 \pm 0.15$ & $0.23 \pm 0.07$ \\
\hline 8 & $2.19 \pm 0.12$ & $1.75 \pm 0.12$ & $0.98 \pm 0.14$ \\
\hline 15 & $3.26 \pm 0.16$ & $2.45 \pm 0.21$ & $1.87 \pm 0.21$ \\
\hline 22 & $6.35 \pm 0.14$ & $4.98 \pm 0.32$ & $2.93 \pm 0.15$ \\
\hline 30 & $10.06 \pm 0.31$ & $10.02 \pm 0.47$ & $6.12 \pm 0.47$ \\
\hline 40 & $23.92 \pm 0.22$ & $21.17 \pm 0.38$ & $10.96 \pm 0.38$ \\
\hline
\end{tabular}




\subsection{References}

1. R. Sen, J. Escorihuela, M. M. J. Smulders, H. Zuilhof, Langmuir 2016, 32, 3412.

2. Gaussian 09, Revision D.01, M. J. Frisch, G. W. Trucks, H. B. Schlegel, G. E. Scuseria, M. A. Robb, J. R. Cheeseman, G. Scalmani, V. Barone, B. Mennucci, G. A. Petersson, H. Nakatsuji, M. Caricato, X. Li, H. P. Hratchian, A. F. Izmaylov, J. Bloino, G. Zheng, J. L. Sonnenberg, M. Hada, M. Ehara, K. Toyota, R. Fukuda, J. Hasegawa, M. Ishida, T. Nakajima, Y. Honda, O. Kitao, H. Nakai, T. Vreven, J. A. Montgomery, Jr., J. E. Peralta, F. Ogliaro, M. Bearpark, J. J. Heyd, E. Brothers, K. N. Kudin, V. N. Staroverov, T. Keith, R. Kobayashi, J. Normand, K. Raghavachari, A. Rendell, J. C. Burant, S. S. Iyengar, J. Tomasi, M. Cossi, N. Rega, J. M. Millam, M. Klene, J. E. Knox, J. B. Cross, V. Bakken, C. Adamo, J. Jaramillo, R. Gomperts, R. E. Stratmann, O. Yazyev, A. J. Austin, R. Cammi, C. Pomelli, J. W. Ochterski, R. L. Martin, K. Morokuma, V. G. Zakrzewski, G. A. Voth, P. Salvador, J. J. Dannenberg, S. Dapprich, A. D. Daniels, O. Farkas, J. B. Foresman, J. V. Ortiz, J. Cioslowski, D. J. Fox, Gaussian, Inc., Wallingford CT, 2013.

3. Spartan '14 Windows, Wavefunction, Inc., Irvine, CA, USA, 2014. See also: http://www.wavefun.com

4. J. M. Hooker, E. W. Kovacs, M. B. Francis, J. Am. Chem. Soc. 2004, 126, 3718 .

5. M. A. Caipa Campos, J. M. J. Paulusse, H. Zuilhof, Chem. Commun. 2010, 46, 5512 .

6. M. T. Gokmen, J. Brassinne, R. A. Prasath, F. E. Du Prez, Chem. Commun. 2011, 47,4652 .

7. J. Guo, G. Chen, X. Ning, M. A. Wolfert, X. Li, B. Xu , G. J. Boons, Chem. Eur. J. $2010,16,13360$.

8. A. Kuzmin, A. Poloukhtine, M. A. Wolfert, V. V. Popik, Bioconjugate Chem. 2010, 21, 2076-2085. 
9. R. J. Williams, I. A. Barker, R. K. O'Reilly, A. P. Dove, ACS Macro Lett. 2012, 1, 1285. 


\section{Appendix 3}

\section{Approach Matters: The Kinetics of Interfacial Inverse-Electron Demand Diels-Alder Reactions}

For full Supporting Information including Cartesian coordinates look at online ESI available in Chemistry - A European Journal available asap.

\section{Table of Contents}

1. Material and Methods. $18 \mathrm{C}$

2. Chemical Synthesis. 185

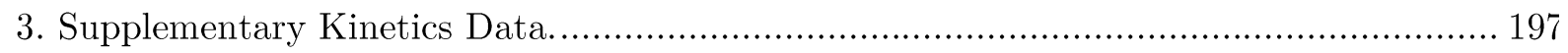

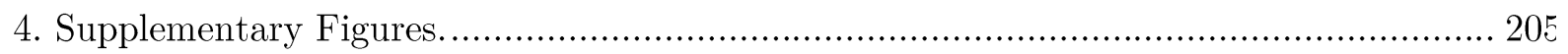

5. Supplementary XPS Spectra (all calculated errors are average of 5 samples)............... 207

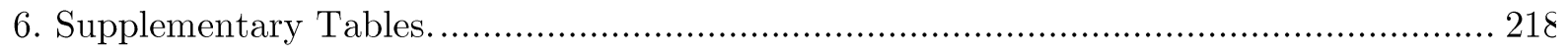

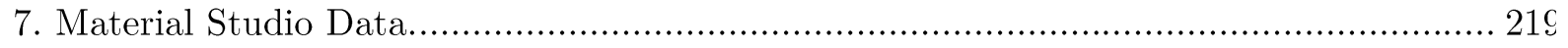

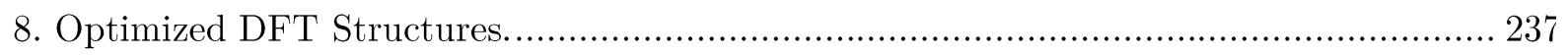

9. Density Functional Theory (DFT) for XPS C1s Calculation ..................................... 24

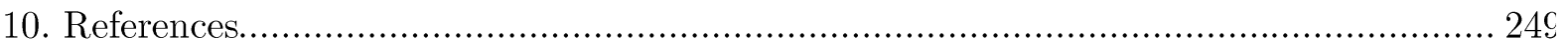




\section{Materials and Methods}

Preparation of phosphonic acid monolayers. $2 \times 1 \mathrm{~cm}$ Al slides were sonicated in hexane for $15 \mathrm{~min}$ followed by wiping with lint-free cotton swabs (Texwipe, NC, USA) to remove the polymer protection layer on top and remove any residual glue. The surfaces were chemically activated by immersion in 1:1 (v/v) $37 \% \mathrm{HCl}-\mathrm{MeOH}$ mixture for $5 \mathrm{~min}$, followed by washing with copious amounts of water and 2-propanol. The activated surfaces were then immersed into $\mathrm{N}_{2}$ filled vials of $5 \mathrm{mM}$ solution of $3: 1$ ratio of phosphonic acid derivatives (octylphosphonic acid and 12-aminododecylphosphonic acid hydrochloride salt for "free" $\mathrm{ME}(\mathrm{m}=7, \mathrm{n}=3) ;$ hexadecylphosphonic acid and 6aminohexylphosphonic acid hydrochloride salt for "buried" $\mathrm{ME}(\mathrm{m}=1, \mathrm{n}=11)$ mixture in 2-propanol, heated to $50^{\circ} \mathrm{C}$ for $5 \mathrm{~min}$, and then left undisturbed for 5 $\mathrm{h}$ at room temperature to obtain self-assembled mixed monolayers.

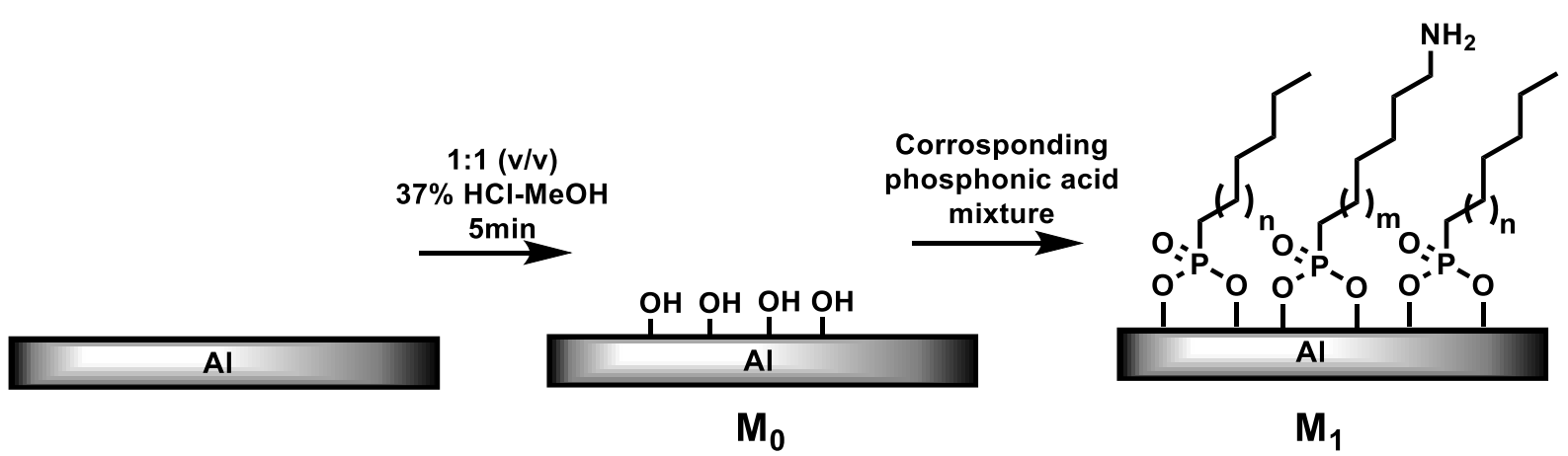

Then surfaces were taken out and sonicated successively for 5 min with 2 propanol, acetone and $\mathrm{CH}_{2} \mathrm{Cl}_{2}$. The surfaces were finally cleaned with $\mathrm{CH}_{2} \mathrm{Cl}_{2}$, air dried and stored under $\mathrm{N}_{2}$ atmosphere. From static water contact angle (SCA) measurements, it was found that the reaction was complete after $5 \mathrm{~h}$, yielding monolayers with 28-30\% C as determined by XPS. Substantially longer reaction times $(16 \mathrm{~h})$ contributed to the formation of undesirable multilayers $(42-44 \% \mathrm{C} 1 \mathrm{~s})$.

Preparation of exo-norbornene terminated monolayers $\left(\mathrm{M}_{2}\right)$. Amineterminated monolayers $\left(\mathbf{M}_{\mathbf{1}}\right)$ were stirred with $50 \mathrm{mM}$ 1,1'-carbonyldiimidazole 
(CDI) solution in water for $1 \mathrm{~h}$ to yield acyl imidazole activated surfaces. These surfaces were immediately reacted with $50 \mathrm{mM}$ exo-5-norbornene-2-methanol solution in DCE. This resulted in carbamate bond formation and covalent tethering of the exo-norbornene on the surface in $16 \mathrm{~h}$. The samples were sonicated and washed with copious amounts of $\mathrm{CH}_{2} \mathrm{Cl}_{2}$, dried and stored under nitrogen atmosphere.
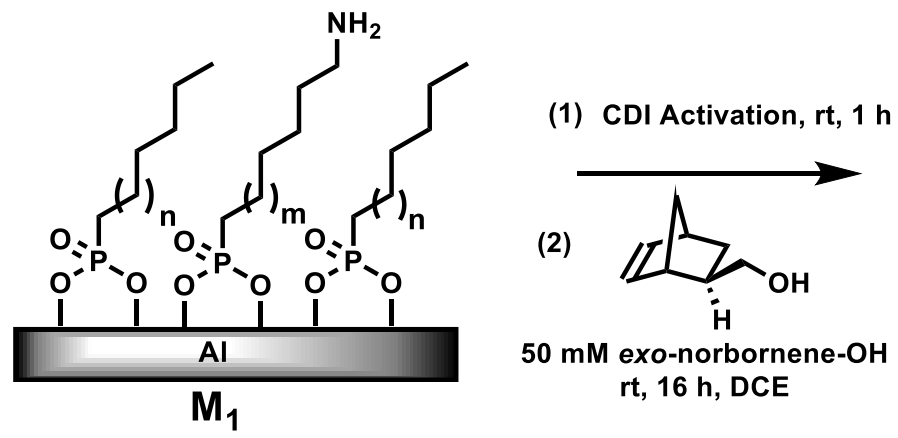

rt, $16 \mathrm{~h}, \mathrm{DCE}$
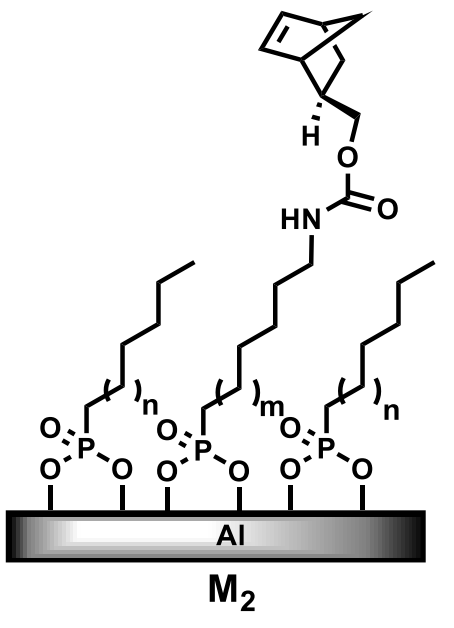

Preparation of endo-norbornene terminated monolayers $\left(\mathrm{M}_{3}\right)$. Aminoterminated monolayers $\left(\mathbf{M}_{\mathbf{1}}\right)$ were stirred with $50 \mathrm{mM}$ aqueous solution of 1,1'carbonyldiimidazole (CDI) for $1 \mathrm{~h}$ to yield acyl imidazole-activated surfaces. These surfaces were immediately reacted with $50 \mathrm{mM}$ endo-5-norbornene-2-methanol solution in DCE. This resulted in covalent tethering of the endo-norbornene on the surface via carbamate bond in $16 \mathrm{~h}$. The samples were sonicated and washed with copious amounts of $\mathrm{CH}_{2} \mathrm{Cl}_{2}$, dried and stored under nitrogen atmosphere. 


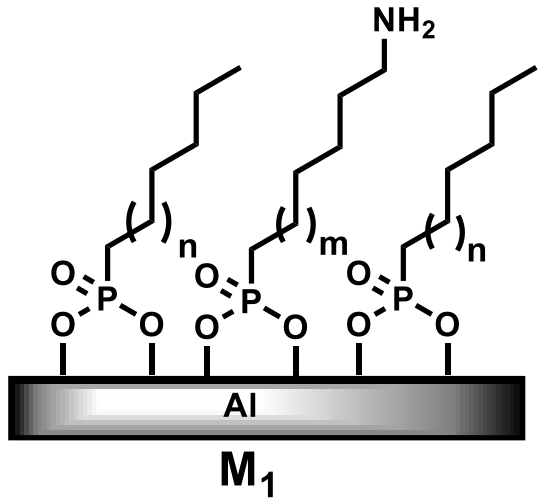

(1)

(2)

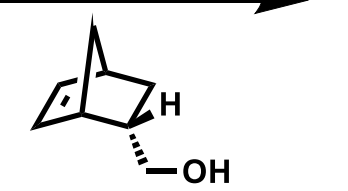

$50 \mathrm{mM}$ endo-norbornene-OH rt, $16 \mathrm{~h}, \mathrm{DCE}$

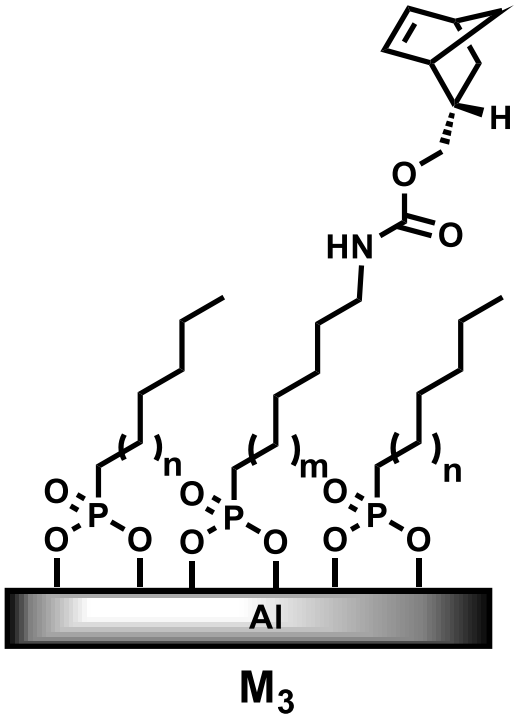

Preparation of tetrazine terminated monolayers $\left(\mathbf{M}_{4}\right)$. Amine-terminated monolayers $\left(\mathbf{M}_{1}\right)$ were stirred with $50 \mathrm{mM}$ aqueous solution of 1,1'carbonyldiimidazole (CDI) for $1 \mathrm{~h}$ to yield acyl imidazole-activated surfaces. These surfaces were immediately reacted with $50 \mathrm{mM}$ (4-(6-methyl-1,2,4,5-tetrazin-3yl)phenyl)methanamine, $\mathbf{1}$ solution in DCE. This resulted in covalent tethering of the tetrazine on the surface via urea bond in $16 \mathrm{~h}$. The samples were sonicated and washed with copious amounts of $\mathrm{CH}_{2} \mathrm{Cl}_{2}$, dried and stored under nitrogen atmosphere.

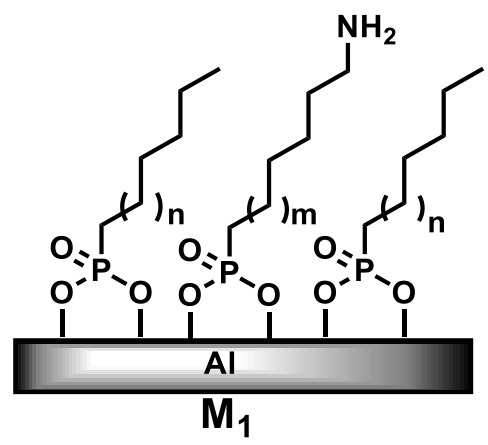

(1) CDI Coupling, $\mathrm{H}_{2} \mathrm{O}, \mathrm{rt}, 1 \mathrm{~h}$<smiles>CCc1ccc(-c2nnc(C)nn2)cc1</smiles>

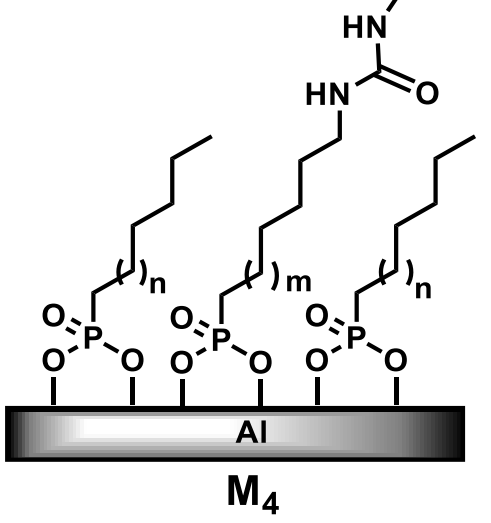

General method for IEDDA reaction on exo-/endo-norbornene reaction on surface: Both free and buried exo-/endo-norbornene-terminated surfaces were 
reacted with a 4-(6-methyl-1,2,4,5-tetrazin-3-yl)benzyl 4-(trifluoromethyl)benzoate solution, 3 in $\mathrm{DCE}$ at $30^{\circ} \mathrm{C}$. The reaction was stirred at a constant speed using a magnetic bead and stirrer and all samples were loaded in a specially constructed Teflon holder to ensure rigorous reproducibility between samples. Samples were immersed into the solution for a set period of reaction time and immediately taken out and washed with copious amounts of DCM. The samples were further sonicated in DCM to remove any physisorbed species for $15 \mathrm{~min}$, dried under a dry nitrogen stream and stored for further analysis in a sealed vial.
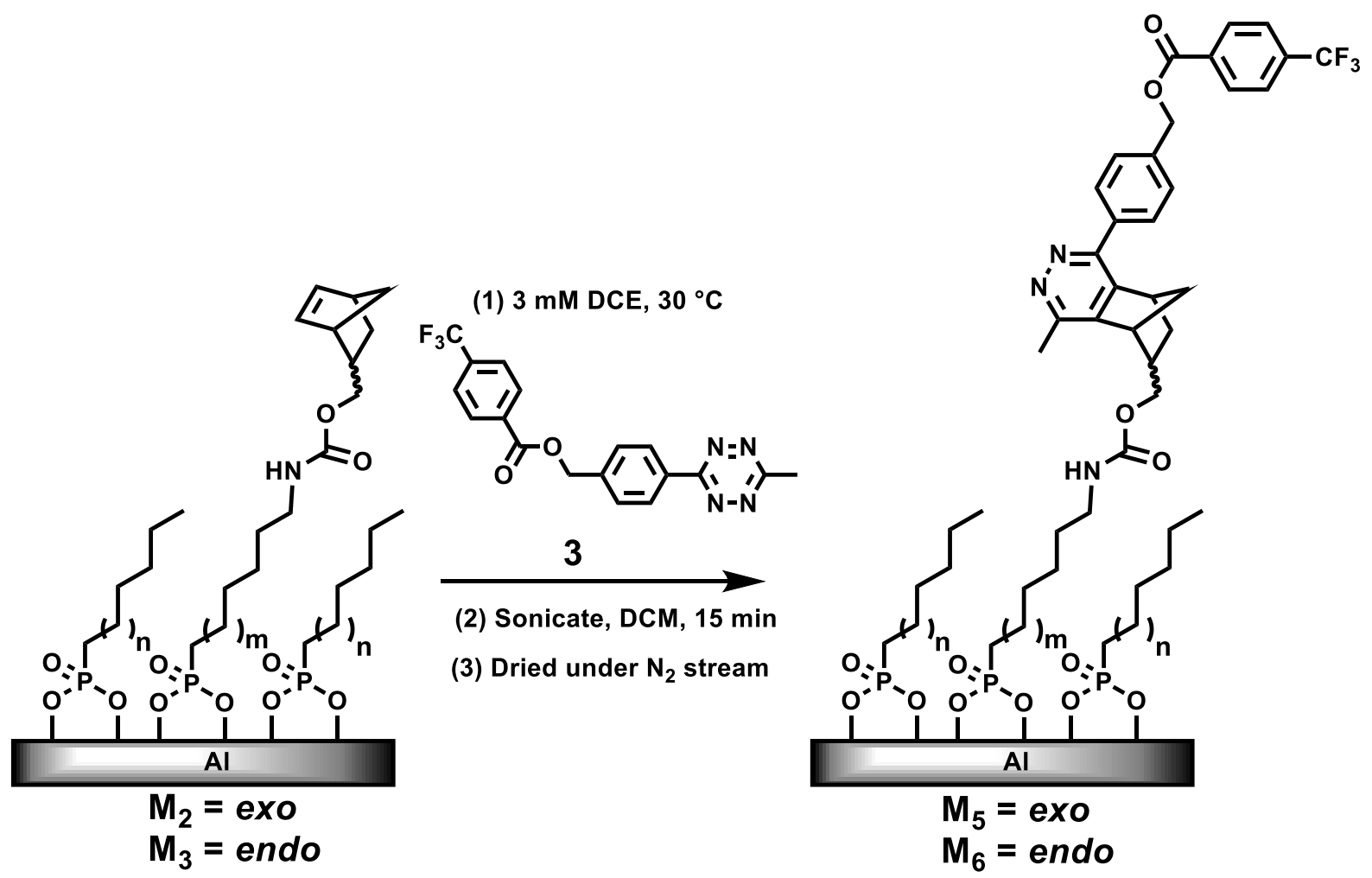

General method for IEDDA reaction on tetrazine terminated on surface:

Both "free" and "buried" tetrazine-terminated surfaces were reacted with $((1 R, 2 S, 4 R)-$ bicyclo[2.2.1]hept-5-en-2-yl)methyl $\quad$ 4-(trifluoromethyl)benzoate $\quad[4$, exo-norbornene tag molecule] or $((1 R, 2 R, 4 R)$-bicyclo[2.2.1]hept-5-en-2-yl)methyl 4(trifluoromethyl)benzoate [6, endo-norbornene tag molecule] solution in DCE at 30 ${ }^{\circ} \mathrm{C}$. The reaction was stirred at a constant speed using a magnetic bead and stirrer and all samples were loaded in a specially constructed Teflon holder to ensure rigorous reproducibility between samples. Samples were immersed into above said 
solution for a set period of reaction time and immediately taken out and washed with copious amounts of DCM. The samples were further sonicated in DCM to remove any physisorbed species for $15 \mathrm{~min}$, dried under a dry nitrogen stream and stored for further analysis in a sealed vial.

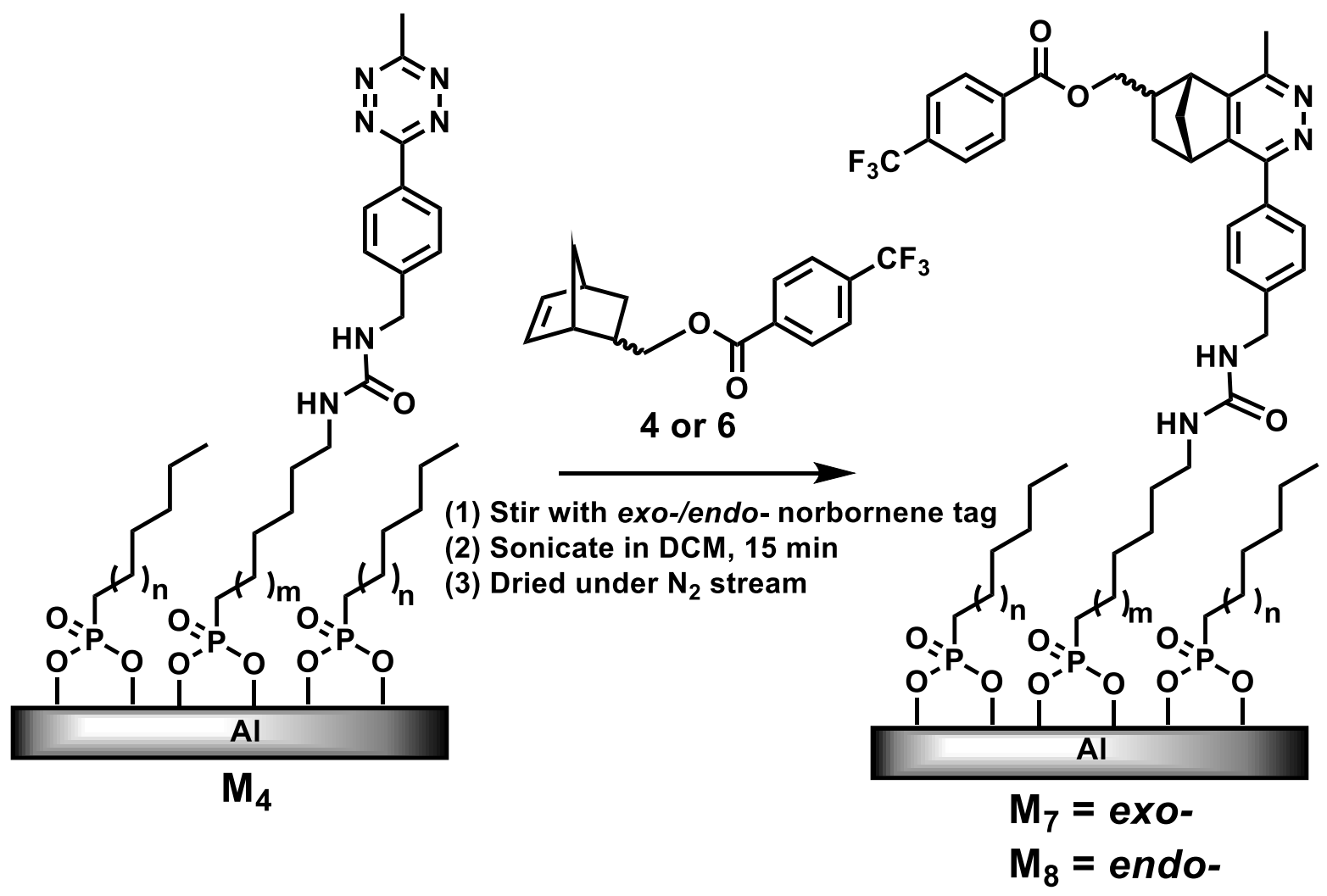




\section{Chemical Synthesis}

2.1 General Remarks. Unless stated otherwise, solvents and dry solvents like dichloromethane, methanol and isopropanol were purchased from Sigma-Aldrich. Unless stated otherwise all of these chemicals were used without further purification.

2.2. Reaction Handling. Unless stated otherwise all non-aqueous reactions were performed in dried glassware under an atmosphere of argon. All flasks were equipped with rubber septa and reactants were handled using standard Schlenk techniques. Temperatures above the room temperature refer to oil bath temperatures which were controlled by a thermostat. Reactions were magnetically stirred.

2.3. ${ }^{1} \mathbf{H}-\mathbf{N M R}$ spectra were recorded at room temperature on a Bruker-400 spectrometer with ${ }^{1} \mathrm{H}$ operating frequency of $400 \mathrm{MHz}$. Unless stated otherwise all spectra were recorded at room temperature in $\mathrm{CDCl}_{3}$ and $\mathrm{DMSO}-d_{6}$ and all chemical shifts are given in $\delta$ units relative to the residual solvent [central line of singlet: $\delta_{\mathrm{H}}=$ $7.27 \mathrm{ppm}\left(\mathrm{CDCl}_{3}\right)$ and $\left.2.50 \mathrm{ppm}\left(\mathrm{DMSO}-d_{6}\right)\right]$. Analysis followed first order and the following abbreviations were used throughout: $\mathrm{s}=$ singlet, br. s. $=$ broad singlet, $\mathrm{d}=$ doublet, $\mathrm{t}=$ triplet, $\mathrm{q}=$ quartet, quin $=$ quintet, sxt $=$ sextet, sept $=$ spt, $\mathrm{dd}=$ doublet of doublet, $\mathrm{dt}=$ doublet of triplet, $\mathrm{ddd}=$ doblet of doublet of doublet, $\mathrm{ddt}=$ doblet of doublet of triplet, $\mathrm{m}=$ multiplet, $\mathrm{mc}=$ centred multiplet. Coupling constants $(J)$ are given in Hertz $(\mathrm{Hz})$.

2.4. ${ }^{13} \mathbf{C}-\mathbf{N M R}$ spectra were recorded at room temperature on a Bruker-400 spectrometer with ${ }^{13} \mathrm{C}$ operating frequency of $101 \mathrm{MHz}$. Unless stated otherwise all spectra were recorded at room temperature in $\mathrm{CDCl}_{3}$ and $\mathrm{DMSO}-d_{6}$ and all chemical shifts are given in $\delta$ units relative to the residual solvent [central line of triplet: $\delta_{\mathrm{C}}=$ $77.2 \mathrm{ppm}\left(\mathrm{CDCl}_{3}\right)$ and heptet $\left.\delta_{\mathrm{C}}=39.5 \mathrm{ppm}\left(\mathrm{DMSO}-d_{6}\right)\right]$. The following abbreviation was used throughout: $\mathrm{s}=$ singlet, $\mathrm{d}=$ doublet, $\mathrm{dd}=$ doublet of doublet. If no coupling constants are given, the multiplicity refers to ${ }^{1} \mathrm{H}-$ decoupled spectra.

2.5. Synthesis of 3-methyl-6-phenyl methylamine-1,2,4,5-tetrazine, 1. 


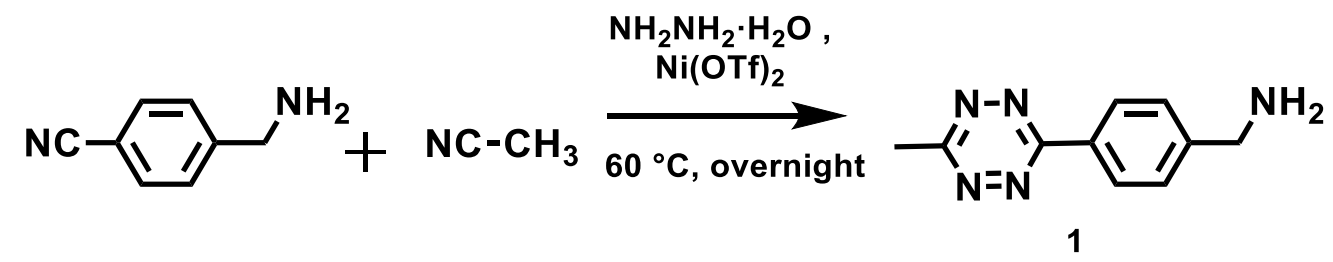

Metal-catalyzed tetrazine synthesis described elsewhere ${ }^{7}$ was applied to synthesize 3 methyl-6-phenyl methylamine-1,2,4,5-tetrazine. Briefly, 4-(aminomethyl) benzonitrile hydrochloride $(1.50 \mathrm{~g}, 8.9 \mathrm{mmol}), \mathrm{Ni}(\mathrm{OTf})_{2}(1.59 \mathrm{~g}, 4.45 \mathrm{mmol})$ and acetonitrile $(4.7 \mathrm{~mL}, 89 \mathrm{mmol})$ were added in a $250 \mathrm{~mL}$ round flask, followed adding $60 \% \mathrm{NH}_{2} \mathrm{NH}_{2} \cdot \mathrm{H}_{2} \mathrm{O}(30 \mathrm{~mL})$, under $\mathrm{N}_{2}$ flow. The mixture was stirred at $60{ }^{\circ} \mathrm{C}$ for 16 $\mathrm{h}$ and allowed to cool to room temperature. Sodium nitrite $(14.6 \mathrm{~g}, 212 \mathrm{mmol})$ in 25 $\mathrm{mL}$ of water was slowly added to the reaction followed by $5 \mathrm{M} \mathrm{HCl}$ until gas evolution ceased ( $\mathrm{pH} 3$ ), in an ice bath. The product was extracted into ethyl acetate and purified by silica column chromatography $(\mathrm{MeOH}: \mathrm{DCM}=1: 9)$ as a red solid. Yield: $0.56 \mathrm{~g}(27 \%) .{ }^{1} \mathrm{H}-\mathrm{NMR}\left(400 \mathrm{MHz}, \mathrm{DMSO}-d_{6}\right), \delta 2.99(3 \mathrm{H}, \mathrm{s}), 4.15(2 \mathrm{H}, \mathrm{s})$, $7.71(2 \mathrm{H}, \mathrm{d}, \mathrm{J}=8 \mathrm{~Hz}), 8.49(2 \mathrm{H}, \mathrm{d}, \mathrm{J}=8 \mathrm{~Hz}) \cdot{ }^{13} \mathrm{C}-\mathrm{NMR}\left(101 \mathrm{MHz}, \mathrm{DMSO}-d_{6}\right), \delta 20.8$, $42.3,127.6,129.5,131.8,139.2,162.3,167.2$. 
<smiles>Cc1nnc(-c2ccc(CN)cc2)nn1</smiles>

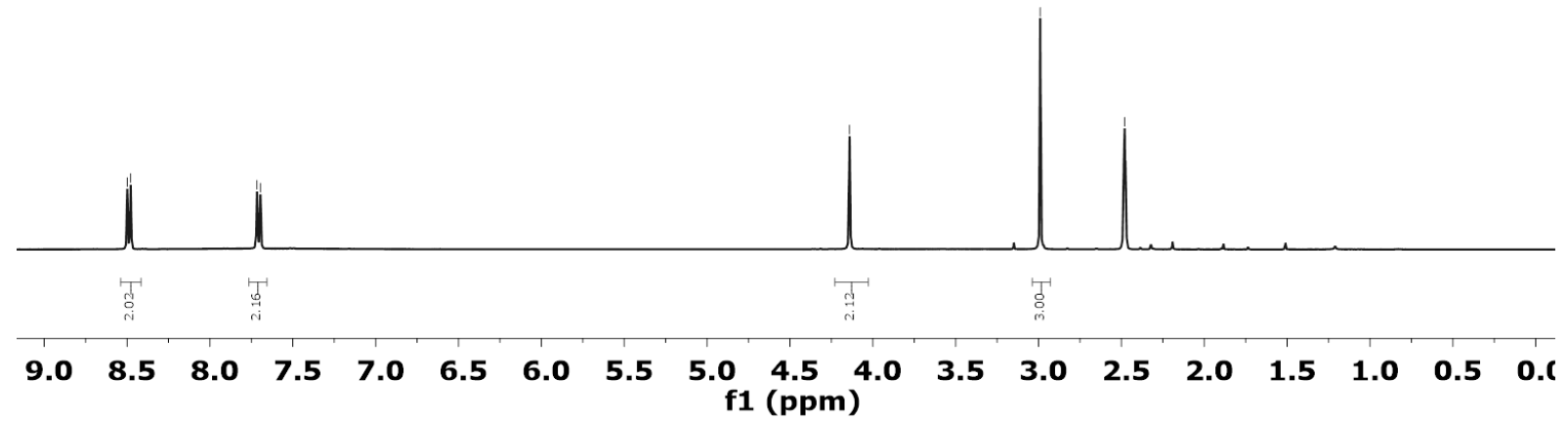

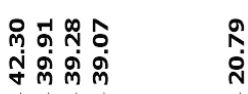

$\mathrm{H}_{2} \mathrm{~N}$

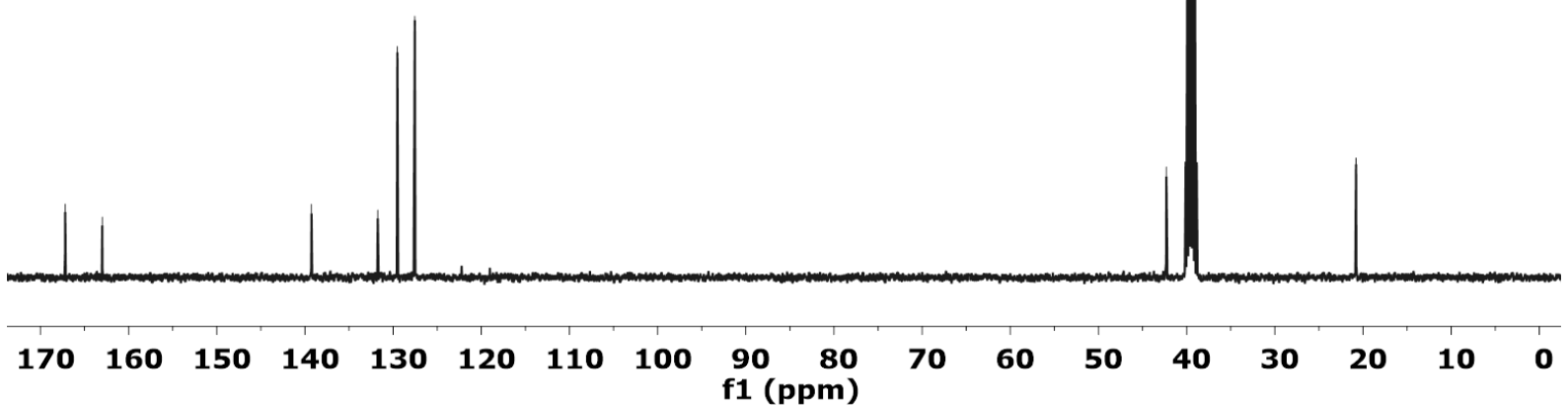


2.6. Synthesis of 3-methyl-6-phenyl methanol-1,2,4,5-tetrazine, 2.

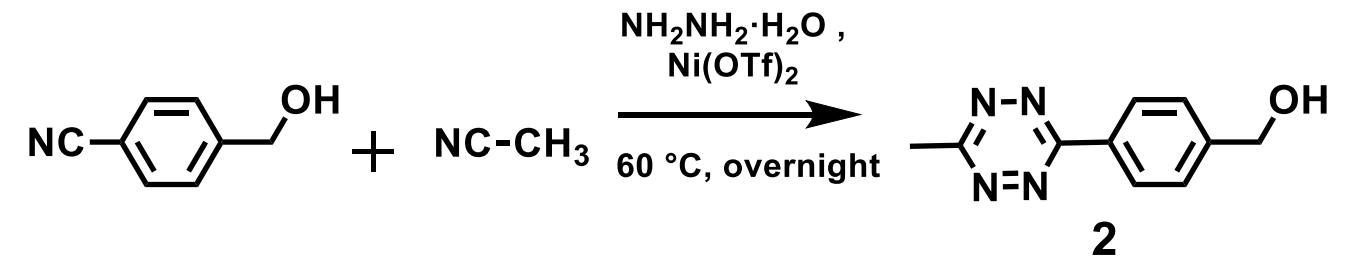

3-methyl-6-phenyl methanol-1,2,4,5-tetrazine was synthesized as per protocol described elsewhere. ${ }^{7}$ Briefly, 4-cyanobenzylalcohol (0.51 g, $\left.3.75 \mathrm{mmol}\right), \mathrm{Ni}(\mathrm{OTf})_{2}$ $(0.67 \mathrm{~g}, 1.88 \mathrm{mmol})$ and acetonitrile $(1.97 \mathrm{~mL}, 37.6 \mathrm{mmol})$ were added in a $100 \mathrm{~mL}$ round flask, followed adding $60 \% \mathrm{NH}_{2} \mathrm{NH}_{2} \cdot \mathrm{H}_{2} \mathrm{O}(12 \mathrm{~mL})$, under $\mathrm{N}_{2}$ flow. The mixture stirred at $60{ }^{\circ} \mathrm{C}$ for $24 \mathrm{~h}$ and allowed to cool to room temperature. Sodium nitrite $(6.35 \mathrm{~g}, 92.0 \mathrm{mmol})$ in $14 \mathrm{~mL}$ water was slowly added to the reaction followed by careful addition of $5 \mathrm{M} \mathrm{HCl}$ until gas evolution ceased. ( $\mathrm{pH} 3$ ), in an ice bath with stirring. The product was extracted with ethyl acetate and organic phase was dried by sodium sulfate. The product was purified using silica column chromatography $($ EtOAc $:$ heptane $=1: 2.8)$ as a red solid. Yield $=0.33 \mathrm{~g}(41 \%) .{ }^{1} \mathrm{H}-\mathrm{NMR}(400$ $\left.\mathrm{MHz}, \mathrm{CDCl}_{3}\right), \delta 3.08(3 \mathrm{H}, \mathrm{s}), 4.82(2 \mathrm{H}, \mathrm{d}), 7.57(2 \mathrm{H}, \mathrm{d}, \mathrm{J}=8 \mathrm{~Hz}), 8.55(2 \mathrm{H}, \mathrm{d}, \mathrm{J}=8 \mathrm{~Hz})$. ${ }^{13} \mathrm{C}-\mathrm{NMR}\left(101 \mathrm{MHz}, \mathrm{CDCl}_{3}\right), \delta$ 21.1, 64.7, 127.4, 128.1, 130.9, 145.6, 163.9, 167.2. 


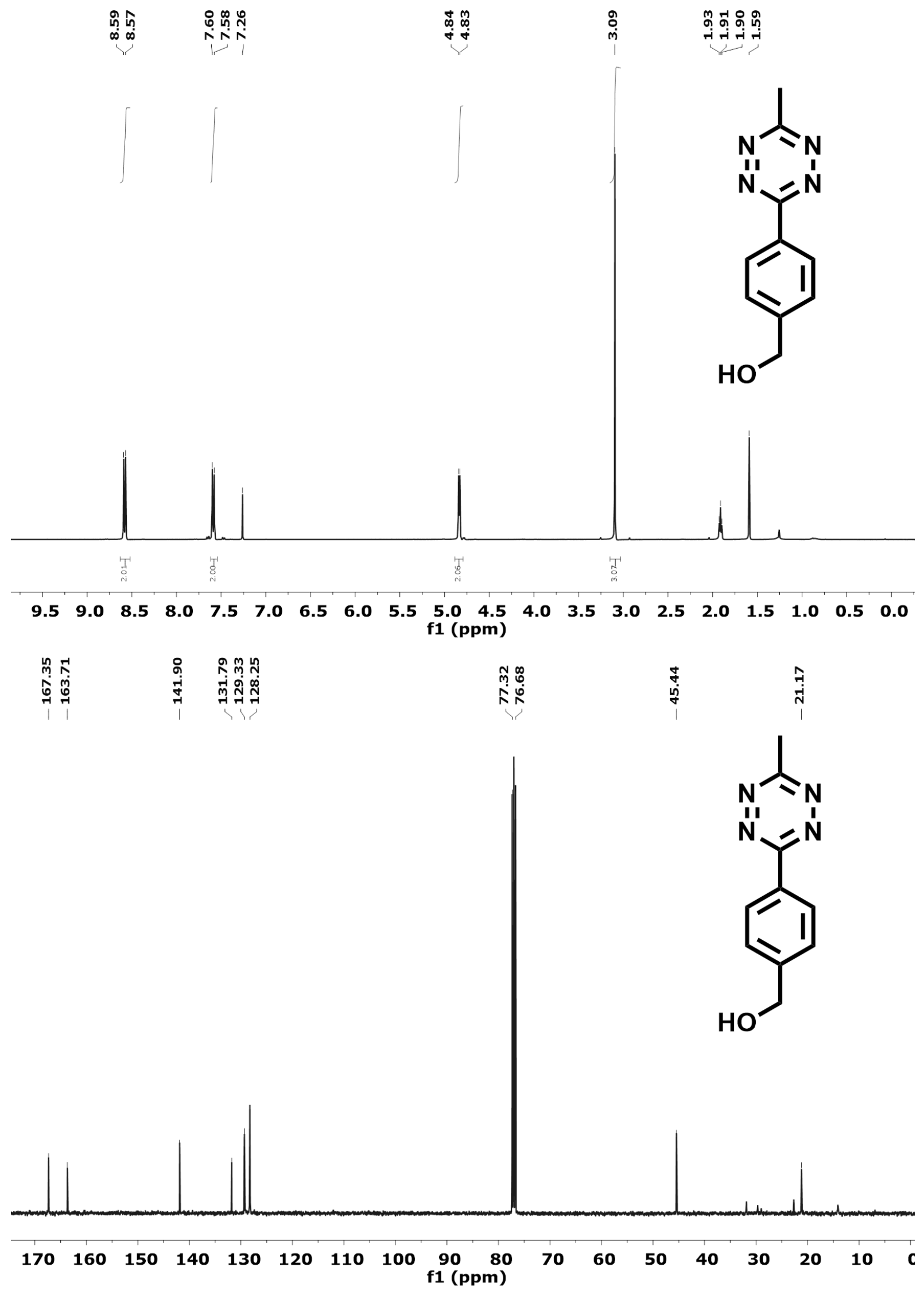


2.7. Synthesis of $\quad[3-(3-$ methyl-1,2,4,5-tetrazine $)]$ benzyl $\quad(4-$ (trifluoromethyl)benzoate, 3.

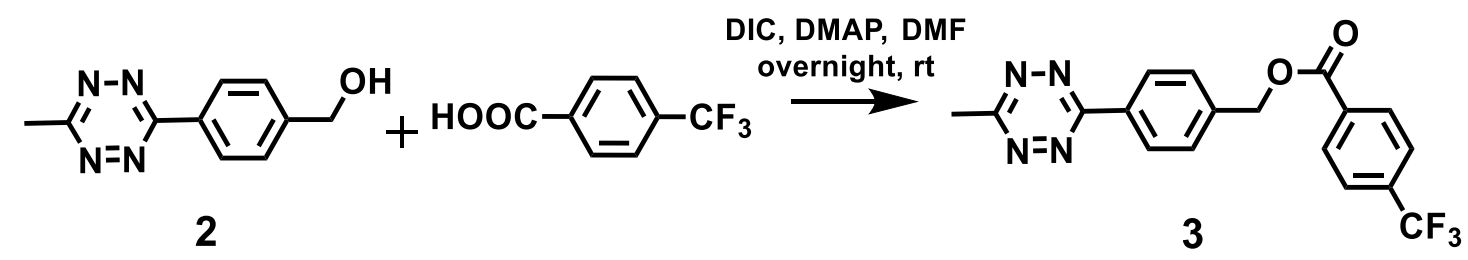

4-(Trifluoromethyl)benzoic acid (0.27 g, $1.38 \mathrm{mmol})$, DMAP (0.01 g, $0.123 \mathrm{mmol}$ ), $2.5 \mathrm{~mL}$ DMF, DIC $(0.18 \mathrm{~mL}, 1.18 \mathrm{mmol})$ and compound 2 (0.21 g, $0.98 \mathrm{mmol})$ were successively added and allowed to react at room temperature for $17 \mathrm{~h}$. DMF was evaporated by rotatory evaporation under reduced pressure. The mixture was extracted with EtOAc and organic phase was washed with brine, dried with sodium sulfate and concentrated by rotatory evaporation to yield an oil, which was purified using silica column chromatography (EtOAc : heptane =1:4) to obtain a red solid. Yield $=0.22 \mathrm{~g}(57 \%) .{ }^{1} \mathrm{H}-\mathrm{NMR}\left(400 \mathrm{MHz}, \mathrm{CDCl}_{3}\right), \delta 3.11(3 \mathrm{H}, \mathrm{s}), 5.51(2 \mathrm{H}, \mathrm{s}), 7.67$ $(2 \mathrm{H}, \mathrm{d}, \mathrm{J}=8 \mathrm{~Hz}), 7.73(2 \mathrm{H}, \mathrm{d}, \mathrm{J}=8 \mathrm{~Hz}), 8.22(2 \mathrm{H}, \mathrm{d}, \mathrm{J}=8 \mathrm{~Hz}), 8.63(2 \mathrm{H}, \mathrm{d}, \mathrm{J}=8 \mathrm{~Hz}) \cdot{ }^{13} \mathrm{C}-$ NMR (101 MHz, $\mathrm{CDCl}_{3}$ ), $\delta ~ 21.2,66.5,125.5,128.3,128.4,128.7,130.2,131.9,132.5$, $133.1,140.2,163.8,165.1,167.4$. 

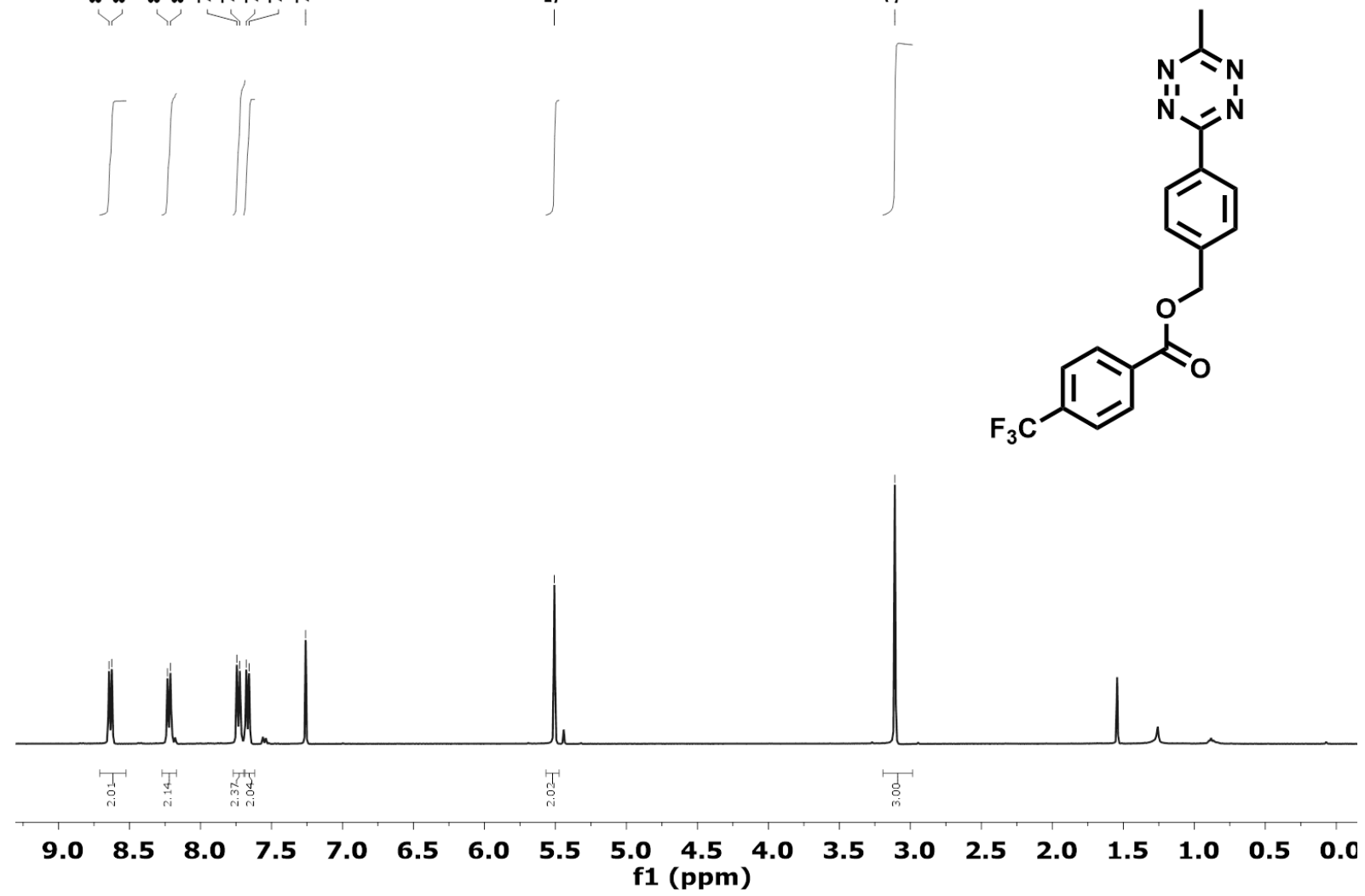

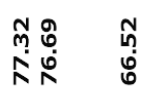

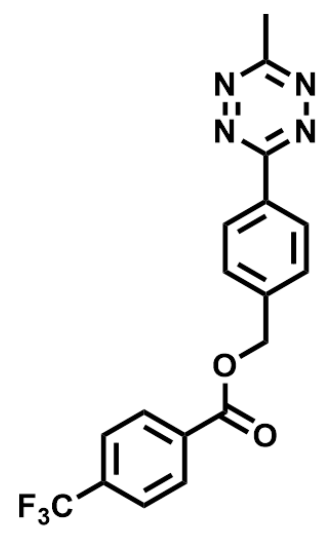

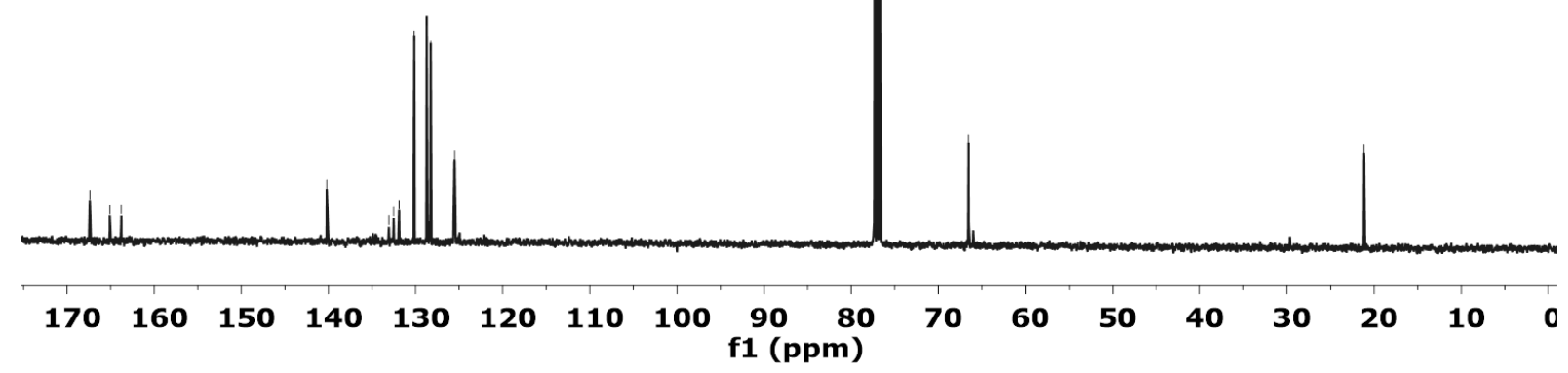

2.8. Synthesis of $((1 \mathrm{R}, 2 \mathrm{~S}, 4 \mathrm{R})-$ bicyclo[2.2.1]hept-5-en-2-yl)methyl $\quad 4-$ (trifluoromethyl)benzoate, 4. 


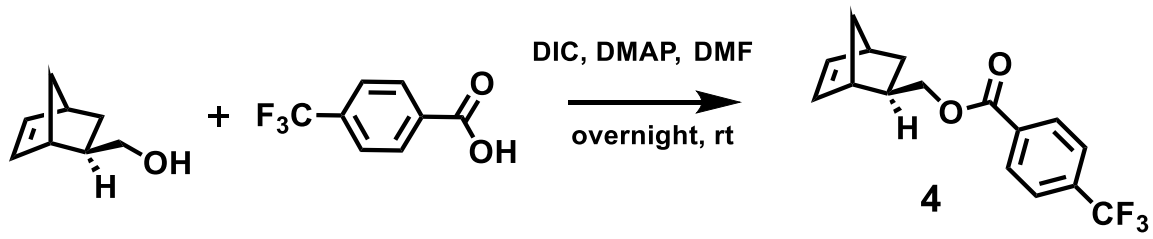

4-(Trifluoromethyl)benzoic acid (0.43 g, $2.25 \mathrm{mmol})$, DMAP (0.024 g, $0.190 \mathrm{mmol}), 4$ $\mathrm{mL}$ DMF, DIC (0.3 mL, $1.93 \mathrm{mmol})$ and ((1R,2S,4R)-bicyclo[2.2.1]hept-5-en-2yl)methanol $(0.19 \mathrm{~mL}, 1.61 \mathrm{mmol})$, were successively added in a $100 \mathrm{~mL}$ rounded flask, and allowed to react for $16 \mathrm{~h}$. DMF was removed using a rotary evaporator under reduced pressure. The mixture was extracted with EtOAc and organic phase was washed by brine, dried by sodium sulfate. The product was purified using silica column chromatography (EtOAc : heptane $=1: 4)$ as white solid. Yield $=0.35 \mathrm{~g}$ (69\%). ${ }^{1} \mathrm{H}-\mathrm{NMR}\left(400 \mathrm{MHz}, \mathrm{CDCl}_{3}\right), \delta 1.23-1.40(4 \mathrm{H}, \mathrm{m}), 1.88(1 \mathrm{H}, \mathrm{m}), 2.80(1 \mathrm{H}, \mathrm{s})$, $2.87(1 \mathrm{H}, \mathrm{s}), 4.25(1 \mathrm{H}, \mathrm{m}), 4.43(1 \mathrm{H}, \mathrm{m}), 6.11(2 \mathrm{H}, \mathrm{m}), 7.70(2 \mathrm{H}, \mathrm{d}, \mathrm{J}=8 \mathrm{~Hz}), 8.16(2 \mathrm{H}$, d, J=8Hz). ${ }^{13} \mathrm{C}-\mathrm{NMR}\left(101 \mathrm{MHz}, \mathrm{CDCl}_{3}\right), \delta$ 29.6, 38.1, 41.6, 43.72, 45.0, 69.6, 122.3, 125.4, 130.0, 133.7, 134.52, 136.1, 137.0, 165.4.
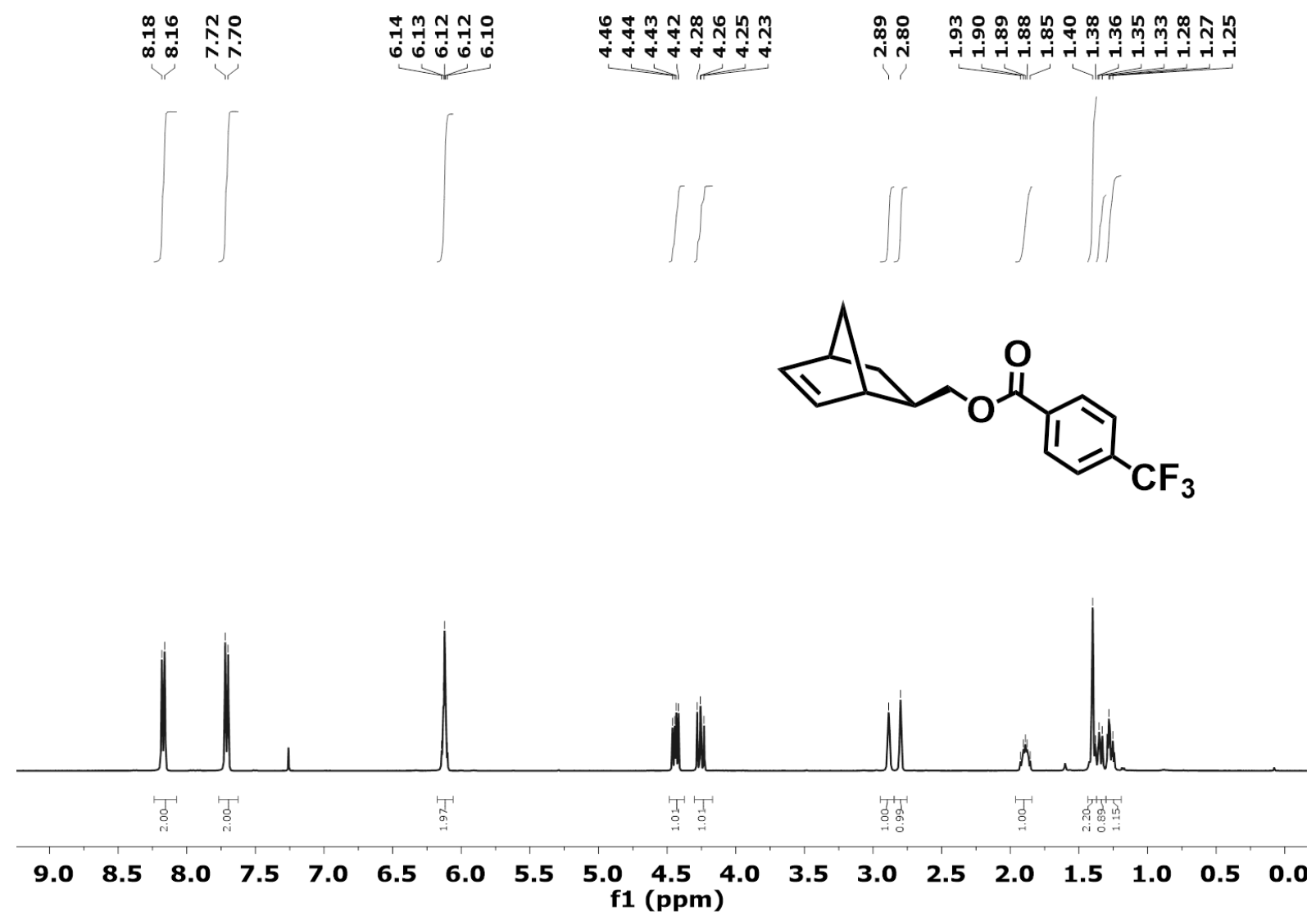


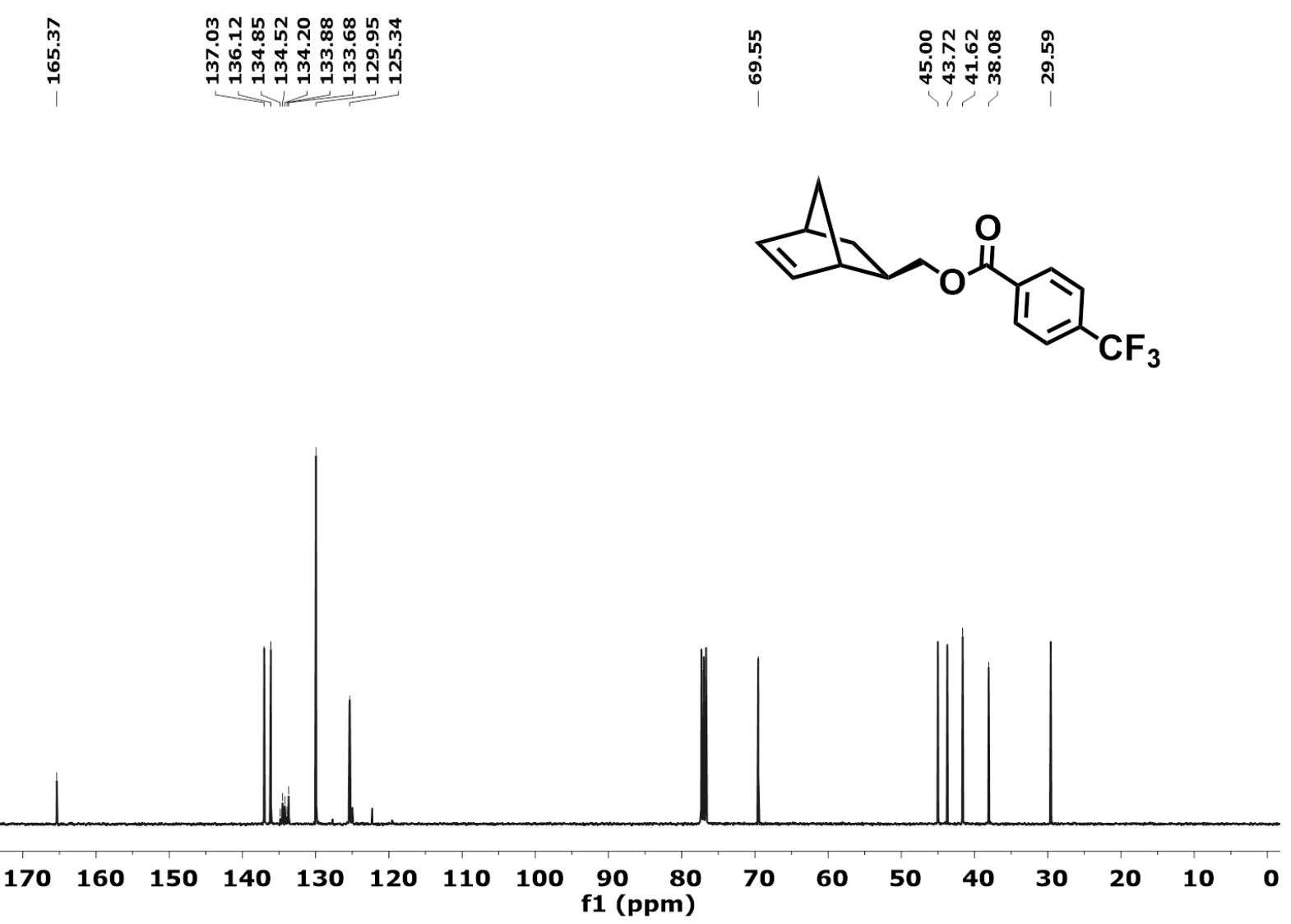


Synthesis of $((1 \mathrm{~S}, 2 \mathrm{~S}, 4 \mathrm{~S})-$ bicyclo[2.2.1]hept-5-en-2-yl)methanol, 5.

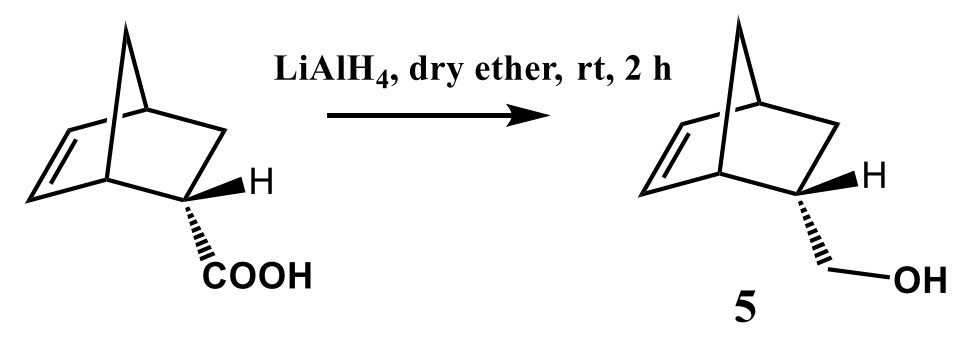

A commercially available mixture of endo- and exo-5-norbornenecarboxylic acid, was subjected to column chromatography (heptane : EtOAc $=1: 4$ ) to obtain the endo-norbornene carboxylic acid. (1S,2S,4S)-bicyclo[2.2.1]hept-5-ene-2-carboxylic acid $(206.6 \mathrm{mg}, 1.5 \mathrm{mmol})$ was mixed with $\mathrm{LiAlH}_{4}(113.5 \mathrm{mg}, 3.0 \mathrm{mmol})$ in $20 \mathrm{~mL}$ dry ether for $2 \mathrm{~h}$. The reaction was quenched with water and the organic product was extracted into ether followed by recovery of the product by evaporating the solvent under reduced pressure. Yield $=0.15 \mathrm{~g}(81 \%) .{ }^{1} \mathrm{H}-\mathrm{NMR}\left(400 \mathrm{MHz}, \mathrm{CDCl}_{3}\right) \delta 6.15$ $(\mathrm{dd}, \mathrm{J}=5.7,3.0 \mathrm{~Hz}, 1 \mathrm{H}), 5.97(\mathrm{dd}, \mathrm{J}=5.7,2.9 \mathrm{~Hz}, 1 \mathrm{H}), 3.40(\mathrm{dd}, \mathrm{J}=10.4,6.5 \mathrm{~Hz}$, $1 \mathrm{H}), 3.26(\mathrm{dd}, \mathrm{J}=10.4,8.9 \mathrm{~Hz}, 1 \mathrm{H}), 2.93(\mathrm{~s}, 1 \mathrm{H}), 2.81(\mathrm{~s}, 1 \mathrm{H}), 2.29$ (ddt, J = 9.2, $6.6,4.5 \mathrm{~Hz}, 1 \mathrm{H}), 1.82(\mathrm{ddd}, \mathrm{J}=11.6,9.2,3.8 \mathrm{~Hz}, 1 \mathrm{H}), 1.48-1.41(\mathrm{~m}, 1 \mathrm{H}), 1.27$ (d, J $=8.2 \mathrm{~Hz}, 1 \mathrm{H}), 0.55-0.49(\mathrm{~m}, 1 \mathrm{H})$. 

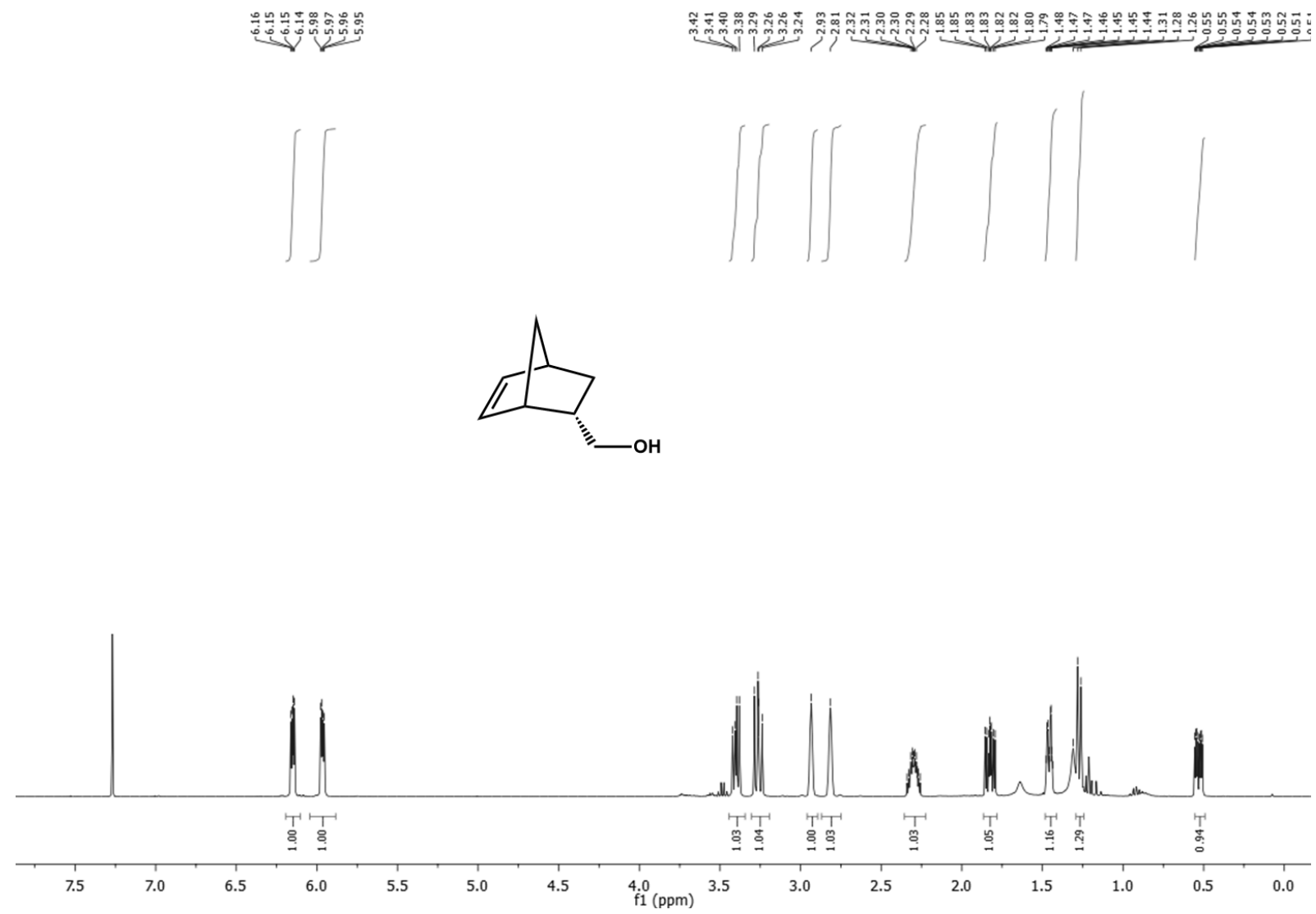

Synthesis of $\quad((1 \mathrm{~S}, 2 \mathrm{~S}, 4 \mathrm{~S})-$ bicyclo[2.2.1]hept-5-en-2-yl)methyl $\quad 4-$ (trifluoromethyl)benzoate, 6 .

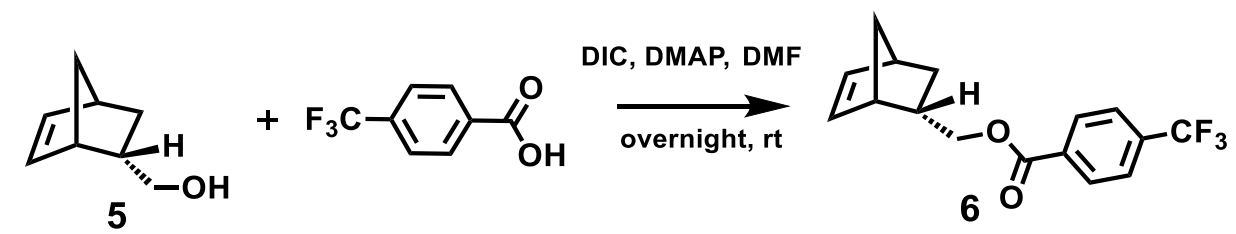

4-(Trifluoromethyl)benzoic acid (0.43 g, $2.25 \mathrm{mmol}$ ), DMAP (0.024 g, $0.19 \mathrm{mmol}), 4$ $\mathrm{mL}$ DMF, DIC (0.3 mL, $1.93 \mathrm{mmol})$ and ((1S,2S,4S)-bicyclo[2.2.1]hept-5-en-2yl)methanol, $\mathbf{5},(0.19 \mathrm{~mL}, 1.61 \mathrm{mmol})$, were successively added in a $100 \mathrm{~mL}$ rounded flask, and allowed to react for $24 \mathrm{~h}$. DMF was removed using a rotary evaporator under reduced pressure. The mixture was extracted with EtOAc and organic phase was washed by brine, dried by sodium sulfate. The product was purified using silica column chromatography (EtOAc $:$ heptane $=1: 4)$ as white solid. Yield $=0.14 \mathrm{~g}$. $(33 \%){ }^{1} \mathrm{H}-\mathrm{NMR}\left(400 \mathrm{MHz}, \mathrm{CDCl}_{3}\right), \delta 8.17(\mathrm{~d}, \mathrm{~J}=7.6 \mathrm{~Hz}, 2 \mathrm{H}), 7.72(\mathrm{~d}, \mathrm{~J}=7.5 \mathrm{~Hz}$, 
$2 \mathrm{H}), 6.22(\mathrm{~s}, 1 \mathrm{H}), 6.01(\mathrm{~s}, 1 \mathrm{H}), 4.21-4.07(\mathrm{~m}, 1 \mathrm{H}), 3.96(\mathrm{t}, \mathrm{J}=9.9 \mathrm{~Hz}, 1 \mathrm{H}), 2.98(\mathrm{~s}$, 1H), $2.87(\mathrm{~s}, 1 \mathrm{H}), 2.57(\mathrm{~s}, 1 \mathrm{H}), 1.93(\mathrm{t}, \mathrm{J}=10.1 \mathrm{~Hz}, 1 \mathrm{H}), 1.51$ (d, J = 7.9 Hz, 1H), $1.32(\mathrm{~d}, \mathrm{~J}=7.8 \mathrm{~Hz}, 1 \mathrm{H}), 0.67(\mathrm{~d}, \mathrm{~J}=10.2 \mathrm{~Hz}, 1 \mathrm{H}) \cdot{ }^{13} \mathrm{C}-\mathrm{NMR}\left(101 \mathrm{MHz}, \mathrm{CDCl}_{3}\right), \delta$ $165.30,137.81,134.50,134.18,133.78,132.07,129.94,125.35,125.01,125.01,122.30$, 68.91, 49.44, 43.99, 42.24, 37.89, 29.69, 28.98.
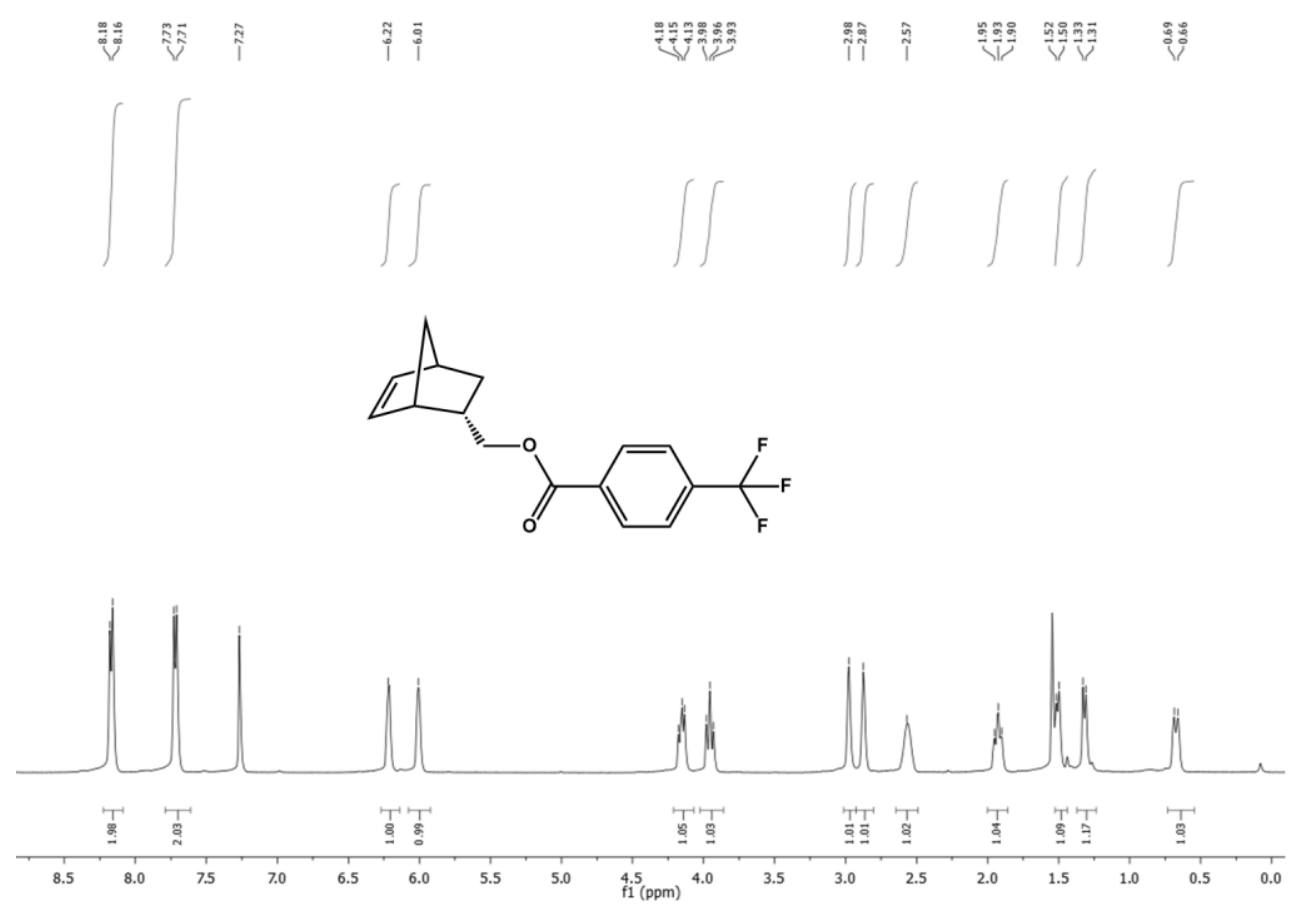

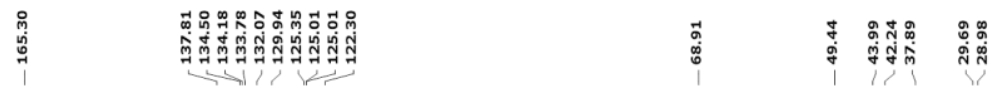

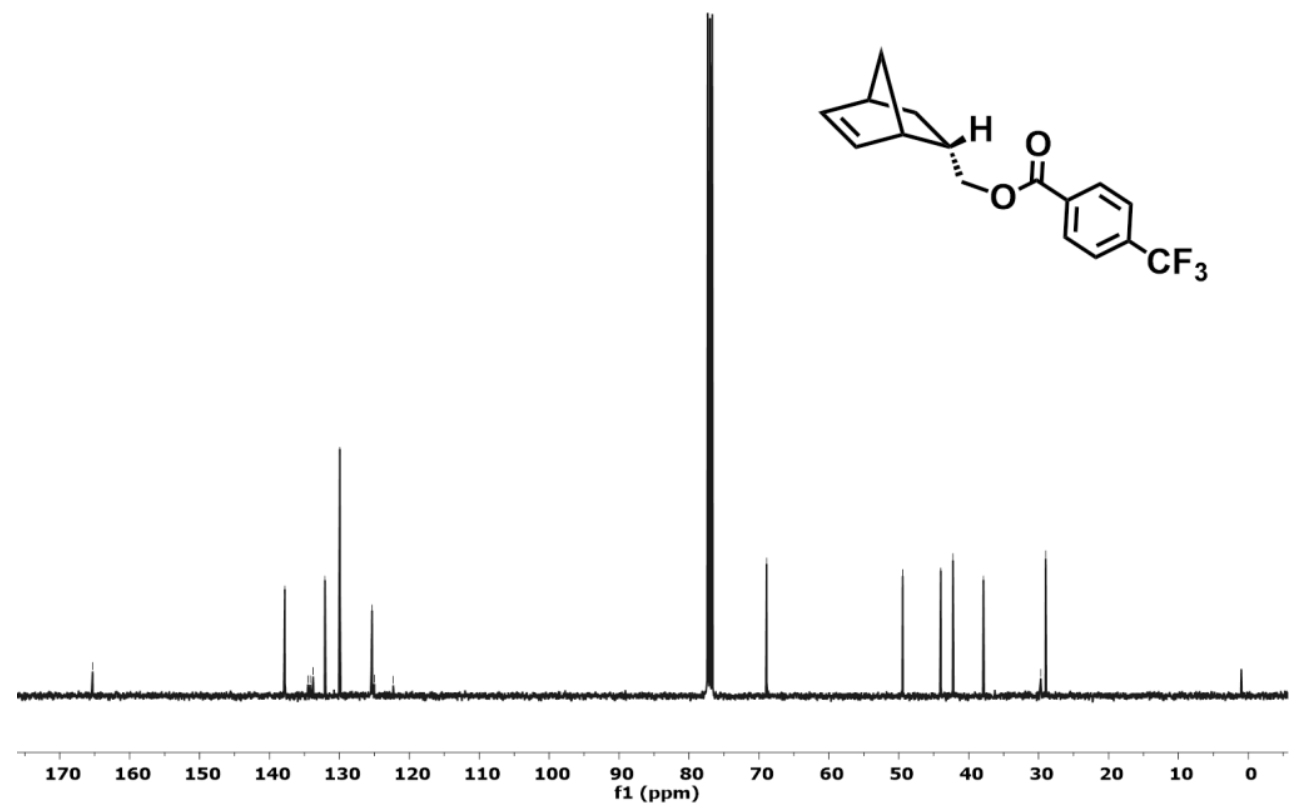




\section{Supplementary Kinetics Data.}

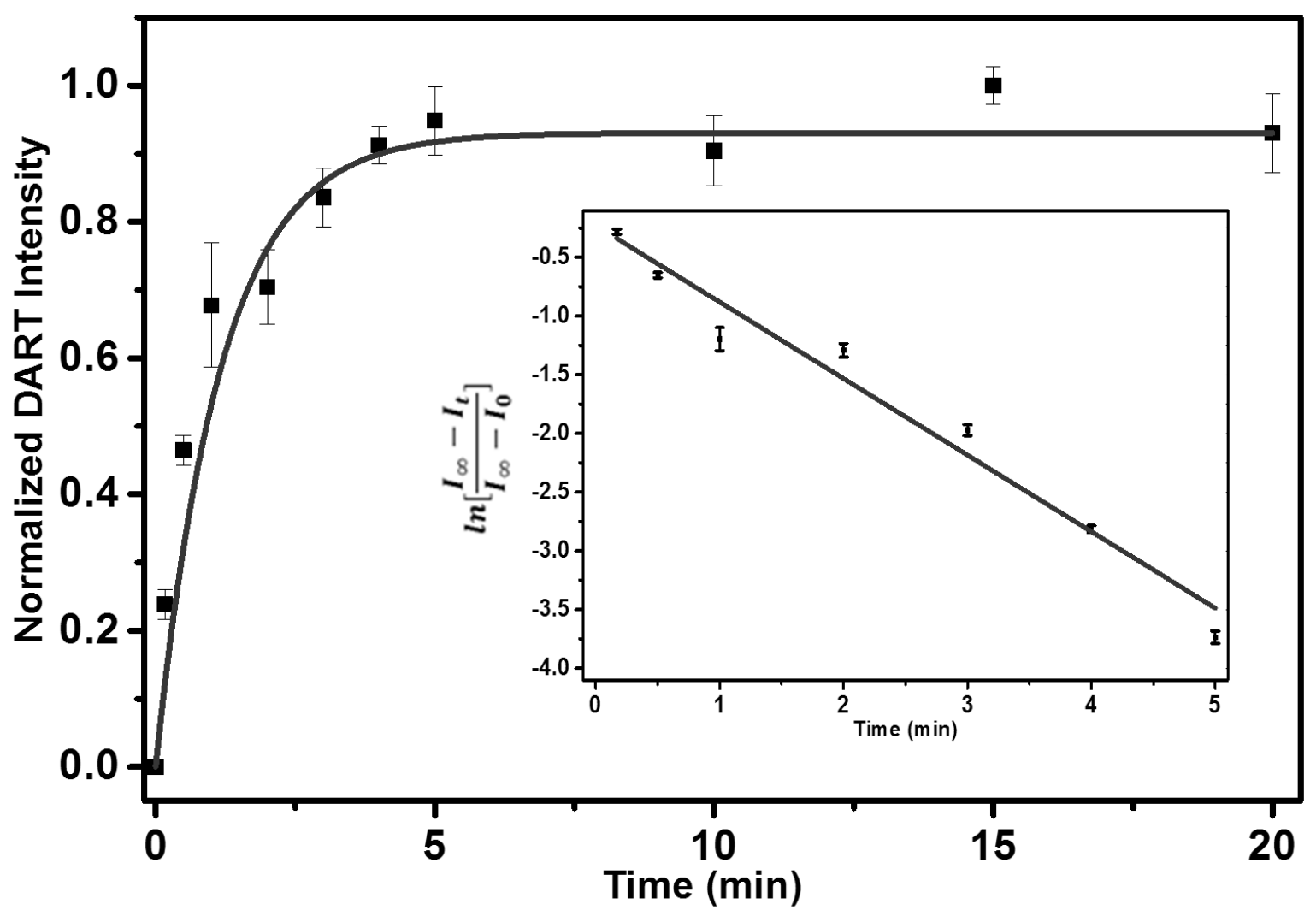

Figure S3.1. Sigmoidal plot of normalized integrated DART-HRMS intensity vs time (min) for "free" exo-norbornene-terminated surface $\left(\mathbf{M}_{2}\right)$ reacting with tetrazine tag, 3 in DCE solution at $30{ }^{\circ} \mathrm{C}$. Inset: Linear plot of $\ln \left|\left(I_{\infty}-I_{t}\right) /\left(I_{\infty}-I_{0}\right)\right|$ vs time (min), the slope of which is the pseudo-first order reaction rate $\left(k^{\prime}\right)$ obtained by a least-squares fit $\left(\mathrm{R}^{2}>0.95\right)$. (Each time point is an average of hexaplet samples.) The second--order rate constant is derived from here using the concentration of the other agent $(3.0 \mathrm{mM})$. 


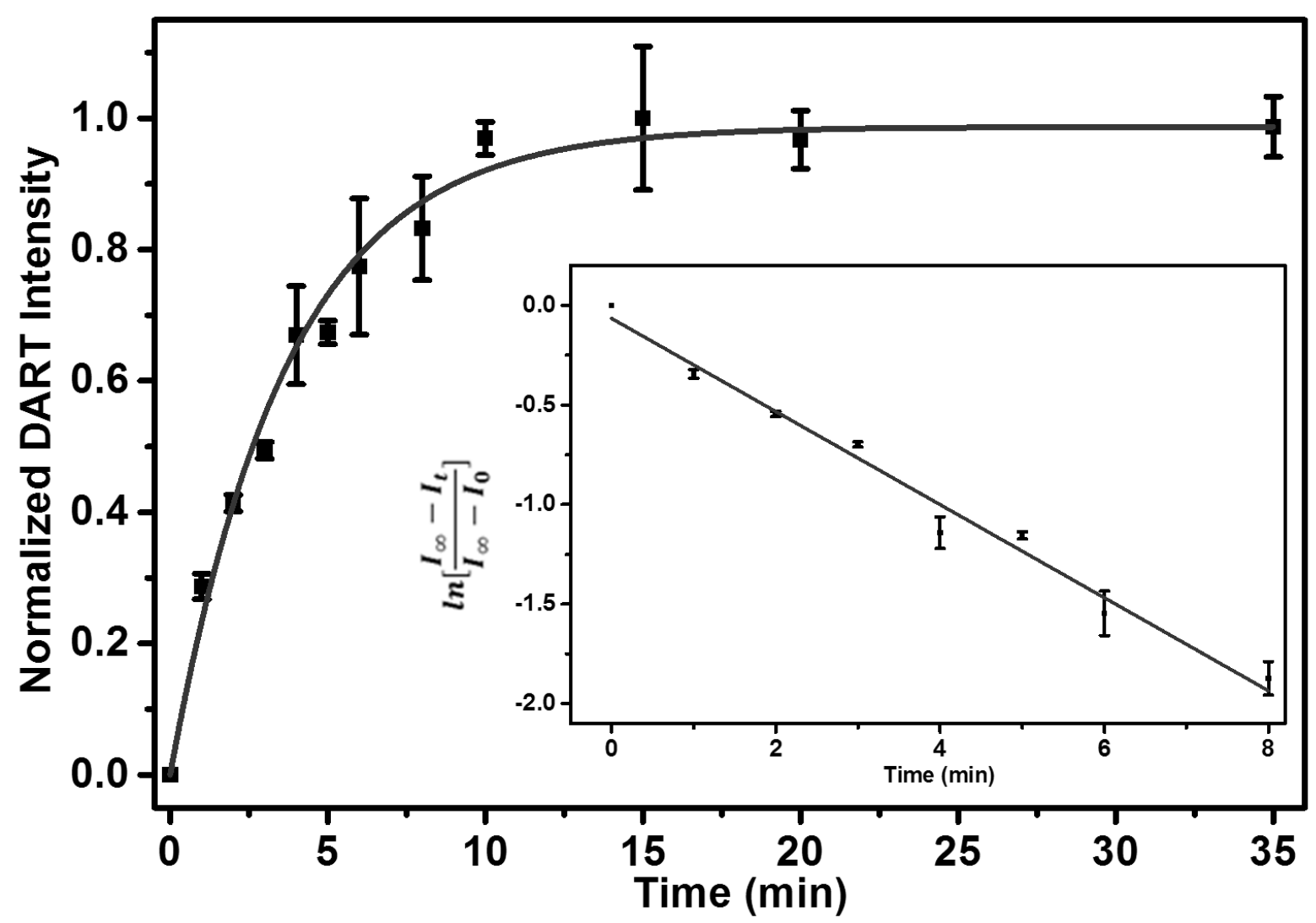

Figure S3.2. Sigmoidal plot of normalized integrated DART-HRMS intensity vs time (min) for "buried" exo-norbornene-terminated surface $\left(\mathbf{M}_{2}\right)$ reacting with tetrazine tag, 3 in DCE solution at $30^{\circ} \mathrm{C}$. Inset: Linear plot of $\ln \left|\left(I_{\infty}-I_{t}\right) /\left(I_{\infty}-I_{0}\right)\right|$ vs time (min), the slope of which is the pseudo - first order reaction rate $\left(\mathrm{k}^{\prime}\right)$ obtained by a least-squares fit $\left(\mathrm{R}^{2}>0.95\right)$. (Each time point is an average of hexaplet samples.) The second-order rate constant is derived from here using the concentration of the other agent $(3.0 \mathrm{mM})$. 


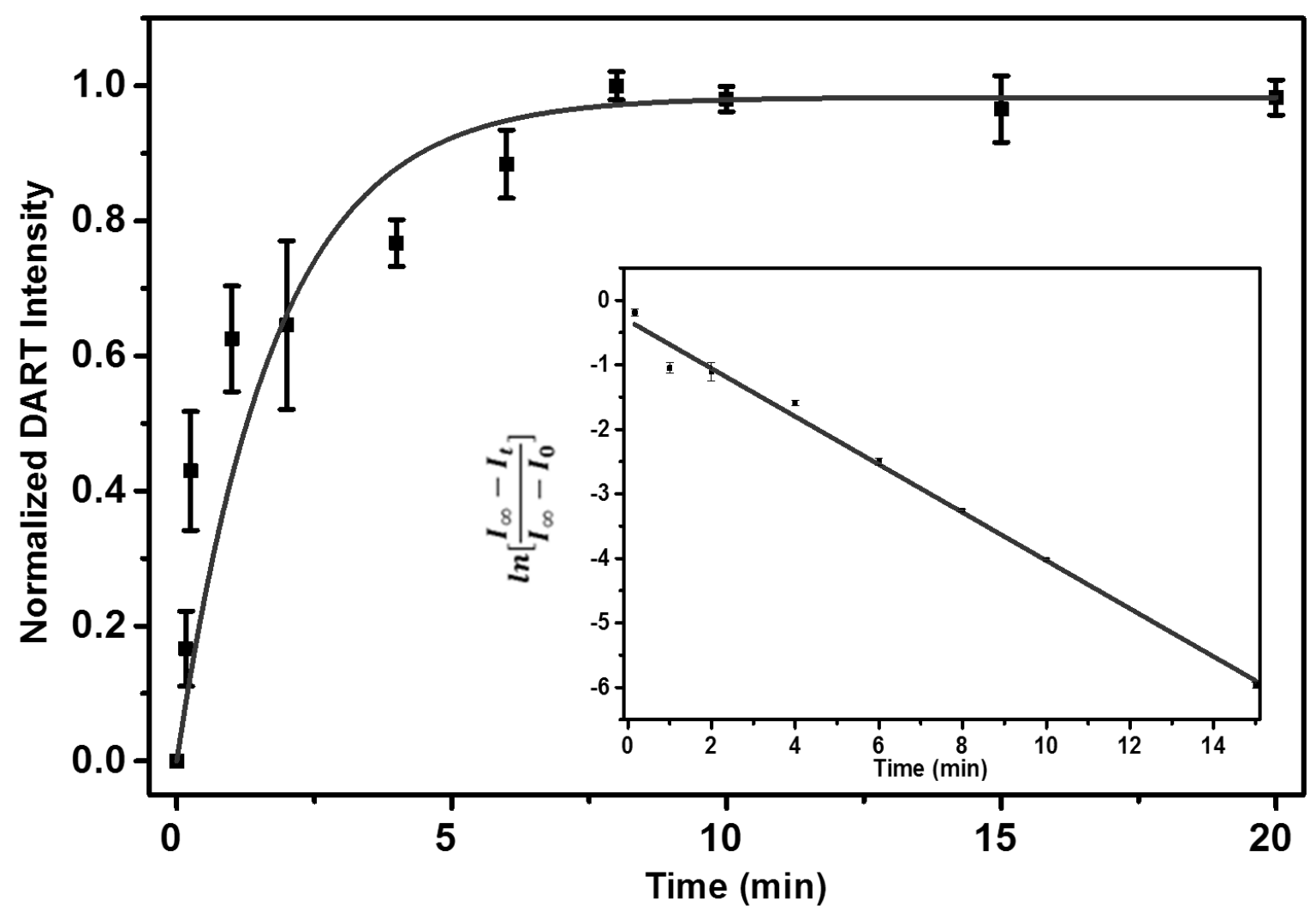

Figure S3.3. Sigmoidal plot of normalized integrated DART-HRMS intensity vs time (min) for "free" endo-norbornene-terminated surface $\left(\mathbf{M}_{\mathbf{3}}\right)$ reacting with tetrazine tag, 3 in DCE solution at $30{ }^{\circ} \mathrm{C}$. Inset: Linear plot of $\ln \left|\left(I_{\infty}-I_{t}\right) /\left(I_{\infty}-I_{0}\right)\right|$ vs time (min), the slope of which is the pseudo-first order reaction rate $\left(\mathrm{k}^{\prime}\right)$ obtained by a least-squares fit $\left(\mathrm{R}^{2}>0.95\right)$. (Each time point is an average of hexaplet samples.) The second-order rate constant is derived from here using the concentration of the other agent $(3.0 \mathrm{mM})$. 


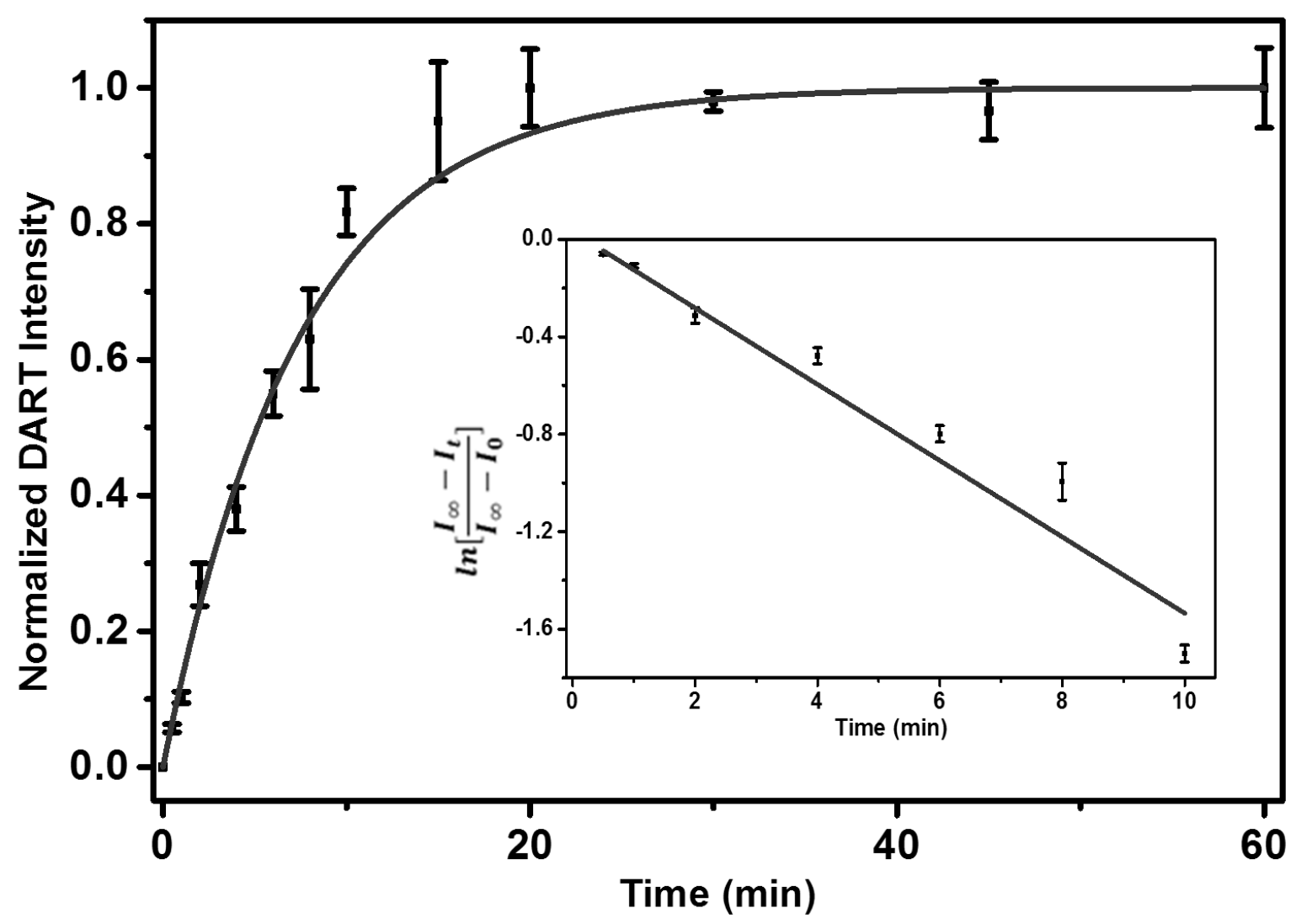

Figure S3.4. Sigmoidal plot of normalized integrated DART-HRMS intensity vs time (min) for "buried" endo-norbornene-terminated surface $\left(\mathbf{M}_{3}\right)$ reacting with tetrazine tag, 3 in DCE solution at $30^{\circ} \mathrm{C}$. Inset: Linear plot of $\ln \left|\left(I_{\infty}-I_{t}\right) /\left(I_{\infty}-I_{0}\right)\right|$ vs time (min), the slope of which is the pseudo - first order reaction rate $\left(\mathrm{k}^{\prime}\right)$ obtained by a least-squares fit $\left(\mathrm{R}^{2}>0.95\right)$. (Each time point is an average of hexaplet samples.) The second-order rate constant is derived from here using the concentration of the other agent $(3.0 \mathrm{mM})$. 


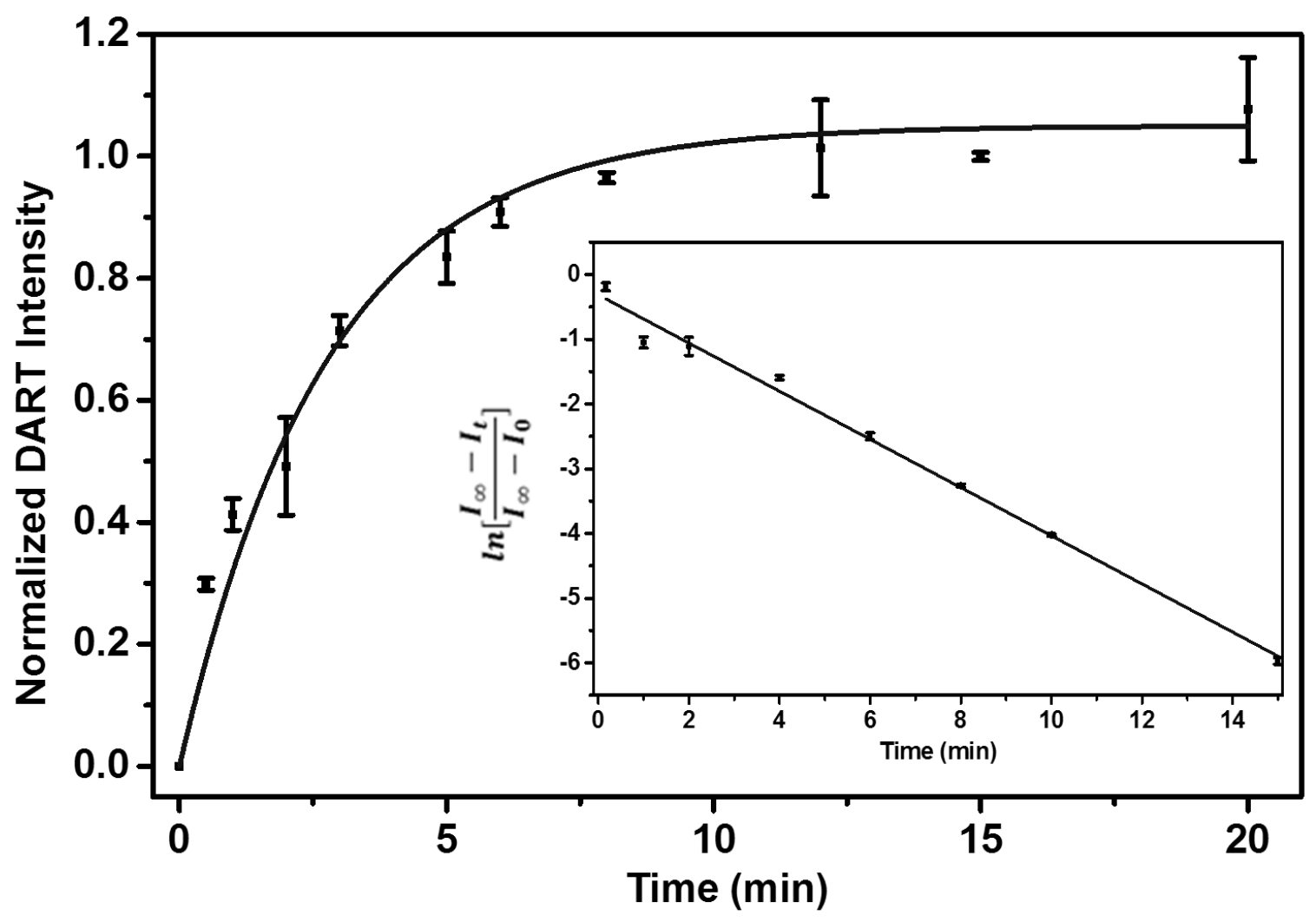

Figure S3.5. Kinetic plot of "free" tetrazine-terminated surface $\left(\mathbf{M}_{4}\right)$ reacting with exo-norbornene tag, 4 in DCE solution at $30{ }^{\circ} \mathrm{C}$. Inset: Linear plot of $\ln \mid\left(I_{\infty}-I_{t}\right) /$ $\left(I_{\infty}-I_{0}\right) \mid$ vs time (min), the slope of which is the pseudo-first order reaction rate $\left(\mathrm{k}^{\prime}\right)$ obtained by a least-squares fit $\left(\mathrm{R}^{2}>0.95\right)$. (Each time point is an average of hexaplet samples.) The second-order rate constant is derived from here using the concentration of the other agent $(3.0 \mathrm{mM})$. 


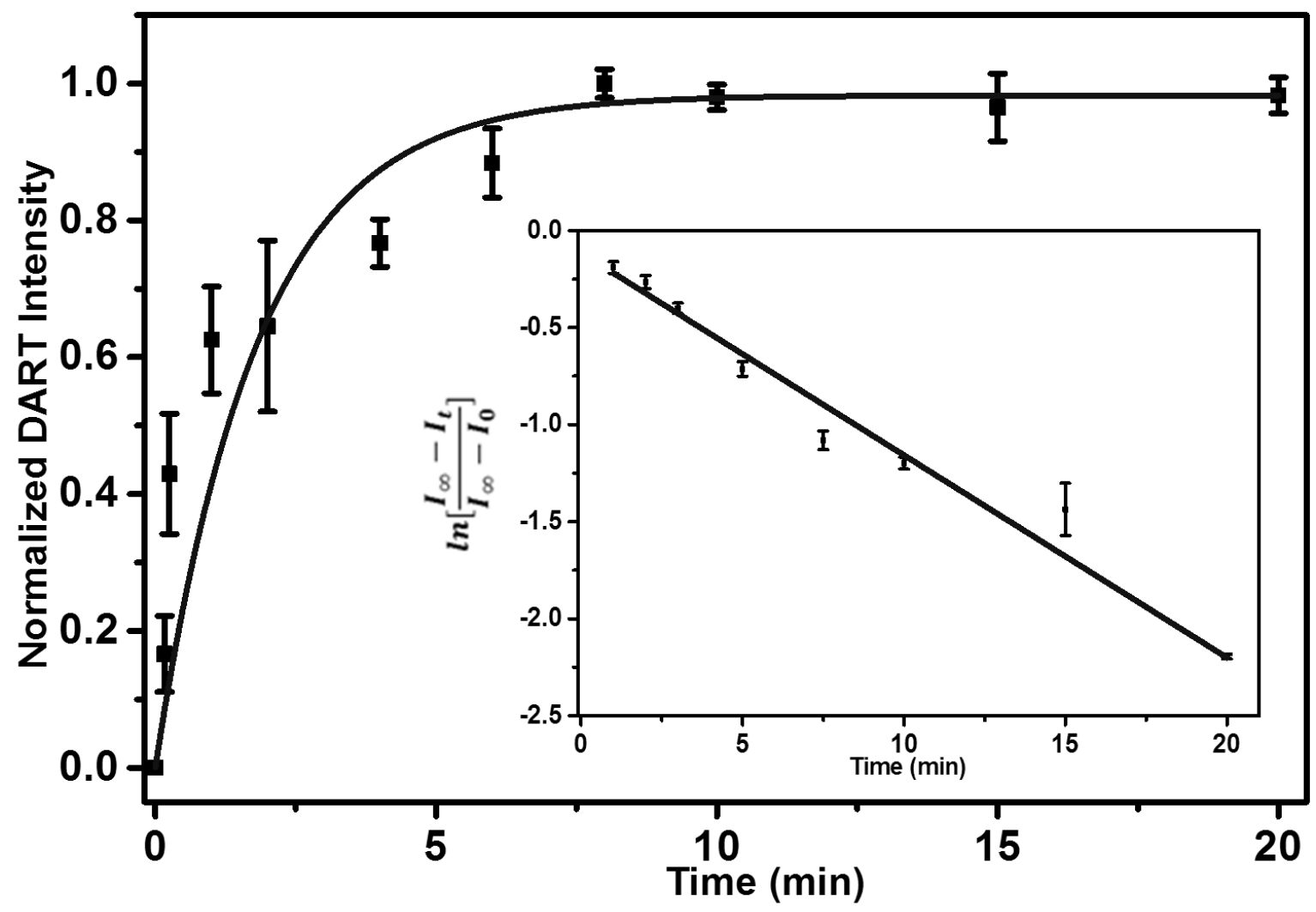

Figure S3.6. Sigmoidal plot of normalized integrated DART-HRMS intensity vs time (min) for "buried" tetrazine-terminated surface $\left(\mathbf{M}_{\mathbf{4}}\right)$ reacting with exonorbornene tag, 4 in DCE solution at $30{ }^{\circ} \mathrm{C}$. Inset: Linear plot of $\ln \mid\left(I_{\infty}-I_{t}\right) /\left(I_{\infty}-\right.$ $\left.I_{0}\right) \mid$ vs time (min), the slope of which is the pseudo-first order reaction rate $\left(\mathrm{k}^{\prime}\right)$ obtained by a least-squares fit $\left(\mathrm{R}^{2}>0.95\right)$. (Each time point is an average of hexaplet samples.) The second-order rate constant is derived from here using the concentration of the other agent $(3.0 \mathrm{mM})$. 


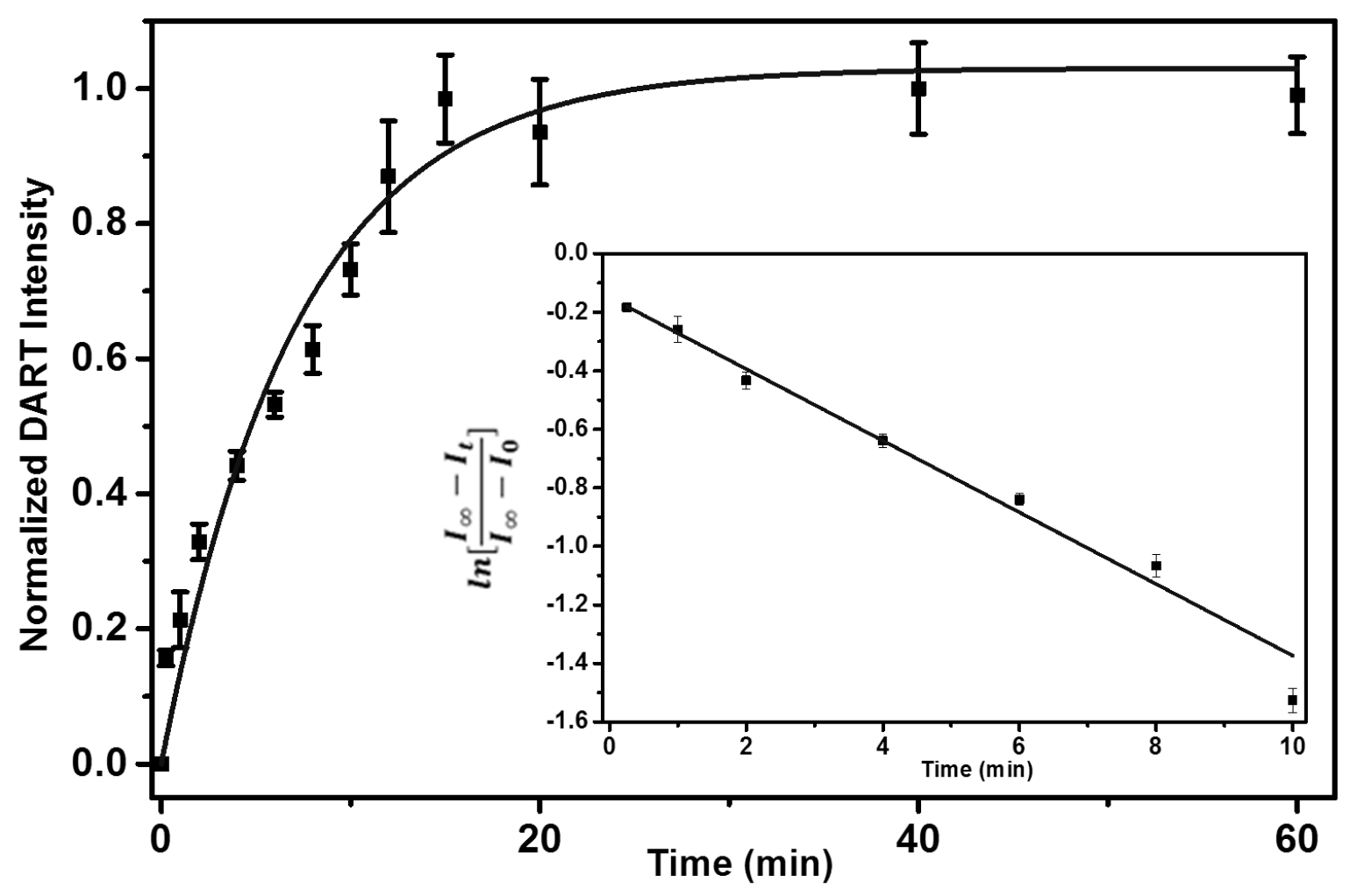

Figure S3.7. Sigmoidal plot of normalized integrated DART-HRMS intensity vs time (min) for "free" tetrazine-terminated surface $\left(\mathbf{M}_{4}\right)$ reacting with endonorbornene tag, 6 in DCE solution at $30{ }^{\circ} \mathrm{C}$. Inset: Linear plot of $\ln \mid\left(I_{\infty}-I_{t}\right) /\left(I_{\infty}-\right.$ $\left.I_{0}\right) \mid$ vs time (min), the slope of which is the pseudo-first order reaction rate $\left(\mathrm{k}^{\prime}\right)$ obtained by a least-squares fit $\left(\mathrm{R}^{2}>0.95\right)$. (Each time point is an average of hexaplet samples.) The second-order rate constant is derived from here using the concentration of the other agent $(3.0 \mathrm{mM})$. 


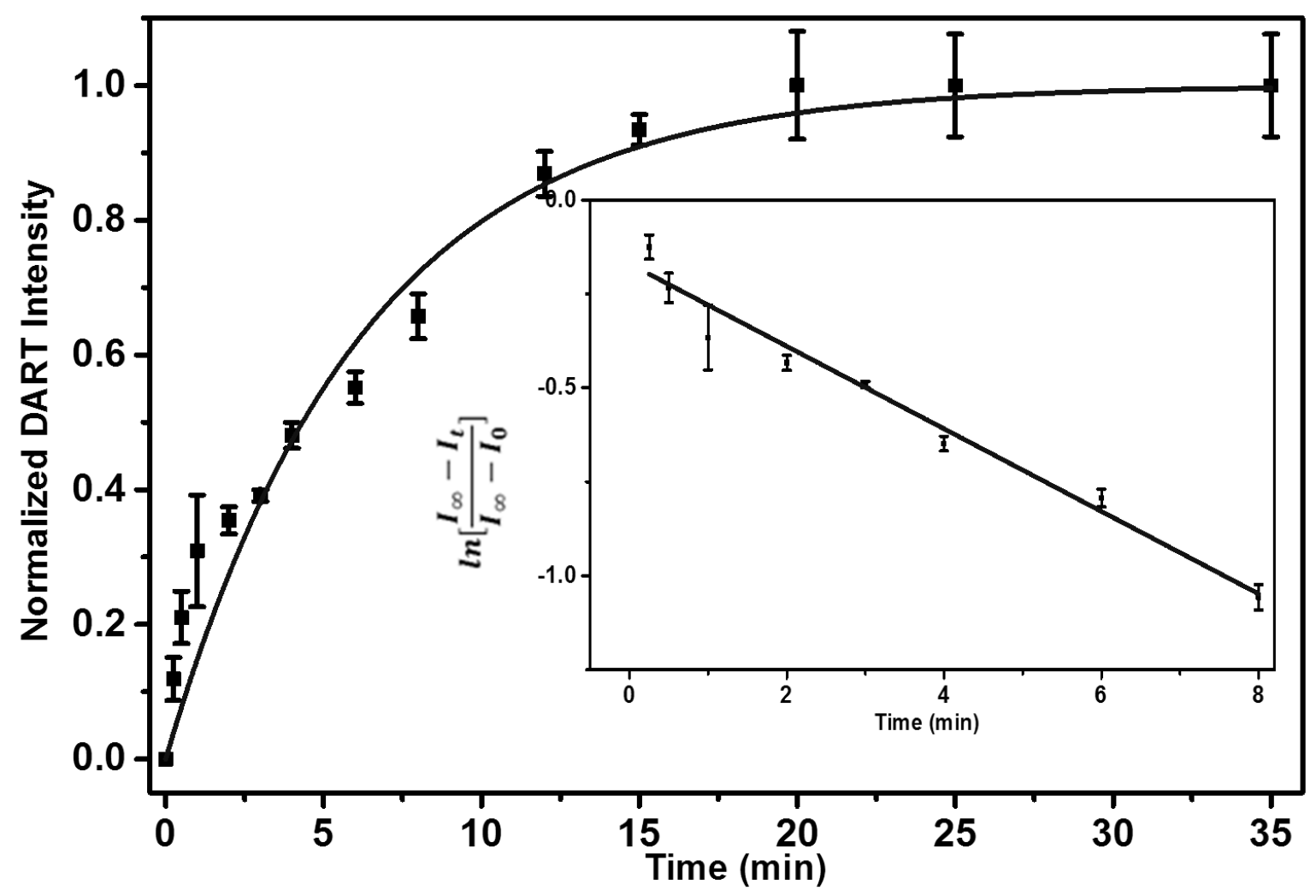

Figure S3.8. Sigmoidal plot of normalized integrated DART-HRMS intensity vs time (min) for "buried" tetrazine-terminated surface $\left(\mathbf{M}_{\mathbf{4}}\right)$ reacting with endonorbornene tag, 6 in DCE solution at $30{ }^{\circ} \mathrm{C}$. Inset: Linear plot of $\ln \mid\left(I_{\infty}-I_{t}\right) /\left(I_{\infty}-\right.$ $\left.I_{0}\right) \mid$ vs time (min), the slope of which is the pseudo-first order reaction rate $\left(\mathrm{k}^{\prime}\right)$ obtained by a least-squares fit $\left(\mathrm{R}^{2}>0.95\right)$. (Each time point is an average of hexaplet samples.) The second-order rate constant is derived from here using the concentration of the other agent $(3.0 \mathrm{mM})$. 


\section{Supplementary Figures.}

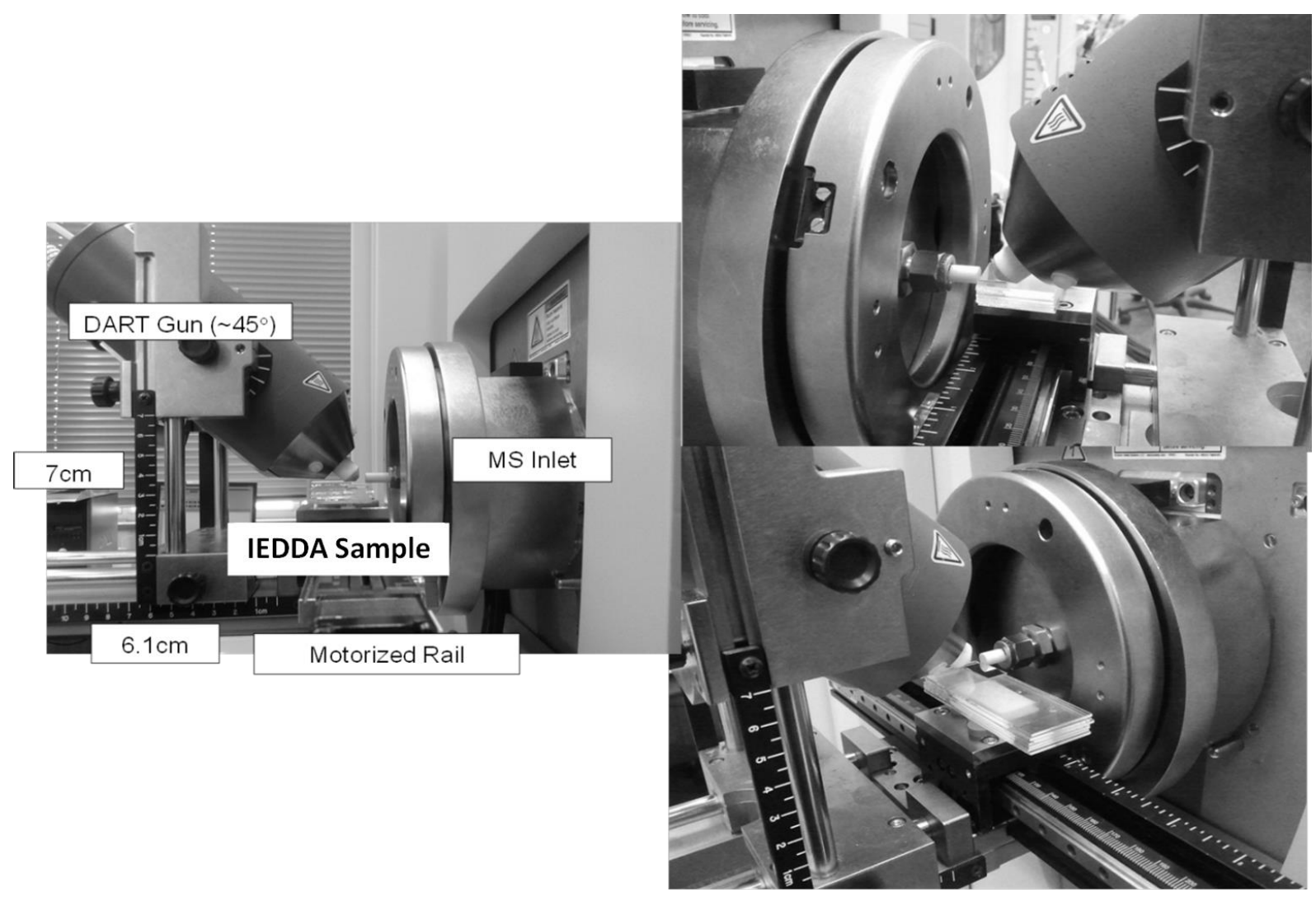

Figure S4.1. Pictures of DART setup for the kinetic analysis of organic surface reactions.

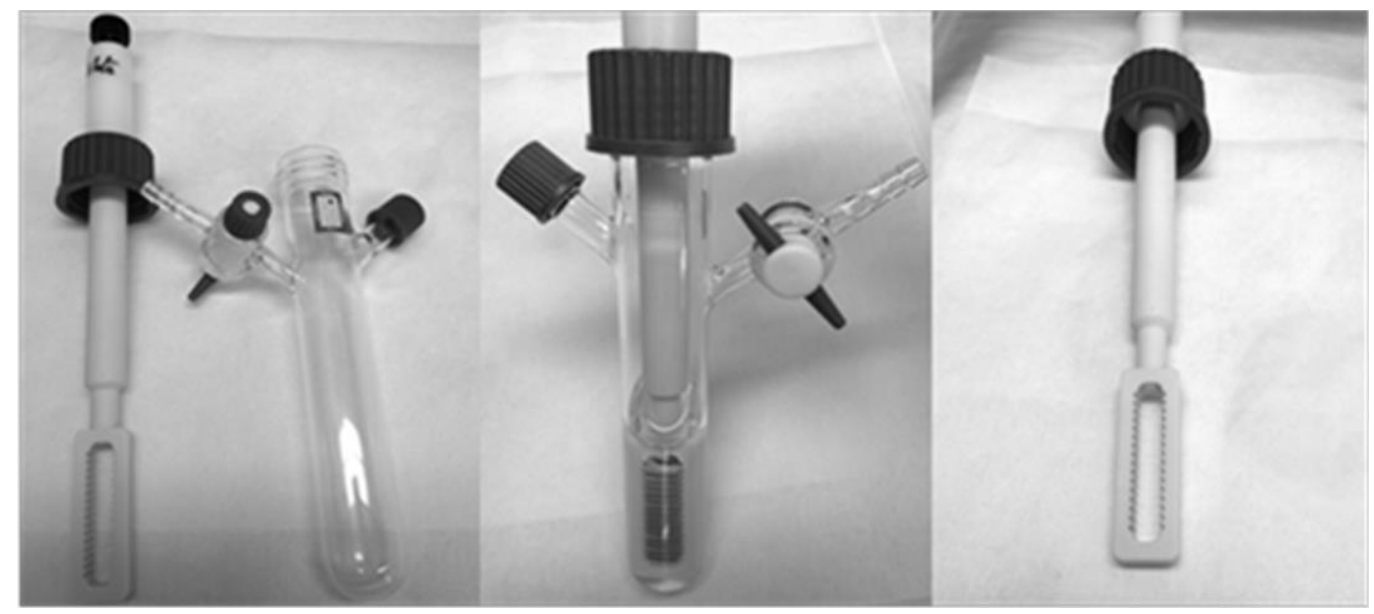

Figure S4.2. Image of the sample holder (16 slots). 


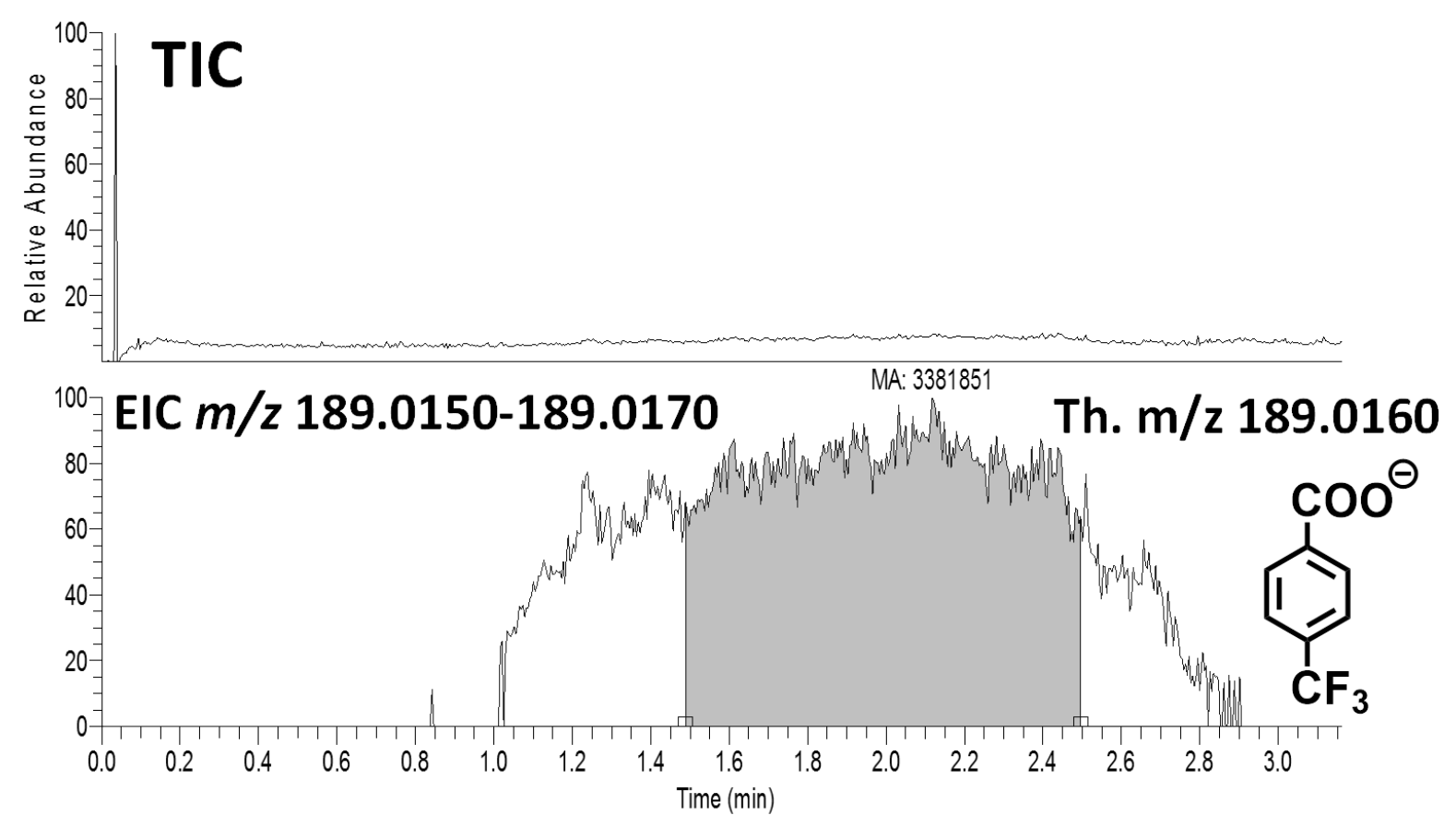

Figure S4.3. Representative spectra of a typical DART-HRMS measurement. Top: Total Ion Current (TIC); bottom: Extracted ion chromatogram (EIC) of $10 \mathrm{mmu}$ window around the theoretical $m / z$ of the ion of interest.

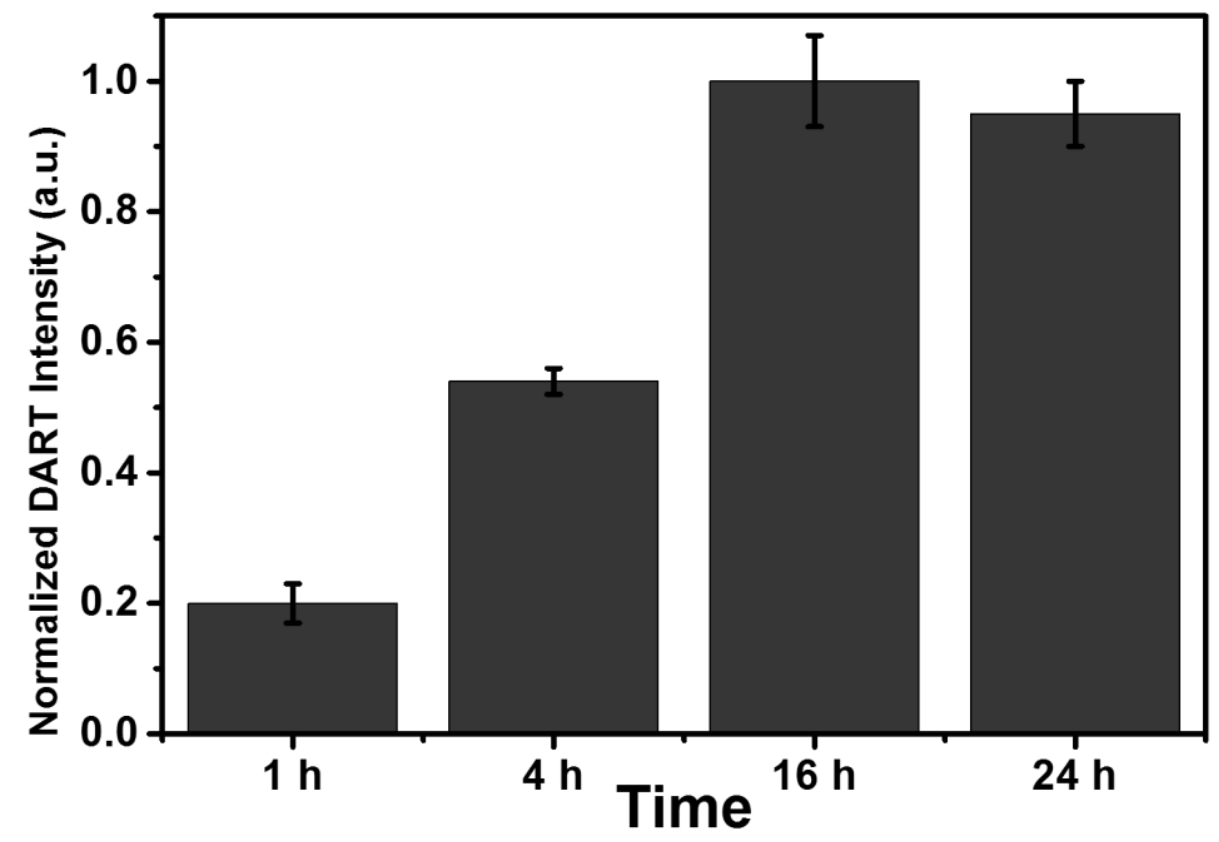

Figure S4.4. Normalized DART-HRMS intensity of MS tag with $m / z 189.0163$ after IEDDA reaction (for $20 \mathrm{~min}$ ) on $\mathbf{M}_{2}$ surfaces. The CDI-activated $\mathbf{M}_{1}$ samples were stirred in a $50 \mathrm{mM}$ solution of exo-norbornene- $\mathrm{OH}$ for different times followed by stirring in a $3.0 \mathrm{mM}$ DCE solution of $\mathbf{3}$ and analyzed by DART for MS tag. 


\section{Supplementary XPS Spectra}

Note: all statistics (average, standard deviation) of the XPS data are derived from 6 independent samples in each case.

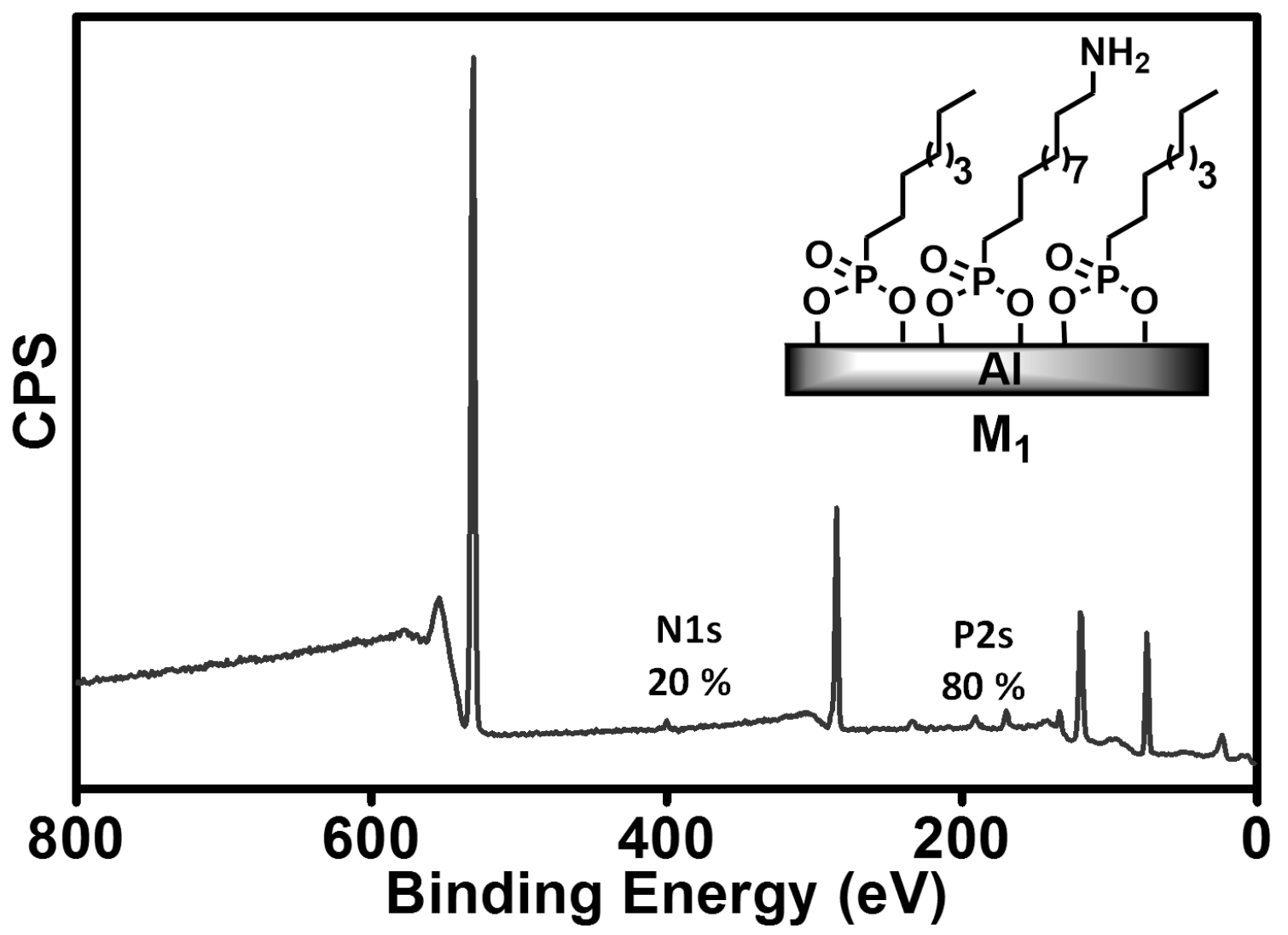

Figure S5.1. XPS wide scan of $\mathbf{M}_{1}$ modified Al surface for "free" microenvironment.

Theoretical $\mathrm{N} / \mathrm{P}$ ratio $=1 / 4=0.25$

Experimental $\mathrm{N} / \mathrm{P}=0.25 \pm 0.02$

$\Rightarrow 3: 1$ alkyl-amino terminated monolayer indeed obtained. 


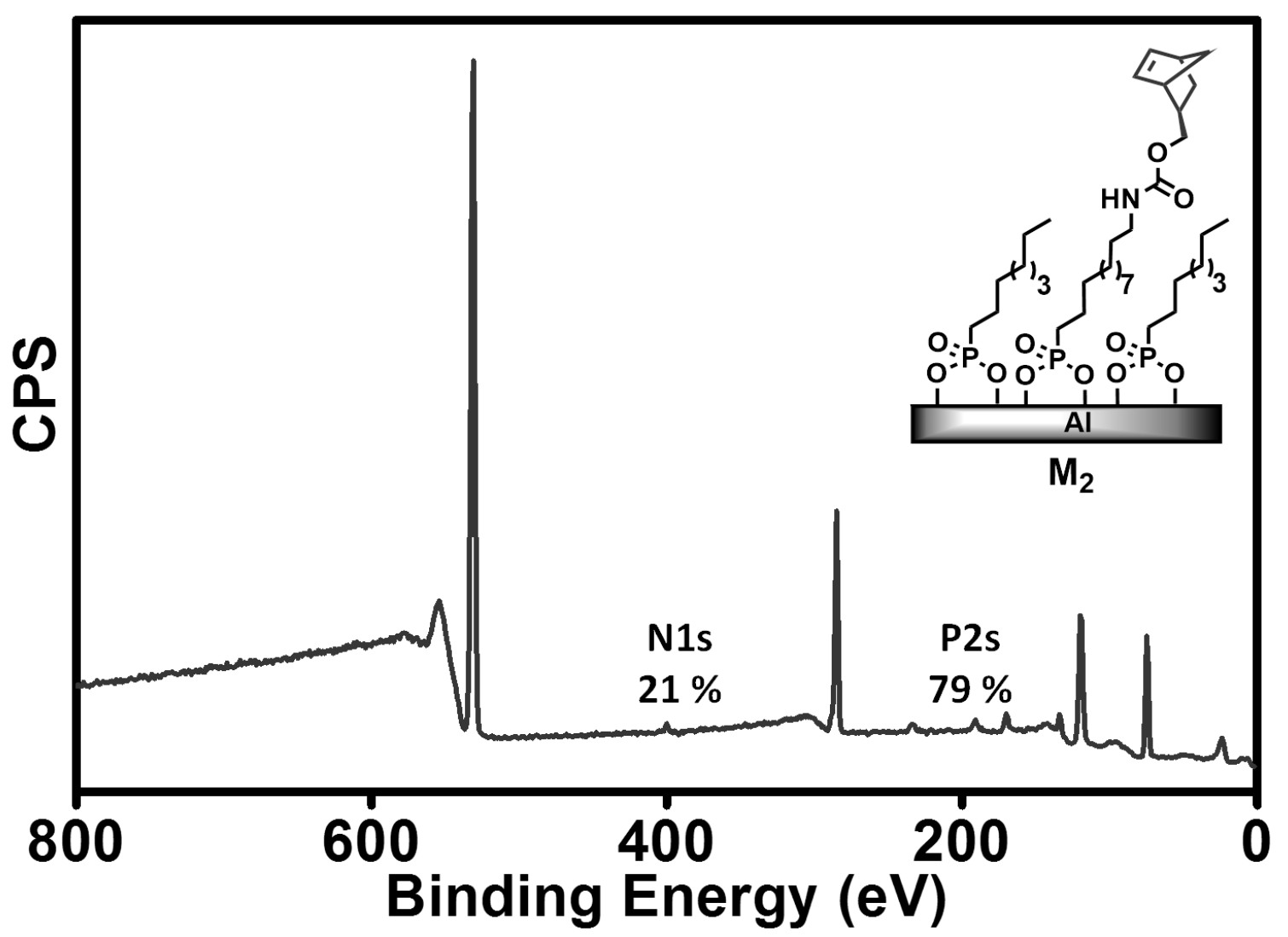

Figure S5.2. XPS wide scan of $\mathbf{M}_{2}$ modified Al surface for "free" microenvironment.

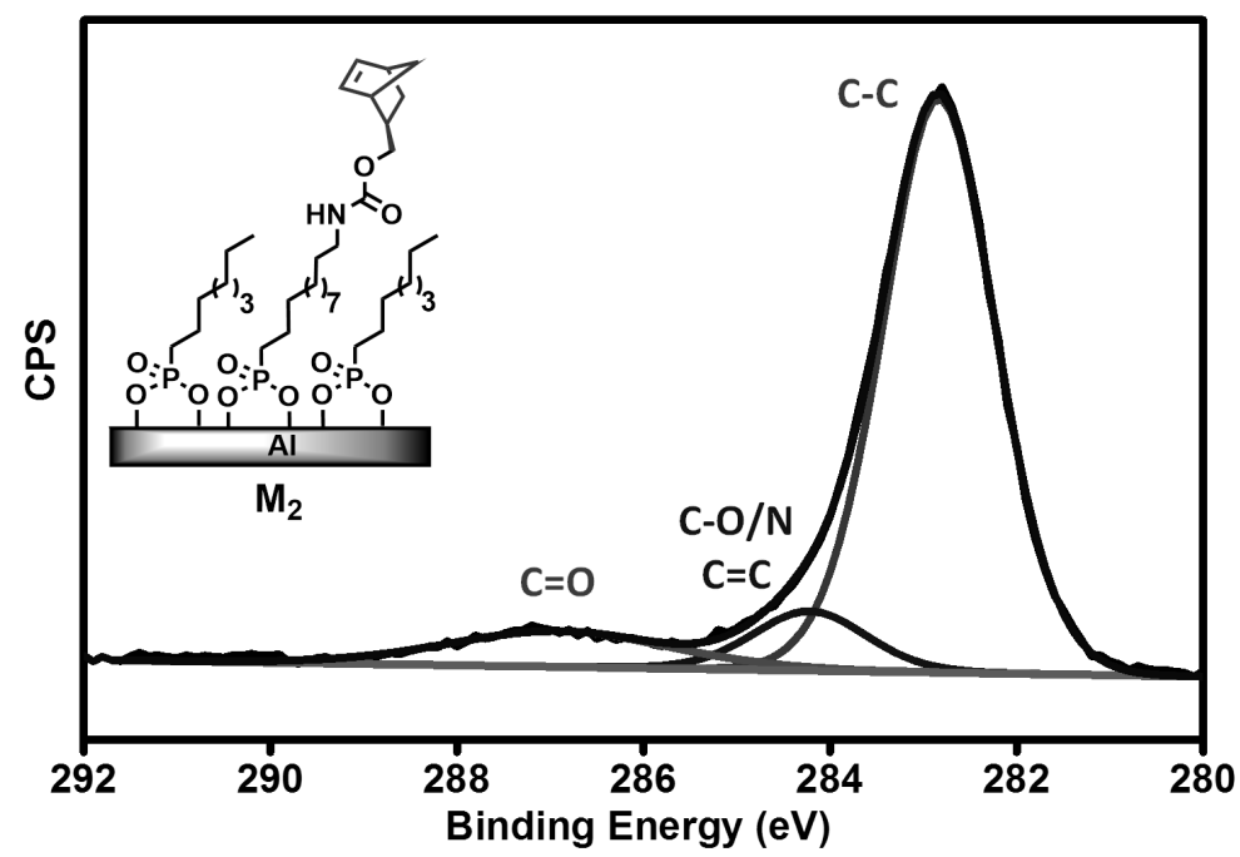

Figure S5.3. XPS C1s narrow scan of $\mathbf{M}_{2}$ surface showing clear carbamate peak at $287.0 \mathrm{eV}$. 


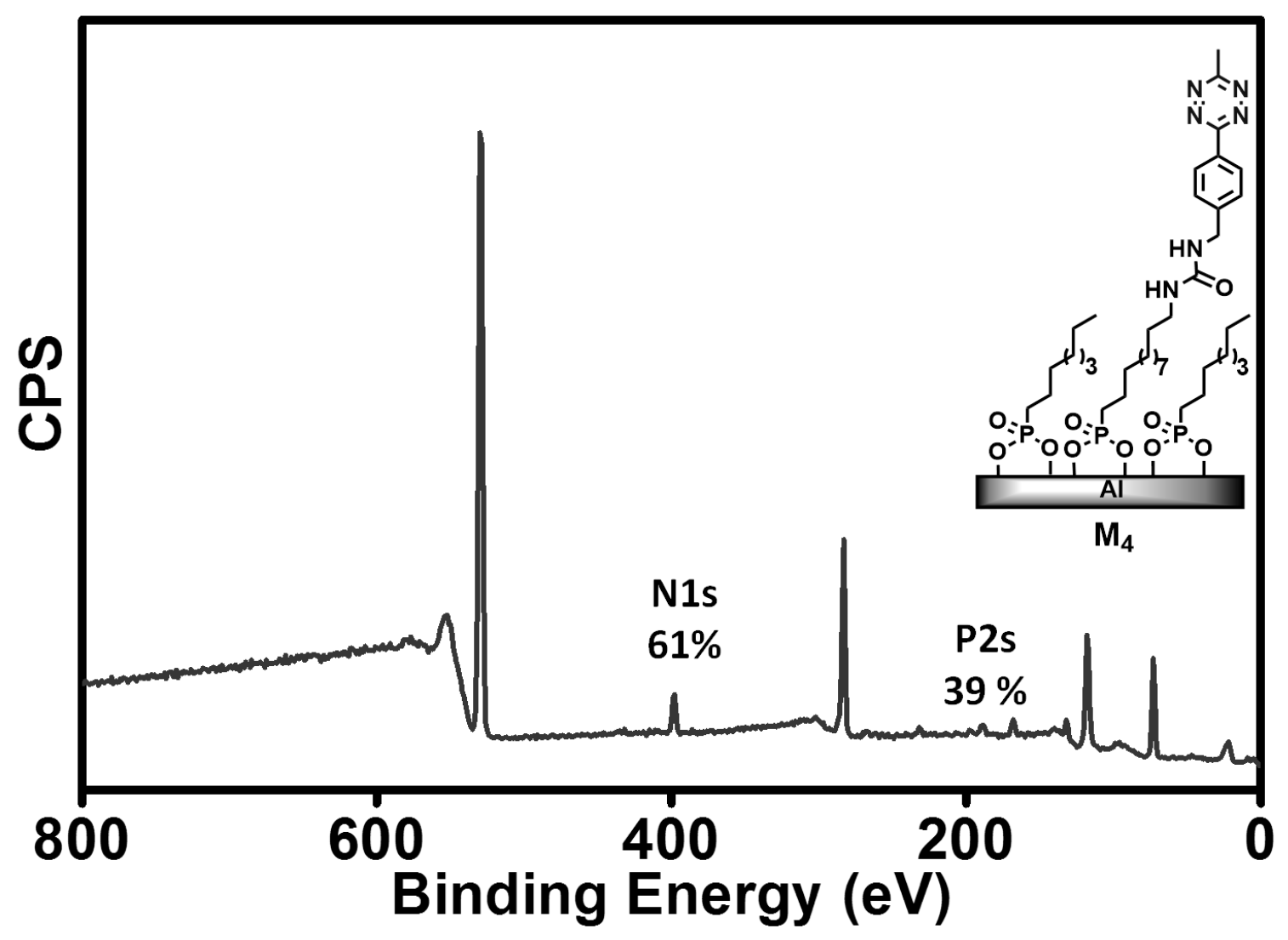

Figure S5.4. XPS wide scan of $\mathbf{M}_{4}$ surface for "free" microenvironment.

Th. $\mathrm{N} / \mathrm{P}=6 / 4=1.50$

Expt. N/P $=1.5 \pm 0.1$

Surface yield $=100 \pm 6 \%$ 


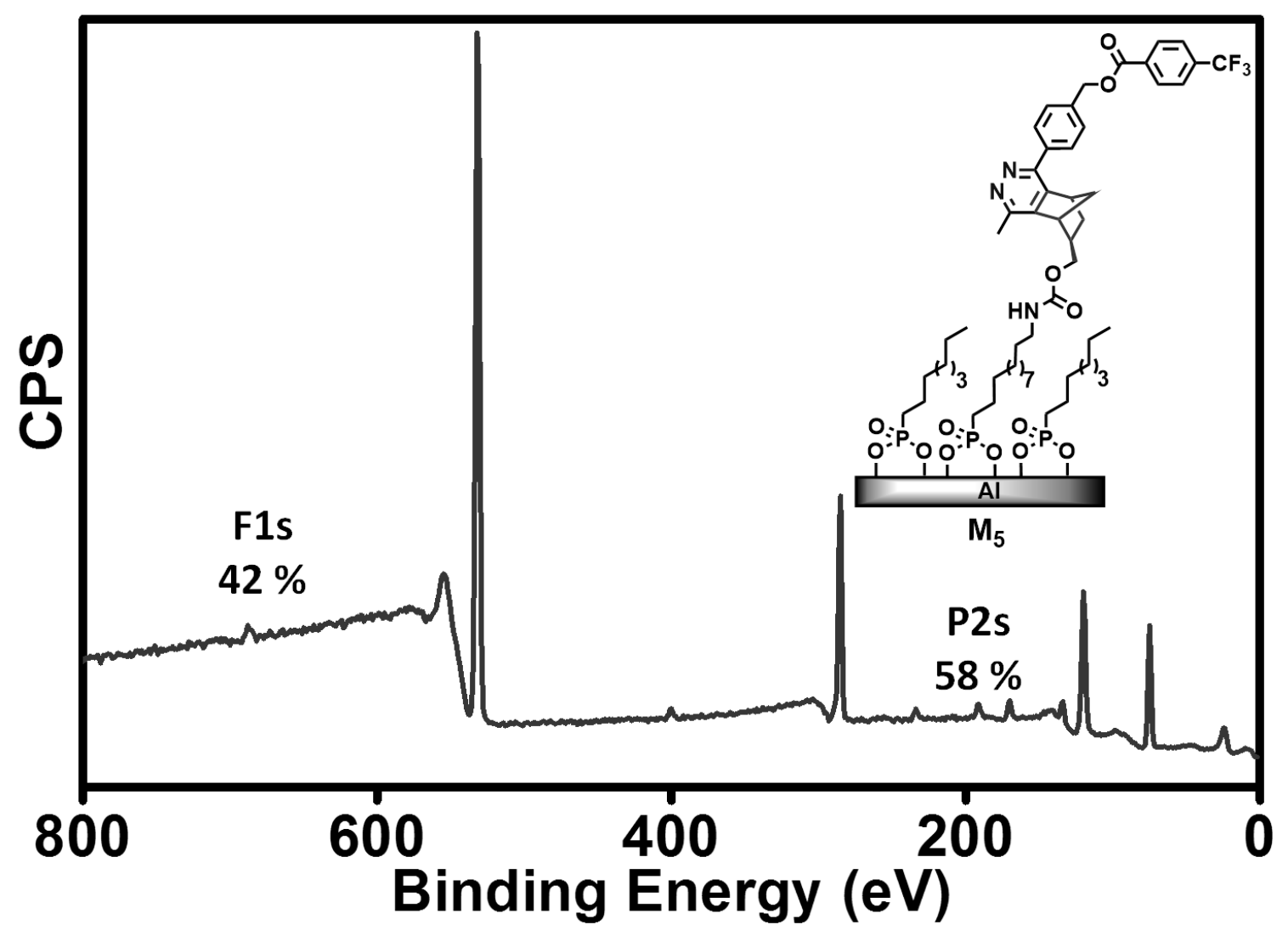

Figure S5.5. Wide scan of IEDDA cycloaddition products $\left(\mathbf{M}_{\mathbf{5}}\right)$ between exonorbornene-terminated surface and tetrazine tag molecule, $\mathbf{3}$ for "free" microenvironment.

Th. $\mathrm{F} / \mathrm{P}=3 / 4=0.75$

Expt. $\mathrm{F} / \mathrm{P}=0.76 \pm 0.02$

Surface yield $=$ Quantitative 


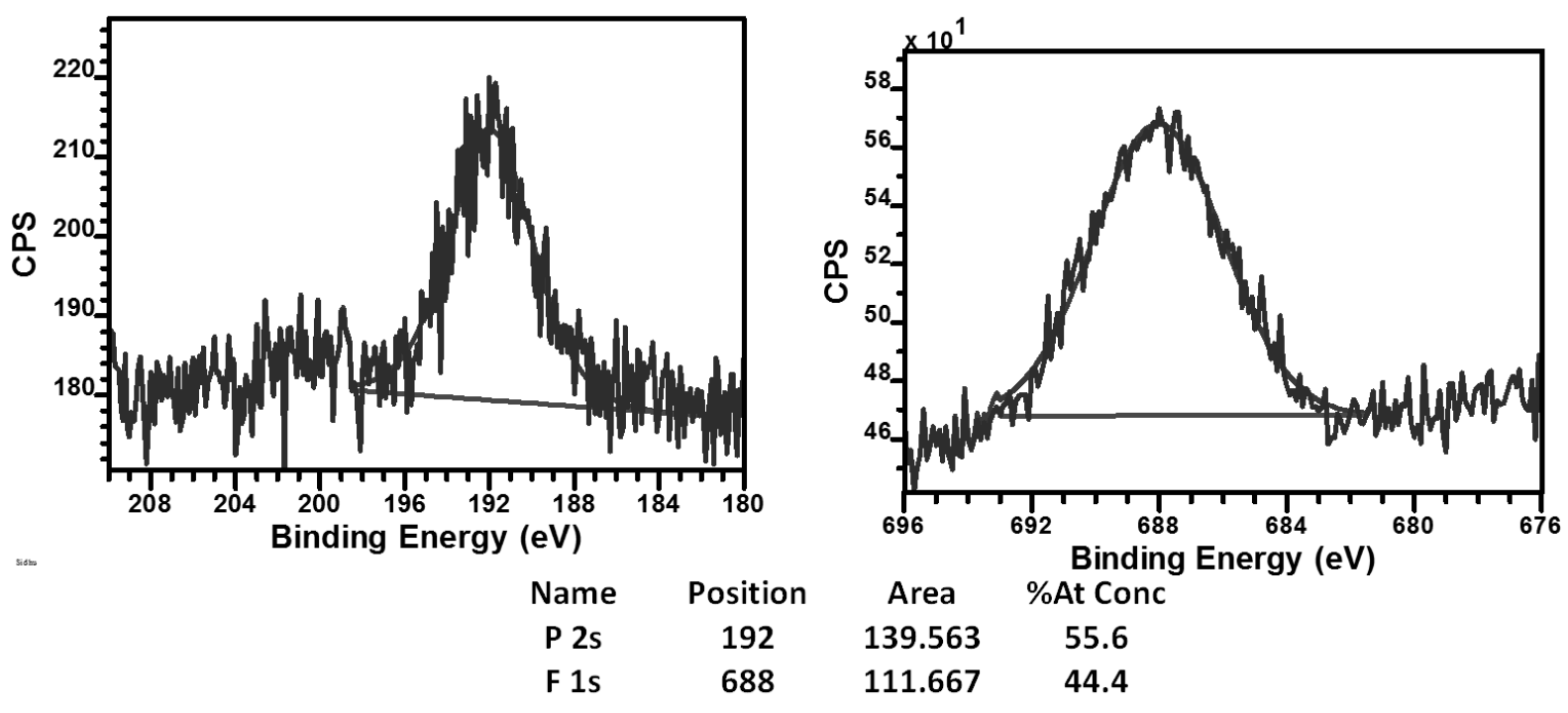

Figure S5.6. F1s and P2s narrow scans of IEDDA cycloaddition products $\left(\mathbf{M}_{5}\right)$ between endo-norbornene-terminated surface and tetrazine tag molecule, $\mathbf{3}$ for "free" microenvironment.

Th. $\mathrm{F} / \mathrm{P}=3 / 4=0.75$

Expt. $\mathrm{F} / \mathrm{P}=0.77 \pm 0.02$

Surface yield $=$ Quantitative 


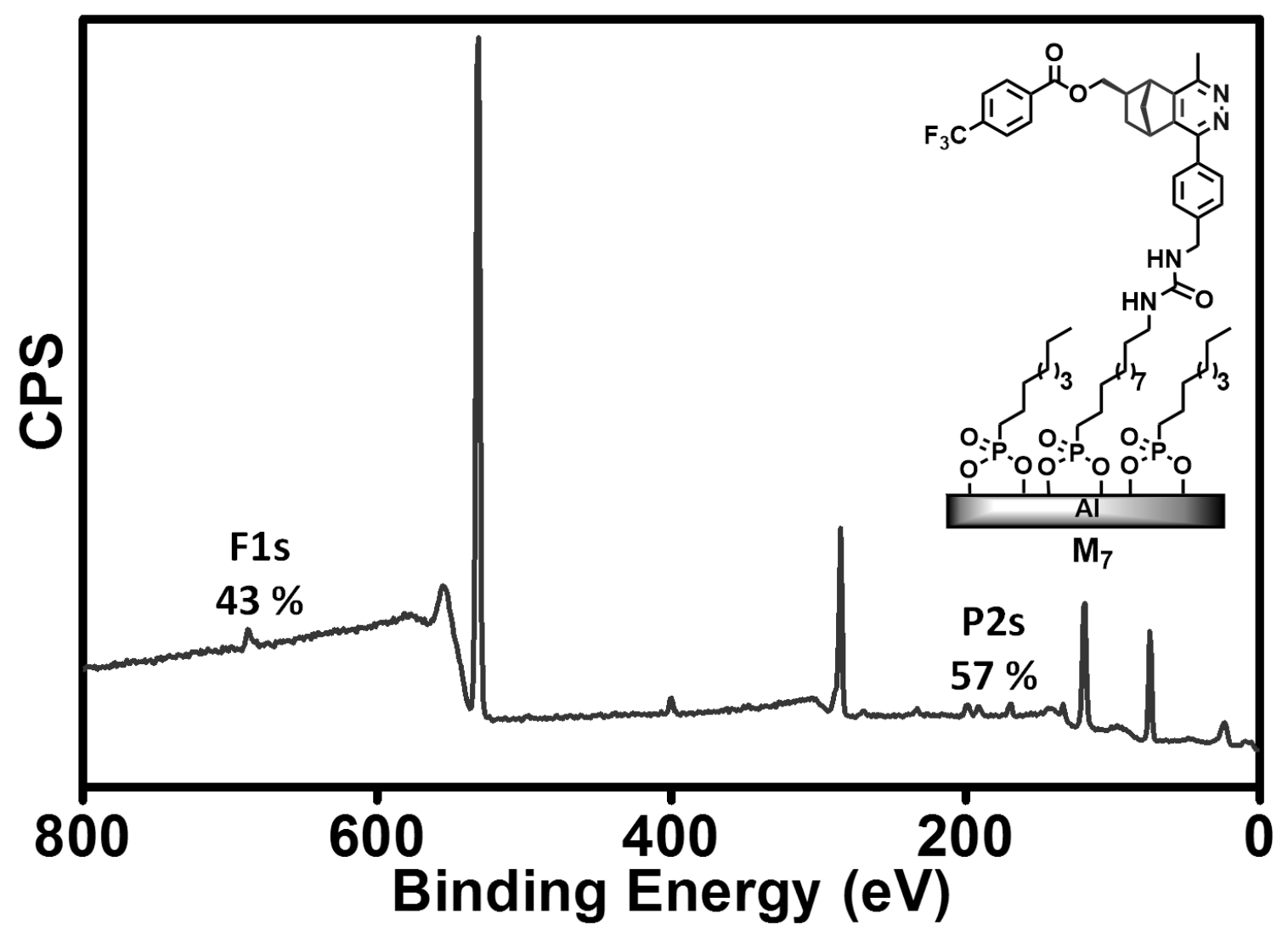

Figure S5.7. Wide scan of IEDDA cycloaddition products $\left(\mathbf{M}_{7}\right)$ between tetrazine $_{(\text {surf.) }}$ and exo-norbornene tag molecule, 4.

Th. $\mathrm{F} / \mathrm{P}=3 / 4=0.75$

Expt. $\mathrm{F} / \mathrm{P}=0.75 \pm 0.02$

Surface yield = Quantitative 


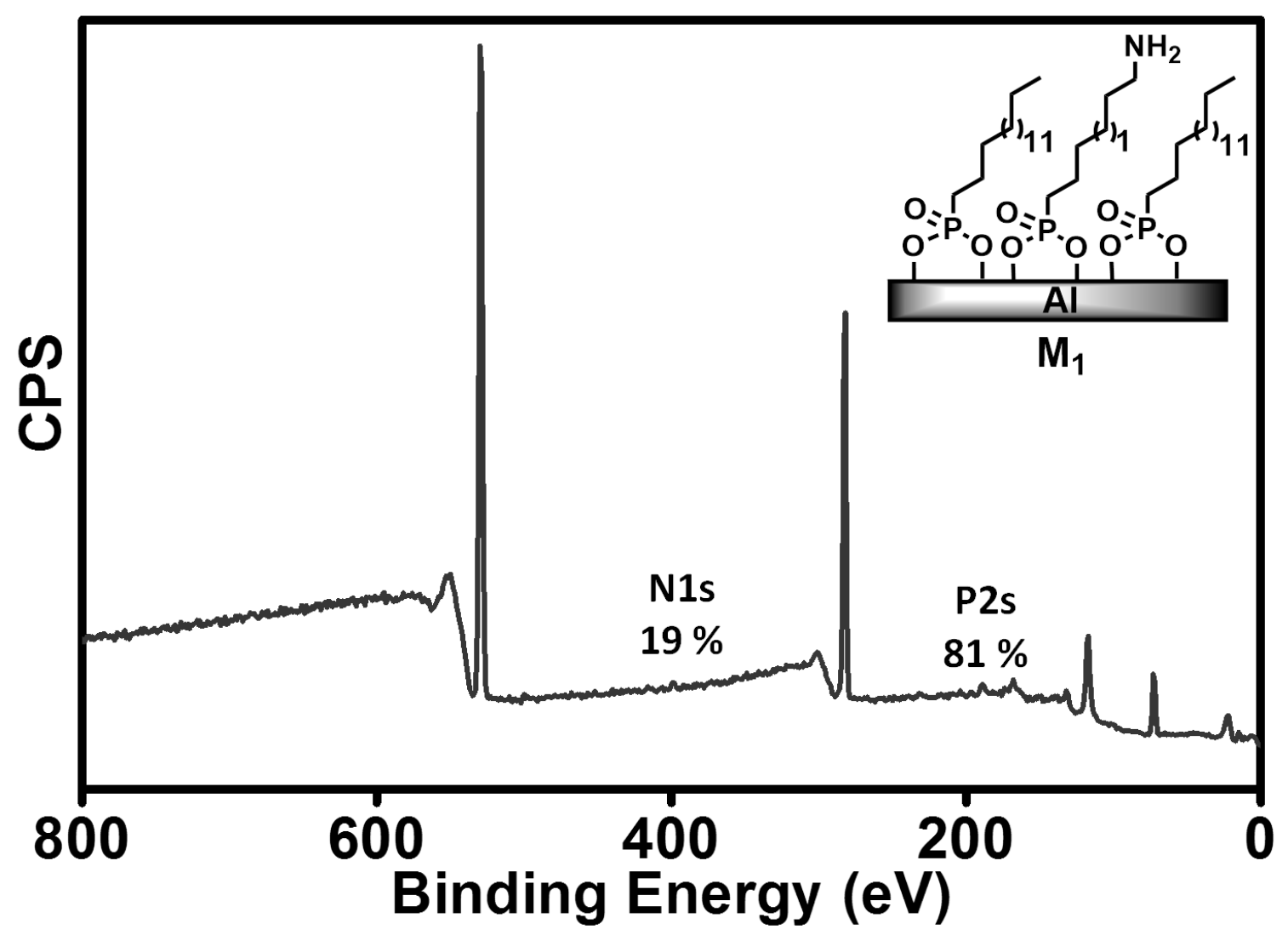

Figure S5.8. XPS wide scan of $\mathbf{M}_{1}-$ modified Al surface for "buried" microenvironment.

Theoretical $\mathrm{N} / \mathrm{P}$ ratio $=1 / 4=0.25$

Experimental $\mathrm{N} / \mathrm{P}=0.26 \pm 0.02$ 


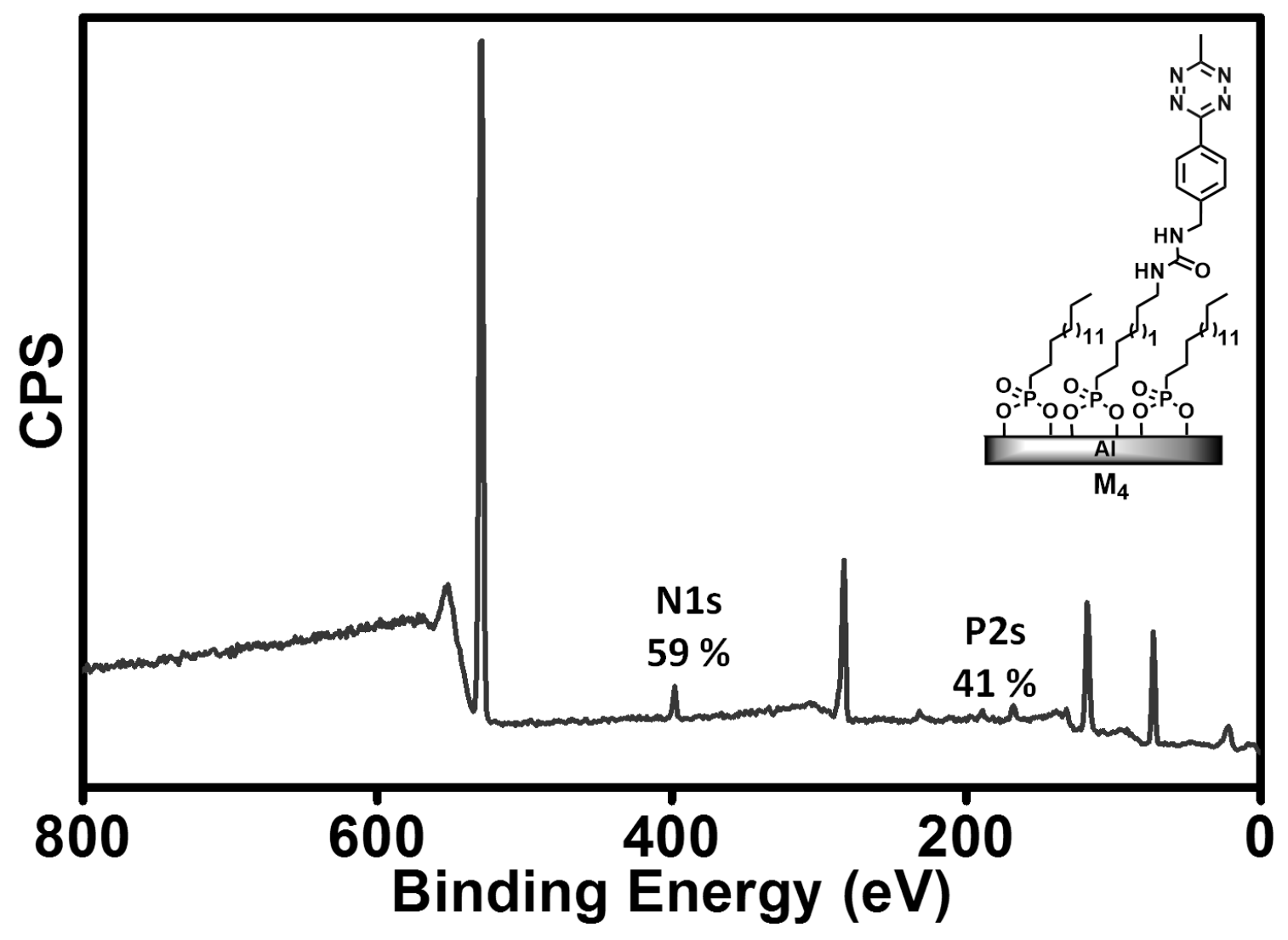

Figure S5.9. XPS wide scan of $\mathbf{M}_{4}$ surface for "buried" microenvironment.

Th. $\mathrm{N} / \mathrm{P}=6 / 4=1.50$

Expt. N/P $=1.4 \pm 0.1$

Surface yield $=95 \pm 5 \%$ 


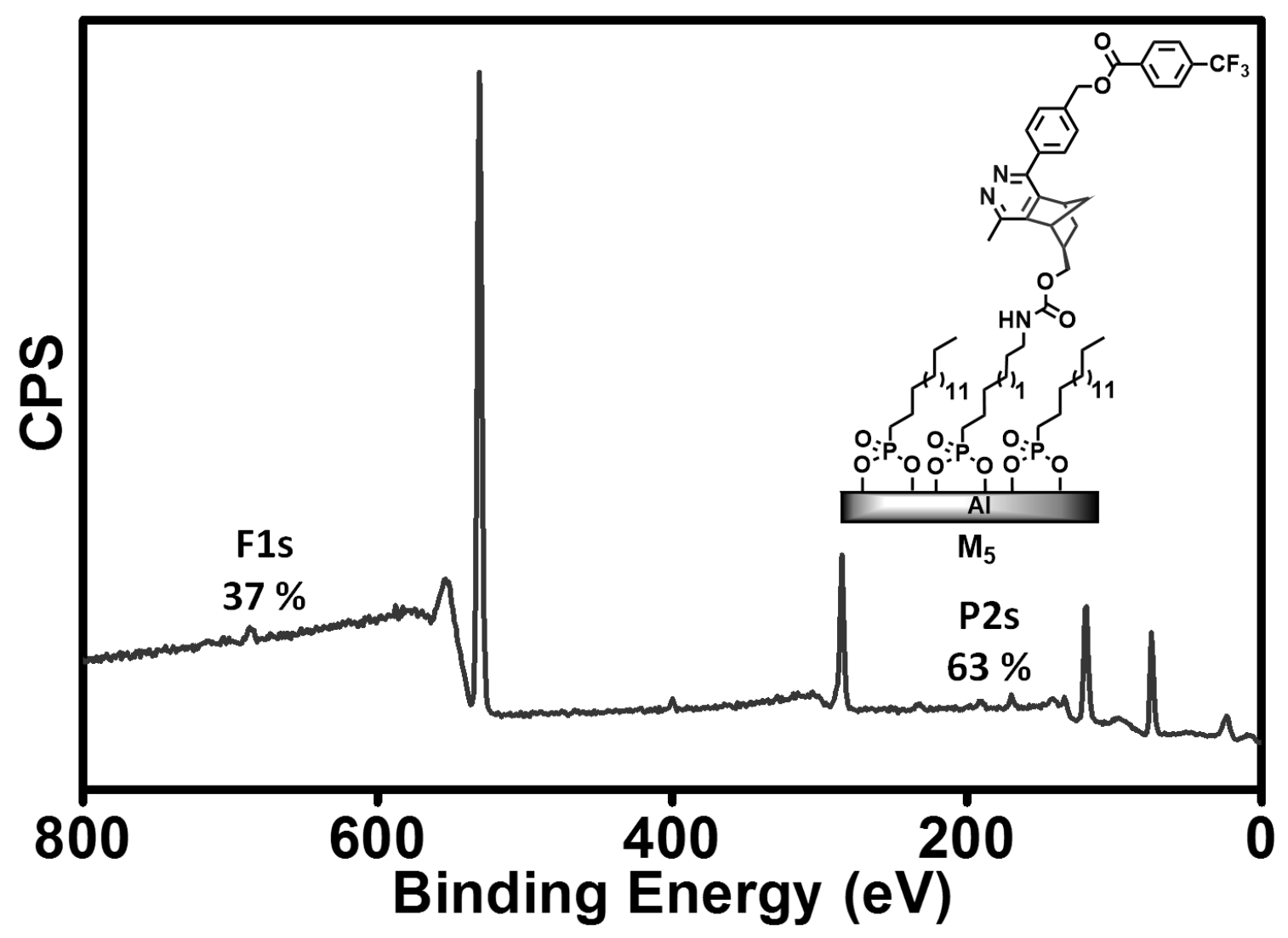

Figure S5.10. Wide scan of IEDDA cycloaddition products $\left(\mathbf{M}_{5}\right)$ between exonorbornene $_{\text {(surf.) }}$ and tetrazine tag molecule, 3 for "buried" microenvironment after 15 min reaction.

Th. $\mathrm{F} / \mathrm{P}=3 / 4=0.75$

Expt. $\mathrm{F} / \mathrm{P}=0.59 \pm 0.02$

Surface yield $=80 \pm 5 \%$ 


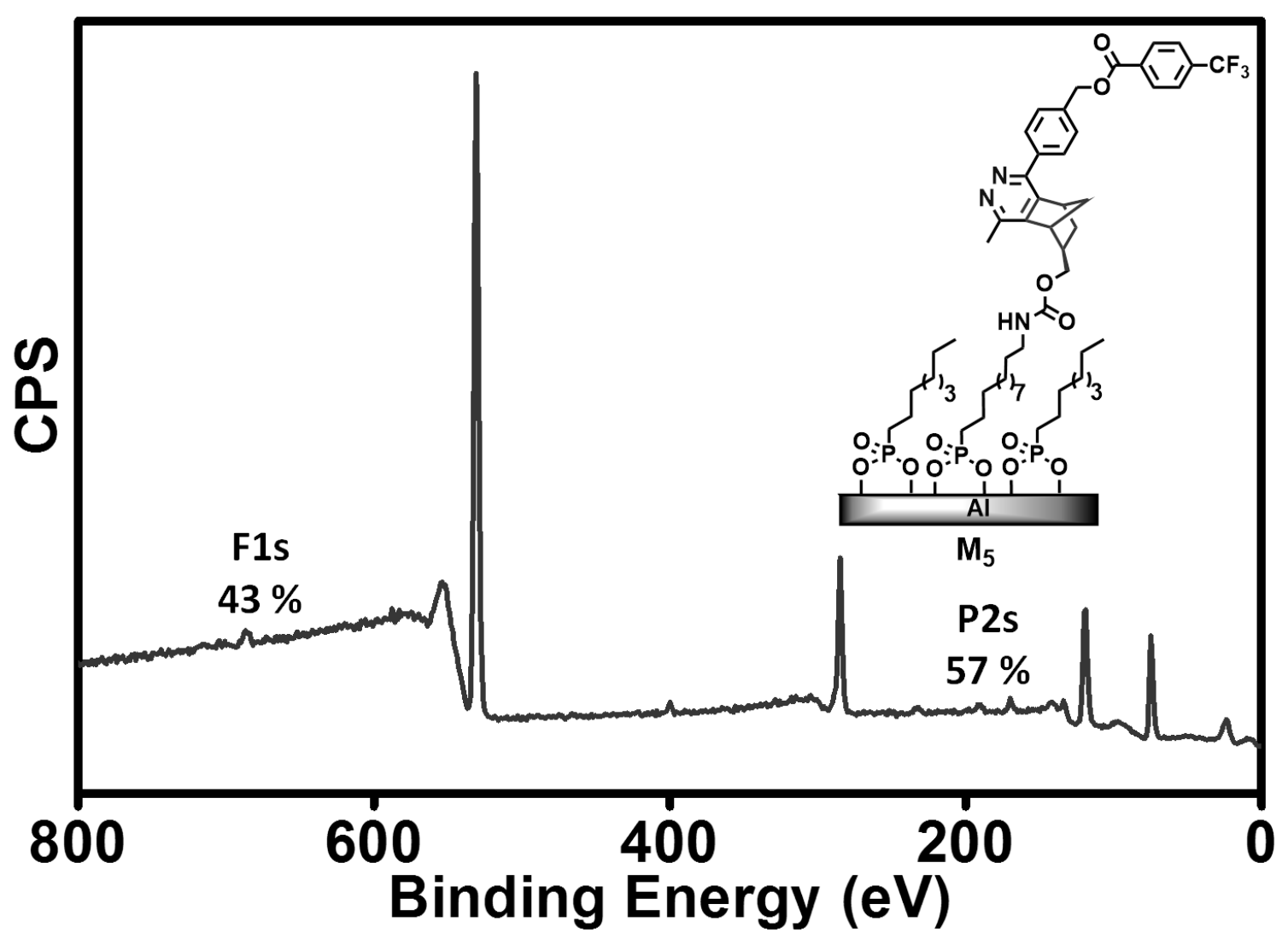

Figure S5.11. Wide scan of IEDDA cycloaddition products $\left(\mathbf{M}_{5}\right)$ between exonorbornene $_{\text {(surf.) }}$ and tetrazine tag molecule, 3 for "buried" microenvironment after $1 \mathrm{~h}$ reaction time.

Th. $\mathrm{F} / \mathrm{P}=3 / 4=0.75$

Expt. $\mathrm{F} / \mathrm{P}=0.70 \pm 0.04$

Surface yield $=94 \pm 6 \%$ 


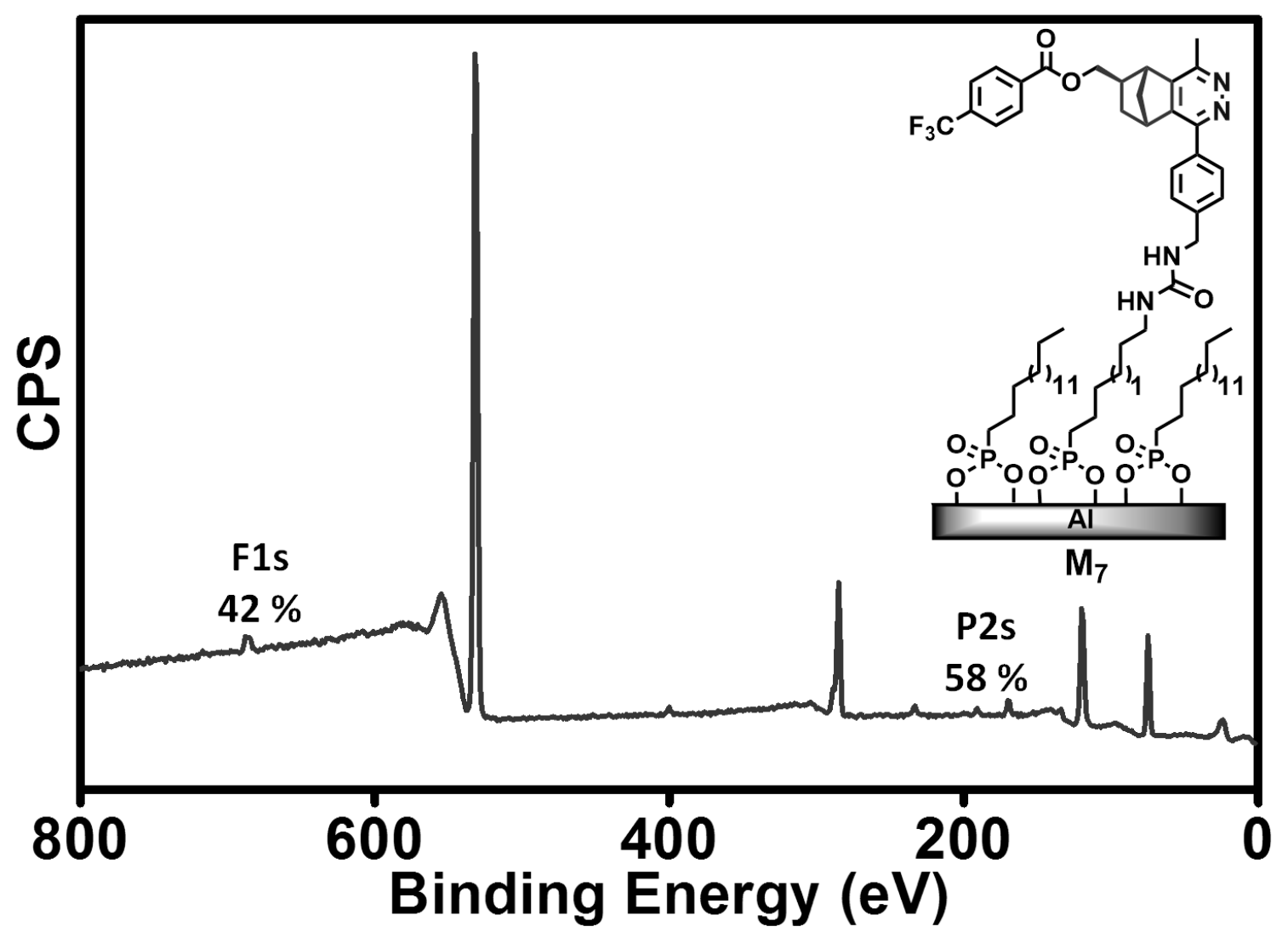

Figure S5.12. Wide scan of IEDDA cycloaddition products $\left(\mathbf{M}_{7}\right)$ between tetrazine $_{(\text {surf.) }}$ and exo-norbornene tag molecule, 4 "buried" microenvironment after 1 h.

Th. $\mathrm{F} / \mathrm{P}=3 / 4=0.75$

Expt. $\mathrm{F} / \mathrm{P}=0.64 \pm 0.07$

Surface yield $=85 \pm 10 \%$ 


\section{Supplementary Tables.}

Table S6.1. Collected kinetic plots of tetrazine-norbornene IEDDA reaction for "free" or "buried" microenvironment (all $\mathrm{R}^{2}>0.95$ ).

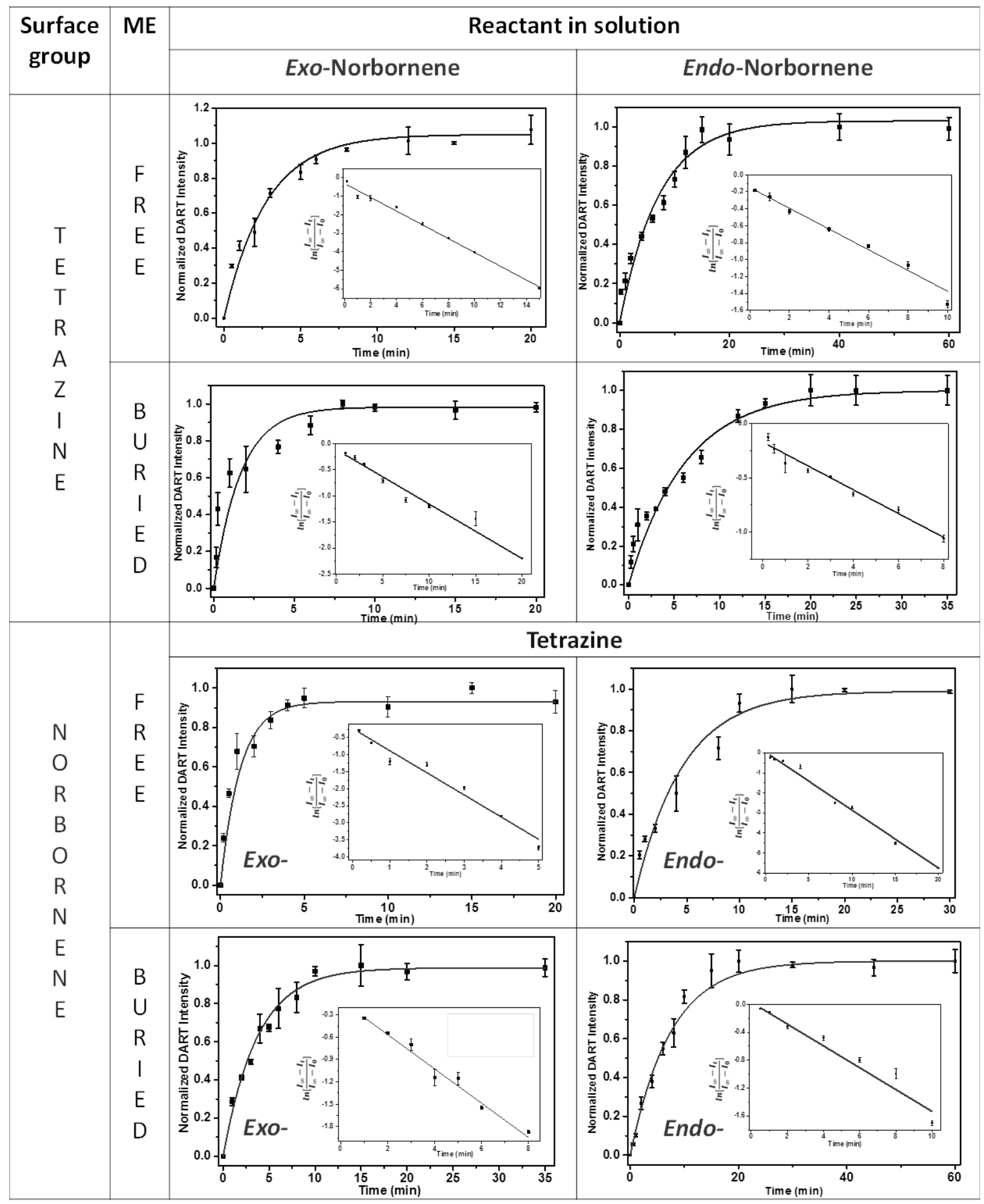




\section{Material Studio Data.}

Figure S7.1. Al oxide $\left(\mathrm{Al}_{2} \mathrm{O}_{3}\right)$ surface in bulk.

All colors used are standard throughout $($ Grey $=\mathrm{C}$, white $=\mathrm{H}$, purple $=\mathrm{P}$, pink $=$ $\mathrm{Al}$, red $=\mathrm{O}$, fluorine $=$ cyan). PLEASE SEE FULL ONLINE ESI FOR COLOR IMAGES.

[Any incomplete fragments and atoms are part of larger structures protruding from the adjacent box].

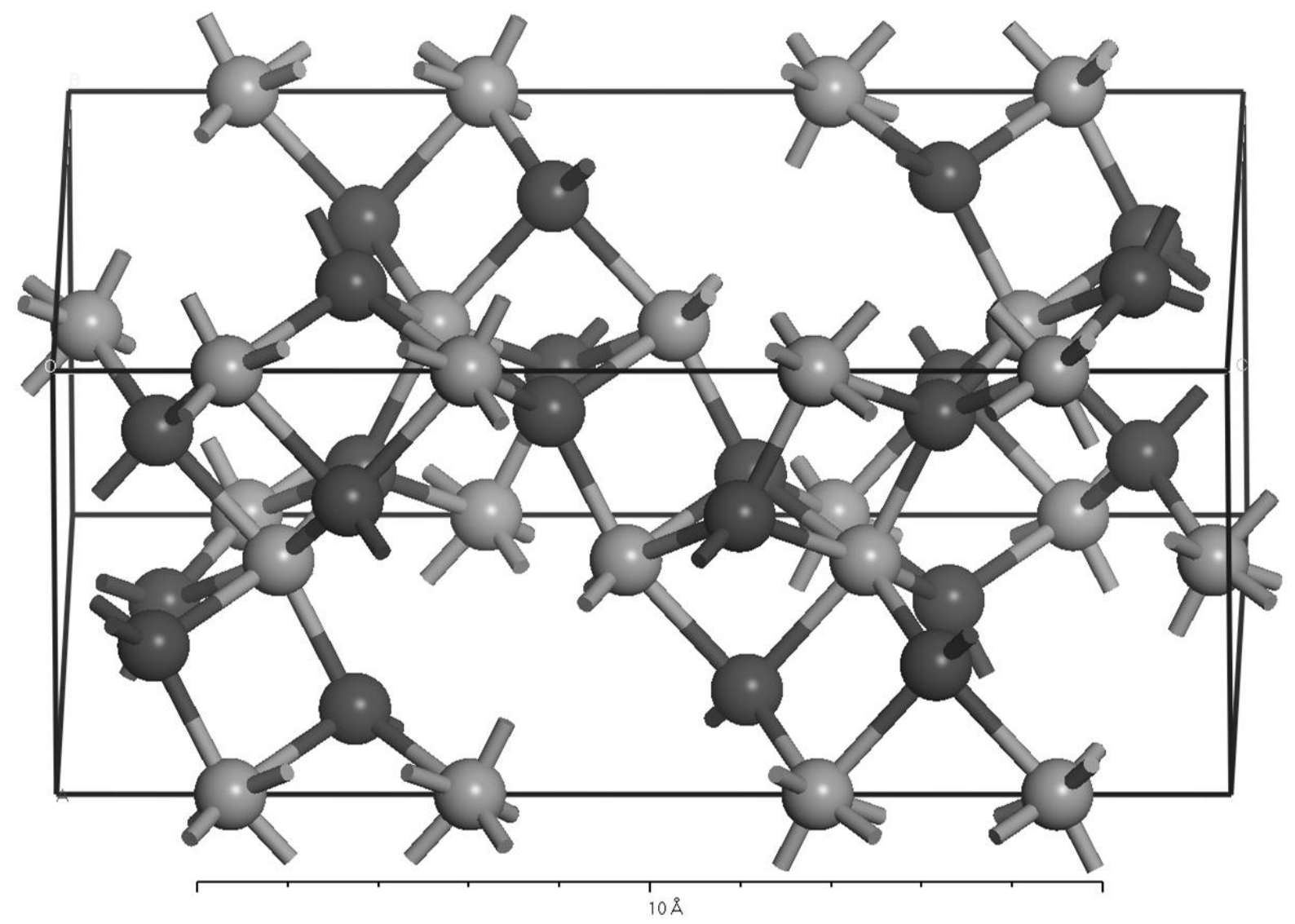

$\mathrm{Al}_{2} \mathrm{O}_{3}$ were cleaved along $(\mathrm{h} \mathrm{k} \mathrm{l})=\left(\begin{array}{lll}-1 & 0 & 0\end{array}\right)$ to build oxidic aluminum surfaces.

Thickness fractional $=1$

Thickness $(\AA)=4.121$

Cap bonds on Top $=\mathrm{O}$ ( oxygen $)$ 
Figure S7.2. Side view of cleaved $\mathrm{Al}_{2} \mathrm{O}_{3} \quad$ surface.

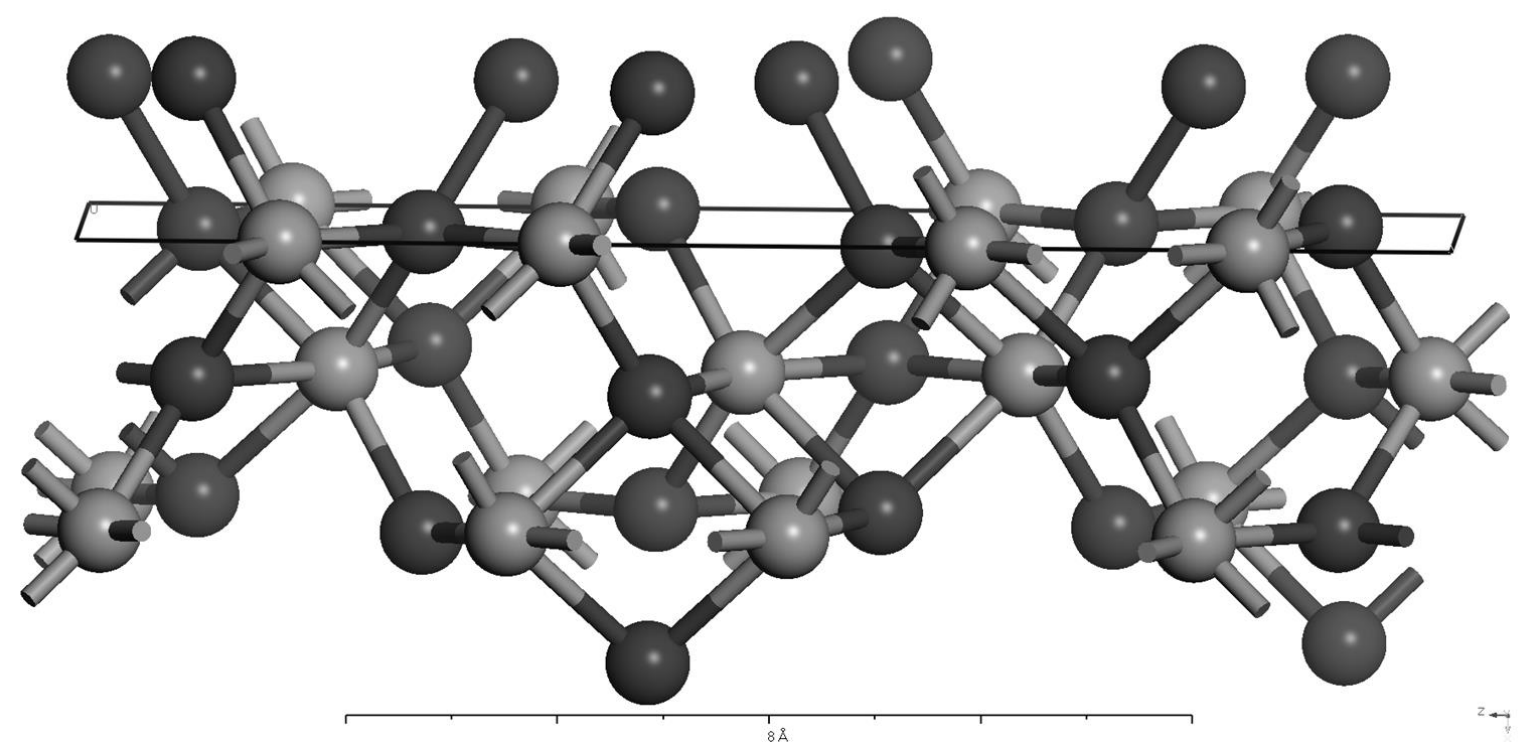

Figure S7.3. Dimensions of vacuum slab above $\mathrm{Al}_{2} \mathrm{O}_{3}$ surface for modelling alkyl groups and later IEDDA fragments.

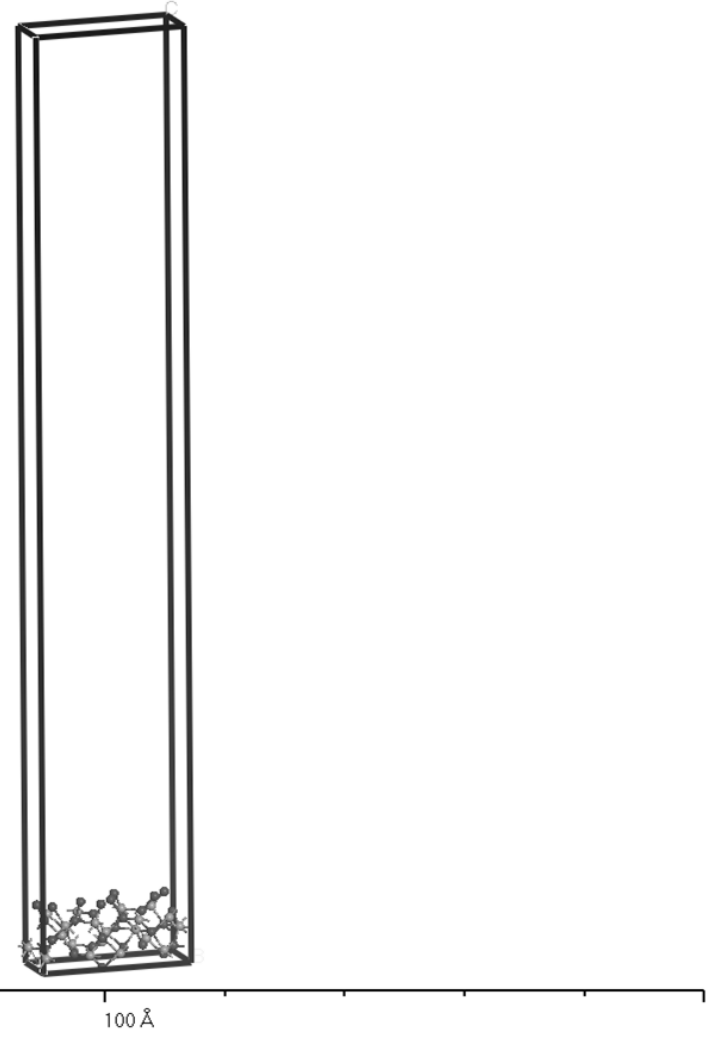




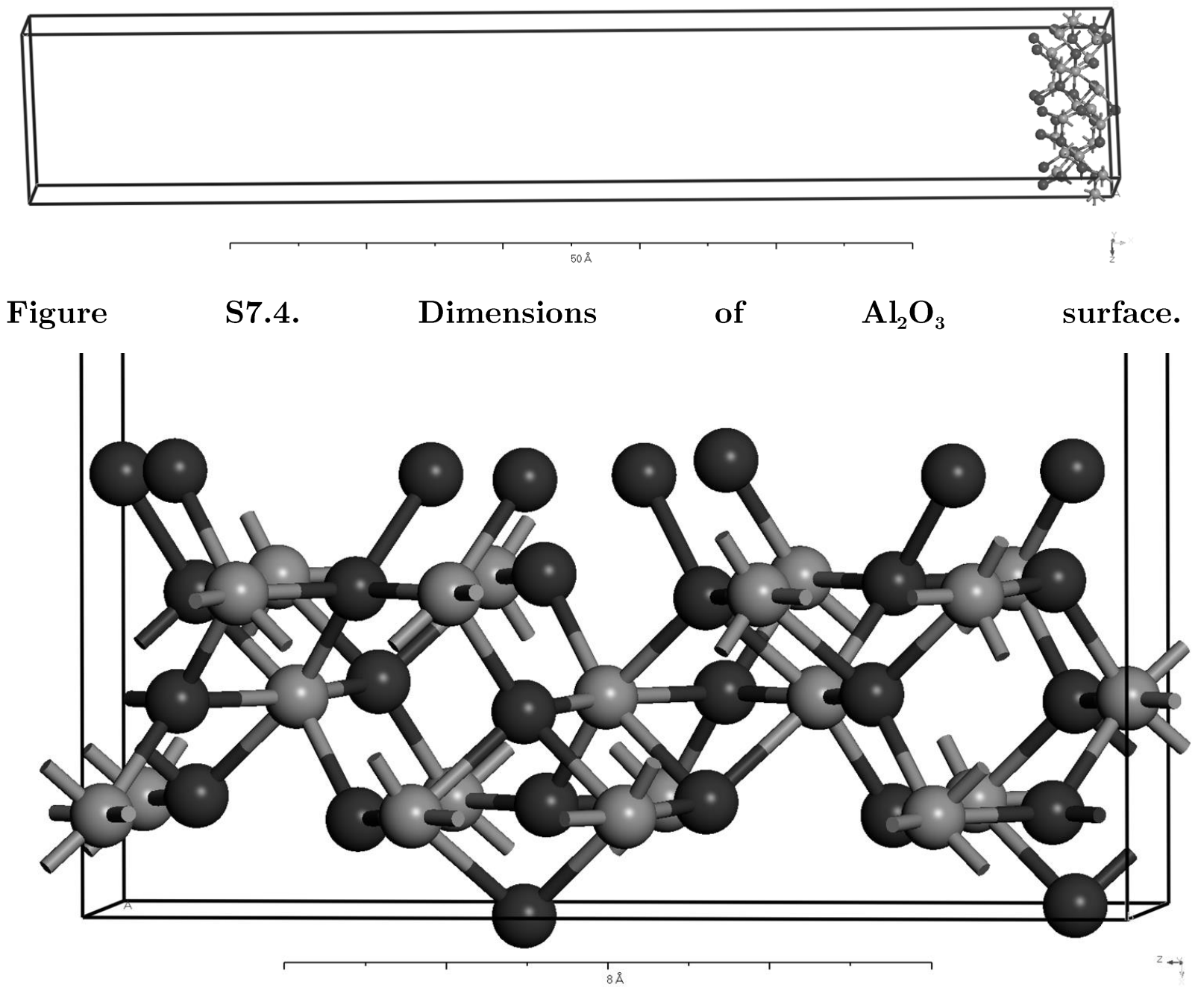

Vacuum Slab Build Dimensions

Vacuum orientation C (axis)

Vacuum thickness $=74.5048 \AA$

Crystal thickness $=80.00 \AA$ 
Figure S7.5. Side view of octylphosphonic acid on $\mathrm{Al}_{2} \mathrm{O}_{3}$ surface (with dimensions).

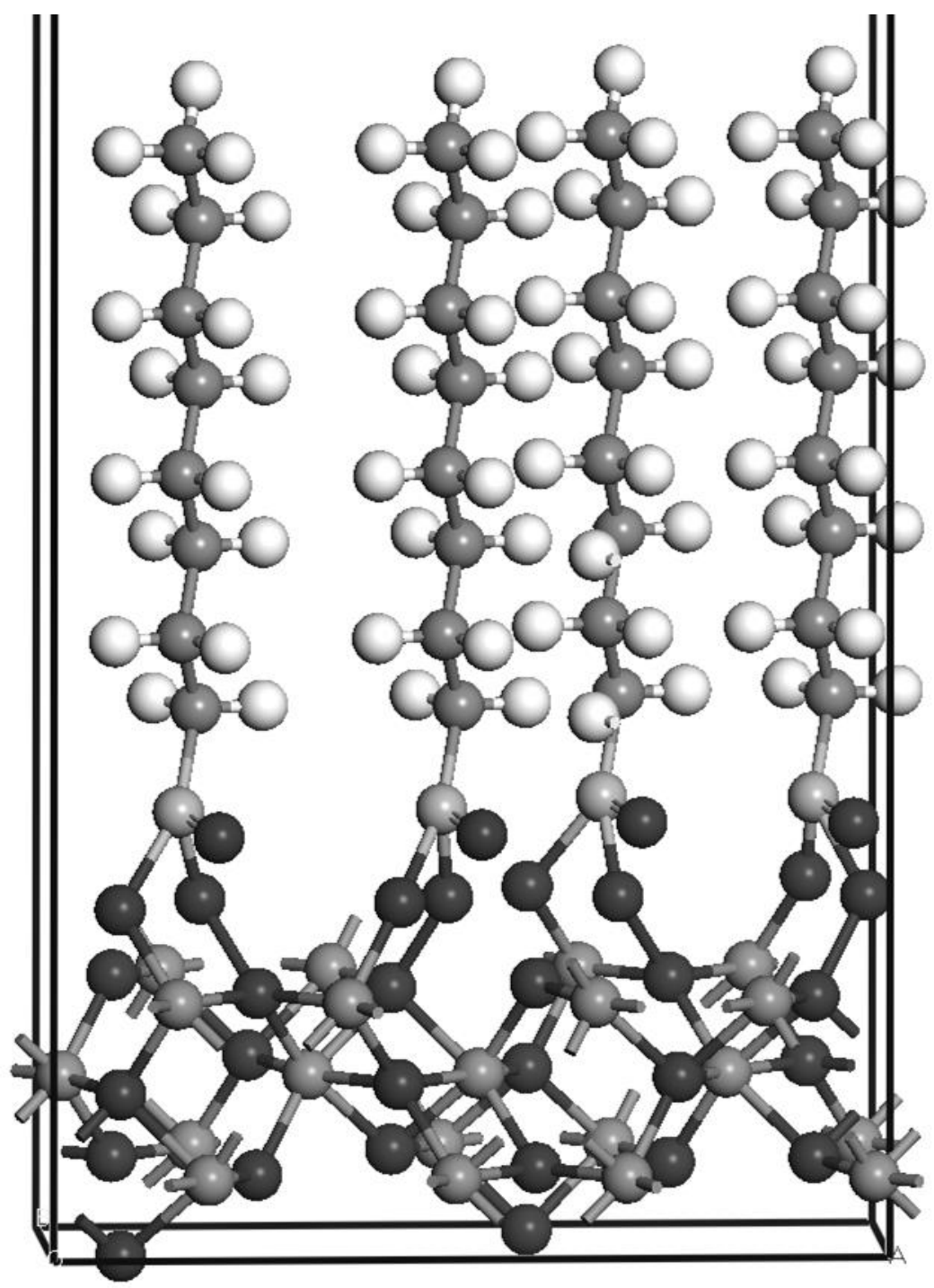

$20 \AA$ 
Figure S7.6. Top view of octylphosphonic acid on $\mathrm{Al}_{2} \mathrm{O}_{3}$ surface (with dimensions).

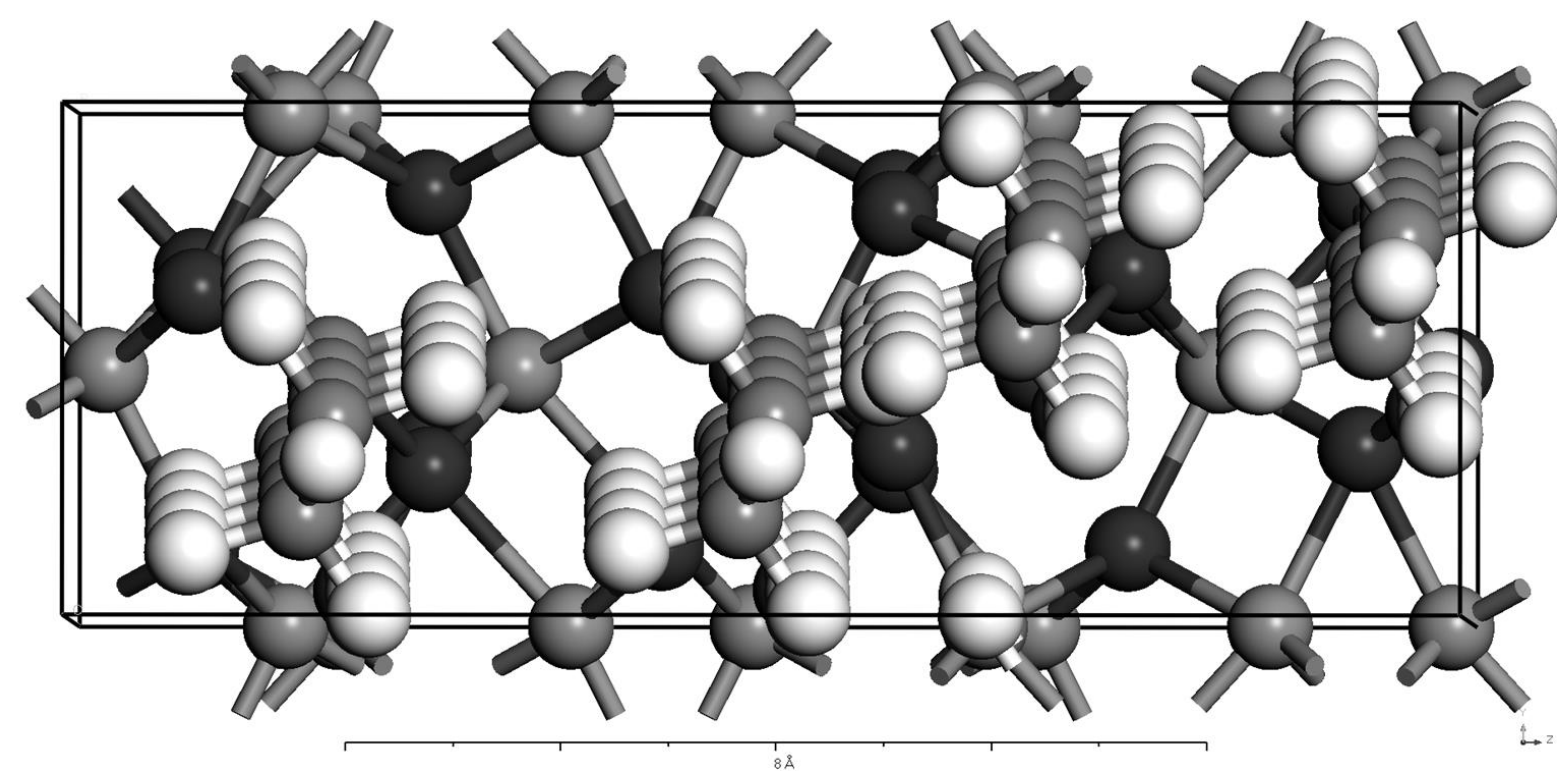

Figure S7.7. Top view of a supercell $(3 \times 5)$ of $-\mathrm{OH}$ terminated $\mathrm{Al}_{2} \mathrm{O}_{3}$ surface. No phosphonic acid attached $(0 \%$ coverage, hydrogens of $-\mathrm{OH}$ highlighted with yellow, other oxygen atoms (red) are part of the $\mathrm{Al}_{2} \mathrm{O}_{3}$ surface and are unavailable to undergo reaction).

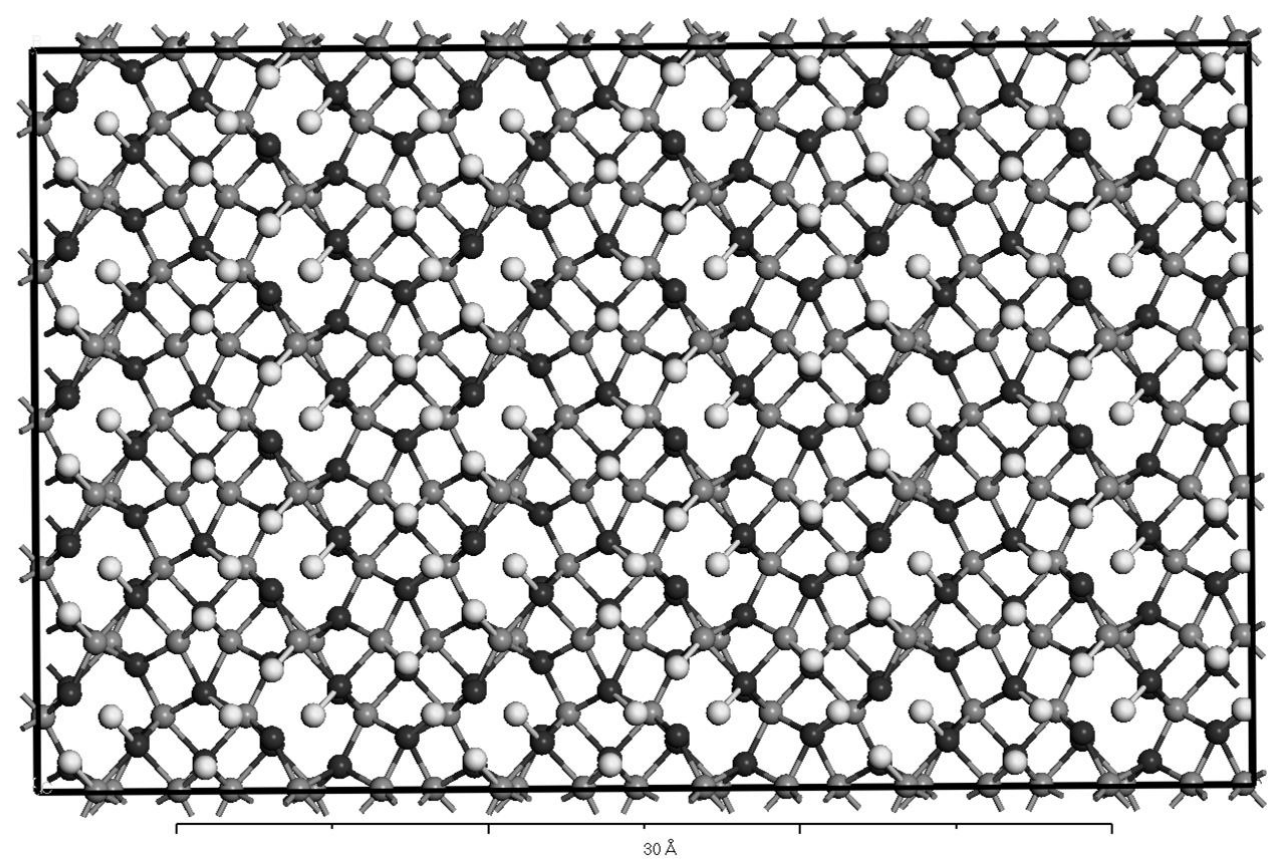


Figure S7.8. Top view of a supercell $(3 \times 5)$ of octyl phosphonic acid (100\% coverage).

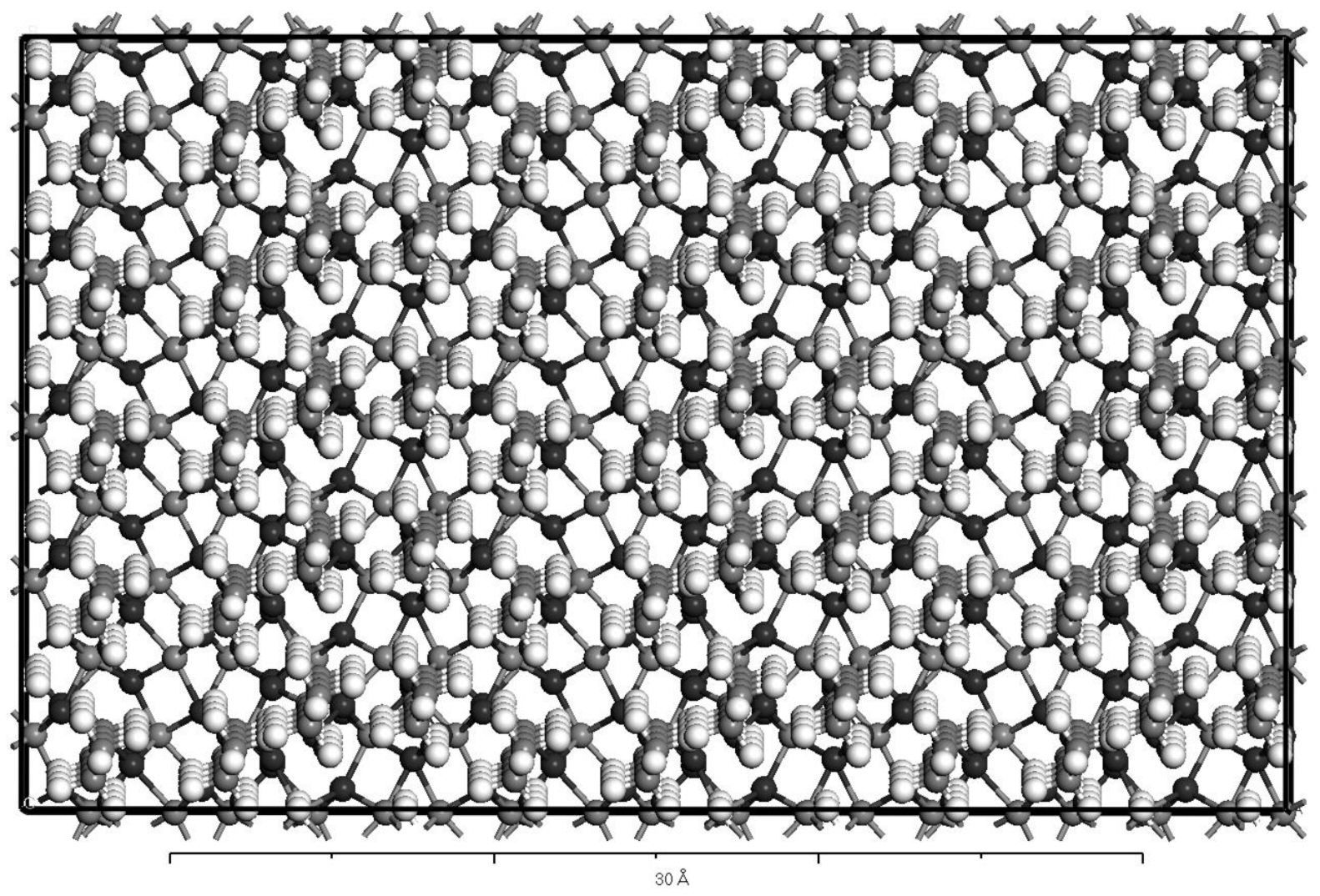


Figure S7.9. Schematic representation of positioning of octyl phosphonic acid groups at the surface to obtain different \% coverages with 'random' positioning (3 different subsets of each \% coverage were used to maximize randomization). (Red balls $=-\mathrm{OH}$ attachment, no attachment; Black balls $=$ site of octylphosphonic acid)

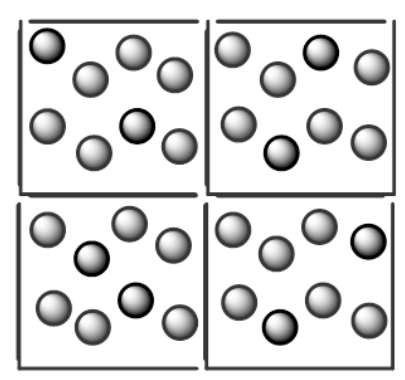

$25 \% \mathrm{~A}$

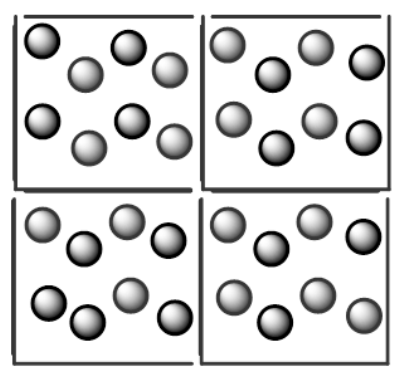

$50 \% \mathrm{~A}$

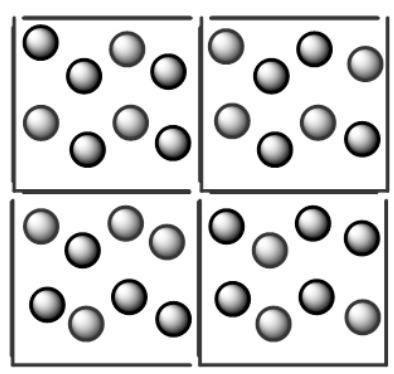

$56.2 \% \mathrm{~A}$

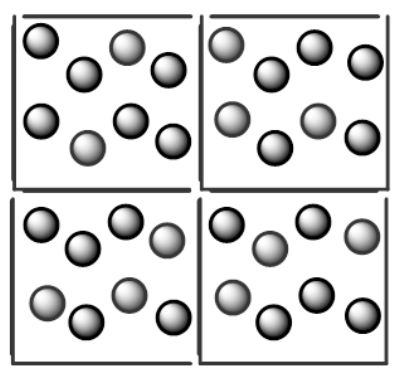

$65.6 \% \mathrm{~A}$

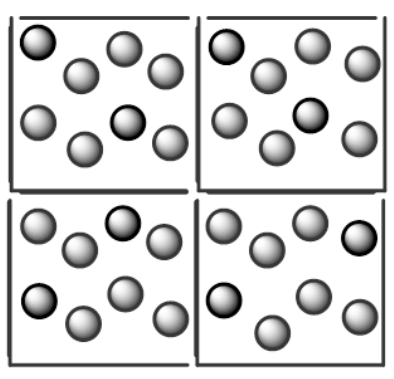

$25 \% \mathrm{~B}$

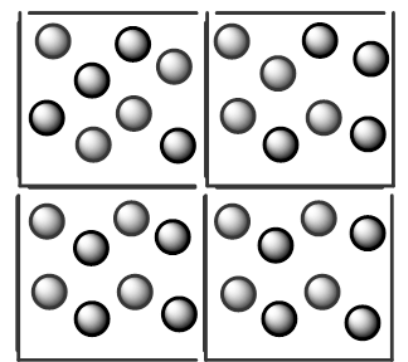

$50 \% \mathrm{~B}$

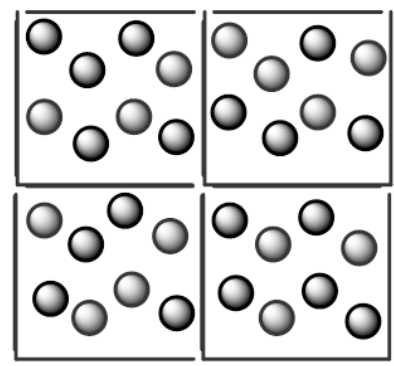

$56.2 \% \mathrm{~B}$

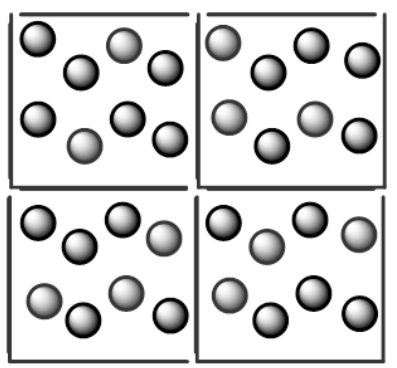

$65.6 \% \mathrm{~B}$

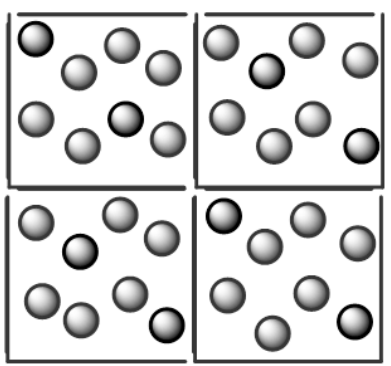

$25 \% \mathrm{C}$
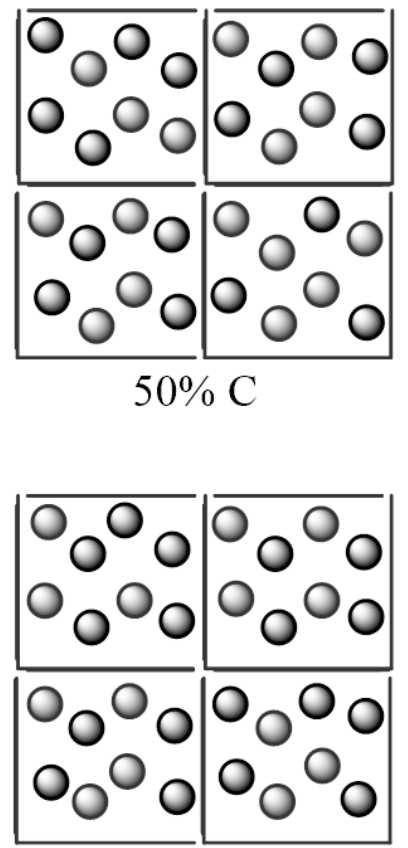

$56.2 \% \mathrm{C}$

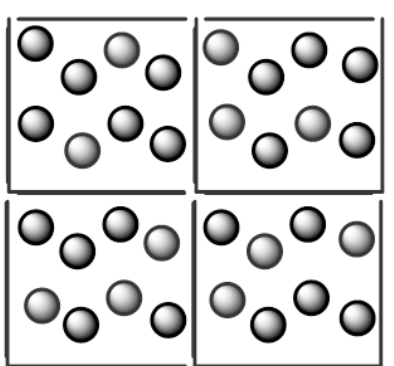

$65.6 \% \mathrm{C}$ 
Figure S7.10. Plot of packing energy vs different surface coverages. Minimum energy denotes maximum stability. [All calculations were done on a supercell $(8 \times 16)$ with 512 chains].

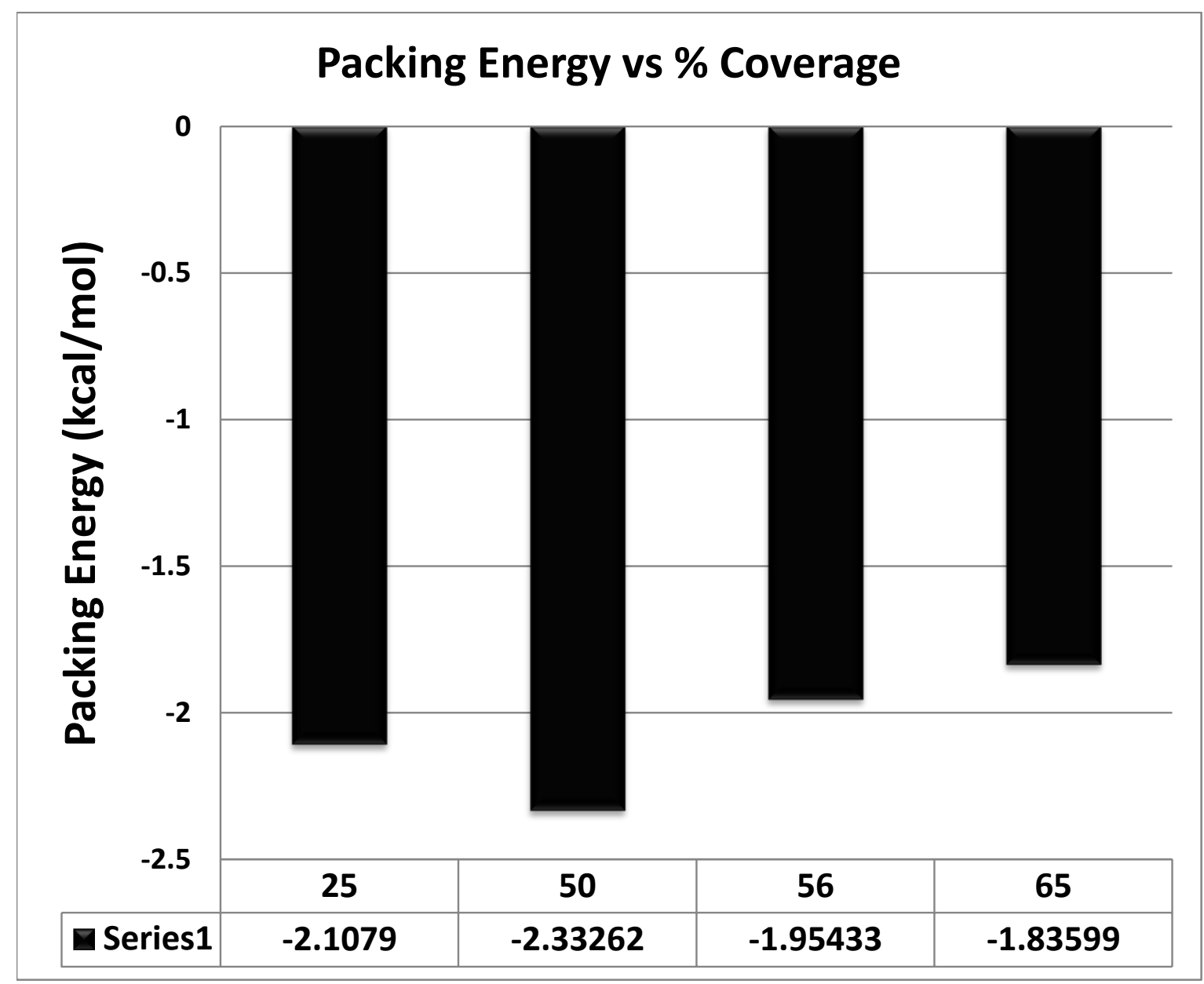


Figure S7.11. Schematic depiction of the most stable configuration for a supercell $(8 \times 16)$ with 512 chains $(50 \% \mathrm{~B})$.

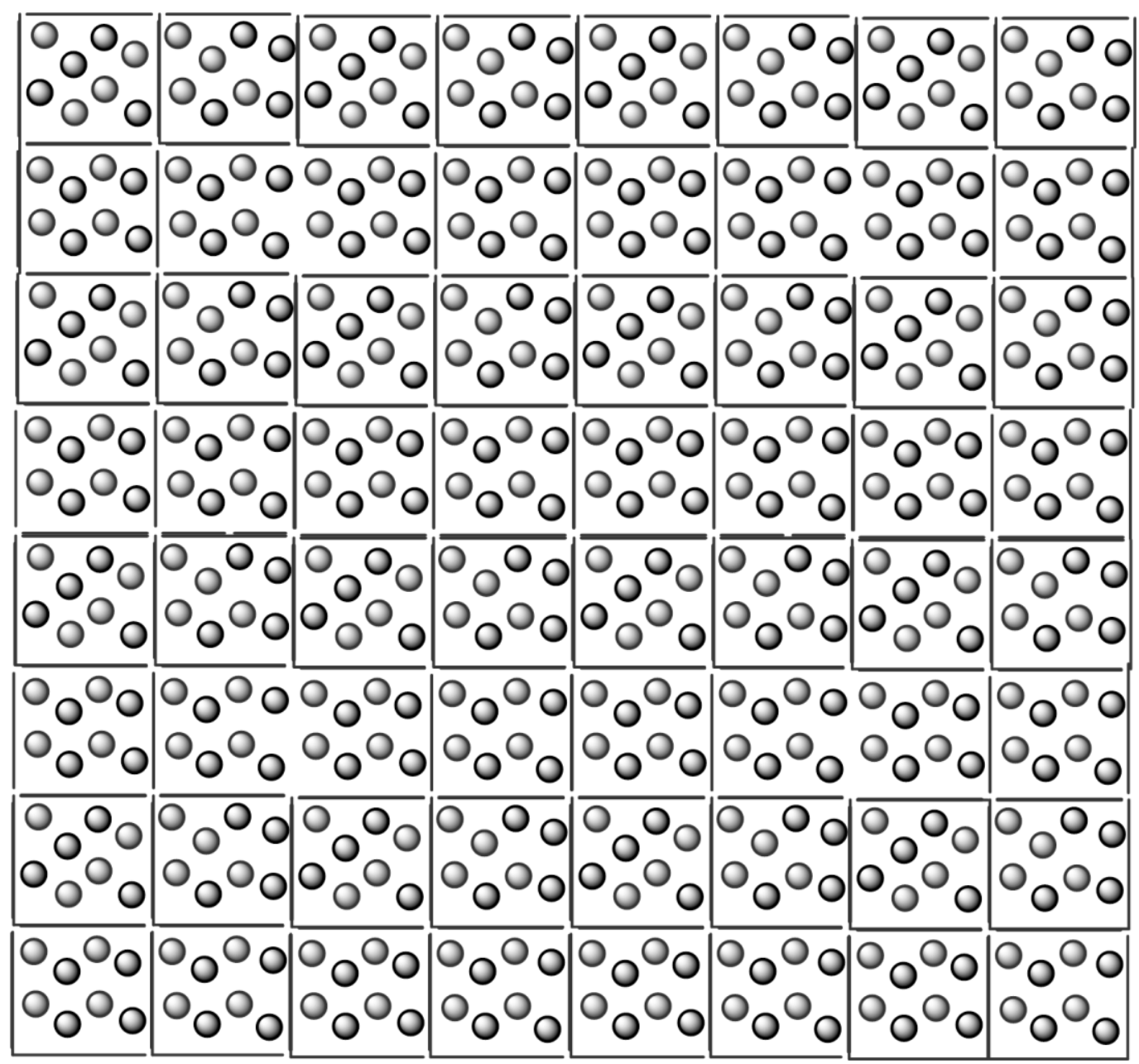


Figure S7.12. Molecular model of $50 \% \mathrm{~B}\left(1^{\text {st }}=\right.$ tilted, $2^{\text {nd }}=$ across view $)$ supercell $(8 \times 16)$ after energy minimization. The picture shows the octylphosphonic acid chains attached randomly to the $\mathrm{Al}_{2} \mathrm{O}_{3}$ surface in a $50 \%$ overall coverage.
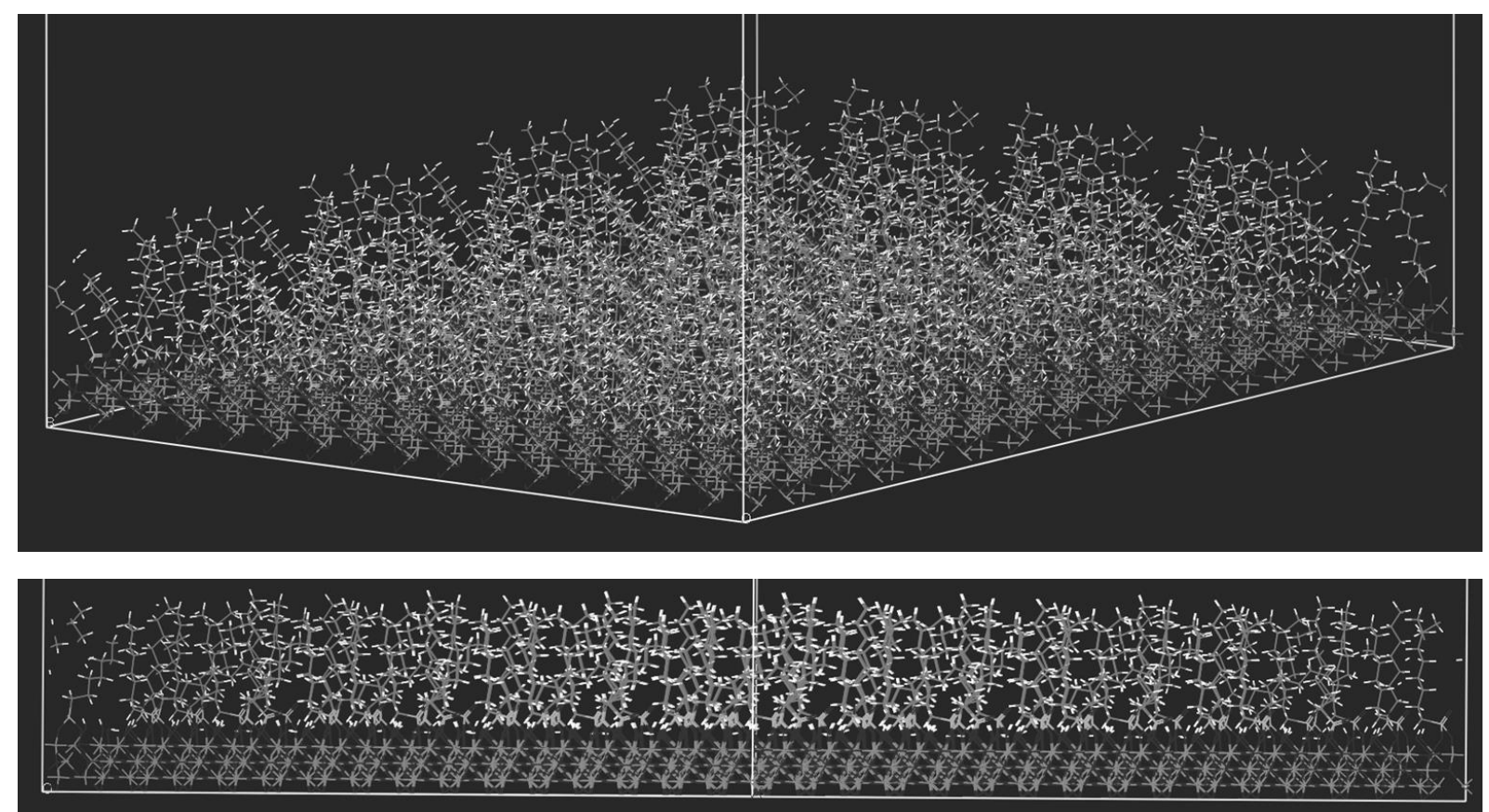
Figure S7.13. Schematic depiction of an alkyl:amine-terminated (3:1) configuration on the $50 \%$ B coverage system (blue balls shows site of exonorbornene or tetrazine attachment).

Note: The reactive groups or alkyl chains are placed randomly at different positions to reduce any bias. Same configuration was kept in either case to ensure similar steric environment).

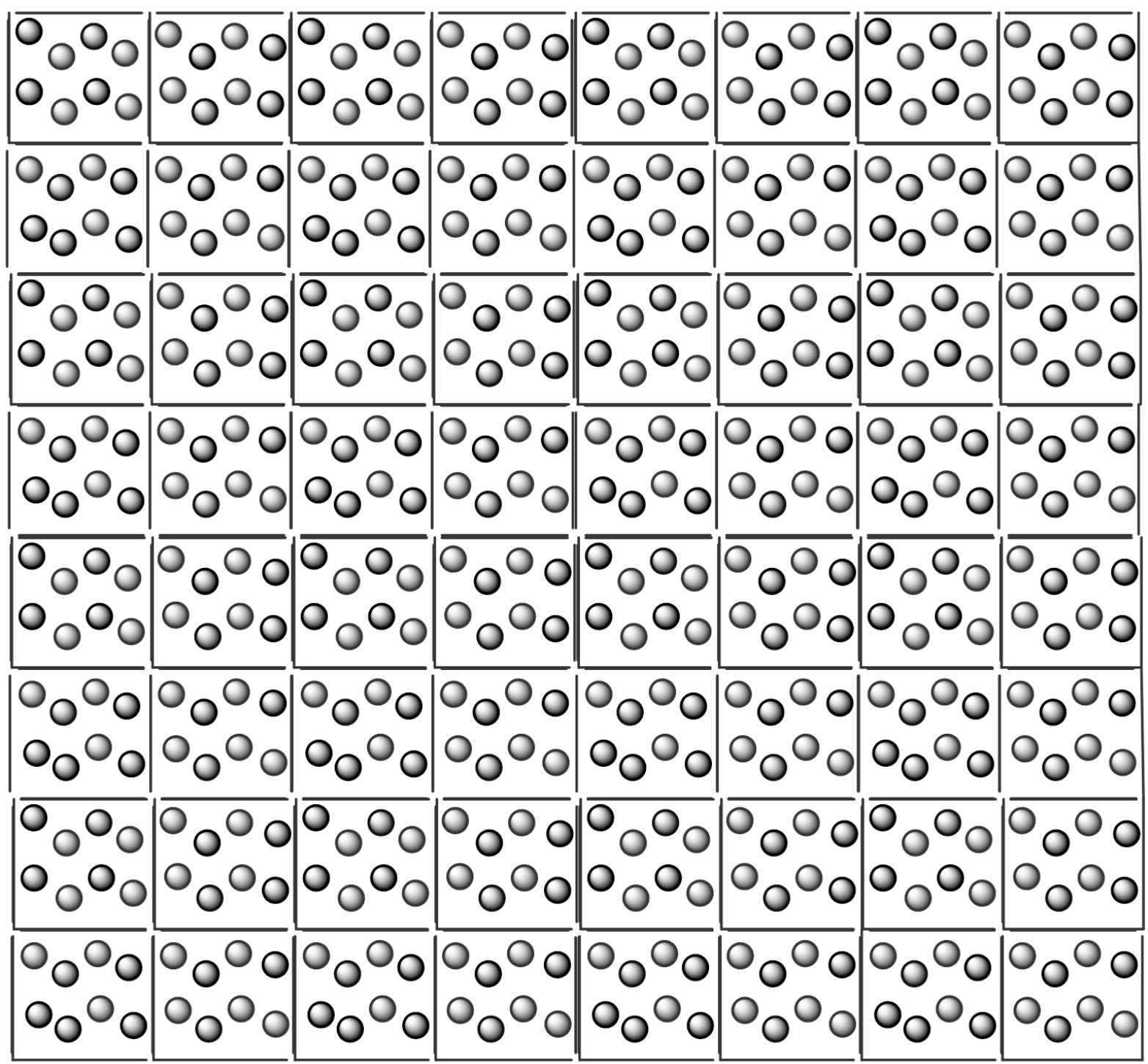


Figure S7.14. Molecular model of $50 \% \mathrm{~B}\left(1^{\text {st }}=\right.$ side, $2^{\text {nd }}=$ across, $3^{\text {rd }}=$ tilted view) supercell $(8 \times 16)$ with attached exo-norbornene:alkyl $(1: 3)$ after the initial energy minimization.

Note: The picture depicts the orientation of norbornene groups with respect to the surface before performing molecular dynamics. The repetitive orientation are the result of the way the structure is built, but not indicative of chemically real orientation. The MD part is thus essential to 'shake up' the structures, and after geometry optimization of the structures resulting from there, chemically relevant structures that depict the actual degree of ordering/clustering can be obtained.
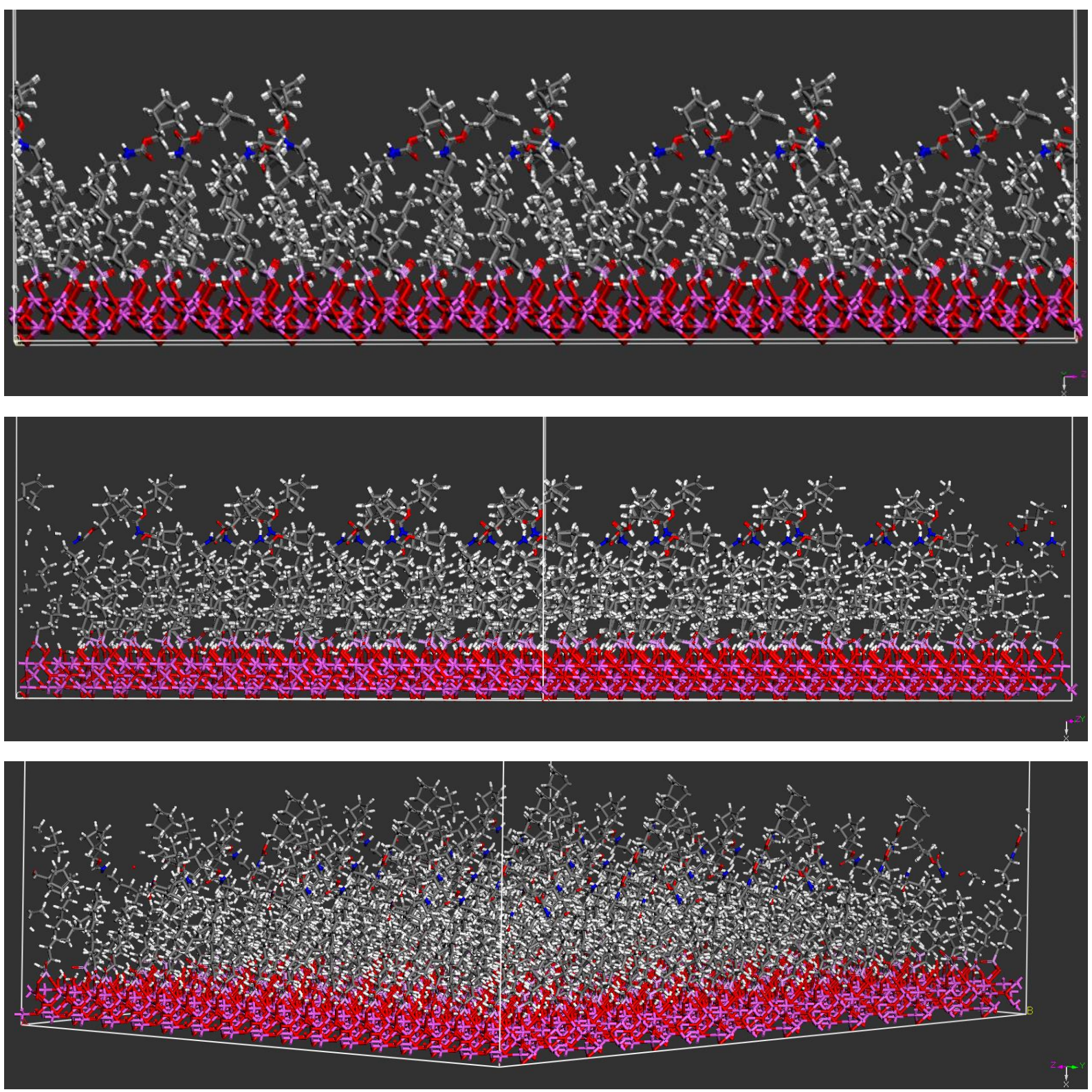
Figure S7.15. (a) Protocol for Molecular Dynamics in Materials Studio on Al surface. (b) Molecular model of $50 \% \mathrm{~B}\left(1^{\text {st }}=\operatorname{side}, 2^{\text {nd }}=\right.$ across, $3^{\text {rd }}=$ tilted view) supercell $(8 \times 16)$ with attached exo-norbornene: alkyl $(1: 3)$ after performing molecular dynamics at $773 \mathrm{~K}$ and subsequent geometry optimization. The picture depicts the orientation of norbornene groups with respect to the surface.

\section{a)}
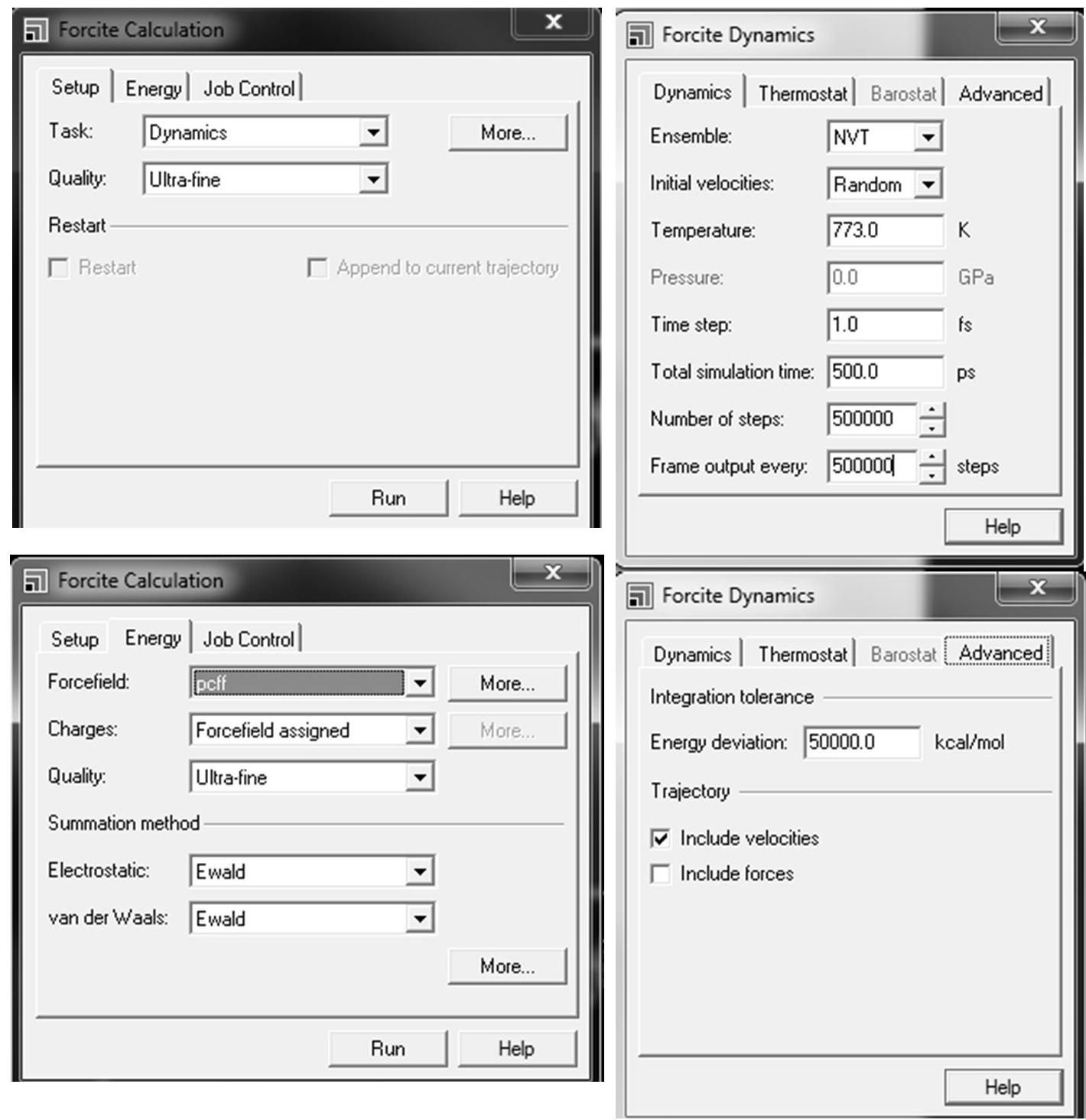

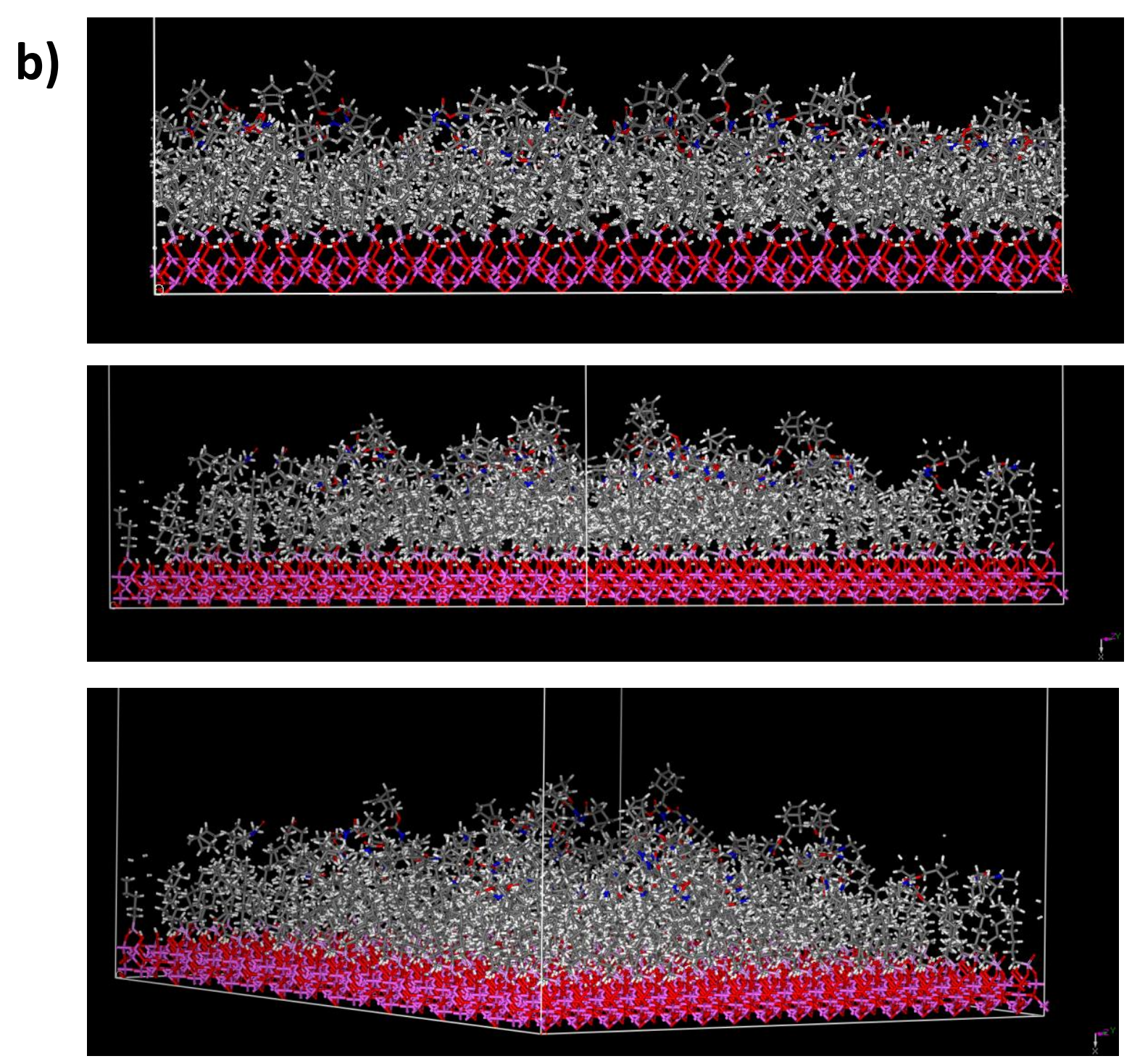
Figure S7.16. Molecular model of $50 \% \mathrm{~B}\left(1^{\text {st }}=\operatorname{side}, 2^{\text {nd }}=\operatorname{across}, 3^{\text {rd }}=\right.$ tilted view) supercell $(8 \times 16)$ with attached tetrazine:alkyl (1:3) after initial energy minimization. [The picture depicts the orientation of tetrazine groups with respect to the surface before performing molecular dynamics.]
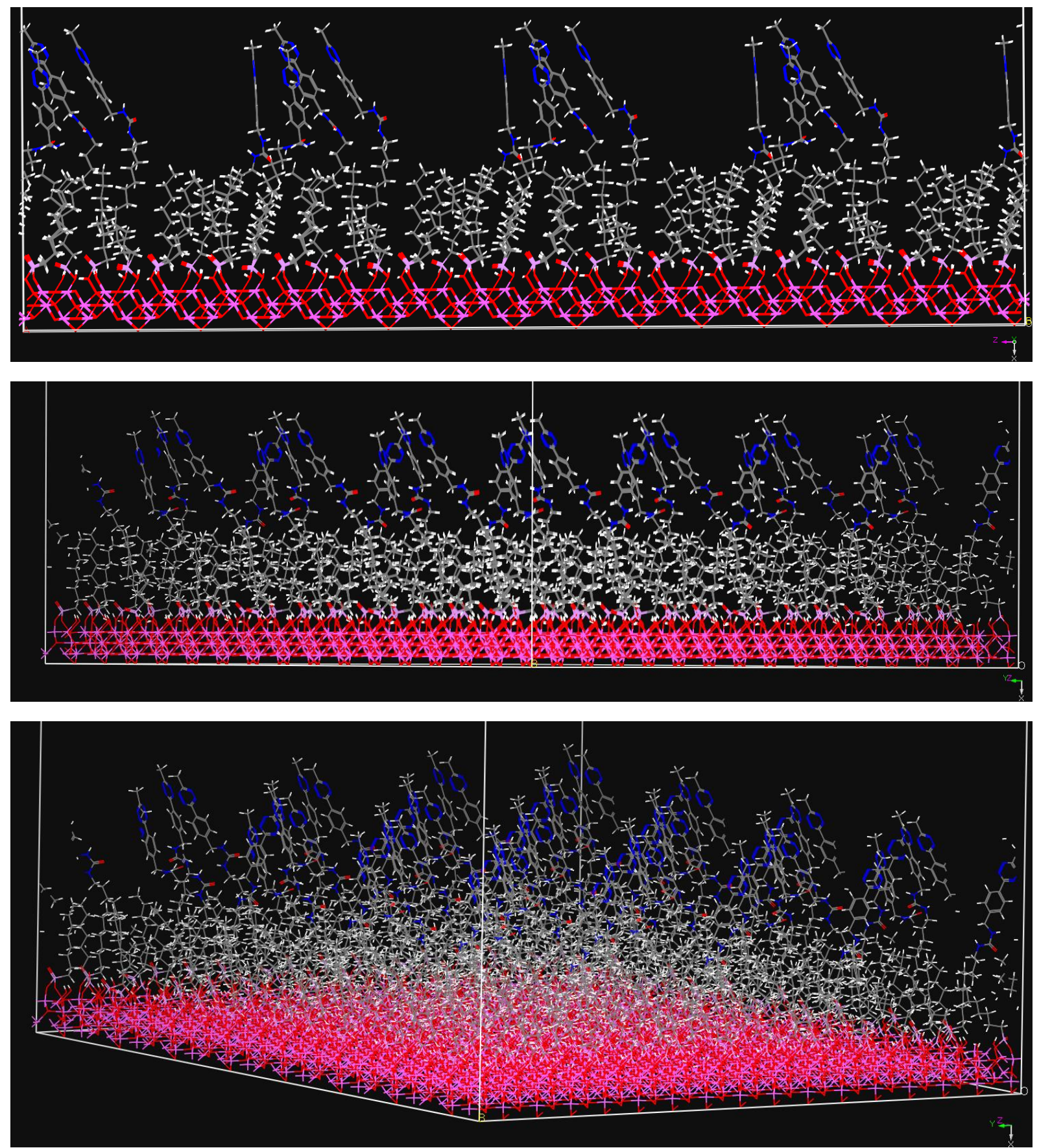
Figure S7.17. Molecular model of $50 \% \mathrm{~B}\left(1^{\text {st }}=\operatorname{side}, 2^{\text {nd }}=\operatorname{across}, 3^{\text {rd }}=\right.$ tilted view) supercell $(8 \times 16)$ with attached tetrazine:alkyl (1:3) after performing molecular dynamics at $773 \mathrm{~K}$ and subsequent geometry optimizations. The picture depicts the orientation of tetrazine groups with respect to the surface.
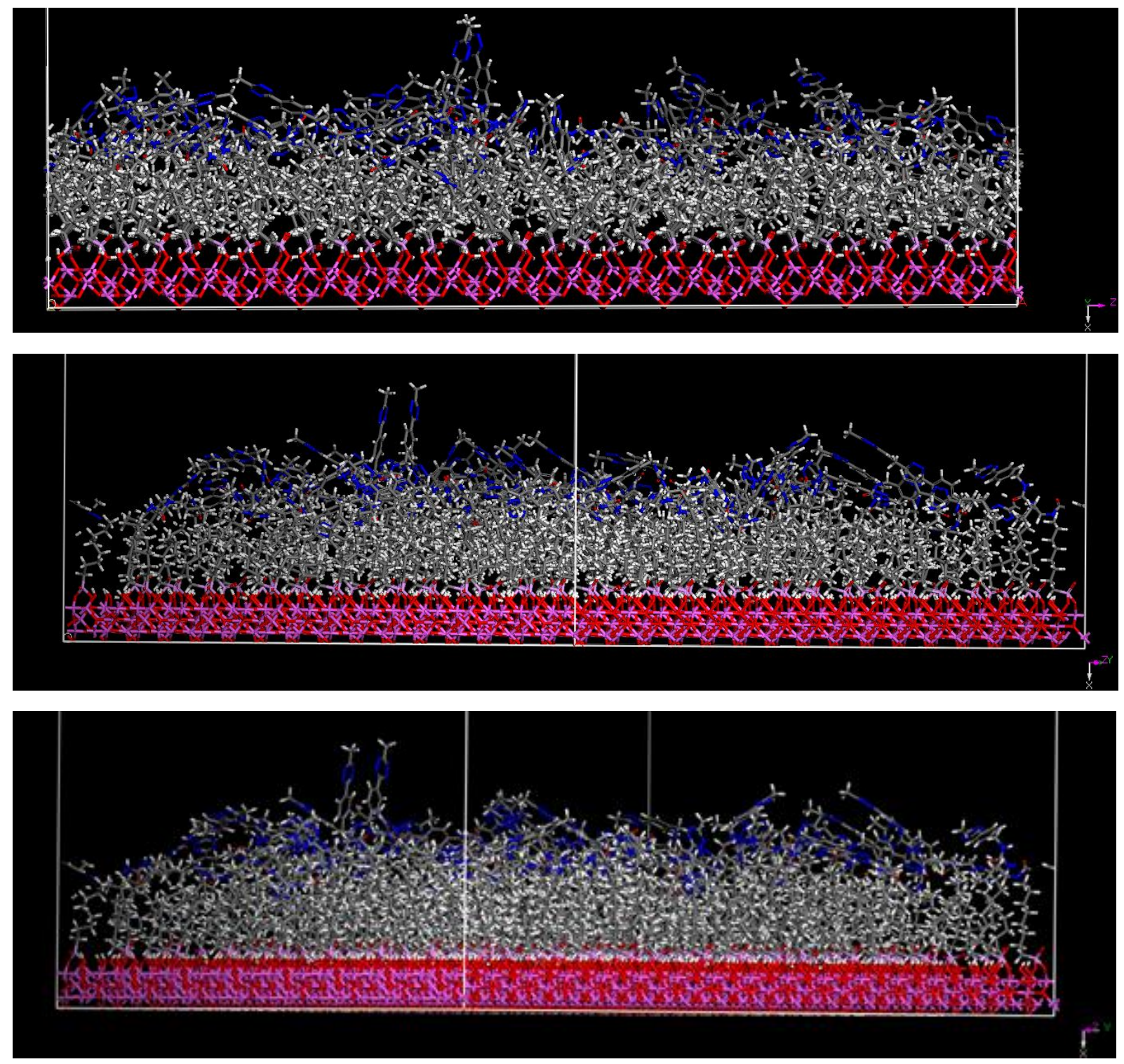
Figure S7.18. Top view of a cluster of exo-norbornene obtained from a supercell $(8 \times 16)$ for exo-norbornene:alkyl (1:3) after performing molecular dynamics at $773 \mathrm{~K}$ and subsequent energy minimization (Figure S7.15b) displayed for understanding surface approach. [All surface atoms, alkyl linkers and microenvironment ignored for simplicity]

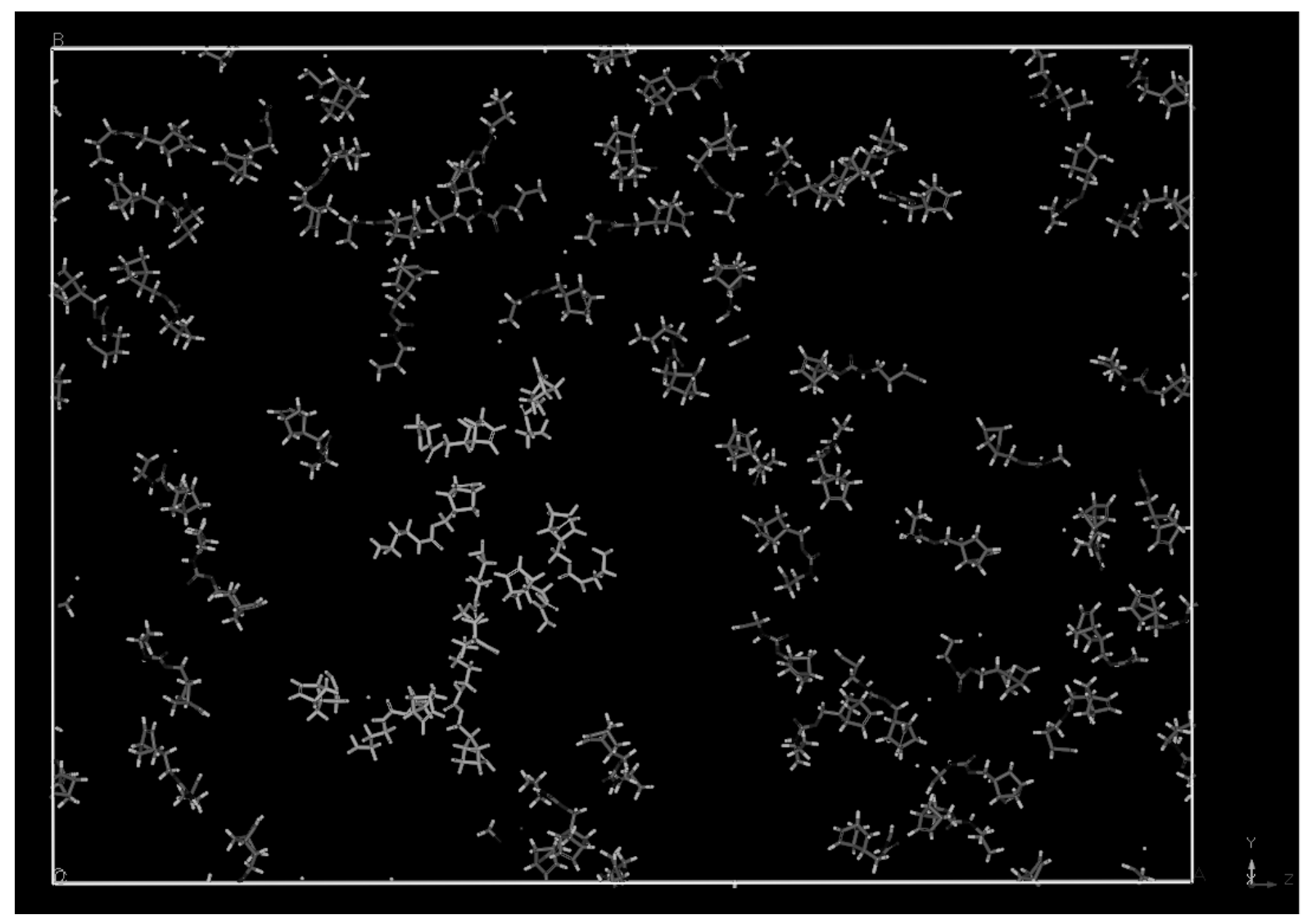


Figure S7.19. Top view of a cluster of tetrazine obtained from a supercell $(8 \times 16)$ for tetrazine:alkyl (1:3) after performing molecular dynamics at $773 \mathrm{~K}$ and subsequent energy minimization (Figure S7.17) displayed for understanding surface approach. [All surface atoms, alkyl linkers and microenvironment ignored for simplicity]

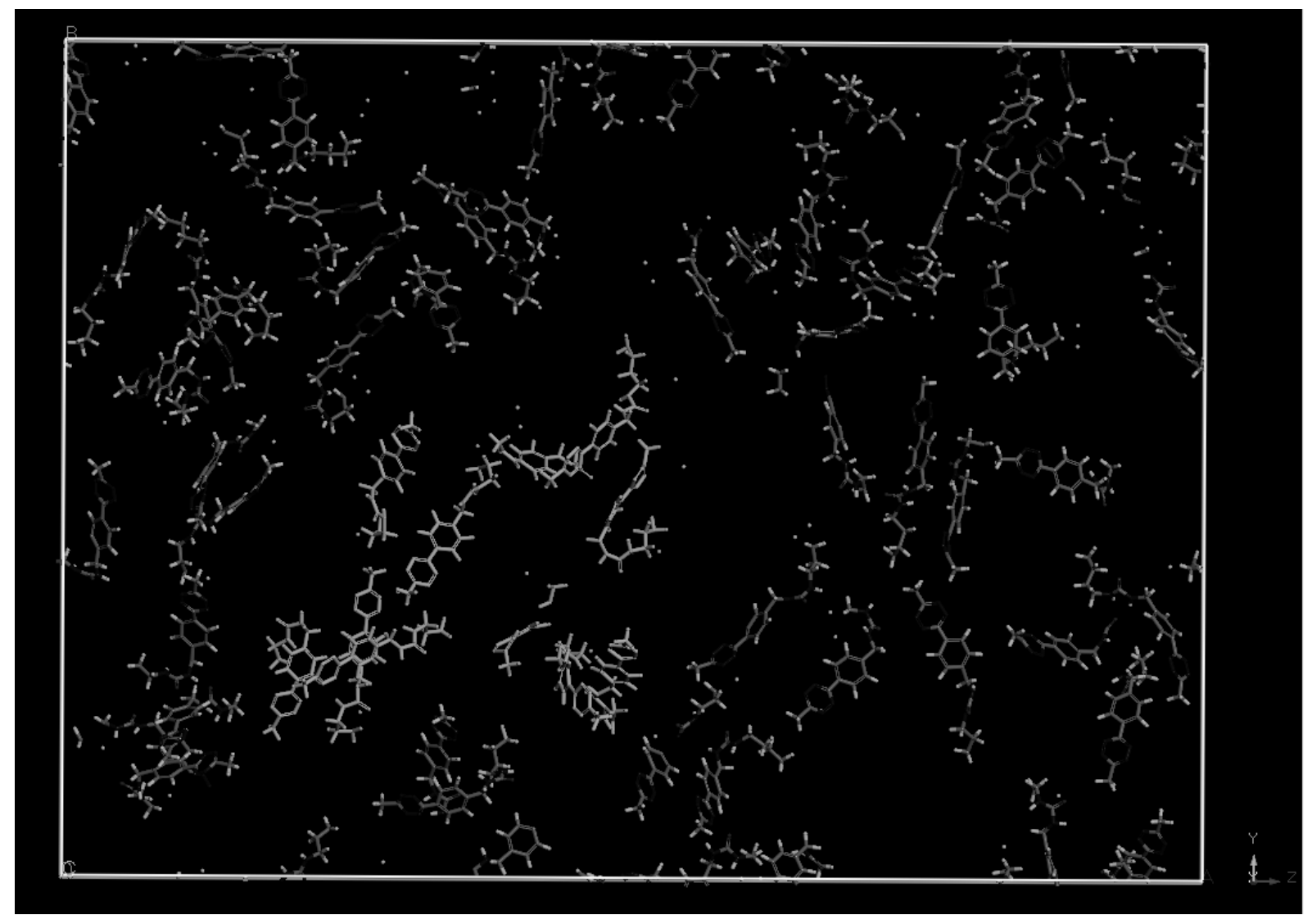


8. Optimized DFT Structures.

Reactant complex bearing tetrazine with $\mathrm{CF}_{3}-$ tag (exo-norbornene on surface)

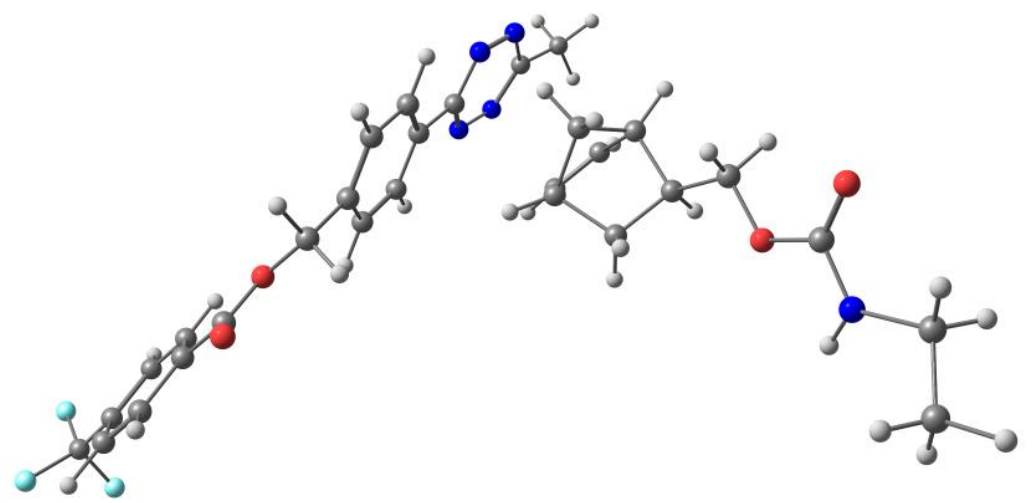

Reactant complex bearing tetrazine with $\mathrm{CF}_{3}-$ tag (endo-norbornene on surface)

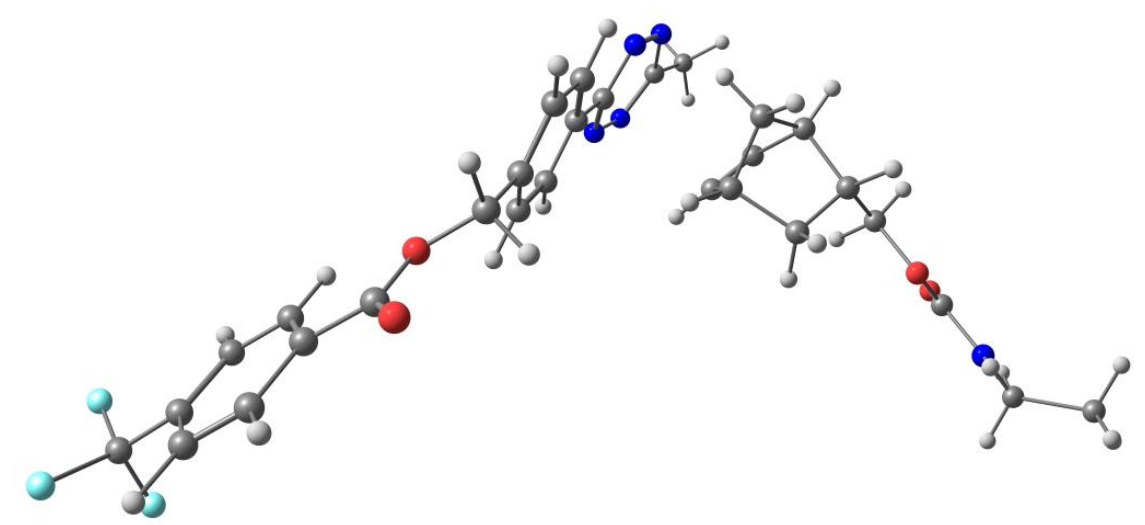

TS with tetrazine bearing $\mathrm{CF}_{3}-\operatorname{tag}$ (exo-norbornene on surface)

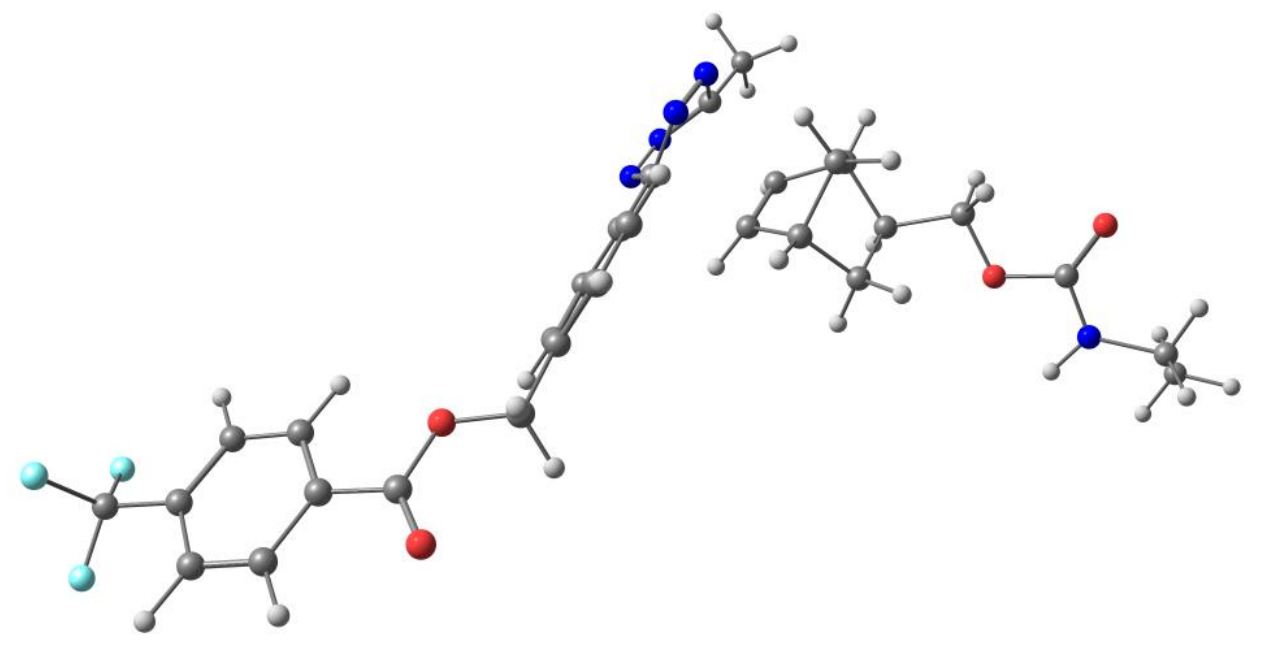


TS with tetrazine bearing $\mathrm{CF}_{3}-\mathrm{tag}$ (endo-norbornene on surface)

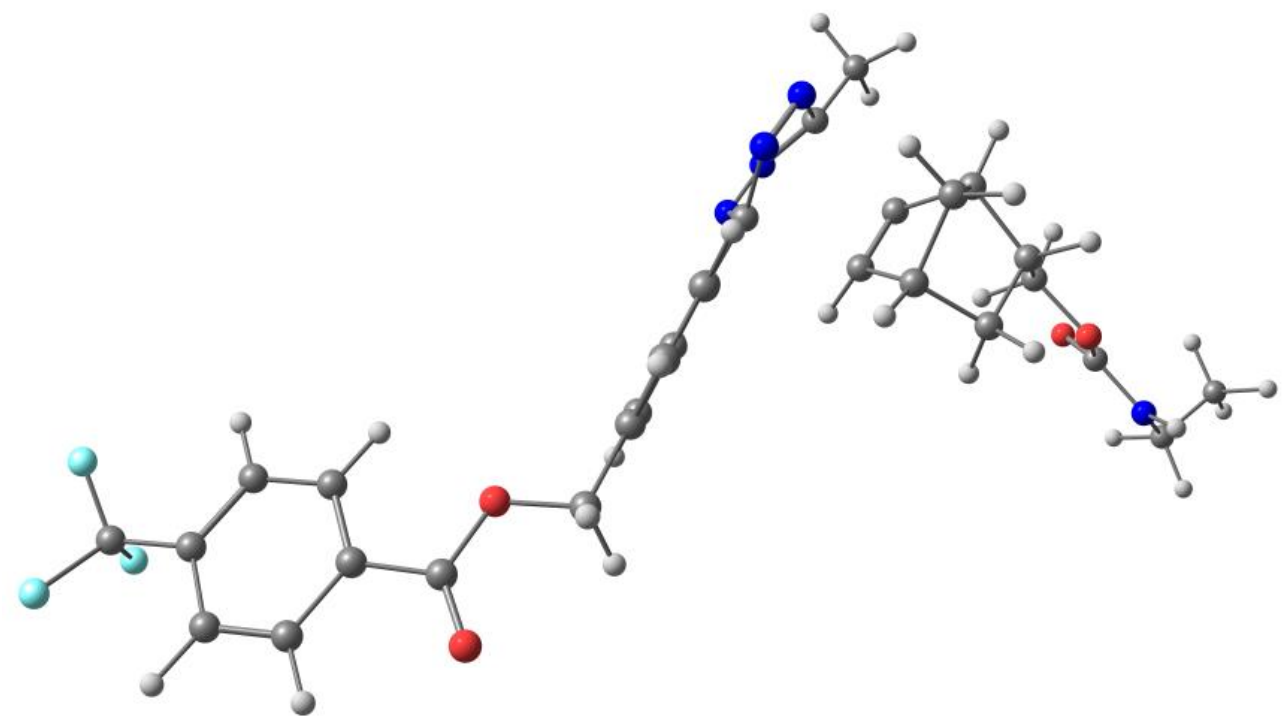

Product with tetrazine bearing $\mathrm{CF}_{3}-$ tag (exo-norbornene on surface)

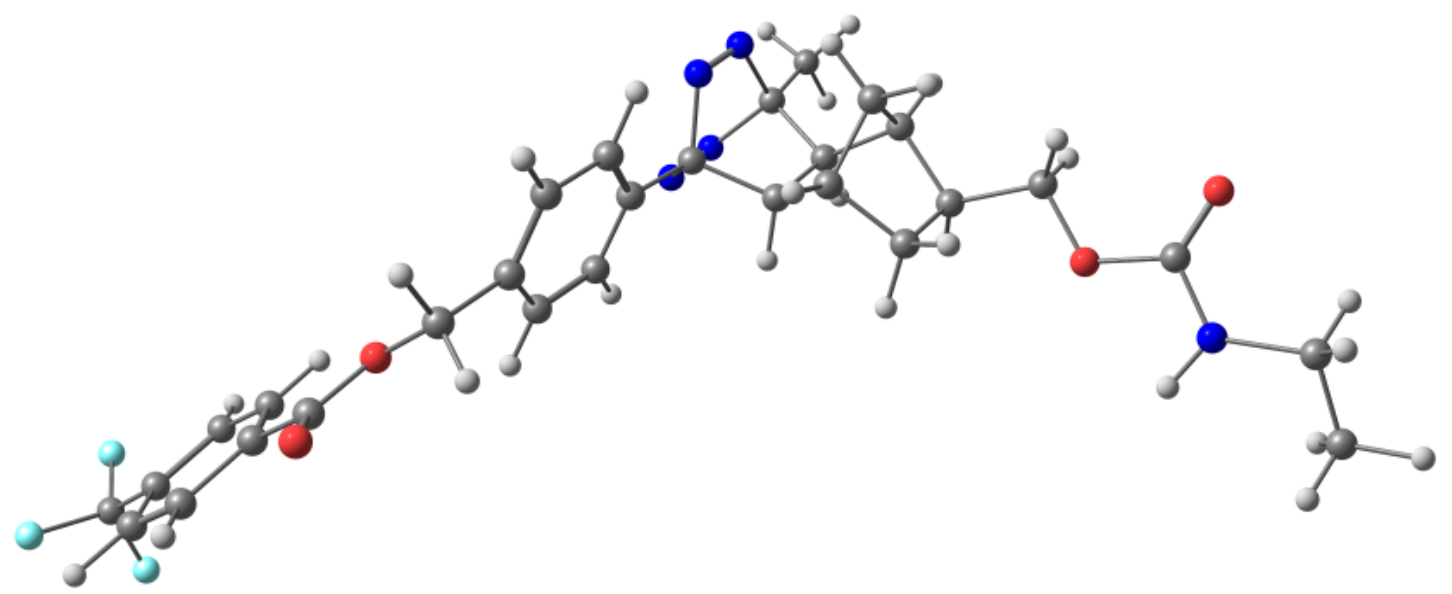

Product with tetrazine bearing $\mathrm{CF}_{3}-$ tag (endo-norbornene on surface)

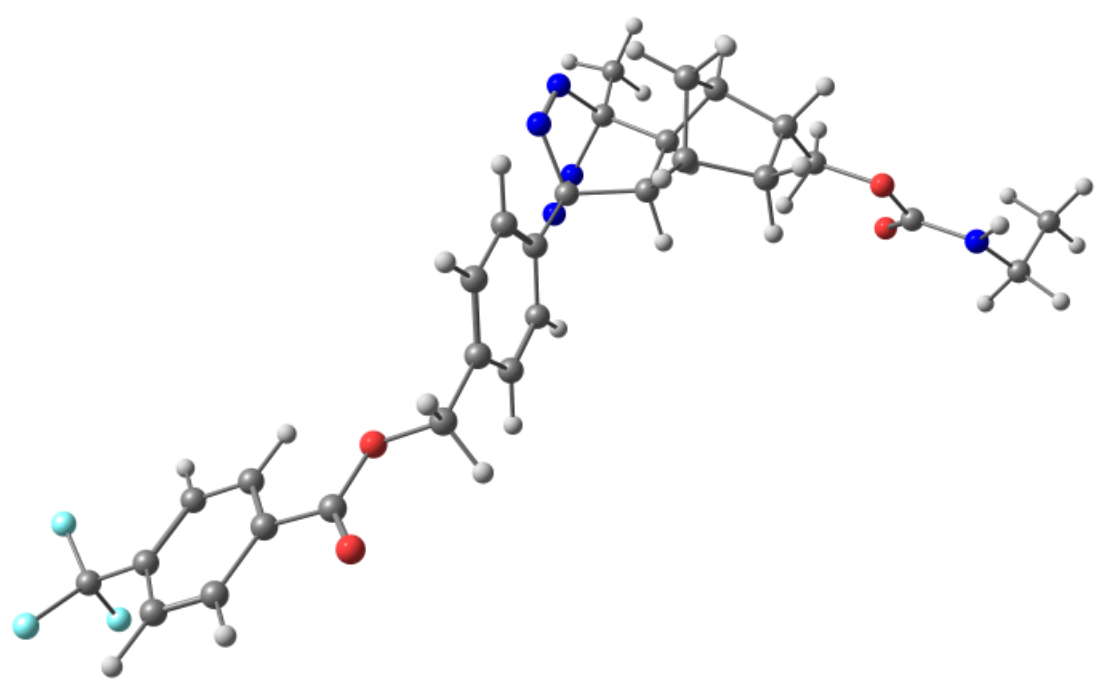


Reactant complex with exo-NBN with $\mathrm{CF}_{3}-$ tag (tetrazine on surface)

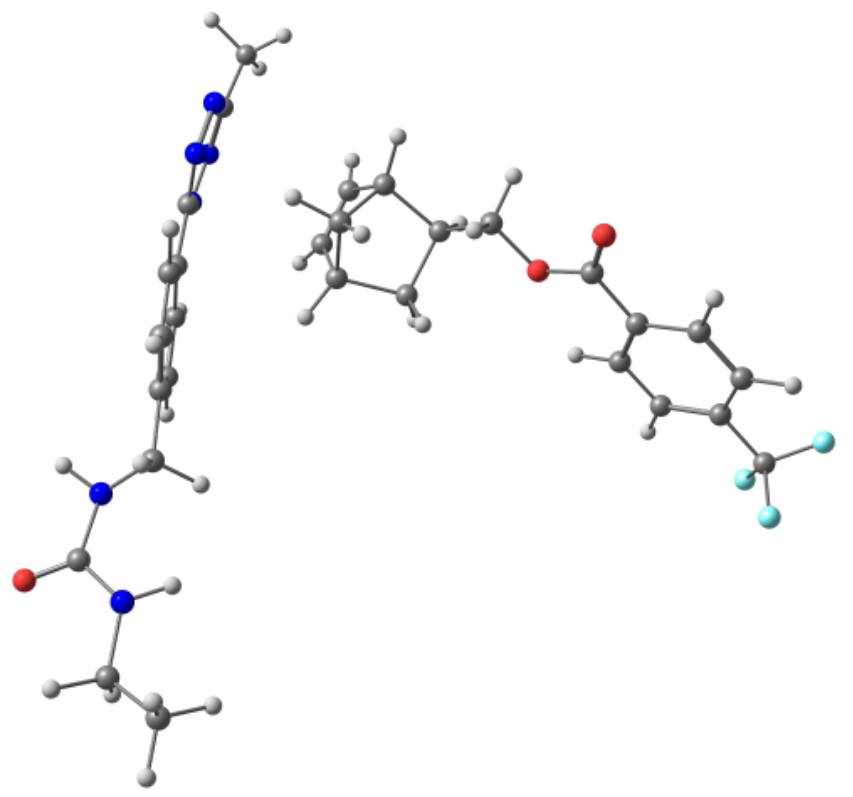

Reactant complex with endo-NBN with $\mathrm{CF}_{3}-$ tag (tetrazine on surface)

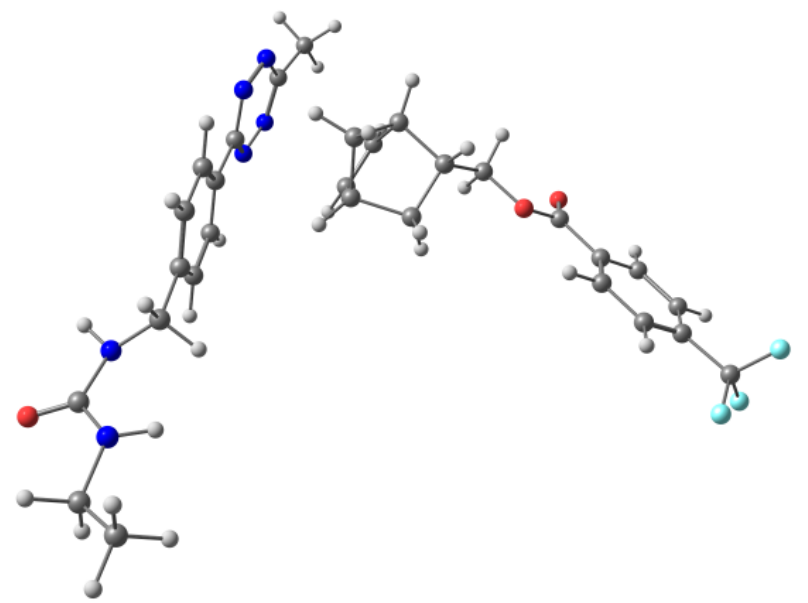

TS with exo-NBN with $\mathrm{CF}_{3}-$ tag (tetrazine on surface)

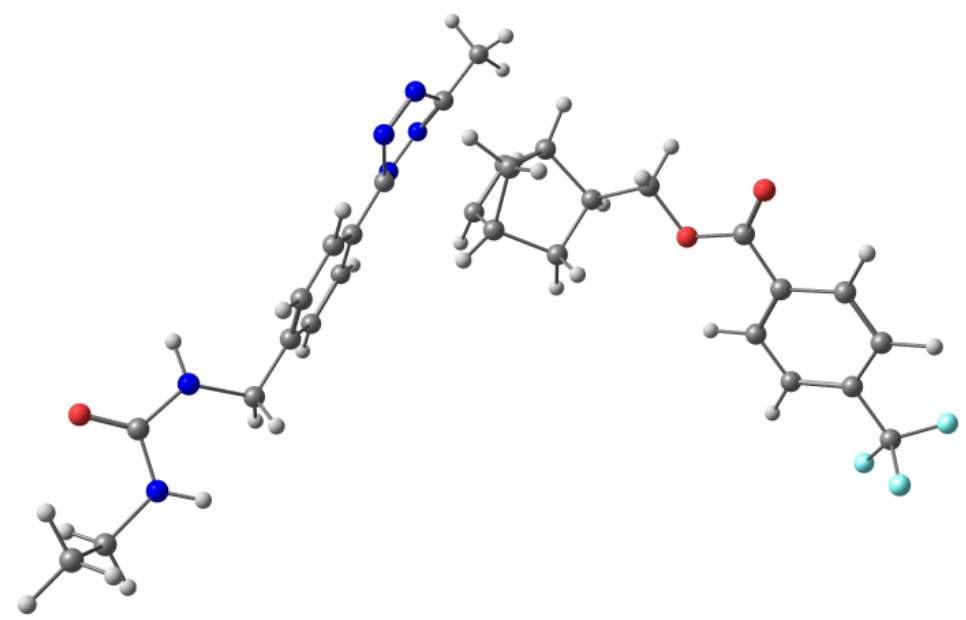


TS with endo-NBN with $\mathrm{CF}_{3}-$ tag (tetrazine on surface)

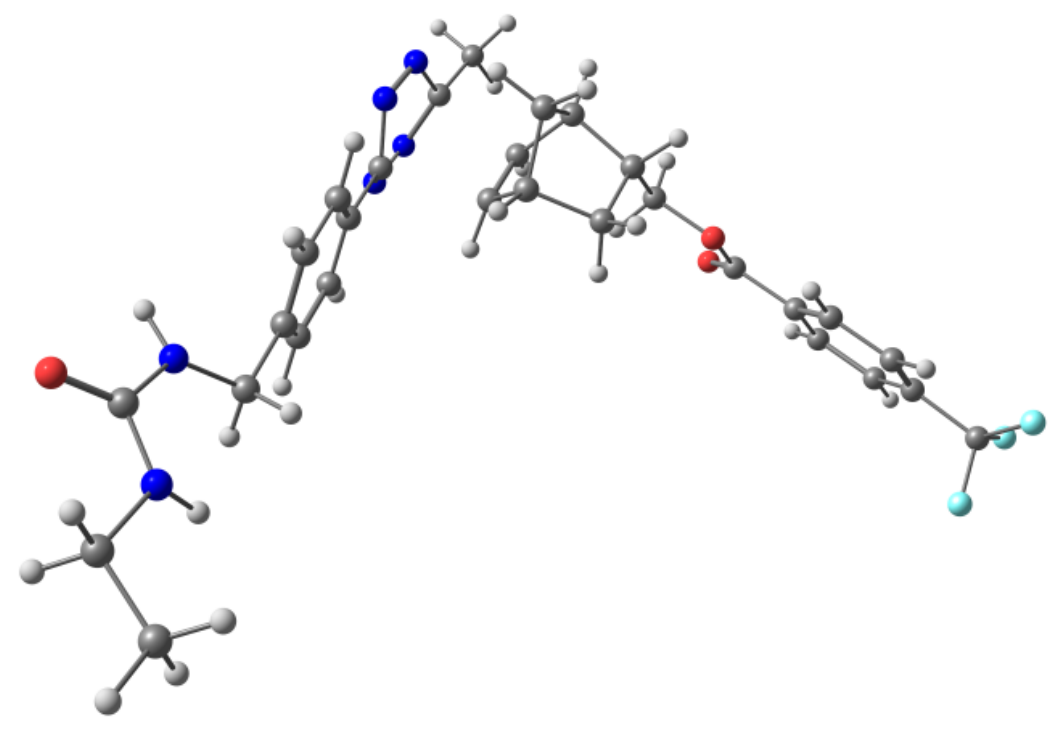

Product with exo-NBN with $\mathrm{CF}_{3}-$ tag (tetrazine on surface)

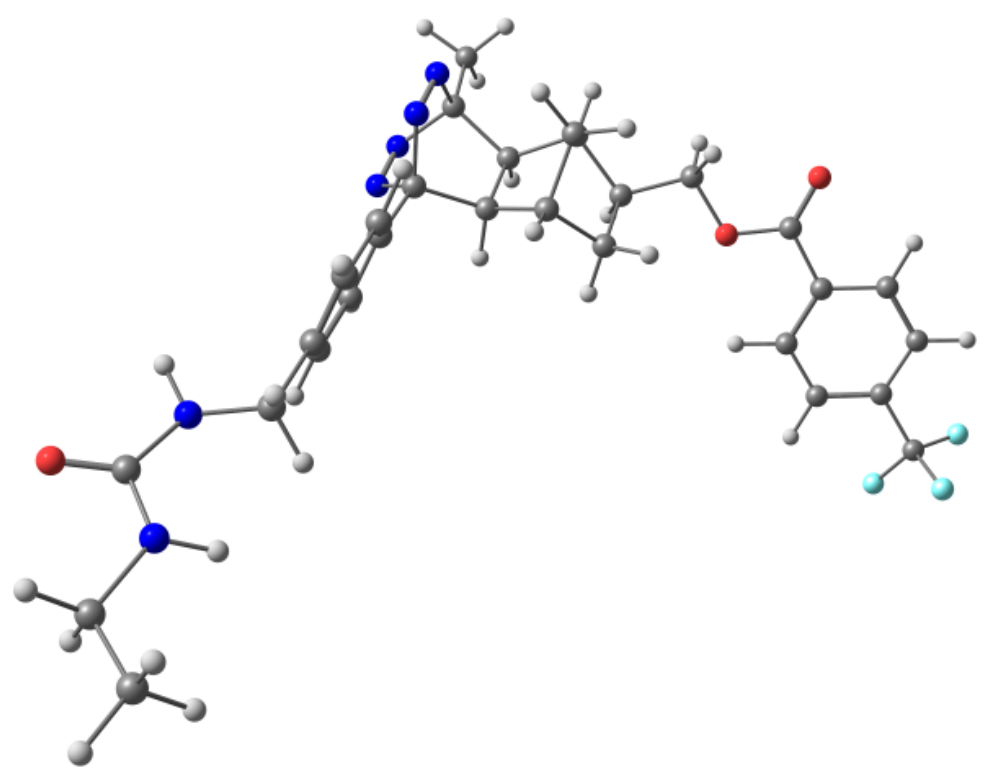

Product with endo-NBN with $\mathrm{CF}_{3}-$ tag (tetrazine on surface)

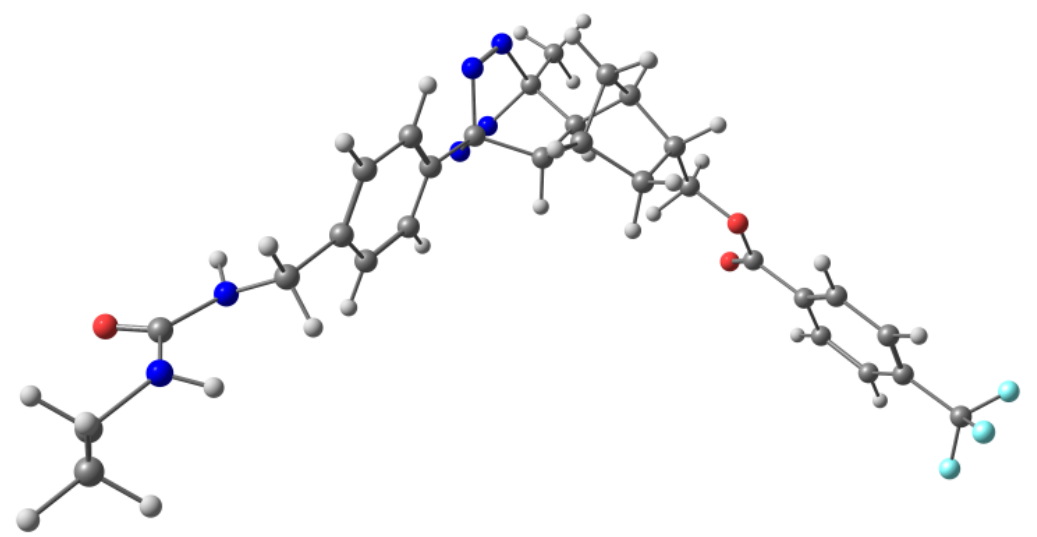


TS exo-NBN with anti $\mathrm{N}_{2}$ elimination (tetrazine on surface)

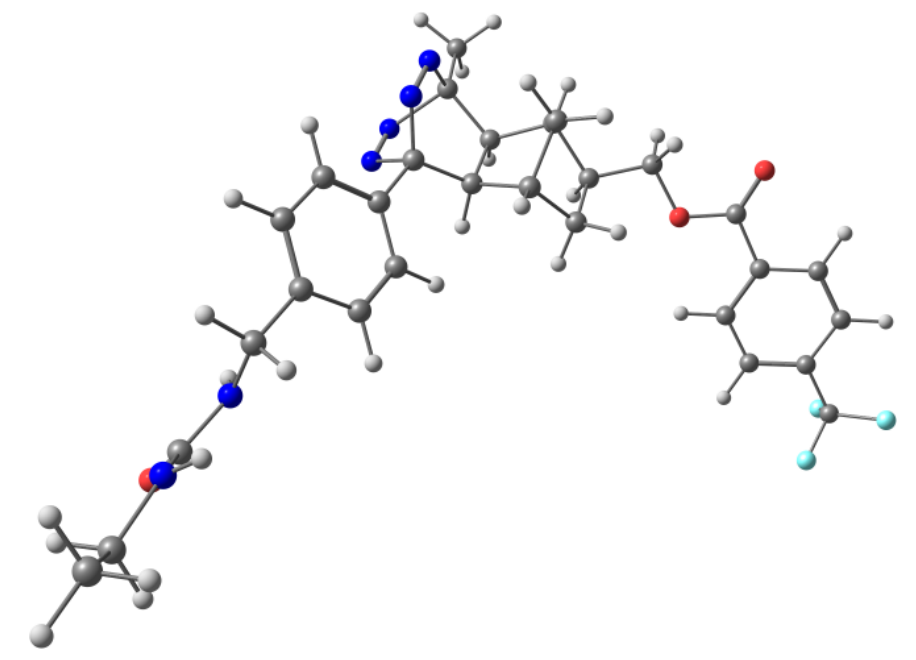

TS exo-NBN with syn $-\mathrm{N}_{2}$ elimination (tetrazine on surface)

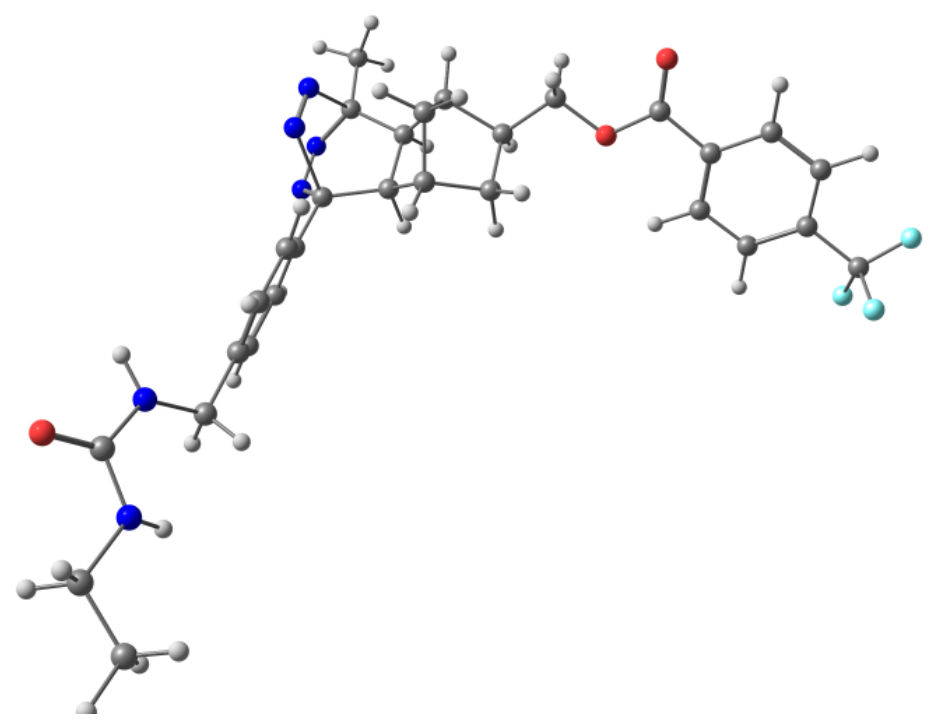

TS endo-NBN with anti $-\mathrm{N}_{2}$ elimination (tetrazine on surface)

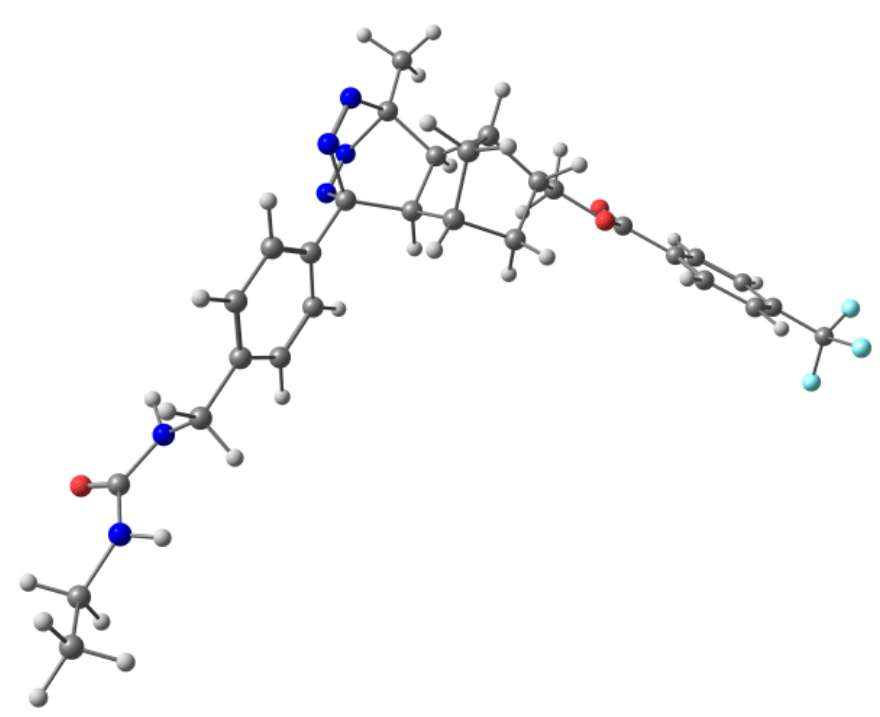


TS endo-NBN with syn $\mathrm{N}_{2}$ elimination (tetrazine on surface)

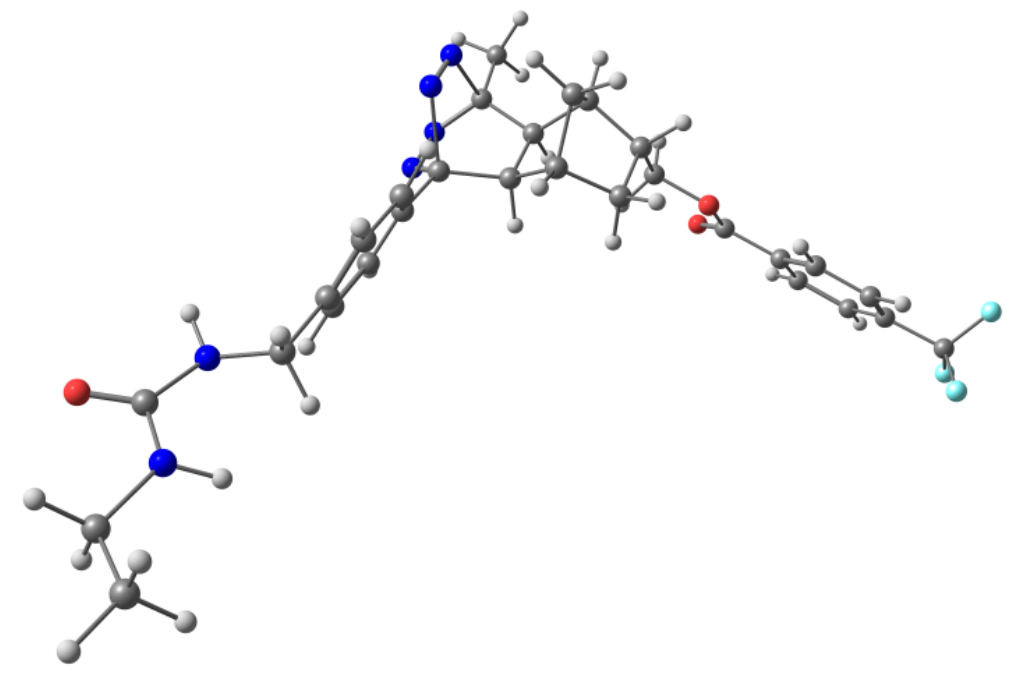

Product exo anti (tetrazine on surface)

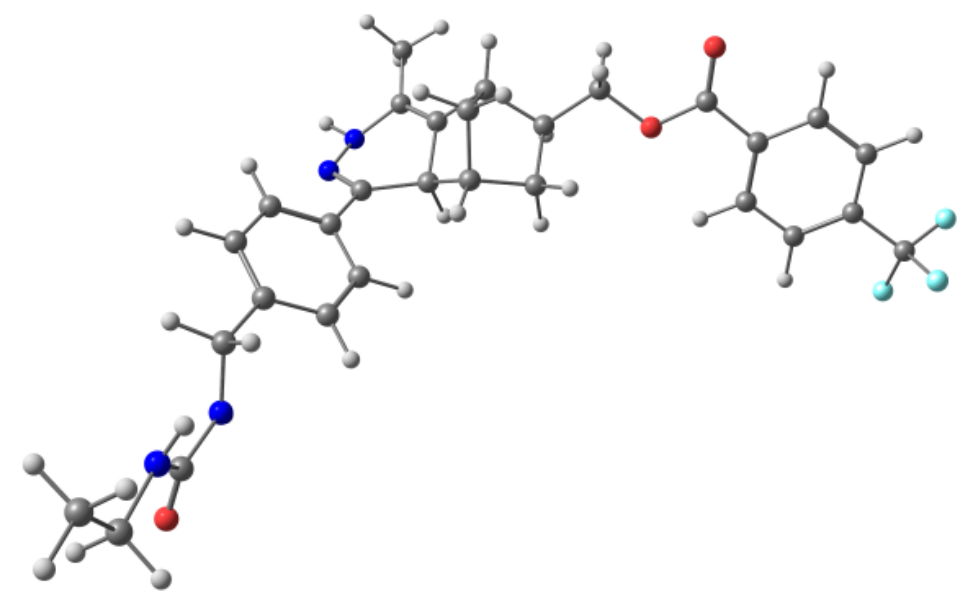

Product exo syn (tetrazine on surface)

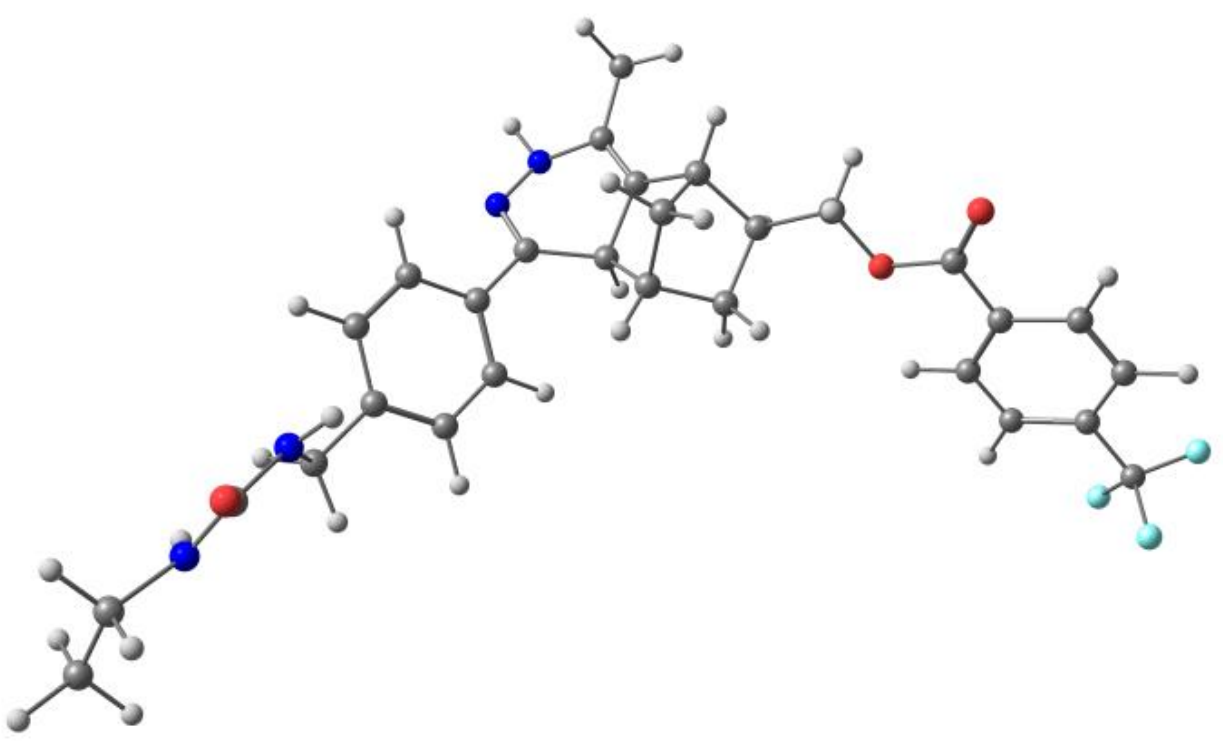


Product endo anti (tetrazine on surface)

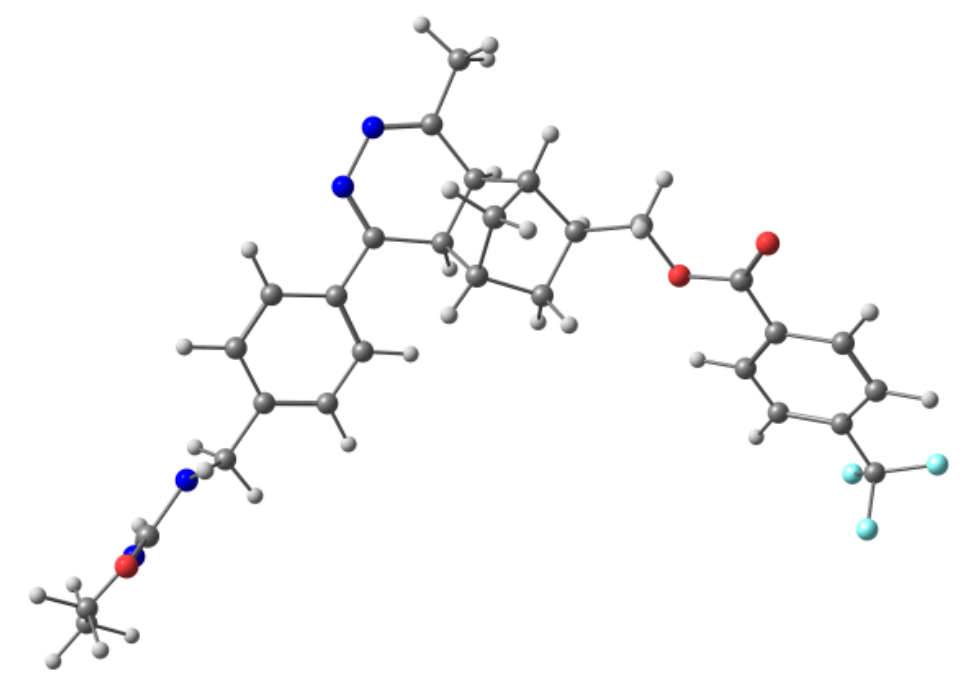

Product endo syn (tetrazine on surface)

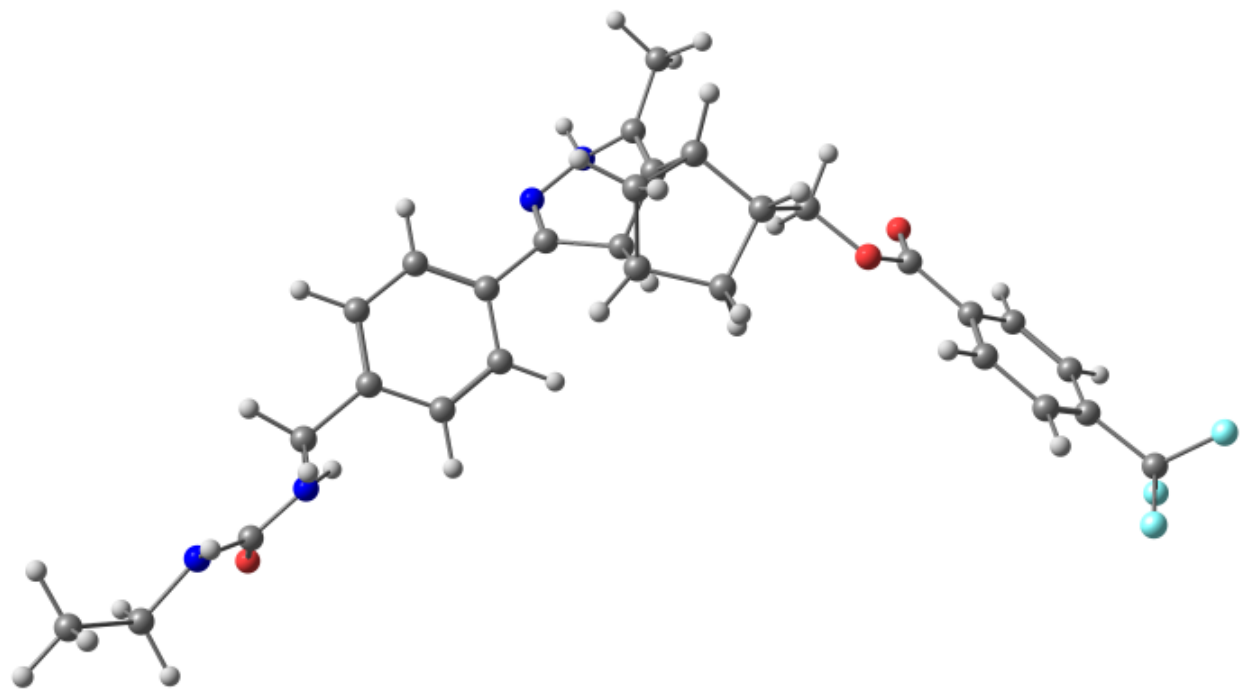

Tautomerization exo anti product (tetrazine on surface)

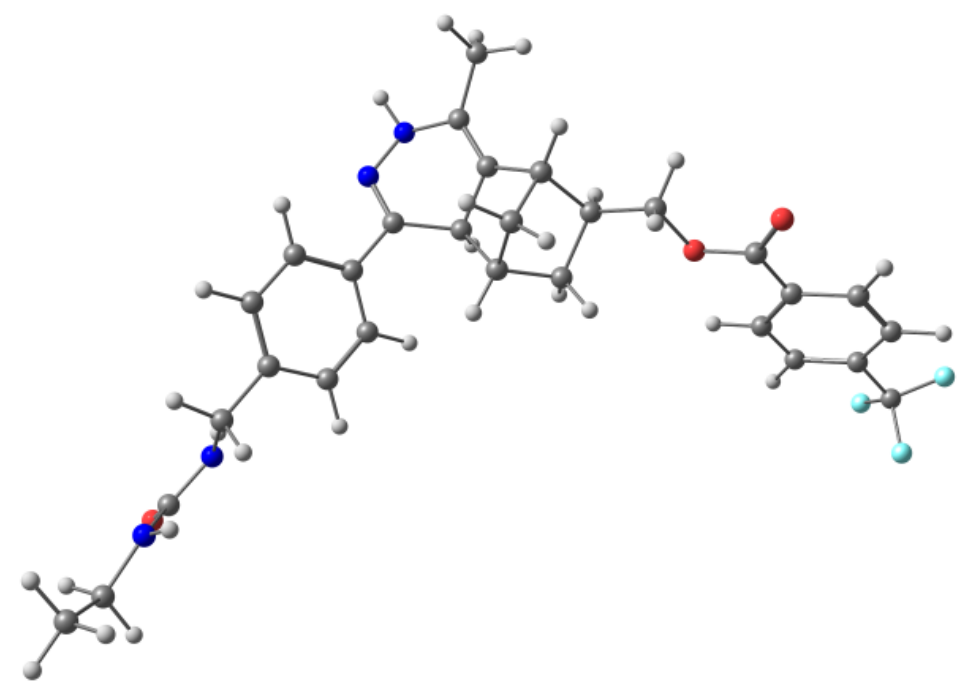


Tautomerization exo syn product(tetrazine on surface)

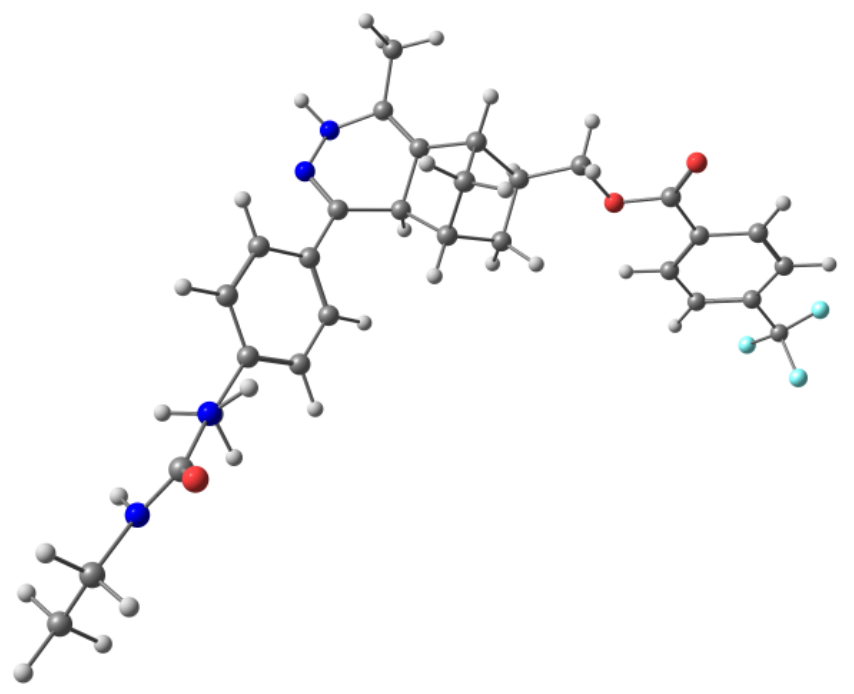

Tautomerization endo anti product (tetrazine on surface)

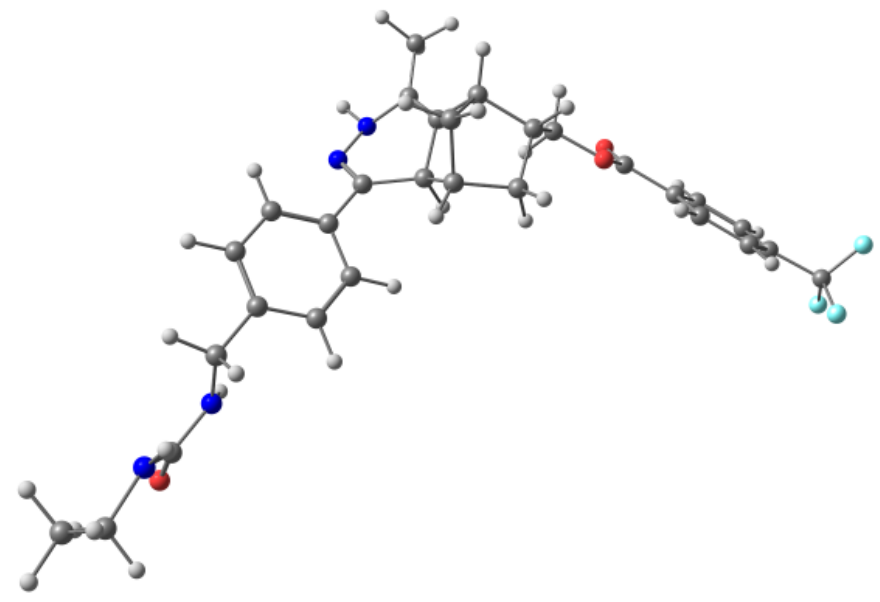

Tautomerization endo syn product (tetrazine on surface)

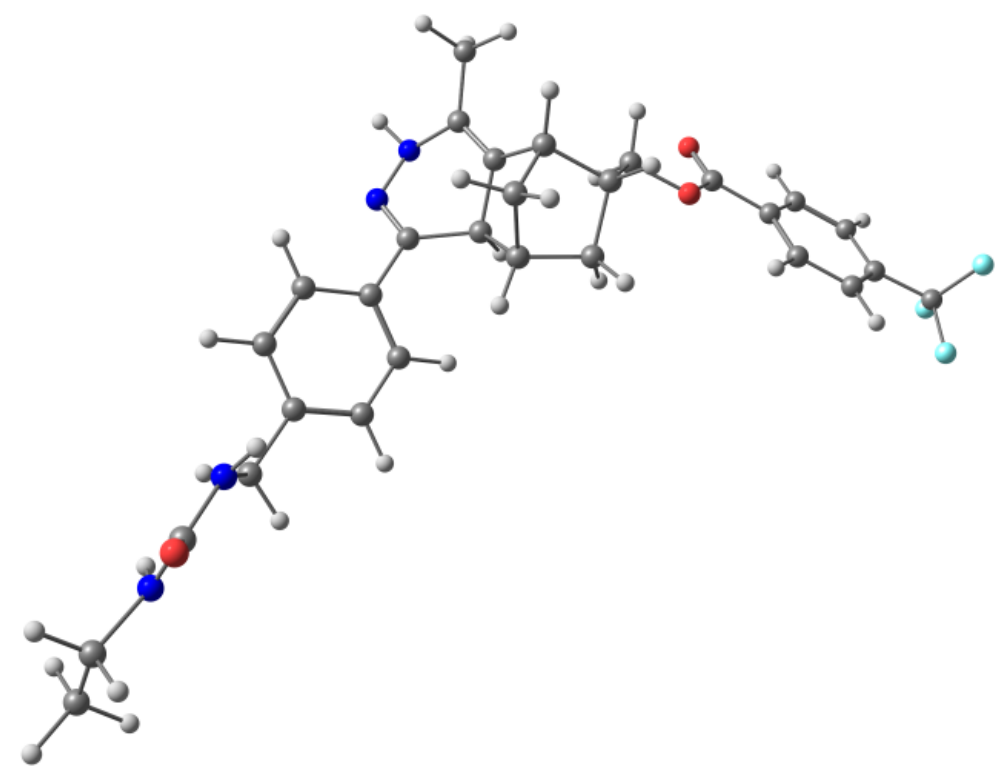




\section{Density Functional Theory (DFT) for XPS C1s Calculation}

Electronic core level calculations (ECC) were used to simulate core levels of C 1s XPS spectra. All ECC were done with the GAUSSIAN09 program. ${ }^{2}$ The effect of the bulk substrate on Al-O-P bound monolayers was mimicked by attaching the organic species to a $\mathrm{Al}\left(\mathrm{OH}_{2}\right)_{2}-$ moiety. The geometries of the different systems were optimized at the B3LYP/6-311G(d,p) level of theory. Natural bond orbital (NBO) analysis (Glendening, E. D.; Reed, A. E.; Carpenter, J. E.; Weinhold, F. NBO, Version 3.1) was employed to obtain the core orbital energies. The simulated XPS spectra were used to facilitate the peak fitting procedure for overlapping contributions in the experimental XPS data.

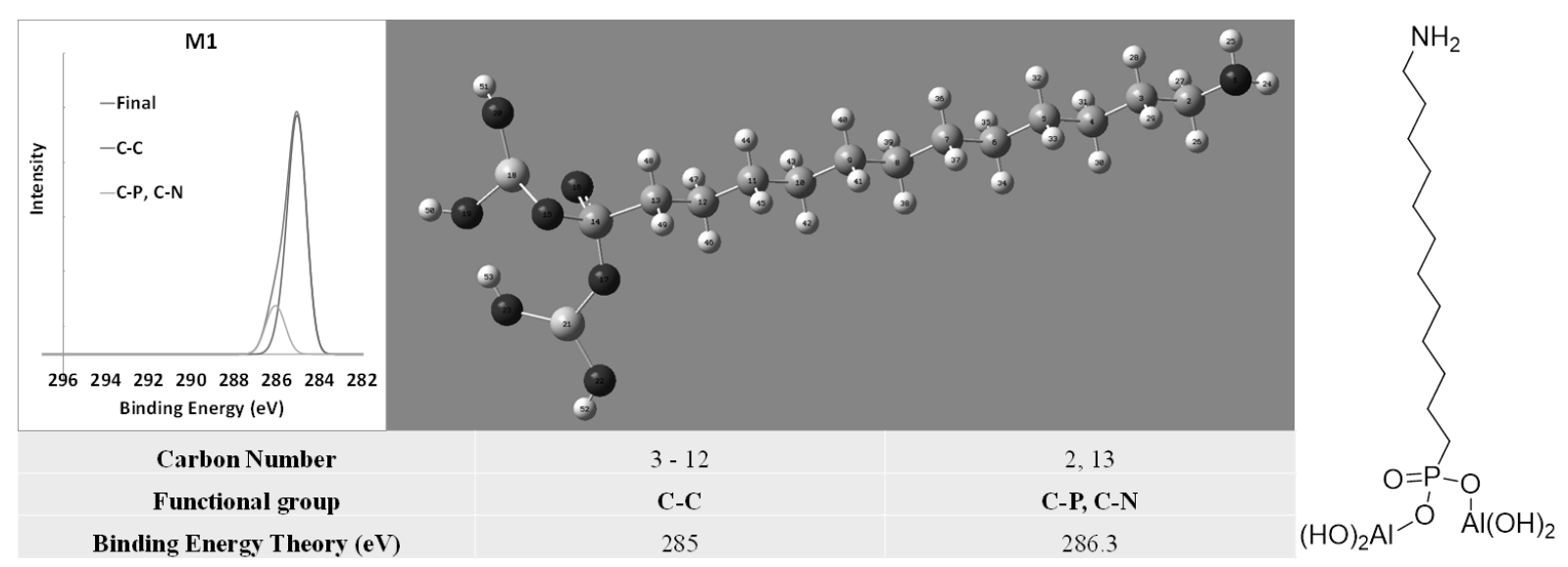

Figure S9.1. Core level C1s XPS spectra (left) for the amine terminated monolayer, simulation based on DFT calculations of the C1s core level energies for the different functional group binding energy shown in table (bottom), and [middle (Gaussview) and right(ChemDraw)) structure of model compound used for the calculation of binding energies. 


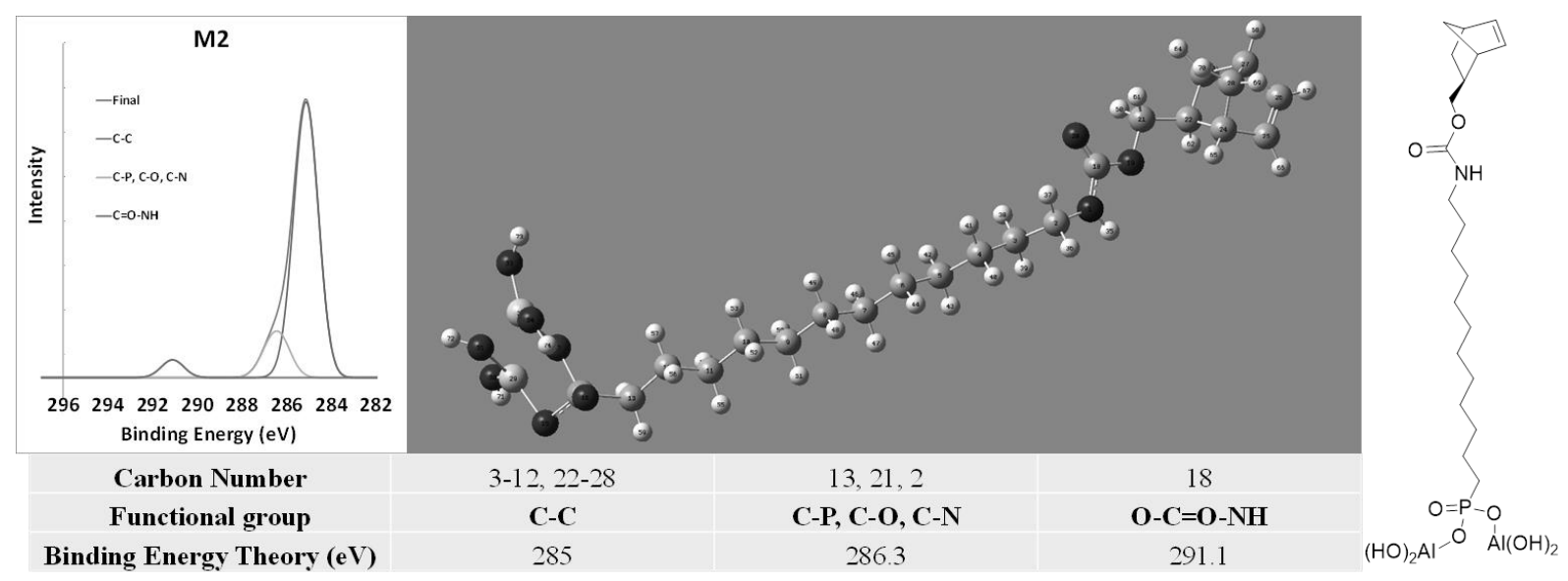

Figure S9.2. Core level C1s XPS spectra (left) for the exo-norbornene termination on amine monolayer, simulation based on DFT calculations of the C1s core level energies for the different functional group binding energy shown in table (bottom), and [middle (Gaussview) and right(ChemDraw)) structure of model compound used for the calculation of binding energies.

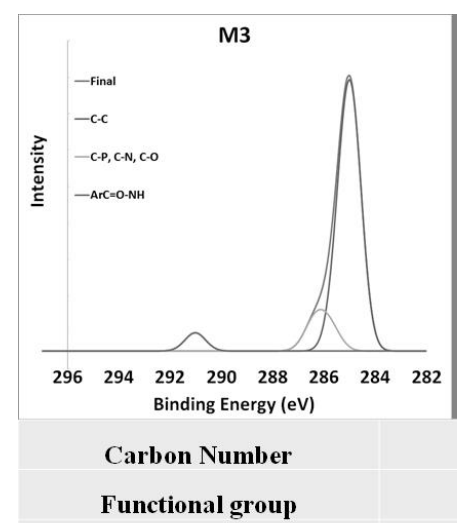

Binding Energy Theory (eV)

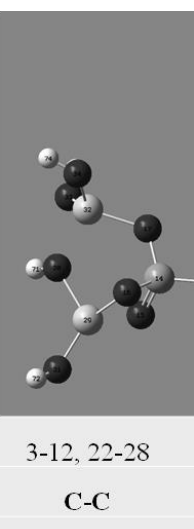

285

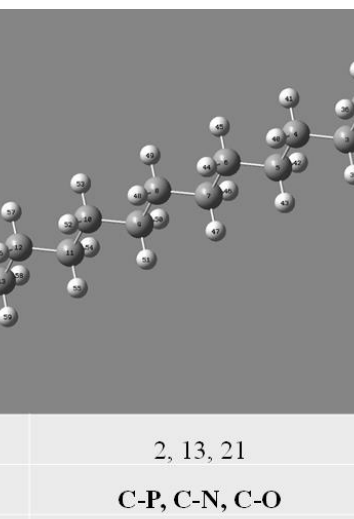

286.2
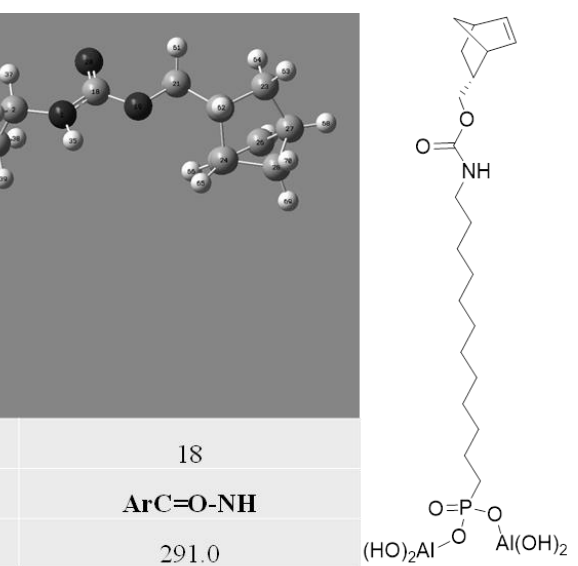

Figure S9.3. Core level C1s XPS spectra (left) for the endo-norbornene termination on amine monolayer, simulation based on DFT calculations of the C1s core level energies for the different functional group binding energy shown in table (bottom), and [middle (Gaussview) and right(ChemDraw)) structure of model compound used for the calculation of binding energies. 


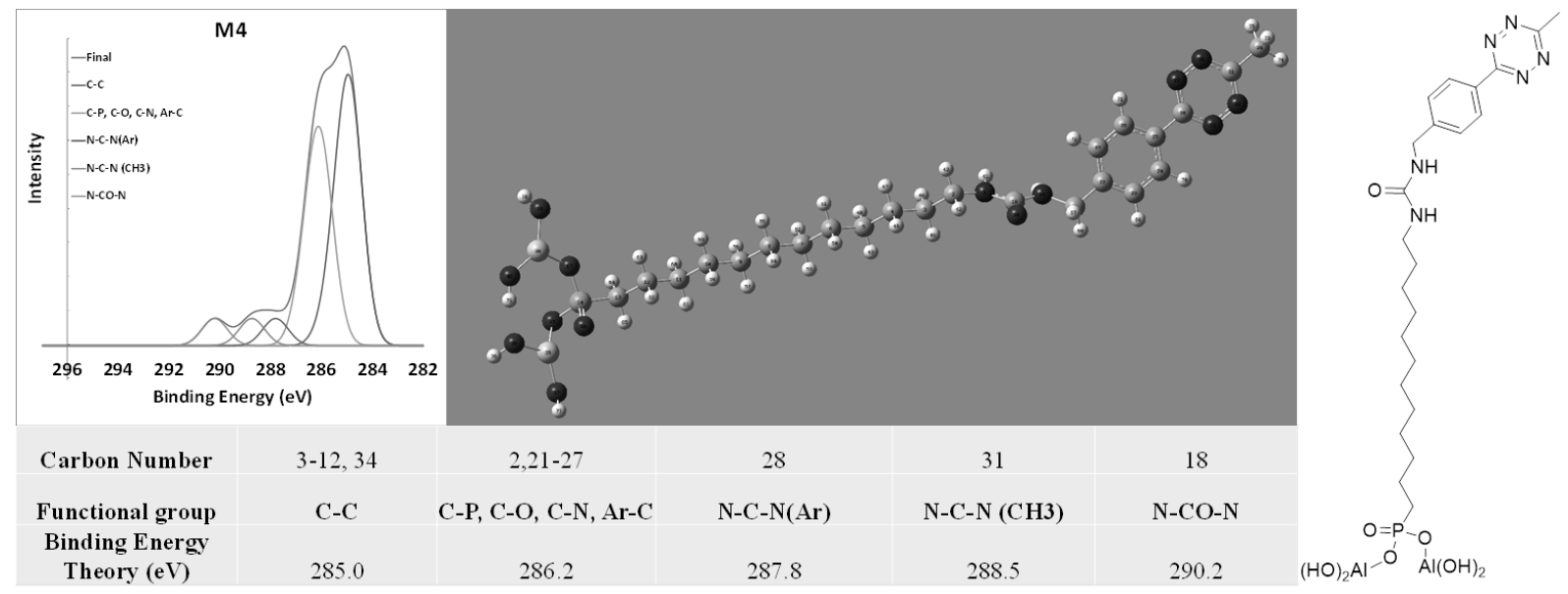

Figure S9.4. Core level C1s XPS spectra (left) for the tetrazine termination on amine monolayer, simulation based on DFT calculations of the C1s core level energies for the different functional group binding energy shown in table (bottom), and [middle (Gaussview) and right (ChemDraw)) structure of model compound used for the calculation of binding energies.

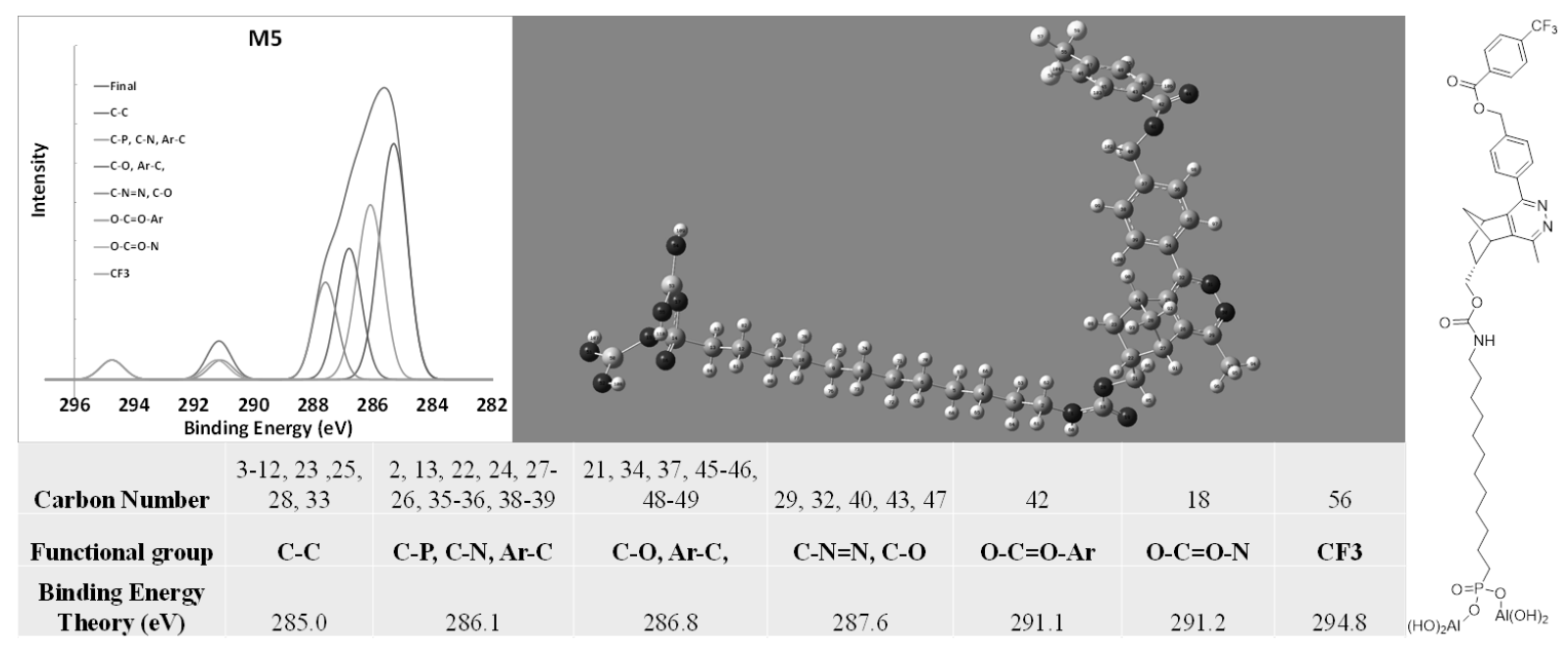

Figure S9.5. Core level C1s XPS spectra (left) for the IEDDA cycloadduct for endo-norbornene termination on amine monolayer, simulation based on DFT calculations of the $\mathrm{C} 1 \mathrm{~s}$ core level energies for the different functional group binding energy shown in table (bottom), and [middle (Gaussview) and right (ChemDraw)) structure of model compound used for the calculation of binding energies. 


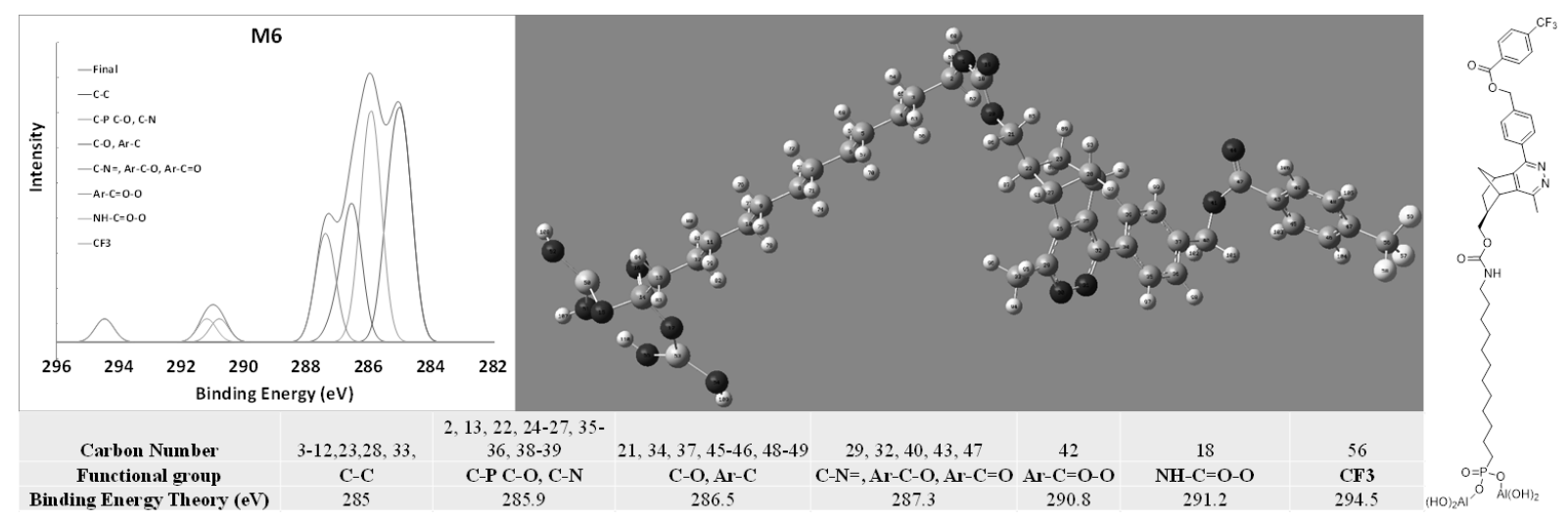

Figure S9.6. Core level C1s XPS spectra (left) for the IEDDA cycloadduct for exonorbornene termination on amine monolayer, simulation based on DFT calculations of the C1s core level energies for the different functional group binding energy shown in table (bottom), and [middle (Gaussview) and right (ChemDraw)) structure of model compound used for the calculation of binding energies.

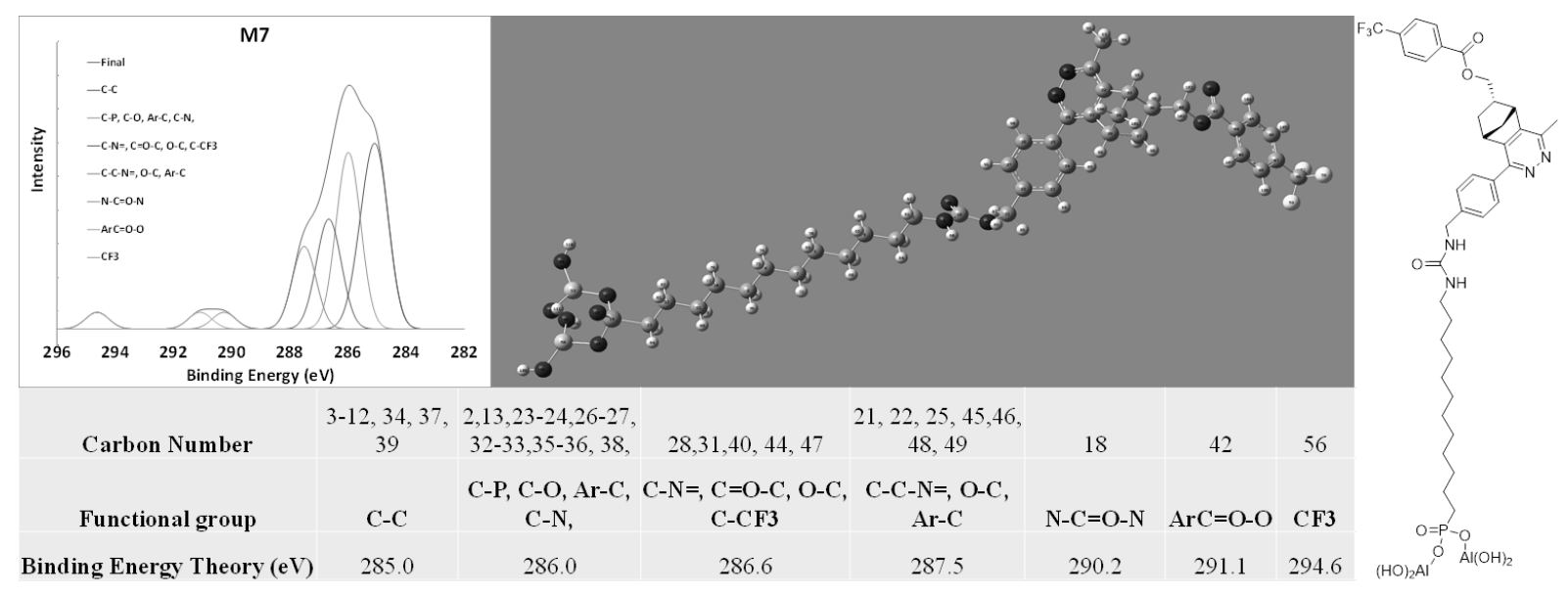

Figure S9.7. Core level C1s XPS spectra (left) for the IEDDA cycloadduct for endo-norbornene termination on amine monolayer, simulation based on DFT calculations of the C1s core level energies for the different functional group binding energy shown in table (bottom), and [middle (Gaussview) and right (ChemDraw)) structure of model compound used for the calculation of binding energies. 


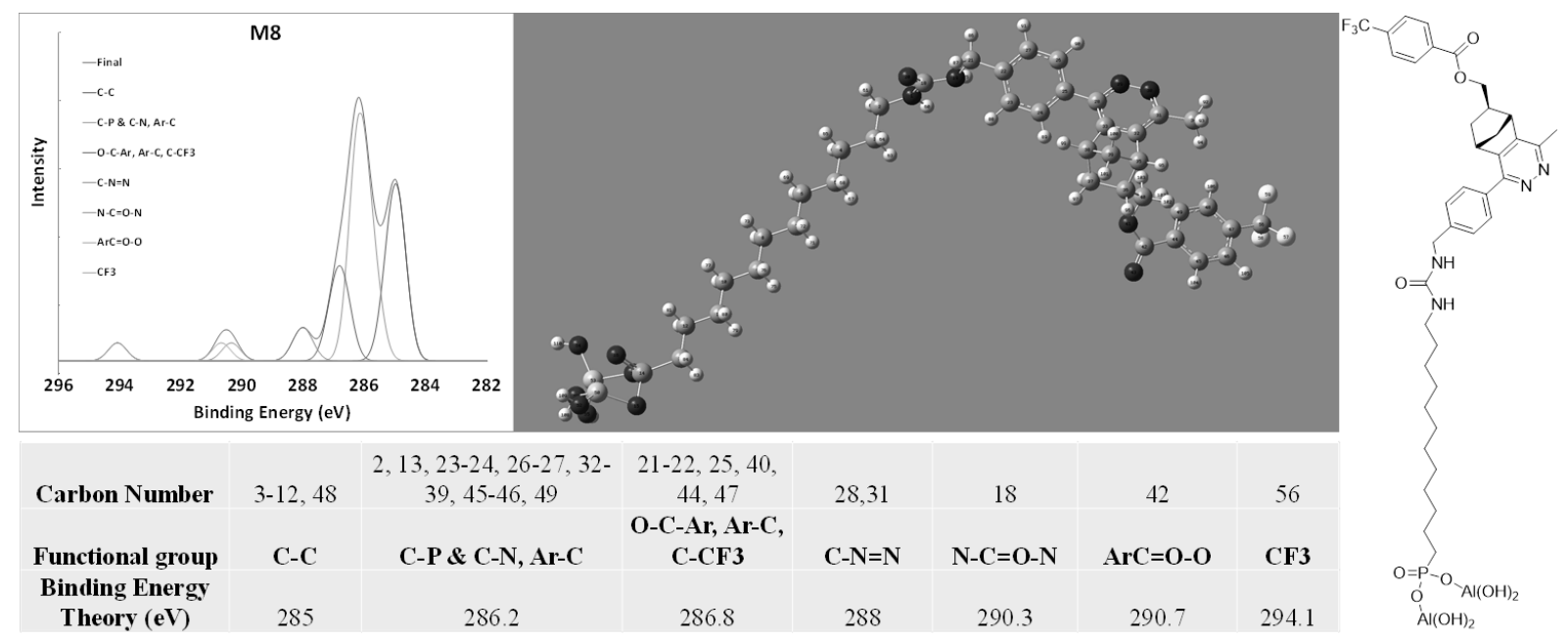

Figure S9.8. Core level C1s XPS spectra (left) for the IEDDA cycloadduct for exonorbornene termination on amine monolayer, simulation based on DFT calculations of the C1s core level energies for the different functional group binding energy shown in table (bottom), and [middle (Gaussview) and right(ChemDraw)) structure of model compound used for the calculation of binding energies.

\section{References}

(1) Sen, R.; Escorihuela, J.; Smulders, M. M. J.; Zuilhof, H. Langmuir 2016, 32, 3412.

(2) Frisch, M. J.; Trucks, G. W.; Schlegel, H. B.; Scuseria, G. E.; Robb, M. A.; Cheeseman, J. R.; Scalmani, G.; Barone, V.; Mennucci, B.; Petersson, G. A.; Nakatsuji, H.; Caricato, M.; Li, X.; Hratchian, H. P.; Izmaylov, A. F.; Bloino, J.; Zheng, G.; Sonnenberg, J. L.; Hada, M.; Ehara, M.; Toyota, K.; Fukuda, R.; Hasegawa, J.; Ishida, M.; Nakajima, T.; Honda, Y.; Kitao, O.; Nakai, H.; Vreven, T.; Montgomery Jr., J. A.; Peralta, J. E.; Ogliaro, F.; Bearpark, M. J.; Heyd, J.; Brothers, E. N.; Kudin, K. N.; Staroverov, V. N.; Kobayashi, R.; Normand, J.; Raghavachari, K.; Rendell, A. P.; Burant, J. C.; Iyengar, S. S.; Tomasi, J.; Cossi, M.; Rega, N.; Millam, N. J.; Klene, M.; Knox, J. E.; Cross, J. B.; Bakken, V.; Adamo, C.; Jaramillo, J.; Gomperts, R.; Stratmann, R. E.; Yazyev, O.; Austin, A. J.; Cammi, R.; Pomelli, C.; Ochterski, J. W.; Martin, R. L.; Morokuma, K.; Zakrzewski, V. G.; 
Voth, G. A.; Salvador, P.; Dannenberg, J. J.; Dapprich, S.; Daniels, A. D.; Farkas, Ö.; Foresman, J. B.; Ortiz, J. V.; Cioslowski, J.; Fox, D. J.; Gaussian, Inc.: Wallingford, CT, USA, 2009.

(3) Zhao, Y.; Truhlar, D. G. Theoretical Chemistry Accounts 2008, 120, 215.

(4) Liu, F.; Paton, R. S.; Kim, S.; Liang, Y.; Houk, K. N. Journal of the American Chemical Society 2013, 135, 15642.

(5) Törk, L.; Jiménez-Osés, G.; Doubleday, C.; Liu, F.; Houk, K. N. Journal of the American Chemical Society 2015, 13\%, 4749.

(6) Gonzalez, C.; Schlegel, H. B. The Journal of Physical Chemistry 1990, 94,5523 .

(7) Yang, J.; Karver, M. R.; Li, W.; Sahu, S.; Devaraj, N. K. Angew. Chem. Int. Ed. 2012, 51, 5222. 


\section{Appendix 4}

\section{Ultrathin Covalently Bound Organic Layers on}

Mica: Formation of Atomically Flat Bio-

functionalizable Surfaces

For full Supporting Information including Cartesian coordinates look at online ESI available in Angewandte Chemie International website at $\underline{\text { anie201701301-sup }-0001-\text { misc information.pdf }}$

\section{Table of Contents}

4.1. Methods.

4.2. Chemical Synthesis......

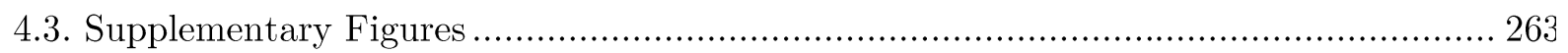

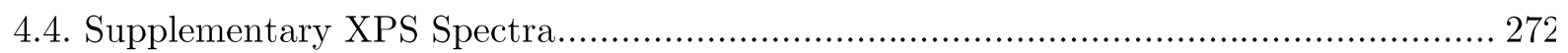

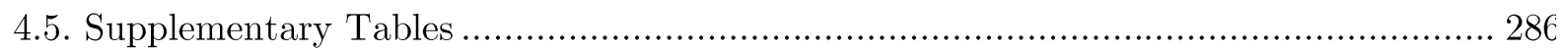

4.6. DFT simulations for XPS C1s narrow scan assignments.................................. $28 \varepsilon$ 


\subsection{Methods}

Materials. Unless otherwise specified, all chemicals were used as received without further purification. Muscovite mica surfaces (Grade V-1, $12 \mathrm{~mm}$ diameter $\times 0.15$ mm thick) were purchased from SPI supplies. 2,3-dihydroxy benzoic acid (DHBA), N-Boc-1,6-hexanediamine, trifluoroacetic acid (TFA), 1H-imidazole-1-sulfonyl azide- $\mathrm{HCl}$ salt, copper sulphate pentahydrate, 1,1'-carbonyldiimidazole, 2,3dihydroxyl benzylamine hydrobromide, sodium periodate, 2,2'-azino-bis $(3-$ ethylbenzothiazoline-6-sulfonic acid) diammonium salt $\left(\right.$ ABTS $\left.^{2-}\right), \quad$ Fe(III)protoporphyrin IX chloride, hydrogen peroxide, potassium carbonate, PBS buffer, ethyl acetate, methanol, hexane, acetone, dichloromethane (DCM) and 2-propanol were purchased from Sigma-Aldrich. For rinsing, solution preparation and contact angle measurements, Milli-Q water (resistivity $18.3 \mathrm{M} \Omega \mathrm{cm}$ ) was used. Oligonucleotides were purchased from Integrated DNA Technologies (IDT), Belgium. Static Water Contact Angle (SCA) Measurements. The wettability of the modified surfaces was determined by automated static water contact angle measurements with a Krüss DSA 100 goniometer (volume of the drop of deionized water was $3.0 \mu \mathrm{L}$ ). The reported values are the average of at least six droplets, and the relative error is less than $\pm 1^{\circ}$.

X-ray Photoelectron Spectroscopy (XPS) Measurements. The XPS analysis of surfaces was performed using a JPS-9200 photoelectron spectrometer (JEOL, Japan). Survey and narrow scan spectra were obtained under UHV conditions using monochromatic $\mathrm{Al} \mathrm{K} \alpha \mathrm{X}$-ray radiation at $12 \mathrm{kV}$ and $20 \mathrm{~mA}$, and an analyser pass energy of $50 \mathrm{eV}$ for wide scans and $10 \mathrm{eV}$ for narrow scans. The emitted electrons were collected at $10^{\circ}$ from the surface normal (take-off angle relative to the surface normal $10^{\circ}$ ). All XPS spectra were evaluated by using Casa XPS software (version 2.3.15). Survey spectra were corrected with linear background before fitting, whereas high-resolution spectra were corrected with linear background. Atomic area ratios were determined after a baseline correction and normalizing the peak area ratios by 
the corresponding atomic sensitivity factors (1.00 for C1s, 1.80 for N1s, 2.93 for O1s, 4.43 for F1s, 1.18 for P2s and 0.75 for Al2s, 5.03 for Br3p, 2.27 for K2s).

DART-HRMS Measurements. Analysis of the modified mica surfaces were performed using a DART-SVP ion source (Ion-Sense, Saugus, MA, USA) coupled to a Q-Exactive orbitrap high-resolution mass spectrometer (Thermo Fisher Scientific, San Jose, CA), mounted on a motorized rail travelling at $0.2 \mathrm{~mm} / \mathrm{s}$. Thermo Scientific Xcalibur software (V2.1.0.1139) was used for data acquisition and processing. The measurements were performed in negative mode at $450{ }^{\circ} \mathrm{C}$ using a scan range of $\mathrm{m} / \mathrm{z} 180.0$ - 345.0, a mass resolution of 70,000 (FWHM) at a scan rate of $1 \mathrm{~Hz}$. The ion trap was tuned with $0.1 \mathrm{mg} / \mathrm{mL}$ methanol solution of quinine $(\mathrm{m} / \mathrm{z}$ 323.41 in negative mode) and optimized. The DART source was positioned $6.1 \mathrm{~cm}$ on the horizontal scale, $7 \mathrm{~cm}$ on the vertical scale with an angle of $45^{\circ}$, such that it is around $1 \mathrm{~mm}$ above the surface (Fig. S1). The distance from the surface to the ceramic tube is minimized by placing them at the edge of the moving rail so that maximum of the $p-\mathrm{CF}_{3}$ benzoate ion and $p-\mathrm{C}_{4} \mathrm{~F}_{9}$ benzoate ions $(m / z 189.016$ and 339.0062 , respectively) would enter the MS.

Atomic Force Microscopy (AFM) Imaging. AFM imaging was performed under air using a Digital Instruments NanoScope V Multimode Scanning Probe Microscopy with a noncontact silicon nitride cantilever with a stiffness of $0.58 \mathrm{~N} / \mathrm{m}$ (Veeco Metrology, CA) at a scan speed of $1 \mu \mathrm{m} / \mathrm{s}$, in the Scan-assist imaging mode. Images were flattened with a third-order flattening procedure using Nanoscope Analysis software (v1.5)

Preparation of modified mica samples $\left[\mathbf{M}_{0}-\mathbf{M}_{1}\right]$. Mica samples were washed with copious amounts of acetone, methanol and dichloromethane and dried under a stream of nitrogen gas. Samples were then left undisturbed for $16 \mathrm{~h}$ in $5 \mathrm{mM}$ solution of 1 in PBS buffer ( $\mathrm{pH}$ 7.4) in sealed vials under nitrogen atmosphere. Both sides of the mica were found to be identically modified without preference. Multiple samples 
(up to 25) could be simultaneously modified in this manner. The samples were sonicated in water for $15 \mathrm{~min}$ after the modification and stored for future applications.

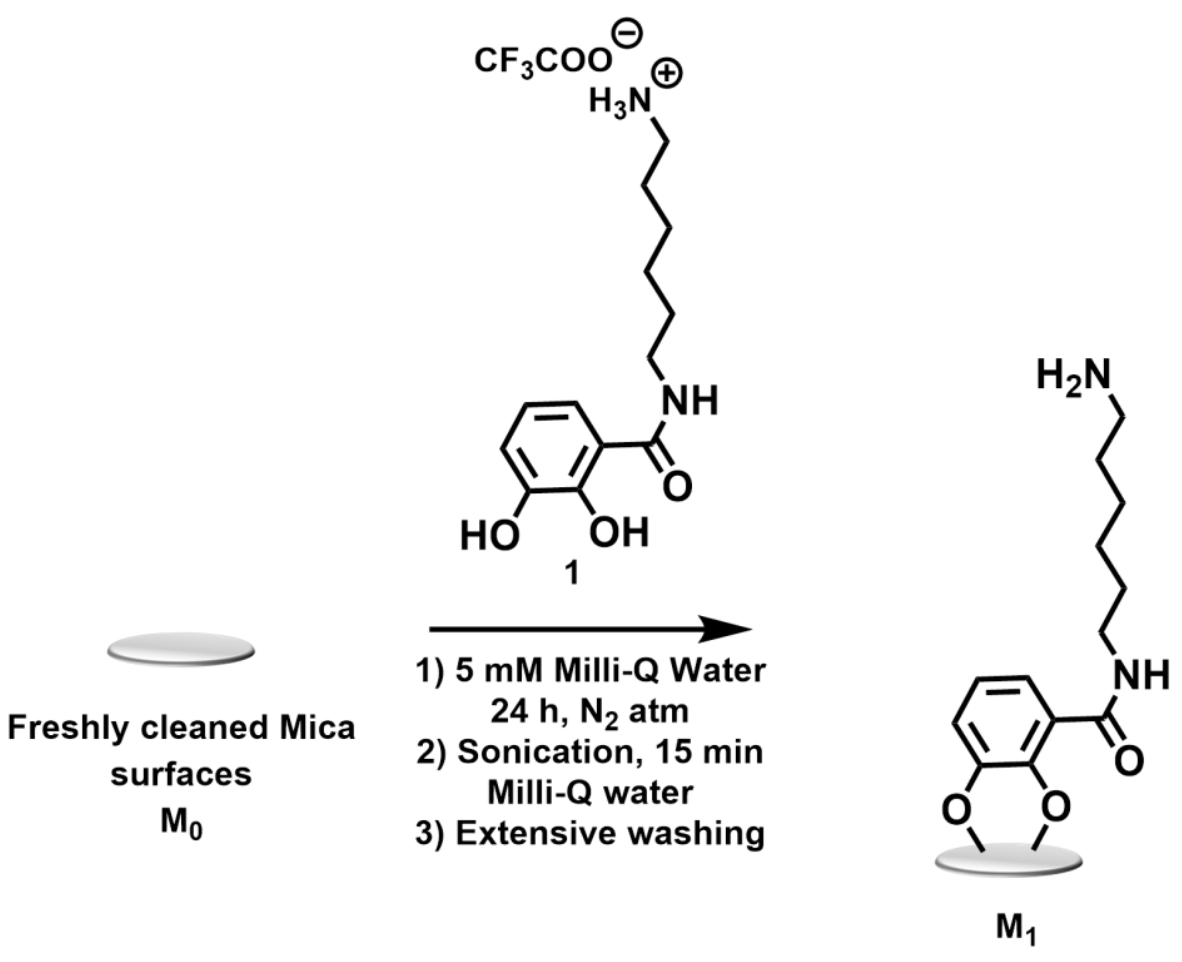

Scheme 1. Attachment of surface anchor 1 to a freshly cleaned mica sample.

4.1.1 Preparation of azide-terminated surface. $\mathbf{M}_{1}$ samples were stirred with a $50 \mathrm{mM}$ solution of $1 \mathrm{H}$-imidazole-1-sulfonyl azide- $\mathrm{HCl}$ salt, $\mathrm{CuSO}_{4} .5 \mathrm{H}_{2} \mathrm{O}$ and $\mathrm{K}_{2} \mathrm{CO}_{3}$ in water for $2 \mathrm{~h}$, followed by sonication in water for $15 \mathrm{~min}$, to afford azideterminated samples $\mathbf{M}_{2}$. The samples were stored under nitrogen atmosphere for further use.

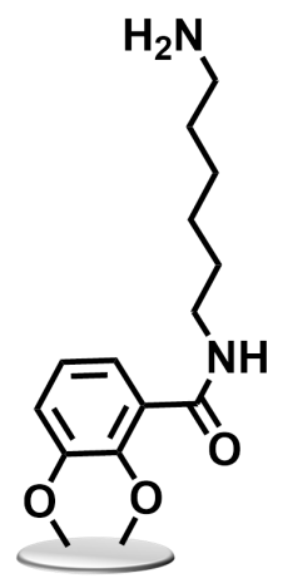

$M_{1}$
(1) $50 \mathrm{mM}$ 1H-imidazole-1-sulfonyl azide- $\mathrm{HCl}$ salt $\mathrm{CuSO}_{4} .5 \mathrm{H}_{2} \mathrm{O}, \mathrm{K}_{2} \mathrm{CO}_{3}, \mathrm{H}_{2} \mathrm{O}, 2 \mathrm{~h}$

(2) Sonication in water, $15 \mathrm{~min}$

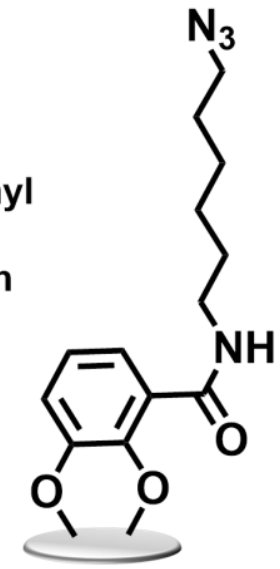

$M_{2}$ 
Scheme 2. Conversion of amine-terminated surface anchor 1 to azide-terminated layers.

4.1.2 Interfacial SPAAC on azide-terminated surface. Azide-terminated samples $\mathbf{M}_{2}$ were stirred $16 \mathrm{~h}$ with endo-BCN- $\mathrm{C}_{4} \mathrm{~F}_{9} \mathrm{MS}$ tag, $\mathbf{2}$, in DCE solvent at 22 ${ }^{\circ} \mathrm{C}$, followed by sonication in dichloromethane for $15 \mathrm{~min}$. The samples were dried under a stream of nitrogen and used for XPS and DART-HRMS measurements. The blue tag connected by aromatic ester linkage with endo-BCN, $\mathbf{2}$, is used to determine the yield on the surface of the SPAAC reaction independently by XPS (F1s signal at $686.0 \mathrm{eV})$ and DART-HRMS $(m / z 339.0062)$.

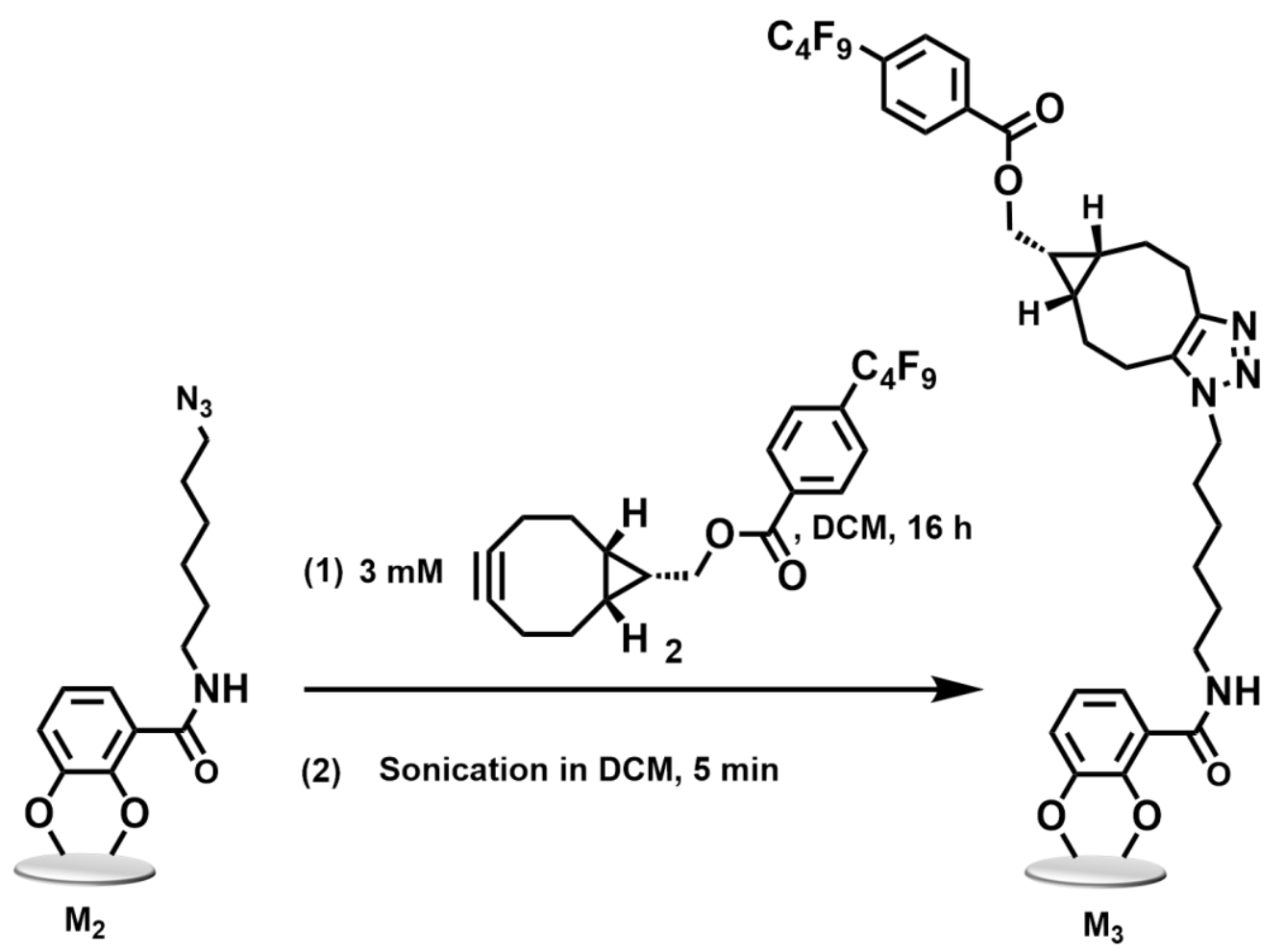

Scheme 3. Interfacial SPAAC reaction between an azide-terminated mica surface and endo-BCN 2 in solution.

4.1.3 Preparation of endo-BCN terminated surfaces Amine-terminated mica was activated using 1,1'-carbonyldiimidazole (50 mM) in water for $1 \mathrm{~h}$. The activated surfaces were linked with endo- $\mathrm{BCN}-\mathrm{OH}, \mathbf{3}$, via carbamate linkage. 


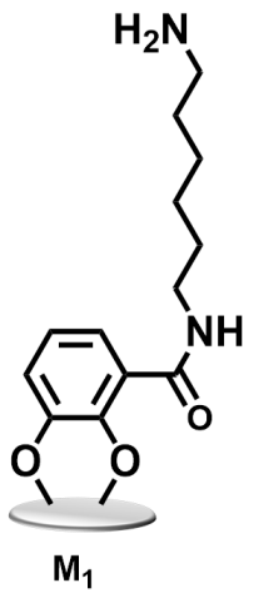

(1) $\mathrm{CDI}, 1 \mathrm{~h}, \mathrm{H}_{2} \mathrm{O}$

(2)

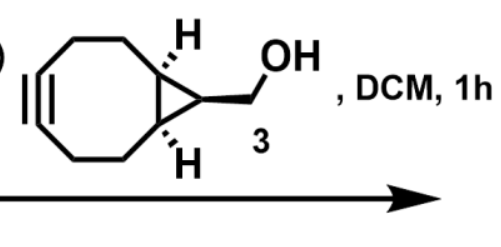

(3) Sonication, DCM, $15 \mathrm{~min}$

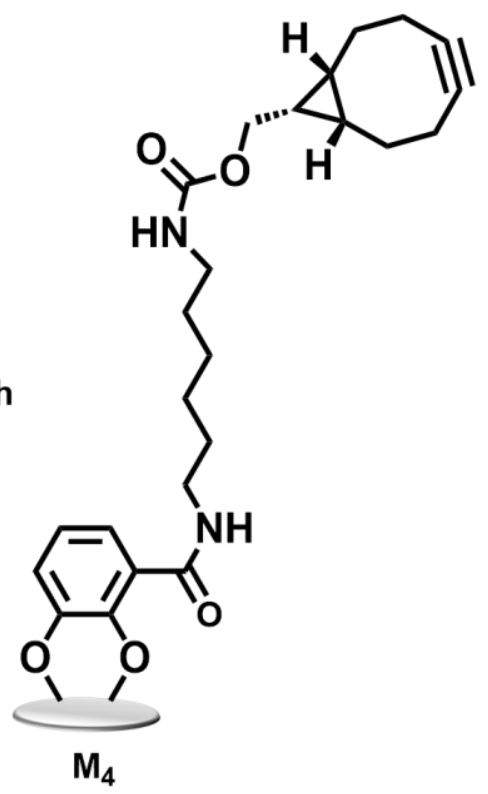

Scheme 4. Preparation of endo-BCN-terminated layers on mica.

4.1.4 Interfacial SPAAC on endo-BCN-terminated surface. Freshly prepared endo-BCN-terminated samples were stirred $16 \mathrm{~h}$ with $3 \mathrm{mM}$ azideterminated counterpart in dichloromethane solution. The azide moiety was coupled with fluorinated tag via aromatic ester bond, which allowed for independent verification of surface yield by XPS (F1s signal at $686.0 \mathrm{eV})$ and DART-HRMS $(\mathrm{m} / z$ 189.016).

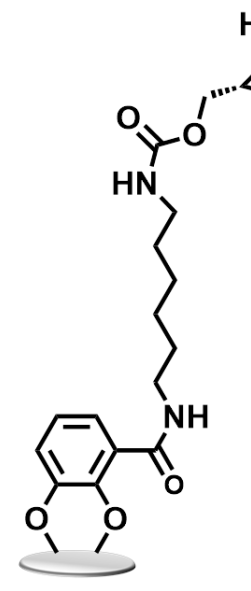

$\mathrm{M}_{4}$

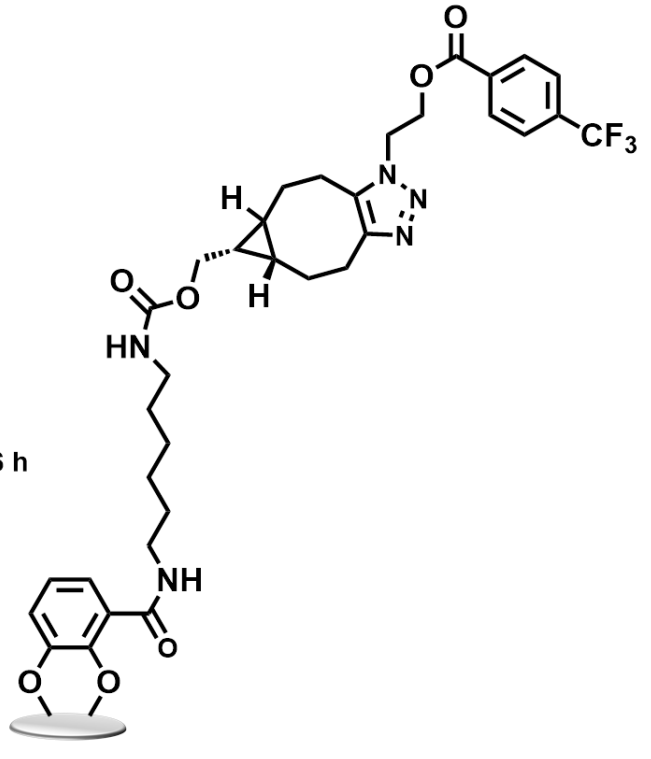

$\mathrm{M}_{5}$

Scheme 5. Interfacial SPAAC reaction between endo-BCN-terminated surfaces and azide counterpart $\mathbf{4}$ in solution. 
4.1.5 Interfacial SPOCQ reaction on mica. Amine terminated mica surfaces $\mathbf{M}_{1}$ were activated by CDI coupling as described earlier and stirred with 2,3dihydroxybenzylamine hydrobromide, to afford catechol terminated layers. These were oxidized to 1,2-dihydroxy quinones by oxidation with aqueous $50 \mathrm{mM} \mathrm{NaIO}_{4}$ solution in $30 \mathrm{~min}$. The samples were washed with copious amounts of water followed by sonication for $15 \mathrm{~min}$ in methanol. The resulting surfaces were then stirred with $\mathbf{2}$ to get the SPOCQ cycloaddition product in $4 \mathrm{~h}$ with excellent surface yields. Blank measurements were carried out rigorously to ensure no false positives resulting from physisorption and to prove the orthogonality of the reaction.

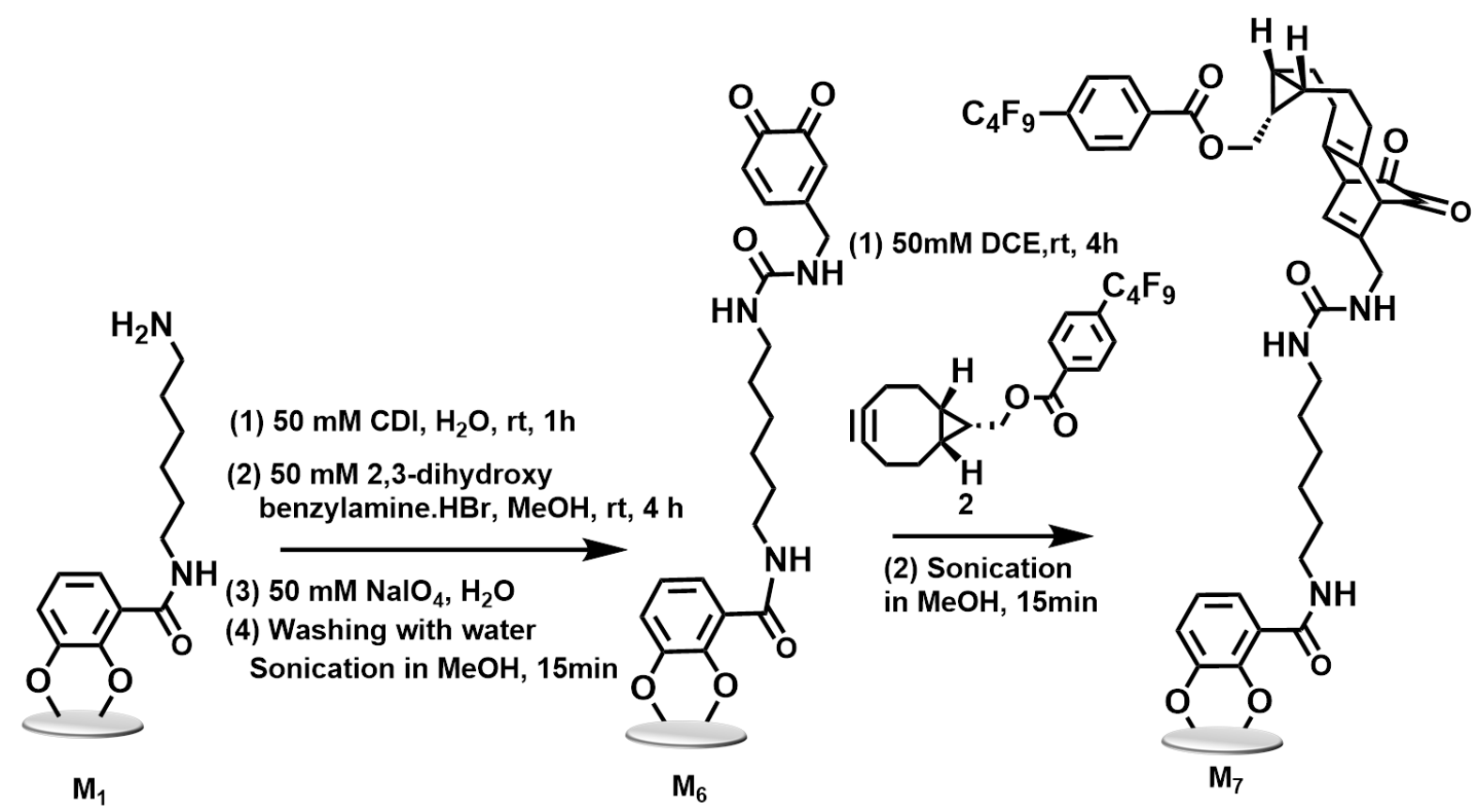

Scheme 6. Interfacial SPOCQ reaction between surfacial $1,2-$ quinones $\left[\mathbf{M}_{6}\right]$ and endo-BCN counterpart, 2 , in solution.

\subsubsection{Proof that sonication techniques are adequate to remove physisorbed} moieties. To ensure that all signals are entirely due to covalently bound moieties alone, all surfaces were sonicated in corresponding solvents for $15 \mathrm{~min}$. A stepwise control experiment was carried out in which endo-BCN-terminated surfaces $\mathbf{M}_{4}$ was reacted with azide terminated counterpart with MS tag $4(m / z 189.016)$ followed by sonication in dichloromethane for $15 \mathrm{~min}$. These surfaces were subsequently stirred in 
endo-BCN with MS tag $2(\mathrm{~m} / z$ 339.0062) and sonicated again. The surfaces were subject to DART-HRMS to see if any MS-tag 2 could be detected, which would only be possible if any residual azide remained physisorbed on the surface after the sonication process. Since no trace of MS tag $\mathbf{2}$ could be detected, (Figure S6) we concluded that the sonication time and solvent chosen is adequate to remove physisorbed moieties.

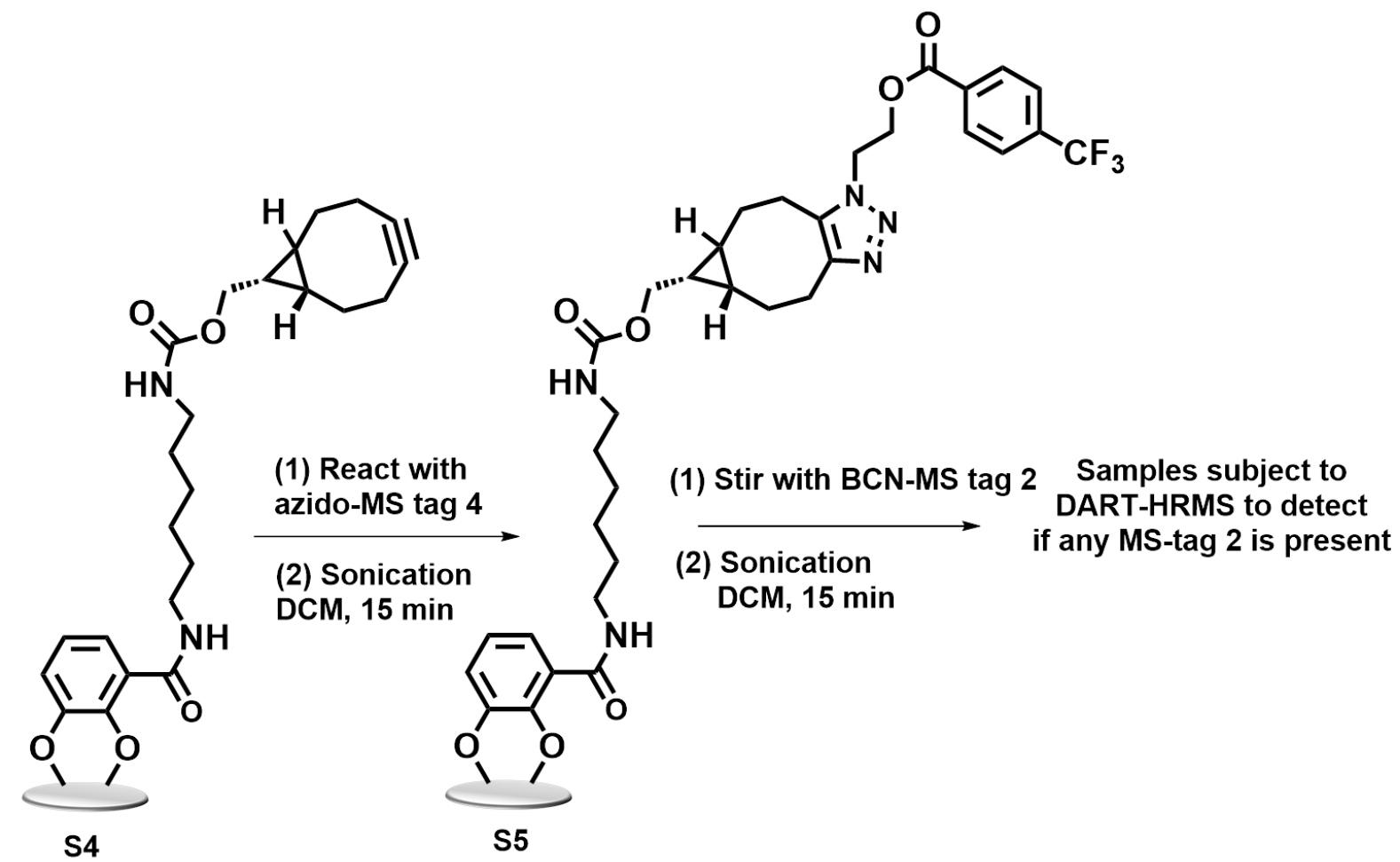

Scheme 7. Control experiment designed to detect if sonication time and solvent is adequate enough to remove all traces of physisorbed moieties after the modification. 
4.1.7 Formation of DNA minicircles. $\mathbf{M}_{1}$ surfaces were activated through CDI coupling as described earlier. Activated samples were stirred overnight in $100 \mu \mathrm{M}$ aqueous solution of $\mathbf{D} 1$. The samples were sonicated for $10 \mathrm{~min}$ in water to remove physisorbed DNA molecules. Subsequent hybridization was performed with $10 \mu \mathrm{M}$ aqueous solution of D2-D9 overnight (16 h). The samples were carefully sonicated in water and immediately used for AFM analysis.

\subsubsection{Formation of EAD2 DNAzyme and oxidative activity on mica. $\mathrm{M}_{1}$} surfaces were activated through CDI coupling as described earlier. Activated samples were stirred overnight in $100 \mu \mathrm{M}$ aqueous solution of EAD2. This was followed by formation of Heme-G quadruplex by stirring the samples in a $10 \mathrm{mM}$ solution of Fe(III)-protoporphyrin IX chloride (Tris-HCl, pH 8) containing $10 \mathrm{mM} \mathrm{KCl}$ for $1 \mathrm{~h}$. Samples were washed with water and sonicated before use. Peroxidation reaction was carried out at $25{ }^{\circ} \mathrm{C}$ in $100 \mu \mathrm{M}$ PBS (pH 6.0) with $5 \mu \mathrm{M}$ ABTS diammonium salt and $5 \mu \mathrm{M} \mathrm{H}_{2} \mathrm{O}_{2}$. The modified mica samples were placed in a sealed vial and solutions of ABTS $^{2-}$ and the $\mathrm{H}_{2} \mathrm{O}_{2}$ were mixed on a mechanical stirred. Formation of the oxidized product $\left(\mathrm{ABTS}^{-}{ }^{\bullet}\right.$ ) was monitored by UV-Vis analysis $(200-500 \mathrm{~nm})$.of the solution at different time points $(5,15,30$ and $60 \mathrm{~min})$. Blank experiments were carried out to ensure the catalytic activity was due to the DNAzymes. 


\subsection{Chemical Synthesis}

4.2.1 General Remarks. Unless stated otherwise, solvents and dry solvents like dichloromethane, methanol and isopropanol were purchased from Sigma-Aldrich. Unless stated otherwise all of these chemicals were used without further purification.

4.2.2. Reaction Handling. Unless stated otherwise all non-aqueous reactions were performed in dried glassware under an atmosphere of argon. All flasks were equipped with rubber septa and reactants were handled using standard Schlenk techniques. Temperatures above the room temperature refer to oil bath temperatures which were controlled by a thermostat. Reactions were magnetically stirred.

4.2.3. $1 \mathbf{H}-\mathbf{N M R}$ spectra were recorded at room temperature on a Bruker AVB-400 spectrometer with ${ }^{1} \mathrm{H}$ operating frequency of $400 \mathrm{MHz}$. Unless stated otherwise all spectra were recorded at room temperature in $\mathrm{CDCl}_{3}$ and all chemical shifts are given in $\delta$ units relative to the residual $\mathrm{CHCl}_{3}$ (central line of singlet: $\delta_{\mathrm{H}}=7.27 \mathrm{ppm}$ ). Analyses followed first order and the following abbreviations were used throughout: s $=$ singlet, br. $\mathrm{s} .=$ broad singlet, $\mathrm{d}=$ doublet, $\mathrm{t}=$ triplet, $\mathrm{q}=$ quartet, quin $=$ quintet, sxt $=$ sextet, sept $=\mathrm{spt}, \mathrm{dd}=$ doublet of doublet, $\mathrm{dt}=$ doublet of triplet, $\mathrm{m}$ $=$ multiplet, $\mathrm{mc}=$ centered multiplet. Coupling constants $(J)$ are given in Hertz $(\mathrm{Hz})$.

4.2.4. 13C-NMR spectra were recorded at room temperature on a Bruker AVB400 spectrometer with ${ }^{13} \mathrm{C}$ operating frequency of $101 \mathrm{MHz}$. Unless stated otherwise all spectra were recorded at room temperature in $\mathrm{CDCl}_{3}$ and all chemical shifts are given in $\delta$ units relative to the $\mathrm{CDCl}_{3}$ (central line of triplet: $\delta_{\mathrm{C}}=77.0 \mathrm{ppm}$ ). The following abbreviation was used throughout: $\mathrm{s}=$ singlet, $\mathrm{d}=$ doublet, $\mathrm{dd}=$ doublet of doublet. If no coupling constants are given, the multiplicity refers to the ${ }^{1} \mathrm{H}-$ decoupled spectra. 


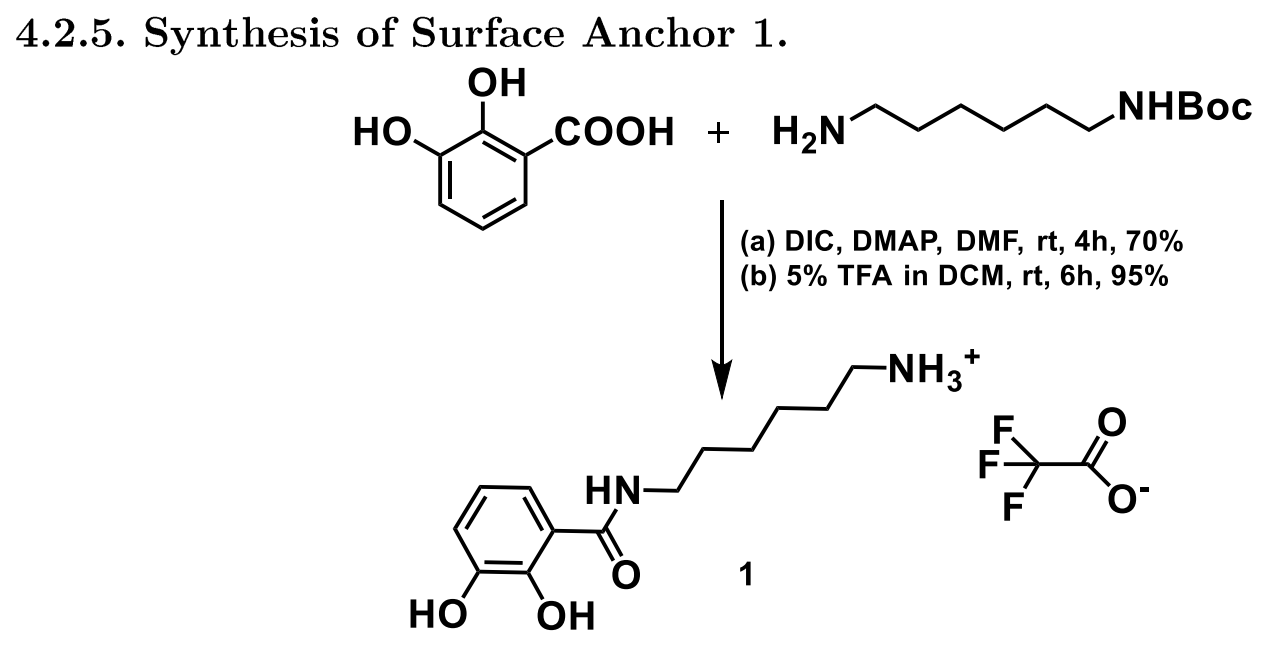

2,3-Dihydroxybenzoic acid (500 $\mathrm{mg}, 3.24 \mathrm{mmol}$ ) was weighed and suspended in $3 \mathrm{~mL}$ anhydrous DMF followed by addition of DIC $(0.5 \mathrm{~mL}, 3.50 \mathrm{mmol})$, triethylamine (1 $\mathrm{mL}, 7.13 \mathrm{mmol}$ ) and DMAP (39 $\mathrm{mg}, 0.32 \mathrm{mmol})$. This was followed by addition of $\mathrm{N}$-Boc 1,6-diaminohexane $(902 \mathrm{mg}, 3.5 \mathrm{mmol})$ and allowed to react for $3 \mathrm{~h}$ at room temperature, under Ar atmosphere. The reaction was monitored by TLC until completion. The solvent was removed under reduced pressure and the resultant residue was purified by column chromatography (EtOAc: Heptane 50:50 v/v) as a colorless oil, (yield: 0.798 g, 70\%) and immediately used. ${ }^{1} \mathrm{H}$ NMR (400 $\mathrm{MHz}, \mathrm{CDCl}_{3}$ ) $\delta 7.03(\mathrm{dd}, \mathrm{J}=7.9,1.3 \mathrm{~Hz}, 1 \mathrm{H}), 7.01(\mathrm{dd}, \mathrm{J}=7.8,1.3 \mathrm{~Hz}, 1 \mathrm{H}), 6.73(\mathrm{t}, \mathrm{J}=8.0 \mathrm{~Hz}$, $1 \mathrm{H}), 4.62(\mathrm{t}, \mathrm{J}=6.2 \mathrm{~Hz}, 1 \mathrm{H}), 3.42(\mathrm{q}, \mathrm{J}=6.6 \mathrm{~Hz}, 1 \mathrm{H}), 3.13(\mathrm{q}, \mathrm{J}=6.6 \mathrm{~Hz}, 1 \mathrm{H}), 1.60$ $(\mathrm{d}, \mathrm{J}=6.9 \mathrm{~Hz}, 1 \mathrm{H}), 1.46(\mathrm{~d}, \mathrm{~J}=6.8 \mathrm{~Hz}, 1 \mathrm{H}), 1.44(\mathrm{~s}, 6 \mathrm{H}), 1.36(\mathrm{dd}, \mathrm{J}=5.3,3.2 \mathrm{~Hz}$, $1 \mathrm{H})$.

Boc-deprotection was performed immediately using 5\% TFA in DCM (3mL) for $4 \mathrm{~h}$. The solvent was removed under vacuum and the residue was washed with HPLC grade hexane to yield a brown solid (yield: $0.788 \mathrm{~g}, 95 \%) \cdot{ }^{1} \mathrm{H} \mathrm{NMR}\left(400 \mathrm{MHz}, \mathrm{D}_{2} \mathrm{O}\right.$ ) $\delta 7.12(\mathrm{~d}, \mathrm{~J}=8.0 \mathrm{~Hz}, 1 \mathrm{H}), 6.98(\mathrm{~d}, \mathrm{~J}=7.9 \mathrm{~Hz}, 1 \mathrm{H}), 6.78(\mathrm{~d}, \mathrm{~J}=8.0 \mathrm{~Hz}, 1 \mathrm{H}), 3.29$ $(\mathrm{t}, \mathrm{J}=7.0 \mathrm{~Hz}, 2 \mathrm{H}), 2.89(\mathrm{t}, \mathrm{J}=7.6 \mathrm{~Hz}, 2 \mathrm{H}), 1.65-1.41(\mathrm{~m}, 3 \mathrm{H}), 1.32(\mathrm{p}, \mathrm{J}=3.5$ $\mathrm{Hz}, 4 \mathrm{H}) ;{ }^{13} \mathrm{C} \mathrm{NMR}\left(101 \mathrm{MHz}, \mathrm{D}_{2} \mathrm{O}\right) \delta 170.15,147.50,144.85,119.69,119.55,118.82$, $116.73,39.76,39.59,28.38,26.85,25.84,25.47$. 

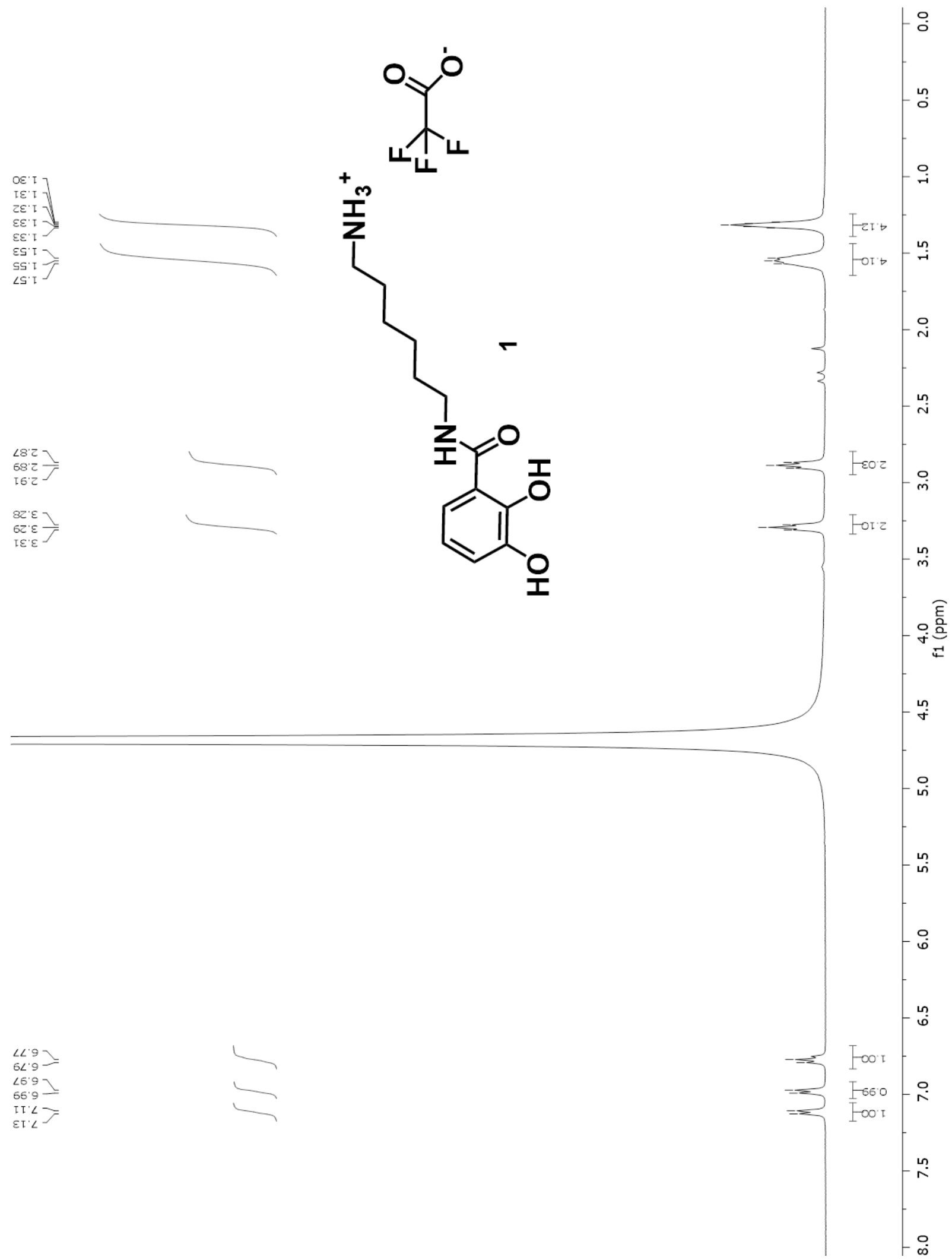

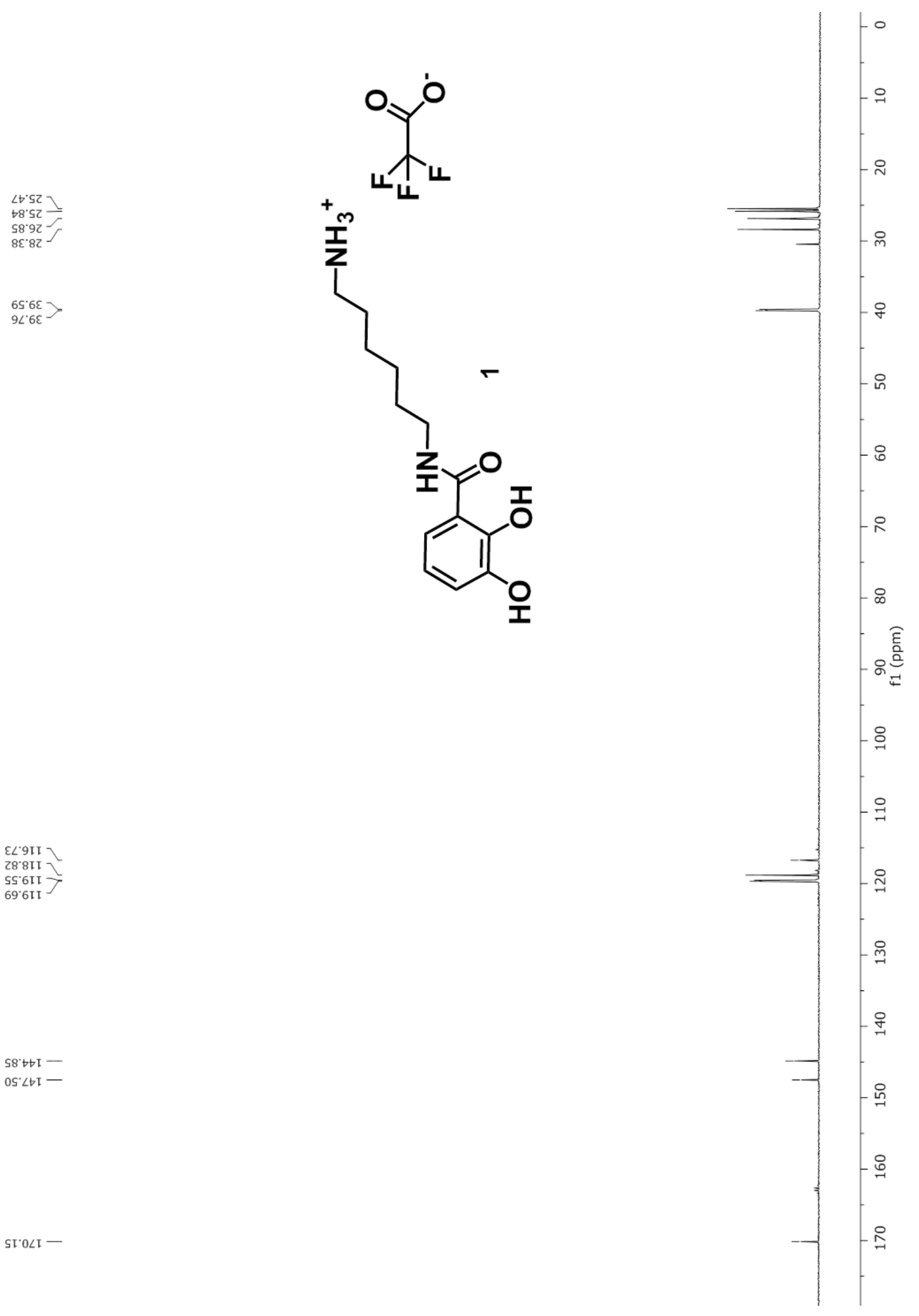

$\left.\begin{array}{l}\varepsilon L^{\circ} 9 \mathrm{II} \\ 28^{\circ} 8 \mathrm{II} \\ \mathrm{C}^{\circ} 6 \mathrm{II}\end{array}\right]$

SS.6II

s8't十I -

$0 S^{\circ} \angle b I$

SI. $0 \angle I-$ 


\subsubsection{Synthesis of 2-azidoethanol from 2-bromoethanol.

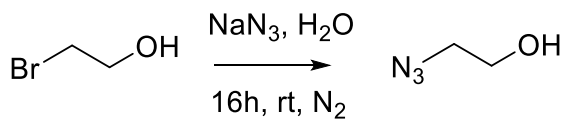

Sodium azide (15.6 g, $0.24 \mathrm{~mol}$ ) was dissolved in $100 \mathrm{~mL}$ milliQ water, followed by the addition of $5.67 \mathrm{~mL}$ of 2 -bromoethanol $(10 \mathrm{~g}, 0.08 \mathrm{~mol})$. The mixture was heated at $80{ }^{\circ} \mathrm{C}$ overnight under $\mathrm{N}_{2}$ flow, during which the color changed from yellow to orange. The product was extracted four times with $75 \mathrm{~mL}$ diethyl ether. The combined organic fractions were dried and evaporated under reduced pressure. The product was obtained as a clear oil. Yield: $54 \%$. The ${ }^{1} \mathrm{H}-\mathrm{NMR}$ spectrum was in accordance with literature values (Giorgio et al. Molecular Pharmaceutics 2013, 10 (3), 975-978). ${ }^{1} \mathrm{H}-\mathrm{NMR}\left(400 \mathrm{MHz}, \mathrm{CDCl}_{3}\right) \delta 3.75(\mathrm{~m}, 2 \mathrm{H}), 3.42(\mathrm{t}, \mathrm{J}=5.0 \mathrm{~Hz}, 2 \mathrm{H})$, $2.66(\mathrm{~s}, 1 \mathrm{H})$.

\subsubsection{Synthesis of 2-azidoethyl 4-(trifluoromethyl)benzoate (4).}<smiles>NCCOC(OCCN)C(=O)OCC(=O)c1ccc(C(F)(F)F)cc1</smiles>

4-(Trifluoromethyl)benzoic acid (13.0 g, $68.8 \mathrm{mmol}$ ) was transferred to a threenecked round bottom flask (100 mL) dicyclohexylcarbodiimide (DCC) (13.60 g, 66.02 mmol) and 4-dimethylaminopyridine (DMAP) (4.00 g, $33.33 \mathrm{mmol})$ dissolved in 100 $\mathrm{mL}$ DCM were added to the flask. The reaction mixture was stirred for $30 \mathrm{~min}$ before adding 2-azidoethanol $(6.66 \mathrm{~g}, 65.55 \mathrm{mmol})$. The final reaction mixture was left stirring overnight at room temperature. The reaction mixture was then filtered and evaporated under reduced pressure, forming a yellow oil. The crude product was purified by column chromatography 10:90 ethyl acetate : hexane as solvent. Yield: $35 \% .{ }^{1} \mathrm{H}-\mathrm{NMR}\left(400 \mathrm{~Hz}, \mathrm{CDCl}_{3}\right) \delta 8.19(\mathrm{~d}, \mathrm{~J}=8.4 \mathrm{~Hz}, 2 \mathrm{H}), 7.74(\mathrm{~d}, \mathrm{~J}=8.0 \mathrm{~Hz}, 2 \mathrm{H})$, $4.54(\mathrm{t}, \mathrm{J}=5.2 \mathrm{~Hz}, 2 \mathrm{H}), 3.63(\mathrm{t}, \mathrm{J}=5.2 \mathrm{~Hz}, 2 \mathrm{H}) .{ }^{13} \mathrm{C}-\mathrm{NMR}\left(101 \mathrm{~Hz}, \mathrm{CDCl}_{3}\right) \delta$ $165.02,135.27,132.74,130.15,127.64,125.47,64.09,49.85$. 


\subsection{Supplementary Figures}

Supplementary Figure S1. Height profile of bare mica $\left(\mathbf{M}_{0}\right)$ showing roughness of $0.1 \pm 0.01 \mathrm{~nm}$ across six random profiles drawn across the surface.

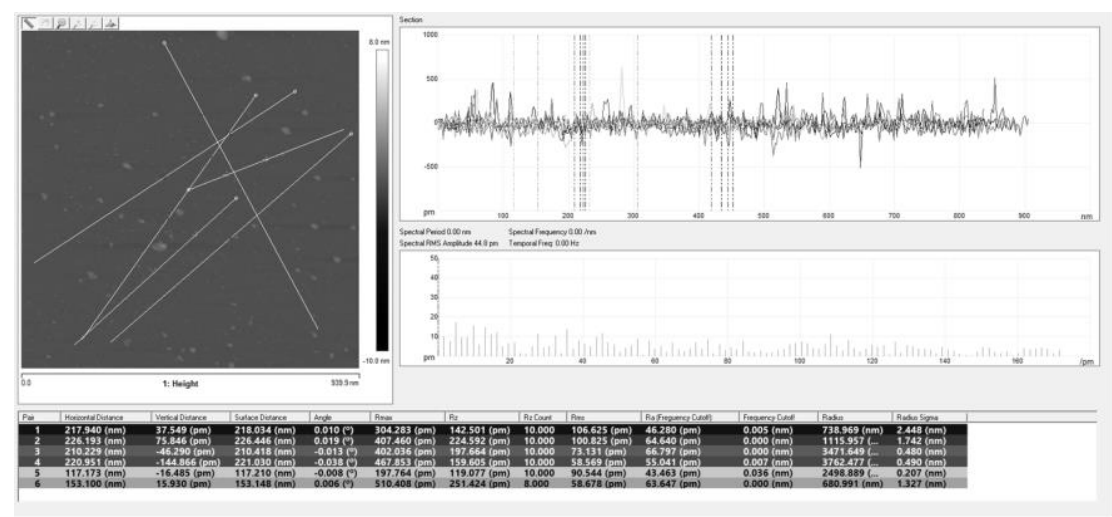

Supplementary Figure S2. Thickness of covalently bound layers of 1 as estimated across a hole on the surface of modified mica $\mathbf{M}_{1}$.

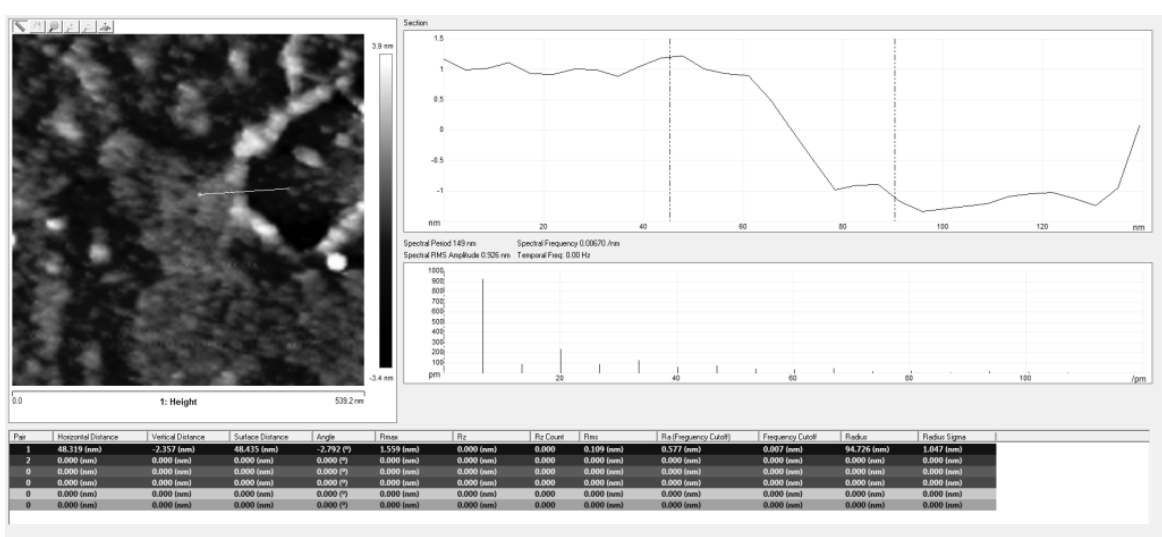

Supplementary Figure S3. Height profile of $\mathbf{M}_{1}$ mica surface showing a roughness of $0.45 \pm 0.1 \mathrm{~nm}$ across six random profiles drawn across the surface.

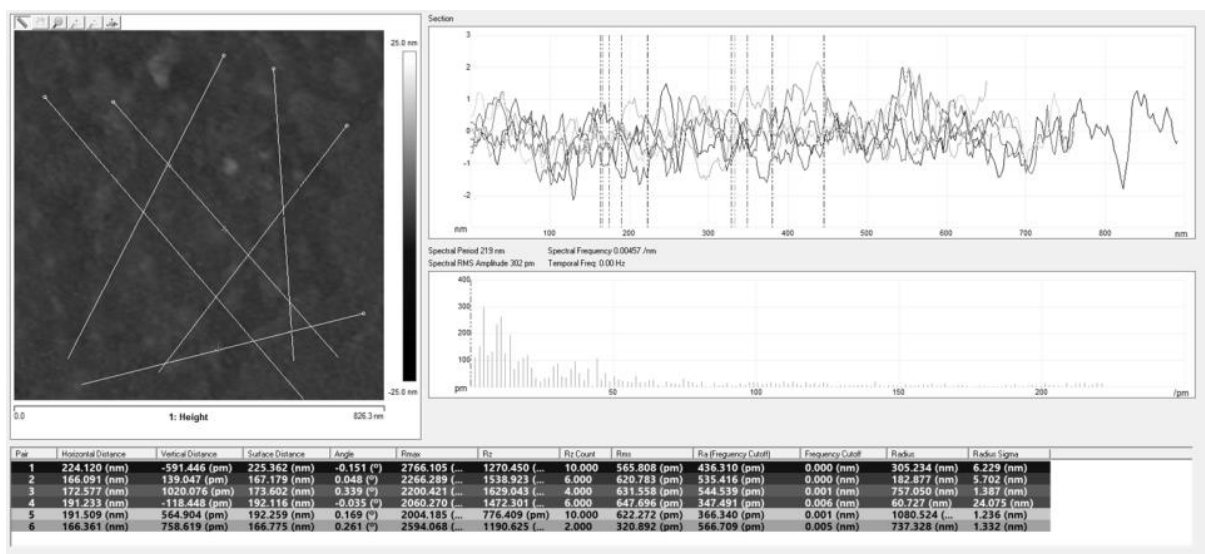


Supplementary Figure S4. DART-HRMS extracted ion chronogram (EIC) (mass window $m / z 339.0052-339.0072$ ) of MS tag 4 (th. mass 339.0062) of two MS scans of SPAAC-modified mica.

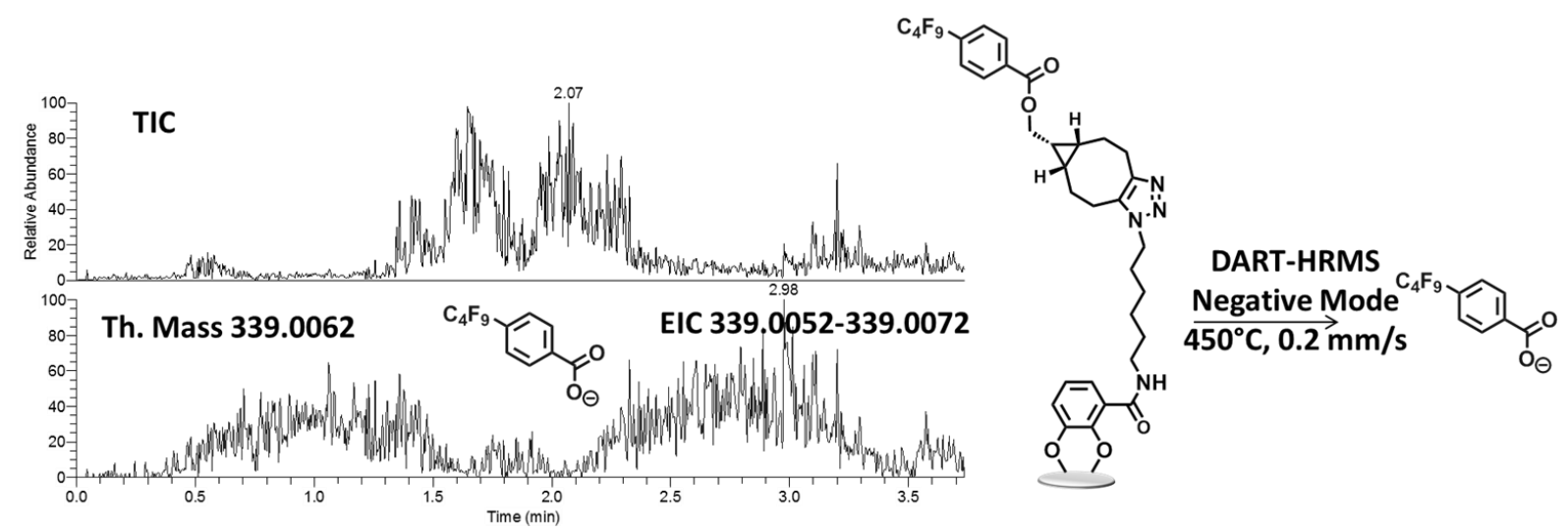

Supplementary Figure S5. DART-HRMS extracted ion chronogram (EIC) (mass window $m / z$ 189.0150-189.0170) of MS tag 2 (th. mass 189.0160) of SPAACmodified mica $\mathbf{M}_{5}$.
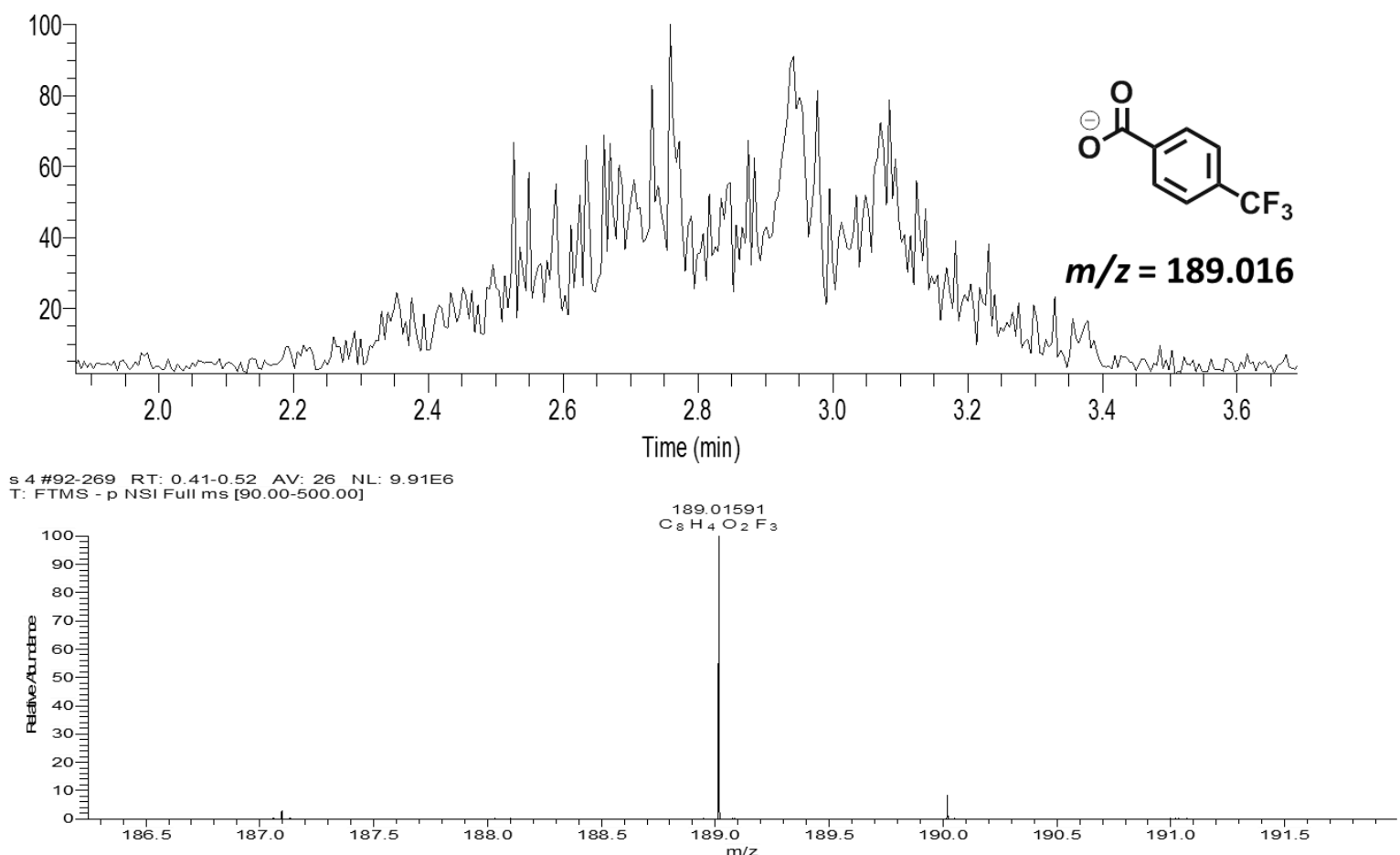
Supplementary Figure S6. Cross-over control experiment: DART-HRMS showing no trace of MS tag $\mathbf{2}(\mathrm{m} / z 339.0062)$, which would have resulted if any physisorbed azide remained on the surface after sonication.
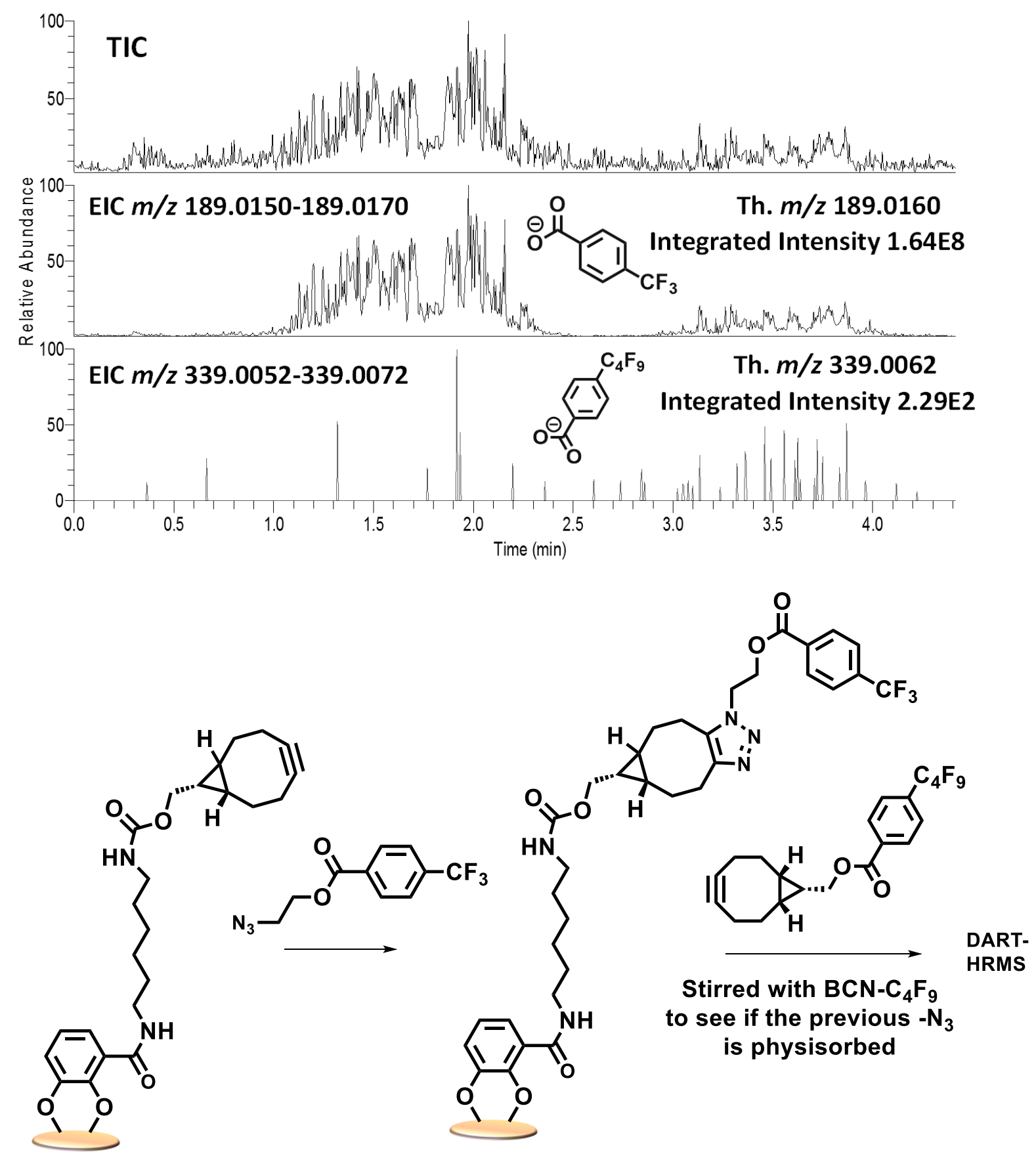

*Sonication in DCM 15 min in between each step 
Supplementary Figure S7. 2D-height AFM image of DNA anchor D1 on mica.

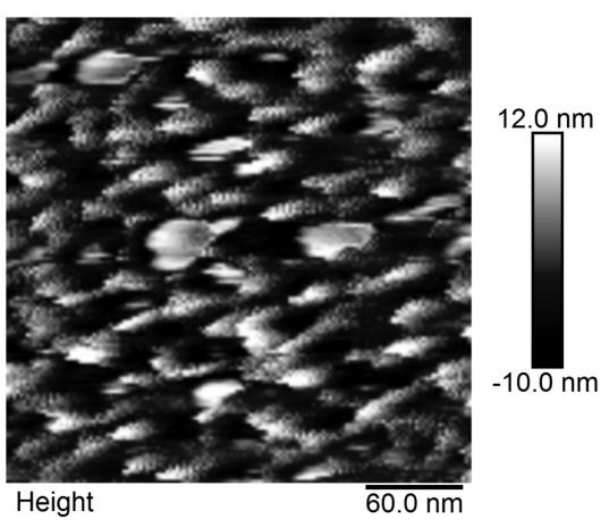

Supplementary Figure S8. Height profile of mica modified with DNA mini-circles showing roughness of $0.9 \pm 0.2 \mathrm{~nm}$ across six random profiles drawn across the surface.

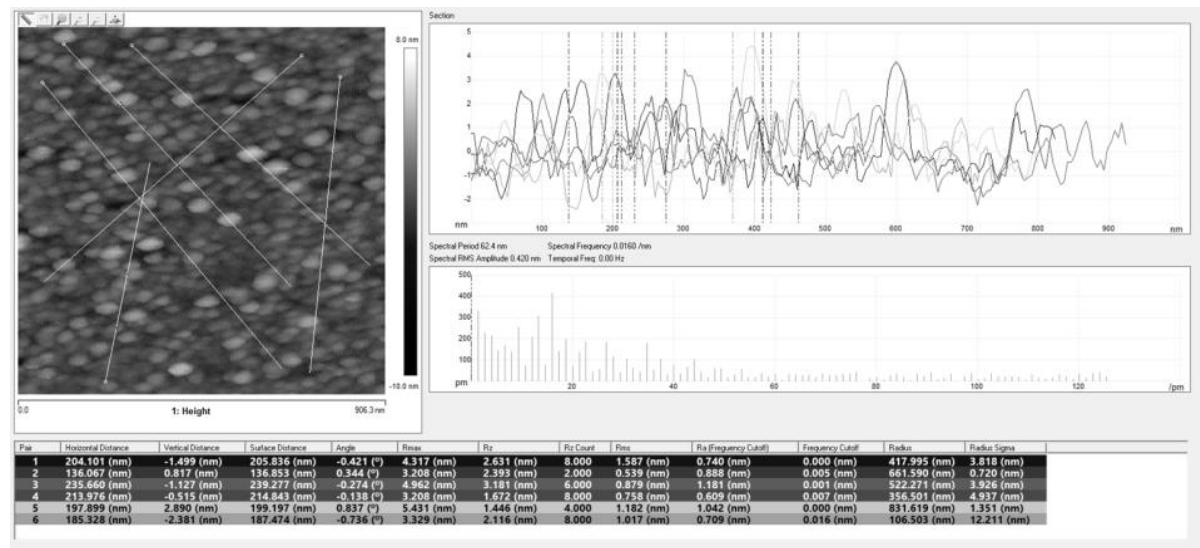

Supplementary Figure S9. Height image of bare mica, surface anchor-modified mica and mica with DNA minicircles (2D and 3D models).
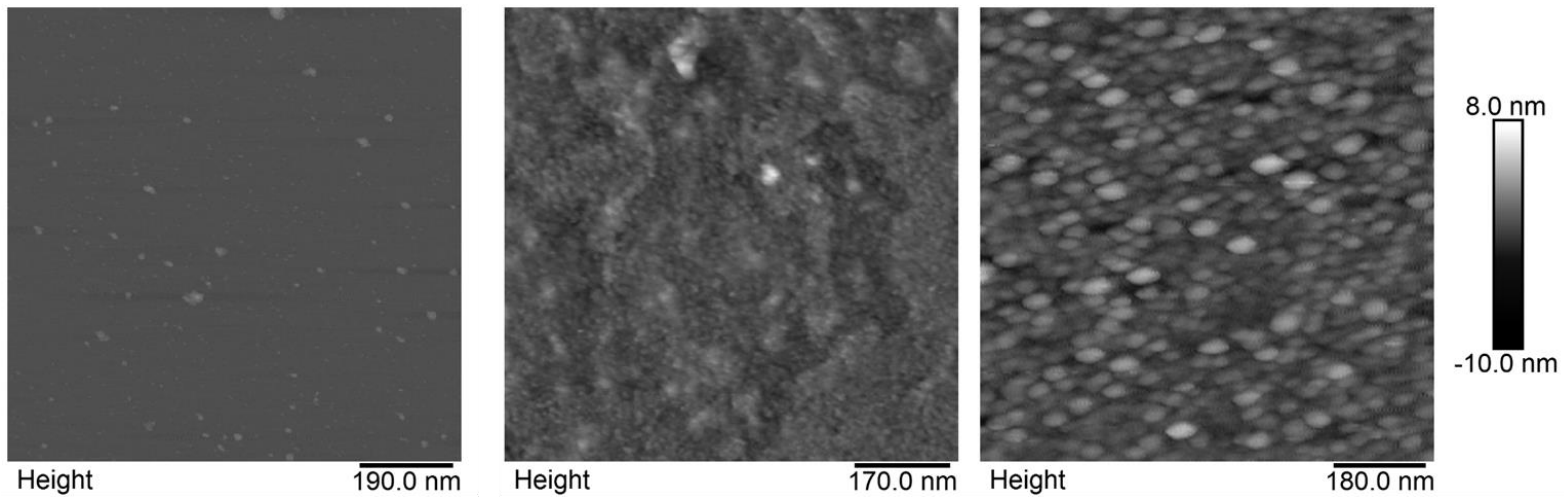


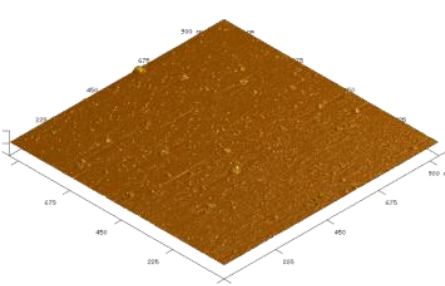

Bare mica

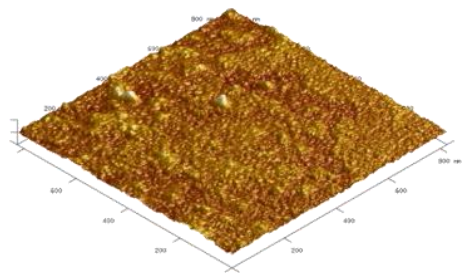

Catechol modified mica

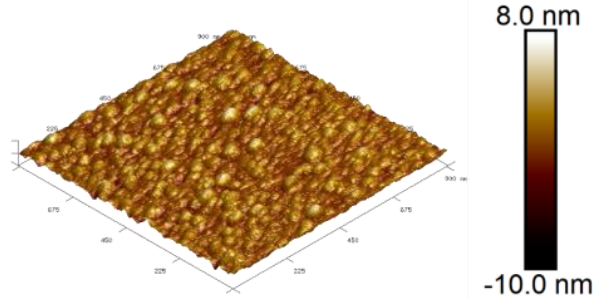

Mica with DNA

minicircles

Supplementary Figure S10. 3D in-phase image of different minicircles which show consistent internal diameter in agreement with theoretical value.

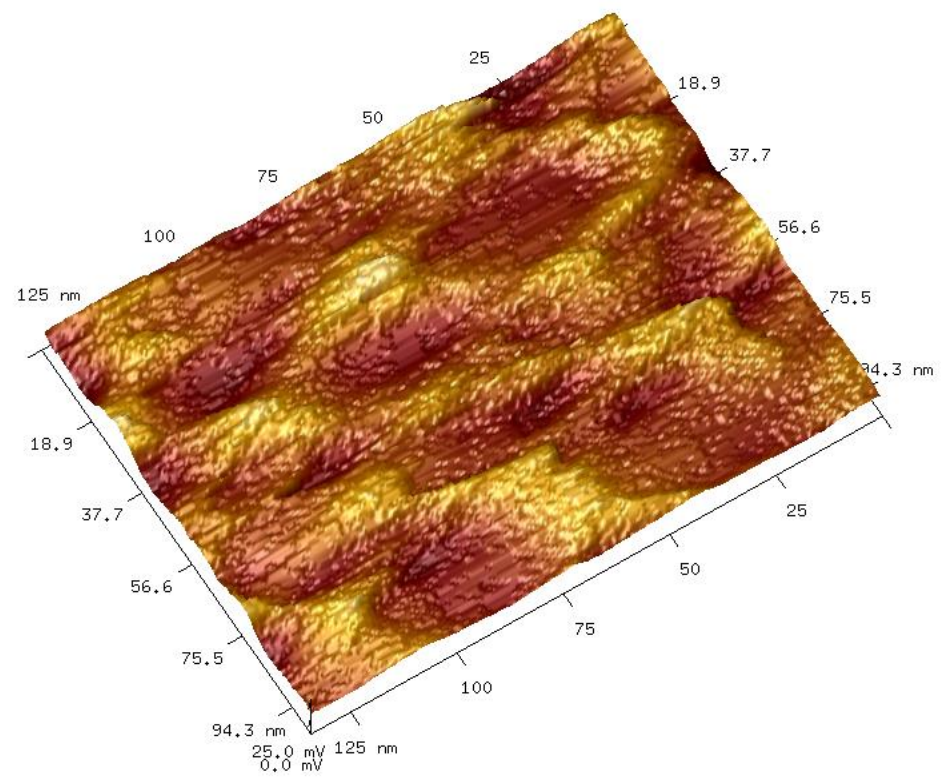

Supplementary Figure S11. In-phase images of bare mica, surface anchor modified mica and mica with immobilized DNA mini-circles (2D and 3D image).
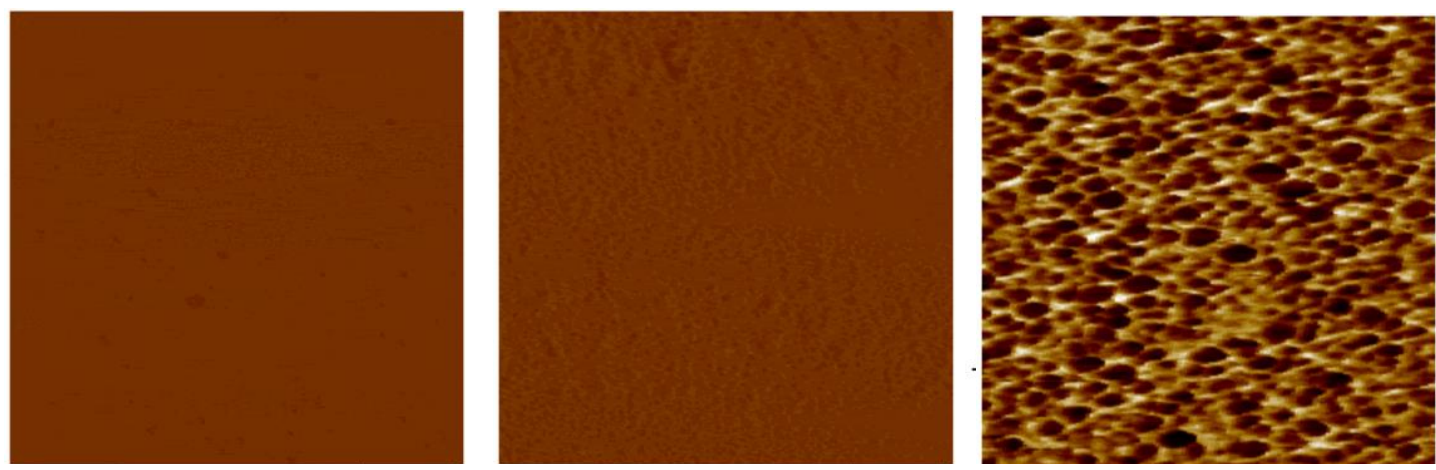

$25.0 \mathrm{mV}$ 


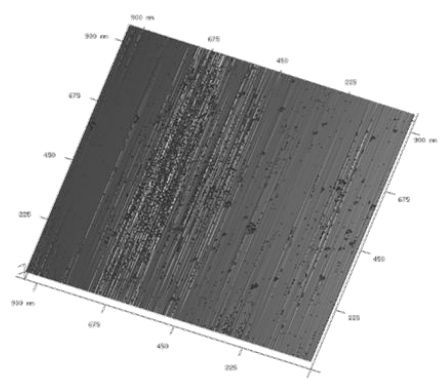

Bare mica

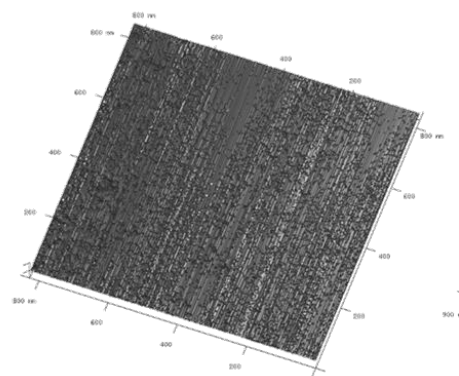

Catechol modified mica

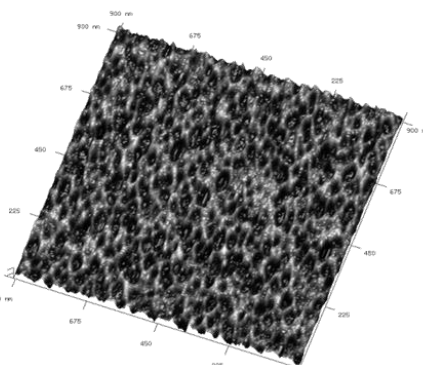

Mica with DNA minicircles

Supplementary Figure S12. Profile across 2D AFM image of different mini-circles which show consistent internal diameter in agreement with theoretical value of 18 nm.
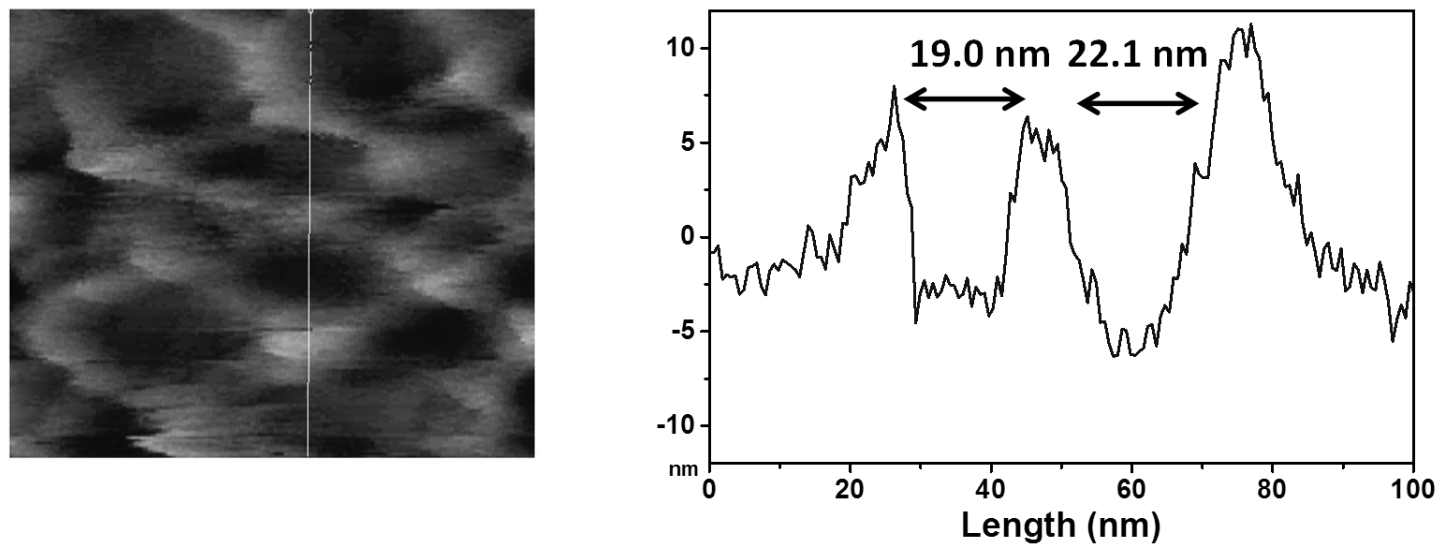

Supplementary Figure S13. 2D-height AFM profile image of single DNA minicircle with six random lines drawn across it showing a consistent height of $4 \mathrm{~nm}$.

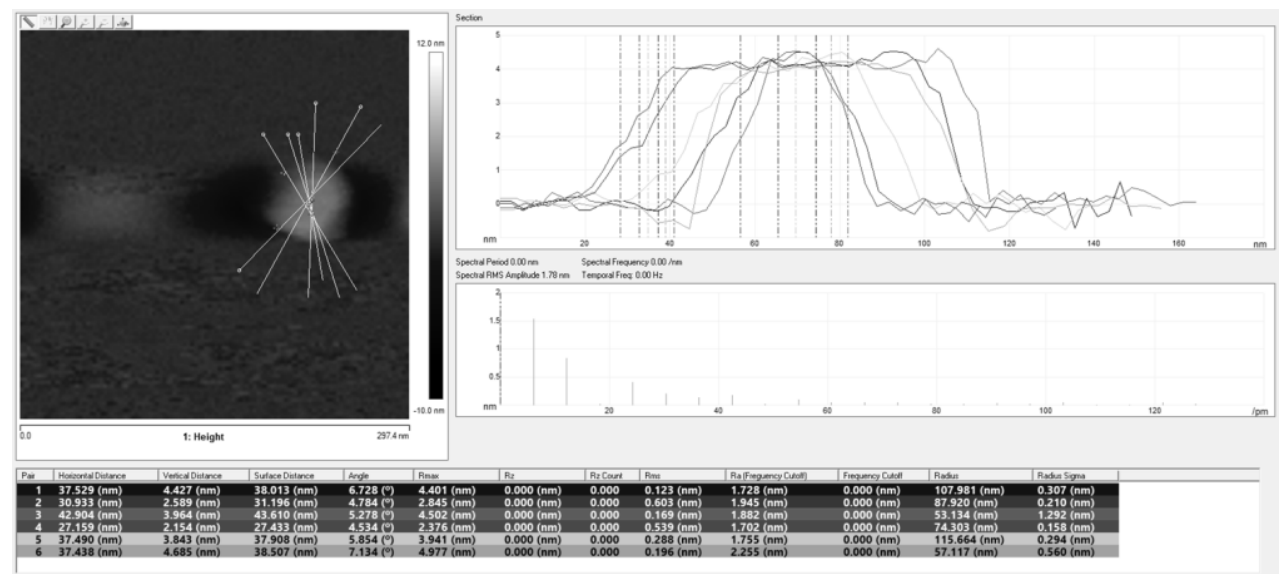

Supplementary Figure S14. 3D-height AFM image of incomplete DNA minicircles on mica obtained at lower concentration (100-fold dilution). 


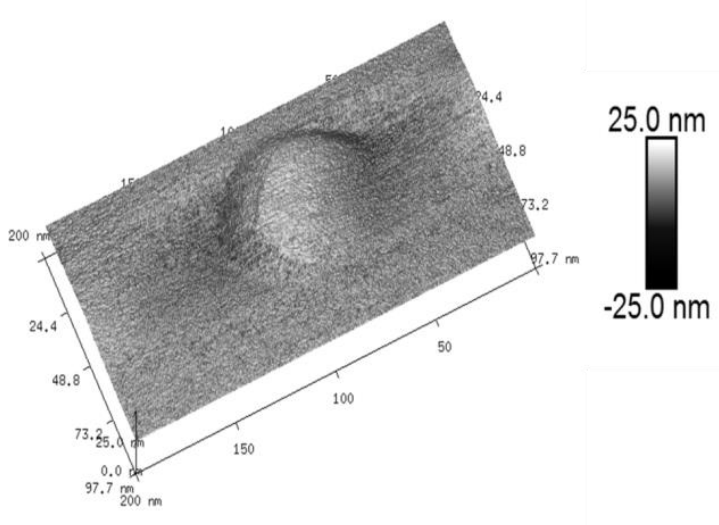

Supplementary Figure S15. 3D-height AFM image other DNA minicircles obtained at lower concentration (100-fold dilution).

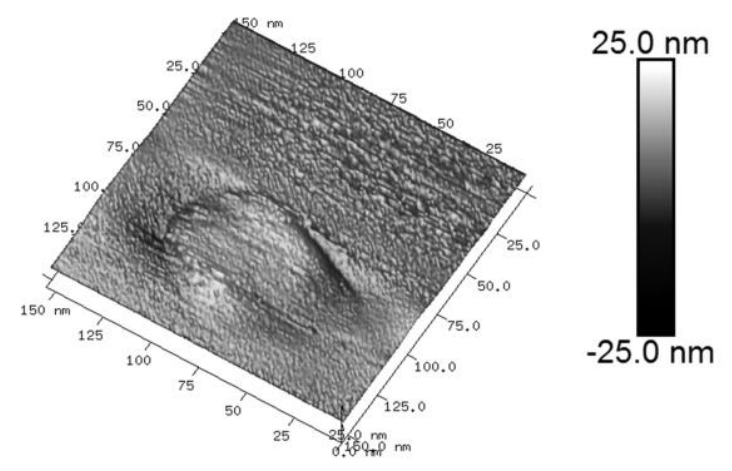

Height

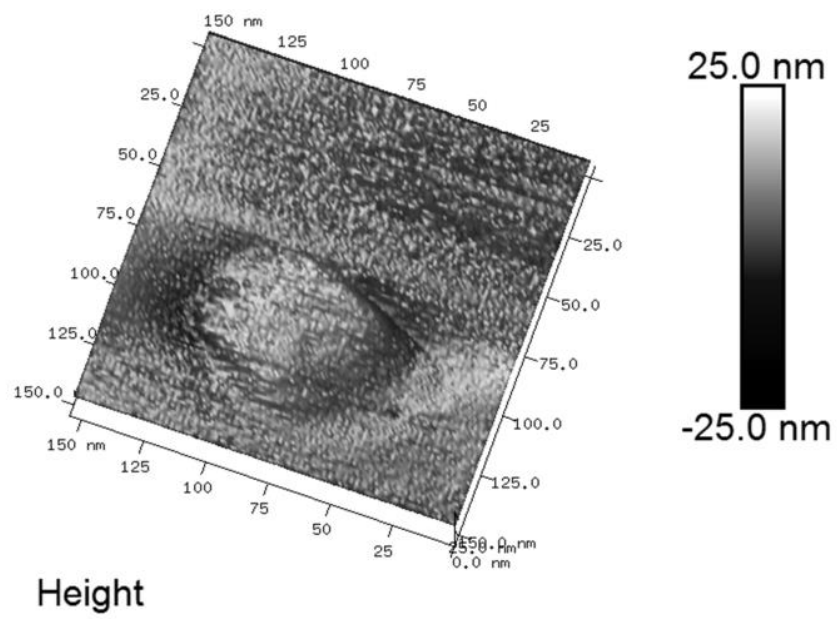

Supplementary Figure S16. 2D-height AFM image and profile of control experiment for DNA minicircles. 

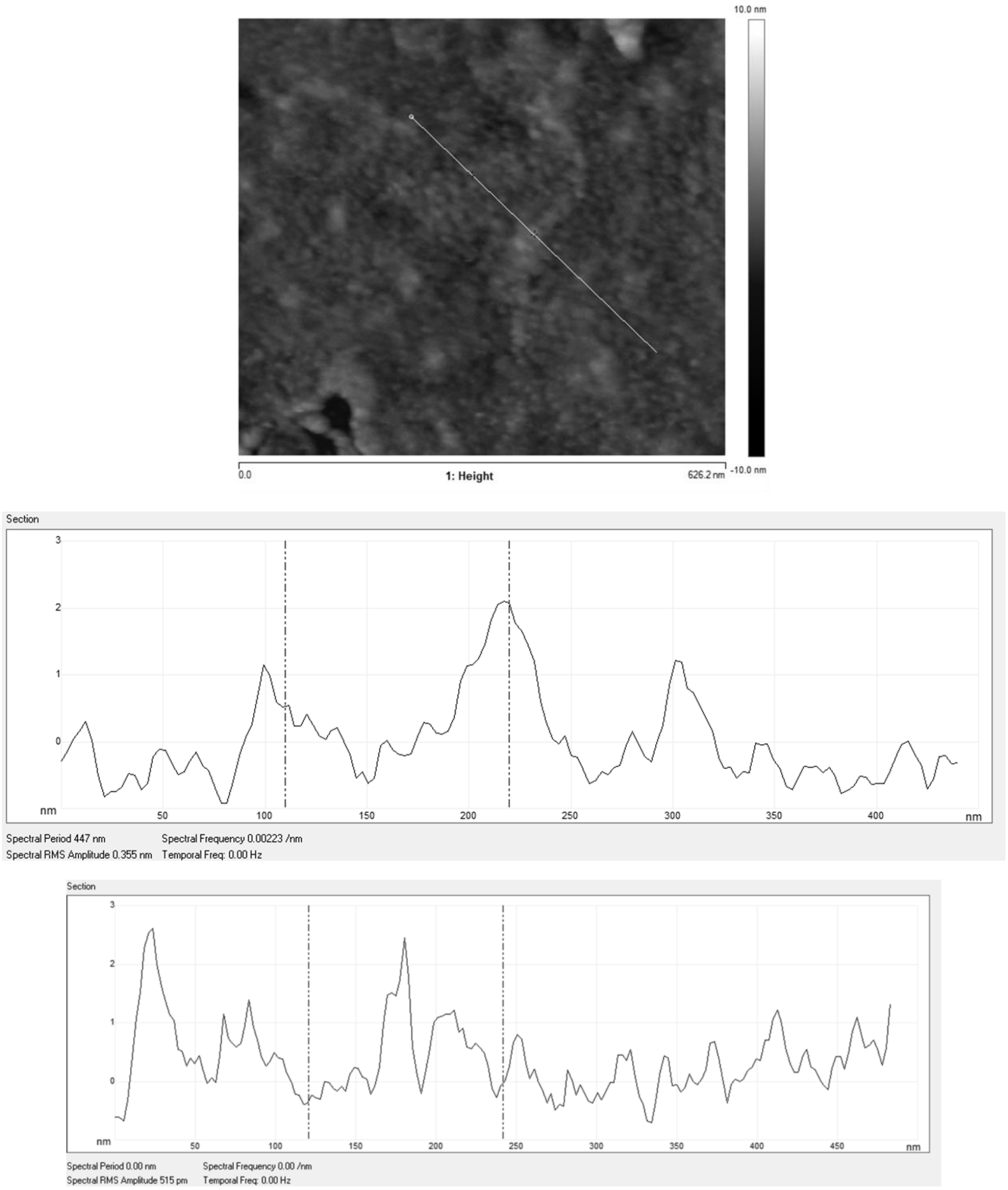

Supplementary Figure S17. DART-HRMS extracted ion chronogram (EIC)

(mass window $m / z 339.0052-339.0072$ ) of MS tag 2 (th. mass 339.0062) of nine scans of SPAAC-modified mica. 


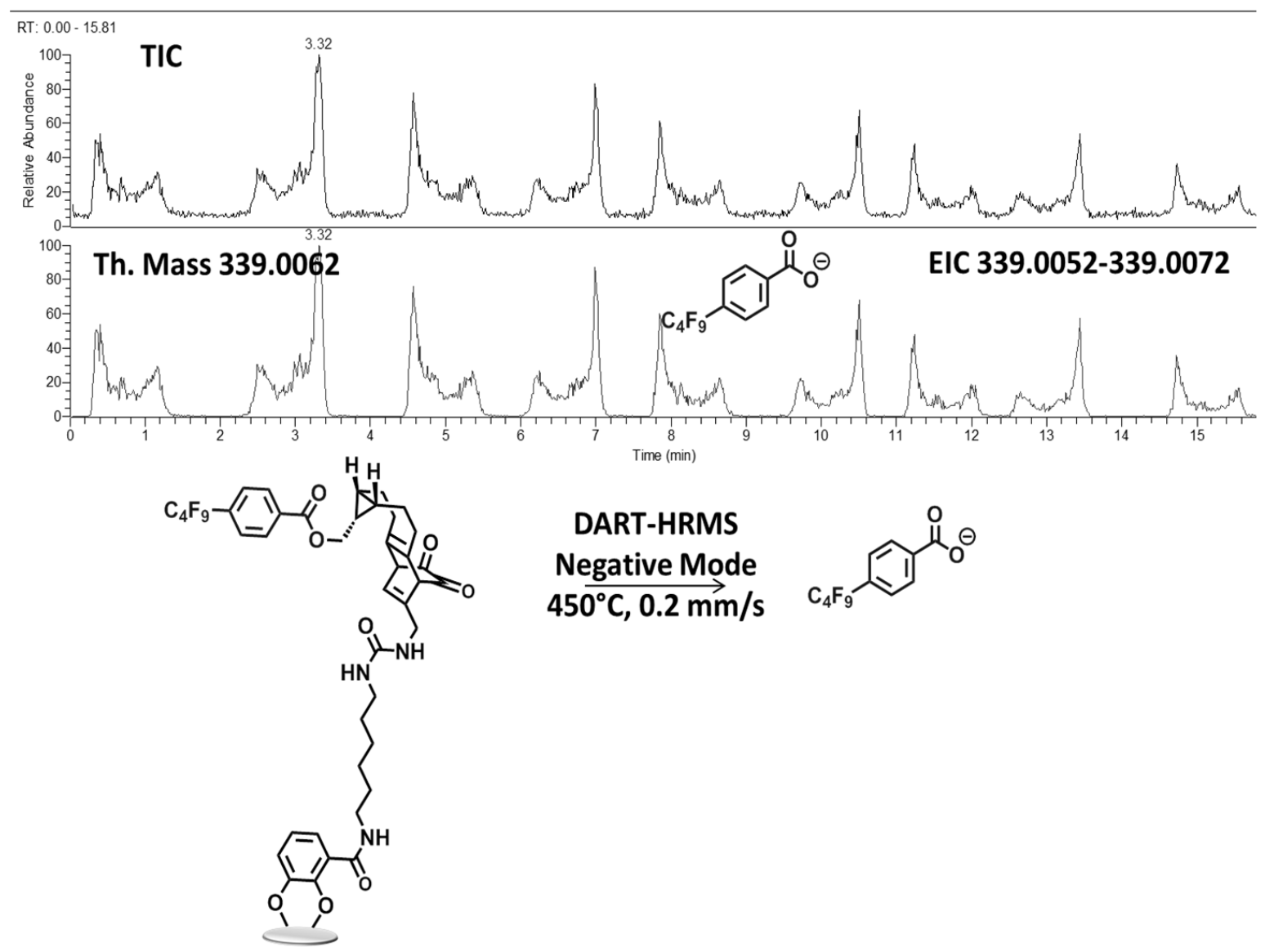


4.4. Supplementary XPS Spectra

S4.1 XPS Wide scan of bare mica $\left(M_{0}\right)$.

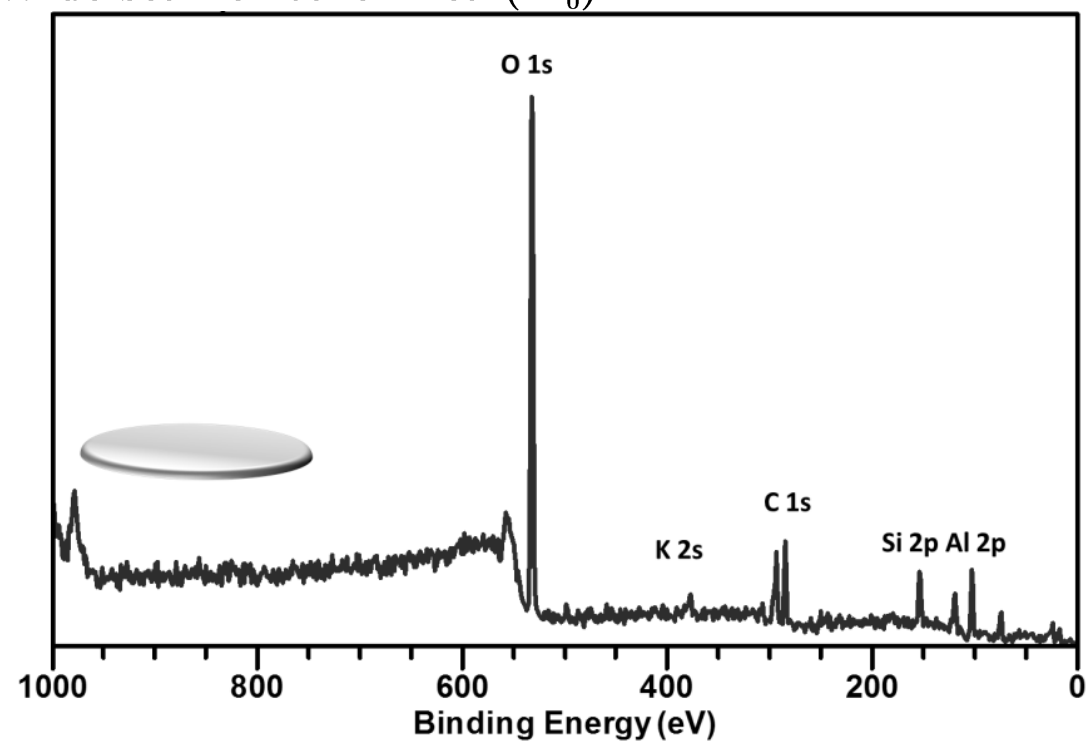

S4.2 XPS Wide scan of modified mica by $1\left(M_{1}\right)$.

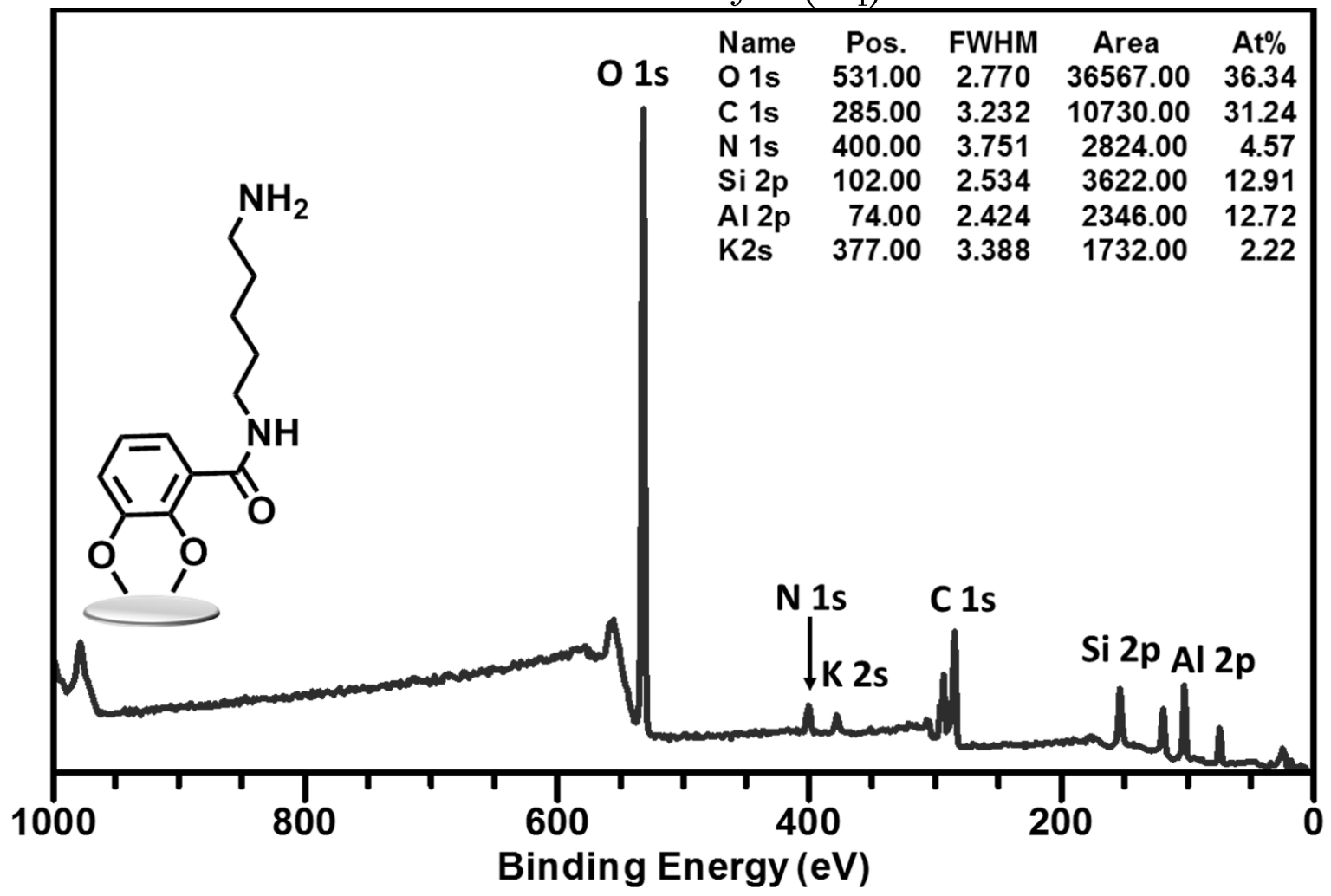

Experimental ratio $(\mathrm{C} / \mathrm{N})=31.24 / 4.57=6.8$

Theoretical ratio $(\mathrm{C} / \mathrm{N})=13 / 2=6.5$ 
S4.3 C1s XPS narrow scan of $M_{1}-$ modified mica.

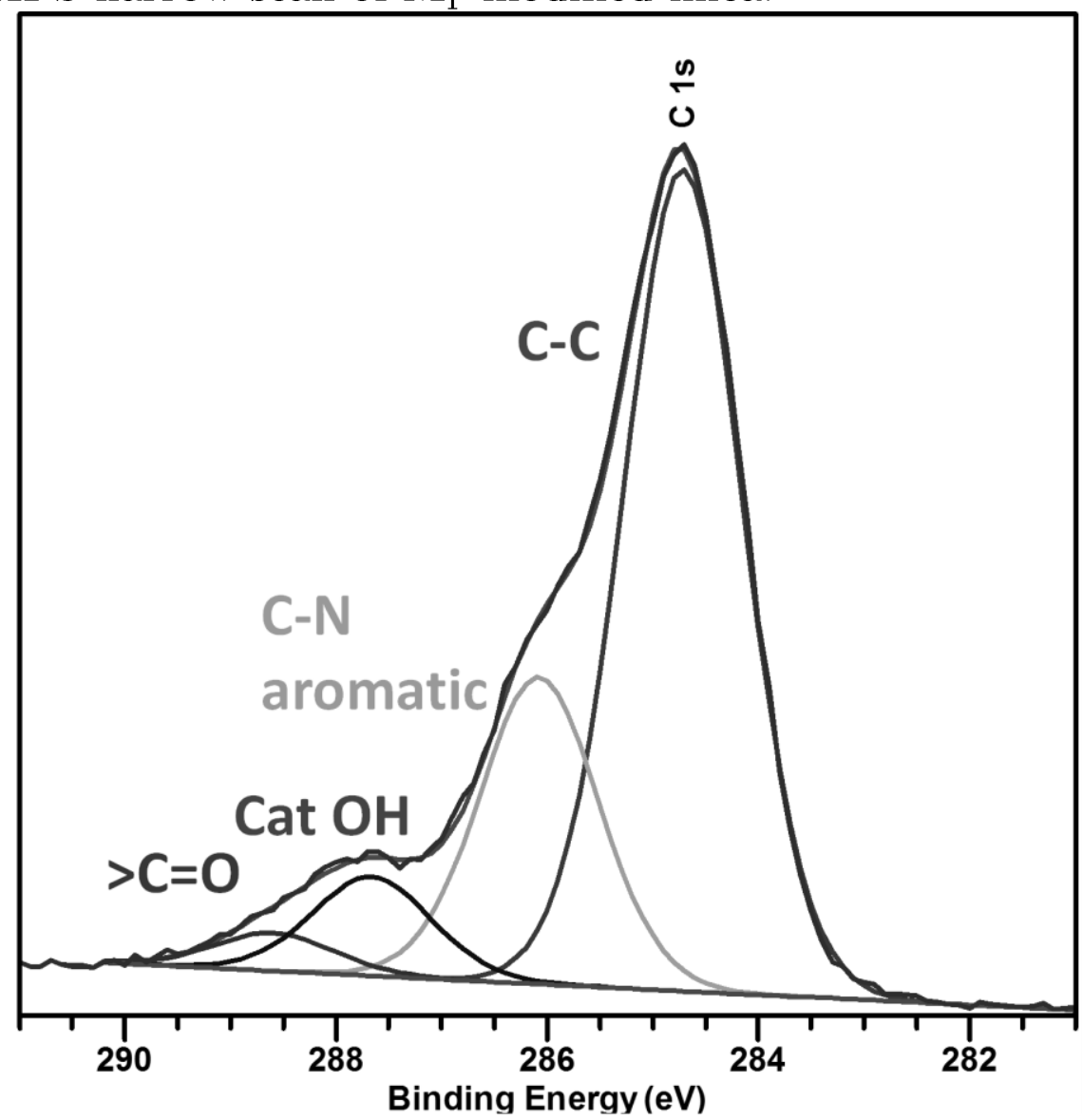

S4.4 XPS wide scan of azide-terminated surface.

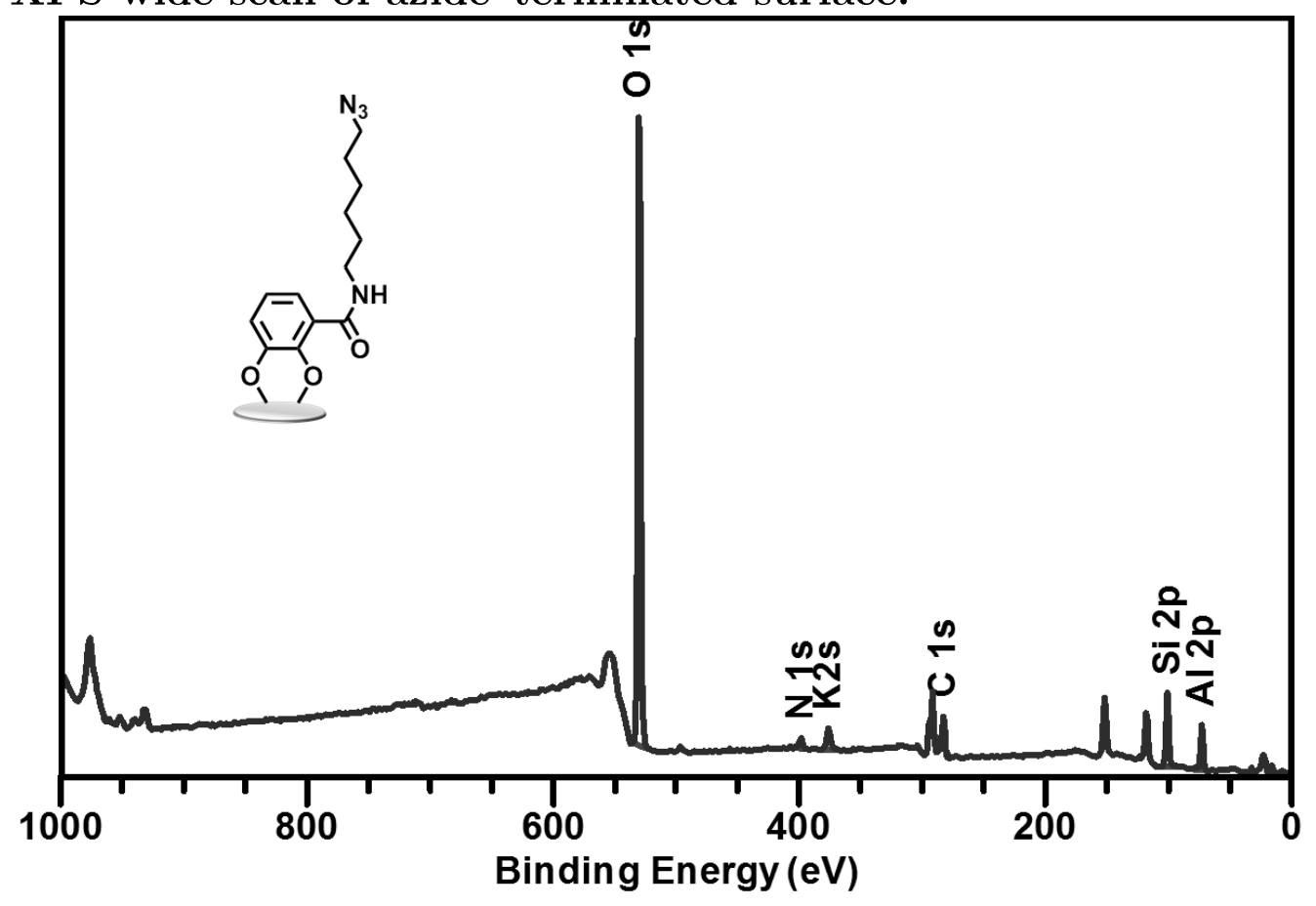


S4.5 XPS N1s narrow scan of azide-terminated surface.

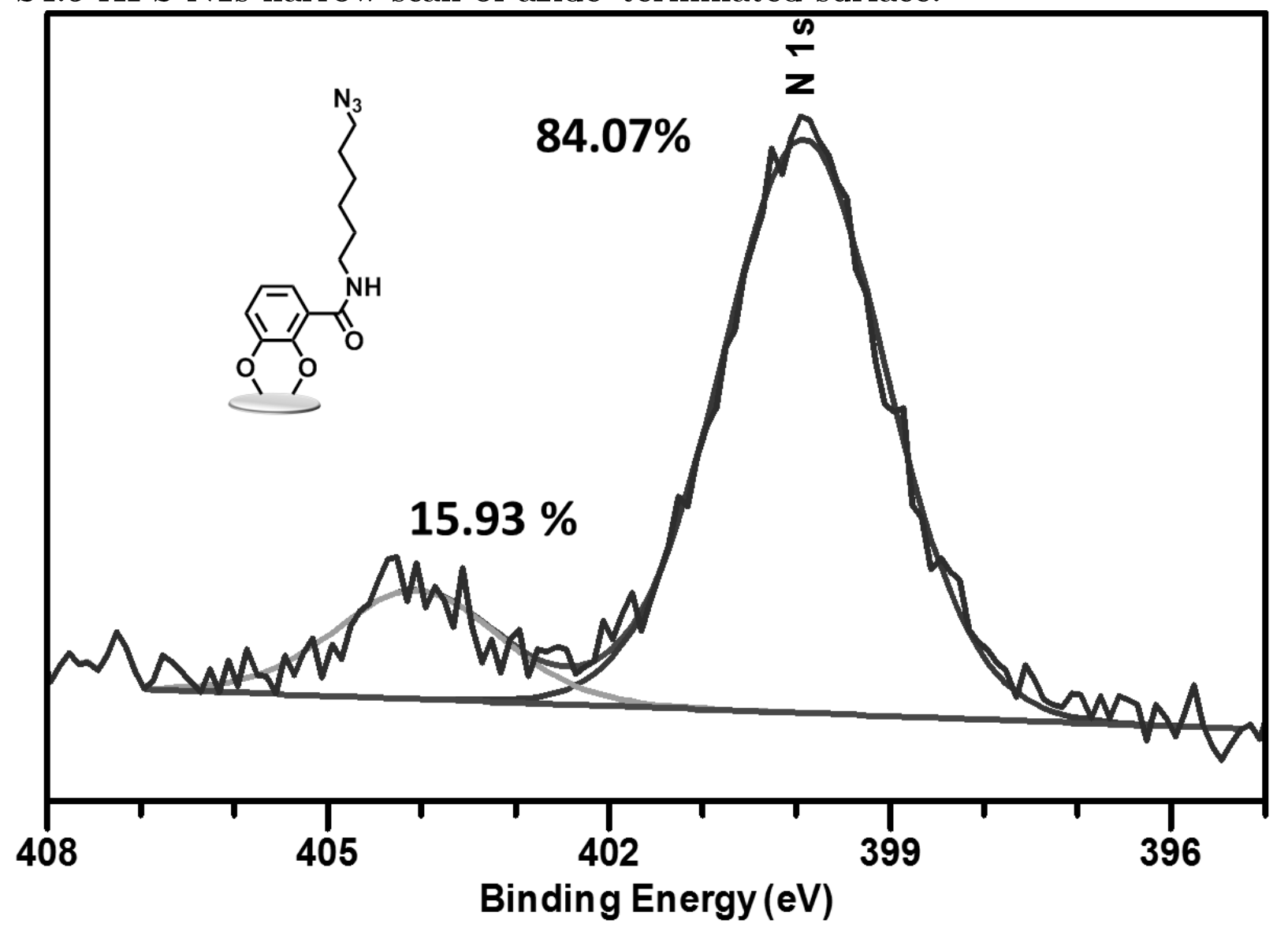

Th. ratio between middle azide $\mathrm{N}$ and other kinds of $\mathrm{N}=1 / 3$ (assuming $100 \%$

conversion of every amine termination with bottom $\alpha$-amide linker $\mathrm{N}$ atom intact).

Obtained ratio $=15.93 / 84.07=0.19$

$\%$ yield $=0.19 / 0.33=57.5=\sim 60$ 
S4.6 XPS Wide Scan of SPAAC on azide-terminated surface and calculation of surface yield.

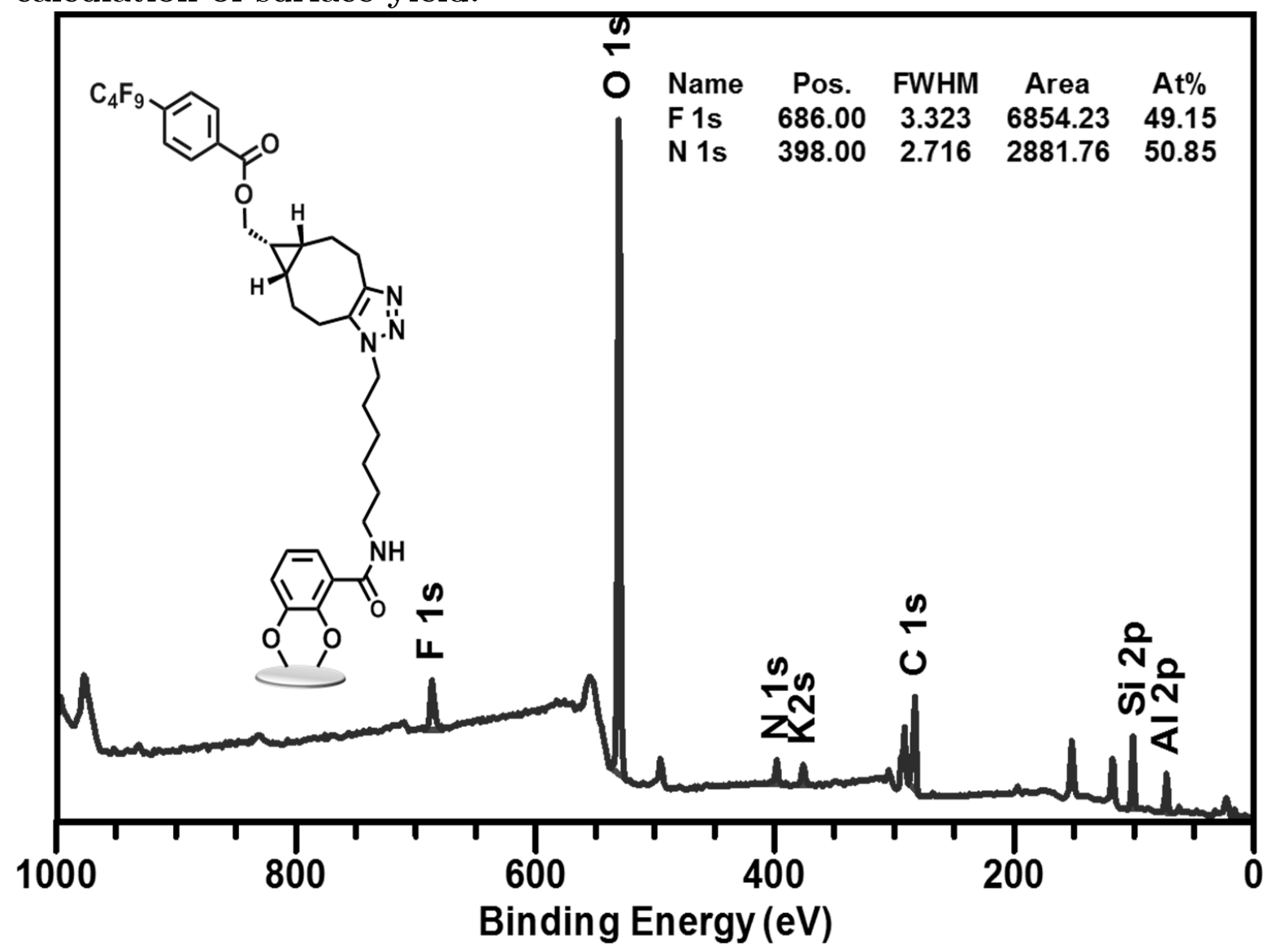

As inferred from the N1s narrow scan of azide-terminated surface, only $60 \%$ of the surfacial amines were converted to azides which are capable of further reaction with solution BCN counterpart.

$\% \mathrm{~N}$ capable of reaction $=50.85 * 0.60=31.02$

Experimental ratio $(\mathrm{F} / \mathrm{N})=49.05 / 31.02=1.51$

Theoretical ratio $(\mathrm{F} / \mathrm{N})=9 / 3=3$

$\%$ yield $=(1.51 / 3.0) * 100=52$ 
S4.7 XPS C1s narrow scans of SPAAC cycloadduct $\left(\mathrm{M}_{3}\right)$ with azide initially on the surface $\left(M_{2}\right)$.

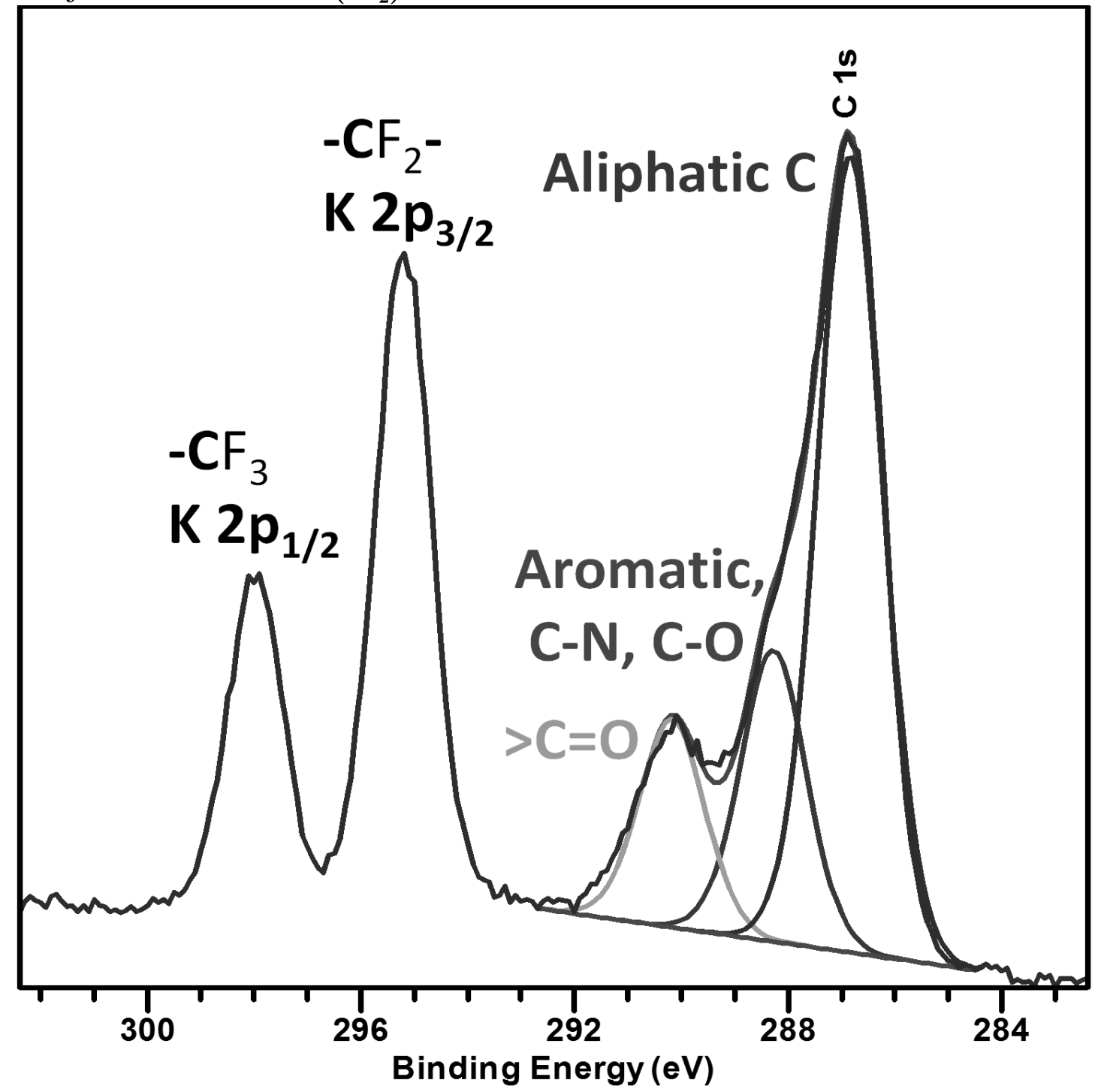


S4.8 XPS N1s narrow scans of SPAAC cycloadduct $\left(\mathrm{M}_{3}\right)$ with azide initially on the surface $\left(M_{2}\right)$.

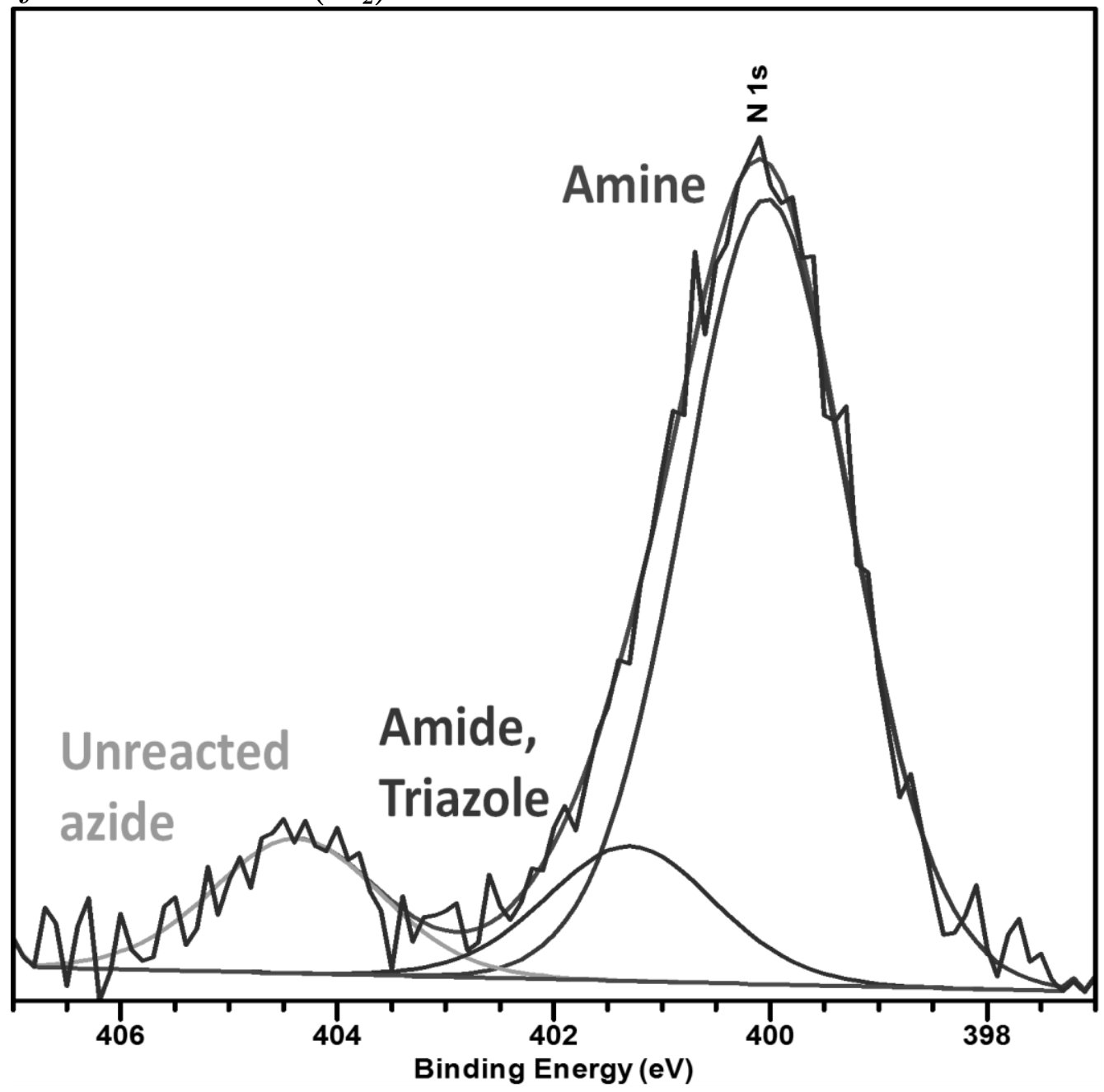

Initial number of N1s $\left(\mathbf{M}_{1}\right)=2$

After $60 \%$ Azide conversion $\left(\mathbf{M}_{\mathbf{2}}\right)=1+0.4+\left(3^{*} 0.60\right)=3.2$

After $51 \%$ SPAAC $\left(\mathbf{M}_{3}\right)$ : Total number of N1s $=1+0.4+\left(3^{*} 0.60\right)=3.2$. 
S4.9 XPS C1s Narrow Scan of BCN immobilized on mica surface.

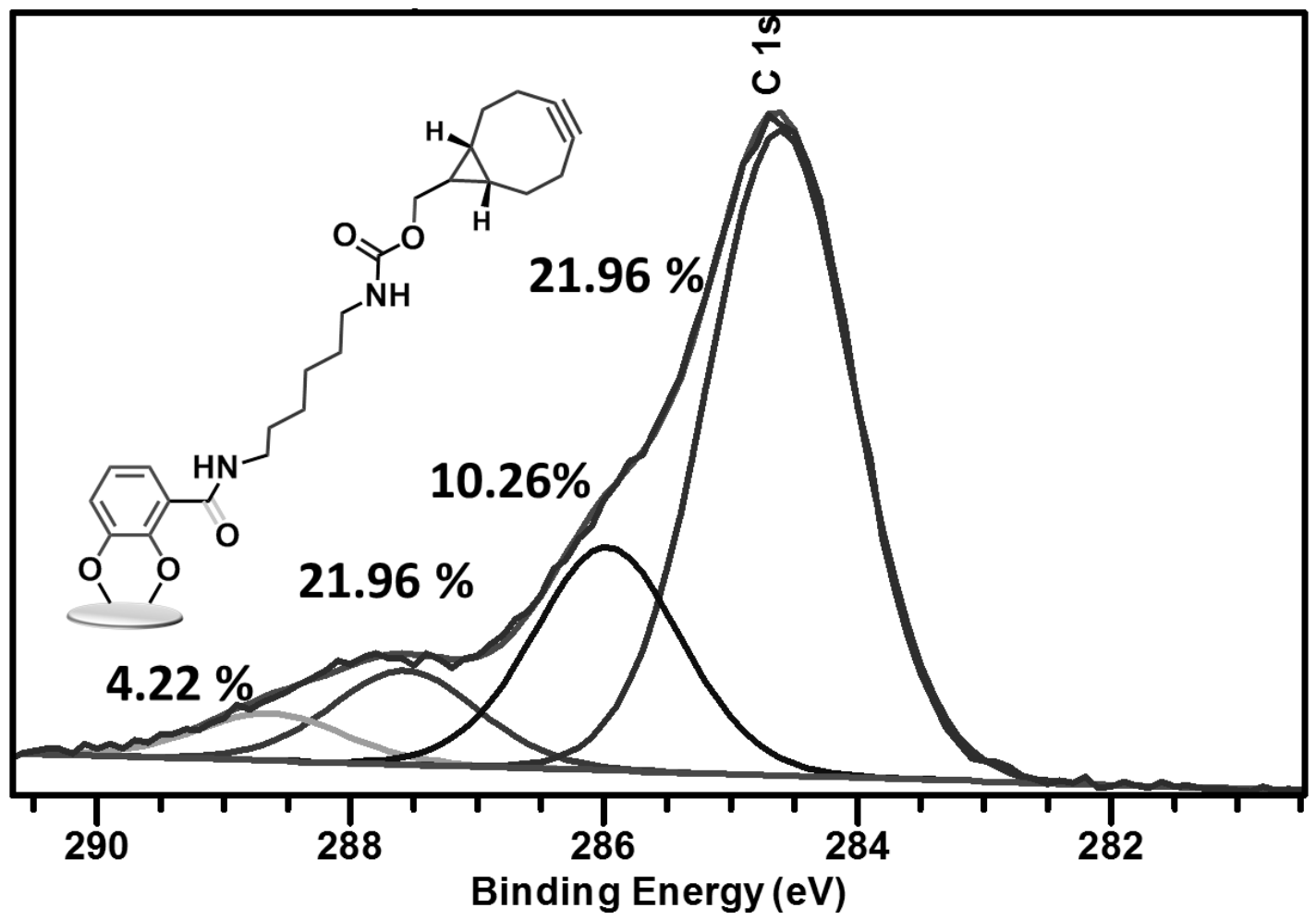

S4.10 XPS wide scan of the inverse SPAAC cycloadduct with BCN $\left(M_{5}\right)$ immobilized on mica surface.

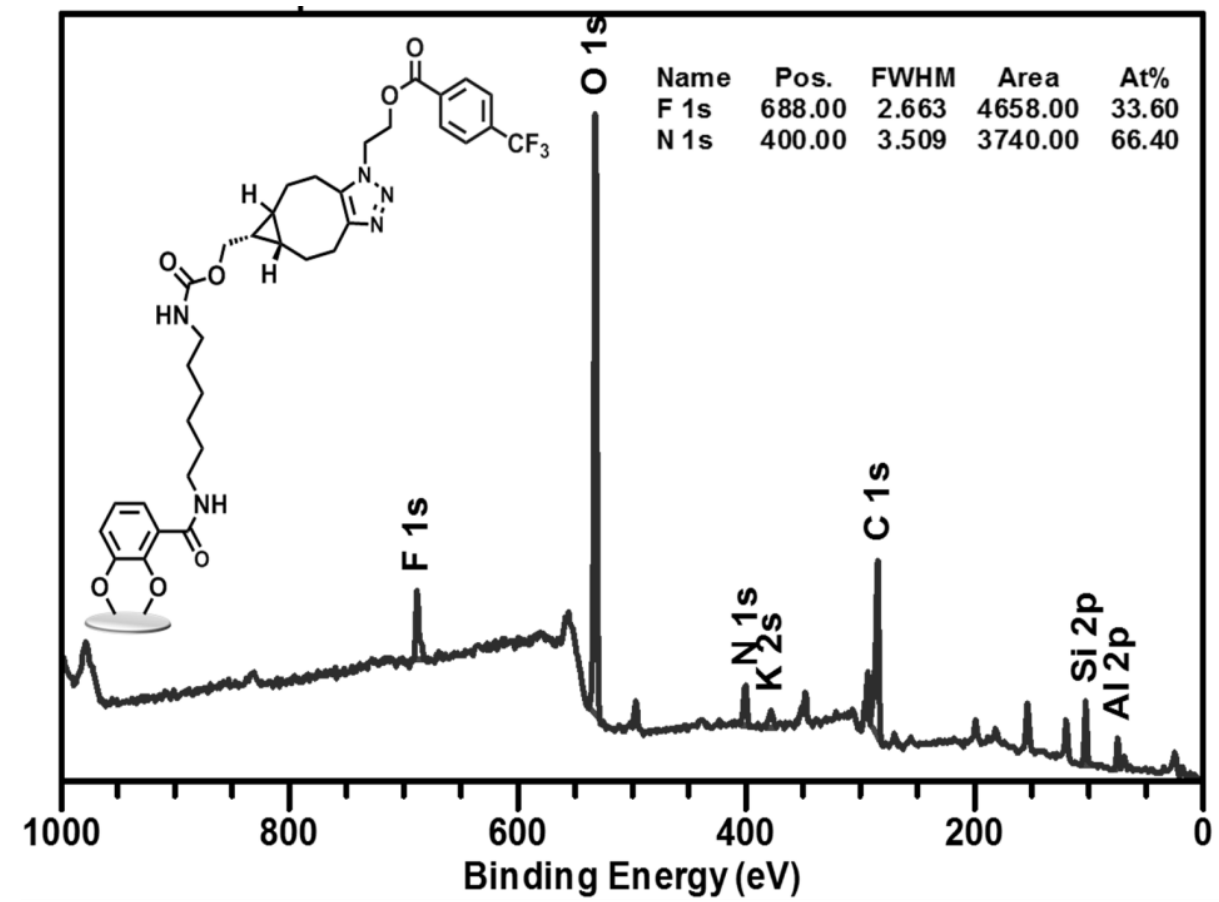

Theoretical ratio $(\mathrm{F} / \mathrm{N})=3 / 5=0.6$

$\%$ yield $=(0.5 / 0.6) * 100=83.3$ 
S4.11 XPS N1s narrow scans of the inverse SPAAC cycloadduct $\left(M_{5}\right)$ with BCN initially on the surface $\left(M_{4}\right)$.

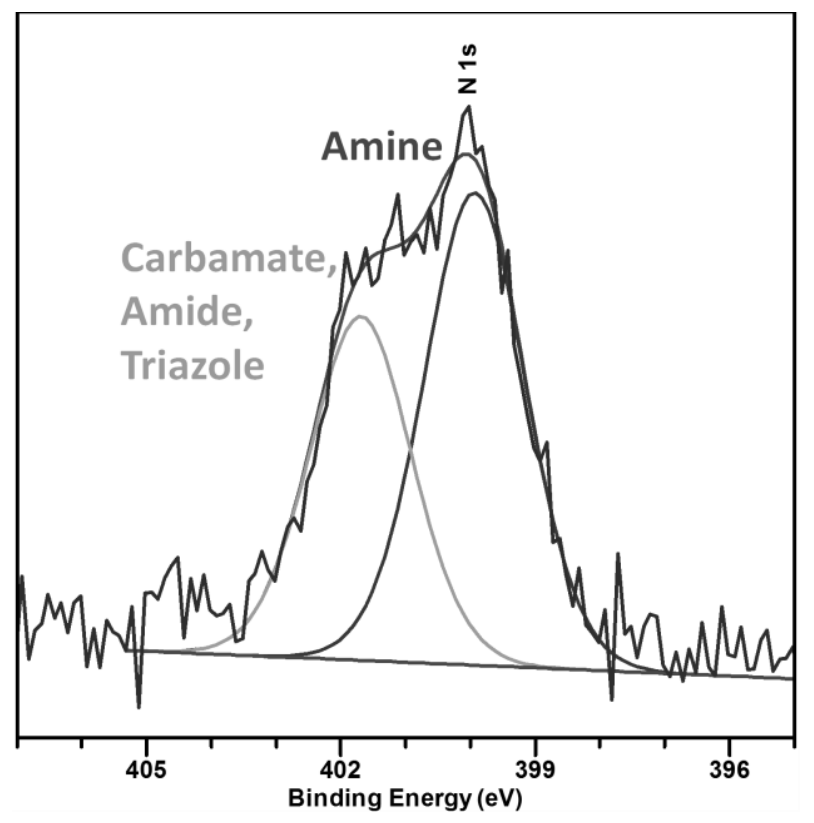

Initial number of N1s $\left(\mathbf{M}_{\mathbf{4}}\right)=2$

After $83 \% \operatorname{SPAAC}\left(\mathbf{M}_{5}\right)$ : Total number of N1s $=2+\left(0.83^{*} 3\right)=4.49$

S4.12 XPS C1s narrow scans of the inverse SPAAC cycloadduct $\left(M_{5}\right)$ with BCN initially on the surface $\left(M_{4}\right)$.

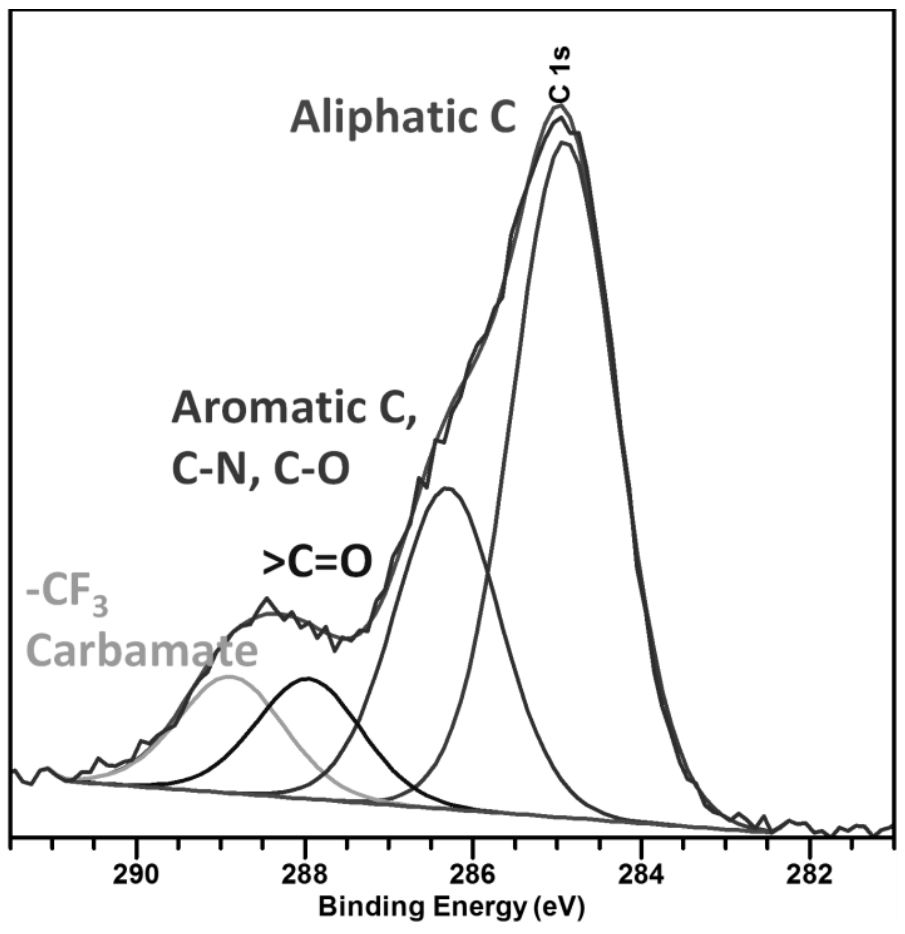


S4.13 XPS wide scan of quinone-terminated surface.

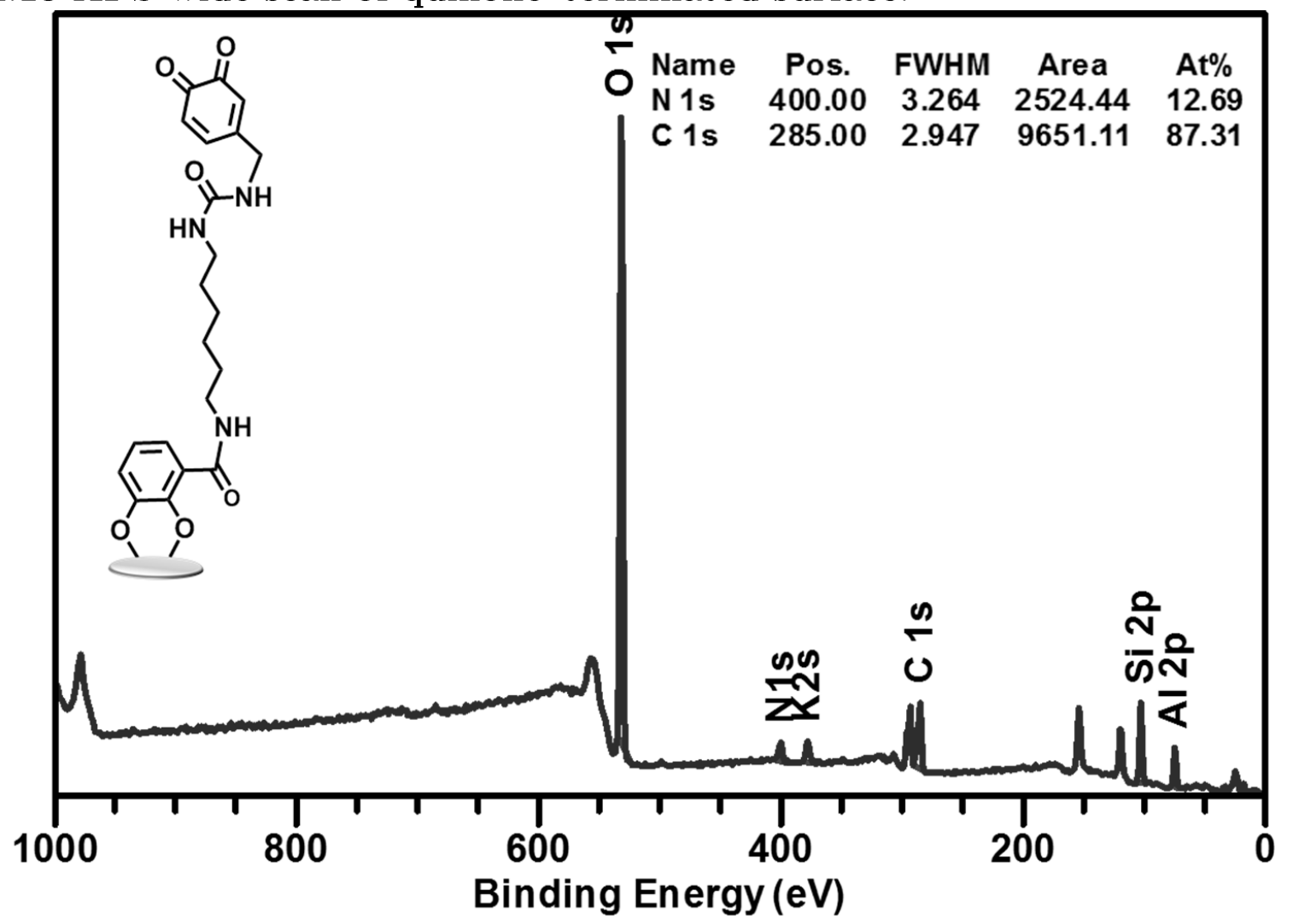

Experimental ratio $(\mathrm{C} / \mathrm{N})=87.31 / 12.69=6.9$

Theoretical ratio $(\mathrm{C} / \mathrm{N})=21 / 3=7$

$\%$ yield quinone on surface $=100$ 
S4.14 XPS wide scan of SPOCQ cycloadduct with BCN immobilized on mica surface $\left(M_{7}\right)$.

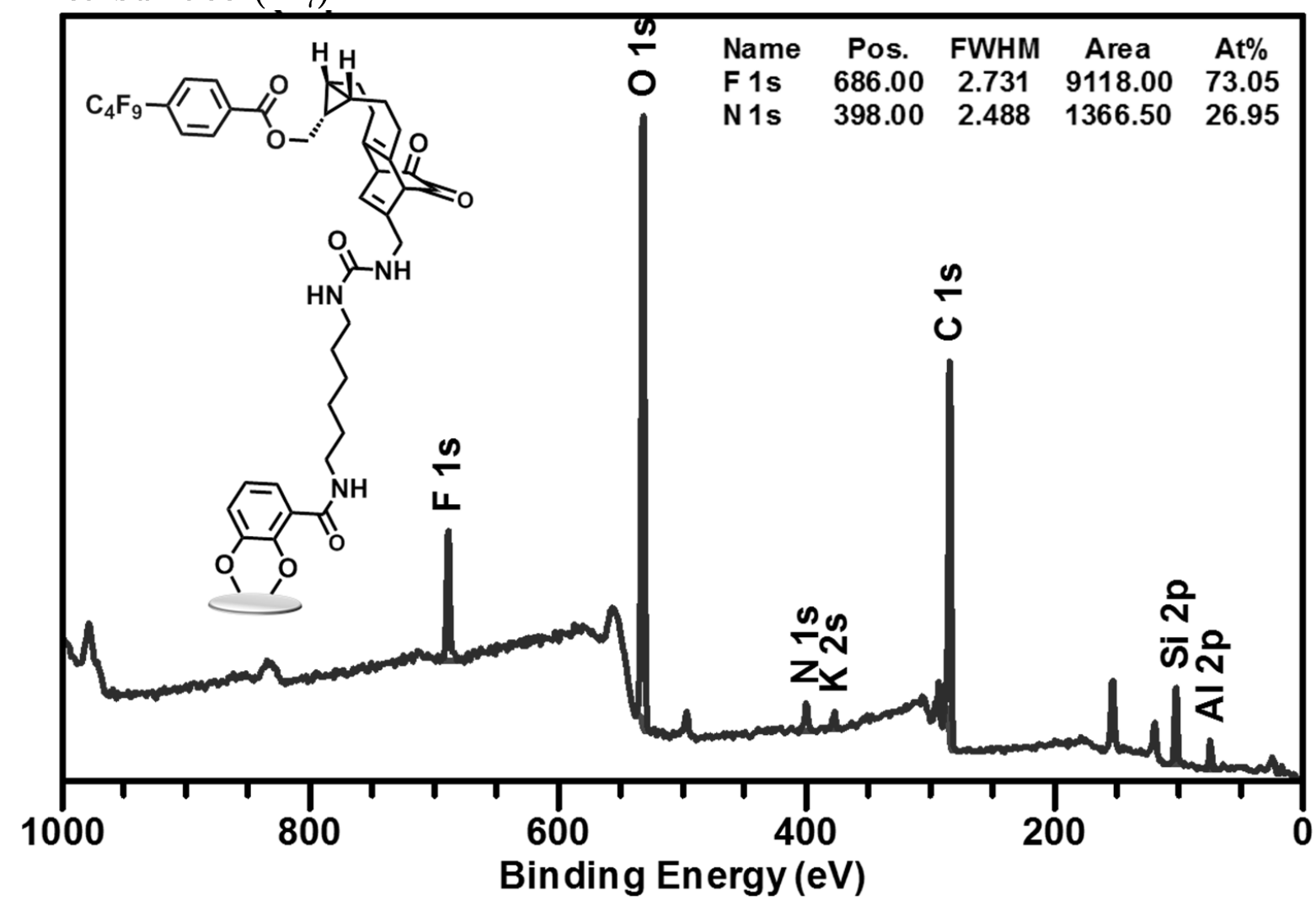

Experimental ratio $(\mathrm{F} / \mathrm{N})=73.05 / 26.95=2.71$

Theoretical ratio $(\mathrm{F} / \mathrm{N})=9 / 3=3.0$

$\%$ yield quinone on surface $=90.3$ 
S4.15 XPS C1s narrow scan of SPOCQ cycloadduct $\left(M_{7}\right)$ on surface.

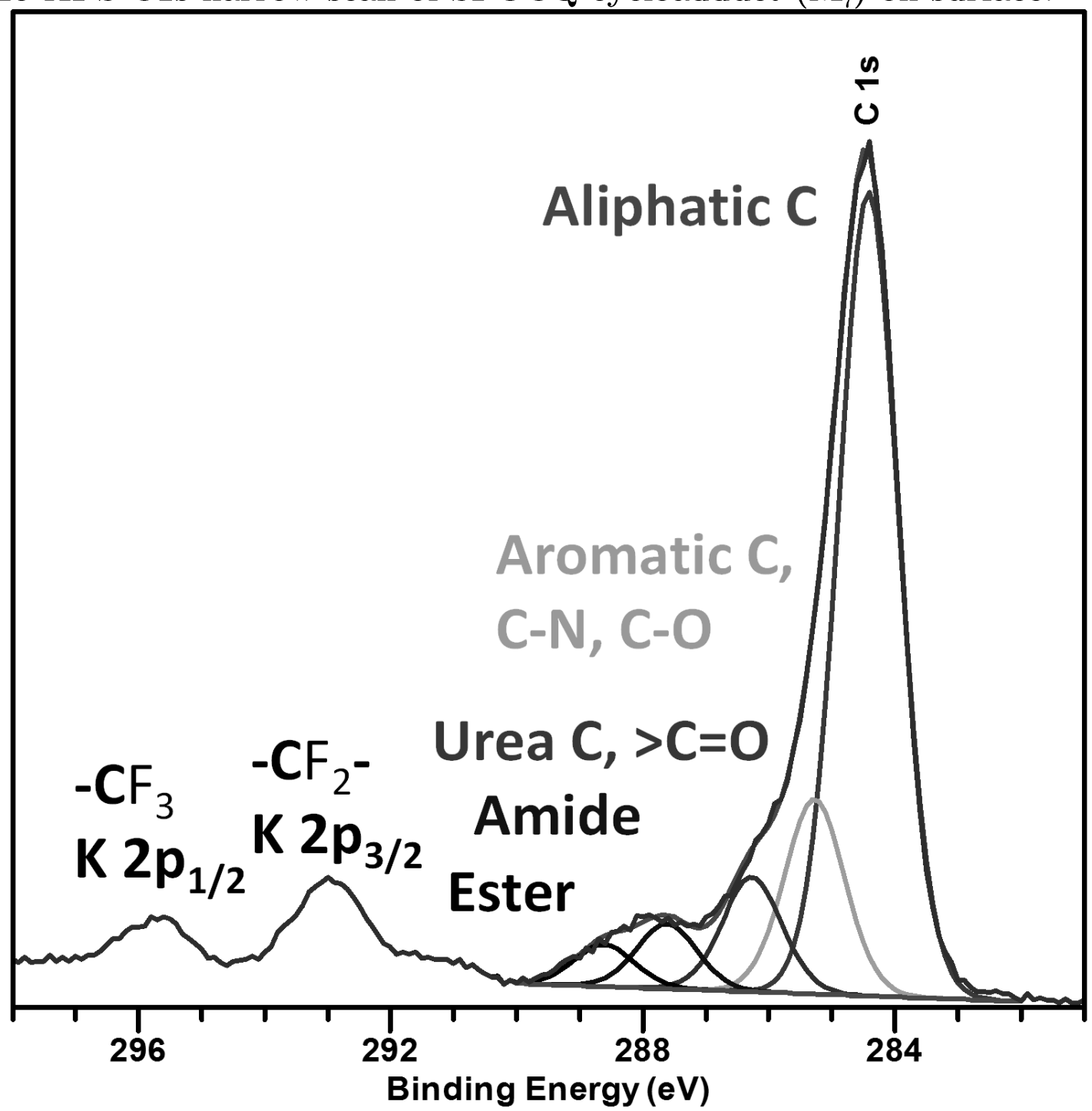


S4.16 XPS N1s narrow scan of SPOCQ cycloadduct $\left(M_{7}\right)$ on surface.

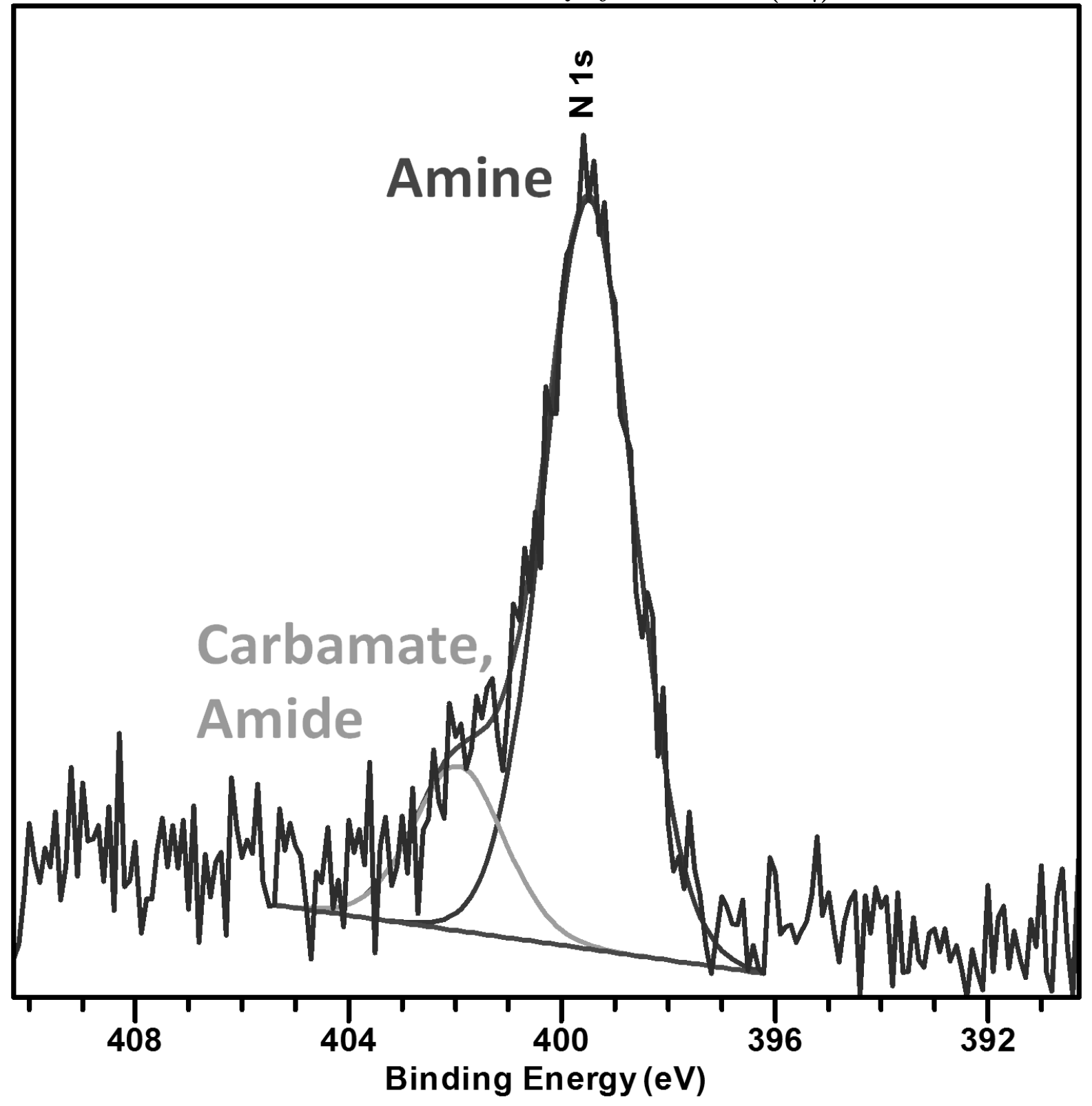


S4.17 Wide scan XPS spectra of EAD2 immobilized on mica surface (inset: Fe2p narrow spectra of the EAD2-based hGQ DNAzymefunctionalized mica).

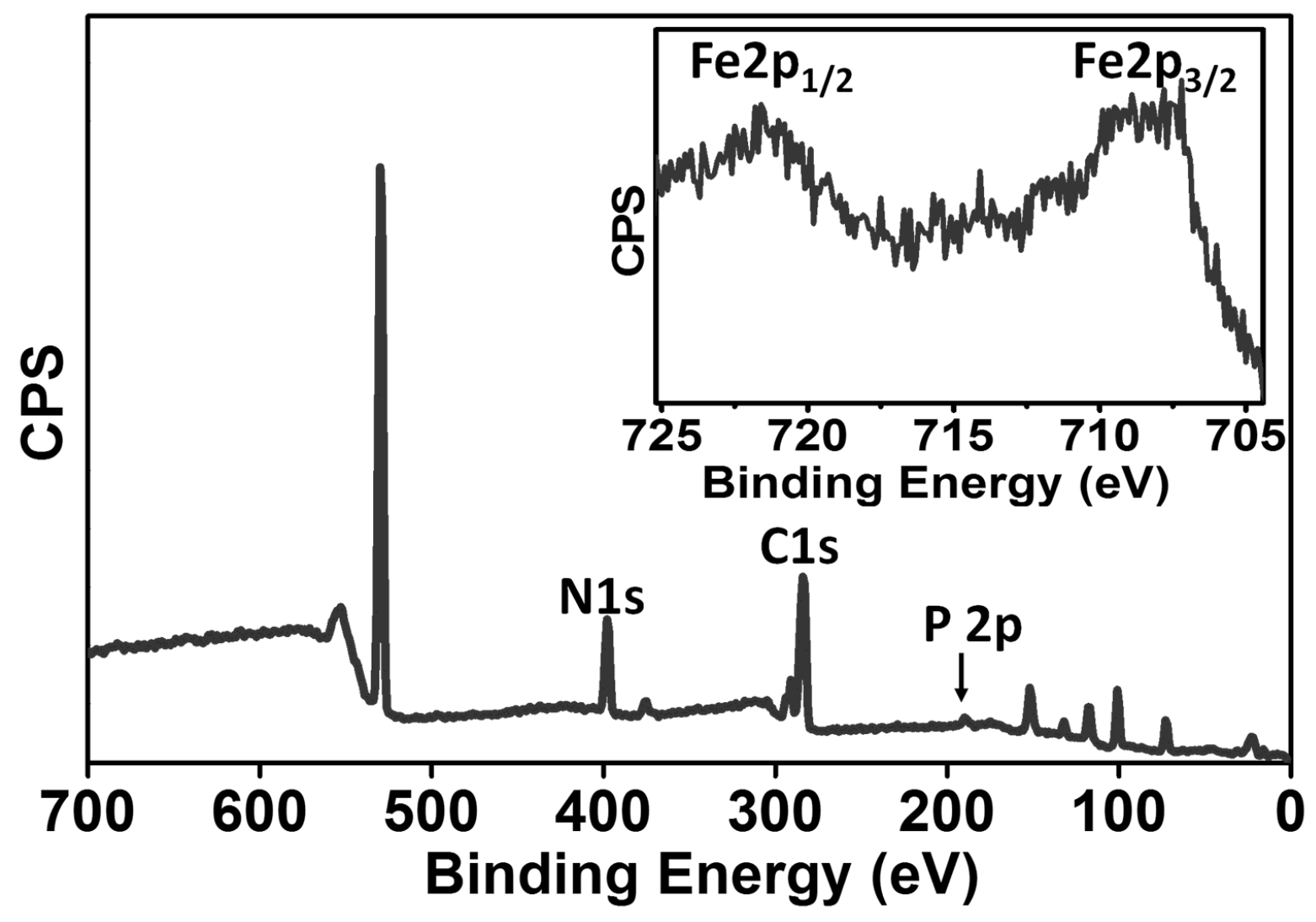


S4.18 XPS C1s narrow scan of EAD2 DNA immobilized on surface.

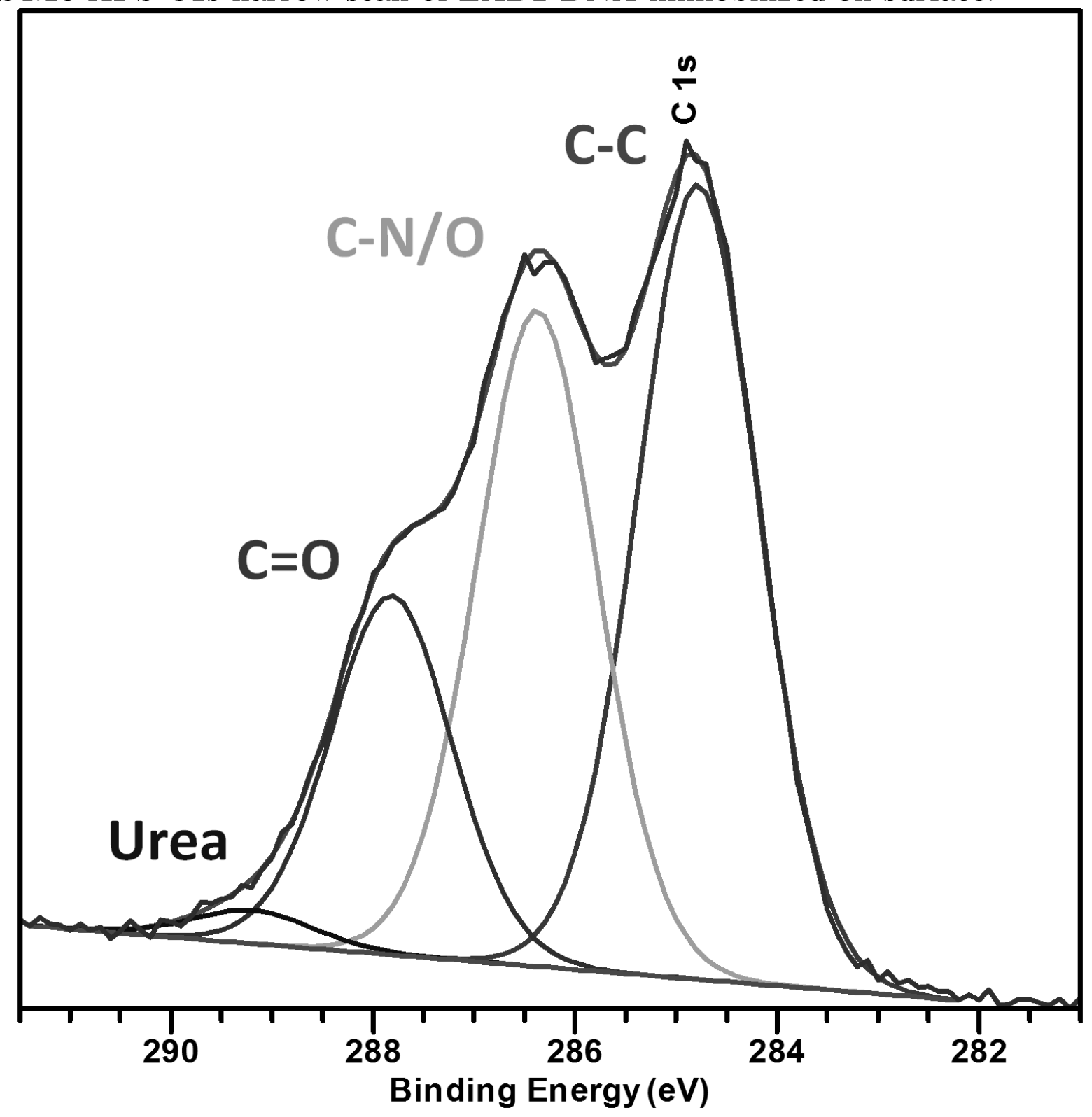




\subsection{Supplementary Tables}

Supplementary Table S1. Control experiments to show importance of each modular part of surface anchor $\mathbf{1}$, thus showing the synergy between them in achieving covalent modification of mica.

\section{SCA Data}

\begin{tabular}{|c|c|c|}
\hline Type of modification & SCA $\left({ }^{\circ}\right)$ after $1 \mathrm{~h}$ & SCA Picture \\
\hline Bare Mica $\left(\mathrm{M}_{0}\right)$ & $<15$ & \\
\hline$M_{1}$ & $\begin{array}{c}55 \\
(24 h)\end{array}$ & \\
\hline $\begin{array}{l}\text { Neutralized } \\
\text { Catechol- } \mathrm{C}_{6} \text {-amine }\end{array}$ & $\begin{array}{c}<15 \\
-\mathrm{NH}_{3}{ }^{+} \text {necessary condition }\end{array}$ & \\
\hline & $\begin{array}{c}<15 \\
-\mathrm{NH}_{3}{ }^{+} \text {necessary condition }\end{array}$ & \\
\hline $\begin{array}{l}\mathrm{NH}_{3}^{+} \\
\mathrm{Cl}^{-}\end{array}$ & $\begin{array}{l}25 \\
\text { Amide necessary condition }\end{array}$ & \\
\hline & $\begin{array}{l}\qquad<15 \\
\text { Amide necessary condition }\end{array}$ & \\
\hline
\end{tabular}


Supplementary Table S2. Different DNA sequences immobilized on mica surface.

\begin{tabular}{|c|c|}
\hline DNA & Sequence \\
\hline D1 & 5'-ATAATTACTA/5iUniAmM/CATGTCTGCG-3' \\
\hline D2 & $\begin{array}{c}\text { 5'-AGAGATTTTTATATATTTTTCGCAGACATG } \\
\text { ATAGTAATTATTAGAGATTTTTATAT-3' }\end{array}$ \\
\hline D3 & 5'-AAAATATATAAAAATCTCTA-3' \\
\hline D4 & 5'-AAAAATATATAAAAA-3' \\
\hline D5 & $\begin{array}{l}\text { 5'-ATTTTTTAGAGATTTTTATATATTTTT } \\
\text { TAGAGATTTTTATATATTTTTTAGAGATT-3' }\end{array}$ \\
\hline D6 & $\begin{array}{l}\text { 5'-ATAAAAATCTCTAAAAAATATATAAAAAT } \\
\text { CTCTAAAAAATATATAAAAATCTCTAA-3' }\end{array}$ \\
\hline D7 & $\begin{array}{l}\text { 5'-TTTATATATTTTTTAGAGATTTTTATATA } \\
\text { TTTTTTAGAGATTTTTATATATTTTTT-3' }\end{array}$ \\
\hline D8 & $\begin{array}{l}\text { 5'-CTCTAAAAAATATATAAAAATCTCTAAAA } \\
\text { AATATATAAAAATCTCTAAAAAATAT-3' }\end{array}$ \\
\hline GQ-anchor & 5'-/5AmMC12/GCGGAGGCG-3' \\
\hline GQ-Wire & $5^{\prime}-\mathrm{GCGGAGGCG-3'}$ \\
\hline EAD2 & 5'-/5AmMC12/CTGGGAGGGAGGGAGGGA-3' \\
\hline
\end{tabular}


6. DFT (B3LYP/6-311+G(d,p)) simulations for XPS C1s narrow scan assignments.

6.1 DFT simulation of XPS C1s narrow scan of $\mathrm{M}_{1}$ surface.
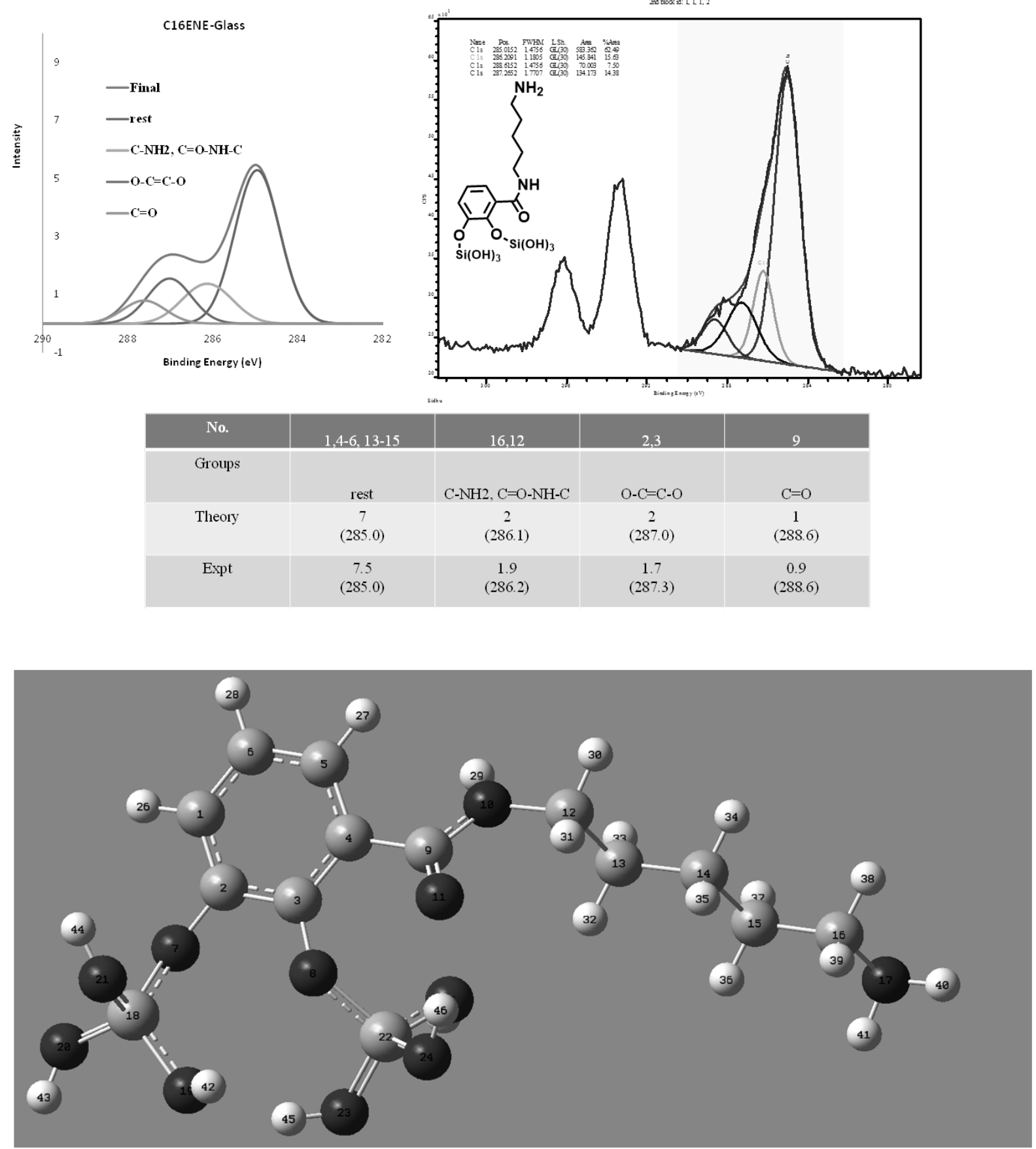

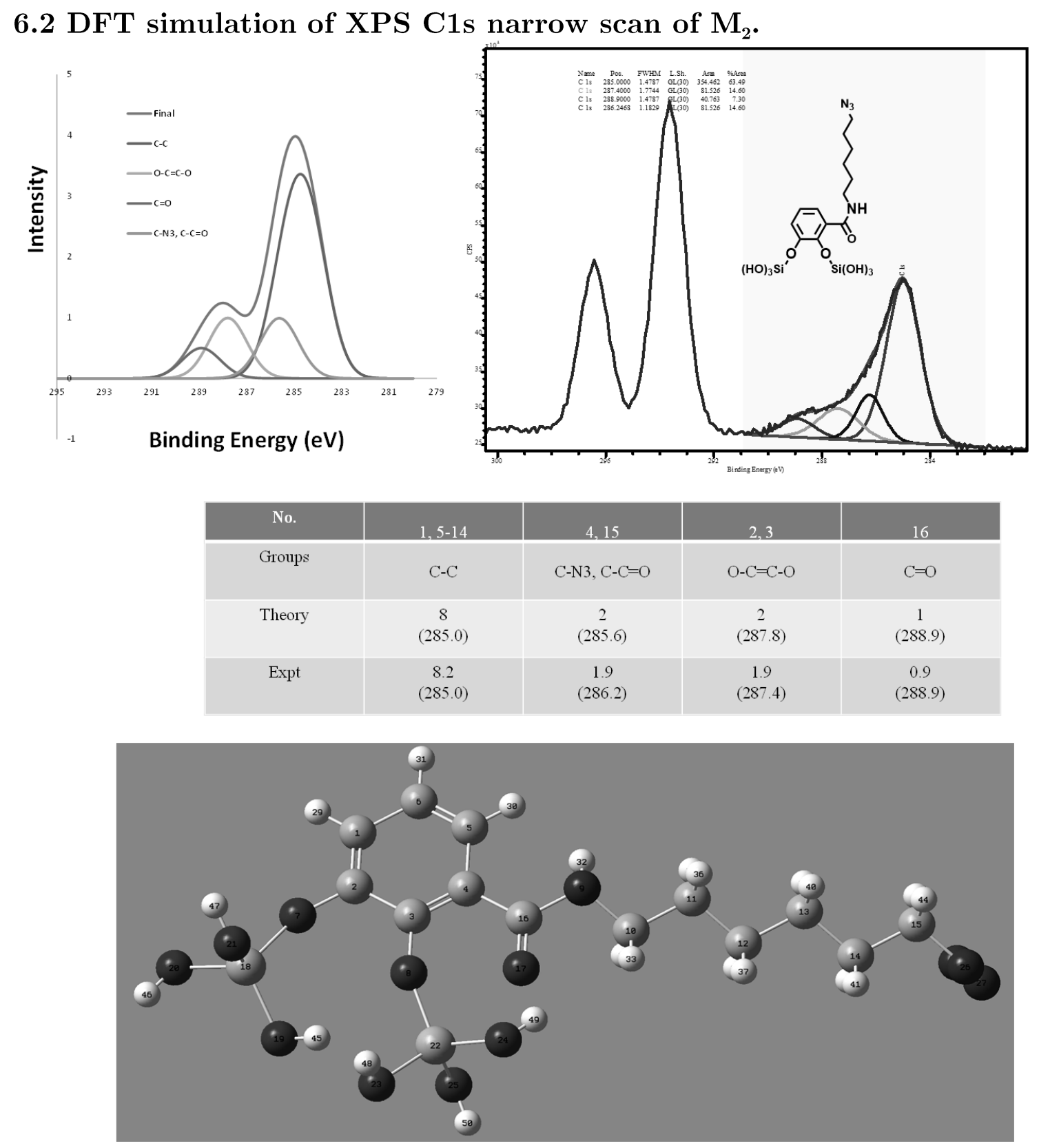
6.3 DFT simulation of XPS C1s narrow scan of M3.
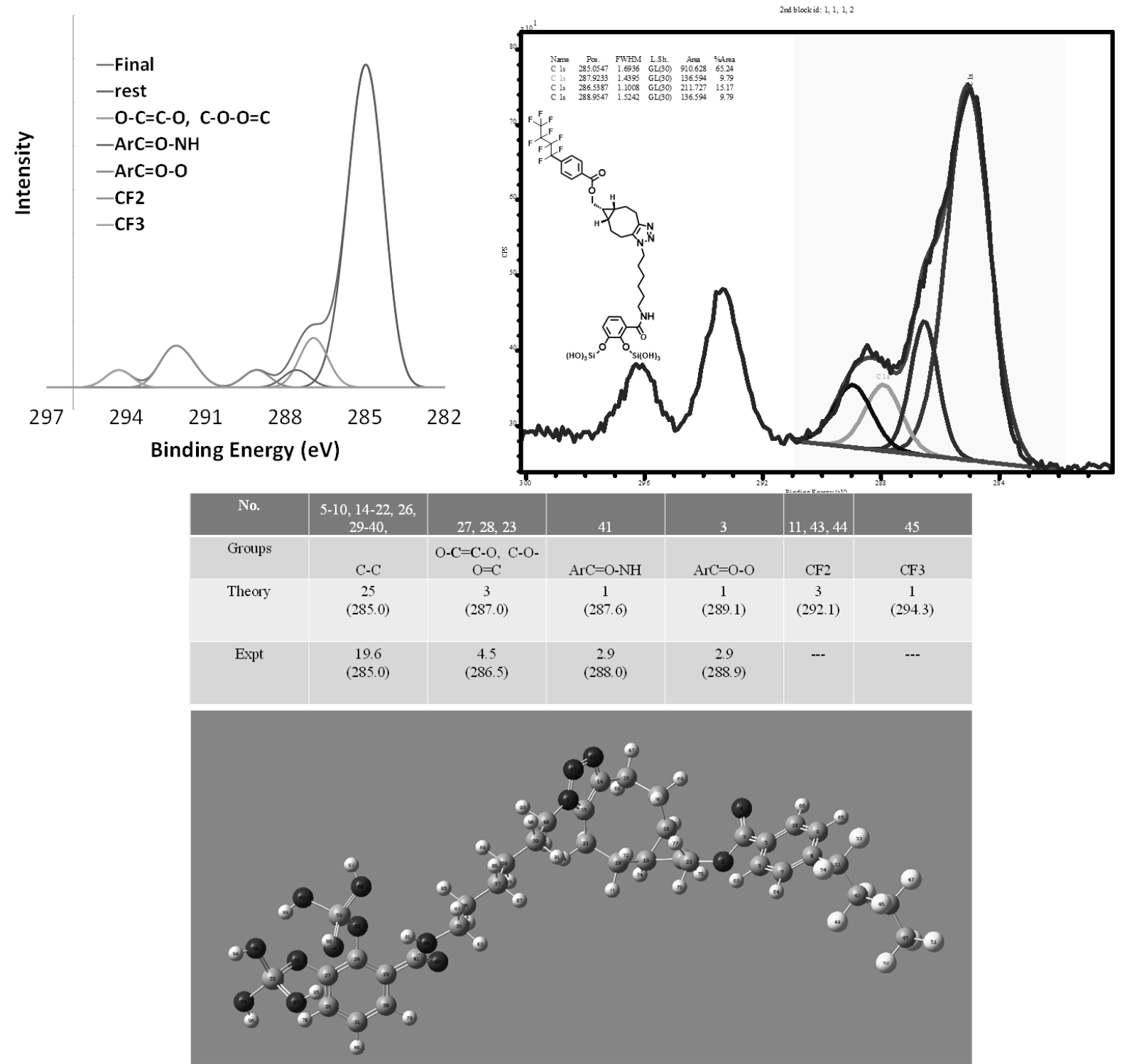
6.4 DFT simulation of XPS C1s narrow scan of $M_{4}$. $\mathrm{BCN}$ on mica surface (S4)
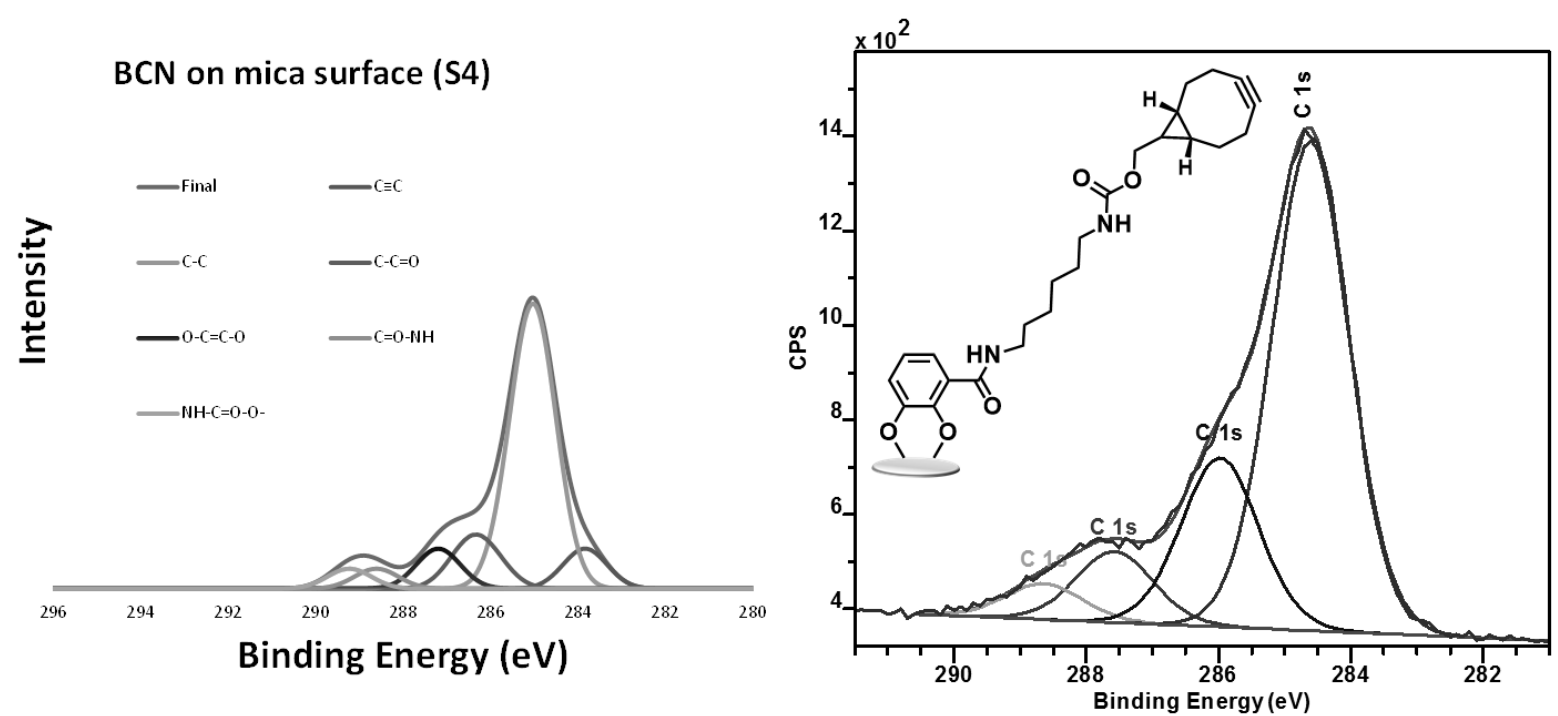

\begin{tabular}{|c|c|c|c|c|c|c|}
\hline No. & 30.31 & $\begin{array}{c}1,4-6,13-16,25- \\
29,32-33\end{array}$ & $17,24,12$ & 2,3 & 18 & 21. \\
\hline Groups & $\mathrm{C} \equiv \mathrm{C}$ & $\mathrm{C}-\mathrm{C}$ & $\begin{array}{c}\mathrm{C}-\mathrm{NH}-\mathrm{C}=\mathrm{O}-\mathrm{O}-\mathrm{C} \\
\mathrm{C}=\mathrm{O}-\mathrm{NH}-\mathrm{C}\end{array}$ & $\mathrm{O}-\mathrm{C}=\mathrm{C}-\mathrm{O}$ & $\mathrm{C}=\mathrm{O}-\mathrm{NH}$ & $\mathrm{NH}-\mathrm{C}=\mathrm{O}-\mathrm{O}-$ \\
\hline Theory & $\begin{array}{c}2 \\
(283.8)\end{array}$ & $\begin{array}{c}15 \\
(285.0)\end{array}$ & $\begin{array}{c}3 \\
(286.3)\end{array}$ & $\begin{array}{c}2 \\
(287.2)\end{array}$ & $\begin{array}{c}1 \\
(288.8)\end{array}$ & $\begin{array}{c}1 \\
(289.2)\end{array}$ \\
\hline Expt & \multicolumn{2}{|c|}{$\begin{array}{c}16.5 \\
(285.0)\end{array}$} & $\begin{array}{c}4.1 \\
(286.4)\end{array}$ & $\begin{array}{c}2.0 \\
(287.9)\end{array}$ & \multicolumn{2}{|c|}{$\begin{array}{c}1.3 \\
(289.0)\end{array}$} \\
\hline
\end{tabular}

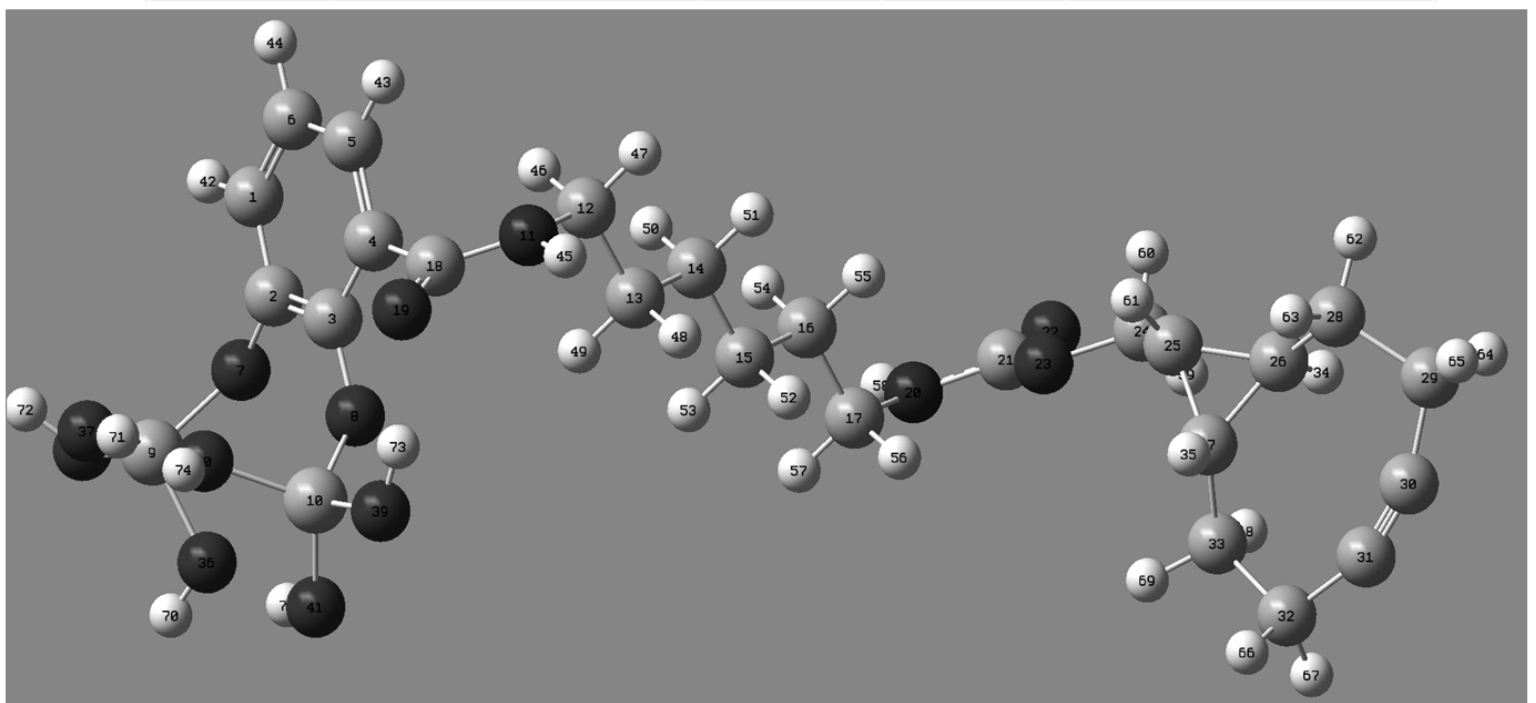


6.5 DFT simulation of XPS C1s narrow scan of $M_{5}$.
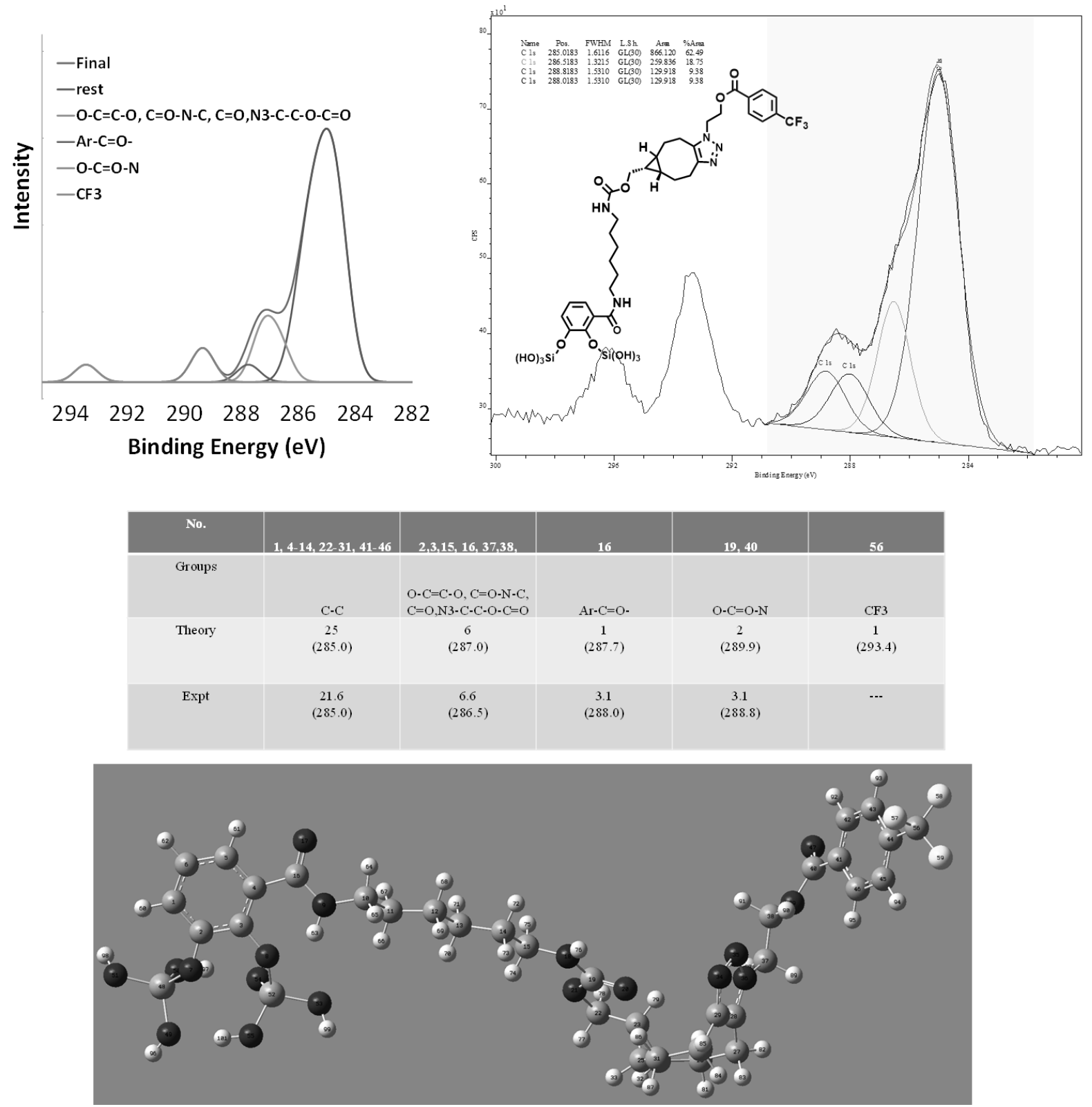
6.6 DFT simulation of XPS C1s narrow scan of $\mathrm{M}_{6}$.

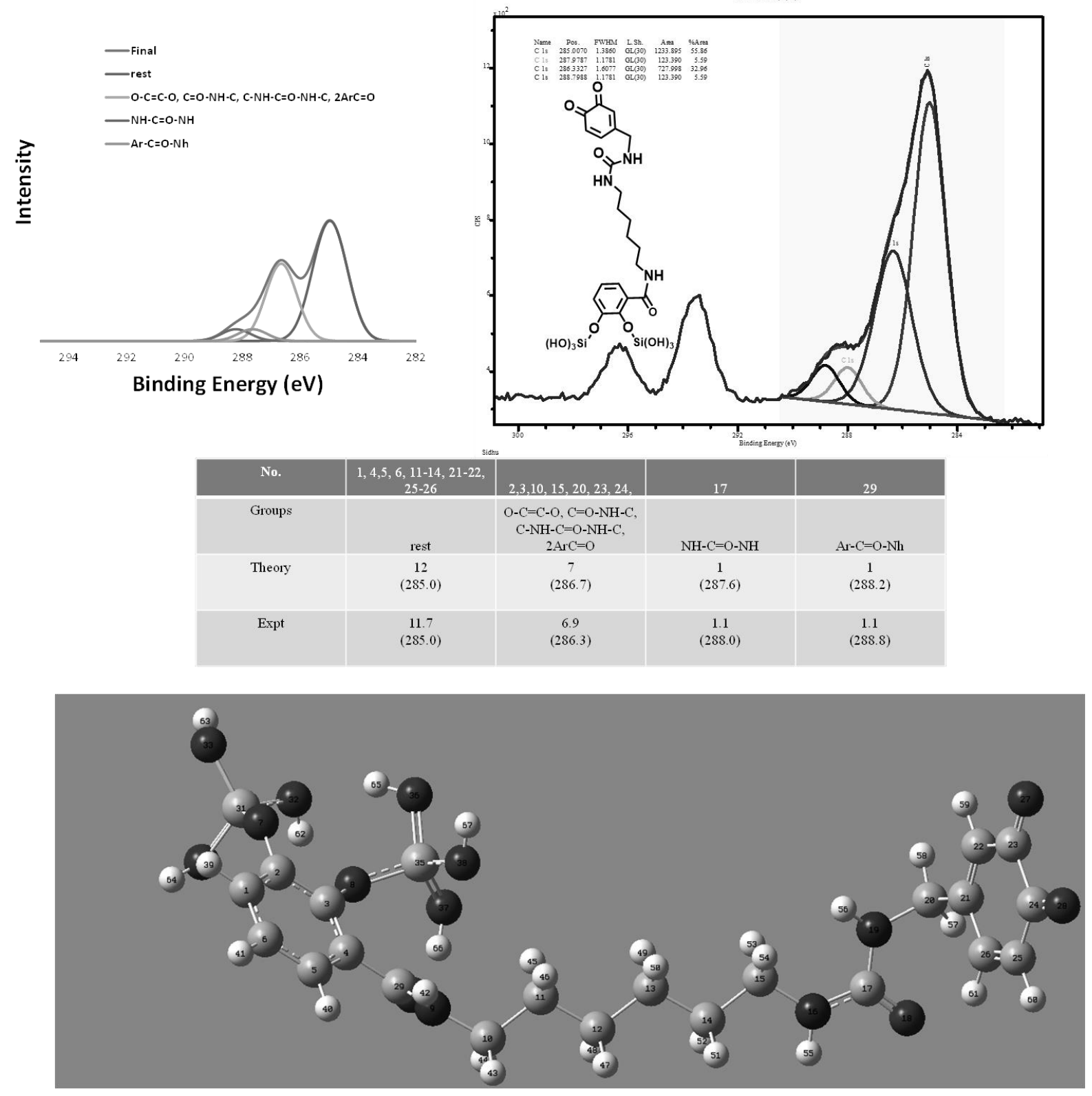


6.7 DFT simulation of XPS C1s narrow scan of $M_{7}$.
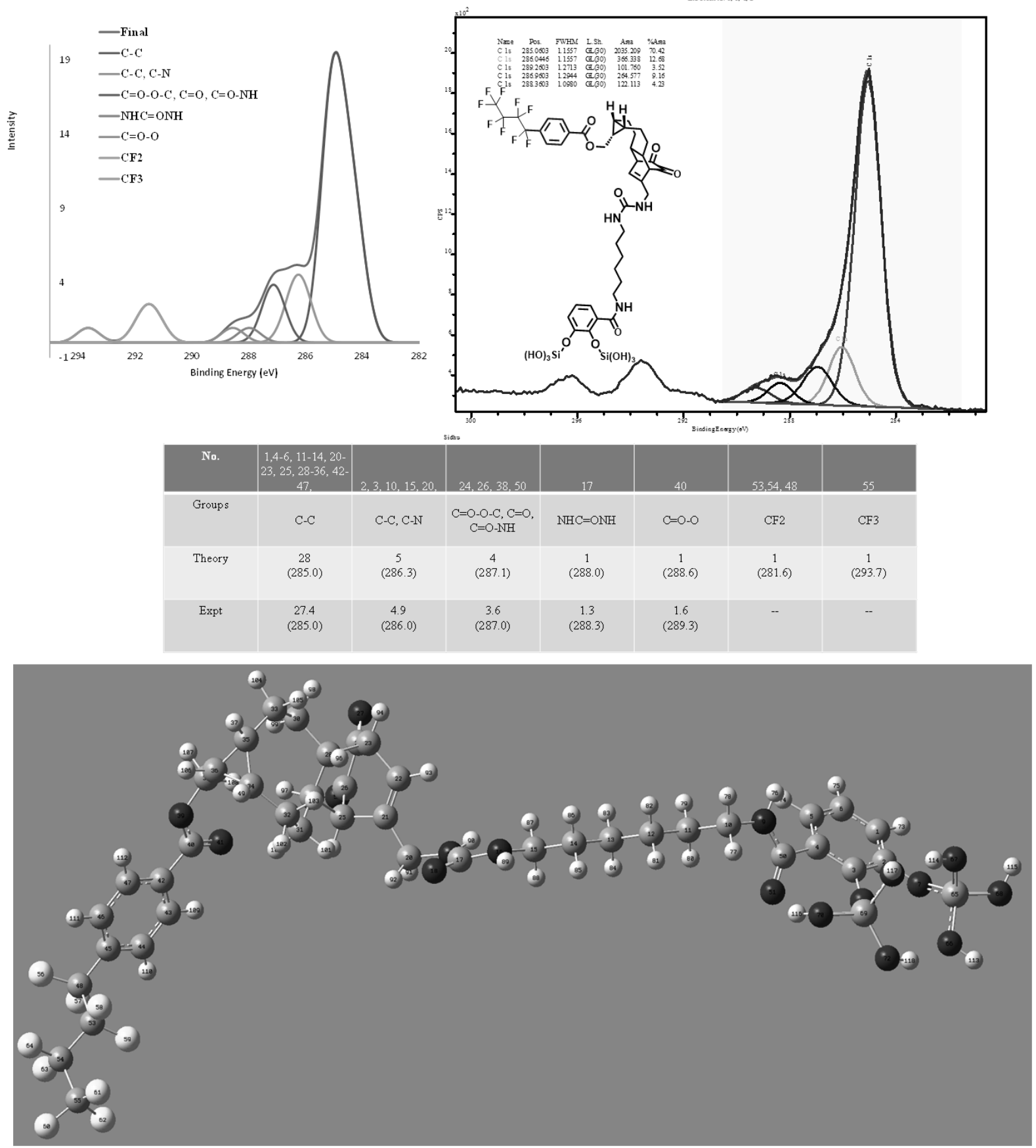


\section{Iist of Publications}

- "Local Light-Induced Modification of the Inside of Microfluidic Glass Chips". Rui R. Carvalho, Sidharam P. Pujari, Stefanie C. Lange, Rickdeb Sen, Elwin X. Vrouwe, and Han Zuilhof. Langmuir 2016, 32 (10), 2389-2398.

- "Use of Ambient Ionization High-Resolution Mass Spectrometry for the Kinetic Analysis of Organic Surface Reactions". Rickdeb Sen ${ }^{\ddagger}$, Jorge Escorihuela ${ }^{\ddagger}$, Maarten M. J. Smulders, and Han Zuilhof. Langmuir, 2016, 32 (14), 3412-3419.

- "Rapid and complete surface modification with strain-promoted oxidationcontrolled cyclooctyne-1,2-quinone cycloaddition (SPOCQ)". Rickdeb Sen Jorge Escorihuela Ed. 2017, 56, 3299-3303.

- "Ultrathin Covalently Bound Organic Layers on Mica: Formation of Atomically Flat Biofunctionalizable Surfaces". Rickdeb Sen, Digvijay Gahtory ${ }^{\ddagger}$, Floris L. van Delft and Han Zuilhof. Angew. Chem. Int. Ed. 2017, $56,4130-4134$

- "Approach Matters: The Kinetics of Interfacial Inverse-Electron Demand Diels-Alder Reactions". Rickdeb Sen, Digvijay Gahtory ${ }^{\ddagger}$, Jorge Escorihuela, and Han Zuilhof. Chem. Eur. J, 2017, doi:10.1002/chem.201703103.

- "Surface-Bound Quadruple H-bonded Dimers: Formation and Exchange Kinetics” Digvijay Gahtory ${ }^{\ddagger}$, Rickdeb Sen ${ }^{\ddagger}$, Maarten M. J. Smulders and Han Zuilhof". Faraday Discussions, 2017, Accepted. 
These authors contributed equally to the publication. 


\section{Bverview of completed training activities}

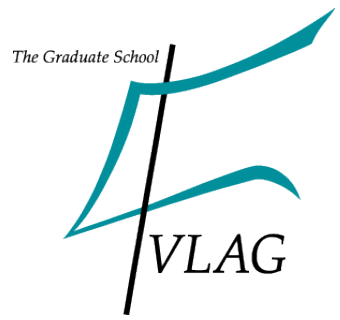

Discipline specific activities (courses, workshops, symposia, summer schools etc.)

NWO Lunteren Analytical meeting Netherlands (Poster)

NWO Lunteren Organic Chemistry meeting Netherlands (Poster)

LC-MS workshop organized by JSB (Seminar)

CHAINS (Poster)

NVMS 50th Anniversary Congress (Poster)

Wageningen symposium on Organic Chemistry,

Royal Netherlands Society of Chemistry (KNCV)

Wageningen symposium on Organic Chemistry,

Royal Netherlands Society of Chemistry (KNCV)

(Seminar)

Advanced Chemistry
Lunteren, The Netherlands

2013

Lunteren, The Netherlands

2014-2015

Wageningen, The Netherlands

2014

Veldhoven, The Netherlands

2013-2016

Rolduc, The Netherlands

2015

Wageningen, The Netherlands

2013-2016

Wageningen, The Netherlands

2016

ORC

2012-2016 
General courses (language courses, presentation courses, statistics, etc.)

VLAG PhD Week

Baarlo

2013

VLAG course: Mobilizing your Scientific Network

Wageningen

2014

VLAG course: Techniques in Scientific Writing

Wageningen

2014

VLAG course: Endnote Introduction

Wageningen

2014

VLAG course: Career Perspectives

Wageningen

2016

Optionals (participation in discussion groups, PhD excursions, MSc courses, etc.)

PhD trip ORC Switzerland and Germany $17 / 4 / 2013$ to $26 / 4 / 13$

$\mathrm{ORC}$

2013

PhD trip ORC Canada

$\mathrm{ORC}$

2015

Group meetings

ORC

2012-2016

Colloquia

$\mathrm{ORC}$

2012-2016

Preparing $\mathrm{PhD}$ research proposal

VLAG/ORC

2012-2016 


\title{
Acknowledgements
}

\author{
"I will either find a way or I will make one". \\ -Hannibal Barca (247-182 BCE) \\ (Carthaginian General)
}

Well...it's done. A journey started long ago is finally completed. All the lofty and daunting mountains, all the forests lovely dark and deep, we had to surmount are now behind us and I stand in the lush valley looking back at the struggles along the way and the key people who made this entire thing possible. It was not an easy struggle and I would like to take this moment to thank people who stood by me during the darkest of hours, at the most desperate of times when the call went out to hold firm the line.

Beyond everything and despite it all, all thanks go to my parents. Thank you ma and thank you baba. You are the pillars upon which I could build myself and get to where I am. An indomitable castle is only as strong as its foundation and its architect. Thank you, for all the sleepless nights, all your prayers and all your encouragement... I could go to a good school, get a great education and be a thinking/rational individual because of your education. Ma, your stories and poems planted within me a seed which has today matured. Your lullabies put me to sleep and it was your inspiration which caused me to "arise, awake up and stop not till the goal is reached". The best moments were of us watching the classic movies of Satyajit Ray and Akira Kurosawa and reading Rabindranath Tagore along with other world classics. Thank you for teaching me everything from "Where go the boats (by RLS)" to linear algebra, of which I unabashedly confess the former gave me the greater joy. Thank you for the support baba, I will always remember your kind words, encouragement and the all the things you did for me so that I didn't have to do it. Thank you for all the queues you weathered in the Indian sun so that I could study 
in the shade. It is not possible for me to dictate in words what all those efforts mean to me. I would also take this moment to thank my dearest sister, Samriddhi Saha. Dear didi, it's been sixteen years since you left us. I dedicate this thesis to you because this is what you would have wanted to see me be the most. Thanks for your advice to "study hard and never smoke or drink"; it guided me to the man I am today. Wish you were here to see me graduate and perhaps we will meet again in another life...

Opportunities rarely ever knock on one's doors twice but in my case it did happen. Here is where my promoter and very dearest guide Dr. Han Zuilhof came in. Dear Han, thanks for the opportunity! Thanks for the support and your belief in me. We shared many a scientific discussions together; we built up our little idea one aluminium sample at a time. At the end there were over 5000 expended $\mathrm{Al}$ samples but a concrete thesis. Every data point which came in and fit our sigmoidal graph, we built up our theories around. It took time to figure it out. The first two years were not easy at all and all those challenges (which we have laid to rest behind us) seemed so insurmountable! I thank you for thinking me capable of dealing with it and can thank you for the complete thesis. However, I choose to thank you more for the first two years when the going was tough and it was not going anywhere. This is the time when an unattached promoter would simply surrender, quit and desert. You took a decision- a harder path, not the easier one... This one would involve a lot of thinking, a lot of ideas and new approaches, a lot of cave-ins and a lot of collapses of our theories. You chose to be on the same little boat along with me, being thrown about in the choppy seas against conjured phantoms of "DART-HRMS is not reproducible". However, we were two of a kind. All these raging storms we weathered together but you kept the direction steady and the goal in sight at all times. We continued at an albeit slower pace as compared to my peers but it was a steady pace. The uphill climb had just began and only the persistent would be victorious. Those 
first two years were a hard time...I just got a new house and in the breaks of working in the lab and laying my room floors, came the idea of the "new approach". That was $4^{\text {th }}$ April, 2014 (Day 479 of 1461 contract days), one year plus of my PhD gone and we rebooted and started afresh, all the old would be forgotten and with a new energy we started up again. I am glad you allowed me to work on this new idea although it was a huge risk as we would leave behind the old without a care and embark on an entirely new approach with no guarantee of success. This choice and various others showed me what a great scientist and supervisor you are. I thoroughly enjoyed every moment of my $\mathrm{PhD}$ and in every step of the way you were there, guiding the boat and calming the rough waters which stood between us and the eventual goal. However, the idea worked, we were on our way...but not quite yet...we needed another catalyst...

October 2014-Introduction to Dr. Jorge Escorihuela. Spaniard, post-doctoral fellow at ORC, avid watcher of movies and like myself a fan of chemistry. Since, a $\mathrm{PhD}$ is "strain-promoted" we can call our immediate friendship and collaboration a "click reaction". We got to work! An idea is only as good as its execution. We identified the problems and ironed out the details. We got protocols standardized and the study of SPAAC kinetics began. The WUR workshop (special thanks to Mr. Andre Sanders for hand-cutting thousands of samples) couldn't provide us with $\mathrm{Al}$ samples quick enough. I had the DART running and you worked on the XPS inexhaustibly. Exciting times... With that out of the way we blazed through SPOCQ with relative ease and finally we collaborated again on the IEDDA. Your DFT calculations were the final clue and the whole picture came together. I am very happy for all the work and will always remember you fondly wherever you might be. I do have a special message for you though "good luck". (Albanian accent)

Tough times forge strong friendships, strong friendships tide tough times. This is where Digvijay Gahtory or DJ comes in. This one was "strain-promoted" too. DJ 
is the synthesis guy, the whiz with molecules and the commander of the columns. Whenever that one molecule which always evaded my limited synthetic skills came about you were there to catch it for me. The surface anchor, the IEDDA tags and surface attachment ideas you and I were all over the place! We spent a lot of time in the lab working and in our office talking about science, our chemistry and great many topics of immense and utterly vital significance. I loved those debates, I loved the information exchange, I discovered a lot I didn't know and we both got enriched. We found new surface strategies, we put things on and off the surface, pushed the DART and XPS to their limits, we planned and executed projects and on a hot summer's day when the sun shines with a benevolent fervor we found time to barbeque and debate some more. Be it science, history, group theory, the beginnings of the universe, politics and what not, nothing escaped our debates and no surface was left unturned. I am very happy we had such a fruitful collaboration and I look forward to more.

Speaking of click reactions, there was another during these four years: meeting Medea...my dearest wife. I cannot thank you enough, words will falter, phrases shall quiver, music shall rend asunder. You are the calm which comes to a tiny hamlet obscure in the mountains ravaged by a passing tempest. For me you are the angel who stopped a war...that is what you are to me. You are my peace, the stillness in my soul and the meaning of life. After the thousand storms which strove to break my resolve you are the light of dawn which gave hope that victory is imminent. There are several memories we have shared together. I will forever remember our walks in the snow-cloaked realms of the tundra, surrounded by foreboding pines on either side, gazing at the northern lights as they danced for our pleasure alone high in the heavens. I cannot describe them all here, tomes will run short, records will not suffice, and these memories are ours and forever shall remain. Through you I also met Arie and Lize, doting in-laws and lifelong friends, thanks for all your love and caring. 
I would then like to thank all the past and present PhDs and post-docs of ORC, you were an amazing bunch! It was a nice working atmosphere and I loved teaching with your guys around it was always a very nice vibe. We taught 660 hours each one of us (if not more) but your presence made it special...I don't remember any of those 660 hours at all. () Don't worry too much that is a compliment. Special thanks to you Rui for the wonderful soup and medicine you brought along when I was sick in September 2014.

I would like to thank all the professors (Maurice, Michel, Teris, Bauke, Floris, Maarten, Louis and Cees) for the Advanced Chemistry courses. Thanks to Maarten for help in plotting the first order reactions and all the help in my first publications. Thank you very much Bauke for the DNA designs in the mica paper along with its amazing frontispiece which you designed while I was away getting married in India. Special thanks goes to Floris van Delft for ideas for my $2^{\text {nd }}$ and $3^{\text {rd }}$ publication (SPOCQ and mica) both of which ended up in Angewandte Chemie (100\% strike rate!). I would also like to thank all the technicians (especially Sidhu). Thanks to all the lab mates and post-docs that made it a nice research atmosphere. I would like to thank my students, Hent, Robbert and Qiaona. I had a very pleasant time with all of you. I would also thank Dr. Sujoy Roy Chowdhury, Dr. Dipak Chatterjee and Dr. Chandan Saha for laying the groundwork of chemistry and mathematics during my student life. I have learned a lot from you all and am still utilizing your imparted knowledge to this day.

If any of you have questions or want to drop by, feel free to do so at my immortal email rickdeb.sen@iitdalumni.com, I would love to hear from you. Signing out from this phase of my life and on to the next!

This is Rickdeb Sen

Your friendly neighborhood surface chemist. 


\section{About the Author}

Rickdeb Sen was born in Kolkata, "The City of Joy", West Bengal, INDIA, on $31^{\text {st }}$ August, 1984. He completed his Bachelor in Chemistry (BSc; 2003-2007) with Honours from Calcutta University. He then pursued Masters in Chemistry (MSc; 2007-2009) from the prestigious Indian Institute of Technology, New Delhi (IIT-Delhi) after securing an All India Rank of

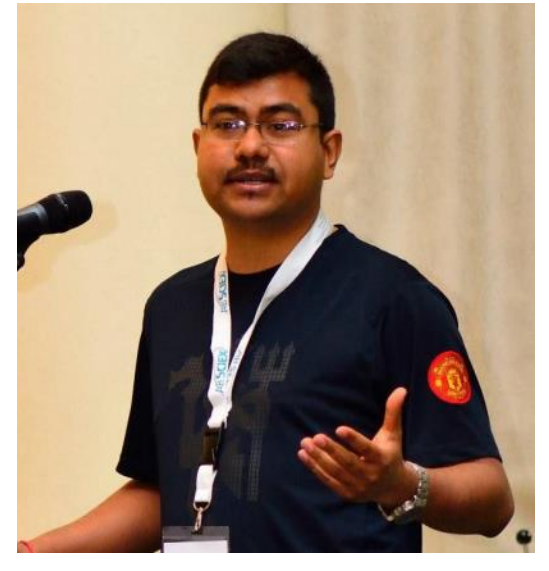
115 in the Joint Entrance to Masters (JAM) Examination. During this time, he was also attained All India Rank $17^{\text {th }}$ in the Graduate Aptitude Test for Engineers (GATE) in 2008 and obtained top scores in Graduate Record Examinations (General and Subject Tests).

He started his $\mathrm{PhD}$ research under the guidance of Dr. Han Zuilhof at Wageningen University, The Netherlands from December 2012. He is highly interested in surface chemistry, mass spectrometry and understanding mechanisms of interfacial reactions. His time as researcher at Wageningen University resulted in four first-author papers and several fruitful collaborations. In his free time, Rickdeb enjoys reading history, debating and swimming. Any conversation on a wide-ranging plethora of topics may be discussed with him at ease over a cup of Darjeeling tea. 
The research and training presented in this thesis was financially supported by The Netherlands Organization for Scientific Research (NWO) for funding via ECHO project number 712.012.006 and VENI grant 722.012.005. This material reflects only the author's views and he is thus not liable for any use that may be made of the information contained therein.

Financial support from Wageningen University for printing this thesis is gratefully acknowledged. 